\title{
Transdiagnostic approaches to mental health
}

Citation for published version (APA):

Rauschenberg, C. (2021). Transdiagnostic approaches to mental health: linking adversity, cognition, candidate mechanisms, and novel digital interventions. [Doctoral Thesis, Maastricht University]. Ridderprint. https://doi.org/10.26481/dis.20211217cr

Document status and date:

Published: 01/01/2021

DOI:

$10.26481 /$ dis.20211217cr

Document Version:

Publisher's PDF, also known as Version of record

\section{Please check the document version of this publication:}

- A submitted manuscript is the version of the article upon submission and before peer-review. There can be important differences between the submitted version and the official published version of record.

People interested in the research are advised to contact the author for the final version of the publication, or visit the DOI to the publisher's website.

- The final author version and the galley proof are versions of the publication after peer review.

- The final published version features the final layout of the paper including the volume, issue and page numbers.

Link to publication

\footnotetext{
General rights rights.

- You may freely distribute the URL identifying the publication in the public portal. please follow below link for the End User Agreement:

www.umlib.nl/taverne-license

Take down policy

If you believe that this document breaches copyright please contact us at:

repository@maastrichtuniversity.nl

providing details and we will investigate your claim.
}

Copyright and moral rights for the publications made accessible in the public portal are retained by the authors and/or other copyright owners and it is a condition of accessing publications that users recognise and abide by the legal requirements associated with these

- Users may download and print one copy of any publication from the public portal for the purpose of private study or research.

- You may not further distribute the material or use it for any profit-making activity or commercial gain

If the publication is distributed under the terms of Article $25 \mathrm{fa}$ of the Dutch Copyright Act, indicated by the "Taverne" license above, 


\section{Transdiagnostic approaches to mental health}

Linking adversity, cognition, candidate mechanisms, and novel digital interventions

Christian Rauschenberg 
ISBN:

978-94-6416-943-0

Artwork:

Lutz Henning Krietenbrink | @lutzhenningk

Cover design Lay-out: $\quad$ Publiss | www.publiss.nl

Print:

Ridderprint | www.ridderprint.nl

(c) Copyright 2021: Christian Rauschenberg, The Netherlands

All rights reserved. No part of this publication may be reproduced, stored in a retrieval system, or transmitted in any form or by any means, electronic, mechanical, by photocopying, recording, or otherwise, without the prior written permission of the author. 


\title{
Transdiagnostic approaches to mental health \\ Linking adversity, cognition, candidate mechanisms, and novel digital interventions
}

\author{
DISSERTATION \\ to obtain the degree of Doctor at the Maastricht University, \\ on the authority of the Rector Magnificus,
}

Prof. dr. Rianne M. Letschert in accordance with the decision of the Board of Deans, to be defended in public

on Friday 17 December 2021 at 12:00 hours

by 


\section{Promotor}

Prof. dr. J. van Os, Utrecht University Medical Centre

\section{Co-promotor}

Prof. dr. U. Reininghaus, Heidelberg University

\section{Assessment Committee}

Prof. dr. P.A.E.G. Delespaul (chair)

Dr. F. van Dael

Prof. dr. N. Jacobs, Open University

Prof. dr. L. Krabbendam, Vrije Universiteit Amsterdam

Dr. S. J. W. Verhagen

The research presented in this thesis was conducted at the School for Mental Health and Neuroscience, Department of Psychiatry and Neuropsychology, Maastricht University, Maastricht, the Netherlands, the Department of Public Mental Health, Central Institute of Mental Health, Heidelberg University, Mannheim, Germany, and the Department of Psychology, Institute of Psychiatry, Psychology and Neuroscience, King's College London, London, United Kingdom. The research was funded by the Mutsaers Foundation, Maastricht University, Dutch Ministry of Health, Welfare and Sport, Netherlands Organization for Health Research and Development, Dutch Research Council, German Research Foundation, and the Federal Ministry of Science, Education and Culture of the state of BadenWürttemberg, Germany. 
PARANIMFEN

Dr. Clara Snijders

Lex Westbroek 


\section{Table of contents}

Chapter 1 General introduction, thesis aims, and outline 9

PART 1: $\quad$ Cognition and mechanisms in transdiagnostic phenotypes

Chapter 2 Reasoning bias, working memory performance, and a transdiagnostic phenotype of affective disturbances and psychotic experiences in the general population

Chapter 3 The jumping to conclusions reasoning bias as a cognitive factor contributing to psychosis progression and persistence: fin dings from NEMESIS-2

Chapter 4 Stress sensitivity as a putative mechanism linking childhood trauma and psychopathology in youth's daily life

Chapter 5 Bullying victimization and stress sensitivity in help-seeking youth: fin dings from an experience sampling study

Chapter 6 Negative life events and stress sensitivity in youth's daily life: 195 an ecological momentary assessment study

PART 2: Digital interventions in public mental health provision

Chapter 7 An ecological momentary compassion-focused intervention for enhancing resilience in help-seeking youth: a pilot study

Chapter 8 Digital interventions for psychosis 267

Chapter 9 Social isolation, mental health, and use of digital 295 interventions in youthduring the COVID-19 pandemic: a nationally representative survey

Chapter 10 Evidence synthesis of digital Interventions to mitigate the negative impact of the COVID-19 pandemic on public mental health: a rapid meta-review

Chapter 11 General discussion 
APPENDIX

$\begin{array}{ll}\text { Impact paragraph } & 420\end{array}$

$\begin{array}{ll}\text { Curriculum Vitae } & 425\end{array}$

List of publications 426

$\begin{array}{ll}\text { Acknowledgements } & 431\end{array}$ 

CHAPTER 1

General introduction 


\section{The complex and multifaceted nature of mental health}

Mental disorders are among the strongest contributors to the global burden of disease - next to cardiovascular and infectious diseases, cancer, and neonatal disorders [1, 2] - and have been found to be the second leading cause of death in young age groups [3]. Mental health's central role in public health has gained global recognition in recent years, as evidenced by its formal inclusion in the United Nation's Sustainable Development Goals [4] and the World Health Organization's goal of universal health coverage [5]. Nevertheless, many people with mental health problems do not receive adequate treatment and the perceived stigma associated with mental health problems remains high $[6,7]$, preventing many individuals from seeking help or to openly talk about their mental health. Thus, understanding key determinants and underlying mechanisms in order to improve prediction of their onset, course, and outcome, as well as developing and implementing novel intervention strategies remain some of society's greatest challenges.

Psychopathologies have a complex aetiology, differ greatly in their phenomenology, and typically first appear during childhood, adolescence, and early adulthood (e.g., around half of all mental disorders emerge before the age of 15 and around three fourth by the age of 18) [8,9]. They are often characterized by transitional staging processes from subclinical to clinical severity [10], as highlighted by clinical staging models [11, 12], and have an estimated lifetime prevalence of around 18-36\% [8, $13,14]$. It is therefore crucial to disrupt illness trajectories at a developmentally early stage [15-17] and to further expand the reach of, and developing innovative strategies for, mental health services, including the provision of easily accessible, person-centred, non-stigmatizing mental health care as well as mental health promotion and prevention programs in diverse communities and settings [18-20].

Despite decades of extensive biopsychosocial research on mental health and significant individual suffering and societal costs, contemporary aetiological models for most psychopathologies continue to fall short of accurately predicting disease onset or determining individual pathways to poor mental health, including whether signs and symptoms will improve or worsen over time [21-25]. Moreover, many individuals continue to have residual symptoms after treatment completion and relapse rates are staggering high, especially for severe mental disorders [26]. In fact, in contrast to many other medical disciplines (e.g., oncology, cardiology), 
there are no reliable biomarkers that can be used to accurately index the risk for, or the presence of, mental disorders $[27,28]$. The sheer complexity of studied constructs, which continues to grow as methodological sophistication increases, appears to significantly complicate, rather than facilitate, significant advancements in the development of more effective non-pharmacological and pharmacological treatments [29-33]. Thus, even with intensive global research efforts, the likelihood that a person in need of care will feel better following treatment - perhaps the most valid of all success criteria - has remained relatively stable in recent years for most mental disorders [34, 35].

Some of the challenges mental health research is currently facing may be explained in part by fundamental problems with the way the dominant traditional medical model conceptualizes mental disorders [21, 29, 36]. This involves the persistent belief that mental disorders (1) are classifiable disease entities that differ markedly from one another and are unambiguous in their phenotypic representation and biological correlates, (2) are associated with disease-specific mechanisms and risk factors, (3) are qualitatively distinct from subclinical expressions of poor mental health, and that (4) findings on mechanisms and outcomes originated from experimental studies are ecologically valid and generalizable to mental health problems in real-life contexts and everyday situations. In contrast to these views, however, accumulating evidence lends evidence that mental disorders share a high degree of overlap at the (poly-)genetic [37-39], biological systems [40-42], and symptom level [24, 43-46], that most socio-environmental risk factors are nonspecifically impacting mental health outcomes [47-50], and findings from research labs do not always translate to real-world settings [51-54].

To help address some of these challenges, efforts have been made to (i) understand psychopathologies more dimensionally, cutting across traditional diagnostic boundaries described in the Diagnostic and Statistical Manual of Mental Disorders (DSM) and International Classification of Diseases (ICD) [55-57], (ii) advocate and systematically study concepts of mental ill-health continua and spectra and the clinical utility of staging models [12, 58-60], (iii) investigate the effects of exposure to socio-environmental risk and resilience factors on transdiagnostic extended phenotypes [61-63], (iv) identify transdiagnostic candidate mechanisms which have been found to be non-specifically associated with a range of mental health outcomes [23, 24, 46, 64], (v) examine how mental health problems unfold in, and 
are associated with, specific contexts in the flow of daily life to increase ecological validity and generalisability, enabling ecological translation of findings to reallife contexts [51, 52, 65-67], and (vi) develop, evaluate, and implement novel transdiagnostic intervention strategies encompassing the full spectrum of public mental health provision (i.e., mental health promotion, prevention, and treatment of mental disorders) $[65,68,69]$.

This thesis builds on these recent developments in order to extend the body of existing knowledge. The theoretical foundation described in this chapter lays the groundwork for the remainder of thesis.

\section{The extended transdiagnostic psychosis phenotype}

In line with developments towards a more dimensional understanding of mental disorders, the extended and transdiagnostic psychosis phenotype has been proposed $[46,57]$. This has been based on findings that psychotic experiences - the subclinical expression of psychotic symptoms, which includes subtle manifestations of delusional ideations, hallucinations or thought problems - are common in the general population [46, 70-72] and frequently co-occur with, or are preceded by, forms of affective dysregulation (e.g., depression, anxiety) [7376]. This also corroborates findings on frequent comorbidity between affective disorders and psychosis spectrum disorders in diverse clinical settings [73] as well as findings on overlapping genetic liability [38]. Psychotic experiences have also been found to be bidirectionally associated with other mental disorders [77], suggesting that they may not only represent an indicator of early psychosis in some cases, but also an important risk marker for a wide range of non-psychotic mental disorders [23, 76-78].

Contemporary models of psychosis emphasize the role of cognitive factors, including biases, schemas, and cognitive deficits, in illness trajectories by implying their contribution to the transformation of experiences of aberrant salience (i.e., characterized by placing inappropriate significance on neutral events, external objects, or internal representations) into psychotic symptoms [79-81]. More specifically, individuals' perception of, and responses to, these excessively vivid experiences are thought to be influenced by cognitive factors and may therefore be critical for the transition from subclinical to clinical severity [81-84]. Cognitive factors have also been found to influence the perception of events as threatening 
and externally caused $[83,85]$, further strengthening their potential role in the exacerbation of psychotic symptoms over time $[86,87]$. Thus, seeing and interpreting the world around us is heavily influenced by cognitive factors, which are influenced by a multitude of biopsychosocial risk and resilience factors.

One of the most comprehensively studied cognitive factor in psychosis is the jumping to conclusions (JTC) bias [88-90]. The JTC bias is characterised by making hasty decisions based on insufficient information and has consistently been found to occur more often in individuals with subclinical or clinical psychosis when compared to comparison subjects and individuals with other mental health conditions, including affective disorders [90]. This suggests that the JTC bias may be specifically association with psychosis and not with frequently co-occurring affective dysregulations. Further, it has been proposed that the JTC bias may contribute to the formation and persistence of delusional ideations, but recent meta-analytic evidence has been inconsistent [91]. Similarly, cognitive deficits, such as a decreased working memory performance, have been shown to be more likely to occur in individuals with psychosis as compared to controls $[92,93]$, while there is, in contrast to the JTC bias, no evidence supporting the notion of specificity as cognitive deficits have been shown to be associated with various psychopathological domains [94, 95].

However, it is currently unknown whether these well-established cognitive factors for psychosis are also associated with a transdiagnostic psychosis phenotype of co-occurring affective dysregulation and psychotic experiences and whether the JTC bias contributes to the progression and persistence of psychosis over time, as proposed by contemporary aetiological models [81, 83].

\section{Adverse childhood experiences and mental health}

In addition to cognitive factors, exposure to adversity has been found to be associated with poor mental health outcomes. Most prominently, the effects of adverse childhood experiences (ACEs) on mental health have extensively been studied over the recent years. ACEs, a specific type of socio-environmental risk, are defined as highly stressful events or situations that occur early in life and are potentially traumatic. They may occur as a single traumatic event (e.g., negative life events like a severe car accident) or as ongoing threats (e.g., bullying victimization in school settings or sexually abusive family members) that jeopardize a young person's safety, trust, and bodily integrity and, thus, require significant social, emotional, 
neurobiological, and behavioural adaptation [96]. There has been large body of research robustly showing that exposure to ACEs, including childhood trauma and bullying victimization, is associated with a 2 to 3 times increased risk for developing a range of mental disorders, including depression [97, 98] and psychosis [99-102]. Although earlier work proposed specific adversity-psychopathology (e.g., more intrusive forms of childhood trauma may be more strongly linked to an increased risk for experiencing delusional ideations as compared to other outcomes) as well as time-of-exposure-outcome (e.g., effects on mental health are more severe if exposure occurs in early childhood) associations, more recent studies suggest that effects of ACEs appear to be non-specific, such that any form of ACEs, and at any time during childhood, is associated with a range immediate and prolonged poor mental health [101, 103-106]. There are some studies, however, which provide some evidence for time sensitivity of exposure on outcome [107-110]. There has also been strong evidence of dose-response relationships and cumulative effects, implicating that higher levels as well as greater numbers of exposure to ACEs progressively increase the likelihood of developing mental health problems [101, 111-114]. Studies have also demonstrated that exposure to ACEs mostly clusters in a relatively small number of individuals, leading to high rates of poly-victimization in those exposed to any form of ACEs [115-118]. The experience of ACEs is also prevalent in the society, ranging from an estimated lifetime prevalence of around $30 \%$ for bullying victimization [119] to around $12 \%$ for sexual abuse [120], although prevalence estimates strongly differ by type, sex, and country.

\section{Stress sensitive as a candidate mechanism linking adversity and mental health}

While there is compelling evidence that cognitive factors and ACEs are associated with mental disorders, little is known about underlying mechanisms, especially in the realm of youth mental health. It is quite unlikely, however, that the various types of ACEs are linked to as many different mechanisms. It is more likely that there are shared common pathways, or candidate mechanisms, which can be studied across various levels of investigation (e.g., biological, behavioural, cognitive). The most widely studied mechanism is behavioural sensitization [121], which posits that repeated exposure to ACEs leads to a gradual increase of individuals' stress response to subsequent adversities as well as minor stressors in daily life. Hence, 
stress sensitivity, sometimes also referred to as stress reactivity, may be a putative mechanism linking exposure to ACEs and mental health outcomes and may be contributing to a vicious cycle of poor mental health outcomes over time [121].

There are several ways to investigate whether individuals who were exposed to ACEs are more sensitive towards stress. On the behavioural level, the Experience Sampling Method (ESM) [122] or Ecological Momentary Assessment (EMA) [123] can be used. ESM is a powerful self-report diary technique [52] which allows for assessing appraisals of subjective experiences, symptoms, and minor stressful events in daily life with high ecological validity. This method is particularly well suited to test the proposition that those exposed to ACEs have a higher stress sensitivity by investigating whether exposure to ACEs is associated with increased sensitivity to stress. More specifically, in a frequently used ESM study design, individuals are prompted 10 times a day over 6 consecutive days to fill in short questionnaires on their smartphone or personal digital assistant. This fine-grained ESM-data (i.e., up to 60 assessment points per person when using described sampling scheme) can be used to approximate individuals' stress sensitivity by looking at individual's affective response (e.g., increase in negative affect) to minor daily stressors (e.g., event-related or social stress) in daily life [124].

There have been several studies that have investigated whether individuals' response to stress is modified by prior exposure to ACEs. Overall, these findings consistently suggest that individuals with depression, an at-risk mental state for psychosis, and psychotic disorders who were exposed to childhood trauma (e.g., physical abuse) or negative life events (e.g., parental separation) showed an elevated stress sensitivity in daily life [125-127]. This supports the notion that stress sensitivity may be an important transdiagnostic mechanism linking adversity and mental health outcomes in diverse clinical samples. However, there have been no studies that specifically investigated the effects of bullying victimization on stress sensitivity in help-seeking individuals and evidence of the effects of negative life events on stress sensitivity is scarce. Moreover, all studies examining the effects of various types of ACEs on stress sensitivity have exclusively included samples of help-seeking adults. As a result, it is currently unknown whether reported findings also generalize to young help-seeking individuals at developmentally early stages of psychopathology. 


\section{From candidate mechanism to novel treatment targets}

As exemplified by reported findings, ESM is a powerful tool to investigate how adversity and other forms of socio-environmental risk may impact mental health outcomes in daily life through transdiagnostic candidate mechanisms. There have been recent efforts to translate these findings to inform novel intervention strategies, most notably Ecological Momentary Interventions (EMIs) [128]. EMIs are a promising approach for targeting transdiagnostic candidate mechanisms, including stress sensitivity [68], and mental health outcomes in daily life using mobile devices. More specifically, while principles of ESM emphasize that psychological experiences, behaviours, and outcomes are highly dynamic and best understood and studied in everyday contexts, EMIs purposefully build on these theoretical foundations by inferring that mechanisms and outcomes may also be best modifiable in everyday contexts and situations, outside clinicians' office [65] (See Figure 1).

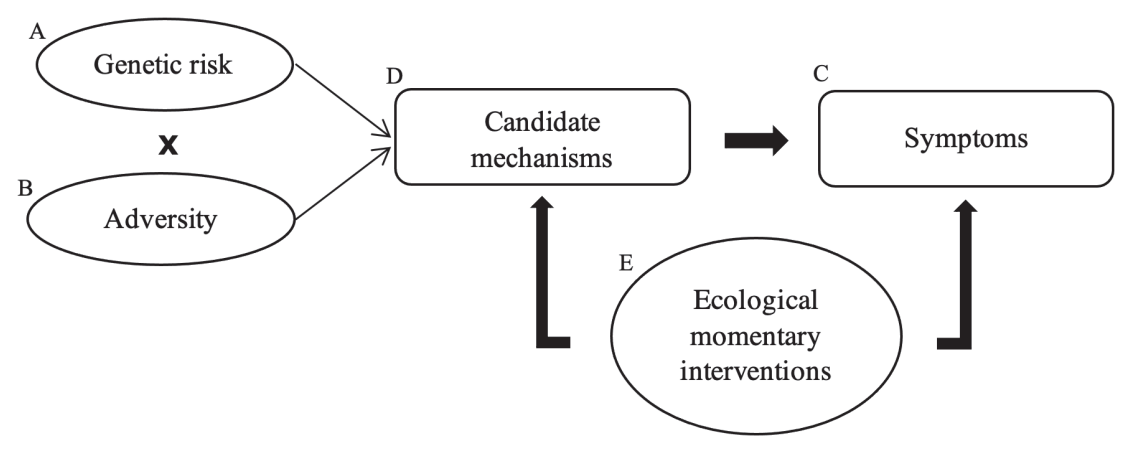

Figure 1. Simplified schema of how adversity impacts mental health through biopsychosocial candidate mechanisms. Genetic risk (A) and adversity (B), and their interaction (X), impact on symptoms (C) through candidate mechanisms (D). This may be directly targeted on the behavioural level using ecological momentary interventions (E) in real-life contexts by using cutting-edge digital technologies.

Most EMIs that have been developed and evaluated to date have been delivered using dedicates applications on the smartphone [129-132]. Thus, as rates of smartphone ownership are high and in close proximity to users, EMIs allow various possibilities to reach individuals in their natural environments in which mental health problems occur [65] and to directly test ecological interventionist causal models [66]. They also enable adaptive, real-time, and real-world delivery 
of intervention components in daily life at the right time and context by real-time processing of ESM data [65]. However, there is currently only very limited evidence that have demonstrated the feasibility and safety of EMIs and initial therapeutic effects on candidate mechanisms and mental health outcomes in young helpseeking individuals.

\section{The recent surge of digital intervention}

The rapid technological advances have not only contributed to the development and evaluation of EMIs, a specific type of digital interventions [68], but also a plethora other forms of technology enabled mental health services [133], which offer ample of possibilities for the digitalisation and personalisation of mental health care [134]. In this context, telemedical and internet-based (eHealth) interventions and smartphone-based mobile health (mHealth) interventions play a particularly important role as they enable low-threshold and real-time delivery of evidence-based psychological interventions that can be tailored to individual needs and preferences $[65,67,135]$. Moreover, over the recent years, other technologies have gained traction, including wearables and virtual reality (VR) devices, that are increasingly being used for the delivery of digital interventions for the prevention and treatment of various mental disorders as well as mental health promotion [136-138]. These numerous types of digital interventions could contribute to further personalise existing mental health services, thereby improving clinical and social outcomes and lowering direct and indirect costs. However, the number of studies that have synthesized the available evidence across the whole spectrum of public mental health provision is very limited.

\section{COVID-19 pandemic and public mental health}

In the first quarter of 2020, the majority of European countries implemented a variety of public health measures to combat the spread of the SARS-CoV-2 coronavirus. Physical distancing and quarantine have been among the most effective measures used to reduce infection rates. However, these preventive measures may have negative consequences on public mental health [139]. Digital inventions may be particularly helpful for providing mental health promotion and prevention programs at the population level and to ensure continued mental health care [67]. However, the evidence whether public health measures 
have negative psychosocial consequences on public mental health remains inconclusive, particularly among youth, and evidence-based recommendations on the use of existing eHealth and mHealth interventions developed and evaluated in recent years are currently lacking, potentially impeding the development and implementation of digital strategies for public mental health. This strongly hinders the evidence-based development and implementation of digital strategies for improving public mental health during this unparalleled public health crisis [67].

\section{Aims and outline of this thesis}

The overall aim of this thesis is twofold. First, the thesis aims to investigate how adverse childhood experiences, cognitive factors, and candidate mechanisms confer risk for developing transdiagnostic phenotypes of depression, anxiety, and psychosis (Part 1). Second, the thesis aims to explore to what extend digital interventions hold promise for, and are currently being used to, alleviating mental health burden in public mental health provision (i.e., mental health promotion, prevention of, and treatment for mental disorder) (Part 2).

More specifically, the studies presented in this thesis investigated the following aims:

\section{Part 1}

Chapter 2 investigates associations of the jumping to conclusions bias and decreased working memory performance with co-occurring affective disturbances and psychotic experiences in the general population.

Chapter $\mathbf{3}$ extends the knowledge base of chapter $\mathbf{2}$ and further investigates whether the JTC bias contributes to psychosis progression and persistence in the general population.

Chapters 4, 5, and 6 examine whether the exposure to various types of ACEs, that is, (i) childhood trauma, (ii) bullying victimisation, and (iii) negative life events, amplifies individuals' sensitivity to stress in a sample, of help-seeking adolescents and young adults with high levels of depressive, anxiety and psychotic symptoms, their biological siblings, and comparison subjects. 


\section{Part 2}

Chapter 7 builds on findings of preceding chapters on the role of stress sensitivity in the realm of youth mental health and investigates, in an uncontrolled phase I pilot study, the feasibility, safety, and initial therapeutic effects of a novel, accessible, transdiagnostic, ecological momentary, compassion-focused intervention for improving emotional resilience to stress ('EMIcompass') in help-seeking youth with psychotic, depressive, and/or anxiety symptoms.

Chapter 8 explores the clinical potential of emerging digital technologies, such as smartphones, smartwatches, fitness trackers and head-mounted displays, for helping individuals with subclinical expressions of psychosis as well as psychosis spectrum disorder.

Chapters 9 and 10 examine the COVID-19 pandemic and its consequences on youth mental health as well as the use of digital technologies. More specifically, Chapter 9 investigates the associations between social isolation, COVID-19-related cognitive preoccupation, worries, and anxiety, objective social risk indicators, and psychological distress, as well as use of, and attitude toward, mobile health (mHealth) interventions in youth. Chapter $\mathbf{1 0}$ systematically synthesises the theoretical and empirical base, user perspective, safety, effectiveness, and costeffectiveness of digital interventions related to public mental health provision (i.e., mental health promotion, prevention, and treatment of mental disorders) that may help to reduce the consequences of the COVID-19 pandemic. 


\section{References}

1. Whiteford, H.A., et al., Global burden of disease attributable to mental and substance use disorders: findings from the Global Burden of Disease Study 2010. The Lancet, 2013. 382(9904): p. 1575-1586.

2. Vigo, D., G. Thornicroft, and R. Atun, Estimating the true global burden of mental illness. Lancet Psychiatry, 2016. 3(2): p. 171-8.

3. Naghavi, M. and C. Global Burden of Disease Self-Harm, Global, regional, and national burden of suicide mortality 1990 to 2016: systematic analysis for the Global Burden of Disease Study 2016. BMJ, 2019. 364: p. 194.

4. United Nations General Assembly, Sustainable development goals. SDGs Transform Our World, 2015. 2030.

5. World Health Organization, The WHO special initiative for mental health (2019-2023): universal health coverage for mental health. 2019, World Health Organization.

6. Franz, L., et al., Stigma and treatment delay in first-episode psychosis: a grounded theory study. Early Interv Psychiatry, 2010. 4(1): p. 47-56.

7. Henderson, C., S. Evans-Lacko, and G. Thornicroft, Mental illness stigma, help seeking, and public health programs. Am J Public Health, 2013. 103(5): p. 777-80.

8. Kessler, R.C., et al., Lifetime prevalence and age-of-onset distributions of mental disorders in the World Health Organization's World Mental Health Survey Initiative. World Psychiatry, 2007. 6(3): p. 168-76.

9. Kim-Cohen, J., et al., Prior juvenile diagnoses in adults with mental disorder: developmental follow-back of a prospective-longitudinal cohort. Arch Gen Psychiatry, 2003. 60(7): p. 709-17.

10. Kessler, R.C., et al., Lifetime prevalence and age-of-onset distributions of DSM-IV disorders in the National Comorbidity Survey Replication. Arch Gen Psychiatry, 2005. 62(6): p. 593-602.

11. McGorry, P.D., et al., Clinical staging: a heuristic and practical strategy for new research and better health and social outcomes for psychotic and related mood disorders. Can J Psychiatry, 2010. 55(8): p. 486-97.

12. Shah, J.L., et al., Transdiagnostic clinical staging in youth mental health: a first international consensus statement. World Psychiatry, 2020. 19(2): p. 233-242.

13. de Graaf, R., et al., Prevalence of mental disorders and trends from 1996 to 2009. Results from the Netherlands Mental Health Survey and Incidence Study-2. Soc Psychiatry Psychiatr Epidemiol, 2012. 47(2): p. 203-13.

14. Merikangas, K.R., et al., Lifetime prevalence of mental disorders in U.S. adolescents: results from the National Comorbidity Survey Replication--Adolescent Supplement (NCS-A). J Am Acad Child Adolesc Psychiatry, 2010. 49(10): p. 980-9.

15. McGorry, P. and J. van Os, Redeeming diagnosis in psychiatry: timing versus specificity. Lancet, 2013. 381(9863): p. 343-5.

16. McGorry, P. and B. Nelson, Why We Need a Transdiagnostic Staging Approach to Emerging Psychopathology, Early Diagnosis, and Treatment. JAMA Psychiatry, 2016. 73(3): p. 191-2. 
17. Malla, A., et al., From early intervention in psychosis to youth mental health reform: a review of the evolution and transformation of mental health services for young people. Soc Psychiatry Psychiatr Epidemiol, 2016. 51(3): p. 319-26.

18. Chekroud, A.M. and A. Trugerman, The Opportunity for Exercise to Improve Population Mental Health. JAMA Psychiatry, 2019. 76(11): p. 1206-1207.

19. Eaton, W.W. and M.D. Fallin, Public mental health. 2019: Oxford University Press.

20. Forbes, M.K., R.M. Rapee, and R.F. Krueger, Opportunities for the prevention of mental disorders by reducing general psychopathology in early childhood. Behav Res Ther, 2019. 119: p. 103411.

21. van Os, J., et al., The evidence-based group-level symptom-reduction model as the organizing principle for mental health care: time for change? World Psychiatry, 2019. 18(1): p. 88-96.

22. Borsboom, D., A. Cramer, and A. Kalis, Brain disorders? Not really... Why network structures block reductionism in psychopathology research. Behav Brain Sci, 2018: p. 1-54.

23. McGorry, P.D., et al., Beyond the "at risk mental state" concept: transitioning to transdiagnostic psychiatry. World Psychiatry, 2018. 17(2): p. 133-142.

24. Nelson, B., et al., Moving From Static to Dynamic Models of the Onset of Mental Disorder: A Review. JAMA Psychiatry, 2017. 74(5): p. 528-534.

25. Guloksuz, S., et al., The Complexities of Evaluating the Exposome in Psychiatry: A DataDriven Illustration of Challenges and Some Propositions for Amendments. Schizophr Bull, 2018. 44(6): p. 1175-1179.

26. Buckman, J.E.J., et al., Risk factors for relapse and recurrence of depression in adults and how they operate: A four-phase systematic review and meta-synthesis. Clin Psychol Rev, 2018. 64: p. 13-38.

27. Quinlan, E.B., et al., Identifying biological markers for improved precision medicine in psychiatry. Mol Psychiatry, 2020. 25(2): p. 243-253.

28. Belbasis, L., et al., Risk factors and peripheral biomarkers for schizophrenia spectrum disorders: an umbrella review of meta-analyses. Acta Psychiatr Scand, 2018. 137(2): p. 88-97.

29. Kendler, K.S., The dappled nature of causes of psychiatric illness: replacing the organicfunctional/hardware-software dichotomy with empirically based pluralism. Mol Psychiatry, 2012. 17(4): p. 377-88.

30. Wray, N.R., et al., Common Disease Is More Complex Than Implied by the Core Gene Omnigenic Model. Cell, 2018. 173(7): p. 1573-1580.

31. Wigman, J.T., et al., Exploring the underlying structure of mental disorders: crossdiagnostic differences and similarities from a network perspective using both a topdown and a bottom-up approach. Psychol Med, 2015. 45(11): p. 2375-87.

32. Saxe, G.N., et al., A Complex Systems Approach to Causal Discovery in Psychiatry. PLoS One, 2016. 11(3): p. e0151174.

33. Boyle, E.A., Y.I. Li, and J.K. Pritchard, An Expanded View of Complex Traits: From Polygenic to Omnigenic. Cell, 2017. 169(7): p. 1177-1186.

34. Kilbourne, A.M., et al., Measuring and improving the quality of mental health care: a global perspective. World Psychiatry, 2018. 17(1): p. 30-38. 
35. Lake, J. and M.S. Turner, Urgent Need for Improved Mental Health Care and a More Collaborative Model of Care. Perm J, 2017. 21: p. 17-024.

36. Craddock, N. and M.J. Owen, The Kraepelinian dichotomy - going, going... but still not gone. Br J Psychiatry, 2010. 196(2): p. 92-5.

37. Cross-Disorder Group of the Psychiatric Genomics, C., et al., Genetic relationship between five psychiatric disorders estimated from genome-wide SNPs. Nat Genet, 2013. 45(9): p. 984-94.

38. Anttila, V., et al., Analysis of shared heritability in common disorders of the brain. Science, 2018. 360(6395).

39. Jones, H.J., et al., Investigating the genetic architecture of general and specific psychopathology in adolescence. Transl Psychiatry, 2018. 8(1): p. 145.

40. Sprooten, E., et al., Addressing reverse inference in psychiatric neuroimaging: Metaanalyses of task-related brain activation in common mental disorders. Hum Brain Mapp, 2017. 38(4): p. 1846-1864.

41. Janiri, D., et al., Shared Neural Phenotypes for Mood and Anxiety Disorders: A Metaanalysis of 226 Task-Related Functional Imaging Studies. JAMA Psychiatry, 2020. 77(2): p. $172-179$.

42. Stein, F., et al., Psychopathological Syndromes Across Affective and Psychotic Disorders Correlate With Gray Matter Volumes. Schizophr Bull, 2021.

43. Caspi, A., et al., The p Factor: One General Psychopathology Factor in the Structure of Psychiatric Disorders? Clin Psychol Sci, 2014. 2(2): p. 119-137.

44. Borsboom, D., A network theory of mental disorders. World Psychiatry, 2017. 16(1): p. 5-13.

45. Reininghaus, U., et al., Transdiagnostic dimensions of psychosis in the BipolarSchizophrenia Network on Intermediate Phenotypes (B-SNIP). World Psychiatry, 2019. 18(1): p. 67-76.

46. van Os, J. and U. Reininghaus, Psychosis as a transdiagnostic and extended phenotype in the general population. World Psychiatry, 2016. 15(2): p. 118-24.

47. Hartmann, J.A., et al., Pluripotential Risk and Clinical Staging: Theoretical Considerations and Preliminary Data From a Transdiagnostic Risk Identification Approach. Front Psychiatry, 2020. 11: p<. 553578.

48. Kraan, T.C., et al., Child Maltreatment and Clinical Outcome in Individuals at Ultra-High Risk for Psychosis in the EU-GEI High Risk Study. Schizophr Bull, 2018. 44(3): p. 584-592.

49. Heleniak, C., et al., Childhood Maltreatment Exposure and Disruptions in Emotion Regulation: A Transdiagnostic Pathway to Adolescent Internalizing and Externalizing Psychopathology. Cognit Ther Res, 2016. 40(3): p. 394-415.

50. Pries, L.K., et al., Evidence That Environmental and Familial Risks for Psychosis Additively Impact a Multidimensional Subthreshold Psychosis Syndrome. Schizophr Bull, 2018. 44(4): p. 710-719.

51. Myin-Germeys, I., et al., Experience sampling research in psychopathology: opening the black box of daily life. Psychol Med, 2009. 39(9): p. 1533-47.

52. Myin-Germeys, I., et al., Experience sampling methodology in mental health research: new insights and technical developments. World Psychiatry, 2018. 17(2): p. 123-132. 
53. Hernaus, D., et al., Psychotic reactivity to daily life stress and the dopamine system: a study combining experience sampling and [18F]fallypride positron emission tomography. J Abnorm Psychol, 2015. 124(1): p. 27-37.

54. Myin-Germeys, I., et al., Subtle fluctuations in psychotic phenomena as functional states of abnormal dopamine reactivity in individuals at risk. Biol Psychiatry, 2005. 58(2): p. 105-10.

55. Reininghaus, U., et al., Evaluation of the validity and utility of a transdiagnostic psychosis dimension encompassing schizophrenia and bipolar disorder. Br J Psychiatry, 2016. 209(2): p. 107-13.

56. Reininghaus, U., S. Priebe, and R.P. Bentall, Testing the psychopathology of psychosis: evidence for a general psychosis dimension. Schizophr Bull, 2013. 39(4): p. 884-95.

57. van Os, J. and R.J. Linscott, Introduction: The extended psychosis phenotype-relationship with schizophrenia and with ultrahigh risk status for psychosis. Schizophr Bull, 2012. 38(2): p. 227-30.

58. Kotov, R., et al., Validity and utility of Hierarchical Taxonomy of Psychopathology (HiTOP): I. Psychosis superspectrum. World Psychiatry, 2020. 19(2): p. 151-172.

59. Hartmann, J.A., et al., Broad clinical high-risk mental state (CHARMS): Methodology of a cohort study validating criteria for pluripotent risk. Early Interv Psychiatry, 2019. 13(3): p. 379-386.

60. van Os, J., The dynamics of subthreshold psychopathology: implications for diagnosis and treatment. Am J Psychiatry, 2013. 170(7): p. 695-8.

61. Quattrone, D., et al., Transdiagnostic dimensions of psychopathology at first episode psychosis: findings from the multinational EU-GEl study. Psychol Med, 2019. 49(8): p. 1378-1391.

62. van Os, J., et al., Replicated evidence that endophenotypic expression of schizophrenia polygenic risk is greater in healthy siblings of patients compared to controls, suggesting gene-environment interaction. The EUGEl study. Psychol Med, 2020. 50(11): p. 18841897.

63. Cosgrave, J., et al., Do environmental risk factors for the development of psychosis distribute differently across dimensionally assessed psychotic experiences? Transl Psychiatry, 2021. 11(1): p. 226.

64. Nolen-Hoeksema, S. and E.R. Watkins, A Heuristic for Developing Transdiagnostic Models of Psychopathology: Explaining Multifinality and Divergent Trajectories. Perspect Psychol Sci, 2011. 6(6): p. 589-609.

65. Reininghaus, U., [Ecological Momentary Interventions in Psychiatry: The Momentum for Change in Daily Social Context]. Psychiatr Prax, 2018. 45(2): p. 59-61.

66. Reininghaus, U., C.A. Depp, and I. Myin-Germeys, Ecological Interventionist Causal Models in Psychosis: Targeting Psychological Mechanisms in Daily Life. Schizophr Bull, 2016. 42(2): p. 264-9.

67. Reininghaus, U. and C. Rauschenberg, Digitale Public Mental Health Ansätze zur Verminderung der psychosozialen Folgen der COVID-19 Pandemie. 2020: Policy Brief, COVID-19 Public Health Network.

68. Myin-Germeys, I., et al., Ecological momentary interventions in psychiatry. Curr Opin Psychiatry, 2016. 29(4): p. 258-63. 
69. Hariman, K., A. Ventriglio, and D. Bhugra, The Future of Digital Psychiatry. Curr Psychiatry Rep, 2019. 21(9): p. 88.

70. Kelleher, I., et al., Psychotic experiences in the population: Association with functioning and mental distress. Schizophr Res, 2015. 165(1): p. 9-14.

71. McGrath, J.J., et al., Psychotic Experiences in the General Population: A Cross-National Analysis Based on 31,261 Respondents From 18 Countries. JAMA Psychiatry, 2015. 72(7): p. 697-705.

72. Wusten, C., et al., Psychotic Experiences and Related Distress: A Cross-national Comparison and Network Analysis Based on 7141 Participants From 13 Countries. Schizophr Bull, 2018. 44(6): p. 1185-1194.

73. Wigman, J.T., et al., Evidence that psychotic symptoms are prevalent in disorders of anxiety and depression, impacting on illness onset, risk, and severity-implications for diagnosis and ultra-high risk research. Schizophr Bull, 2012. 38(2): p. 247-57.

74. Varghese, D., et al., Psychotic-like experiences in major depression and anxiety disorders: a population-based survey in young adults. Schizophr Bull, 2011. 37(2): p. 389-93.

75. Rimvall, M.K., et al., Psychotic experiences from preadolescence to adolescence: when should we be worried about adolescent risk behaviors? Eur Child Adolesc Psychiatry, 2020. 29(9): p. 1251-1264.

76. Kelleher, I., et al., Clinicopathological significance of psychotic experiences in nonpsychotic young people: evidence from four population-based studies. Br J Psychiatry, 2012. 201(1): p. 26-32.

77. McGrath, J.J., et al., The Bidirectional Associations Between Psychotic Experiences and DSM-IV Mental Disorders. Am J Psychiatry, 2016. 173(10): p. 997-1006.

78. Bhavsar, V., et al., Psychotic experiences, psychiatric comorbidity and mental health need in the general population: a cross-sectional and cohort study in Southeast London. Psychol Med, 2021. 51(1): p. 147-157.

79. Howes, O.D. and S. Kapur, The dopamine hypothesis of schizophrenia: version III--the final common pathway. Schizophr Bull, 2009. 35(3): p. 549-62.

80. Reininghaus, U., et al., Stress Sensitivity, Aberrant Salience, and Threat Anticipation in Early Psychosis: An Experience Sampling Study. Schizophr Bull, 2016. 42(3): p. 712-22.

81. Howes, O.D. and R.M. Murray, Schizophrenia: an integrated sociodevelopmentalcognitive model. Lancet, 2014. 383(9929): p. 1677-1687.

82. Garety, P.A., et al., A cognitive model of the positive symptoms of psychosis. Psychol Med, 2001. 31(2): p. 189-95.

83. Freeman, D., Persecutory delusions: a cognitive perspective on understanding and treatment. Lancet Psychiatry, 2016. 3(7): p. 685-92.

84. Garety, P.A. and D. Freeman, Cognitive approaches to delusions: A critical review of theories and evidence. British Journal of Clinical Psychology, 1999. 38(2): p. 113-154.

85. Garety, P.A., et al., Implications for neurobiological research of cognitive models of psychosis: a theoretical paper. Psychol Med, 2007. 37(10): p. 1377-91.

86. Brown, P., F. Waite, and D. Freeman, 'Twisting the lion's tail': Manipulationist tests of causation for psychological mechanisms in the occurrence of delusions and hallucinations. Clin Psychol Rev, 2019. 68: p. 25-37. 
87. Ward, T. and P.A. Garety, Fast and slow thinking in distressing delusions: A review of the literature and implications for targeted therapy. Schizophr Res, 2019. 203: p. 80-87.

88. Ross, R.M., et al., Jumping to Conclusions About the Beads Task? A Meta-analysis of Delusional Ideation and Data-Gathering. Schizophr Bull, 2015. 41(5): p. 1183-91.

89. Dudley, R., et al., Psychosis, Delusions and the "Jumping to Conclusions" Reasoning Bias: A Systematic Review and Meta-analysis. Schizophr Bull, 2016. 42(3): p. 652-65.

90. So, S.H., et al., 'Jumping to conclusions' data-gathering bias in psychosis and other psychiatric disorders - Two meta-analyses of comparisons between patients and healthy individuals. Clin Psychol Rev, 2016. 46: p. 151-67.

91. Fine, C., et al., Hopping, skipping or jumping to conclusions? Clarifying the role of the JTC bias in delusions. Cogn Neuropsychiatry, 2007. 12(1): p. 46-77.

92. Grossman, M., et al., Comparison of the neurocognitive profiles of individuals with elevated psychotic or depressive symptoms. Early Interv Psychiatry, 2019. 13(4): p. 928934.

93. Kelleher, I., et al., Neurocognition in the extended psychosis phenotype: performance of a community sample of adolescents with psychotic symptoms on the MATRICS neurocognitive battery. Schizophr Bull, 2013. 39(5): p. 1018-26.

94. McGrath, L.M., et al., Extending the 'cross-disorder' relevance of executive functions to dimensional neuropsychiatric traits in youth. J Child Psychol Psychiatry, 2016. 57(4): p. 462-71.

95. Owens, M., et al., When does anxiety help or hinder cognitive test performance? The role of working memory capacity. Br J Psychol, 2014. 105(1): p. 92-101.

96. Association, A.P. and A.P. Association, DSM 5. American Psychiatric Association, 2013. 70.

97. LeMoult, J., et al., Meta-analysis: Exposure to Early Life Stress and Risk for Depression in Childhood and Adolescence. J Am Acad Child Adolesc Psychiatry, 2020. 59(7): p. 842855.

98. Selous, C., et al., Adverse childhood experiences and adult mood problems: evidence from a five-decade prospective birth cohort. Psychol Med, 2020. 50(14): p. 2444-2451.

99. Varese, F., et al., Childhood adversities increase the risk of psychosis: a meta-analysis of patient-control, prospective- and cross-sectional cohort studies. Schizophr Bull, 2012. 38(4): p. 661-71.

100. Beards, S., et al., Life events and psychosis: a review and meta-analysis. Schizophr Bull, 2013. 39(4): p. 740-7.

101. Croft, J., et al., Association of Trauma Type, Age of Exposure, and Frequency in Childhood and Adolescence With Psychotic Experiences in Early Adulthood. JAMA Psychiatry, 2019. 76(1): p. 79-86.

102. Bell, C.J., et al., Childhood abuse and psychotic experiences in adulthood: findings from a 35-year longitudinal study. Br J Psychiatry, 2019. 214(3): p. 153-158.

103. van Nierop, M., et al., Psychopathological mechanisms linking childhood traumatic experiences to risk of psychotic symptoms: analysis of a large, representative population-based sample. Schizophr Bull, 2014. 40 Suppl 2: p. S123-30. 
104. Paul, S.E., et al., The intergenerational transmission of childhood maltreatment: Nonspecificity of maltreatment type and associations with borderline personality pathology. Dev Psychopathol, 2019. 31(3): p. 1157-1171.

105. Misiak, B., et al., Toward a unified theory of childhood trauma and psychosis: A comprehensive review of epidemiological, clinical, neuropsychological and biological findings. Neurosci Biobehav Rev, 2017. 75: p. 393-406.

106. Gibson, L.E., L.B. Alloy, and L.M. Ellman, Trauma and the psychosis spectrum: A review of symptom specificity and explanatory mechanisms. Clin Psychol Rev, 2016. 49: p. 92105.

107. Morgan, C., et al., Threat, hostility and violence in childhood and later psychotic disorder: population-based case-control study. Br J Psychiatry, 2020. 217(4): p. 575-582.

108. Bentall, R.P., et al., From adversity to psychosis: pathways and mechanisms from specific adversities to specific symptoms. Soc Psychiatry Psychiatr Epidemiol, 2014. 49(7): p. 1011-22.

109. Arseneault, L., et al., Childhood trauma and children's emerging psychotic symptoms: A genetically sensitive longitudinal cohort study. Am J Psychiatry, 2011. 168(1): p. 65-72.

110. Alameda, L., et al., A systematic review on mediators between adversity and psychosis: potential targets for treatment. Psychol Med, 2020. 50(12): p. 1966-1976.

111. Elmore, A.L. and E. Crouch, The Association of Adverse Childhood Experiences With Anxiety and Depression for Children and Youth, 8 to 17 Years of Age. Acad Pediatr, 2020. 20(5): p. 600-608.

112. Bjorkenstam, E., et al., Cumulative exposure to childhood adversity, and treated attention deficit/hyperactivity disorder: a cohort study of 543650 adolescents and young adults in Sweden. Psychol Med, 2018. 48(3): p. 498-507.

113. Stilo, S.A., et al., Further evidence of a cumulative effect of social disadvantage on risk of psychosis. Psychol Med, 2017. 47(5): p. 913-924.

114. Newbury, J., et al., Cumulative Effects of Neighborhood Social Adversity and Personal Crime Victimization on Adolescent Psychotic Experiences. Schizophr Bull, 2018. 44(2): p. 348-358.

115. Lereya, S.T., et al., Adult mental health consequences of peer bullying and maltreatment in childhood: two cohorts in two countries. Lancet Psychiatry, 2015. 2(6): p. 524-31.

116. Arseneault, L., L. Bowes, and S. Shakoor, Bullying victimization in youths and mental health problems: 'much ado about nothing'? Psychol Med, 2010. 40(5): p. 717-29.

117. Turner, H.A., D. Finkelhor, and R. Ormrod, Poly-victimization in a national sample of children and youth. Am J Prev Med, 2010. 38(3): p. 323-30.

118. Finkelhor, D., R.K. Ormrod, and H.A. Turner, Poly-victimization: a neglected component in child victimization. Child Abuse Negl, 2007. 31(1): p. 7-26.

119. Stanaway, J.D., et al., Global, regional, and national comparative risk assessment of 84 behavioural, environmental and occupational, and metabolic risks or clusters of risks for 195 countries and territories, 1990-2017: a systematic analysis for the Global Burden of Disease Study 2017. The Lancet, 2018. 392(10159): p. 1923-1994.

120. Stoltenborgh, M., et al., A global perspective on child sexual abuse: meta-analysis of prevalence around the world. Child Maltreat, 2011. 16(2): p. 79-101. 
121. Collip, D., I. Myin-Germeys, and J. Van Os, Does the concept of "sensitization" provide a plausible mechanism for the putative link between the environment and schizophrenia? Schizophr Bull, 2008. 34(2): p. 220-5.

122. Csikszentmihalyi, M. and R. Larson, Validity and reliability of the experience-sampling method, in Flow and the foundations of positive psychology. 2014, Springer. p. 35-54.

123. Shiffman, S., A.A. Stone, and M.R. Hufford, Ecological momentary assessment. Annu Rev Clin Psychol, 2008. 4: p. 1-32.

124. Myin-Germeys, I., et al., Emotional reactivity to daily life stress in psychosis. Arch Gen Psychiatry, 2001. 58(12): p. 1137-44.

125. Wichers, M., et al., Mechanisms of gene-environment interactions in depression: evidence that genes potentiate multiple sources of adversity. Psychol Med, 2009. 39(7): p. 1077-86.

126. Lardinois, M., et al., Childhood trauma and increased stress sensitivity in psychosis. Acta Psychiatr Scand, 2011. 123(1): p. 28-35.

127. Reininghaus, U., et al., Psychological processes underlying the association between childhood trauma and psychosis in daily life: an experience sampling study. Psychol Med, 2016. 46(13): p. 2799-813.

128. Heron, K.E. and J.M. Smyth, Ecological momentary interventions: incorporating mobile technology into psychosocial and health behaviour treatments. $\mathrm{Br} J$ Health Psychol, 2010. 15(Pt 1): p. 1-39.

129. Vaessen, T., et al., ACT in daily life in early psychosis: an ecological momentary intervention approach. Psychosis-Psychological Social and Integrative Approaches, 2019. 11(2): p. 93-104.

130. Bastiaansen, J.A., et al., An evaluation of the efficacy of two add-on ecological momentary intervention modules for depression in a pragmatic randomized controlled trial (ZELF-i). Psychol Med, 2020: p. 1-10.

131. Balaskas, A., et al., Ecological momentary interventions for mental health: A scoping review. PLoS One, 2021. 16(3): p. e0248152.

132. van Aubel, E., et al., Blended care in the treatment of subthreshold symptoms of depression and psychosis in emerging adults: A randomised controlled trial of Acceptance and Commitment Therapy in Daily-Life (ACT-DL). Behav Res Ther, 2020. 128: p. 103592.

133. Mohr, D.C., et al., The behavioral intervention technology model: an integrated conceptual and technological framework for eHealth and mHealth interventions. J Med Internet Res, 2014. 16(6): p. e146.

134. Hollis, C., et al., Technological innovations in mental healthcare: harnessing the digital revolution. Br J Psychiatry, 2015. 206(4): p. 263-5.

135. Schick, A., et al., The effects of a novel, accessible, transdiagnostic ecological momentary intervention for improving resilience in youth (EMIcompass): study protocol for a randomized controlled trial. 2020: PsyArXiv.

136. Valmaggia, L.R., F. Day, and M. Rus-Calafell, Using virtual reality to investigate psychological processes and mechanisms associated with the onset and maintenance of psychosis: a systematic review. Soc Psychiatry Psychiatr Epidemiol, 2016. 51(7): p. 921-36. 
137. Freeman, D., et al., Virtual reality in the assessment, understanding, and treatment of mental health disorders. Psychol Med, 2017. 47(14): p. 2393-2400.

138. Maher, N.A., et al., Passive data collection and use in healthcare: A systematic review of ethical issues. Int J Med Inform, 2019. 129: p. 242-247.

139. Holmes, E.A., et al., Multidisciplinary research priorities for the COVID-19 pandemic: a call for action for mental health science. Lancet Psychiatry, 2020. 7(6): p. 547-560. 



\section{PART 1}

Cognition and mechanisms in transdiagnostic phenotypes 



\section{CHAPTER 2}

\section{Reasoning bias, working memory performance, and a transdiagnostic phenotype of affective disturbances and psychotic experiences in the general population}

Ulrich Reininghaus ${ }^{*}$, Christian Rauschenberg*, Margreet ten Have, Ron de Graaf, Saskia van Dorsselaer, Claudia J. P. Simons, Nicole Gunther, Lotta-Katrin Pries, Sinan Guloksuz, Rajiv Radhakrishnan, Maarten Bak, and Jim van Os

* These authors share first authorship 


\section{Abstract}

Background: The jumping to conclusions (JTC) reasoning bias and decreased working memory performance (WMP) are associated with psychosis, but associations with affective disturbances (i.e. depression, anxiety, mania) remain inconclusive. Recent findings also suggest a transdiagnostic phenotype of cooccurring affective disturbances and psychotic experiences (PEs). This study investigated whether JTC bias and decreased WMP are associated with cooccurring affective disturbances and PEs.

Methods: Data were derived from the second Netherlands Mental Health Survey and Incidence Study (NEMESIS-2). Trained interviewers administered the Composite International Diagnostic Interview (CIDI) at three time points in a general population sample $(\mathrm{N}=4618)$. The beads and digit-span task were completed to assess JTC bias and WMP, respectively. CIDI was used to measure affective disturbances and an add-on instrument to measure PEs.

Results: Compared to individuals with neither affective disturbances nor PEs, the JTC bias was more likely to occur in individuals with co-occurring affective disturbances and PEs [moderate psychosis (1-2 PEs): adjusted relative risk ratio (RRR) 1.17, 95\% Cl 0.98-1.41; and high psychosis (3 or more PEs or psychosisrelated help-seeking behaviour): adjusted RRR 1.57, 95\% CI 1.19-2.08], but not with affective disturbances and PEs alone, whereas decreased WMP was more likely in all groups. There was some evidence of a dose-response relationship, as JTC bias and decreased WMP were more likely in individuals with affective disturbances as the level of PEs increased or help-seeking behaviour was reported.

Conclusion: The findings suggest that JTC bias and decreased WMP may contribute to a transdiagnostic phenotype of co-occurring affective disturbances and PEs.

Keywords: Anxiety; depression; cognitive bias; cognitive deficits; jumping to conclusions; mania; psychosis; reasoning bias; transdiagnostic phenotype; working memory 


\section{Introduction}

Psychotic experiences (PEs) are common [1, 2], with an estimated lifetime prevalence of $7 \%$, but transitory for most individuals [3]. However, PEs persist in around 20\% and evolve into a psychotic disorder in about 7\% [3-6]. Recent findings also suggest that PEs frequently co-occur with symptoms of common mental disorders (i.e. depression, anxiety) [7-12] and that the degree of cooccurrence may be influenced by exposure to socio-environmental risk [13-15]. In line with these findings, the polygenic risk score for schizophrenia has been shown to be associated with PEs and affective disturbances in relatives of individuals with psychotic disorder and comparison subjects [16] and developmental psychopathology in children and adolescents [17-19]. This was further supported by a considerable overlap of genetic liability and molecular neuropathology between psychotic disorders and affective disorders [20-26]. Thus, evidence suggest an extended and transdiagnostic psychosis phenotype with temporal and phenomenological continuity across developmental stages of psychotic and affective disorders and shared socio-environmental and genetic risk [1, 2, 27-29].

Contemporary models of psychosis [30-33] propose various risk factors and mechanisms involved in illness onset, including cognitive biases and deficits, but whether these factors are also associated with transdiagnostic phenotypes remains largely under-researched. The jumping to conclusions (JTC) reasoning bias describes the tendency to make hasty decisions based on insufficient information [34] and has been consistently found to occur more often in individuals with subclinical and clinical psychosis [34-37]. Based on cognitive models $[38,39]$, it has been suggested that the JTC bias is particularly involved in the formation and maintenance of delusional ideations [35, 40], with some recent evidence supporting this assumption [34]. Similarly, cognitive deficits have been demonstrated across severity levels of psychosis, which is considered a core finding supporting the neurodevelopmental hypothesis [41,42], with several studies focussing on decreased working memory performance (WMP) as a proxy for cognitive deficits [43-52].

In contrast, there have been inconsistent findings for the presence of both the JTC bias and decreased WMP in non-psychotic disorders. A recent meta-analysis found some evidence that the JTC bias was more likely in individuals with non-psychotic disorders [37]. However, effect sizes varied considerably across studies and those 
of low quality reported the strongest effects. After outliers were excluded from analyses, no effects for depression, anxiety disorders, and obsessive-compulsive disorders were found. Similarly, studies investigating decreased WMP in those with non-psychotic disorders are mixed and the evidence differs largely across domains of psychopathology. There is evidence for decreased WMP in individuals with subclinical and clinical anxiety [53], while findings for depression and mania appear to be more heterogeneous with some studies reporting a lowered [54, 55], and others a similar WMP [56-58] compared to controls.

Taken together, there is robust evidence that JTC bias and decreased WMP are associated with psychosis, but attempts to show similar associations in individuals with affective disturbances have led to mixed results. As there is evidence that affective disturbances and PEs frequently co-occur in general population and clinical samples, an important next step is to investigate whether the JTC bias and decreased WMP contribute to a transdiagnostic phenotype. It is reasonable to assume that risk factors and mechanisms proposed in contemporary models of psychosis [30-33] extend to individuals with affective disturbances if they are accompanied by PEs, which may give rise to generalisability and specificity of recent findings.

\section{Aims and hypotheses}

The aim of the current study was to investigate associations of the JTC bias and decreased WMP with co-occurring affective disturbances and PEs in the general population. More specifically, we tested the following hypotheses: First, compared to individuals with neither affective disturbances nor PEs (group 1), the JTC bias is more likely to occur in those with sole presence of PEs (group 3) and in those with co-occurring affective disturbances and PEs (further stratified into moderate psychosis=1-2 PEs (group 4); and high psychosis=3 or more PEs or psychosisrelated help-seeking behaviour (group 5)), but not in those with sole presence of affective disturbances (group 2). Second, decreased WMP is associated with an increased likelihood of reporting sole presence of affective disturbances (group 2), sole presence of PEs (group 3) and co-occurring affective disturbances and PEs (group 4 and group 5). Third, there is evidence for a dose-response relationship, in which the JTC bias and decreased WMP is more likely to occur in those with affective disturbances as the level of PEs increases or individuals report psychosisrelated help-seeking behaviour (comparing group 5 and group 4). 


\section{Materials and methods}

\section{Sample}

Data were derived from the second Netherlands Mental Health Survey and Incidence Study (NEMESIS-2), a three-wave psychiatric epidemiological cohort study conducted to estimate the prevalence, incidence, and course of psychiatric disorders in the Dutch general population. Based on a multistage, stratified random sampling of households, all respondents were interviewed at home with the Composite International Diagnostic Interview (CIDI) version 3.0 [59-61] and additional questionnaires. Inclusion criteria were: aged 18-65 years. Exclusion criteria were: insufficient command of the Dutch language. The first wave (T0) was performed from November 2007 to July 2009, with a total of 6646 persons interviewed (response rate 65.1\%). This sample was representative for the Dutch general population although younger subjects were slightly underrepresented [62]. For the second wave (T1), performed from November 2010 to June 2012, all T0 respondents were approached. Of these, 5303 individuals were interviewed again (response rate of $80.4 \%$ with those deceased excluded). The attrition rate was not associated with 12-month prevalence of psychopathology at baseline [63]. From November 2013 to June 2015, the third wave (T2) was completed with 4618 persons who were interviewed a third time (response rate of $87.8 \%$ from T1 with those deceased excluded). Again, attrition rate was not associated with the 12-month prevalence of mental disorders at T1, except for alcohol and drug dependence and bipolar disorder [64]. The time between baseline and second follow-up was, on average, 6 years and 6 days. The study was approved by the Medical Ethics Review Committee for Institutions of Mental Health Care. After having been informed about the study aims, respondents provided written informed consent at each wave. The face-to-face interviews were carried out by trained interviewers, who were not clinicians, using a laptop computer. A more detailed description of the methodology is presented elsewhere [62, 65].

\section{Data collection}

\section{Sociodemographic characteristics and socio-environmental factors}

Data on age, sex, level of education, urbanicity, ethnic minority status, cannabis use, and childhood trauma were collected using a sociodemographic schedule, trauma questionnaire, and the CIDI. 


\section{Working memory performance}

At the second wave (T1), participants were asked to complete the digit-span task to assess working memory performance. The procedure and items were based on the Wechsler Adult Intelligence Scale (WAIS-III) [66, 67]. The digit-span task was divided into two parts, consisting of a forward ( 6 items) and backward (6 items) task condition. Both parts were separated by three unrelated interview sections. For each item, participants were asked to repeat two different sequences of digits, spanning from 4 to 9 digits for the forward and 3 to 8 digits for the backward condition. If at least one out of two sequences was repeated correctly, the interviewer moved to the next item. For each completed item, one digit was added to increase difficulty. Scores were based on the number of correct answers and up to 4 (forward condition) and 2 (backward condition) extra points. For study purposes, the sum score was computed and transposed to T0 and T2 as performance at T1 was considered to represent individuals' trait cognitive ability.

\section{JTC bias}

As part of the third wave (T2), the beads task was completed to assess the presence or absence of the JTC bias. The beads task [68] is an experimental task designed to measure individuals' reasoning style under ambiguous conditions. Participants were shown two jars containing red and blue coloured beads in opposite ratios. In this study, the more difficult version of the beads task with a colour ratio of 60:40 beads was used to increase sensitivity to detect JTC bias in a general population sample. The jars as well as all instructions were presented on a computer screen. After both jars were shown and a training session was completed, participants were instructed that all beads are drawn consecutively from one jar and, once presented, are returned to the same jar. After each draw, participants were asked whether they want to make a decision from which jar the beads were drawn or if they would like to see another bead, with the possibility to see up to 10 beads before a decision had to be made. The order of presented beads was predetermined and the dominant colour presented in the training session selected at random. Again, the number of beads drawn at T2 was considered to represent individuals' reasoning style and the values were transposed to TO and T1. Consistent with previous studies [34], JTC bias was defined as making a decision based on two or fewer beads [37]. 


\section{Affective disturbances}

Depression, anxiety, and mania were measured at three time points using core items of the CIDI version 3.0. This measure uses a true-false response format asking for the prevalence of symptoms for various mental disorders, including depressive episode, social phobia, generalised anxiety disorder, and manic episode (e.g. feeling fearful, depressed, experiences of a panic attack). All items are presented in Supplementary Table 1.

\section{Psychotic experiences (PES)}

Studies concluded that earlier versions of the CIDI are not reliable and valid measures for psychotic disorders [69]. Thus, a psychosis measure was constructed based on the section of psychotic symptoms in CIDI 1.1. The instrument consisted of 20 items asking for the lifetime prevalence of PEs (i.e. 15 delusional and 5 hallucinatory experiences). In case PEs were endorsed, participants were asked to state, on a 4-point Likert scale, the frequency, distress, and the impact of PEs on their daily life, including whether they had sought help for these experiences. Sum scores were calculated by adding reported PEs. More details are described elsewhere [13] and used items are reported in Supplementary Table 1.

\section{Grouping absence, presence and co-occurrence of affective disturbances and psychotic experiences}

Individuals were grouped based on answers given on measures assessing depression, anxiety, mania, and PEs. Five groups were generated representing the absence, sole presence, or co-occurrence of affective disturbances and PEs: neither affective disturbances nor PEs (group 1); sole presence of affective disturbances (group 2); sole presence of PEs (group 3); co-occurring affective disturbances and PEs (further stratified into moderate psychosis=1-2 PEs (group 4); and high psychosis $=3$ or more PEs or psychosis-related help-seeking behaviour (group 5)).

\section{Statistical analysis}

All analyses were carried out using STATA version 13.1 [70]. As the digit-span task and beads task were completed at $\mathrm{T} 1$ and $\mathrm{T} 2$, respectively, analyses were performed on samples with differing numbers of observations. First, socio-demographic 
characteristics (i.e. age, sex, education level) and socio-environmental factors (i.e. urbanicity, ethnic minority status, cannabis use, and childhood trauma) were compared across groups using linear regression and chi-square tests as appropriate. Second, to investigate associations of JTC bias (binary variable) and working memory performance (continuous variable) with co-occurring affective disturbances and PEs, the MLOGIT command was used to fit multinomial logistic regression models. The CLUSTER option was used to compute cluster-robust standard errors to correct for clustering of data (i.e. three observations for each individual). Sum scores of the digit-span task were recoded that higher scores represent lower WMP and standardized (mean=0, SD=1). Lastly, relative risk ratios (RRRs) for group status by JTC bias and decreased WMP were compared using the Wald test. All models were adjusted for various a priori defined potential confounders. First, we adjusted for socio-demographic characteristics and socioenvironmental factors and, in models with JTC bias as the independent variable, we also adjusted for WMP.

\section{Results}

\section{Basic sample characteristics}

In total, thqe sample consisted of 4618 participants at the third wave. Of these, 4596 completed the beads task (99.5\%) with 13788 observations for all three timepoints. There were no differences between individuals who completed the beads task and those who did not with regard to socio-demographic characteristics and other variables. The sample characteristics are presented in Table 1. Overall, individuals who reported affective disturbances and/or PEs were more likely to be younger, female, less educated, more often from an ethnic minority group, to have used cannabis regularly at least once during lifetime, and to have experienced childhood trauma before the age of 16 . There were considerable differences between those with sole presence of affective disturbances and co-occurring affective disturbances and PEs in terms of most sample characteristics and socioenvironmental risk factors. Basic characteristics of the sample of individuals who completed the digit-span task at T1 are presented in Supplementary Table 2. 


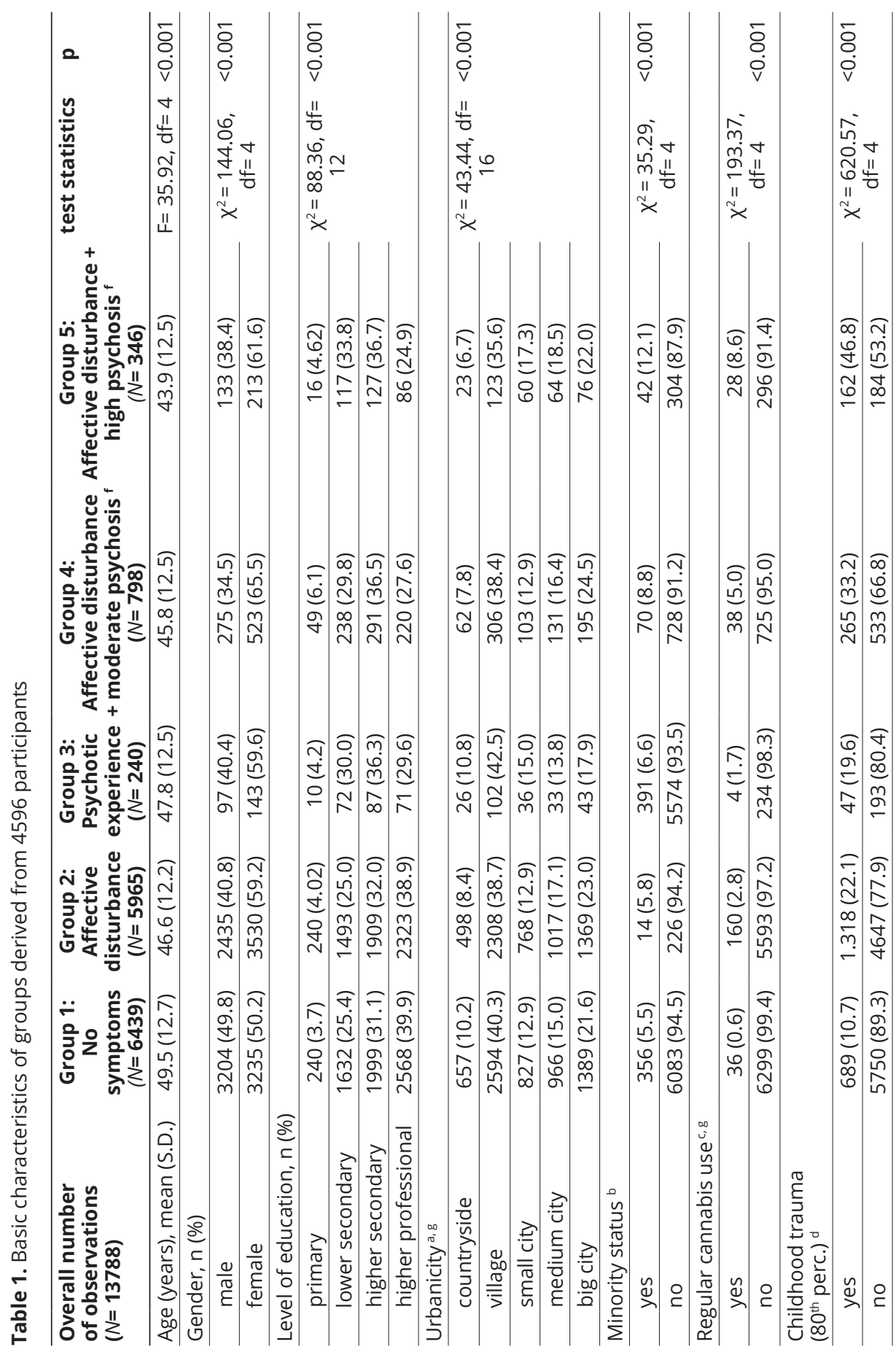




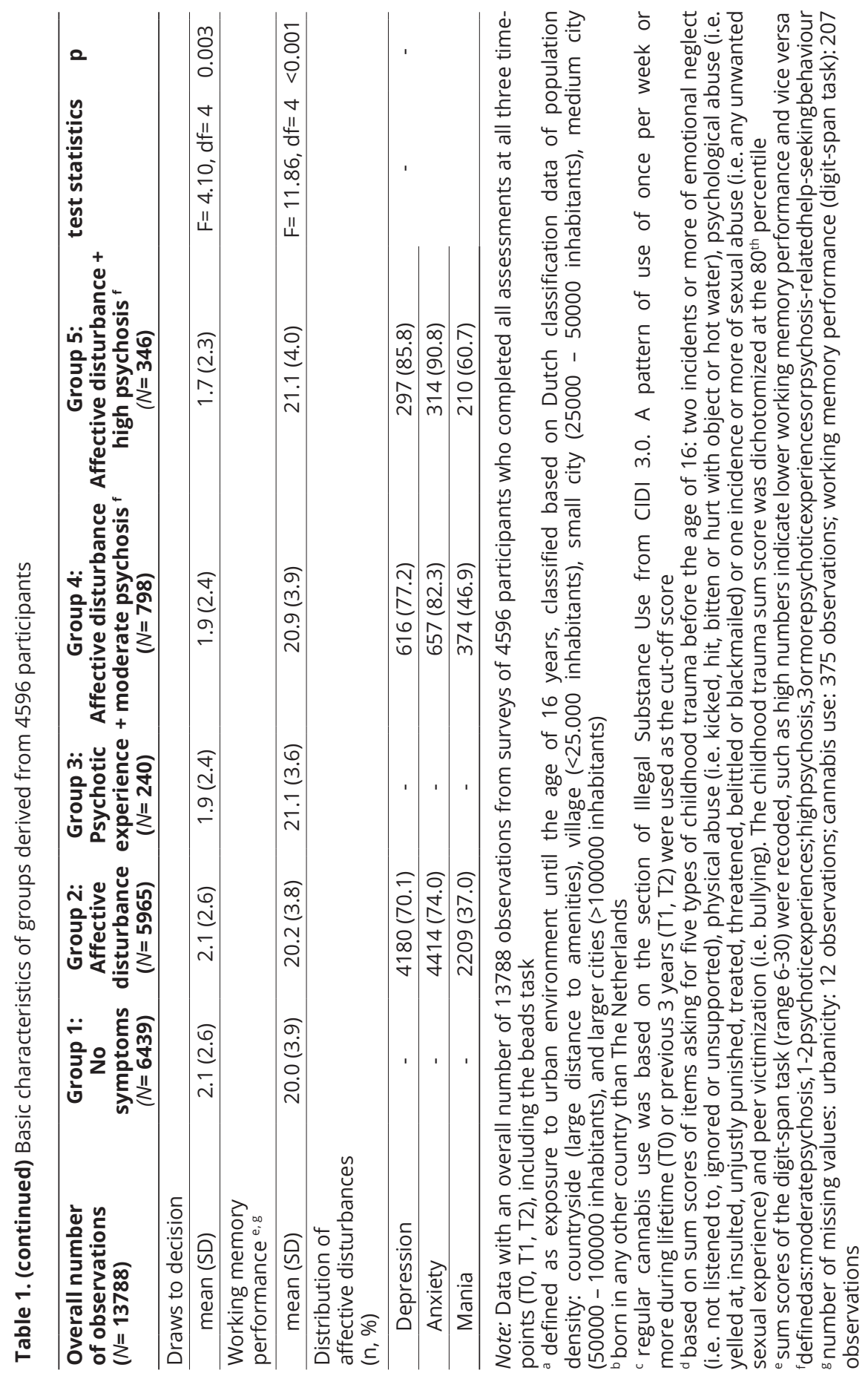




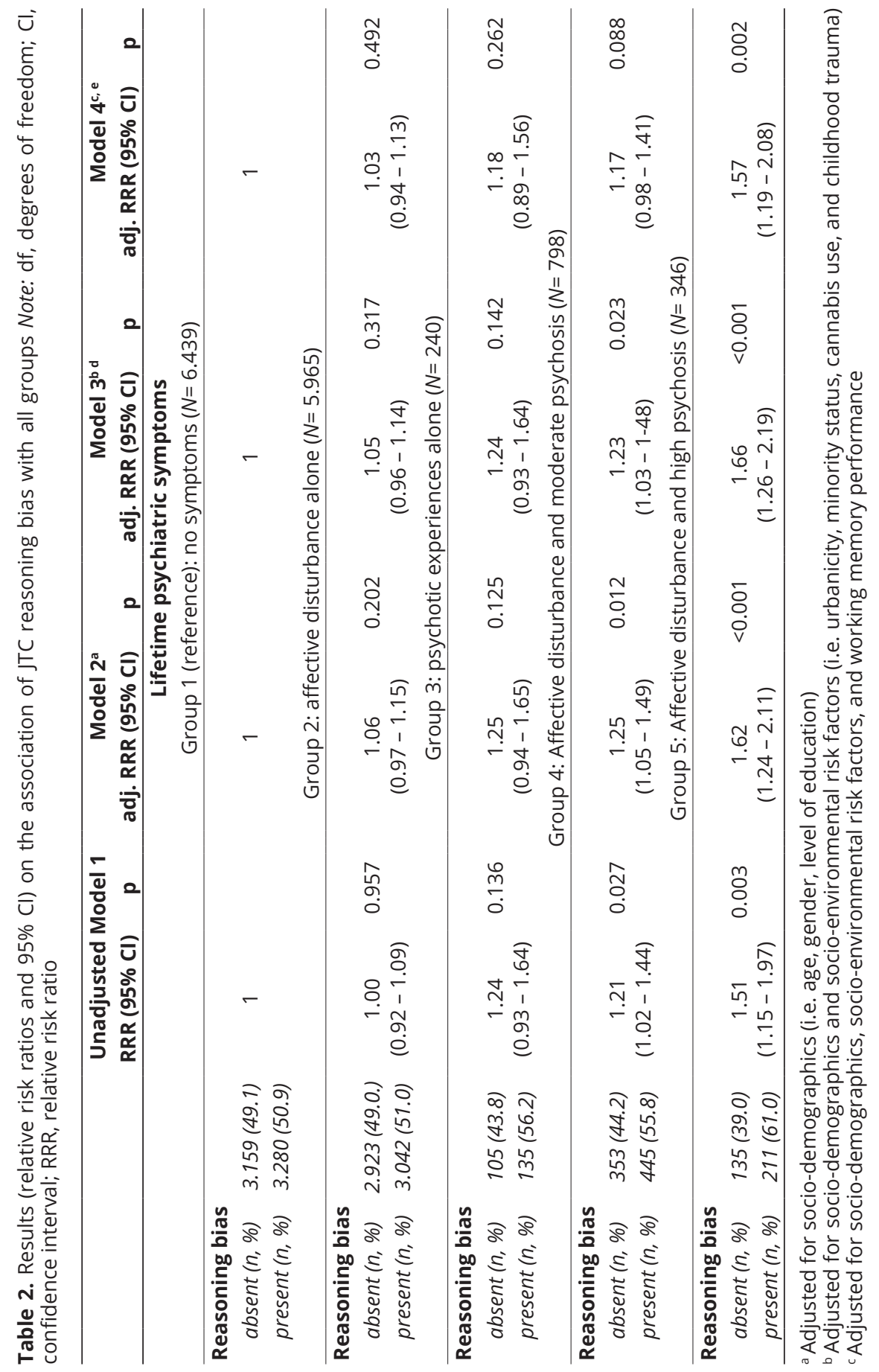


d test for differences in relative risk ratios across groups for Model 3:

\begin{tabular}{lcc}
\hline & $\chi^{2}(\mathbf{d f})$ & $p$ \\
\hline $\begin{array}{l}\text { affective disturbance only vs. } \\
\text { affective disturbance + moderate psychosis }\end{array}$ & $3.46(1)$ & 0.063 \\
\hline $\begin{array}{l}\text { affective disturbance only vs. } \\
\text { affective disturbance + high psychosis }\end{array}$ & $11.23(1)$ & $<0.001$ \\
\hline $\begin{array}{l}\text { affective disturbance + moderate psychosis } \\
\text { affective disturbance + high psychosis }\end{array}$ & $4.10(1)$ & 0.043 \\
\hline
\end{tabular}

e test for differences in relative risk ratios across groups for Model 4:

\begin{tabular}{lcc}
\hline & $\chi^{2}$ (df) & $p$ \\
\hline $\begin{array}{l}\text { affective disturbance only vs. } \\
\text { affective disturbance + moderate psychosis }\end{array}$ & $2.05(1)$ & 0.153 \\
\hline $\begin{array}{l}\text { affective disturbance only vs. } \\
\text { affective disturbance + high psychosis }\end{array}$ & $9.06(1)$ & 0.003 \\
\hline $\begin{array}{l}\text { affective disturbance + moderate psychosis } \\
\text { affective disturbance + high psychosis }\end{array}$ & $3.78(1)$ & 0.052 \\
\hline
\end{tabular}




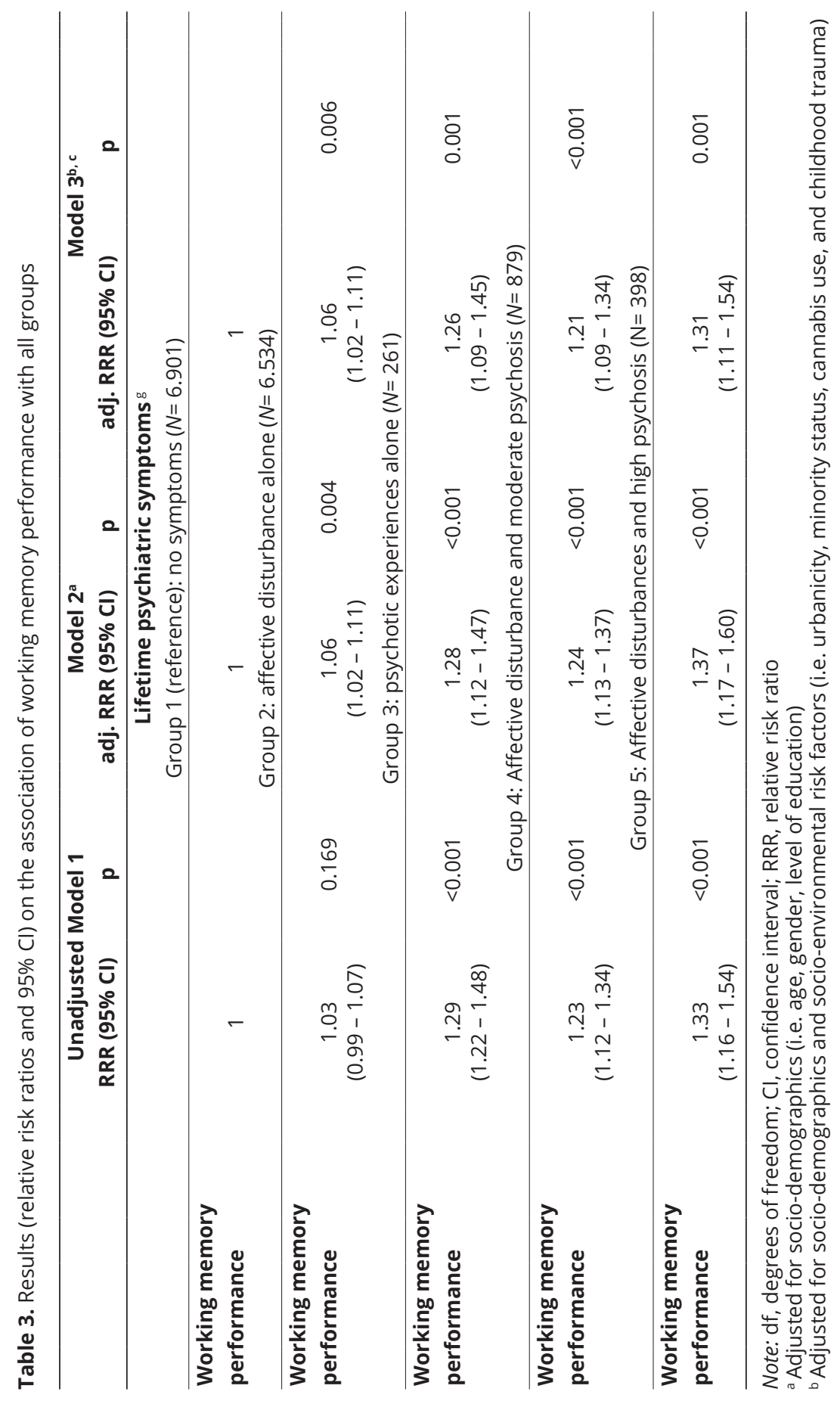


${ }^{c}$ test for differences in relative risk ratios across groups for Model 3:

\begin{tabular}{lcc}
\hline & $\chi^{2}(\mathrm{df})$ & $p$ \\
\hline $\begin{array}{l}\text { affective disturbance only vs. } \\
\text { affective disturbance + moderate psychosis }\end{array}$ & $7.01(1)$ & 0.008 \\
\hline $\begin{array}{l}\text { affective disturbance only vs. } \\
\text { affective disturbance + high psychosis }\end{array}$ & $6.90(1)$ & 0.009 \\
\hline $\begin{array}{l}\text { affective disturbance + moderate psychosis } \\
\text { affective disturbance + high psychosis }\end{array}$ & $0.88(1)$ & 0.349 \\
\hline
\end{tabular}

\section{JTC bias and co-occurring affective disturbances and PEs}

As shown in Table 2 and Figure 1, there was evidence that, compared to individuals with neither affective disturbances nor PEs, JTC bias was more likely to be present in those with co-occurring affective disturbances and PEs (moderate psychosis: $\mathrm{RRR}=1.23,95 \% \mathrm{Cl} 1.03-1.48, p=0.023$; high psychosis: $\mathrm{RRR}=1.66,95 \% \mathrm{Cl} 1.26-2.19$, $p<0.001$ ), but not in those with sole presence of affective disturbances (RRR=1.05, $95 \% \mathrm{Cl} 0.96-1.14, p=0.317$ ) and sole presence of PEs ( $R R R=1.24,95 \% \mathrm{Cl} 0.93-1.64$, $p=0.142$ ) after adjustment for age, sex, education level, urbanicity, ethnic minority status, cannabis use, and childhood trauma. When we additionally adjusted for working memory performance, the associations were attenuated (moderate psychosis: RRR=1.17, 95\% Cl $0.98-1.41, p=0.088$; high psychosis: RRR=1.57, 95\% $\mathrm{Cl} 1.19-2.08, p=0.002)$. When we compared associations across groups, we found no significant differences in group 2 vs. group 4 ( $p=0.153)$, whereas significant differences were apparent in the comparison of group 2 vs. group $5(p=0.003)$ and group 4 vs. 5 ( $p=0.052)$. Model fit statistics are provided in Supplementary Table 3.

\section{Working memory performance and co-occurring affective disturbances and PEs}

As shown in Table 3 and Figure 1, we found evidence that decreased WMP was more likely in individuals with sole presence of, or co-occurring, affective disturbances and PEs (affective disturbances: RRR=1.06, 95\% Cl $1.02-1.11$, $p=0.006$; PEs: RRR=1.26, 95\% Cl 1.09 - 1.45, $p=0.001$; co-occurring affective disturbances and moderate psychosis: RRR=1.21, 95\% Cl 1.09-1.34, $p<0.001$; cooccurring affective disturbances and high psychosis: RRR=1.31, 95\% Cl $1.11-1.54$, $p<0.001$ ) compared to those with neither affective disturbances nor PEs, after adjusting for socio-demographic characteristics and socio-environmental factors. 
When we compared associations across groups, we found significant differences in group 2 vs. group 4 ( $p=0.008$ ) and group 2 vs. group 5 ( $p=0.009)$, but not group 4 vs. group $5(p=0.349)$. Model fit statistics are provided in Supplementary Table 3.

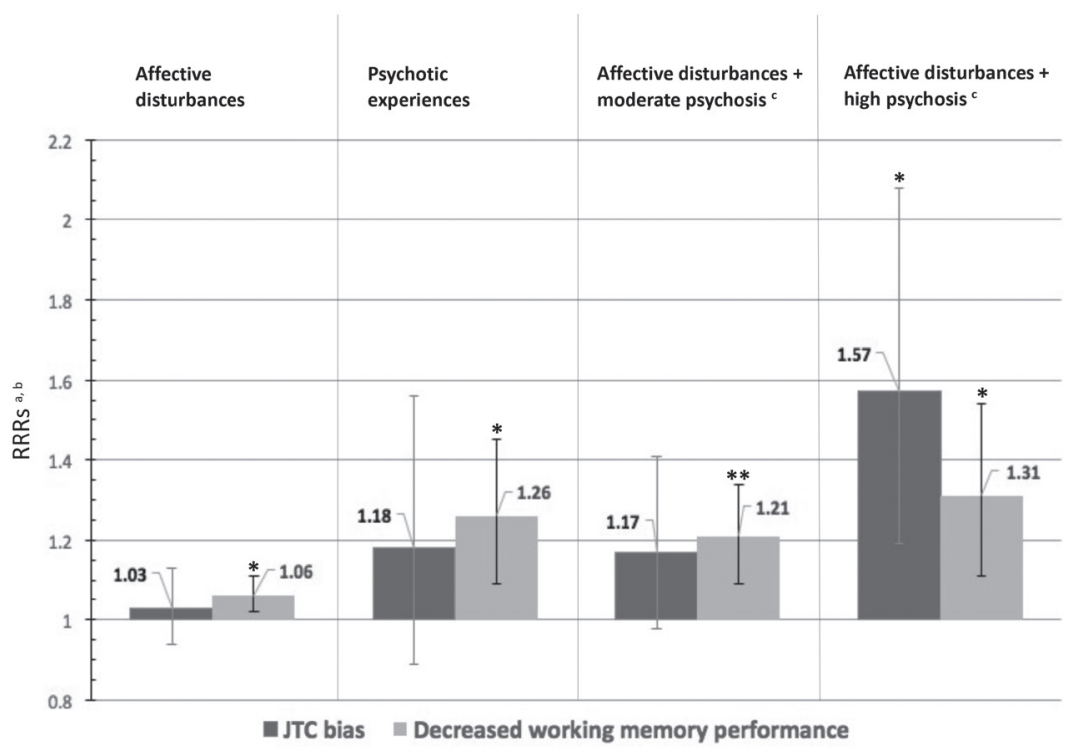

Figure 1. Results on the association of JTC reasoning bias and decreased WMP with all groups Note: RRRs and $95 \% \mathrm{Cl}$ are shown; RRRs, relative risk ratios; Cl, confidence interval; JTC bias, jumping to conclusions reasoning bias, WMP, working memory performance

* $p<0.05, * * p<0.001$

a adjusted for age, gender, level of education, urbanicity, minority status, cannabis use, and childhood trauma, and in models with JTC as independent variable also working memory performance

${ }^{b}$ compared to individuals with neither affective disturbances nor psychotic experiences (reference group RRR $=1$ )

cdefined as: moderate psychosis, 1-2 psychotic experiences; high psychosis, 3 or more psychotic experiences or psychosis-related help-seeking behaviour

\section{Discussion}

\section{Main findings}

This study investigated whether the JTC bias and decreased WMP are associated with a transdiagnostic phenotype of co-occurring affective disturbances and PEs in the general population. First, we found that the JTC bias was more likely to be present in individuals with co-occurring affective disturbances and PEs, but not in those with sole presence of affective disturbances or PEs. There was some 
attenuation of this association when we additionally adjusted for WMP. Second, decreased WMP was associated with an increased likelihood of reporting sole presence of and co-occurring affective disturbances and PEs. Third, there was some evidence of a dose-response relationship, as JTC bias and decreased WMP was progressively more likely to be present in individuals with affective disturbances as the level of PEs increased or psychosis-related help-seeking behaviour was reported, though some inconsistencies were observed for comparisons across groups.

\section{Methodological considerations}

The strength of the current study was that analyses were based on a large population-based cohort study. However, several methodological considerations should be taken into account before interpreting our findings. First, the number of individuals with JTC bias was greater than that reported in other studies [34]. We assume that assessment length may have resulted in fatigue effects and decreased motivation, leading to hastier decisions independent from individuals' reasoning style. However, this does not prevent us from inferring valuable insights given robust RRRs found in the current study. Second, the digit-span and beads task were assessed once during the study period and scores were considered to reflect trait cognitive ability and reasoning style. Ideally, tasks would have been completed at all three time points to calculate more robust estimates. However, assessment burden associated with the inclusion of both tasks at all three assessments was considered to be high and potential benefits comparably low, given reports of low variability for both the JTC bias [71, 72] and WMP [73] over time. Third, cross-sectional modelling of data derived from three waves did not allow for investigating temporality of JTC bias and decreased WMP with psychopathological outcomes. Future studies may employ longitudinal pathway analyses to further investigate the temporality of reported findings. Fourth, the transdiagnostic phenotypes of co-occurring affective disturbances and PEs were computed based on an observational and not a data-driven approach.

\section{Comparison with previous research}

We found that JTC bias is more prevalent in individuals who reported affective disturbances and PEs at least once during their lifetime compared to those who 
experienced neither affective disturbances nor PEs. This adds to recent findings of robust associations between JTC bias and psychosis [34-37] and suggests, for the first time, that JTC bias contributes to a transdiagnostic phenotype in the general population. This association, however, was found to be attenuated when we additionally adjusted for WMP, indicating that cognitive deficits may mediate, to a degree, the manifestation of reasoning style and its impact on mental health. This finding is in line with studies which have demonstrated altered neuropsychological functioning to be associated with JTC bias [74-76]. It has been suggested [43, 77] that JTC bias may partly be explained by difficulties in keeping information in mind but more studies are warranted to further investigate the role of cognitive deficits as an alternative explanation of the association between JTC bias, affective disturbances, and PEs. Echoing recent findings [37], there was no evidence that the JTC bias was more likely to be present in individuals who reported lifetime affective disturbances but not PEs.

When looking at differences across groups, we found some evidence for a doseresponse relationship as the JTC bias was more likely to occur in individuals with affective disturbances as the number of PEs increased or psychosis-related helpseeking behaviour was reported. While these results suggest some degree of specificity of JTC bias for psychosis [37], some inconsistencies were observed. Critically, there was only weak evidence (at trend level) that JTC bias was more likely to be present in individuals with sole presence of PEs. This may be explained by potentially imprecise estimates as a result of the small number of observations in this group - the smallest of all $(N=240)$, which is a notable finding per se given psychosis has long been studied in isolation [27].

Similarly, there was some, albeit less strong, evidence for a dose-response relationship for the association between decreased WMP and affective disturbances and PEs. Interestingly, individuals who reported both affective disturbances and PEs showed a greater decrease in WMP compared to those with affective disturbances but not PEs. However, there were, again, some inconsistencies in group comparisons for this dose-response relationship. The finding of decreased WMP in those with sole presence of and co-occurring affective disturbances and PES suggest that, as has recently been noted [78-81], cognitive deficits may constitute a more broadly distributed vulnerability factor across various (transdiagnostic) psychopathological domains. 
Overall, reported findings point to the need to further investigate whether psychological processes and mechanisms involved in the development and maintenance of psychosis extend to transdiagnostic phenotypes in both general population and clinical samples to overcome shortcomings of focussing only on psychosis and to further corroborate contemporary aetiological models [27, 28]. Studies that do not exclude but purposefully allow for comorbidities are now warranted to facilitate progress in research, treatment, and aetiological models as well as dimensional and transdiagnostic approaches to psychopathology [28, 29, 82,83 ] to achieve the goals set by the Research Domain Criteria [84].

\section{Conclusion}

Our findings suggest that JTC bias and decreased WMP may contribute to a transdiagnostic phenotype of co-occurring affective disturbances and PEs, with some evidence supporting specificity of JTC bias with psychosis. Future studies should further investigate specificity and generalisability of psychological processes and mechanisms to transdiagnostic phenotypes. Further, investigating putative mechanisms involved in the formation and maintenance of transdiagnostic phenotypes may be an important next step for the development of process-based treatment protocols [27, 28, 85-89] to, ultimately, alleviate individual's mental health burden. 


\section{References}

1. van Os, J. and U. Reininghaus, Psychosis as a transdiagnostic and extended phenotype in the general population. World Psychiatry, 2016. 15(2): p. 118-24.

2. van Os, J. and R.J. Linscott, Introduction: The extended psychosis phenotype--relationship with schizophrenia and with ultrahigh risk status for psychosis. Schizophrenia Bulletin, 2012. 38(2): p. 227-30.

3. Linscott, R.J. and J. van Os, An updated and conservative systematic review and metaanalysis of epidemiological evidence on psychotic experiences in children and adults: on the pathway from proneness to persistence to dimensional expression across mental disorders. Psychological Medicine, 2013. 43(6): p. 1133-49.

4. Calkins, M.E., et al., Persistence of psychosis spectrum symptoms in the Philadelphia Neurodevelopmental Cohort: a prospective two-year follow-up. World Psychiatry, 2017. 16(1): p. 62-76.

5. Kaymaz, N., et al., Do subthreshold psychotic experiences predict clinical outcomes in unselected non-help-seeking population-based samples? A systematic review and meta-analysis, enriched with new results. Psychological Medicine, 2012. 42(11): p. 223953.

6. Hanssen, M., et al., The incidence and outcome of subclinical psychotic experiences in the general population. British Journal of Clinical Psychology, 2005. 44(Pt 2): p. 181-91.

7. McGrath, J.J., et al., The Bidirectional Associations Between Psychotic Experiences and DSM-IV Mental Disorders. American Journal of Psychiatry, 2016. 173(10): p. 997-1006.

8. Bebbington, P. and D. Freeman, Transdiagnostic Extension of Delusions: Schizophrenia and Beyond. Schizophrenia Bulletin, 2017. 43(2): p. 273-282.

9. Fusar-Poli, P., et al., Comorbid depressive and anxiety disorders in 509 individuals with an at-risk mental state: impact on psychopathology and transition to psychosis. Schizophrenia Bulletin, 2014. 40(1): p. 120-31.

10. Hartley, S., C. Barrowclough, and G. Haddock, Anxiety and depression in psychosis: a systematic review of associations with positive psychotic symptoms. Acta Psychiatrica Scandinavica, 2013. 128(5): p. 327-46.

11. Varghese, D., et al., Psychotic-like experiences in major depression and anxiety disorders: a population-based survey in young adults. Schizophrenia Bulletin, 2011. 37(2): p. 389-93.

12. Wigman, J.T., et al., Evidence that psychotic symptoms are prevalent in disorders of anxiety and depression, impacting on illness onset, risk, and severity-implications for diagnosis and ultra-high risk research. Schizophrenia Bulletin, 2012. 38(2): p. 247-57.

13. van Nierop, M., et al., Childhood trauma is associated with a specific admixture of affective, anxiety, and psychosis symptoms cutting across traditional diagnostic boundaries. Psychological Medicine, 2015. 45(6): p. 1277-88.

14. Guloksuz, S., et al., Evidence that the presence of psychosis in non-psychotic disorder is environment-dependent and mediated by severity of non-psychotic psychopathology. Psychological Medicine, 2015. 45(11): p. 2389-401.

15. Guloksuz, S., et al., Exposure to environmental factors increases connectivity between symptom domains in the psychopathology network. BMC Psychiatry, 2016. 16: p. 223. 
16. van Os, J., et al., Evidence that polygenic risk for psychotic disorder is expressed in the domain of neurodevelopment, emotion regulation and attribution of salience. Psychological Medicine, 2017. 47(14): p. 2421-2437.

17. Nivard, M.G., et al., Genetic Overlap Between Schizophrenia and Developmental Psychopathology: Longitudinal and Multivariate Polygenic Risk Prediction of Common Psychiatric Traits During Development. Schizophrenia Bulletin, 2017. 43(6): p. 11971207.

18. Jones, H.J., et al., Phenotypic Manifestation of Genetic Risk for Schizophrenia During Adolescence in the General Population. JAMA Psychiatry, 2016. 73(3): p. 221-8.

19. Jansen, P.R., et al., Polygenic scores for schizophrenia and educational attainment are associated with behavioural problems in early childhood in the general population. Journal of Child Psychology and Psychiatry, 2018. 59(1): p. 39-47.

20. Cross-Disorder Group of the Psychiatric Genomics, C., Identification of risk loci with shared effects on five major psychiatric disorders: a genome-wide analysis. Lancet, 2013. 381(9875): p. 1371-1379.

21. Cross-Disorder Group of the Psychiatric Genomics, C., Genetic relationship between five psychiatric disorders estimated from genome-wide SNPs. Nature Genetics, 2013. 45(9): p. 984-94.

22. Cheng, C.M., et al., Co-aggregation of major psychiatric disorders in individuals with firstdegree relatives with schizophrenia: a nationwide population-based study. Molecular Psychiatry, 2017.

23. Martin, J., M.J. Taylor, and P. Lichtenstein, Assessing the evidence for shared genetic risks across psychiatric disorders and traits. Psychological Medicine, 2017: p. 1-16.

24. Allardyce, J., et al., Association Between Schizophrenia-Related Polygenic Liability and the Occurrence and Level of Mood-Incongruent Psychotic Symptoms in Bipolar Disorder. JAMA Psychiatry, 2018. 75(1): p. 28-35.

25. Gandal, M.J., et al., Shared molecular neuropathology across major psychiatric disorders parallels polygenic overlap. Science, 2018. 359(6376): p. 693-697.

26. The Brainstorm Consortium, C., Analysis of shared heritability in common disorders of the brain. Science, 2018. 360(6395).

27. Reininghaus, U., et al., Evaluation of the validity and utility of a transdiagnostic psychosis dimension encompassing schizophrenia and bipolar disorder. British Journal of Psychiatry, 2016. 209(2): p. 107-13.

28. Reininghaus, U., S. Priebe, and R.P. Bentall, Testing the psychopathology of psychosis: evidence for a general psychosis dimension. Schizophrenia Bulletin, 2013. 39(4): p. 884-95.

29. Shevlin, M., et al., The Psychosis Continuum: Testing a Bifactor Model of Psychosis in a General Population Sample. Schizophrenia Bulletin, 2017. 43(1): p. 133-141.

30. Howes, O.D. and R.M. Murray, Schizophrenia: an integrated sociodevelopmentalcognitive model. Lancet, 2014. 383(9929): p. 1677-1687.

31. Howes, O.D., et al., The Role of Genes, Stress, and Dopamine in the Development of Schizophrenia. Biological Psychiatry, 2017. 81(1): p. 9-20.

32. van Os, J., G. Kenis, and B.P. Rutten, The environment and schizophrenia. Nature, 2010. 468(7321): p. 203-12. 
33. Garety, P.A., et al., A cognitive model of the positive symptoms of psychosis. Psychological Medicine, 2001. 31(2): p. 189-95.

34. Dudley, R., et al., Psychosis, Delusions and the "Jumping to Conclusions" Reasoning Bias: A Systematic Review and Meta-analysis. Schizophrenia Bulletin, 2016. 42(3): p. 652-65.

35. Fine, C., et al., Hopping, skipping or jumping to conclusions? Clarifying the role of the JTC bias in delusions. Cognitive Neuropsychiatry, 2007. 12(1): p. 46-77.

36. Ross, R.M., et al., Jumping to Conclusions About the Beads Task? A Meta-analysis of Delusional Ideation and Data-Gathering. Schizophrenia Bulletin, 2015. 41(5): p. 1183-91.

37. So, S.H., et al., 'Jumping to conclusions' data-gathering bias in psychosis and other psychiatric disorders - Two meta-analyses of comparisons between patients and healthy individuals. Clinical Psychology Review, 2016. 46: p. 151-67.

38. Freeman, D., Persecutory delusions: a cognitive perspective on understanding and treatment. Lancet Psychiatry, 2016. 3(7): p. 685-92.

39. Garety, P.A. and D. Freeman, Cognitive approaches to delusions: A critical review of theories and evidence. British Journal of Clinical Psychology, 1999. 38(2): p. 113-154.

40. Freeman, D. and P. Garety, Advances in understanding and treating persecutory delusions: a review. Social Psychiatry and Psychiatric Epidemiology, 2014. 49(8): p. 117989.

41. Weinberger, D.R., The pathogenesis of schizophrenia: a neurodevelopmental theory. The Neurology of Schizophrenia. 1986: Elsevier.

42. Murray, R.M. and S.W. Lewis, Is schizophrenia a neurodevelopmental disorder? 1988.

43. Barch, D.M. and J.M. Sheffield, Cognitive impairments in psychotic disorders: common mechanisms and measurement. World Psychiatry, 2014. 13(3): p. 224-32.

44. Mollon, J., et al., Psychotic Experiences and Neuropsychological Functioning in a Population-based Sample. JAMA Psychiatry, 2016. 73(2): p. 129-38.

45. Wood, S.J., et al., Spatial working memory ability is a marker of risk-for-psychosis. Psychological Medicine, 2003. 33(7): p. 1239-1247.

46. Brewer, W.J., et al., Memory impairments identified in people at ultra-high risk for psychosis who later develop first-episode psychosis. American Journal of Psychiatry, 2005. 162(1): p. 71-8.

47. Lee, J. and S. Park, Working memory impairments in schizophrenia: a meta-analysis. Journal of Abnormal Psychology, 2005. 114(4): p. 599-611.

48. White, T., M. Schmidt, and C. Karatekin, Verbal and visuospatial working memory development and deficits in children and adolescents with schizophrenia. Early Intervention in Psychiatry, 2010. 4(4): p. 305-13.

49. Conklin, H.M., et al., Verbal working memory impairment in schizophrenia patients and their first-degree relatives: Evidence from the digit span task. American Journal of Psychiatry, 2000. 157(2): p. 275-277.

50. Forbes, N.F., et al., Working memory in schizophrenia: a meta-analysis. Psychological Medicine, 2009. 39(6): p. 889-905.

51. Mollon, J. and A. Reichenberg, Cognitive development prior to onset of psychosis. Psychological Medicine, 2018. 48(3): p. 392-403. 
52. Mollon, J., et al., Course of Cognitive Development From Infancy to Early Adulthood in the Psychosis Spectrum. JAMA Psychiatry, 2018.

53. Moran, T.P., Anxiety and working memory capacity: A meta-analysis and narrative review. Psychological Bulletin, 2016. 142(8): p. 831-64.

54. Rose, E.J. and K.P. Ebmeier, Pattern of impaired working memory during major depression. Journal of Affective Disorders, 2006. 90(2-3): p. 149-61.

55. McGrath, J., B. Chapple, and M. Wright, Working memory in schizophrenia and mania: correlation with symptoms during the acute and subacute phases. Acta Psychiatrica Scandinavica, 2001. 103(3): p. 181-8.

56. Larson, E.R., et al., Working memory and inhibitory control among manic and euthymic patients with bipolar disorder. Journal of the International Neuropsychological Society, 2005. 11(02).

57. Walsh, N.D., et al., A longitudinal functional magnetic resonance imaging study of verbal working memory in depression after antidepressant therapy. Biological Psychiatry, 2007. 62(11): p. 1236-43.

58. Scult, M.A., et al., The association between cognitive function and subsequent depression: a systematic review and meta-analysis. Psychological Medicine, 2017. 47(1): p. 1-17.

59. Alonso, J., et al., Sampling and methods of the European Study of the Epidemiology of Mental Disorders (ESEMeD) project. Acta Psychiatrica Scandinavica 2004(420): p. 8-20.

60. Kessler, R.C. and T.B. Ustun, The World Mental Health (WMH) Survey Initiative Version of the World Health Organization (WHO) Composite International Diagnostic Interview (CIDI). Int J Methods Psychiatr Res, 2004. 13(2): p. 93-121.

61. de Graaf, R., Ormel, J., ten Have, M., Burger, H. and Buist-Bouwman, M., Mental disorders and service use in the Netherlands. Results from the European Study of the Epidemiology of Mental Disorders (ESEMeD). The WHO World Mental Health Surveys: global perspectives on the epidemiology of mental disorders. 2008.

62. de Graaf, R., M. Ten Have, and S. van Dorsselaer, The Netherlands Mental Health Survey and Incidence Study-2 (NEMESIS-2): design and methods. International Journal of Methods in Psychiatric Research, 2010. 19(3): p. 125-41.

63. de Graaf, R., et al., Sociodemographic and psychiatric predictors of attrition in a prospective psychiatric epidemiological study among the general population. Result of the Netherlands Mental Health Survey and Incidence Study-2. Comprehensive Psychiatry, 2013. 54(8): p. 1131-9.

64. de Graaf, R., van Dorsselaer, S., Tuithof, M. and ten Have, M., Sociodemographic and psychiatric predictors of attrition in the third wave of the Netherlands Mental Health Survey and Incidence Study-2 (NEMESIS-2). 2015, Utrecht: Trimbos-instituut.

65. de Graaf, R., et al., Prevalence of mental disorders and trends from 1996 to 2009. Results from the Netherlands Mental Health Survey and Incidence Study-2. Social Psychiatry and Psychiatric Epidemiology, 2012. 47(2): p. 203-13.

66. Wechsler, D., WAIS-III: Wechsler Adult Intelligence Scale (3rd ed.) Administration and Scoring Manual. 1997, San Antonio, TX: Psychological Corporation.

67. Wechsler, D., WAIS-III, Nederlandse bewerking: Technische handleiding. 2000: Lisse: Swets \& Zeitlinger B.V. 
68. Phillips, L.D. and W. Edwards, Conservatism in a simple probability inference task. Journal of Experimental Psychology, 1966. 72(3): p. 346-54.

69. Andrews, G. and L. Peters, The psychometric properties of the Composite International Diagnostic Interview. Social Psychiatry and Psychiatric Epidemiology, 1998. 33(2): p. 808.

70. StataCorp. 2013, StataCorp LP: College Station, TX.

71. So, S.H., et al., Jumping to conclusions, a lack of belief flexibility and delusional conviction in psychosis: a longitudinal investigation of the structure, frequency, and relatedness of reasoning biases. Journal of Abnormal Psychology, 2012. 121(1): p. 129-39.

72. Catalan, A., et al., Data Gathering Bias: Trait Vulnerability to Psychotic Symptoms? PLoS One, 2015. 10(7): p. e0132442.

73. Eriksson, J., et al., Neurocognitive Architecture of Working Memory. Neuron, 2015. 88(1): p. 33-46.

74. Gonzalez, L.E., et al., Neuropsychological functioning and jumping to conclusions in recent onset psychosis patients. Schizophrenia Research, 2017.

75. Garety, P., et al., Neuropsychological functioning and jumping to conclusions in delusions. Schizophrenia Research, 2013. 150(2-3): p. 570-4.

76. Falcone, M.A., et al., Jumping to conclusions, neuropsychological functioning, and delusional beliefs in first episode psychosis. Schizophrenia Bulletin, 2015. 41(2): p. 4118.

77. Freeman, D., et al., Understanding jumping to conclusions in patients with persecutory delusions: working memory and intolerance of uncertainty. Psychological Medicine, 2014. 44(14): p. 3017-24.

78. Shanmugan, S., et al., Common and Dissociable Mechanisms of Executive System Dysfunction Across Psychiatric Disorders in Youth. American Journal of Psychiatry, 2016. 173(5): p. 517-26.

79. McGrath, L.M., et al., Extending the 'cross-disorder' relevance of executive functions to dimensional neuropsychiatric traits in youth. Journal of Child Psychology and Psychiatry, 2016. 57(4): p. 462-71.

80. Millan, M.J., et al., Cognitive dysfunction in psychiatric disorders: characteristics, causes and the quest for improved therapy. Nature Reviews Drug Discovery, 2012. 11(2): p. $141-68$.

81. White, L.K., et al., An Evaluation of the Specificity of Executive Function Impairment in Developmental Psychopathology. Journal of the American Academy of Child \& Adolescent Psychiatry, 2017. 56(11): p. 975-982 e3.

82. Kotov, R., et al., The Hierarchical Taxonomy of Psychopathology (HiTOP): A dimensional alternative to traditional nosologies. Journal of Abnormal Psychology, 2017. 126(4): p. 454-477.

83. Caspi, A., et al., The p Factor: One General Psychopathology Factor in the Structure of Psychiatric Disorders? Clinical Psychological Science, 2014. 2(2): p. 119-137.

84. Insel, T., et al., Research domain criteria (RDoC): toward a new classification framework for research on mental disorders. American Journal of Psychiatry, 2010. 167(7): p. 74851. 
85. Moritz, S., et al., Metacognition-augmented cognitive remediation training reduces jumping to conclusions and overconfidence but not neurocognitive deficits in psychosis. Frontiers in Psychology, 2015. 6: p. 1048.

86. Waller, H., et al., Targeting reasoning biases in delusions: a pilot study of the Maudsley Review Training Programme for individuals with persistent, high conviction delusions. Journal of Behavior Therapy and Experimental Psychiatry, 2011. 42(3): p. 414-21.

87. Garety, P.A., et al., SlowMo, a digital therapy targeting reasoning in paranoia, versus treatment as usual in the treatment of people who fear harm from others: study protocol for a randomised controlled trial. Trials, 2017. 18(1): p. 510.

88. Ross, K., et al., A randomized experimental investigation of reasoning training for people with delusions. Schizophrenia Bulletin, 2011. 37(2): p. 324-33.

89. Reininghaus, U., C.A. Depp, and I. Myin-Germeys, Ecological Interventionist Causal Models in Psychosis: Targeting Psychological Mechanisms in Daily Life. Schizophrenia Bulletin, 2016. 42(2): p. 264-9. 


\section{Supplementary material}

Table S1. Items used to measure anxiety, depression, mania, and psychotic experiences

\begin{tabular}{|c|c|}
\hline \multicolumn{2}{|c|}{ Psychopathological domain } \\
\hline \multicolumn{2}{|c|}{$\begin{array}{l}\text { Anxiety } \\
\text { (CIDI 3.0) }\end{array}$} \\
\hline Item 1 & $\begin{array}{l}\text { SC20 "Have you ever in your life had an attack of fear or panic when all } \\
\text { of a sudden you felt very frightened, anxious, or uneasy?" }\end{array}$ \\
\hline Item 2 & $\begin{array}{l}\text { SC20a "Have you ever had an attack when all of a sudden you became } \\
\text { very uncomfortable, either became short of breath, dizzy, nauseous, or } \\
\text { your heart pounded, or you thought that you might lose control, die, or } \\
\text { go crazy?" }\end{array}$ \\
\hline Item 3 & $\begin{array}{l}\text { SC26a "Did you ever have a time in your life when you were a "worrier" } \\
\text { - that is, when you worried a lot more about things than other people } \\
\text { with the same problems as you?" }\end{array}$ \\
\hline Item 4 & $\begin{array}{l}\text { SC26a "Did you ever have a time in your life when you were much } \\
\text { more nervous or anxious than most other people with the same } \\
\text { problems as you?" } \\
\text { SC26ba "Did you ever have a period lasting one month or longer when } \\
\text { you were anxious and worried most days?" }\end{array}$ \\
\hline Item 5 & $\begin{array}{l}\text { SC29a "Was there ever a time in your life when you felt very afraid or } \\
\text { really, really shy with people, like meeting new people, going to parties, } \\
\text { going on a date, or using a public bathroom?" }\end{array}$ \\
\hline
\end{tabular}

Depression

(CIDI 3.0)

Item $1 \quad \mathrm{SC21}$ a "Have you ever in your life had a period lasting several days or longer when most of the day you felt sad, empty or depressed?"

Item $2 \quad S C 22^{a}$ "Have you ever had a period lasting several days or longer when most of the day you were very discouraged about how things were going in your life?"

Item $3 \quad$ SC23a "Have you ever had a period lasting several days or longer when you lost interest in most things you usually enjoy like work, hobbies, and personal relationships?"

Mania

(CIDI 3.0)

Item 1 SC24 "Have you ever had a period lasting four days or longer when you became so happy or excited that you either got into trouble, people worried about you, or a doctor said you were manic?"

Item 2 SC25 "Have you ever had a period lasting four days or longer when most of the time you were very irritable, grumpy, or in a bad mood?" 
Table S1. (continued) Items used to measure anxiety, depression, mania, and psychotic experiences

\begin{tabular}{ll}
\hline Item 1 & "people were spying on you" \\
Item 2 & "people were following you" \\
Item 3 & "you were secretly being tested on" \\
Item 4 & "someone was conspiring against you" \\
Item 5 & "a 'double' had taken the place of a loved one" \\
Item 6 & "someone was reading your mind" \\
Item 7 & "you could hear the thoughts of others" \\
Item 8 & "others could hear your thoughts" \\
Item 9 & "alien thoughts were placed in your head" \\
Item 10 & "someone took thoughts from your head" \\
Item 11 & "special messages were sent to you through media" \\
Item 12 & "you were influenced by strange energies" \\
Item 13 & "you were being controlled by an outer force" \\
Item 14 & "your thoughts were being influenced by machines" \\
Item 15 & "any other delusion reported by subject" \\
Item 16 & "you saw things that no one else could see" \\
Item 17 & "you could hear things that no one else could hear" \\
Item 18 & "your own thoughts were broadcasted" \\
Item 19 & "you smelled strange things, that others could not smell" \\
Item 20 & "you had strange sensations, like being touched when no one was \\
\hline
\end{tabular}

Note: CIDI, Composite International Diagnostic Interview

${ }^{a}$ denotations are based on screening items of the CIDI Version 3.0 


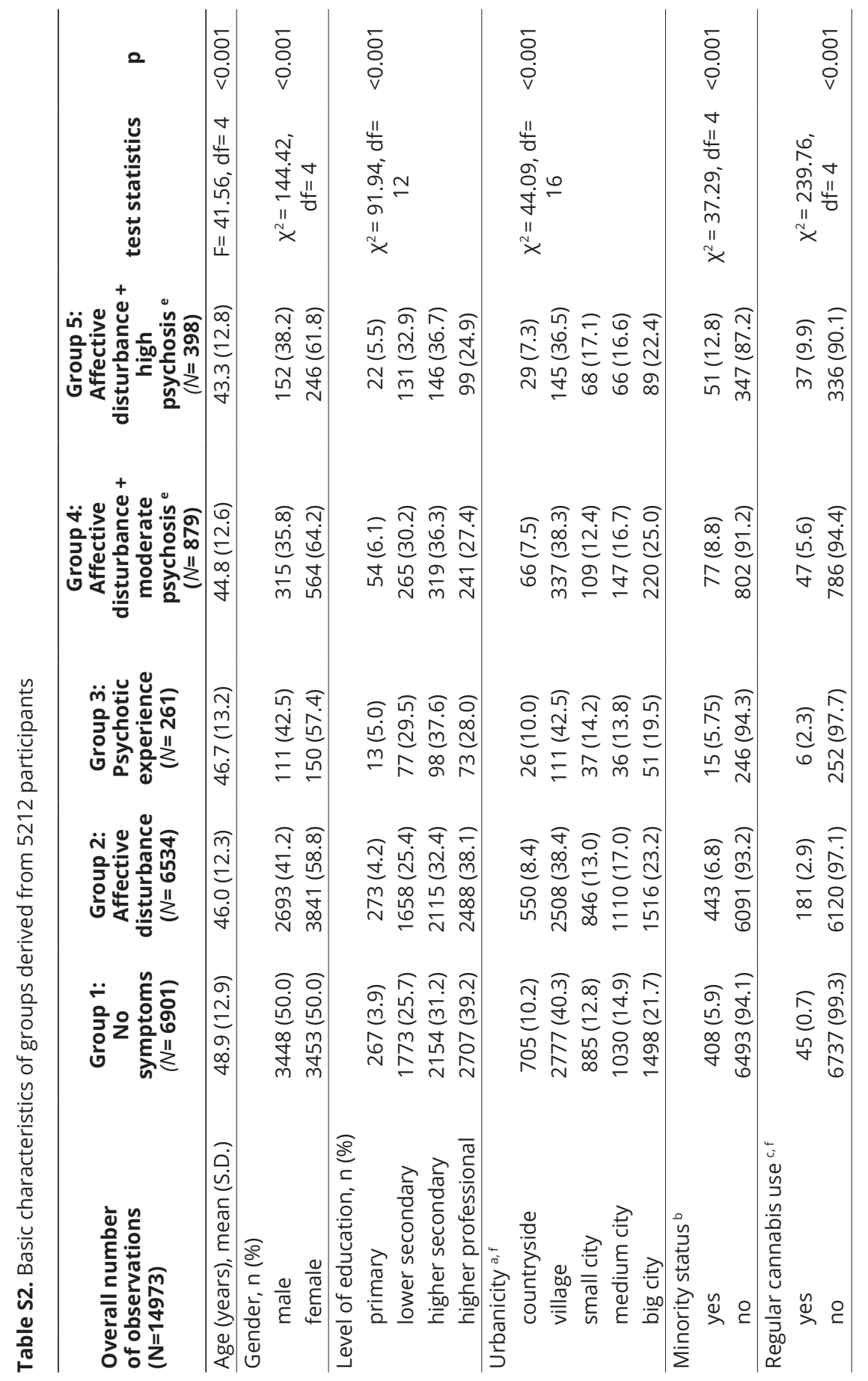




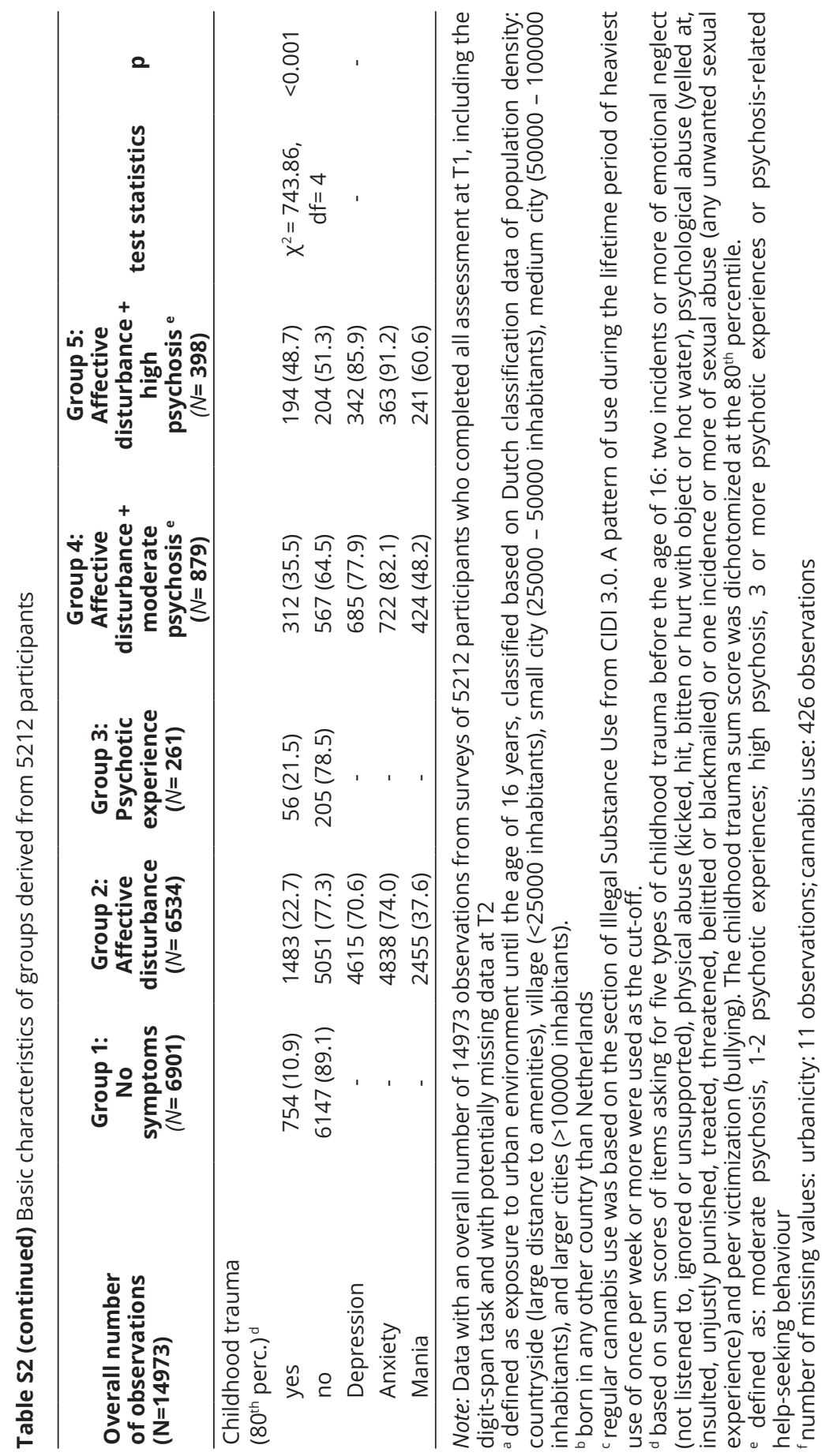


Table S3. Model fit statistics for multinomial logistic regression models

\begin{tabular}{ccccc}
\hline & df & LL & AIC & BIC \\
\hline Models regarding JTC bias & & & & \\
Model 1 & 8 & -13734.56 & 27485.12 & 27545.03 \\
Model 2 & 20 & -13515.73 & 27071.46 & 27221.23 \\
Model 3 & 36 & -13177.58 & 26427.17 & 26696.75 \\
Model 4 & 40 & -13152.55 & 26385.11 & 26684.65 \\
\hline Models regarding WMP & & & & \\
Model 1 & 8 & -15196.59 & 30409.18 & 30469.86 \\
Model 2 & 20 & -14961.39 & 29962.78 & 30114.46 \\
Model 3 & 36 & -14567.11 & 29206.21 & 29479.25 \\
\hline
\end{tabular}

Note: df, degrees of freedom; LL= Log-likelihood; AIC, Akaike Information Criterion; BIC, Bayesian Information Criterion. 



\title{
CHAPTER 3
}

\author{
The jumping to conclusions \\ reasoning bias as a cognitive \\ factor contributing to psychosis \\ progression and persistence: \\ findings from NEMESIS-2
}




\section{Abstract}

Background: Contemporary models of psychosis implicate the importance of affective dysregulation and cognitive factors (e.g. biases and schemas) in the development and maintenance of psychotic symptoms, but studies testing proposed mechanisms remain limited. This study, uniquely using a prospective design, investigated whether the jumping to conclusions (JTC) reasoning bias contributes to psychosis progression and persistence.

Methods: Data were derived from the second Netherlands Mental Health Survey and Incidence Study (NEMESIS-2). The Composite International Diagnostic Interview and an add-on instrument were used to assess affective dysregulation (i.e. depression, anxiety and mania) and psychotic experiences (PEs), respectively. The beads task was used to assess JTC bias. Time series analyses were conducted using data from T1 and T2 ( $\mathrm{N}=8666)$, excluding individuals who reported high psychosis levels at TO.

Results: Although the prospective design resulted in low statistical power, the findings suggest that, compared to those without symptoms, individuals with lifetime affective dysregulation were more likely to progress from low/moderate psychosis levels (state of 'aberrant salience', one or two PEs) at T1 to high psychosis levels ('frank psychosis', three or more PEs or psychosis-related help-seeking behaviour) at T2 if the JTC bias was present [adj. relative risk ratio (RRR): 3.8, 95\% confidence interval $(\mathrm{Cl}) 0.8-18.6, p=0.101]$. Similarly, the JTC bias contributed to the persistence of high psychosis levels (adj. RRR: $12.7,95 \% \mathrm{Cl} 0.7-239.6, \mathrm{p}=$ 0.091).

Conclusions: We found some evidence that the JTC bias may contribute to psychosis progression and persistence in individuals with affective dysregulation. However, well-powered prospective studies are needed to replicate these findings.

Keywords: Cognitive models; jumping to conclusions; persistence; progression; psychosis; psychotic experiences; reasoning bias; transdiagnostic phenotype 


\section{Introduction}

Psychosis spectrum disorders have a complex aetiology and multifaceted phenomenology. Psychotic experiences (PEs), the attenuated subclinical expression of positive psychotic symptoms, are common, with an estimated lifetime prevalence ranging from $5 \%$ to $8 \%$ [1]. PEs are often preceded by, or co-occur with, affective dysregulation (e.g. depression, anxiety) [2], which is in accordance with clinical observations of frequent comorbidity of affective disorders with psychotic disorders as well as substantially overlapping genetic liability [3]. These findings have been taken to suggest a transdiagnostic and extended psychosis phenotype with temporal and phenomenological continuity across developmental stages of psychotic and affective disorders [1]. Importantly, however, PEs are often transitory and neither inherently distressing nor inevitably impairing [4, 5], but persist in around 20\% [6]. Of these, approximately $7 \%$ develop a psychotic disorder [6]. Consequently, there has been an increasing interest in identifying clinically relevant predictors for illness onset [7-9].

Contemporary models of psychosis implicate the importance of cognitive factors (e.g. schemas, biases) in illness trajectories by contributing to transforming experiences of aberrant salience into frank psychosis as well as symptom persistence $[10,11]$. Specifically, individuals' appraisal of, and response to [1214], these excessively vivid and intense experiences, which may be mediated by dysregulated dopaminergic signalling $[10,15]$, are thought to be integral for the development of psychosis-related distress and impairment [11]. The interpretation of experiences as threatening and of external causation [10,11, 16, 17], combined with altered behaviour and cognitive responses, has been shown to be associated with psychosis spectrum disorders [14, 18].

The jumping to conclusions (JTC) reasoning bias is among the most widely studied cognitive biases in psychosis and describes individuals' tendency to make hasty decisions based on insufficient information [19]. It has been consistently found to be associated with subclinical and clinical psychosis [19-22] as well as a transdiagnostic phenotype of co-occurring affective dysregulation and PEs [23], but not with non-psychotic disorders [22]. Thus, the JTC bias may be an important cognitive factor involved in the formation and maintenance of psychosis, especially delusional ideations [18, 24], with some recent meta-analytical evidence 
supporting this assumption [19]. Critically, most studies have investigated associations of JTC bias with psychosis using cross-sectional designs [22] and evidence on proposed specificity of JTC bias with psychosis in individuals with a transdiagnostic phenotype of mental health problems remains very limited [23].

Longitudinal studies investigating the role of JTC bias in psychosis are rare. These studies suggest that JTC bias may be i) predictive for less improvement of psychotic symptoms over time [25], ii) stable despite symptom remission [26, 27], iii) associated with poorer outcomes in people with psychosis [28] as well as antipsychotic treatment response [29], and iv) associated with worse vocational functioning [30]. To our knowledge, no study has directly and prospectively tested the contribution and specificity of JTC bias to psychosis progression and persistence in individuals with co-occurring affective dysregulation, as proposed in recent integrative sociodevelopmental-cognitive models [10].

\section{Aims and hypotheses}

For the present study, we aimed to extend previous research by investigating whether the JTC bias contributes to psychosis progression and persistence in the general population. Specifically, the following hypotheses were tested: First, compared to individuals with neither affective dysregulation nor PEs, the JTC bias is associated with an increased risk to progress from low/moderate psychosis levels (i.e. one or two PEs; hereafter 'state of aberrant salience') at T1 to high psychosis levels (i.e. three or more PEs or psychosis-related help-seeking behaviour; hereafter 'frank psychosis') at T2 in individuals with co-occurring lifetime affective dysregulation. Second, the JTC bias does not contribute to firstoccurrence of frank psychosis at T2 when individuals report sole presence of affective dysregulation at T1. Third, the JTC bias is associated with an increased risk to report persistence of frank psychosis between T1 and T2 in individuals with lifetime affective dysregulation.

\section{Materials and methods}

\section{Sample}

Data were obtained from the second Netherlands Mental Health Survey and Incidence Study (NEMESIS-2), a nationally representative cohort study designed to investigate the incidence, prevalence, course, and outcome of mental disorders. 
For current analyses, we used data of the first three waves (T0-T2), while, in the meantime, the fourth wave (T3) has been completed. A multistage, stratified random sampling of households was applied to ensure representativeness of the sample in terms of age, region, and population density. First, a random sample of 184 of 443 municipalities was drawn, which were stratified by four regions (north, east, south, west) and five levels of population density. The four largest cities (i.e., Amsterdam, Rotterdam, The Hague, Utrecht) were also included. Thus, twentyfour strata were used to stratify the sample. The number of addresses selected per municipality was based on the distribution of the number of inhabitants aged 18-64 years. Secondly, a random sample of households was drawn from these addresses with the same probability to be selected. Finally, an individual aged 18-64 years was included based on the most recent birthday at first contact. The face-to-face interviews were performed at home by trained interviewers, who were not clinicians, using a laptop computer. The Composite International Diagnostic Interview (CIDI) version 3.0 [31] and additional questionnaires were used. The inclusion criterion was: aged 18-65 years. Exclusion criterion was insufficient command of the Dutch language. The first wave (T0) was conducted from November 2007 to July 2009 and enrolled 6646 participants (response rate $65 \cdot 1 \%)$. Although younger subjects were slightly underrepresented, this sample was representative for the Dutch general population [32]. In the second wave (T1), carried out from November 2010 to June 2012, all respondents were approached, and, of these, 5303 individuals were interviewed again (response rate $80.4 \%$ from T0 with those deceased excluded). From November 2013 to June 2015, the third wave (T2) was completed with 4618 persons who were interviewed a third time (response rate $87 \cdot 8 \%$ from T1 with those deceased excluded). Psychopathology reported at T0 (baseline) reflects lifetime prevalence, while symptoms reported at T1 and T2 reflect 3-year interval occurrence (i.e. between T0 and T1 and T1-T2, respectively). Data from T1 and T2 were used for the current study. The Medical Ethics Review Committee for Institutions of Mental Health Care approved the study. More details on the study are provided elsewhere [32].

\section{Data collection}

\section{Sociodemographic characteristics and cognitive alterations}

Data on age, sex, and level of education were assessed in the additional questionnaire. The digit-span task, based on the Wechsler Adult Intelligence Scale 
(WAIS-III) [33], was used to assess working memory performance as a proxy for cognitive alterations.

\section{JTC bias}

The beads task, a probabilistic reasoning task, was completed at the third wave (T2) to assess the presence or absence of JTC bias. The beads task is designed to measure individuals' reasoning style under ambiguous conditions [34]. During the task, individuals were shown two jars with red and blue coloured beads in opposite ratios (i.e. 60 to 40 beads). The jars as well as all instructions were presented on a laptop screen. After a training session, participants were informed that the beads will be drawn consecutively from one jar and, once shown, returned to the same jar. After each draw, participants were asked whether they want to decide from which of the two jars the beads were drawn or if they prefer to see another bead. Although not communicated, participants were allowed to see up to 20 beads before they had to decide. The order of beads was fixed and the colour shown in the training session selected at random. The number of beads drawn at T2 was considered to represent individuals' trait reasoning style. Based on previous studies [19], the presence of JTC bias was defined as making a decision based on two or fewer beads [22].

\section{Affective dysregulation}

Affective dysregulation was assessed at all three timepoints using core items of the CIDI version 3.0. The CIDI measure for core symptoms uses a true-false response format to screen for the prevalence of various mental disorders, including depressive episode, social phobia, generalised anxiety disorder, and manic episode (e.g. feeling fearful, depressed, experiences of a panic attack). For current analyses, a variable was constructed that combines reported affective symptoms (i.e., depression, anxiety, and mania) from all assessment points (T0T2). Thus, the variable affective dysregulation represents both lifetime prevalence (T0) as well as interval occurrence (T0-T1, T1-T2) (binary variable). All items are presented in Supplementary Table 1. 


\section{Psychotic experiences (PEs)}

As earlier studies concluded that the CIDI is not a reliable and valid measures for psychotic disorders, a psychosis measure was constructed based on the psychosis section of the CIDI 1·1. The measure consisted of 20 items asking for PEs (i.e. 15 delusional and five hallucinatory experiences). At T0, the lifetime prevalence was assessed, while at T1 and T2, individuals were asked whether PEs have occurred between assessment points (i.e. interval occurrence). In case PEs were endorsed, participants were asked to state, on a 4-point Likert scale, the frequency, distress, and the impact of PEs on their daily life, including whether they had sought help for these experiences. For current analyses, we used the number of PEs endorsed, irrespective of reported frequency, distress, and impact. Sum scores were calculated by adding reported PEs. The used items are reported in Supplementary Table 1. To determine psychosis-related help-seeking behaviour, the service assessments of the CIDI 3.0 were used.

\section{Grouping absence, presence and co-occurrence of affective dysregulation and psychotic experiences}

In line with previous work [23,35], individuals were grouped based on answers given on measures assessing depression, anxiety, and mania (summarized as affective dysregulation) and PEs. Five groups were generated representing the absence, sole presence, or co-occurrence of lifetime affective dysregulation and PEs: neither affective dysregulation nor PEs (group 1); sole presence of affective dysregulation (group 2); sole presence of PEs (group 3); affective dysregulation and aberrant salience (group 4; one or two PEs); affective dysregulation and frank psychosis (group 5; three or more PEs or psychosis-related help-seeking behaviour).

\section{Statistical analysis}

All analyses were performed using STATA version 13.1 [36]. First, individuals with frank psychosis and any affective dysregulation at T0 (lifetime prevalence) were excluded from analyses $(N=198)$, making sure incident states of frank psychosis at T1 and T2 were identifiable. Second, socio-demographic characteristics (i.e. age, sex, education level) and cognitive alterations (working-memory performance) were compared across groups using linear regression and chi-square tests as 
appropriate. Third, the MLOGIT command was used to fit multinomial logistic regression models with time-lagged variables, while correcting for clustering of data (i.e. two observations for each individual) using the CLUSTER option. In order to test whether the JTC bias contributes to psychosis progression and/ or persistence over time, reported symptoms at T1 (categorical variable with 5 levels), JTC bias (binary variable), and its interaction (psychopathological domains $x$ JTC bias) were added to the model of T2 psychopathological domains as the dependent variable. Relative risk ratios (RRRs) for symptoms progression and persistence were compared using the Wald test. All models were adjusted for various a priori defined potential confounders. First, we adjusted for sociodemographic characteristics (i.e. age, sex, level of education) and, subsequently, for cognitive alterations (i.e. working memory performance). Missing values for exposure, outcome, or covariates were assumed to be missing at random and, thus, individuals were excluded from statistical modelling, but retained for crude RRRs estimates.

\section{Results}

\section{Basic sample characteristics}

In total, the sample consisted of 4618 participants at the third wave. Of these, 4333 completed the beads task, all measures, and did not report lifetime prevalence of co-occurring affective dysregulation and frank psychosis at T0, resulting in 8666 observations combining T1 and T2 (93.8\%). There were no differences between individuals who completed the beads task and other measures and those who did not with regard to socio-demographic characteristics and cognitive alterations. The sample characteristics are presented in Table 1. As shown, individuals who reported affective dysregulation and/or PEs were slightly younger, female, less educated, and had more cognitive alterations. Group differences on variables, including exposure to various socio-environmental risk factors (e.g. childhood trauma, cannabis use, minority status, urbanicity), are reported elsewhere [23, 35] and provided in Table 1. Overall, there were considerable differences when individuals with sole presence of affective dysregulation and co-occurring affective dysregulation and PEs were compared in terms of most sample characteristics, socio-environmental risk factors, and cognitive alterations. A frequency table of reported PEs at T1 is provided in Supplementary Table 2. 


\section{JTC bias and psychosis progression and occurrence}

As shown in Table 2, compared to individuals with neither affective dysregulation nor PEs at both timepoints, those who reported affective dysregulation and a state of aberrant salience at T1 were more likely, albeit below conventional alpha, to report frank psychosis at T2 if the JTC bias was present (adj. RRR: 3·8, 95\% Cl 0.8 - 18.6, $p=0 \cdot 101$ ). In contrast, co-occurrence of affective dysregulation and frank psychosis at T2 was not influenced by JTC bias in individuals who reported sole presence of affective dysregulation at T1 (adj. RRR: $1 \cdot 3,95 \% \mathrm{Cl} 0 \cdot 4-5 \cdot 0, p=0 \cdot 659$ ). 


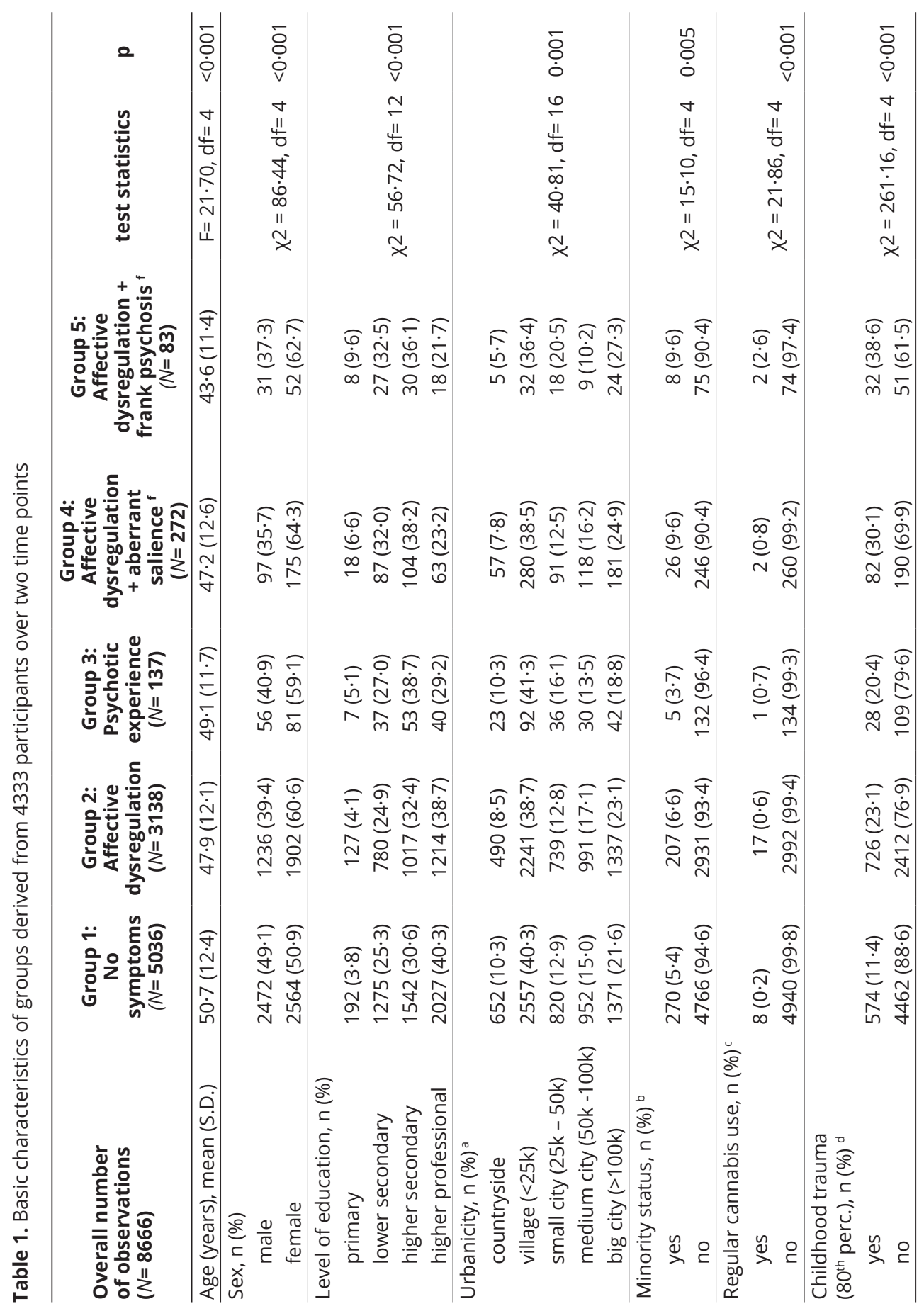




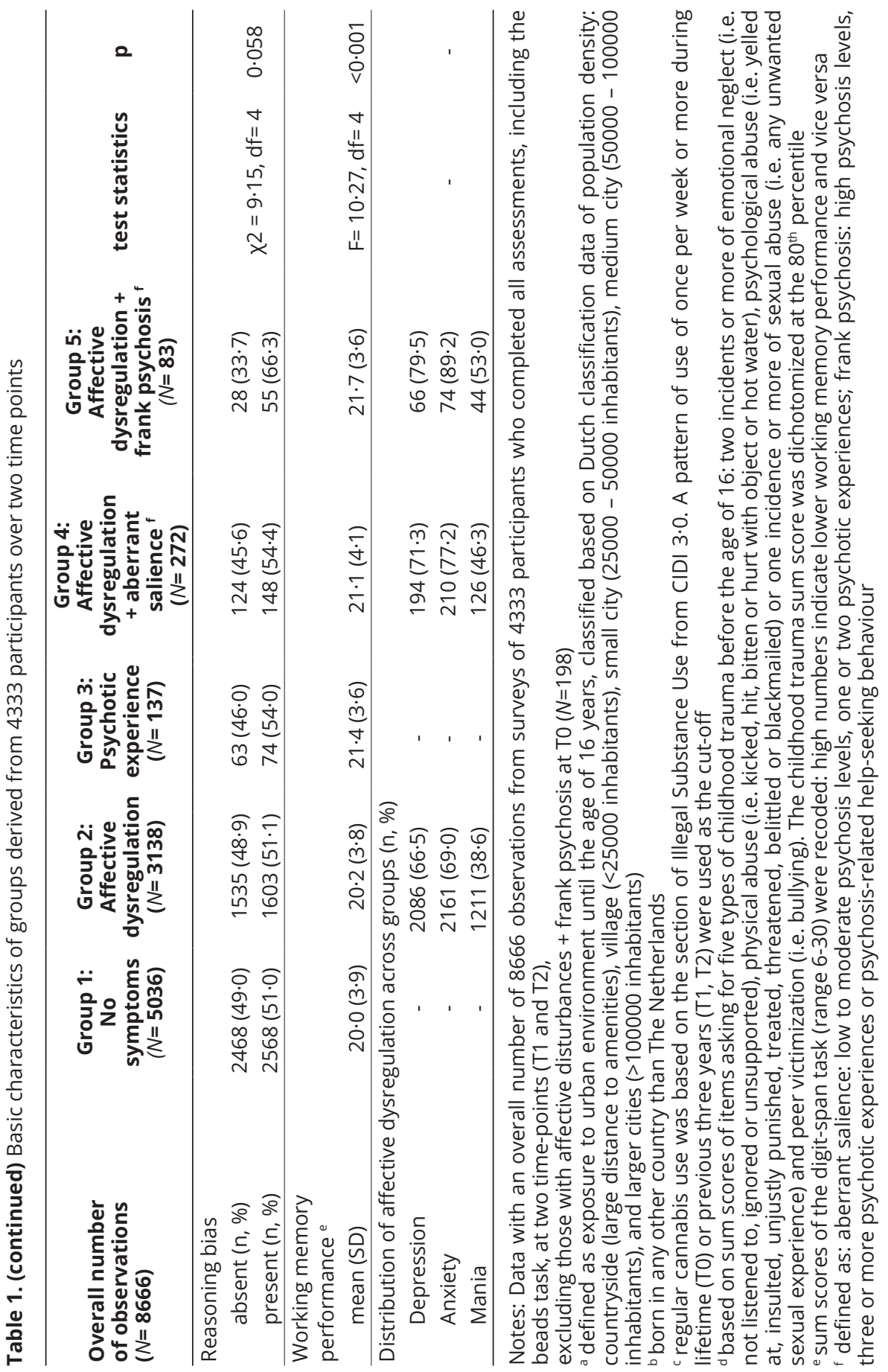




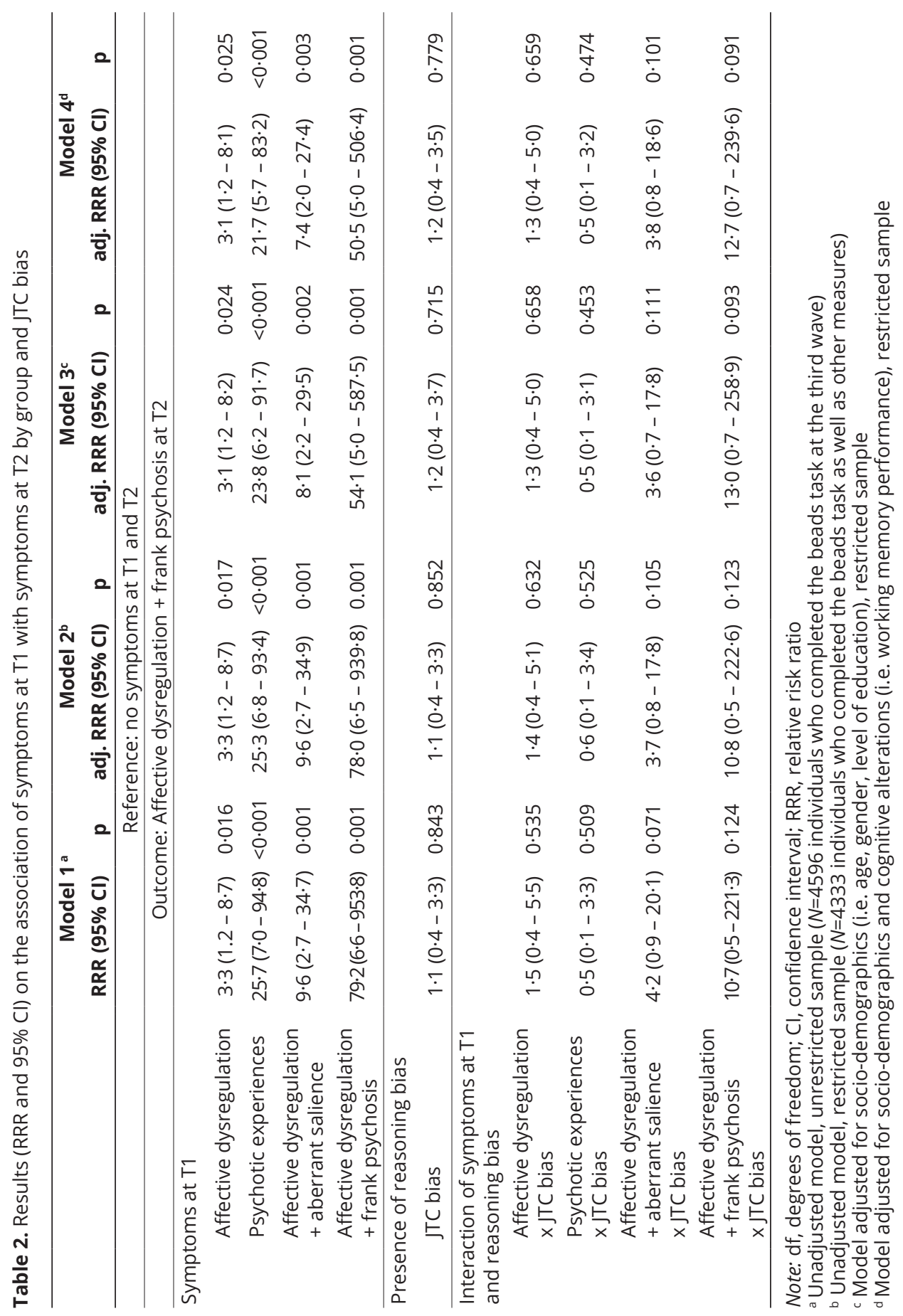




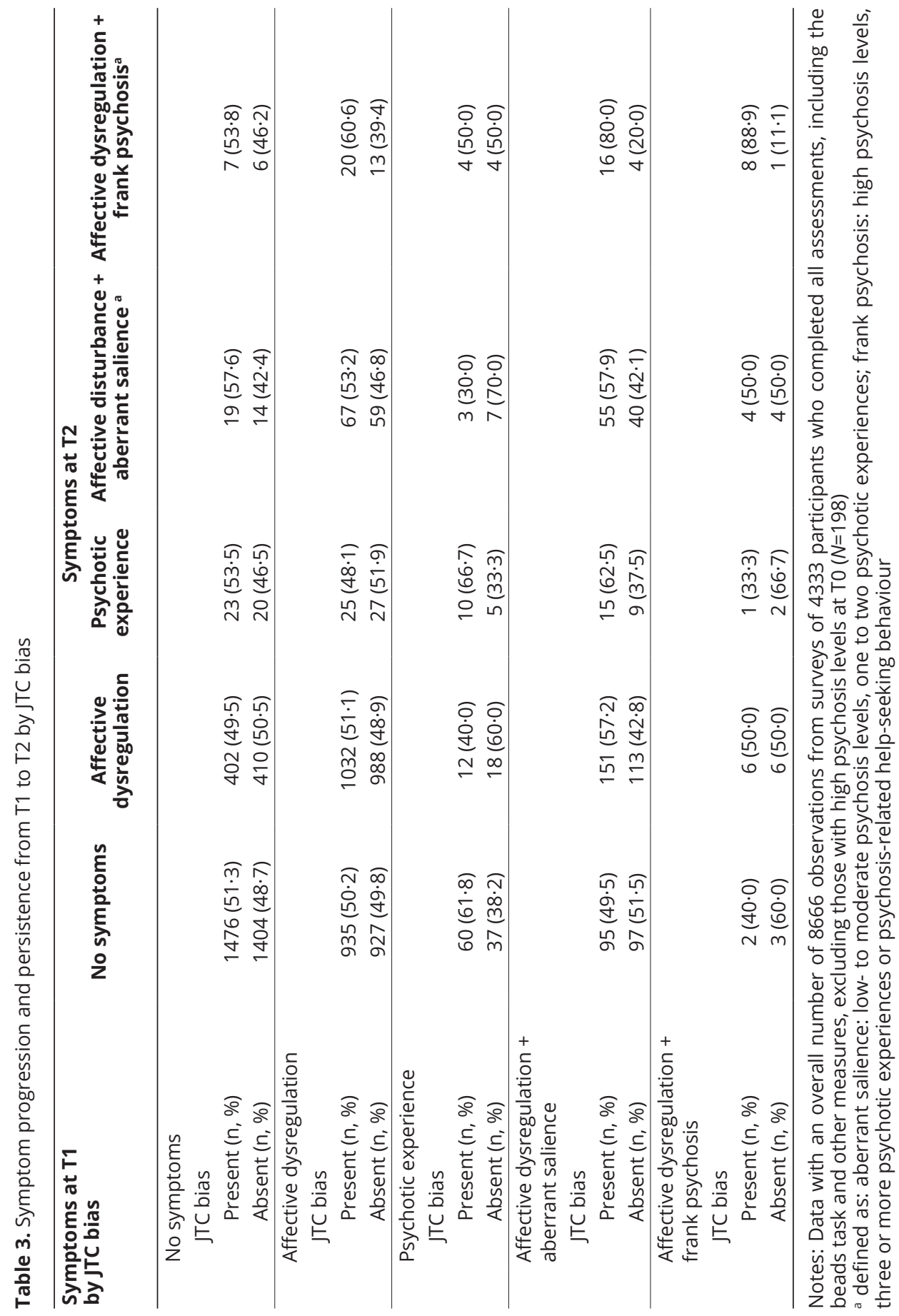




\section{JTC bias and psychosis persistence}

Presence of the JTC bias was associated, albeit below conventional alpha, with an increased risk to maintain frank psychosis at both timepoints in individuals with lifetime affective dysregulation (Table 2: adj. RRR: 12.7, 95\% Cl $0 \cdot 7-239 \cdot 6$, $p=0.091$ ). The associations of all other symptoms at T1 with symptoms at T2 by JTC bias are provided in Supplementary Table 3 and model fit statistics for multinomial logistic regression models in Supplementary Table 4.

\section{Discussion}

\section{Main findings}

This study investigated whether the JTC bias contributes to psychosis progression and persistence in a community sample. Power was low and although associations were not significant at conventional alpha, we interpret findings at the level of clinical evidence rather than arbitrary statistical cut-off, as recommended recently [37] (i.e. most people would still buy a lottery ticket if the probability of winning was $90 \%$ instead of $95 \%$ ). Thus, there was a suggestion that, compared to those who did not report any symptoms, individuals with lifetime affective dysregulation and a state of aberrant salience at T1 were more likely to report frank psychosis at T2 if the JTC bias was present. Similarly, there was a suggestion that the JTC bias contributed to the persistence of frank psychosis over time in individuals with lifetime affective dysregulation. These associations remained largely unchanged after adjusting for demographics (age, sex, education) as well as for cognitive alterations (working memory performance). However, large prospective cohort studies are needed to replicate these findings.

\section{Methodological considerations}

The unique strength of the current study is that the largest data set on JTC-bias to date was used, for the first time, to prospectively investigate the contribution of the JTC bias on psychosis progression and persistence in a longitudinal representative cohort study. However, the following limitations should be considered before interpreting our findings. First, as presented in Table 3, the number of individuals with lifetime affective dysregulation who progressed from a state of aberrant salience at T1 to frank psychosis at T2 or who reported persistence of frank 
psychosis at both timepoints were low ( $N=20$ and $N=9$, respectively), resulting in imprecise estimates. The null hypothesis significance testing paradigm - and the p-value threshold intrinsic to it - is currently strongly debated with widely differing views [38-40]. Thus, reported findings should be considered as suggestive but not conclusive evidence. Well-powered longitudinal cohort studies are needed to replicate reported findings. However, this does not preclude, as argued above, inferring valuable insights given marked differences in the presence of JTC bias comparing respective groups (e.g. in $80 \%$ of individuals who progressed from aberrant salience at T1 to frank psychosis at T2 the JTC bias was present compared to $54 \%$ in those without symptoms at both timepoints). Second, the JTC bias was assessed only once during the study period (T2) and, thus, the presence or absence of JTC bias was conceptualized as individuals' trait reasoning style. Ideally, the beads task would have been completed at more timepoints for more robust estimates and to take potential fluctuation of reasoning style into account. However, assessment burden was considered to be too high and associated benefits too low, especially considering findings of low variability of JTC bias over time [27]. Importantly, however, an uncontrolled study including thirty-one helpseeking individuals with psychosis suggests that JTC bias may vary over time and that these changes may be associated with symptom severity [28]. Thus, more research is needed that specifically investigates stability of JTC bias and potential moderators and mediators of change. Third, although we excluded individuals with lifetime affective dysregulation and frank psychosis at T0, we did not exclude all individuals with psychosis (e.g. low psychosis levels) as resulting stratified groups were considered to be too small to test hypotheses. However, excluding those who have already progressed to high psychosis levels before the study period allowed us to investigate more accurately the role of JTC bias in psychosis progression as psychosis at T1 and T2 reflect first-time interval occurrence. Fourth, we conceptualized low to moderate levels of psychosis (i.e. individuals who endorsed one or two PEs) to represent a state of aberrant salience and high psychosis levels (i.e. individuals who endorsed three or more PEs or reported psychosis-related behaviour) to reflect frank psychosis. As individuals' level of distress and impairment were not directly considered in constructing scores, it is possible that some individuals with high psychosis levels, especially those who did not seek help from mental health services, did not experience any psychosisrelated distress while, conversely, some with low to moderate levels of psychosis 
may experience distress. This would be at variance with established definitions of anomalous experiences of aberrant salience. Thus, our operationalization of distinguishing between individuals with aberrant salience and frank psychosis should be interpreted with caution. However, again, the low number of individuals who reported the emergence of help-seeking behaviour between T0 and T1 or T1 and T2 have prevented us from using this more valid indicator for frank psychosis. Fifth, recent evidence suggests that JTC bias may be a manifestation or consequence of general cognitive impairment and may not represent a specific cognitive factor involved in psychosis progression over time [41]. Thus, adjusting for various domains of individuals' cognitive ability would have been preferable. Critically, only working memory performance has been assessed at the second wave (T1) in NEMESIS-2 and used as a proxy for cognitive deficits to minimize assessment burden. In the current study, however, controlling for working memory performance did not attenuate reported associations (see Model 3 and 4 in Table 2). Sixth, PEs investigated in the current study differed in terms of quality and type (e.g. delusional ideations and hallucinations). As there is some evidence that JTC bias may be specific to the development of delusional ideations, investigating this further in sensitivity analyses would have been important. However, after stratification by type of PEs, group sizes were too small to investigate on specificity of JTC bias in relation to delusional ideations.

\section{Comparison with previous research}

The JTC bias is among the most widely studied cognitive biases in psychosis. However, to our knowledge, no study has prospectively investigated the role of JTC bias in psychosis progression and persistence, testing dominant models of psychosis ontogenesis. The findings of the current study support the suggestion that individuals with JTC bias are more likely to progress from states of aberrant salience to frank psychosis. Thus, JTC bias may not only cross-sectionally be associated with psychosis liability, as consistently shown [19-22], but may also influence the development of more severe psychosis levels or psychosis-related help-seeking behaviour over time. Similarly, the findings support the notion that the JTC bias may contribute to the persistence of frank psychosis. These findings are in accordance with recently proposed, but rarely tested, models of psychosis which have posited the importance of cognitive factors in the development and maintenance of psychosis $[10,15]$. 
Whilst recognizing low power and statistically formally inconclusive findings, we hypothesize that if JTC bias indeed contributes to psychosis progression or persistence then, given high rates of JTC bias in individuals without any symptoms, it is likely that JTC bias adds to or combines with other genetic and socioenvironmental risk factors. For example, an individual who has been exposed to childhood trauma or developmental hazards early in life may experience otherwise irrelevant stimuli as excessively salient, whilst, concurrently, risk exposure may have provoked the development of threat beliefs about the world and others [11]. Consequently, in search for an explanation, the initially nondistressing experiences of aberrant salience may be interpreted, as a secondary process, as threatening and externally caused [11] and, subsequently, lead to more severe psychosis levels and/or the development of help-seeking behaviour. As noted previously [11], the JTC bias may be particularly important during this stage: Individuals' tendency to gather less information to draw conclusions in a standardized cognitive task may translate to real-life situations in the form of hastier decisions about the negative intentions of others and, thus, lowering the probability of processing alternative explanations which may result in stronger delusional convictions. Thus, threat beliefs of salient experiences and associated appraisal processes may be influenced by the presence of JTC bias, especially when combined with low belief flexibility [42], another well-established cognitive factor. These processes may give rise to a vicious circle of increasing psychosis severity and distress. However, this has not been directly demonstrated and should be further investigated in future studies. Also, how JTC bias is associated with the behavioural response to psychosis as well as other cognitive factors like safetyseeking, avoidance, worrying, and unhelpful emotional regulation strategies [10, $11,16]$ should be further investigated.

\section{Conclusion}

There was some evidence that the JTC bias may contribute to psychosis progression and persistence in individuals with lifetime affective dysregulation from the general population. However, large prospective studies are needed to replicate reported findings. An important next step is to further investigate the causal status of JTC bias in the development and maintenance of psychosis in order to inform promising treatment targets [18] and develop process-based treatment protocols that aim to directly manipulate reasoning bias and other cognitive factors [43]. 


\section{References}

1. van Os, J. and U. Reininghaus, Psychosis as a transdiagnostic and extended phenotype in the general population. World Psychiatry, 2016. 15(2): p. 118-24.

2. Wigman, J.T., et al., Evidence that psychotic symptoms are prevalent in disorders of anxiety and depression, impacting on illness onset, risk, and severity-implications for diagnosis and ultra-high risk research. Schizophr Bull, 2012. 38(2): p. 247-57.

3. Brainstorm, C., et al., Analysis of shared heritability in common disorders of the brain. Science, 2018. 360(6395).

4. Wusten, C., et al., Psychotic Experiences and Related Distress: A Cross-national Comparison and Network Analysis Based on 7141 Participants From 13 Countries. Schizophr Bull, 2018. 44(6): p. 1185-1194.

5. Loberg, E.M., et al., Psychosocial characteristics differentiate non-distressing and distressing voices in 10,346 adolescents. Eur Child Adolesc Psychiatry, 2019.

6. Linscott, R.J. and J. van Os, An updated and conservative systematic review and metaanalysis of epidemiological evidence on psychotic experiences in children and adults: on the pathway from proneness to persistence to dimensional expression across mental disorders. Psychol Med, 2013. 43(6): p. 1133-49.

7. Fusar-Poli, P., et al., Transdiagnostic Risk Calculator for the Automatic Detection of Individuals at Risk and the Prediction of Psychosis: Second Replication in an Independent National Health Service Trust. Schizophr Bull, 2018.

8. Hartmann, J.A., et al., At-risk studies and clinical antecedents of psychosis, bipolar disorder and depression: a scoping review in the context of clinical staging. Psychol Med, 2019. 49(2): p. 177-189.

9. Nelson, B., et al., Moving From Static to Dynamic Models of the Onset of Mental Disorder: A Review. JAMA Psychiatry, 2017. 74(5): p. 528-534.

10. Howes, O.D. and R.M. Murray, Schizophrenia: an integrated sociodevelopmentalcognitive model. Lancet, 2014. 383(9929): p. 1677-1687.

11. Freeman, D., Persecutory delusions: a cognitive perspective on understanding and treatment. Lancet Psychiatry, 2016. 3(7): p. 685-92.

12. Peters, E., et al., Clinical relevance of appraisals of persistent psychotic experiences in people with and without a need for care: an experimental study. Lancet Psychiatry, 2017. 4(12): p. 927-936.

13. Garety, P.A. and D. Freeman, Cognitive approaches to delusions: a critical review of theories and evidence. Br J Clin Psychol, 1999. 38 ( Pt 2)(2): p. 113-54.

14. Ward, T., et al., Clinical and theoretical relevance of responses to analogues of psychotic experiences in people with psychotic experiences with and without a need-for-care: an experimental study. Psychol Med, 2019: p. 1-10.

15. Howes, O.D., et al., The Role of Genes, Stress, and Dopamine in the Development of Schizophrenia. Biol Psychiatry, 2017. 81(1): p. 9-20.

16. Garety, P.A., et al., Implications for neurobiological research of cognitive models of psychosis: a theoretical paper. Psychol Med, 2007. 37(10): p. 1377-91.

17. Garety, P.A., et al., A cognitive model of the positive symptoms of psychosis. Psychol Med, 2001. 31(2): p. 189-95. 
18. Brown, P., F. Waite, and D. Freeman, 'Twisting the lion's tail': Manipulationist tests of causation for psychological mechanisms in the occurrence of delusions and hallucinations. Clin Psychol Rev, 2019. 68: p. 25-37.

19. Dudley, R., et al., Psychosis, Delusions and the "Jumping to Conclusions" Reasoning Bias: A Systematic Review and Meta-analysis. Schizophr Bull, 2016. 42(3): p. 652-65.

20. Fine, C., et al., Hopping, skipping or jumping to conclusions? Clarifying the role of the JTC bias in delusions. Cogn Neuropsychiatry, 2007. 12(1): p. 46-77.

21. Ross, R.M., et al., Jumping to Conclusions About the Beads Task? A Meta-analysis of Delusional Ideation and Data-Gathering. Schizophr Bull, 2015. 41(5): p. 1183-91.

22. So, S.H., et al., 'Jumping to conclusions' data-gathering bias in psychosis and other psychiatric disorders - Two meta-analyses of comparisons between patients and healthy individuals. Clin Psychol Rev, 2016. 46: p. 151-67.

23. Reininghaus, U., et al., Reasoning bias, working memory performance and a transdiagnostic phenotype of affective disturbances and psychotic experiences in the general population. Psychol Med, 2019. 49(11): p. 1799-1809.

24. Freeman, D. and P. Garety, Advances in understanding and treating persecutory delusions: a review. Soc Psychiatry Psychiatr Epidemiol, 2014. 49(8): p. 1179-89.

25. Rodriguez, V., et al., Jumping to conclusions at first onset of psychosis predicts longer admissions, more compulsory admissions and police involvement over the next 4 years: the GAP study. Psychol Med, 2018: p. 1-11.

26. Peters, E. and P. Garety, Cognitive functioning in delusions: a longitudinal analysis. Behav Res Ther, 2006. 44(4): p. 481-514.

27. So, S.H., et al., Jumping to conclusions, a lack of belief flexibility and delusional conviction in psychosis: a longitudinal investigation of the structure, frequency, and relatedness of reasoning biases. J Abnorm Psychol, 2012. 121(1): p. 129-139.

28. Dudley, R., et al., 'Jumping to conclusions' in first-episode psychosis: a longitudinal study. Br J Clin Psychol, 2013. 52(4): p. 380-93.

29. Menon, M., R. Mizrahi, and S. Kapur, 'Jumping to conclusions' and delusions in psychosis: relationship and response to treatment. Schizophr Res, 2008. 98(1-3): p. 225-31.

30. Andreou, C., et al., Investigation of the role of the jumping-to-conclusions bias for shortterm functional outcome in schizophrenia. Psychiatry Res, 2014. 218(3): p. 341-7.

31. Kessler, R.C. and T.B. Ustun, The World Mental Health (WMH) Survey Initiative Version of the World Health Organization (WHO) Composite International Diagnostic Interview (CIDI). Int J Methods Psychiatr Res, 2004. 13(2): p. 93-121.

32. de Graaf, R., M. Ten Have, and S. van Dorsselaer, The Netherlands Mental Health Survey and Incidence Study-2 (NEMESIS-2): design and methods. Int J Methods Psychiatr Res, 2010. 19(3): p. 125-41.

33. Wechsler, D., WAIS-III: Wechsler Adult Intelligence Scale (3rd ed.) Administration and Scoring Manual. 1997, San Antonio, TX: Psychological Corporation.

34. Phillips, L.D. and W. Edwards, Conservatism in a simple probability inference task. J Exp Psychol, 1966. 72(3): p. 346-54.

35. Radhakrishnan, R., et al., Interaction between environmental and familial affective risk impacts psychosis admixture in states of affective dysregulation. Psychol Med, 2019. 49(11): p. 1879-1889. 
36. StataCorp. 2013, StataCorp LP: College Station, TX.

37. Amrhein, V., S. Greenland, and B. McShane, Scientists rise up against statistical significance. Nature, 2019. 567(7748): p. 305-307.

38. McShane, B.B., et al., Abandon Statistical Significance. The American Statistician, 2019. 73(sup1): p. 235-245.

39. Ioannidis, J.P.A., The Proposal to Lower P Value Thresholds to .005. JAMA, 2018. 319(14): p. $1429-1430$.

40. Hurlbert, S.H., R.A. Levine, and J. Utts, Coup de Grâce for a Tough Old Bull: "Statistically Significant" Expires. The American Statistician, 2019. 73(sup1): p. 352-357.

41. Tripoli, G., et al., Jumping To Conclusions, General Intelligence, And Psychosis Liability: Findings From The Multi-Centre EU-GEl Case-Control Study. bioRxiv, 2019.

42. Ward, T., et al., Data-Gathering, Belief Flexibility, and Reasoning Across the Psychosis Continuum. Schizophr Bull, 2018. 44(1): p. 126-136.

43. Moritz, S. and T.S. Woodward, Metacognitive training in schizophrenia: from basic research to knowledge translation and intervention. Curr Opin Psychiatry, 2007. 20(6): p. 619-25. 


\section{Supplementary material}

Table S1. Items used to measure anxiety, depression, mania, and psychotic experiences

\begin{tabular}{|c|c|}
\hline \multicolumn{2}{|c|}{ Psychopathological domains } \\
\hline \multicolumn{2}{|c|}{$\begin{array}{l}\text { Anxiety } \\
(\text { CIDI 3.0) }\end{array}$} \\
\hline Item 1 & $\begin{array}{l}\text { SC20a "Have you ever in your life had an attack of fear or panic when } \\
\text { all of a sudden you felt very frightened, anxious, or uneasy?" }\end{array}$ \\
\hline Item 2 & $\begin{array}{l}\text { SC20a "Have you ever had an attack when all of a sudden you became } \\
\text { very uncomfortable, either became short of breath, dizzy, nauseous, or } \\
\text { your heart pounded, or you thought that you might lose control, die, or } \\
\text { go crazy?" }\end{array}$ \\
\hline Item 3 & $\begin{array}{l}\text { SC26a "Did you ever have a time in your life when you were a "worrier" } \\
\text { - that is, when you worried a lot more about things than other people } \\
\text { with the same problems as you?" }\end{array}$ \\
\hline Item 4 & $\begin{array}{l}\text { SC26a "Did you ever have a time in your life when you were much } \\
\text { more nervous or anxious than most other people with the same } \\
\text { problems as you?" } \\
\text { SC26ba "Did you ever have a period lasting one month or longer when } \\
\text { you were anxious and worried most days?" }\end{array}$ \\
\hline Item 5 & $\begin{array}{l}\text { SC29a "Was there ever a time in your life when you felt very afraid or } \\
\text { really, really shy with people, like meeting new people, going to parties, } \\
\text { going on a date, or using a public bathroom?" }\end{array}$ \\
\hline
\end{tabular}

\section{Depression}

(CIDI 3.0)

Item $1 \quad \mathrm{SC21}$ a "Have you ever in your life had a period lasting several days or longer when most of the day you felt sad, empty or depressed?"

Item $2 \quad$ SC22a "Have you ever had a period lasting several days or longer when most of the day you were very discouraged about how things were going in your life?"

Item 3 SC23a "Have you ever had a period lasting several days or longer when you lost interest in most things you usually enjoy like work, hobbies, and personal relationships?"

Mania

(CIDI 3.0)

Item $1 \quad$ SC24a "Have you ever had a period lasting four days or longer when you became so happy or excited that you either got into trouble, people worried about you, or a doctor said you were manic?"

Item $2 \quad \mathrm{SC25}$ "Have you ever had a period lasting four days or longer when most of the time you were very irritable, grumpy, or in a bad mood?" 
Table S1. (continued) Items used to measure anxiety, depression, mania, and psychotic experiences

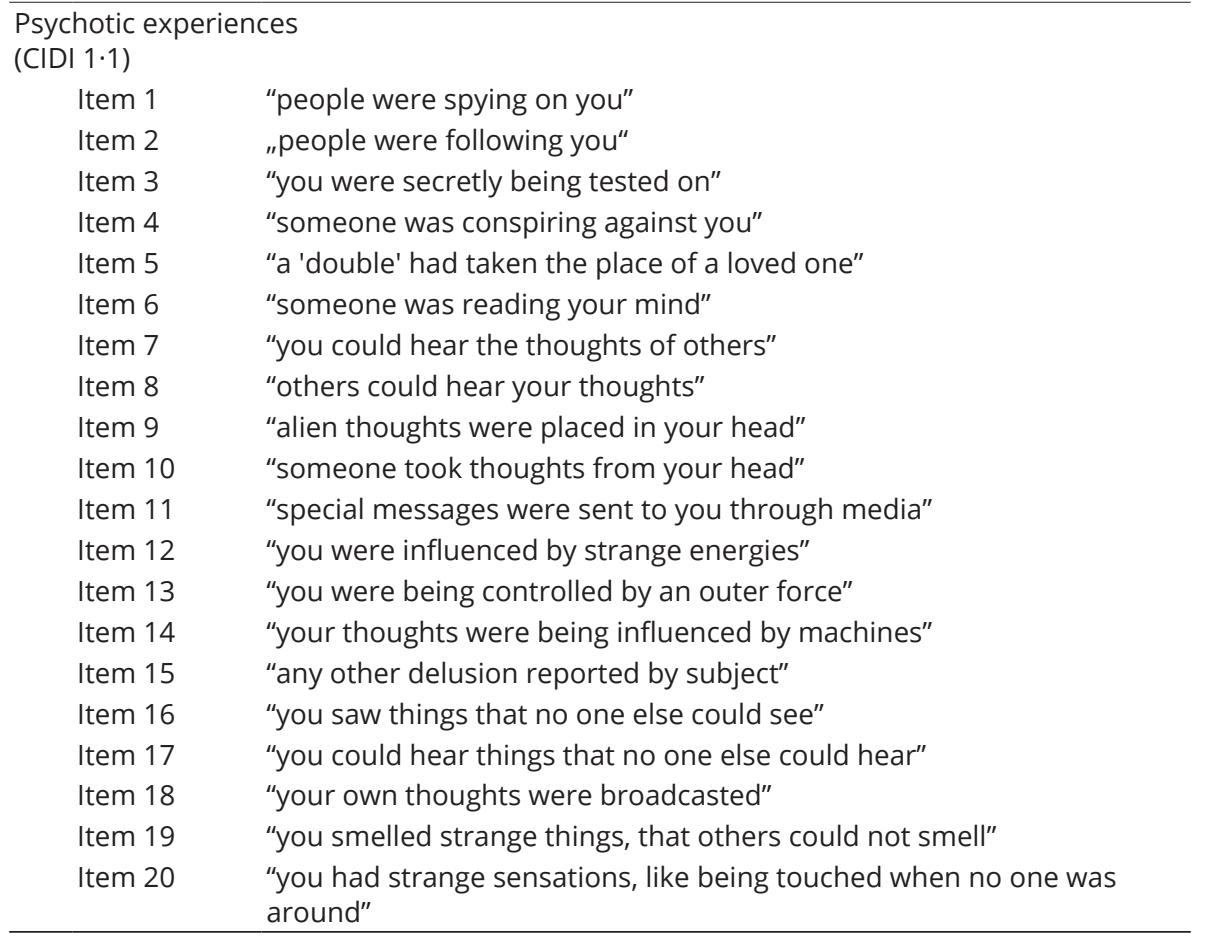

Note: Composite International Diagnostic Interview Version, CIDI

${ }^{a}$ denotations are based on screening items of the CIDI Version 3.0 
Table S2. Frequencies of reported PEs at T1

\begin{tabular}{|c|c|c|}
\hline \multicolumn{2}{|c|}{ Psychotic experiences } & \multirow[t]{2}{*}{$\begin{array}{l}\text { Frequencies at T1 } \\
(\mathrm{n}, \%)\end{array}$} \\
\hline$(\mathrm{CIDI} 1 \cdot 1)$ & & \\
\hline & \multicolumn{2}{|l|}{ "Since our previous interview,... } \\
\hline Item 1 & ...people were spying on you" & $76(1.8)$ \\
\hline Item 2 & ...people were following you" & $21(0.5)$ \\
\hline Item 3 & ...you were secretly being tested on" & $16(0.4)$ \\
\hline Item 4 & ...someone was conspiring against you" & $13(0.3)$ \\
\hline Item 5 & ...a 'double' had taken the place of a loved one" & $1(0.02)$ \\
\hline Item 6 & ...someone was reading your mind" & $18(0.4)$ \\
\hline Item 7 & ...you could hear the thoughts of others" & $31(0.7)$ \\
\hline Item 8 & ...others could hear your thoughts" & $17(0.4)$ \\
\hline Item 9 & ...alien thoughts were placed in your head" & $10(0.2)$ \\
\hline Item 10 & ...someone took thoughts from your head" & $4(0.1)$ \\
\hline Item 11 & $\begin{array}{l}\text {...special messages were sent to you through } \\
\text { media" }\end{array}$ & $5(0.1)$ \\
\hline Item 12 & ...you were influenced by strange energies" & $1(0.02)$ \\
\hline Item 13 & ...you were being controlled by an outer force" & $5(0.1)$ \\
\hline Item 14 & $\begin{array}{l}\text {...your thoughts were being influenced by } \\
\text { machines" }\end{array}$ & $8(0.2)$ \\
\hline Item 15 & ...any other delusion reported by subject" & $21(0.5)$ \\
\hline Item 16 & ...you saw things that no one else could see" & $51(1.2)$ \\
\hline Item 17 & $\begin{array}{l}\text {...you could hear things that no one else could } \\
\text { hear" }\end{array}$ & $27(0.6)$ \\
\hline Item 18 & ...your own thoughts were broadcasted" & $10(0.2)$ \\
\hline Item 19 & $\begin{array}{l}\text {...you smelled strange things, that others could } \\
\text { not smell" }\end{array}$ & $35(0.8)$ \\
\hline Item 20 & $\begin{array}{l}\text {...you had strange sensations, like being touched } \\
\text { when no one was around" }\end{array}$ & $51(1.2)$ \\
\hline
\end{tabular}

Note: Composite International Diagnostic Interview Version, CIDI

a Individuals who reported lifetime prevalence of affective dysregulation and frank psychosis (i.e., more than 3 psychotic experiences or psychosis-related help-seeking behavior) at T0 were excluded from analyses $(N=198)$. Thus, reported frequencies represent interval occurrence of psychotic experiences. 


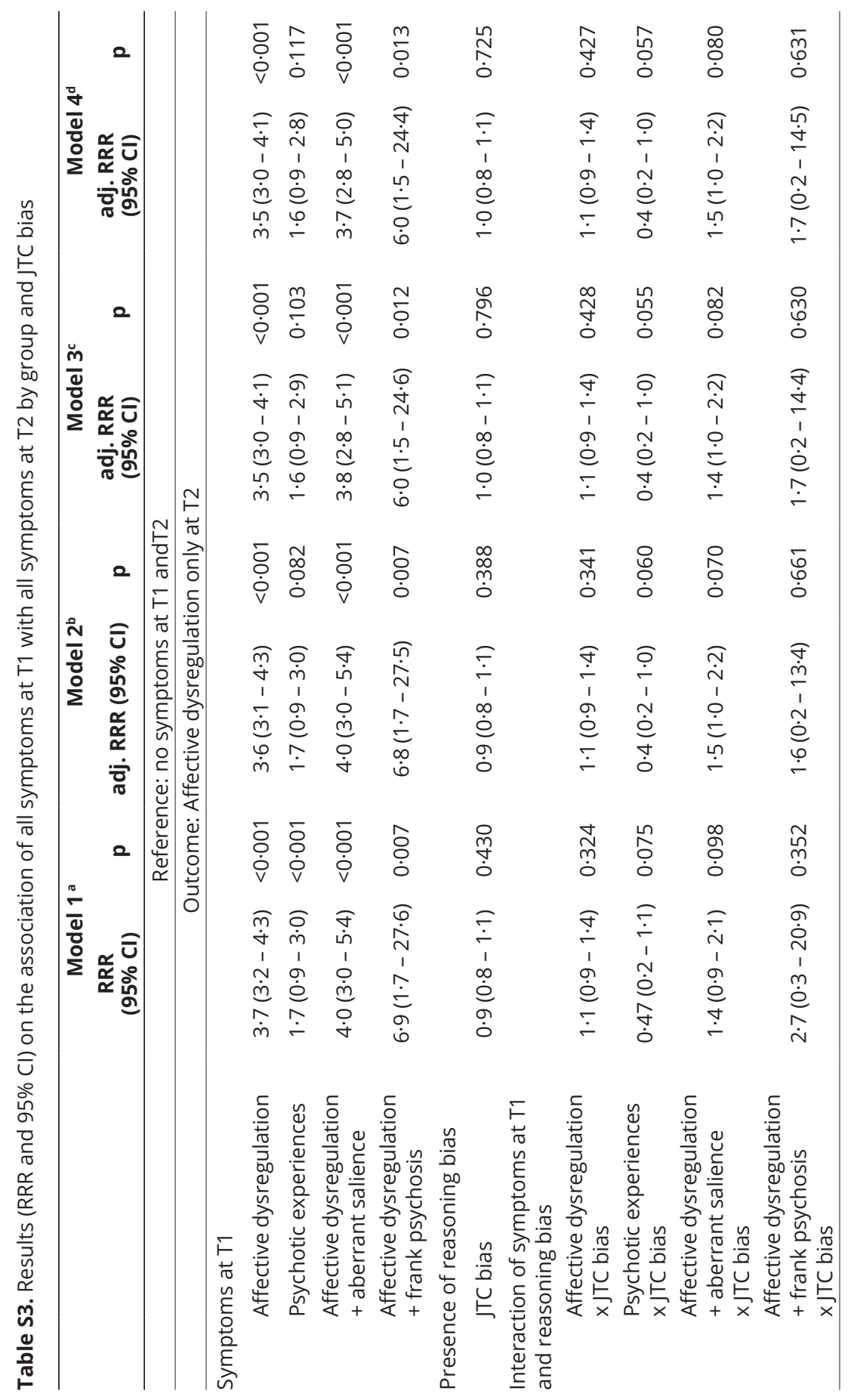




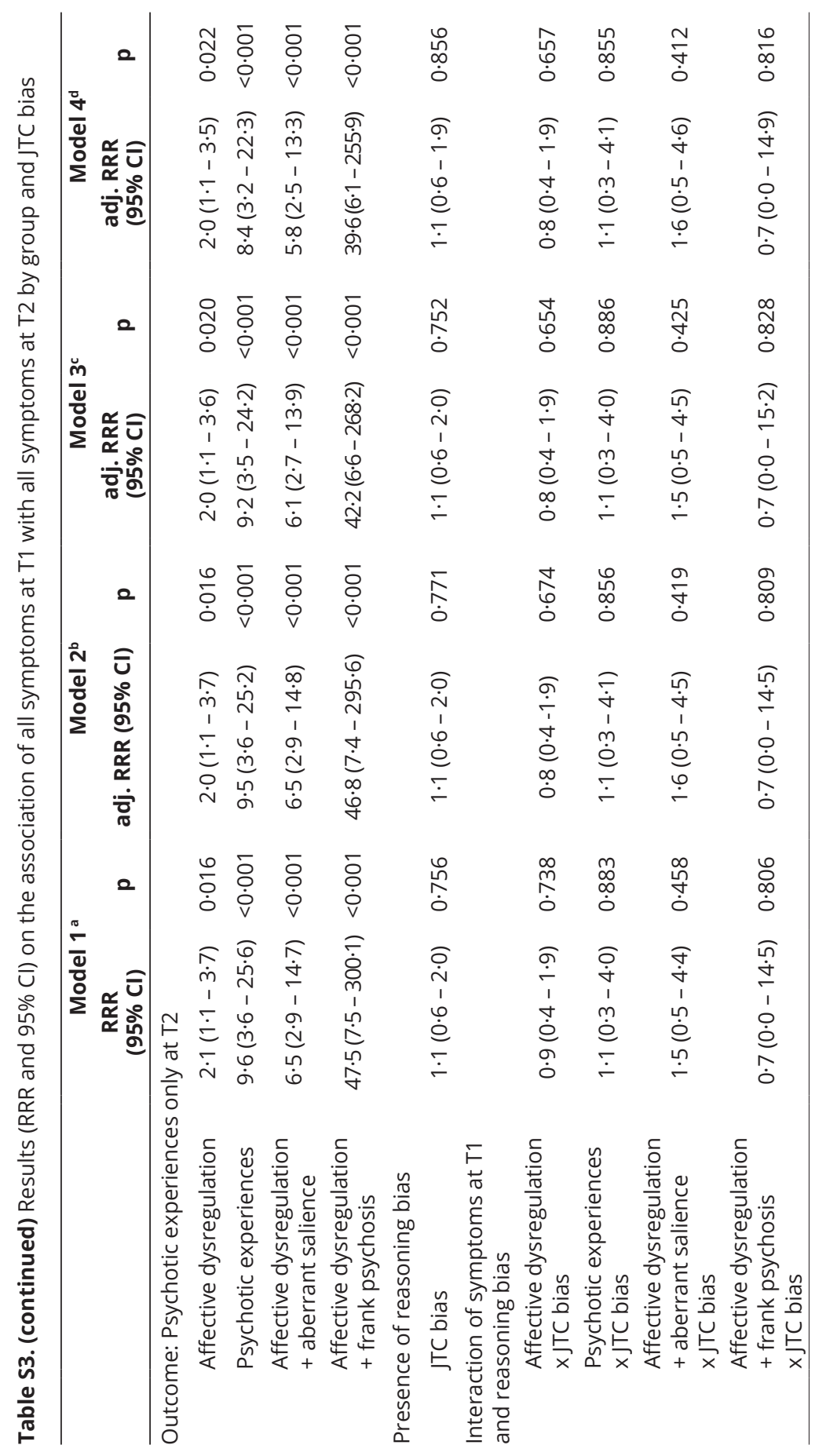




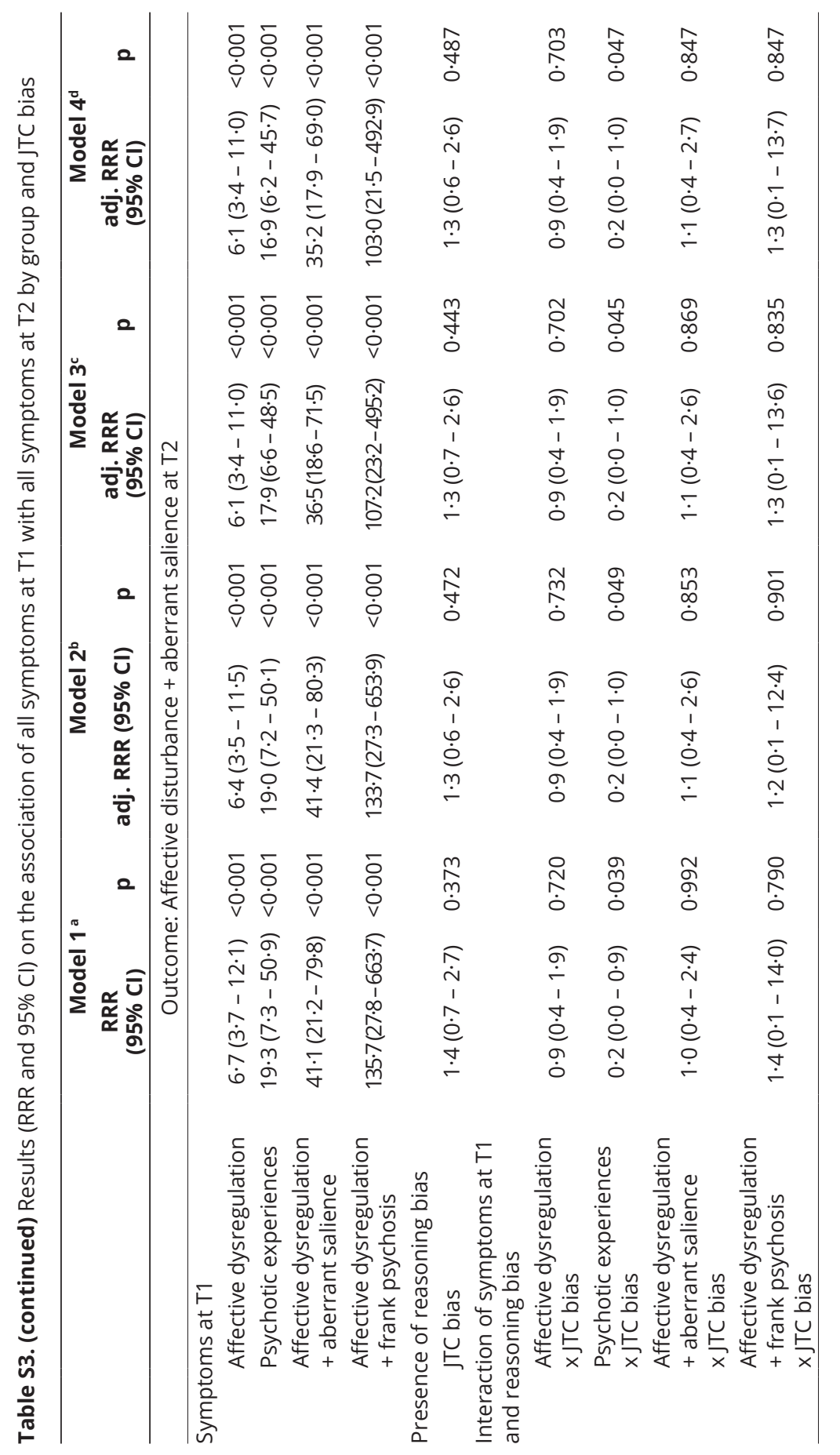




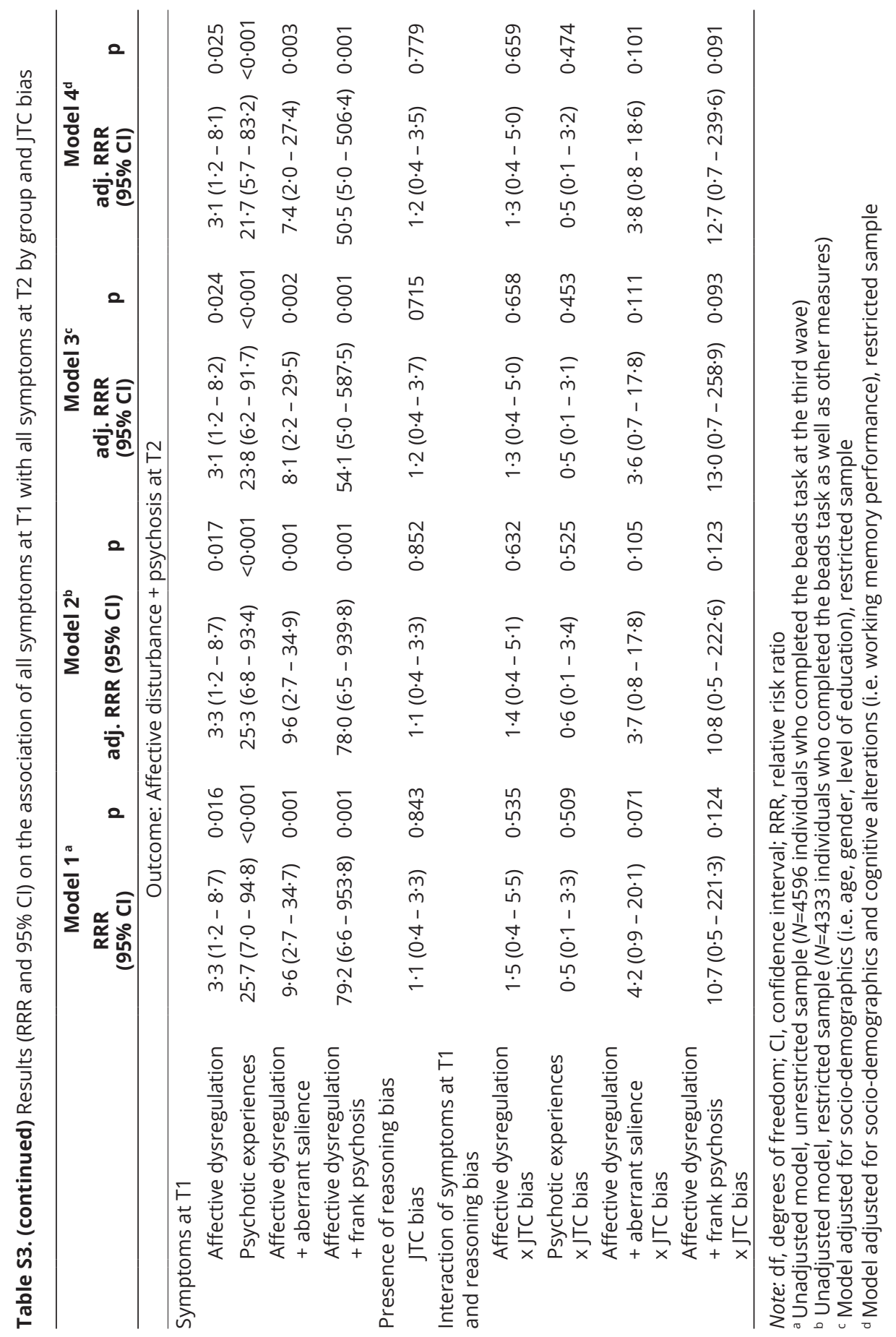


Table S4. Model fit statistics for multinomial logistic regression models

\begin{tabular}{lllllll}
\hline & Observations & df & LL (null) & LL (model) & AIC & BIC \\
\hline Model 1 & 8666 & 40 & -7816.63 & -7152.92 & 14385.85 & 14668.53 \\
\hline Model 2 & 8666 & 52 & -7816.63 & -7077.14 & 14258.28 & 14625.77 \\
\hline Model 3 & 8666 & 56 & -7816.63 & -7062.12 & 14236.24 & 14632.00 \\
\hline
\end{tabular}

Note: $\mathrm{df}$, degrees of freedom; LL= Log-likelihood; AIC, Akaike Information Criterion; BIC, Bayesian Information Criterion. 



\section{CHAPTER 4}

\section{Stress sensitivity as a putative mechanism linking childhood trauma and psychopathology in youth's daily life}

Christian Rauschenberg, Jim van Os, Dimitri Cremers, Matthieu Goedhart, Jan N.M. Schieveld, Ulrich Reininghaus 


\section{Abstract}

Objective: Childhood trauma (CT) is associated with a range of psychopathologies, including psychosis. However, evidence on underlying mechanisms remains limited. The study aimed to investigate whether CT impacts on youth mental health by modifying sensitivity to stress in daily life.

Method: The experience sampling method (ESM) was used to measure momentary stress, negative affect and psychotic experiences in 99 adolescents and young adults (43 help-seeking service users, 16 siblings and 40 controls). Before ESM assessments, CT and depressive, anxiety and psychotic symptoms were assessed.

Results: Stress sensitivity, that is, the association between momentary stress and (i) negative affect and (ii) psychotic experiences, was modified by physical and emotional abuse and, partially, emotional and physical neglect, but not sexual abuse in service users and controls. While there was strong evidence for increased stress sensitivity in service users when high vs. low levels of CT were compared, a pattern of resilience was evident in controls, with attenuated, or no differences in, stress sensitivity in those with high vs. low CT levels. Less consistent findings were observed in siblings.

Conclusions: Stress sensitivity may be an important risk and resilience mechanism through which CT impacts on mental health in youth.

Keywords: trauma; stress; psychopathology; child and adolescent psychiatry; early intervention 


\section{Introduction}

More than $50 \%$ of all life-time cases of mental disorders (based on the Diagnostic and Statistical Manual of Mental Disorders; DSM-IV) appear before the age of 14 (early adolescence) and 75\% before the age of 24 (young adulthood), often characterized by transitional staging processes from subclinical to clinical severity [1]. Thus, disruption of illness trajectories at a developmentally early stage is important $[2,3]$, as reflected in recent calls for a reform of youth mental health services [4]. A crucial step in developing early intervention strategies is to improve our understanding of underlying mechanisms involved [5].

In recent years, evidence has accumulated that subclinical expressions of positive psychotic symptoms are common among the adolescent $[6,7]$ and adult general population [7, 8] associated with a family history of psychotic disorder [7] and linked to an increased risk of developing psychotic [7] and non-psychotic disorders [9]. Recent findings further suggest that subclinical psychotic experiences cooccur with symptoms of common mental disorders (i.e. depression, anxiety) $[10,11]$ supporting observations that, in particular during the early stages of psychopathology, mixed states of symptoms frequently occur that lack diagnostic specificity $[2,5]$. In line with an increased focus on dimensional models of psychopathology [12-18], recent findings have been taken to suggest an extended and transdiagnostic psychosis phenotype with temporal and phenomenological continuity and shared socio-environmental risk factors such as childhood trauma (CT) across developmental stages of psychotic and non-psychotic disorders [11, 17, 19].

Childhood trauma (CT) refers to potentially harmful experiences including sexual, physical, and emotional abuse and physical and emotional neglect [20]. It has been linked to the prevalence and persistence of subclinical psychotic experiences and the onset of psychotic disorders [21-24]. Studies have consistently shown an association between $\mathrm{CT}$ and an increased risk of experiencing psychotic symptoms in adolescence and adulthood, with most evidence supporting a link with sexual [20, 21, 23, 25-27], physical [28-33], and emotional [23, 27] abuse. In addition, CT was found to affect a range of other psychopathologies, including anxiety disorders, major depression, eating disorders, drug disorders, suicidality, and behavioural problems [34-39] and may be associated with an admixture of affective, anxiety and psychotic symptoms [40]. 
Although there is robust evidence linking CT and mental disorders, little is known about underlying mechanisms involved. In current models, CT is thought to make individuals more sensitive to subsequent adversity through enhanced stress sensitivity [28, 41-47]. The experience sampling method (ESM) provides a context-sensitive technique to investigate whether prior experiences of CT amplify stress sensitivity in daily life $[44,48-51]$. Studies using ESM have found that individuals with prior experiences of childhood physical and sexual abuse showed elevated sensitivity towards minor daily stressors in frequent general practitioner attenders [48] and individuals suffering from depression, an at-risk mental state for psychosis, and psychotic disorders [47, 49, 50].

Taken together, high rates of $\mathrm{CT}[52,53]$ and robust links with a range of psychopathologies point to the need to further investigate how CT impacts on mental health at a developmentally early stage of psychopathology. In the current study, we investigated whether CT impacts on youth mental health through an elevated stress sensitivity, characterized by strong emotional reactivity (i.e. increased negative affect) and more intense psychotic experiences in response to minor daily stressors. For this, we recruited a sample of help-seeking adolescents and young adults (service users), their biological siblings, and controls. Siblings of service users have an increased liability to psychopathology and, therefore, constitute an intermediate risk group, in which shared genetic and socioenvironmental risk factors for mental health have been reported [54-56]

\section{Aims and hypotheses of the study}

This study aimed to test the following hypotheses: (1) within groups (service users, siblings, and controls), there is an association between momentary stress (eventrelated, activity-related, and social stress) and (i) negative affect and (ii) psychotic experiences [H1], (2) within groups, the association between momentary stress and (i) negative affect and (ii) psychotic experiences is modified by CT, with greater associations in individuals exposed to high $v$. low levels of various types of abuse and neglect [H2] and, lastly, (3) the difference in magnitude of associations of momentary stress with (i) negative affect and (ii) psychotic experiences between those exposed to high $v$. low levels of CT varies across groups, with greater differences in service users $v$. controls, service users $v$. siblings, and siblings $v$. controls [H3]. 


\section{Materials and methods}

\section{Sample}

We recruited a sample of help-seeking adolescents and young adults (service users), their siblings, and controls. The group of service users were recruited from the Mutsaers foundation (MF) in Limburg, the Netherlands. The MF provides secondary mental health services with a focus on child and adolescent psychiatry. Inclusion criteria were: aged 12-20 years; currently receiving treatment from MF mental health services. Exclusion criteria were: intellectual disability (IQ score below 70); insufficient knowledge of the Dutch language; being diagnosed according to DSM-IV with an Autistic Spectrum Disorder with the exception of Pervasive Developmental Disorder Not Otherwise Specified. In addition, siblings of service users were enrolled. Inclusion criteria were: aged 12-20 years; participation of a biological sibling. Exclusion criteria were the same as in the group of service users with the addition that siblings were excluded if they had a lifetime history of receiving treatment at a mental health service. Lastly, controls were recruited from schools of the same catchment area as MF mental health services. Inclusion criteria were: aged 12-20 years. Exclusion criteria were the same as in the sibling group. Our study was approved by the Medical Ethics Review Committee of Maastricht University Medical Centre in Maastricht, the Netherlands.

\section{Data collection}

\section{Socio-demographic characteristics}

Data on age, gender, ethnicity, level of education were collected using a detailed socio-demographic schedule.

\section{Childhood trauma}

CT was measured using the Dutch version of the short form of the Childhood Trauma Questionnaire (CTQ-SF) [57], adopted for use in adolescents and young adults. The CTQ-SF is an established 28-item self-report questionnaire with five sub-scales (i.e. sexual, physical and emotional abuse as well as physical and emotional neglect). It assesses CT before the age of 16 using a 5-point Likert scale $(1=$ never true, $5=$ very often true). This allows the calculation of mean and categorical severity scores. Sufficient psychometric properties have been demonstrated [58-60]. 


\section{Depressive, anxiety, and psychotic symptoms}

The Beck Depression Inventory (BDI-II) was used to assess depressive symptoms occurring over the past 2 weeks asking 21-items rated on a 4-point scale (0-3) of increasing severity. It consists of 2 factors: negative cognition (9-items) and affective-somatic feelings (12-items). The Community Assessment of Psychic Experiences (CAPE), a self-report instrument with 42-items, was completed to capture the frequency and distress (both rated on a 4-point scale from 0-3) of positive (20-items) and negative (14-items) non-clinical psychotic and depressive (8 items) symptoms. The State-Trait Anxiety Inventory (STAI) [61] was used to measure state and trait anxiety. A Dutch version consisting of 60-items rated on a 4-point Likert scale (1-4) was used [62]. The first part (STAI-DY1) measures trait (20-items) and the second part (STAI-DY2) assesses state (40-items) anxiety. For all measures, good psychometric properties have been reported [62-66].

\section{ESM measures}

Momentary stress (event-related, activity-related and social stress), negative affect, and psychotic experiences were collected using the experience sampling method (ESM), a random time-sampling self-assessment technique. The ESM captures moment-to-moment daily variation in variables in real life outside the research laboratory with high ecological validity $[51,67,68]$. For data acquisition, participants received a personal digital assistant (PsyMate) [69] which beeped 10 times each day on 6 consecutive days at unpredictable moments between 7:30 am and 10:30 pm (scheduled at random within set blocks of time). Event-related, activity-related, and social stress were conceptualized as minor disturbances and distinctive unpleasant events, activities, and social situations occurring in the flow of daily life. Good concurrent validity with other stress measures has been reported $[44,70]$.

Event-related stress was assessed asking participants to report where they were (e.g. home, private room, family/friends, work/school, public place) and the most important event that had happened since the last beep by rating the pleasantness and importance ranging from "very unpleasant" (rating of -3) to "very pleasant" (rating of 3) and "very unimportant" (rating of -3) to "very important" (rating of 3). The coding was reversed that higher ratings indicate higher levels of stress (with ratings of -3 coded as 7 and ratings of 3 coded as 1 ). Activity-related stress 
was measured by asking "What am I doing (just before the beep)" (e.g. resting, smoking, watching TV) and 3 follow-up questions ("I would prefer doing something else", "This activity is difficult for me", "I can do this well" [reversed]) ranging from "not at all" (rating of 1) to "very much" (rating of 7). Social stress was measured by asking participants to specify categorically with whom they were spending time (e.g. nobody, partner, family) and to rate the current social context using the items "I would prefer to be alone", "I find the people I am with pleasant" [reversed], "I feel safe (with these people)" [reversed], and "I feel threatened" (if with someone) or "I like to be alone" [reversed] and "I would prefer to have company" (if alone) ranging from "not at all" (rating of 1) to "very much" (rating of 7).

Negative affect was measured asking participants 5-items to rate the degree of feeling anxious, lonely, insecure, irritated, and down. Psychotic experiences were measured using 8-items asking for mental states associated with psychotic experiences: hallucinations, delusions, and thought problems ("I see things that aren't really there", "I hear things that aren't really there", "I feel suspicious/ paranoid", "I feel harried", "I feel unreal", "My thoughts are influenced by other", "I can't get these thoughts out of my head", "I feel like I am losing control"), both rated on a 7-point Likert scale ranging from "not at all" (rating of 1) to "very much" (rating of 7). High levels of internal consistency and good concurrent validity with interviewer-rated measures of psychotic experiences has been previously reported $[50,71]$.

\section{Statistical analysis}

Basic sample characteristics and ESM aggregate mean scores (i.e. over a period of 6 days for each participant) and standardized BDI-II, CAPE, and STAI-DY1/DY2 scores in service users, their siblings and controls were compared using linear regression and $\chi^{2}$-tests as appropriate. Second, as ESM data has a multilevel structure with multiple observations (level-1) nested within participants (level-2), the 'xtmixed' command in Stata v. 13 [72] was used to fit linear mixed models. Separate models for each momentary stressor (event-related, activity-related, and social stress) as the independent variable and (i) negative affect and (ii) psychotic experiences as the outcome variable were computed, while controlling for potential confounders (i.e. age, gender, ethnicity, level of education). Third, CT was included into the model by adding two-way (stress $x$ trauma, stress $x$ group, 
trauma $x$ group) and three-way (e.g. stress $x$ trauma $x$ group) interaction terms to investigate whether the associations between momentary stress and (i) negative affect and (ii) psychotic experiences were modified by prior exposure to childhood trauma (mean scores of sexual, physical and emotional abuse, and physical and emotional neglect) and group (service users, siblings, and controls). Likelihood ratio tests were calculated to assess improvements in model fit after interaction terms were added. Fourth, the 'lincom' command was used to compute linear combinations of coefficients for testing the hypotheses that, within each group, the association of momentary stress with (i) negative affect and (ii) psychotic experiences was greater in individuals exposed to high $v$. low levels of various forms of CT (+/- 1 S.D. of standardized CTQ scores, mean = 0, S.D. = 1) [73, 74]. Lastly, we investigated whether differences in the magnitude of associations of momentary stress with (i) negative affect and (ii) psychotic experiences between those exposed to high $v$. low levels of various forms of CT are greater in service users $v$. controls, service users $v$. siblings, and siblings $v$. controls.

\section{Results}

\section{Basic sample and clinical characteristics}

In total, the sample consisted of 109 adolescents and young adults eligible to participate in the study. Of these, 99 individuals (43 service users, 16 siblings, 40 controls) completed the ESM with $\geq 20$ valid responses over the 6-day assessment period as well as the CTQ, BDI-II, CAPE, STAI-DY1/DY2. As shown in Table 1, service users, their siblings and controls did not differ in age, gender, or ethnicity. However, service users were less often educated to further or higher educational levels than siblings and controls. In addition to primary diagnosis of specific and non-specific mental disorders with a high proportion of comorbidity (55.8\%) in the group of service users, there was evidence for differences in symptomatology in service users $v$. controls and service users $v$. siblings respectively, with higher levels of depression (BDI-II: $B=0.68, p=0.001 ; B=1.02 p<0.001)$, state $(B=0.46$, $p=0.035$; only some evidence in service users $v$. siblings $B=0.54, p=0.065$ ) and trait $(B=0.64, p=0.003 ; B=0.93, p=0.001)$ anxiety, and negative $(B=0.40, p=0.066$; $B=0.72, p=0.014)$ and positive $(B=0.73, p=0.001 ; B=0.81, p=0.004)$ psychotic-like experiences. Moreover, comparing CT in service users and controls, higher levels of sexual $(B=0.45, p=0.041)$ and emotional $(B=0.60, p=0.006)$ abuse as well as 
higher emotional $(B=0.61, p=0.005)$ and physical $(B=0.59, p=0.006)$ neglect were found, with some evidence of a difference in physical $(B=0.42, p=0.059)$ abuse. Further, emotional $(B=0.73, p=0.011)$ and physical $(B=0.74, p=0.010)$ neglect were markedly higher in service users than siblings, but not significantly, however, in sexual $(B=0.40, p=0.170)$, physical $(B=0.31, p=0.283)$, and emotional $(B=0.48$, $p=0.092$ ) abuse. In addition, we found that physical (intraclass correlation coefficient $(I C C)=0.80,95 \% \mathrm{Cl} 0.47-0.95)$ and emotional $(I C C=0.17,95 \% \mathrm{Cl} 0.00-0.95)$ abuse as well as physical $(I C C=0.48,95 \% \mathrm{Cl} 0.11-0.87)$ and emotional $(I C C=0.27,95 \% \mathrm{Cl} 0.02$ - 0.88) neglect scores, but not sexual (ICC $=0.00,95 \% \mathrm{Cl} 0.00-0.00)$ abuse scores, of service users and their siblings were correlated. There were no differences in various symptom domains and CT between siblings and controls. 
Table 1. Basic sample characteristics

\begin{tabular}{|c|c|c|c|c|c|}
\hline & $\begin{array}{c}\text { Service users } \\
(n=43)\end{array}$ & $\begin{array}{l}\text { Siblings } \\
(n=16)\end{array}$ & $\begin{array}{c}\text { Controls } \\
(n=40)\end{array}$ & Test statistic & $\mathbf{p}$ \\
\hline Age (years), mean (S.D.) & $15.4(1.4)$ & $15.4(2.4)$ & $15.6(2.0)$ & $\mathrm{F}=0.22, \mathrm{df}=2$ & 0.802 \\
\hline \multicolumn{6}{|l|}{ Gender, n (\%) } \\
\hline Male & $17(39.5)$ & $7(43.8)$ & $17(42.5)$ & \multirow{2}{*}{$\chi^{2}=0.12, d f=2$} & \multirow{2}{*}{0.943} \\
\hline Female & $26(60.5)$ & $9(56.2)$ & $23(57.5)$ & & \\
\hline \multicolumn{6}{|l|}{ Ethnicity, n (\%) } \\
\hline White Dutch & $27(62.8)$ & $10(62.5)$ & $25(64.1)$ & \multirow{2}{*}{$\chi^{2}=0.02, \mathrm{df}=2$} & \multirow{2}{*}{0.990} \\
\hline Other & $16(37.2)$ & $6(37.5)$ & $14(35.9)$ & & \\
\hline \multicolumn{6}{|l|}{ Level of education, $n(\%)^{a}$} \\
\hline School & $30(69.8)$ & $7(43.8)$ & $17(42.5)$ & \multirow{3}{*}{$\chi^{2}=9.69, \mathrm{df}=4$} & \multirow{3}{*}{0.046} \\
\hline Further & $13(30.2)$ & $7(43.8)$ & $20(50.0)$ & & \\
\hline Higher & - & $2(12.5)$ & $3(7.5)$ & & \\
\hline \multicolumn{6}{|l|}{ Number of valid beeps } \\
\hline mean (range, min-max) & $44.21(25-59)$ & $42.81(23-57)$ & $44.58(24-58)$ & $F=0.25, d f=2$ & 0.777 \\
\hline \multicolumn{6}{|c|}{ Attempted suicide, $\mathrm{n}(\%)^{\mathrm{b}}$} \\
\hline During last year & $6(14.0)$ & - & - & \multirow[t]{2}{*}{-} & - \\
\hline Before age 17 & $8(18.6)$ & - & - & & \\
\hline \multicolumn{6}{|l|}{ DSM-IV diagnosis, n (\%) } \\
\hline $\begin{array}{l}\text { Pervasive } \\
\text { developmental } \\
\text { disorders NOS }\end{array}$ & $10(23.3)$ & - & $5(12.5)$ & & \\
\hline $\begin{array}{l}\text { Attention-deficit and } \\
\text { disruptive behaviour }\end{array}$ & $6(14.0)$ & $3(18.8)$ & - & & \\
\hline Adjustment disorders & $4(9.3)$ & - & - & & \\
\hline Anxiety disorders & $2(4.7)$ & - & - & & \\
\hline Depressive disorders & $2(4.7)$ & - & - & & \\
\hline $\begin{array}{l}\text { Gender identity } \\
\text { disorders }\end{array}$ & $2(4.7)$ & - & - & & \\
\hline Learning disorders & - & - & $2(5.0)$ & & \\
\hline $\begin{array}{l}\text { Other disorders of } \\
\text { infancy, childhood, or } \\
\text { adolescence }\end{array}$ & $5(11.6)$ & - & - & & \\
\hline $\begin{array}{l}\text { Parent-child relational } \\
\text { problem }\end{array}$ & $5(11.6)$ & $1(6.3)$ & $1(2.5)$ & & \\
\hline Comorbid condition ${ }^{c}$ & $24(55.8)$ & $2(4.7)$ & - & & \\
\hline None & $7(16.3)$ & $12(75.0)$ & $32(80.0)$ & & \\
\hline BDI-II sum scores, mean (S.D. $)^{d}$ & $12.57(9.31)$ & $4.06(3.32)$ & $6.93(7.00)$ & $F=9.25, d f=2$ & $<0.001$ \\
\hline \multicolumn{6}{|c|}{ BDI-II severity scores $\mathrm{n}(\%)$} \\
\hline Minimal & $20(47.6)$ & $15(93.8)$ & $32(80.0)$ & \multirow[t]{4}{*}{$\chi^{2}=16.20, \mathrm{df}=6$} & \multirow[t]{4}{*}{0.013} \\
\hline Mild & $12(28.6)$ & $1(6.3)$ & $5(12.5)$ & & \\
\hline Moderate & $8(19.1)$ & - & $2(5.0)$ & & \\
\hline Severe & $2(4.8)$ & - & $1(2.5)$ & & \\
\hline
\end{tabular}


Table 1. (continued) Basic sample characteristics

\begin{tabular}{|c|c|c|c|c|c|}
\hline & $\begin{array}{l}\text { Service users } \\
(n=43)\end{array}$ & $\begin{array}{l}\text { Siblings } \\
(n=16)\end{array}$ & $\begin{array}{c}\text { Controls } \\
(n=40)\end{array}$ & Test statistic & $\mathbf{p}$ \\
\hline \multicolumn{6}{|c|}{ CAPE sum scores, mean (S.D. $)^{\mathrm{d}}$} \\
\hline Positive & $9.84(9.38)$ & $4.00(3.27)$ & $4.60(3.90)$ & $F=7.67, d f=2$ & $<0.001$ \\
\hline Negative & $9.78(6.93)$ & $5.56(3.90)$ & $7.42(4.83)$ & $F=3.64, d f=2$ & 0.030 \\
\hline Depressive & $7.57(4.00)$ & $4.31(1.78)$ & $4.67(3.39)$ & $F=8.91, d f=2$ & $<0.001$ \\
\hline $\begin{array}{l}\text { STAI-DY1 } \\
\text { mean scores, mean (S.D.) }\end{array}$ & $1.76(0.53)$ & $1.52(0.35)$ & $1.56(0.37)$ & $F=2.95, d f=2$ & 0.057 \\
\hline $\begin{array}{l}\text { STAI-DY2 } \\
\text { mean scores, mean (S.D.) }\end{array}$ & $2.15(0.52)$ & $1.71(0.25)$ & $1.85(0.42)$ & $F=7.75, d f=2$ & $<0.001$ \\
\hline \multicolumn{6}{|l|}{$\begin{array}{l}\text { CTQ mean scores, mean } \\
(\text { S.D. })^{d}\end{array}$} \\
\hline Sexual abuse & $1.29(0.87)$ & $1.05(0.20)$ & $1.02(0.13)$ & $F=2.37, d f=2$ & 0.099 \\
\hline Physical abuse & $1.31(0.77)$ & $1.14(0.32)$ & $1.08(0.23)$ & $F=1.91, d f=2$ & 0.153 \\
\hline Emotional abuse & $1.98(1.05)$ & $1.56(0.45)$ & $1.46(0.65)$ & $F=4.26, d f=2$ & 0.017 \\
\hline Emotional neglect & $2.28(0.95)$ & $1.73(0.36)$ & $1.81(0.51)$ & $F=5.58, d f=2$ & 0.005 \\
\hline Physical neglect & $1.45(0.61)$ & $1.10(0.15)$ & $1.17(0.31)$ & $F=5.39, d f=2$ & 0.006 \\
\hline \multicolumn{6}{|l|}{ CTQ severity scores $\mathrm{n}(\%)$} \\
\hline \multicolumn{6}{|l|}{ Sexual abuse } \\
\hline None/Minimal & $35(83.4)$ & $15(93.8)$ & $39(97.5)$ & $\chi^{2}=7.61, \mathrm{df}=6$ & 0.268 \\
\hline Low & $2(4.8)$ & - & - & & \\
\hline Moderate & $2(4.8)$ & $1(6.3)$ & $1(2.5)$ & & \\
\hline Severe & $3(7.1)$ & - & - & & \\
\hline \multicolumn{6}{|l|}{ Physical abuse } \\
\hline None/Minimal & $36(85.7)$ & $14(87.5)$ & $38(95.0)$ & $\chi^{2}=5.32, \mathrm{df}=6$ & 0.503 \\
\hline Low & $1(2.4)$ & $1(6.3)$ & $1(2.5)$ & & \\
\hline Moderate & $2(4.8)$ & $1(6.3)$ & $1(2.5)$ & & \\
\hline Severe & $3(7.1)$ & - & - & & \\
\hline \multicolumn{6}{|l|}{ Emotional abuse } \\
\hline None/Minimal & $22(52.4)$ & $11(68.8)$ & $34(85.0)$ & $\chi^{2}=11.93, \mathrm{df}=6$ & 0.064 \\
\hline Low & $11(26.2)$ & $4(25.0)$ & $3(7.5)$ & & \\
\hline Moderate & $4(9.5)$ & $1(6.3)$ & $1(2.5)$ & & \\
\hline Severe & $5(11.9)$ & - & $2(5.0)$ & & \\
\hline \multicolumn{6}{|l|}{ Physical neglect } \\
\hline Minimal & $28(66.7)$ & $16(100.0)$ & $37(92.5)$ & $\chi^{2}=14.34, \mathrm{df}=6$ & 0.026 \\
\hline Low & $7(16.7)$ & - & $2(5.0)$ & & \\
\hline Moderate & $4(9.5)$ & - & - & & \\
\hline Severe & $3(7.1)$ & - & $1(2.5)$ & & \\
\hline \multicolumn{6}{|l|}{ Emotional neglect } \\
\hline None/Minimal & $18(42.9)$ & $11(68.8)$ & $23(57.5)$ & $\chi^{2}=13.20, \mathrm{df}=6$ & 0.040 \\
\hline Low & $13(31.0)$ & $5(31.3)$ & $15(37.5)$ & & \\
\hline Moderate & $4(9.5)$ & - & $2(5.0)$ & & \\
\hline Severe & $7(16.7)$ & - & - & & \\
\hline
\end{tabular}


Table 1. (continued) Basic sample characteristics

Note: S.D., standard deviation; $d f$, degrees of freedom; $\beta$, standardized regression coefficients; $\mathrm{Cl}$, confidence interval;

a categories defined as: school (primary education, LBO, MAVO, VMBO), further (MBO, HAVO, $\mathrm{VWO}$ ), and higher (HBO, WO) of the Dutch educational system

${ }^{\mathrm{b}}$ based on self-reports

c consisting of the following diagnostic categories in the group of service users: Additional codes (Parent-child relational problem, 33.3\%; Borderline intellectual functioning, 13.3\%; Neglect of child, 6.7\%), Attention-deficit and disruptive behaviour disorders (10\%), Learning disorders (10\%), Personality Disorders (6.7\%), Mild mental retardation (6.7\%), Anxiety Disorders (3.3\%), Dissociative Disorders (3.3\%), Tic disorders (3.3\%), Amphetamine related disorders (3.3\%)

${ }^{d}$ difference in standardized coefficients across groups:

\begin{tabular}{|c|c|c|c|c|c|c|}
\hline & \multicolumn{2}{|c|}{ Service users v. controls } & \multicolumn{2}{|c|}{ Siblings v. controls } & \multicolumn{2}{|c|}{ Service users v. siblings } \\
\hline & $\beta(95 \% \mathrm{Cl})$ & $\mathbf{p}$ & $\beta(95 \% \mathrm{Cl})$ & $\mathbf{p}$ & $\beta(95 \% \mathrm{Cl})$ & $\mathbf{p}$ \\
\hline BDI- II & $0.68(0.27-1.08)$ & 0.001 & $-0.34(-0.89-0.20)$ & 0.221 & $1.02(0.48-1.56)$ & $<0.001$ \\
\hline \multicolumn{7}{|l|}{ CAPE } \\
\hline Positive & $0.73(0.31-1.14)$ & 0.001 & $-0.08(-0.63-0.47)$ & 0.764 & $0.81(0.26-1.36)$ & 0.004 \\
\hline Negative & $0.40(-0.03-0.83)$ & 0.066 & $-0.32(-0.89-0.25)$ & 0.273 & $0.15-1.29)$ & 0.014 \\
\hline Depressive & $0.77(0.37-1.18)$ & $<0.001$ & $-0.09(-0.64-$ & 0.731 & $0.87(0.33-0.14)$ & 0.002 \\
\hline STAI-DY1 & $0.46(0.03-0.89)$ & 0.035 & $-0.08(-C$ & 0.796 & $0.54(-0.03-1.11)$ & 0.065 \\
\hline STAI-DY2 & $64(0.23-1.05)$ & 0.003 & -0.29 & 0.294 & $0.93(0.39-1.48)$ & 0.001 \\
\hline \multicolumn{7}{|l|}{ CTQ } \\
\hline $\begin{array}{l}\text { Sexual } \\
\text { abuse }\end{array}$ & $0.45(0.02-0.88)$ & 0.041 & $0.05(-0.53-0.63)$ & 0.862 & $0.40(-0.17-0.98)$ & 0.170 \\
\hline $\begin{array}{l}\text { Physical } \\
\text { abuse }\end{array}$ & $0.42(-0.02-0.85)$ & 0.059 & $0.10(-0.48-0.69)$ & 0.721 & $0.31(-0.26-0.89)$ & 0.283 \\
\hline $\begin{array}{l}\text { Emotional } \\
\text { abuse }\end{array}$ & $0.60(0.18-1.03)$ & 0.006 & $0.12(-0.45-0.69)$ & 0.676 & $0.48(-0.08-1.05)$ & 0.092 \\
\hline $\begin{array}{l}\text { Physical } \\
\text { neglect }\end{array}$ & $0.59(0.17-1.01)$ & 0.006 & $-0.15(-0.71-0.41)$ & 0.600 & $0.74(0.18-1.30)$ & 0.010 \\
\hline $\begin{array}{l}\text { Emotional } \\
\text { neglect }\end{array}$ & $0.61(0.19-1.03)$ & 0.005 & $-0.12(-0.68-0.44)$ & 0.673 & $0.73(0.17-1.29)$ & 0.011 \\
\hline
\end{tabular}




\section{Aggregate scores}

Overall, no differences in aggregated scores of various types of momentary stress (event-related, activity-related, and social stress) were found comparing service users and controls as well as siblings and controls (see Table 2). There was some evidence that service users experienced more (i) negative affect $(p=0.077)$ and (ii) psychotic experiences $(p=0.087)$ in their daily life compared to controls; no differences were found between siblings and controls.

\section{Association between stress, negative affect, and psychotic experiences by group [H1]}

Table 3 shows that, within service users and controls, each type of stress was associated with a small to moderate increase in (i) negative affect and (ii) psychotic experiences [H1], but not in siblings (except for association between event-related stress and negative affect). Further, there was evidence for two-way interaction effects of stress $\times$ group on (i) negative affect and (ii) psychotic experiences (all $p<$ $0.05)$ excluding the association between social stress and negative affect $(p=0.188)$. 


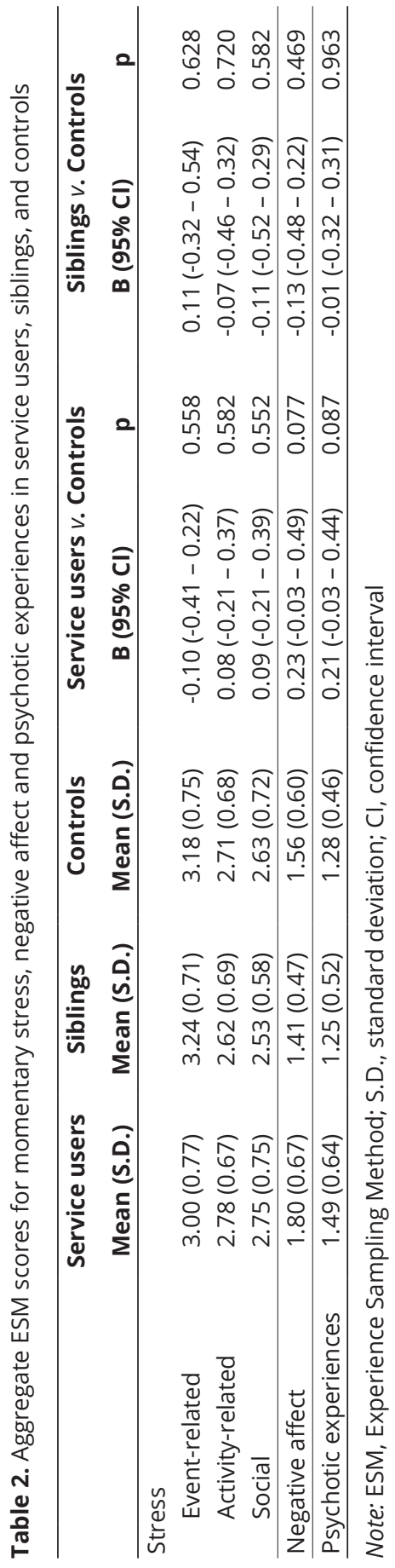




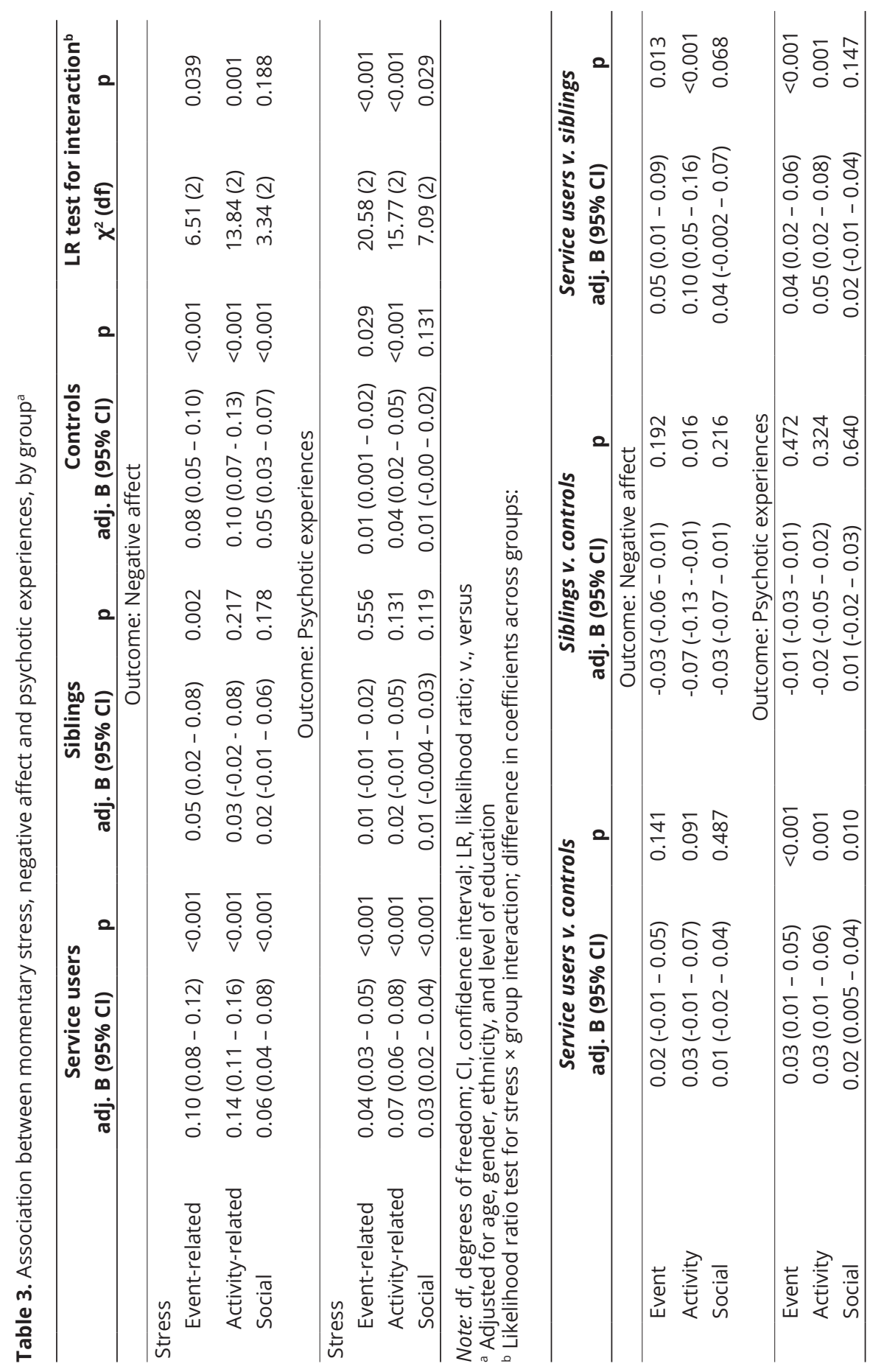




\section{Association between stress, negative affect, and psychotic experiences by sexual, physical and emotional abuse and group [H2 \& H3]}

As presented in Table 4 and 5, there was no evidence that childhood sexual abuse amplified the association of event-related, activity-related and social stress with (i) negative affect and (ii) psychotic experiences in service users, their siblings and controls.

However, there was strong evidence that the association of various types of momentary stress with (i) negative affect and (ii) psychotic experiences was modified by physical and emotional abuse within [H2] and across [H3] groups as indicated by significant 3-way interaction effects described below and illustrated in Supplementary Figures (Figures S1).

\section{Within groups (H2)}

In service users exposed to high $v$. low levels of physical and emotional abuse, event-related $(B=0.13, p<0.001 ; B=0.19, p<0.001)$ and activity-related $(B=0.10$, $p<0.001 ; B=0.15, p<0.001)$ stress were associated with more intense (i) negative affect, and event-related stress $(B=0.06, p<0.001 ; B=0.10, p<0.001)$ was associated with more intense (ii) psychotic experiences. In contrast to service users, activityrelated $(B=-0.16, p=0.033)$ and social $(B=-0.26, p=0.014)$ stress were associated with less intense negative affect in daily life in controls exposed to high $v$. low levels of physical abuse. There was no evidence of effect modification by levels of emotional abuse in controls, except for a greater association between event-related $(B=0.07$, $p=0.014$ ) stress and psychotic experiences in those exposed to high $v$. low levels of emotional abuse. In siblings, there was no evidence that childhood abuse modified associations between momentary stress with (i) negative affect and (ii) psychotic experiences. 


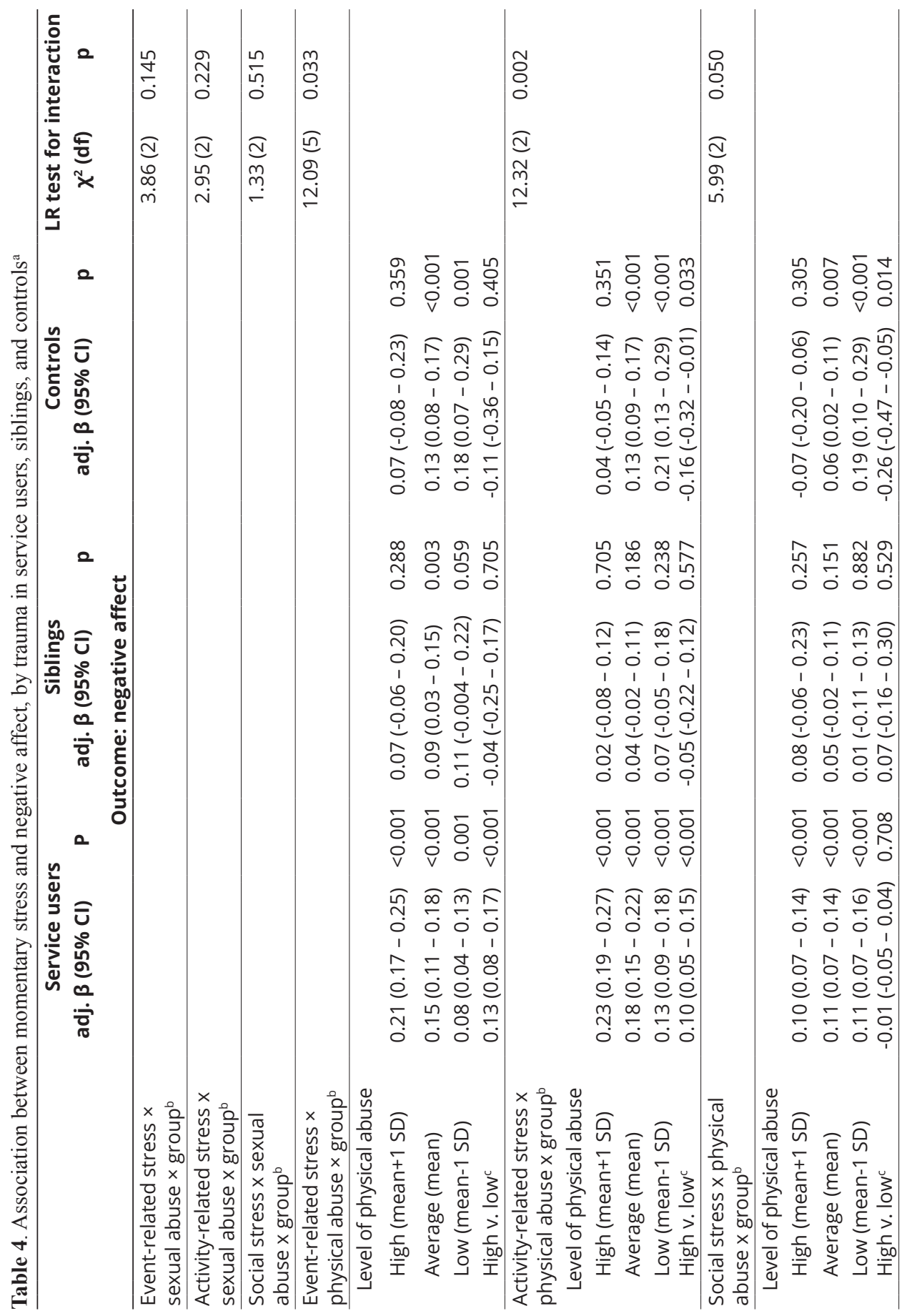




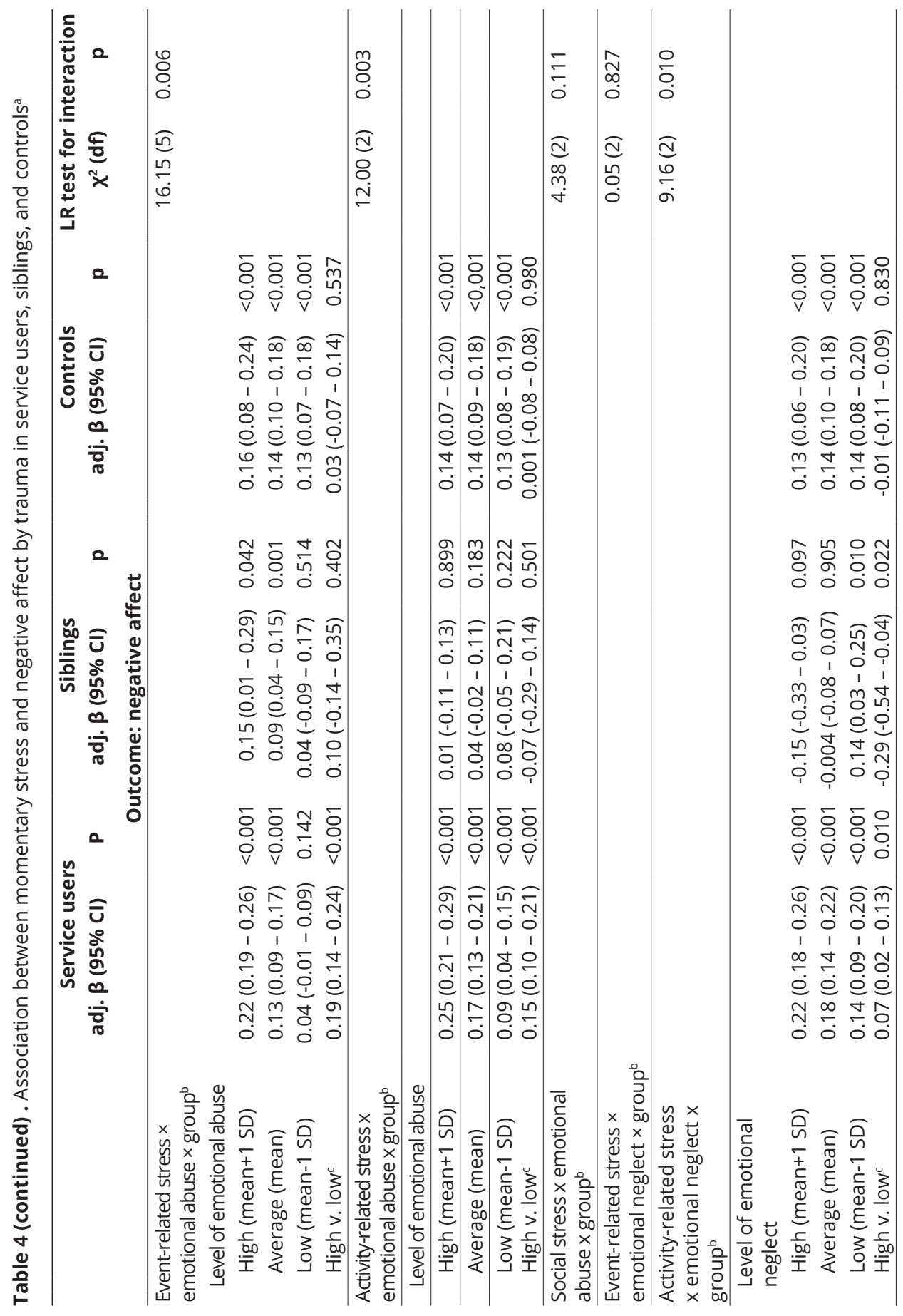




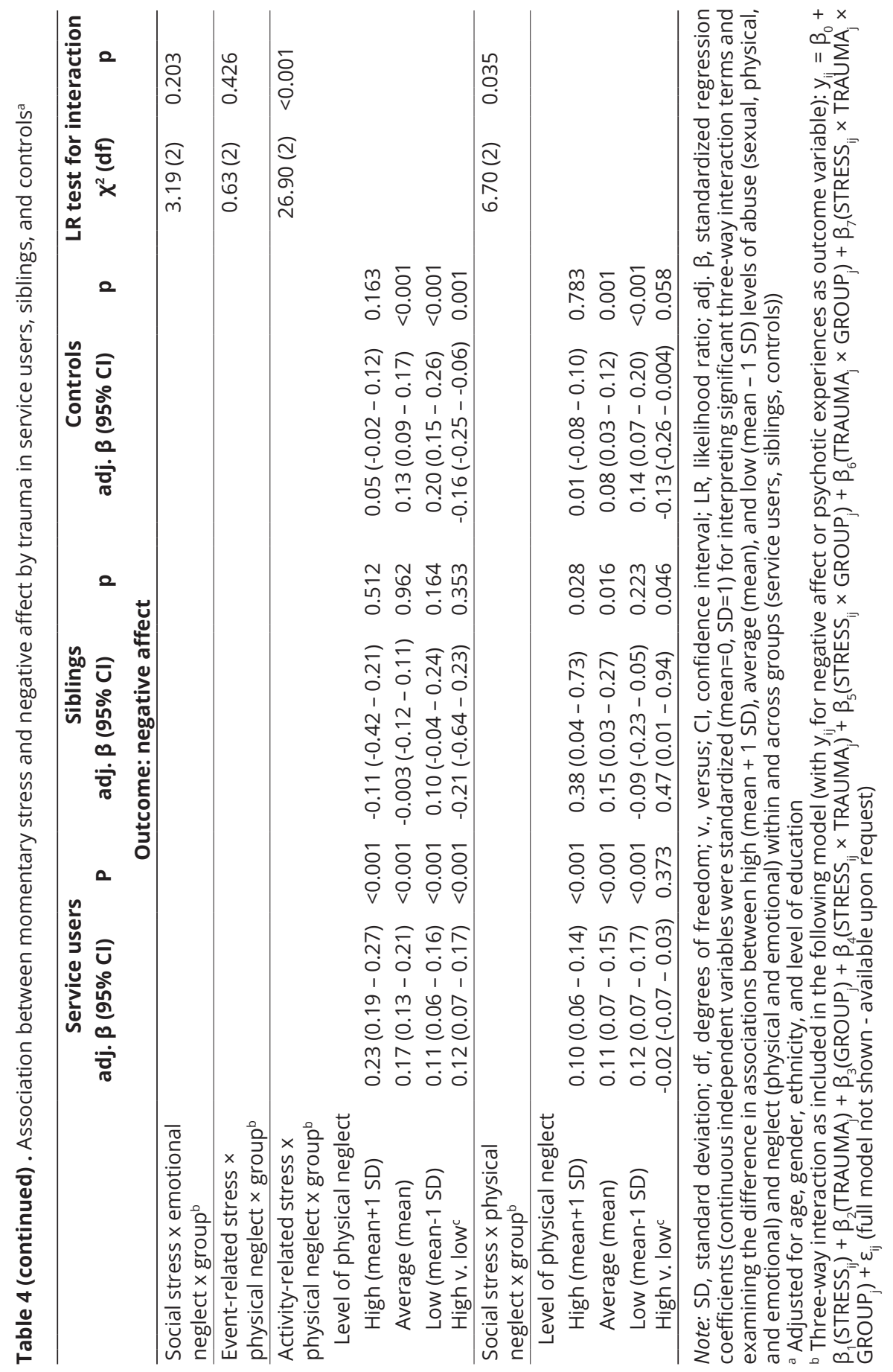




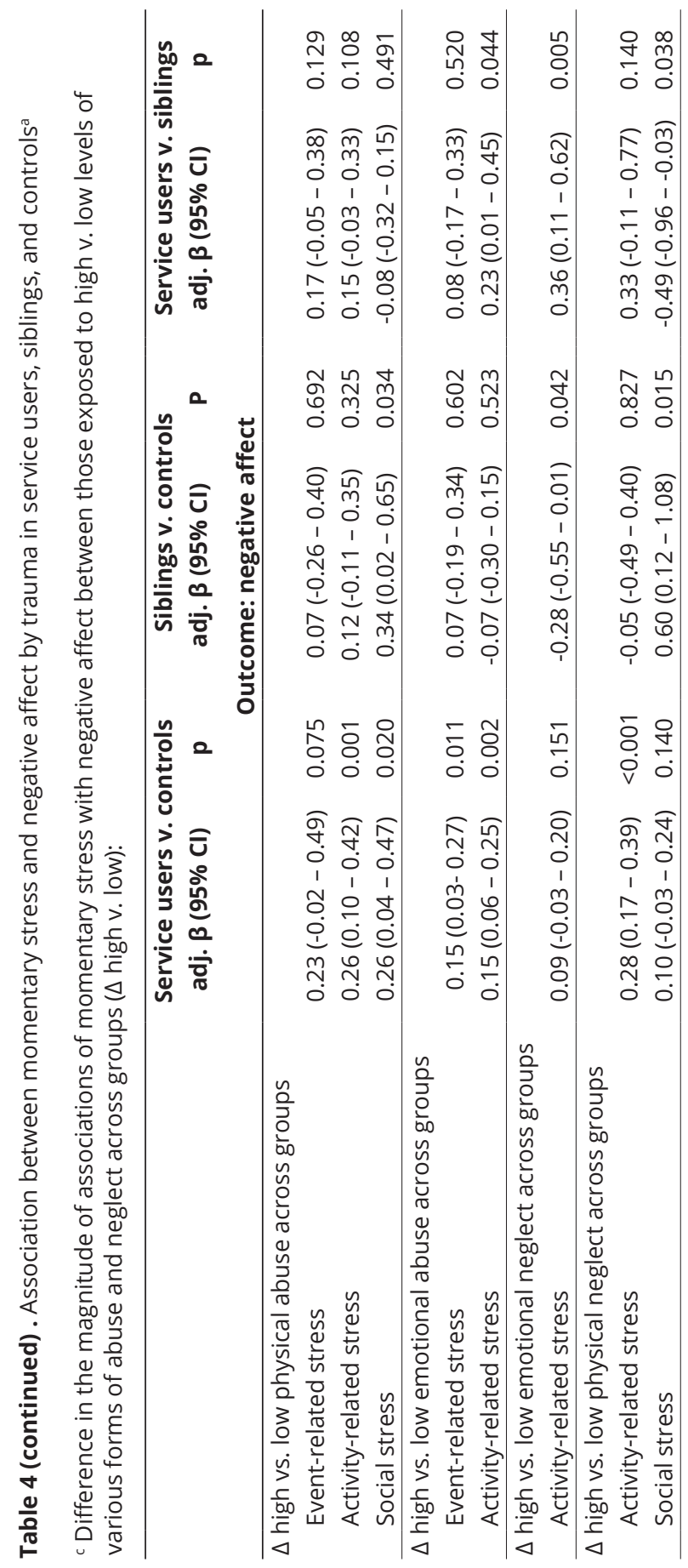




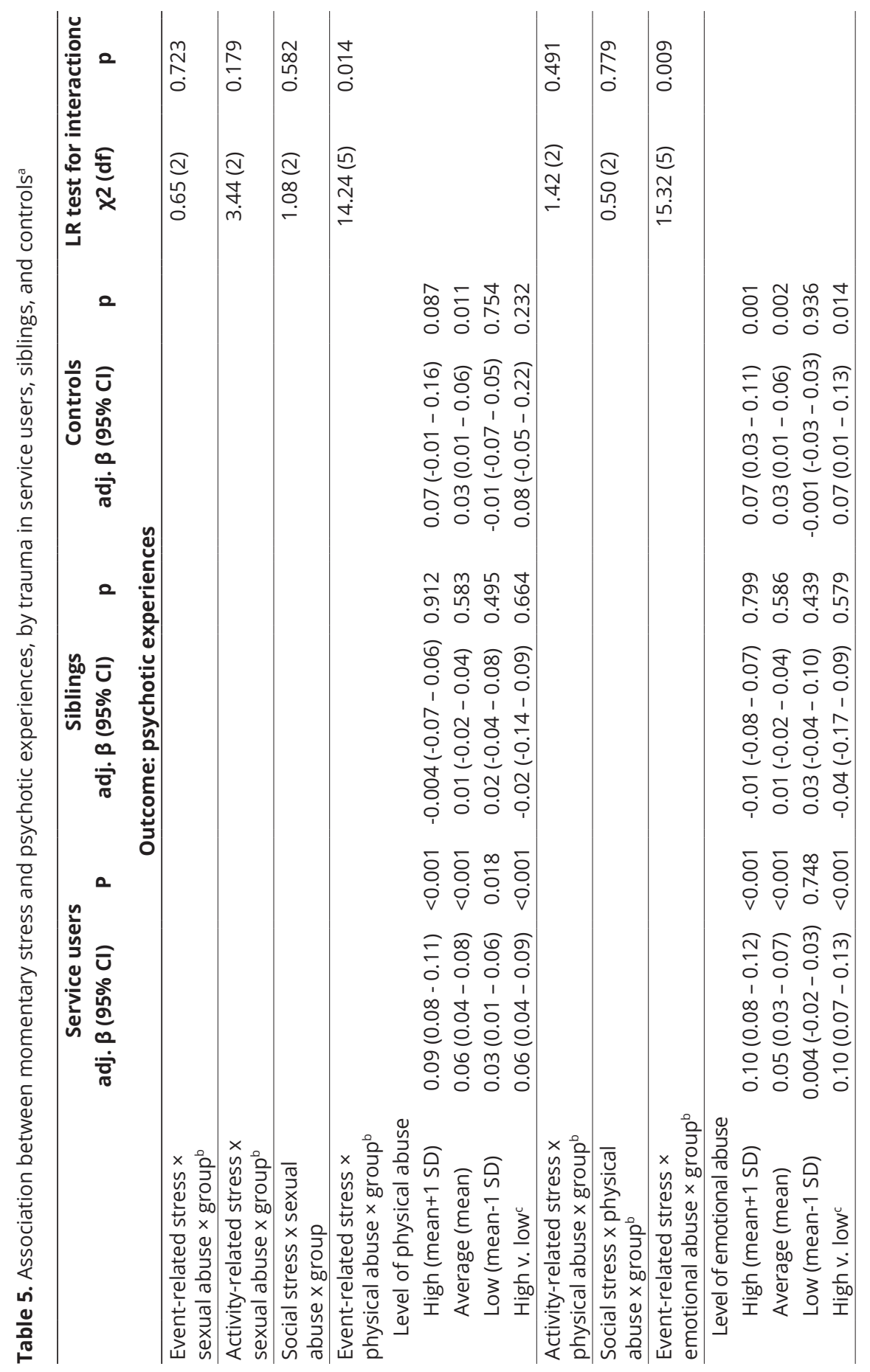




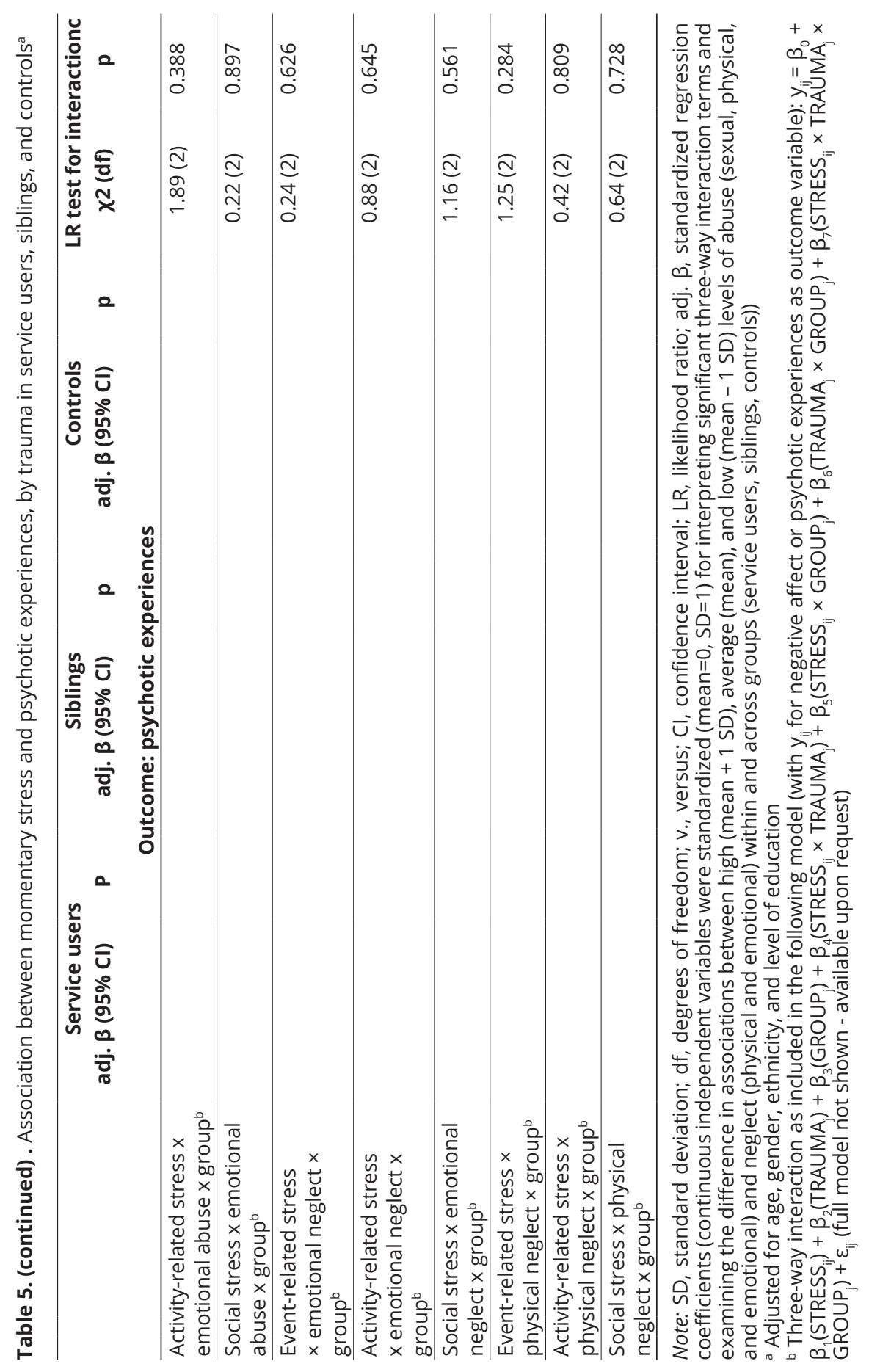




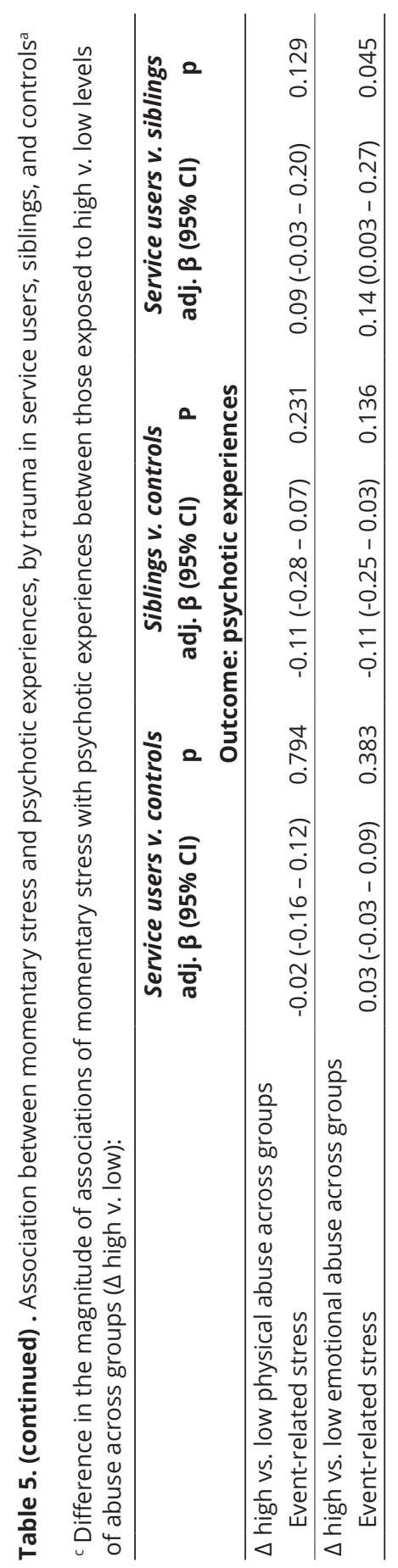




\section{Group comparisons (H3)}

We next investigated differences in magnitude of associations between those exposed to high $v$. low levels of abuse across groups. The difference in magnitude of associations of event-related $(B=0.23, p=0.075 ; B=0.15, p=0.011)$, activityrelated $(B=0.26, p=0.001 ; B=0.15, p=0.002)$, and social $(B=0.26, p=0.020)$ stress with negative affect between those exposed to high $v$. low levels of physical and emotional abuse were greater in service users than in controls. Similarly, the difference in magnitude of associations of social $(B=0.34, p=0.034)$ stress with negative affect between those exposed to high $v$. low levels of physical abuse was greater in siblings than in controls. Also, differences in magnitude of associations of activity-related $(B=0.23, p=0.044)$ stress with (i) negative affect and event-related ( $B=0.14, p=0.045$ ) stress with (ii) psychotic experiences between those exposed to high $v$. low levels of emotional abuse were greater in service users than in siblings.

\section{Association between stress, negative affect, and psychotic experiences by emotional- and physical neglect and group [H2 \& H3]}

As shown in Table 4, the association of various types of momentary stress with negative affect was modified by emotional and physical neglect, as indicated by significant 3-way interaction effects. In contrast, no evidence was found that emotional and physical neglect modified the association between momentary stress and psychotic experiences.

\section{Within groups $(\mathrm{H} 2)$}

Activity-related $(B=0.07, p=0.010 ; B=0.12, p<0.001)$ stress was associated with more intense negative affect in service users exposed to high $v$. low levels of emotional and physical neglect. In contrast, activity-related ( $B=-0.16, p=0.001)$ and social $(B=-0.13, p=0.058)$ stress were associated with less intense negative affect in controls exposed to high $v$. low levels of physical neglect. A mixed pattern of findings emerged in siblings.

\section{Group comparisons (H3)}

We found, across groups, differences in magnitude of associations between those exposed to high $v$. low levels of neglect. The difference in magnitude of associations 
of activity-related $(B=0.28, p<0.001)$ stress with negative affect between those exposed to high v. low levels of physical neglect was greater in service users than in controls. In contrast, a mixed pattern of findings on differences in the magnitude of associations between those exposed to high $v$. low levels of neglect were found in service users $v$. siblings and siblings $v$. controls.

\section{Discussion}

\section{Main findings}

Using a context-sensitive and ecologically valid experience sampling design, we found strong evidence that momentary stress was associated with increased (i) negative affect and (ii) psychotic experiences in service users and controls [H1]. In line with hypotheses [H2 \& H3], prior experiences of childhood trauma (physical and emotional abuse and, partially, emotional and physical neglect, but not sexual abuse) was found to impact on the stress sensitivity in daily life by modifying the association between momentary stress and (i) negative affect and (ii) psychotic experiences within and across service users and controls. While in service users more intense (i) negative affect and (ii) psychotic experiences were found when high $v$. low levels of various types of abuse and neglect were compared, controls showed, intriguingly, either less intense negative affect (evident in physical abuse and neglect) or no marked differences in the magnitude of associations, suggesting more resilience to the detrimental effects of $\mathrm{CT}$ on the stress sensitivity in daily life. A less consistent pattern of findings was evident in siblings.

\section{Methodological considerations}

Several methodological considerations should be considered when interpreting our findings. First, the CTQ used in the study is a retrospective, self-report measure of CT. One concern is that recall bias and cognitive distortion might affect ratings [75]. However, in the current study, the potential impact was minimized due to the lower age of the sample and, thus, the reduced time that had passed between exposure to and assessment of trauma. Similarly, while ESM allows the assessment of variables with high ecological validity, all ESM ratings were based on subjective self-reports. Hence, our findings require triangulation including other levels of investigation (e.g. biological markers and other socio-environmental factors) $[76,77]$. Nevertheless, ESM has recently been found to be a reliable and valid assessment method in a young nonclinical sample [78]. 
Second, ESM may be associated with assessment burden and, especially in the group of service users, selection bias cannot be ruled out. However, through an extensive ESM recruitment procedure there was, overall, a large proportion of participants with a sufficient number of valid responses $(90.8 \%$ with, on average, more than 42 responses in each group). Third, the sample size of the sibling group was small and inconsistent findings might have occurred due to sampling error in this intermediate risk group. Fourth, cross-sectional modelling of ESM data did not allow for investigating temporality of stress, negative affect and psychotic experiences. Time-lagged analyses of ESM data $[79,80]$ are required to investigate temporality as an important criterion to establish causality [81]. Fifth, although several potential confounders (i.e. age, gender, ethnicity, level of education) were assessed, other unmeasured confounders (e.g. direct or indirect measures of genetic risk, other childhood adversities, direct measures of shared genetic and socio-environmental risk of siblings) may have influenced reported findings. Results remained largely unchanged, however, when we controlled for clustering of observations within families (see Supplementary Tables S1 and S2). Last, individuals with a DSM-IV diagnosis and relatively high scores in various psychopathology measures (i.e. BDI-II, CAPE, STAI) were included in the control group. The rationale was to increase ecological validity as diagnoses and symptoms represent general population risk of morbidity and to overcome disadvantages including only a "super-healthy" control group $[82,83]$. Thus, passing the threshold to seek help in mental health services was the a priori defined exclusion criterion. However, when we performed a sensitivity analysis excluding all controls with DSM-IV diagnoses $(N=8)$, findings remained largely unchanged (see Supplementary Tables S3, S4, and S5).

\section{Comparison with previous research}

In recent years, evidence has accumulated that links experiences of abuse and neglect during childhood with the aetiology of a number of psychopathologies, including psychosis. Elevated stress sensitivity, characterized by heightened emotional reactivity and more intense psychotic experiences in response to minor hassles in the flow of daily life, has been proposed to constitute a putative underlying mechanism through which CT impacts on mental health. However, the number of studies conducted to date and the generalisability of recent findings to a help-seeking adolescent and young adult population remained limited. 
The present study found, for the first time, that, in help-seeking adolescents and young adults with, on average, high levels of depressive, anxiety, and psychotic symptoms, prior exposure to CT was associated with increased negative affect and psychotic experiences in response to momentary stress in daily life. This finding is in accordance with the proposition that CT may sensitize individuals to subsequent adversity and adds to recent findings [47-50]. In controls, when high $v$. low levels of CT were compared, momentary stress was, in contrast, associated with either less intense negative affect (evident in physical abuse and physical neglect) or no marked differences in the magnitude of associations. This finding of qualitative interaction in service users and controls represents an important finding, as it suggests that non-help seeking controls with higher levels of CT were less sensitive and more resilient towards minor stress in daily life. In siblings, however, we found no evidence that stress sensitivity was increased in daily life and findings on elevated stress sensitivity in those exposed to CT were less consistent in this group than in service users. This may in part be explained by the lower levels of CT (in particular, physical and emotional neglect) in siblings than in service user. Also, given only some of the siblings will go on to develop a mental disorder, they may have formed a group of resilient and non-resilient individuals, and, thus, only some evidence of elevated stress sensitivity in those with higher levels of CT was observed at a group level. However, the results should be interpreted with caution as the sample size of the sibling group was small and inconsistent, and, thus, findings may have occurred due to sampling error. Consistent with some ESM studies in help-seeking individuals and controls [48, 70, 84], we found no difference in aggregate ESM scores of momentary stress across groups, but elevated sensitivity to momentary stress in service users compared to controls and siblings. This tentatively suggests that it is not higher overall levels of perceived daily stress per se, but increased sensitivity to stress that is of relevance for mental health in help-seeking adolescents and young adults.

Reported findings in controls partly echoes findings in which adult controls exposed to high levels of sexual abuse reported less intense negative affect and psychotic experiences in response to minor socio-environmental stressors [50]. It corroborates, more generally, findings from previous research that a significant proportion of individuals exposed to childhood adversity do show resilience to develop any form of psychopathology and maintain psychosocial functioning [85-87]. Our findings suggest that CT does not inevitably lead to an increased 
sensitization to subsequent adversity. Instead, how CT impacts on mental health through stress sensitivity may depend on both individuals' vulnerability and various protective factors of biopsychosocial nature [87-89]. Several psychological, socioenvironmental, and biological factors have been posited to contribute to offset the detrimental impact of adversity such as strong social networks and secure attachment, high-quality interpersonal relationships, good cognitive, social, and emotional skills, and a lower polygenic risk [85-87].

In line with observations of undefined clinical pictures and non-specific mental health symptoms at a developmentally early stage of psychopathology [2,5], high levels of depressive, anxiety, and psychotic symptoms as well as high proportions of non-specific diagnoses and comorbidity were observed in the group of service users, which may further support previously proposed extended transdiagnostic phenotypes of psychopathology [11, 12, 14, 15,90]. Notably, our study found that CT impacts more consistently on the association between momentary stress and negative affect and, thus, the emotional reactivity in daily life. This may be viewed, while speculative, in the context of proposed affective pathways to psychosis [91, 92] and recent integrated socio-developmental models [41, 43, 88, 93], in which the importance of premorbid anxiety and depressive symptoms and impairments in cognitive, motor, and social domains are posited to be manifest many years before prodromal symptoms and the first psychotic episode. Critically, and despite strong evidence from several studies, sexual abuse was not found to amplify individuals' stress sensitivity in our study. This may, in part, be explained by higher levels of trauma repression (i.e. amnesia for abuse memories) or impeded disclosure [94, 95], indicated by higher levels of self-reported sexual abuse in help-seeking adults compared to help-seeking adolescents and young adults and older and younger community samples [96-99].

Taken together, our findings suggest that stress sensitivity may be an important underlying risk or resilience mechanism of a transdiagnostic phenotype of depression, anxiety, and psychosis through which CT may impact on mental health in youth. While primary prevention of childhood trauma remains the ultimate - but difficult to achieve - goal, there is a need to carefully assess CT in youth mental health services and continue to strengthen the evidence on its impact (including the impact of clustering of CT within families) as basis for developing 
and evaluating evidence-based treatments in order to tackle the consequences of $\mathrm{CT}$ in help-seeking adolescents and young adults.

This may be addressed through developing and evaluating ecological momentary interventions that directly target elevated stress sensitivity in specific contexts in the real-world and real-time through interactive delivery schemes $[100,101]$ to prevent adverse clinical and social outcomes later in life. 


\section{References}

1. Kessler, R.C., et al., Lifetime prevalence and age-of-onset distributions of DSM-IV disorders in the National Comorbidity Survey Replication. Arch Gen Psychiatry, 2005. 62(6): p. 593-602.

2. McGorry, P. and J. van Os, Redeeming diagnosis in psychiatry: timing versus specificity. Lancet, 2013. 381(9863): p. 343-5.

3. McGorry, P. and B. Nelson, Why We Need a Transdiagnostic Staging Approach to Emerging Psychopathology, Early Diagnosis, and Treatment. JAMA Psychiatry, 2016. 73(3): p. 191-2.

4. Malla, A., et al., From early intervention in psychosis to youth mental health reform: a review of the evolution and transformation of mental health services for young people. Soc Psychiatry Psychiatr Epidemiol, 2016. 51(3): p. 319-26.

5. van Os, J., The dynamics of subthreshold psychopathology: implications for diagnosis and treatment. Am J Psychiatry, 2013. 170(7): p. 695-8.

6. Kelleher, I., et al., Prevalence of psychotic symptoms in childhood and adolescence: a systematic review and meta-analysis of population-based studies. Psychol Med, 2012. 42(9): p. 1857-63.

7. Linscott, R.J. and J. van Os, An updated and conservative systematic review and metaanalysis of epidemiological evidence on psychotic experiences in children and adults: on the pathway from proneness to persistence to dimensional expression across mental disorders. Psychol Med, 2013. 43(6): p. 1133-49.

8. McGrath, J.J., et al., Psychotic Experiences in the General Population: A Cross-National Analysis Based on 31,261 Respondents From 18 Countries. JAMA Psychiatry, 2015. 72(7): p. 697-705.

9. Fisher, H.L., et al., Specificity of childhood psychotic symptoms for predicting schizophrenia by 38 years of age: a birth cohort study. Psychol Med, 2013. 43(10): p. 2077-86.

10. Fusar-Poli, P., et al., Comorbid depressive and anxiety disorders in 509 individuals with an at-risk mental state: impact on psychopathology and transition to psychosis. Schizophr Bull, 2014. 40(1): p. 120-31.

11. van Os, J. and U. Reininghaus, Psychosis as a transdiagnostic and extended phenotype in the general population. World Psychiatry, 2016. 15(2): p. 118-24.

12. Caspi, A., et al., The p Factor: One General Psychopathology Factor in the Structure of Psychiatric Disorders? Clin Psychol Sci, 2014. 2(2): p. 119-137.

13. Craddock, N. and M.J. Owen, The Kraepelinian dichotomy - going, going... but still not gone. Br J Psychiatry, 2010. 196(2): p. 92-5.

14. Eaton, N.R., et al., Transdiagnostic factors of psychopathology and substance use disorders: a review. Soc Psychiatry Psychiatr Epidemiol, 2015. 50(2): p. 171-82.

15. Patalay, P., et al., A general psychopathology factor in early adolescence. Br J Psychiatry, 2015. 207(1): p. 15-22.

16. Shevlin, M., et al., The Psychosis Continuum: Testing a Bifactor Model of Psychosis in a General Population Sample. Schizophr Bull, 2017. 43(1): p. 133-141. 
17. Reininghaus, U., et al., Evaluation of the validity and utility of a transdiagnostic psychosis dimension encompassing schizophrenia and bipolar disorder. Br J Psychiatry, 2016. 209(2): p. 107-13.

18. Reininghaus, U., S. Priebe, and R.P. Bentall, Testing the psychopathology of psychosis: evidence for a general psychosis dimension. Schizophr Bull, 2013. 39(4): p. 884-95.

19. van Os, J. and R.J. Linscott, Introduction: The extended psychosis phenotype-relationship with schizophrenia and with ultrahigh risk status for psychosis. Schizophr Bull, 2012. 38(2): p. 227-30.

20. Morgan, C. and H. Fisher, Environment and schizophrenia: environmental factors in schizophrenia: childhood trauma--a critical review. Schizophr Bull, 2007. 33(1): p. 3-10.

21. Matheson, S.L., et al., Childhood adversity in schizophrenia: a systematic meta-analysis. Psychol Med, 2013. 43(2): p. 225-38.

22. Morgan, C. and C. Gayer-Anderson, Childhood adversities and psychosis: evidence, challenges, implications. World Psychiatry, 2016. 15(2): p. 93-102.

23. Varese, F., et al., Childhood adversities increase the risk of psychosis: a meta-analysis of patient-control, prospective- and cross-sectional cohort studies. Schizophr Bull, 2012. 38(4): p. 661-71.

24. Trotta, A., R.M. Murray, and H.L. Fisher, The impact of childhood adversity on the persistence of psychotic symptoms: a systematic review and meta-analysis. Psychol Med, 2015. 45(12): p. 2481-98.

25. Heins, M., et al., Childhood trauma and psychosis: a case-control and case-sibling comparison across different levels of genetic liability, psychopathology, and type of trauma. Am J Psychiatry, 2011. 168(12): p. 1286-94.

26. Holshausen, K., C.R. Bowie, and K.L. Harkness, The Relation of Childhood Maltreatment to Psychotic Symptoms in Adolescents and Young Adults With Depression. J Clin Child Adolesc Psychol, 2016. 45(3): p. 241-7.

27. Daalman, K., et al., Childhood trauma and auditory verbal hallucinations. Psychol Med, 2012. 42(12): p. 2475-84.

28. Bentall, R.P., et al., From adversity to psychosis: pathways and mechanisms from specific adversities to specific symptoms. Soc Psychiatry Psychiatr Epidemiol, 2014. 49(7): p. 1011-22.

29. Harley, M., et al., Cannabis use and childhood trauma interact additively to increase the risk of psychotic symptoms in adolescence. Psychol Med, 2010. 40(10): p. 1627-34.

30. Kelleher, I., et al., Associations between childhood trauma, bullying and psychotic symptoms among a school-based adolescent sample. Br J Psychiatry, 2008. 193(5): p. 378-82.

31. Read, J., et al., Childhood trauma, psychosis and schizophrenia: a literature review with theoretical and clinical implications. Acta Psychiatr Scand, 2005. 112(5): p. 330-50.

32. McGrath, J.J., et al., The association between childhood adversities and subsequent first onset of psychotic experiences: a cross-national analysis of 23998 respondents from 17 countries. Psychol Med, 2017: p. 1-16.

33. Arseneault, L., et al., Childhood trauma and children's emerging psychotic symptoms: A genetically sensitive longitudinal cohort study. Am J Psychiatry, 2011. 168(1): p. 65-72. 
34. Brown, J., et al., Childhood abuse and neglect: specificity of effects on adolescent and young adult depression and suicidality. J Am Acad Child Adolesc Psychiatry, 1999. 38(12): p. 1490-6.

35. Chen, L.P., et al., Sexual abuse and lifetime diagnosis of psychiatric disorders: systematic review and meta-analysis. Mayo Clin Proc, 2010. 85(7): p. 618-29.

36. Gilbert, R., et al., Burden and consequences of child maltreatment in high-income countries. Lancet, 2009. 373(9657): p. 68-81.

37. McLaughlin, K.A., et al., Childhood adversities and adult psychiatric disorders in the national comorbidity survey replication II: associations with persistence of DSM-IV disorders. Arch Gen Psychiatry, 2010. 67(2): p. 124-32.

38. Nanni, V., R. Uher, and A. Danese, Childhood maltreatment predicts unfavorable course of illness and treatment outcome in depression: a meta-analysis. Am J Psychiatry, 2012. 169(2): p. 141-51.

39. Green, J.G., et al., Childhood adversities and adult psychiatric disorders in the national comorbidity survey replication I: associations with first onset of DSM-IV disorders. Arch Gen Psychiatry, 2010. 67(2): p. 113-23.

40. van Nierop, M., et al., Childhood trauma is associated with a specific admixture of affective, anxiety, and psychosis symptoms cutting across traditional diagnostic boundaries. Psychol Med, 2015. 45(6): p. 1277-88.

41. Howes, O.D. and R.M. Murray, Schizophrenia: an integrated sociodevelopmentalcognitive model. Lancet, 2014. 383(9929): p. 1677-87.

42. Morgan, C., et al., Modelling the interplay between childhood and adult adversity in pathways to psychosis: initial evidence from the AESOP study. Psychol Med, 2014. 44(2): p. 407-19.

43. Morgan, C., et al., Migration, ethnicity, and psychosis: toward a sociodevelopmental model. Schizophr Bull, 2010. 36(4): p. 655-64.

44. Myin-Germeys, I., et al., Emotional reactivity to daily life stress in psychosis. Arch Gen Psychiatry, 2001. 58(12): p. 1137-44.

45. Kendler, K.S., J.W. Kuhn, and C.A. Prescott, Childhood sexual abuse, stressful life events and risk for major depression in women. Psychol Med, 2004. 34(8): p. 1475-82.

46. Hammen, C., R. Henry, and S.E. Daley, Depression and sensitization to stressors among young women as a function of childhood adversity. Journal of Consulting and Clinical Psychology, 2000. 68(5): p. 782-787.

47. Wichers, M., et al., Mechanisms of gene-environment interactions in depression: evidence that genes potentiate multiple sources of adversity. Psychol Med, 2009. 39(7): p. 1077-86.

48. Glaser, J.P., et al., Childhood trauma and emotional reactivity to daily life stress in adult frequent attenders of general practitioners. J Psychosom Res, 2006. 61(2): p. 229-36.

49. Lardinois, M., et al., Childhood trauma and increased stress sensitivity in psychosis. Acta Psychiatr Scand, 2011. 123(1): p. 28-35.

50. Reininghaus, U., et al., Psychological processes underlying the association between childhood trauma and psychosis in daily life: an experience sampling study. Psychol Med, 2016. 46(13): p. 2799-813. 
51. Palmier-Claus, J.E., et al., Experience sampling research in individuals with mental illness: reflections and guidance. Acta Psychiatr Scand, 2011. 123(1): p. 12-20.

52. Briere, J. and D.M. Elliott, Prevalence and psychological sequelae of self-reported childhood physical and sexual abuse in a general population sample of men and women. Child Abuse Negl, 2003. 27(10): p. 1205-22.

53. MacMillan, H.L., et al., Prevalence of child physical and sexual abuse in the community. Results from the Ontario Health Supplement. JAMA, 1997. 278(2): p. 131-5.

54. Lichtenstein, P., et al., Common genetic determinants of schizophrenia and bipolar disorder in Swedish families: a population-based study. Lancet, 2009. 373(9659): p. 2349.

55. van Os, J., B.P. Rutten, and R. Poulton, Gene-environment interactions in schizophrenia: review of epidemiological findings and future directions. Schizophr Bull, 2008. 34(6): p. 1066-82.

56. Shih, R.A., P.L. Belmonte, and P.P. Zandi, A review of the evidence from family, twin and adoption studies for a genetic contribution to adult psychiatric disorders. Int Rev Psychiatry, 2004. 16(4): p. 260-83.

57. Bernstein, D., Fink, L, Childhood Trauma Questionnaire: a Retrospective Self-report Manual. 1998, San Antonio, Texas: The Psychological Corporation.

58. Bernstein, D.P., et al., Validity of the Childhood Trauma Questionnaire in an adolescent psychiatric population. J Am Acad Child Adolesc Psychiatry, 1997. 36(3): p. 340-8.

59. Bernstein, D.P., et al., Development and validation of a brief screening version of the Childhood Trauma Questionnaire. Child Abuse Negl, 2003. 27(2): p. 169-90.

60. Thombs, B.D., et al., A validation study of the Dutch Childhood Trauma QuestionnaireShort Form: factor structure, reliability, and known-groups validity. Child Abuse Negl, 2009. 33(8): p. 518-23.

61. Spielberger, C.D., Gorsuch, R.L., Lushene, P.R., Vagg, P.R., \& Jacobs, G.A, Manual for the State-Trait Anxiety Inventory. 1983, Palo Alto, CA: Consulting Psychologists Press.

62. Van der Ploeg, H.M., De Zelf-Beoordelings Vragenlijst (STAI-DY). Tijdschrift voor psychiatrie, 1982. 24(9): p. 576-88.

63. Dozois, D.J.A., K.S. Dobson, and J.L. Ahnberg, A psychometric evaluation of the Beck Depression Inventory - II. Psychological Assessment, 1998. 10(2): p. 83-89.

64. Konings, M., et al., Validity and reliability of the CAPE: a self-report instrument for the measurement of psychotic experiences in the general population. Acta Psychiatr Scand, 2006. 114(1): p. 55-61.

65. Osman, A., et al., Psychometric properties of the Beck Depression Inventory-II in nonclinical adolescent samples. J Clin Psychol, 2008. 64(1): p. 83-102.

66. Osman, A., et al., Reliability and validity of the Beck depression inventory--Il with adolescent psychiatric inpatients. Psychol Assess, 2004. 16(2): p. 120-32.

67. Myin-Germeys, I., et al., Experience sampling research in psychopathology: opening the black box of daily life. Psychol Med, 2009. 39(9): p. 1533-47.

68. Shiffman, S., A.A. Stone, and M.R. Hufford, Ecological momentary assessment. Annu Rev Clin Psychol, 2008. 4(1): p. 1-32. 
69. Myin-Germeys, I., M. Birchwood, and T. Kwapil, From environment to therapy in psychosis: a real-world momentary assessment approach. Schizophr Bull, 2011. 37(2): p. 244-7.

70. Palmier-Claus, J.E., G. Dunn, and S.W. Lewis, Emotional and symptomatic reactivity to stress in individuals at ultra-high risk of developing psychosis. Psychol Med, 2012. 42(5): p. 1003-12.

71. Myin-Germeys, I., P. Delespaul, and J. van Os, Behavioural sensitization to daily life stress in psychosis. Psychol Med, 2005. 35(5): p. 733-41.

72. StataCorp. 2013, StataCorp LP: College Station, TX.

73. Aiken, L.S., West, S. G., Multiple regression: Testing and interpreting interactions. 1991, Thousand Oaks, CA: Sage Publications.

74. Cohen, J., Cohen, P, West, S, Aiken, L, Applied Multiple Regression/Correlation Analysis for the Behavioral Sciences. 2003, Mahwah, NJ: Erlbaum.

75. Maughan, B. and M. Rutter, Retrospective reporting of childhood adversity: issues in assessing long-term recall. J Pers Disord, 1997. 11(1): p. 19-33.

76. Hernaus, D., et al., Psychotic reactivity to daily life stress and the dopamine system: a study combining experience sampling and [18F]fallypride positron emission tomography. J Abnorm Psychol, 2015. 124(1): p. 27-37.

77. Myin-Germeys, I., et al., Subtle fluctuations in psychotic phenomena as functional states of abnormal dopamine reactivity in individuals at risk. Biol Psychiatry, 2005. 58(2): p. 105-10.

78. Cristobal-Narvaez, P., et al., Impact of Adverse Childhood Experiences on Psychotic-Like Symptoms and Stress Reactivity in Daily Life in Nonclinical Young Adults. PLoS One, 2016. 11(4): p. e0153557.

79. Kramer, I., et al., Time-lagged moment-to-moment interplay between negative affect and paranoia: new insights in the affective pathway to psychosis. Schizophr Bull, 2014. 40(2): p. 278-86.

80. Wichers, M., et al., A time-lagged momentary assessment study on daily life physical activity and affect. Health Psychol, 2012. 31(2): p. 135-44.

81. Hill, A.B., The Environment and Disease: Association or Causation? Proc R Soc Med, 1965. 58: p. 295-300.

82. Lee, W., et al., Bias in psychiatric case-control studies: literature survey. Br J Psychiatry, 2007. 190: p. 204-9.

83. Schwartz, S. and E. Susser, The use of well controls: an unhealthy practice in psychiatric research. Psychol Med, 2011. 41(6): p. 1127-31.

84. Myin-Germeys, I., et al., Emotional reactivity to daily life stress in psychosis and affective disorder: an experience sampling study. Acta Psychiatr Scand, 2003. 107(2): p. 124-31.

85. Collishaw, S., et al., Resilience to adult psychopathology following childhood maltreatment: evidence from a community sample. Child Abuse Negl, 2007. 31(3): p. 211-29.

86. Jaffee, S.R., et al., Individual, family, and neighborhood factors distinguish resilient from non-resilient maltreated children: a cumulative stressors model. Child Abuse Negl, 2007. 31(3): p. 231-53. 
87. Rutten, B.P., et al., Resilience in mental health: linking psychological and neurobiological perspectives. Acta Psychiatr Scand, 2013. 128(1): p. 3-20.

88. Owen, M.J., A. Sawa, and P.B. Mortensen, Schizophrenia. The Lancet, 2016. 388(10039): p. 86-97.

89. Belsky, J. and M. Pluess, Beyond diathesis stress: differential susceptibility to environmental influences. Psychol Bull, 2009. 135(6): p. 885-908.

90. Carragher, N., et al., The structure of adolescent psychopathology: a symptom-level analysis. Psychol Med, 2016. 46(5): p. 981-94.

91. Myin-Germeys, I. and J. van Os, Stress-reactivity in psychosis: evidence for an affective pathway to psychosis. Clin Psychol Rev, 2007. 27(4): p. 409-24.

92. Klippel, A., et al., Modeling the Interplay Between Psychological Processes and Adverse, Stressful Contexts and Experiences in Pathways to Psychosis: An Experience Sampling Study. Schizophr Bull, 2017. 43(2): p. 302-315.

93. van Os, J., G. Kenis, and B.P. Rutten, The environment and schizophrenia. Nature, 2010. 468(7321): p. 203-12.

94. Chu, J.A., et al., Memories of childhood abuse: dissociation, amnesia, and corroboration. Am J Psychiatry, 1999. 156(5): p. 749-55.

95. Pope, H.G. and J.I. Hudson, Can memories of childhood sexual abuse be repressed? Psychological Medicine, 1995. 25(01): p. 121.

96. MacDonald, K., et al., Minimization of Childhood Maltreatment Is Common and Consequential: Results from a Large, Multinational Sample Using the Childhood Trauma Questionnaire. PLoS One, 2016. 11(1): p. e0146058.

97. Sahin, S., et al., The history of childhood trauma among individuals with ultra high risk for psychosis is as common as among patients with first-episode schizophrenia. Early Interv Psychiatry, 2013. 7(4): p. 414-20.

98. Paivio, S.C. and K.M. Cramer, Factor structure and reliability of the Childhood Trauma Questionnaire in a Canadian undergraduate student sample. Child Abuse Negl, 2004. 28(8): p. 889-904.

99. Reininghaus, U., et al., Stress Sensitivity, Aberrant Salience, and Threat Anticipation in Early Psychosis: An Experience Sampling Study. Schizophr Bull, 2016. 42(3): p. 712-22.

100. Myin-Germeys, I., et al., Ecological momentary interventions in psychiatry. Curr Opin Psychiatry, 2016. 29(4): p. 258-63.

101. Reininghaus, U., C.A. Depp, and I. Myin-Germeys, Ecological Interventionist Causal Models in Psychosis: Targeting Psychological Mechanisms in Daily Life. Schizophr Bull, 2016. 42(2): p. 264-9. 


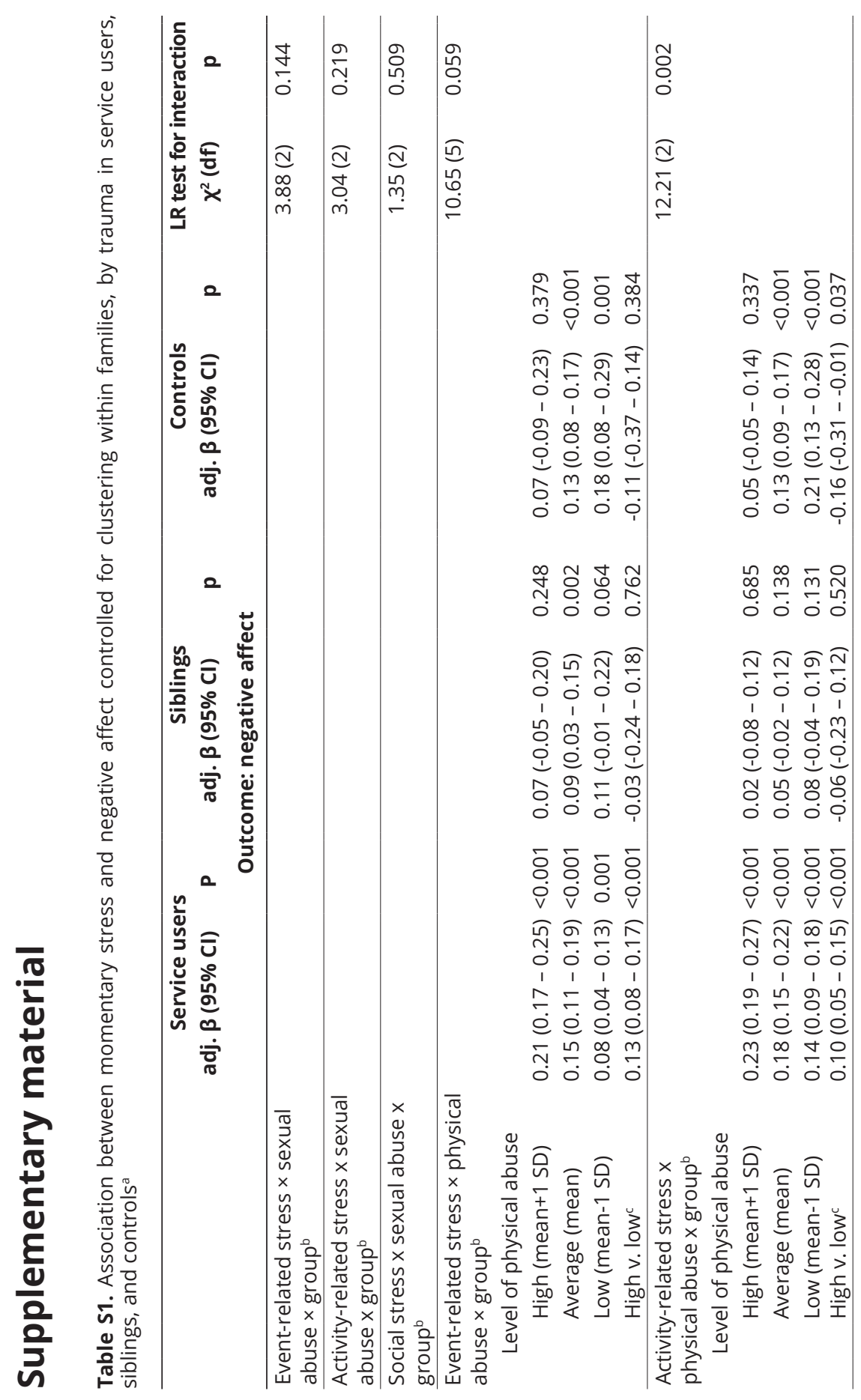




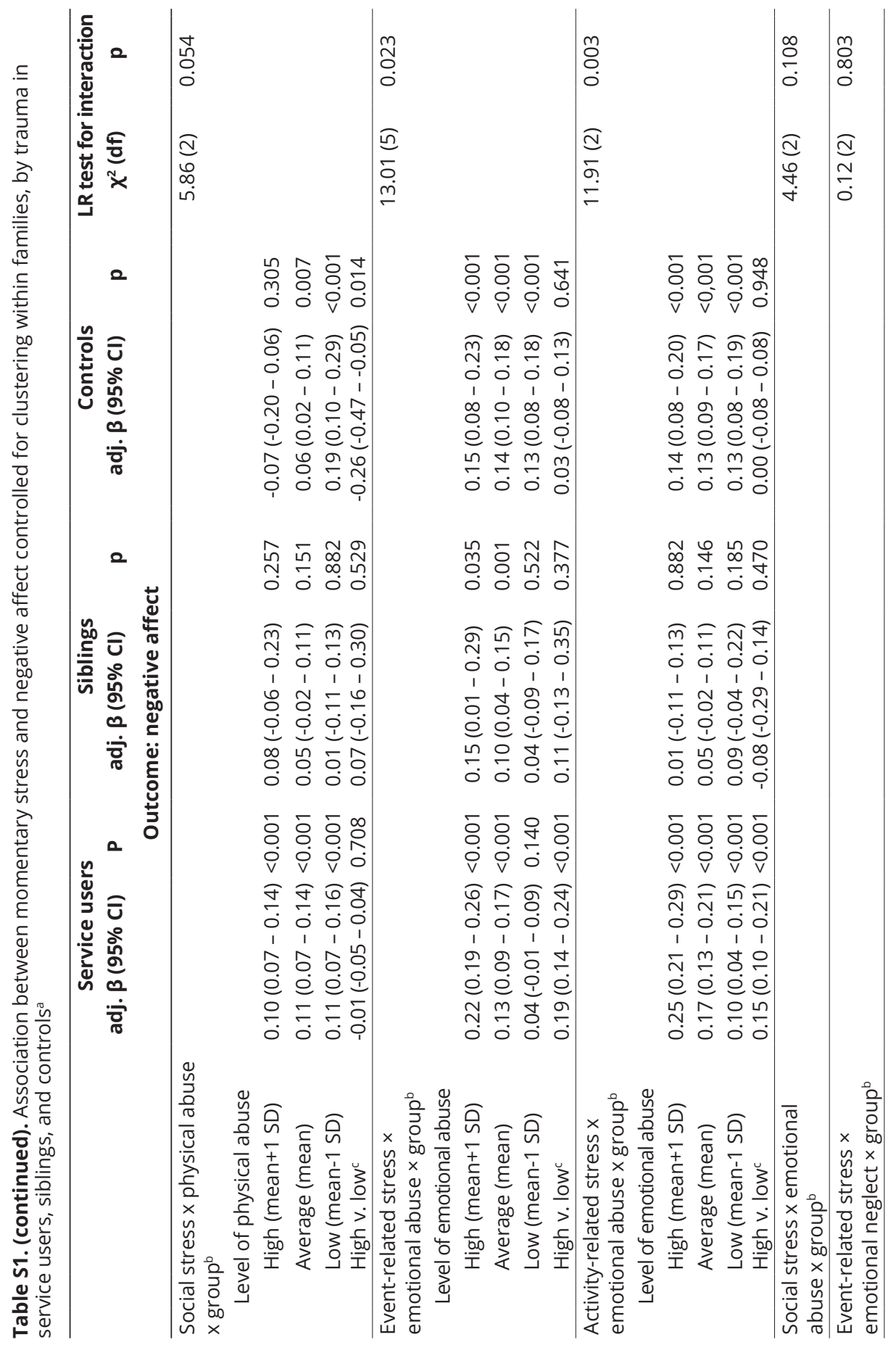




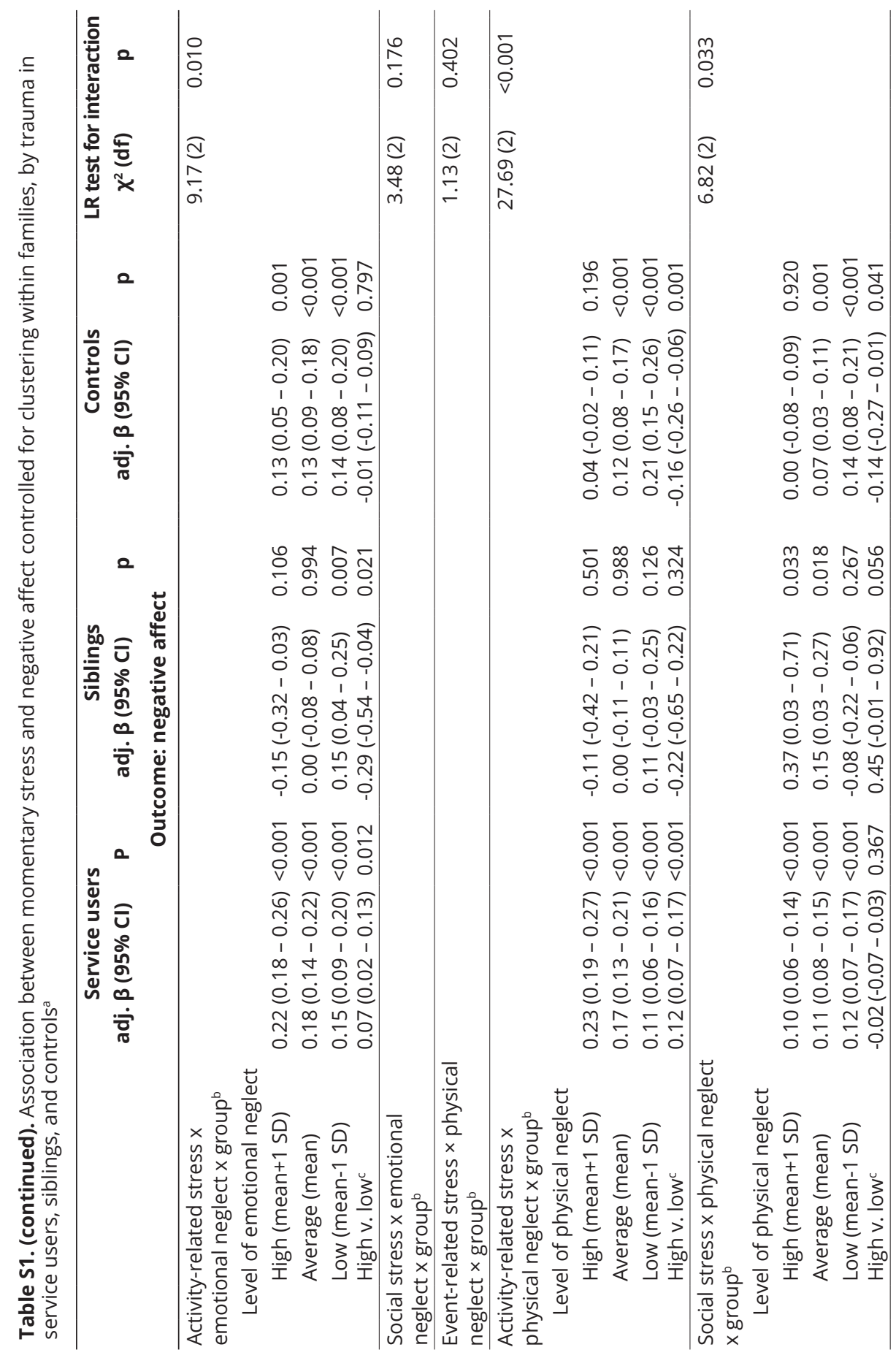




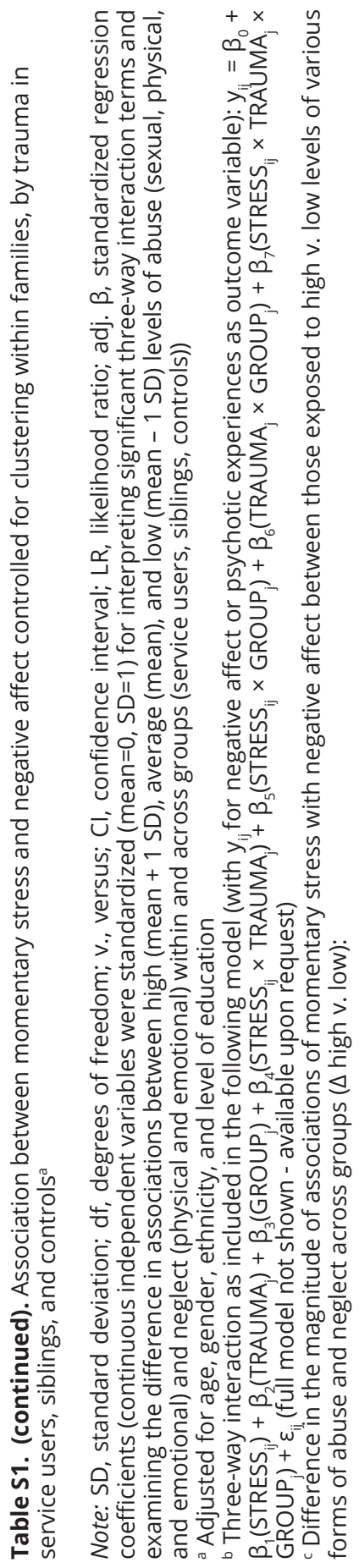




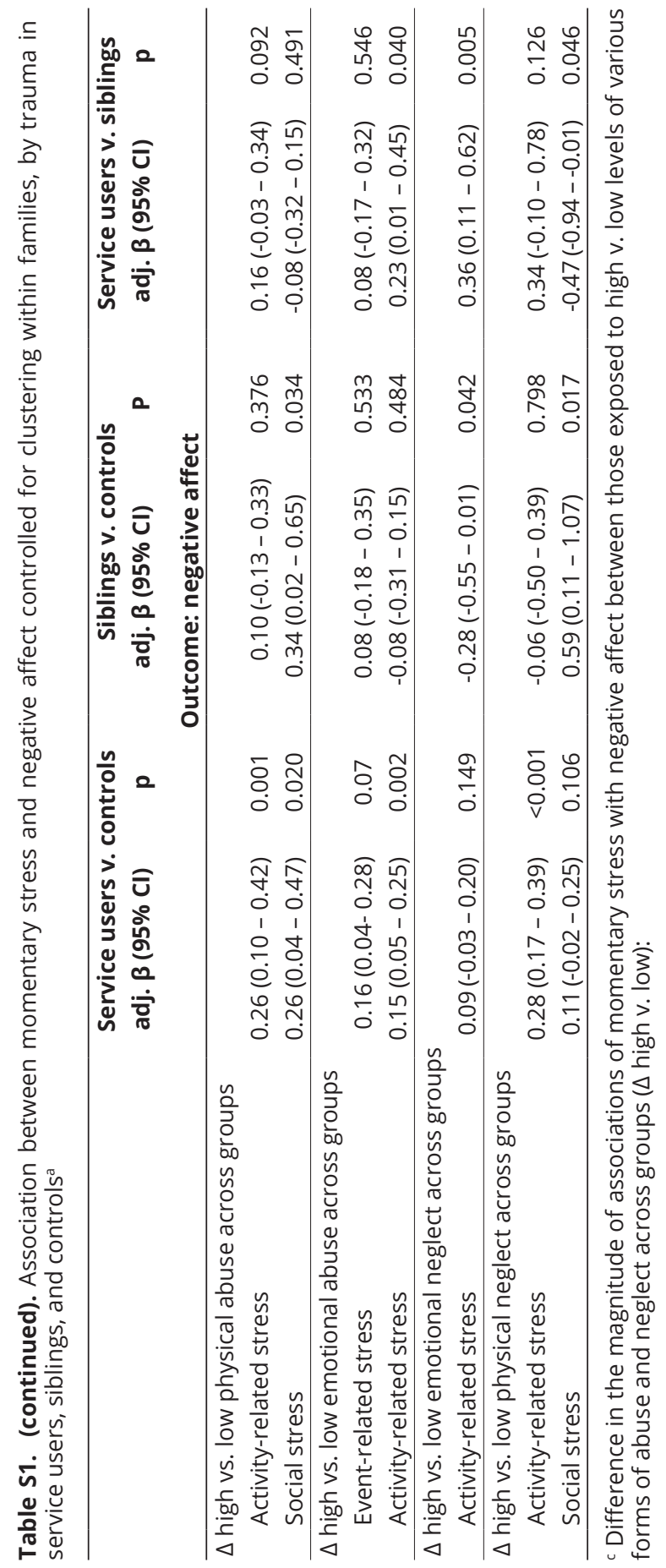




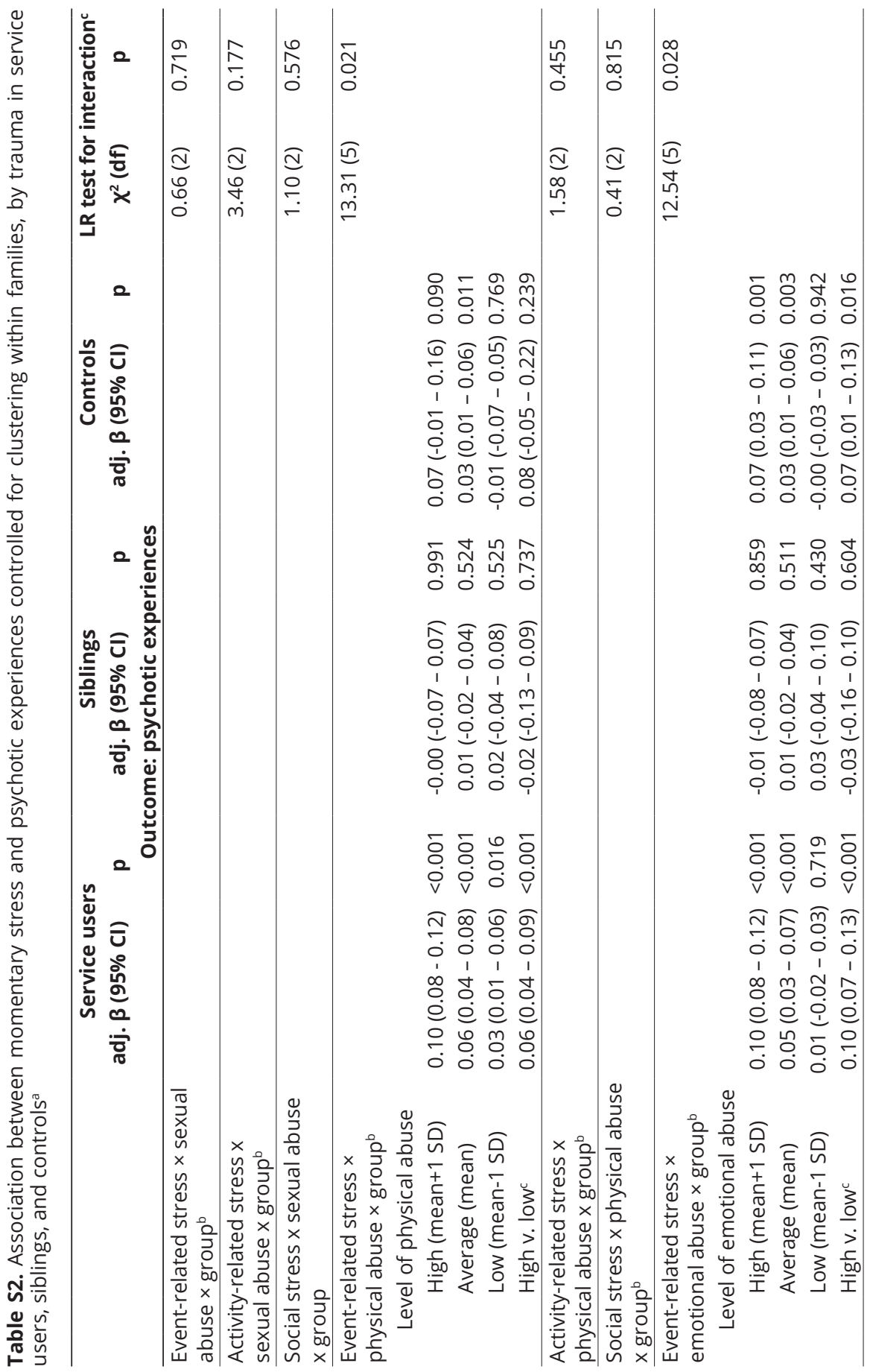




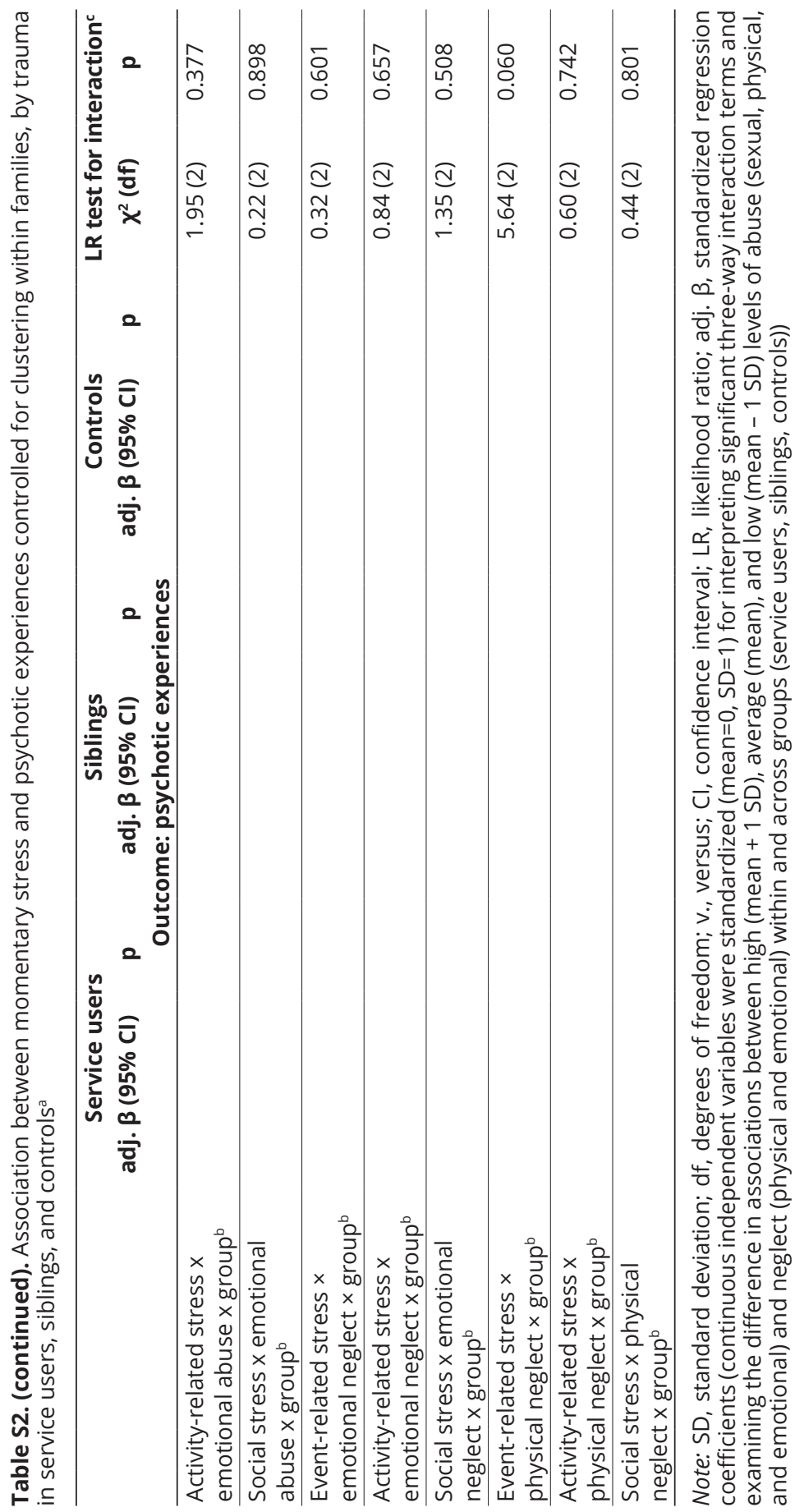




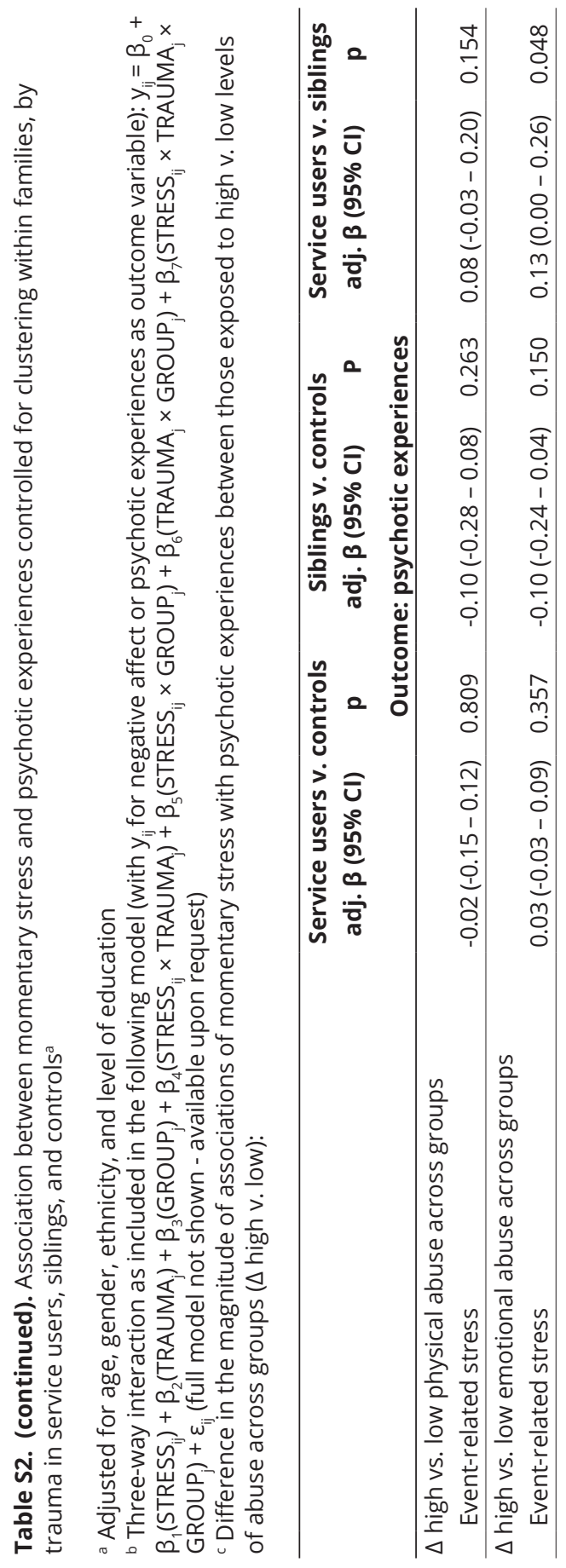




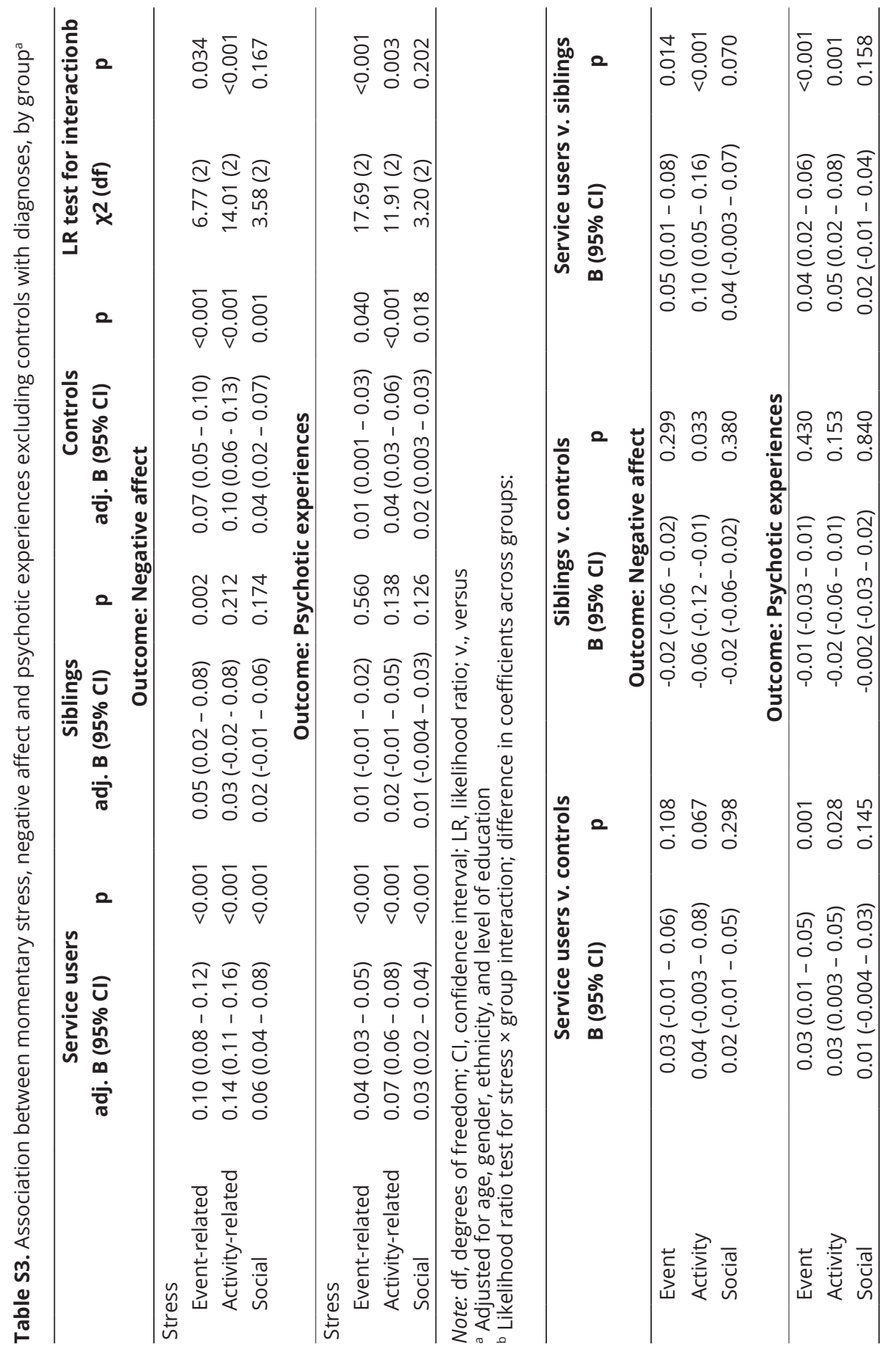




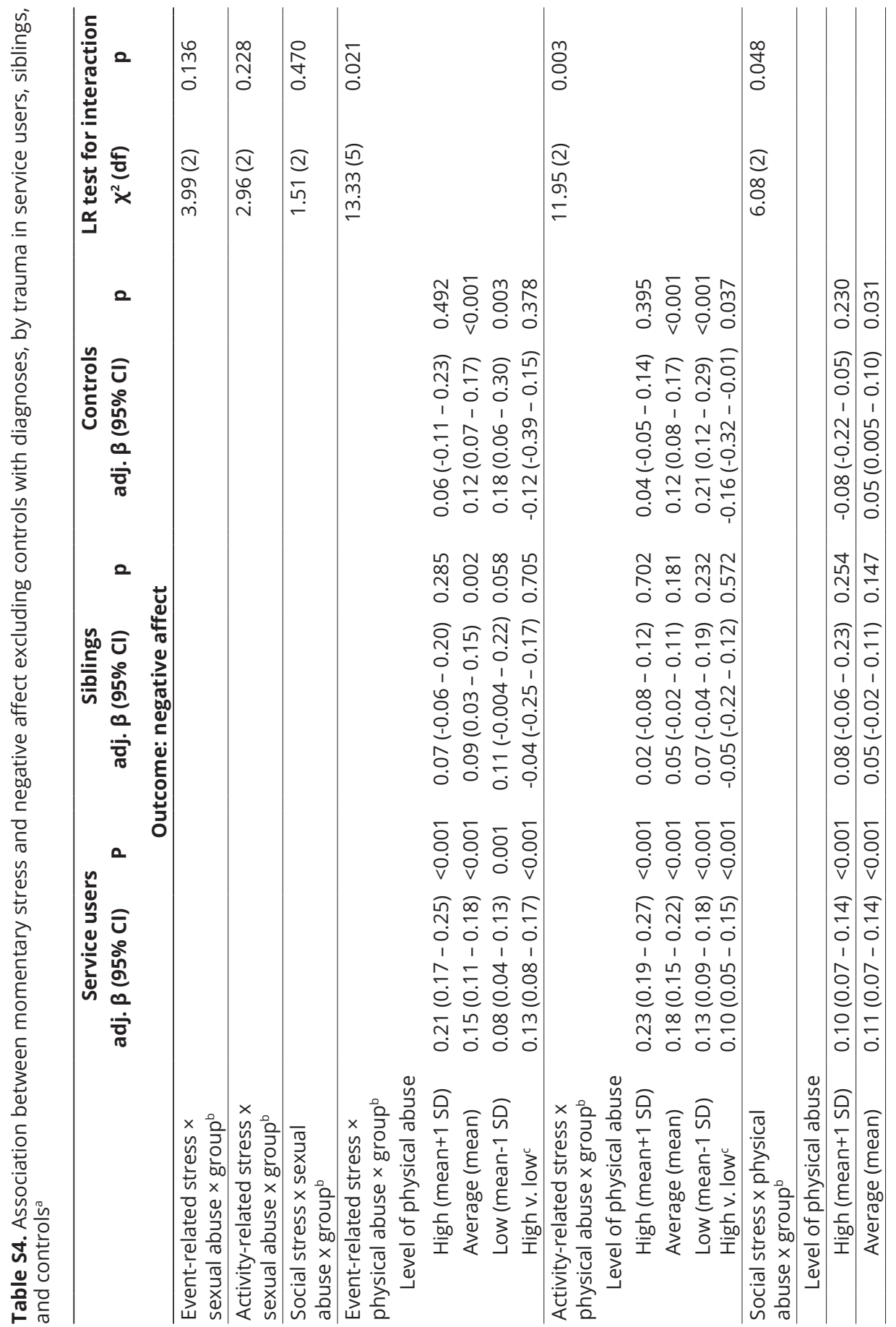




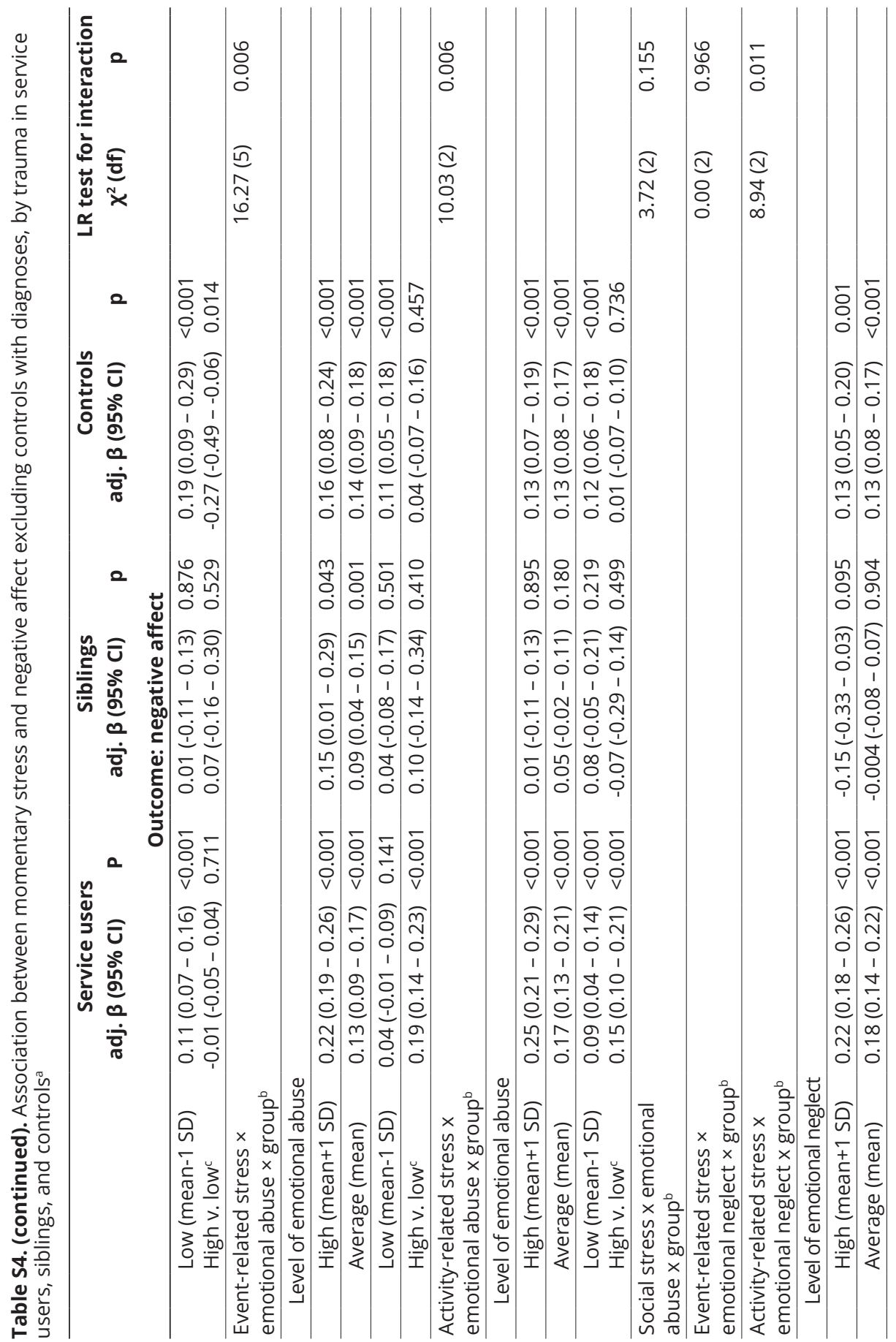




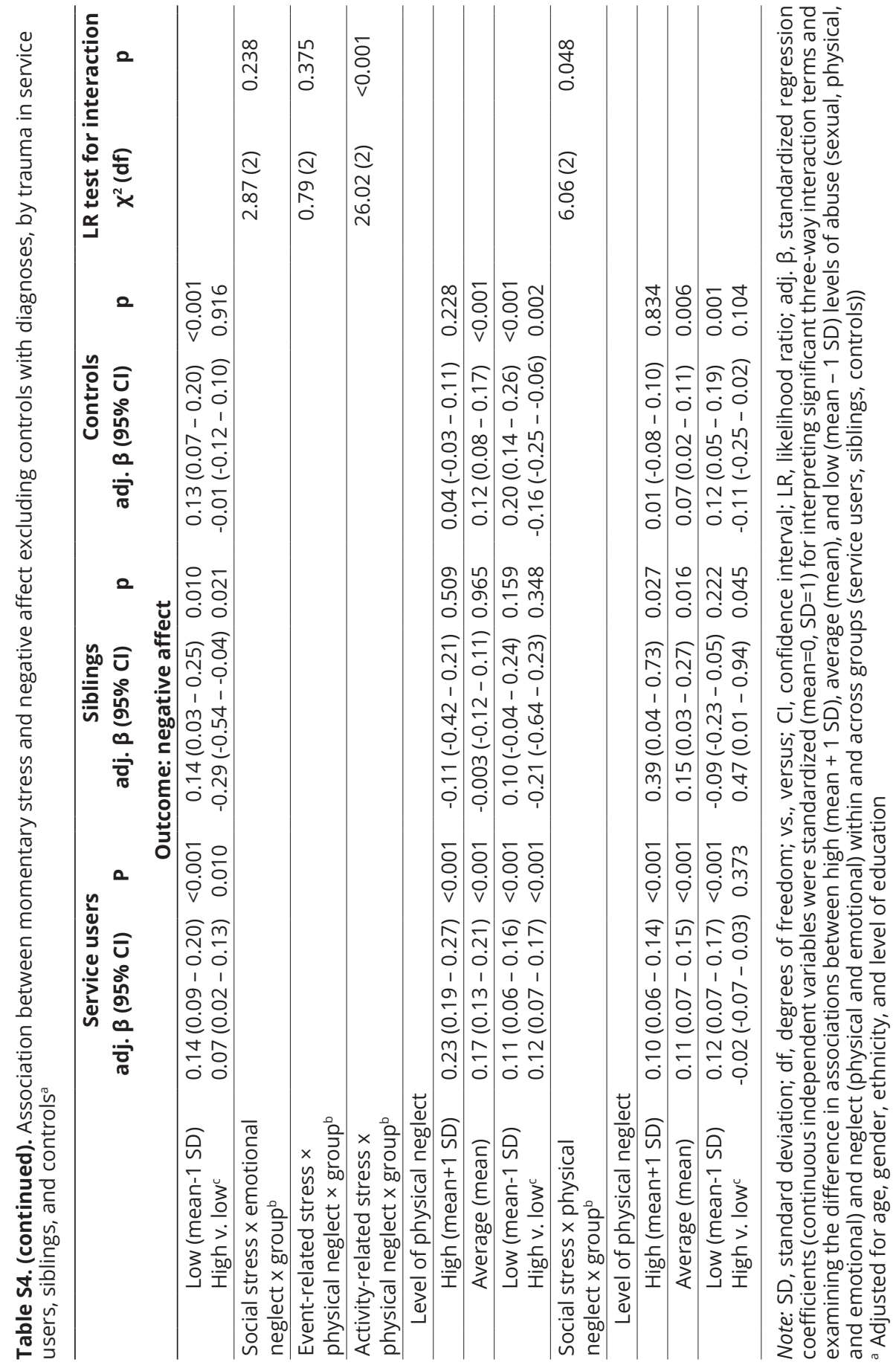




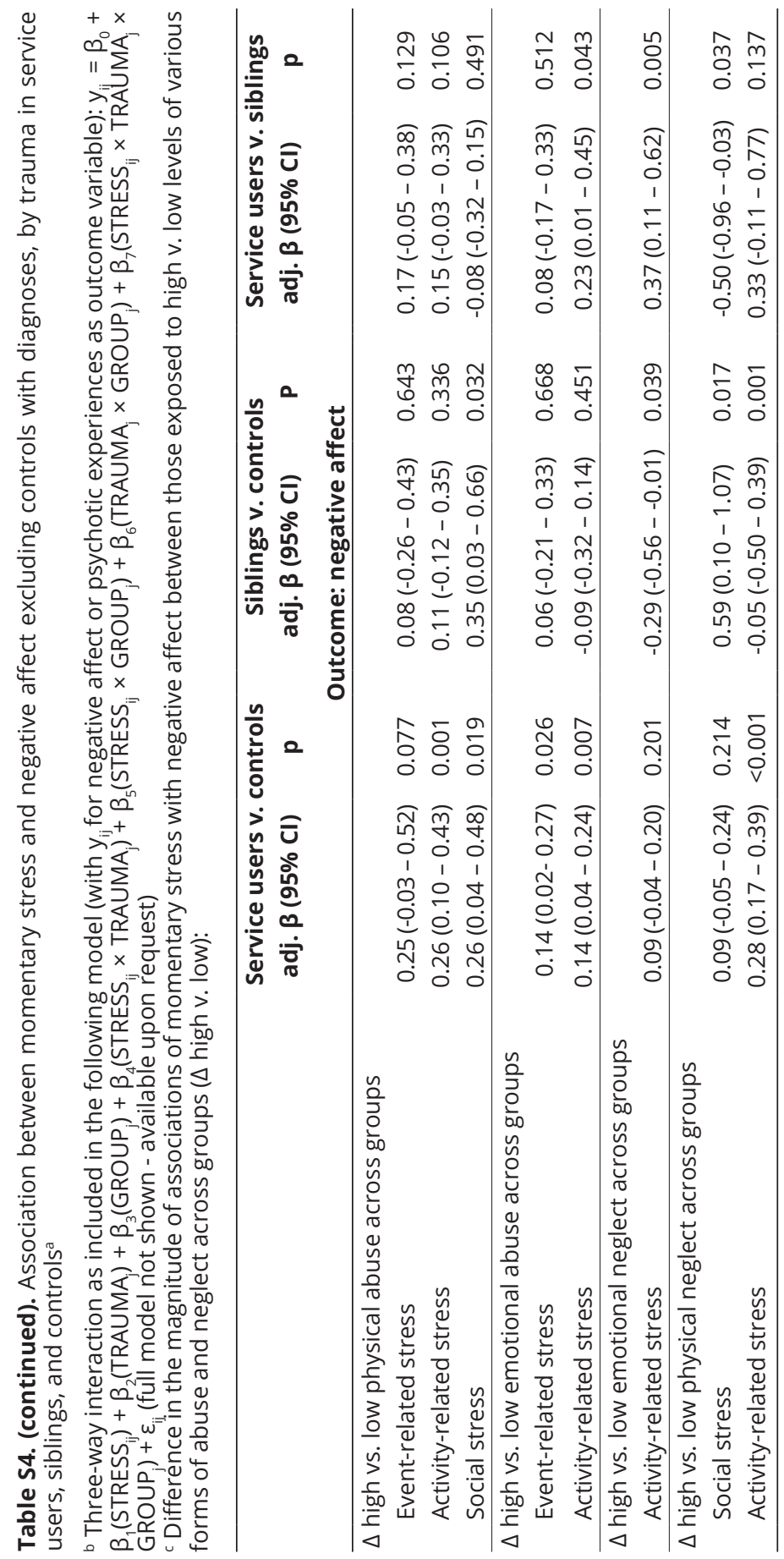




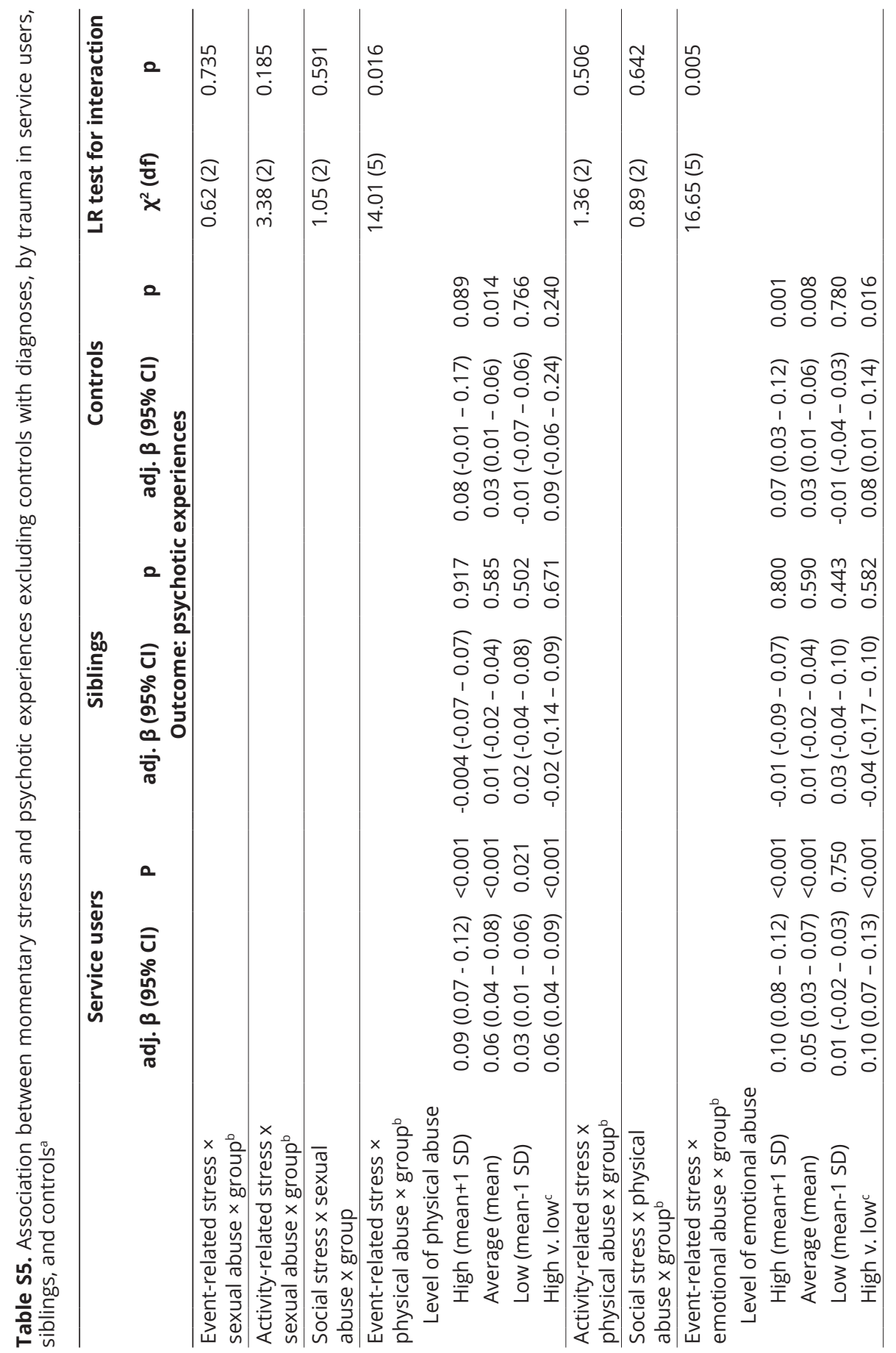




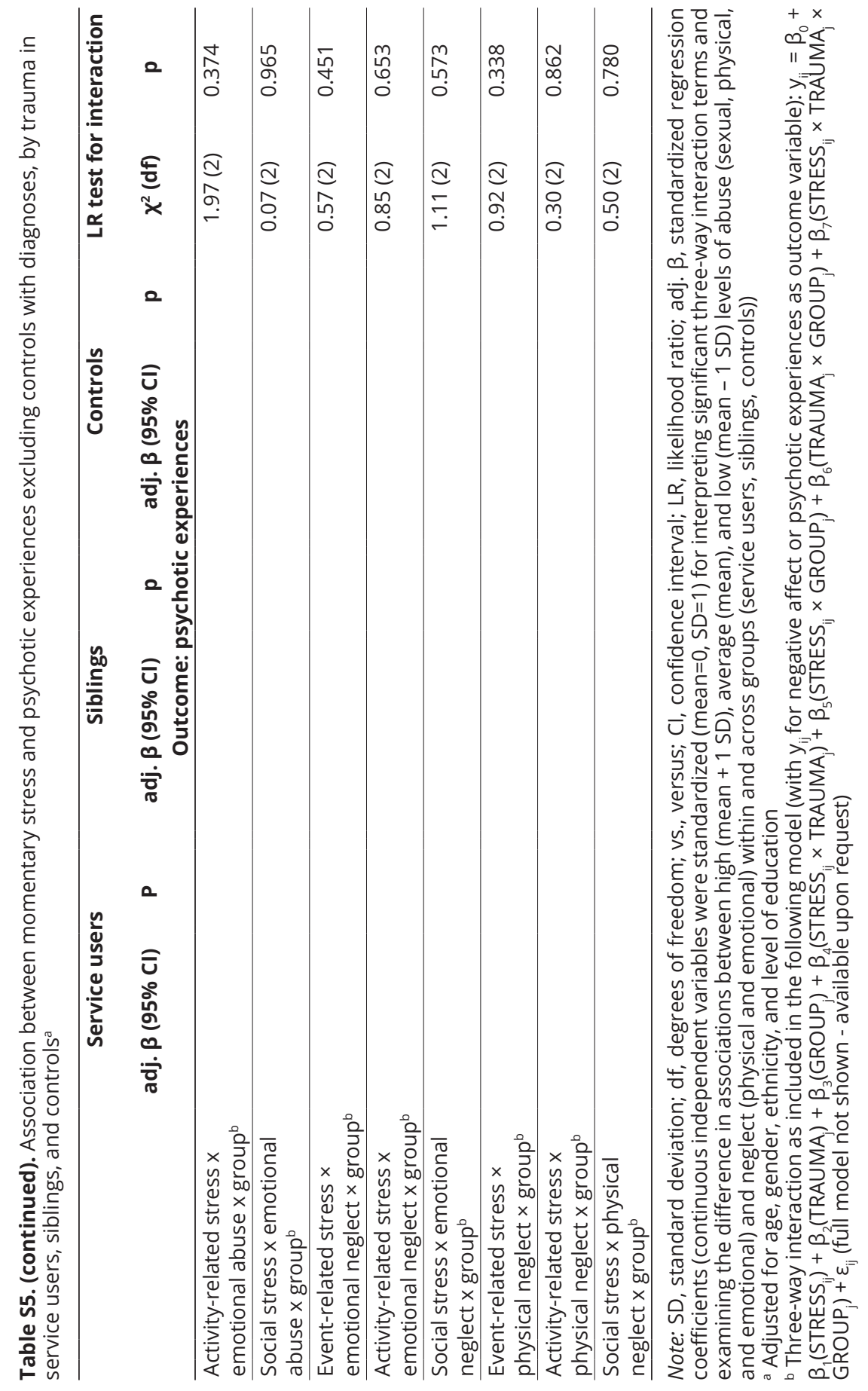




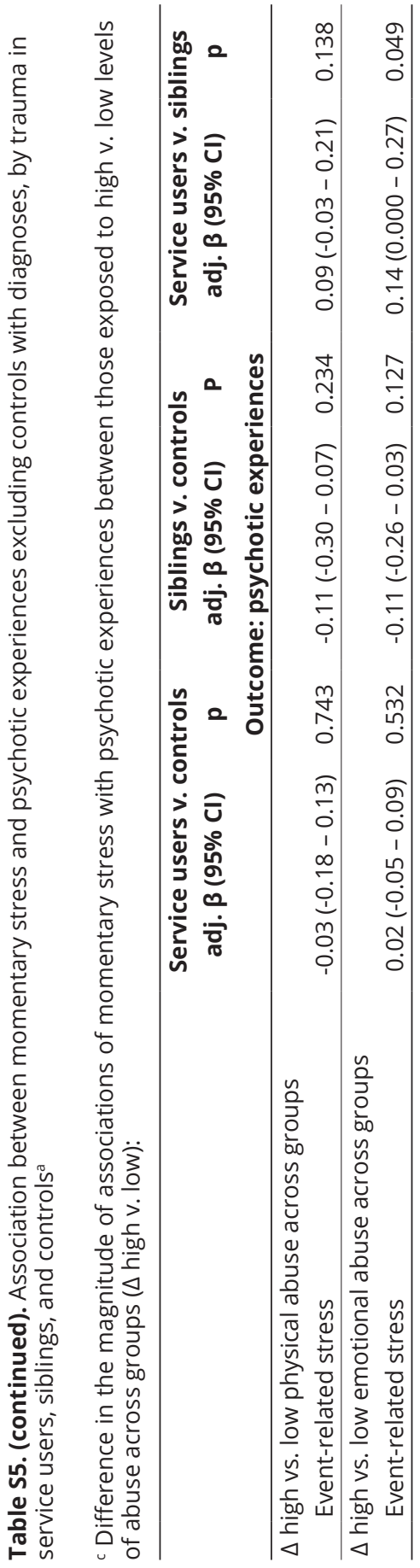



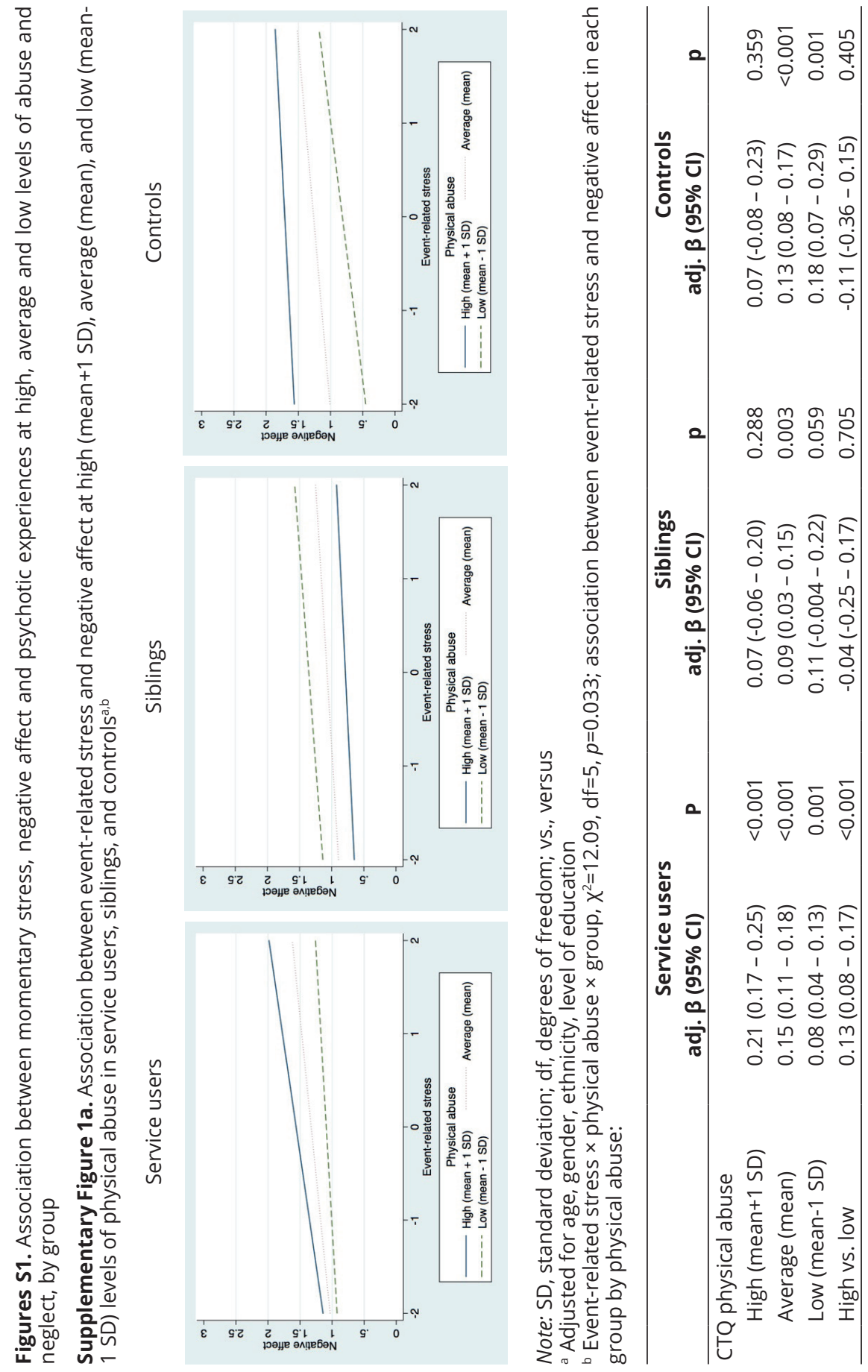

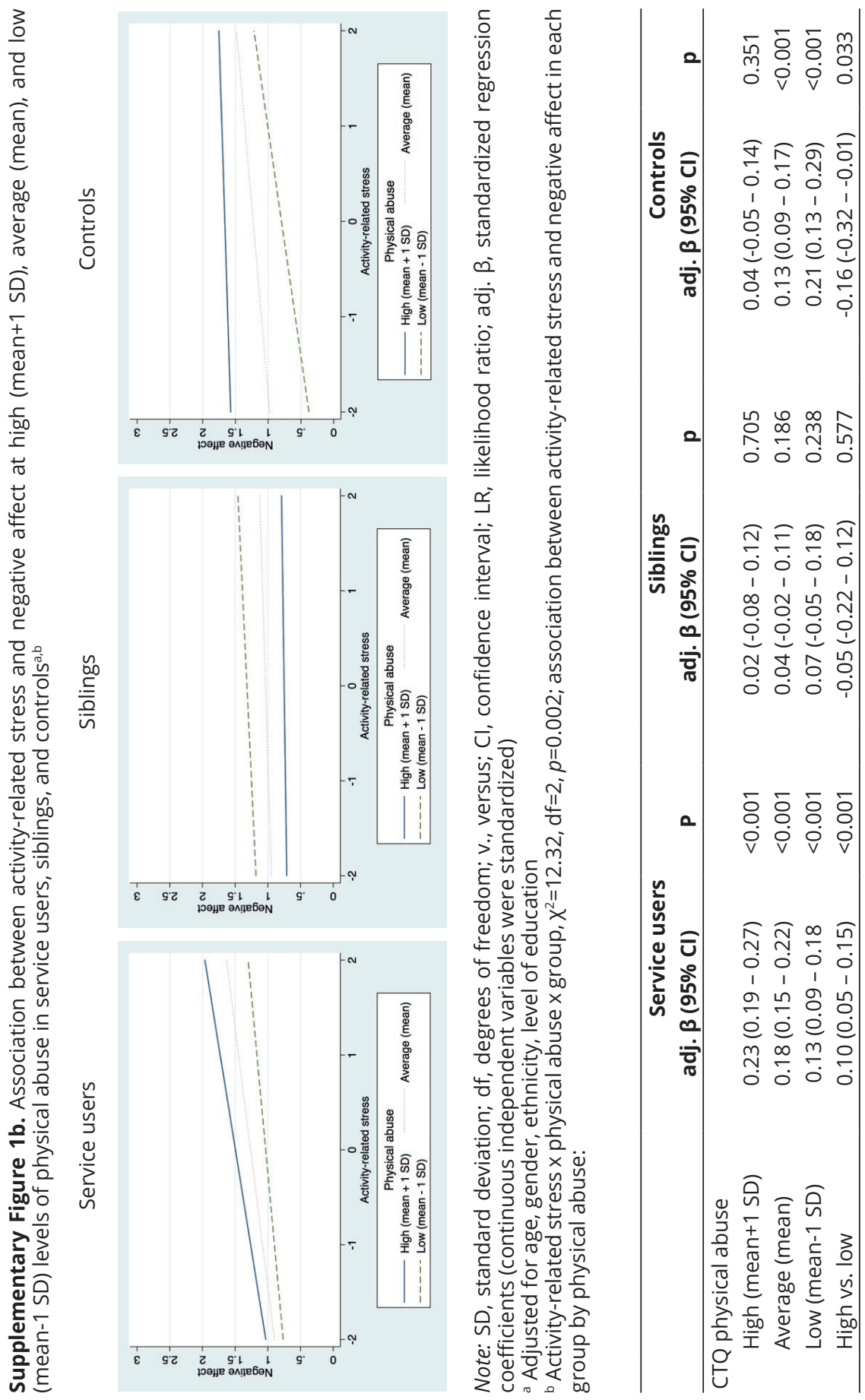

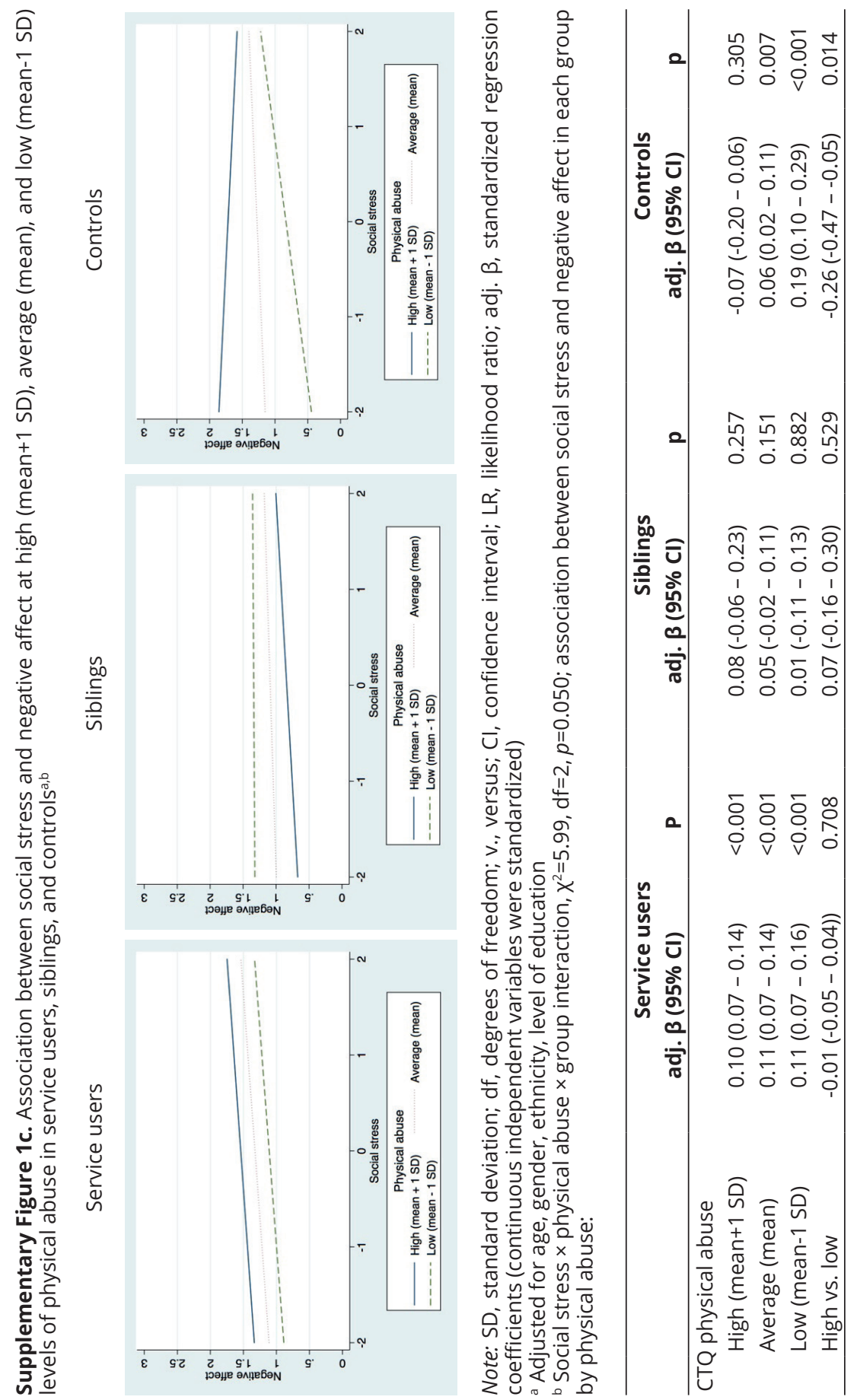

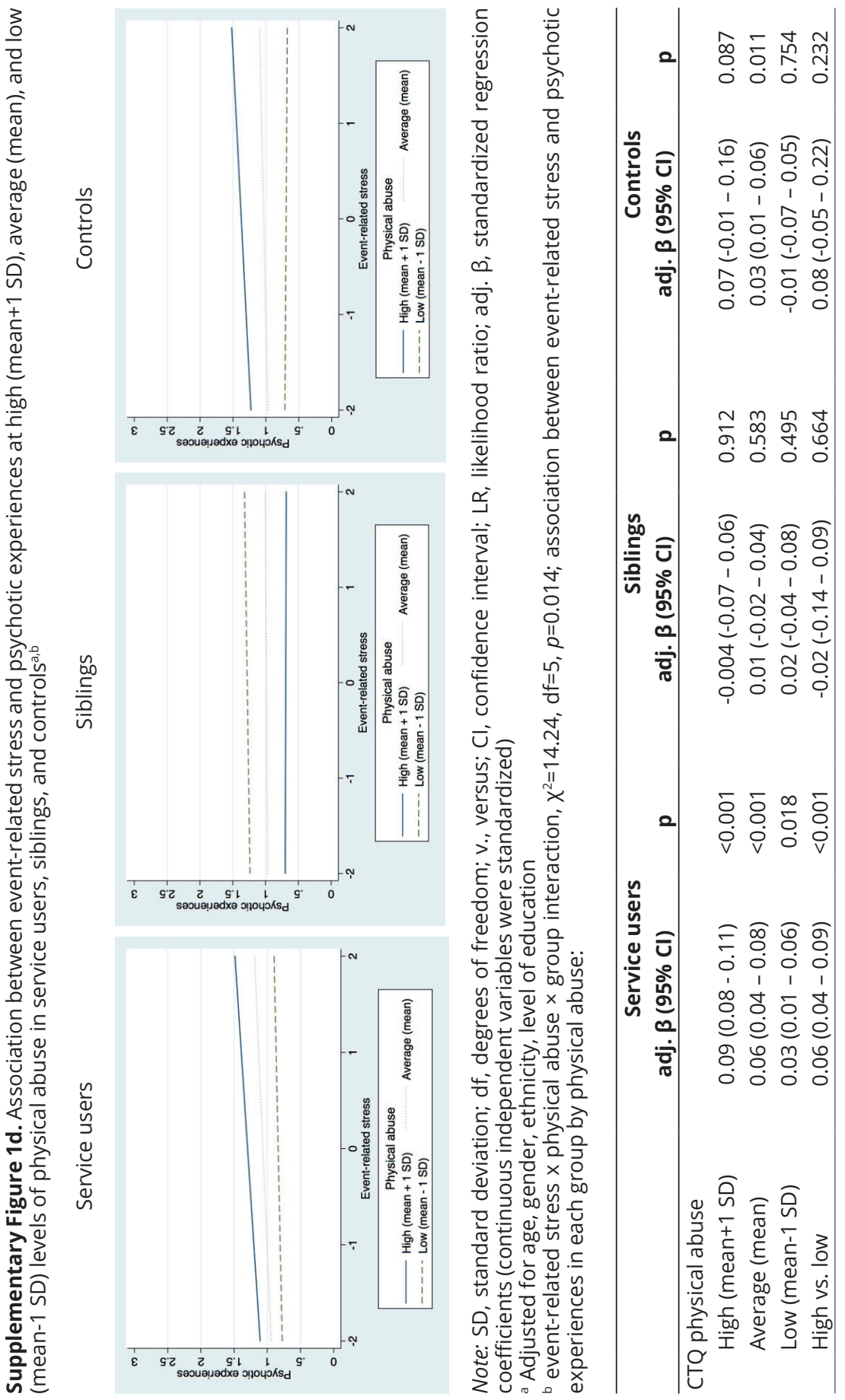


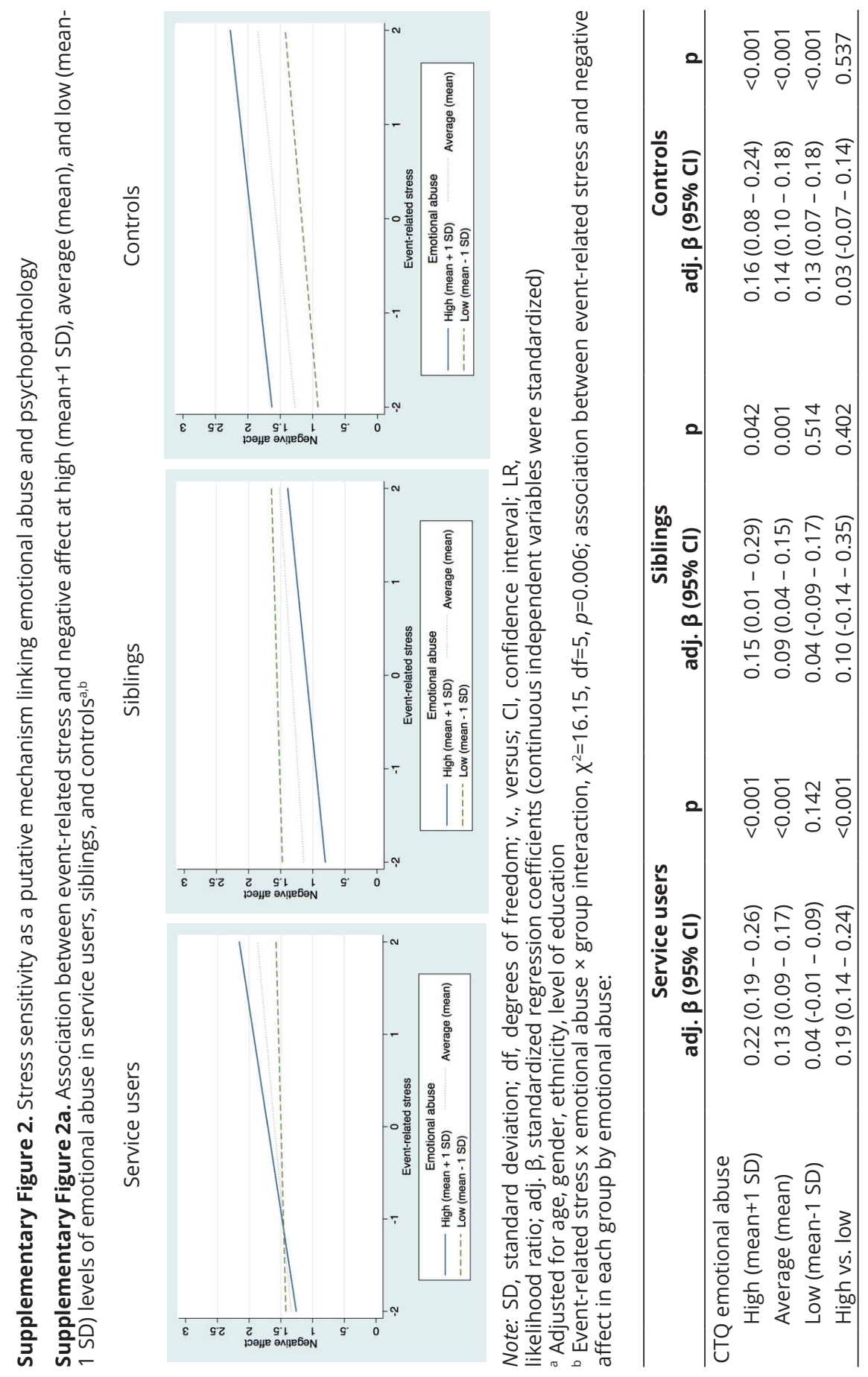



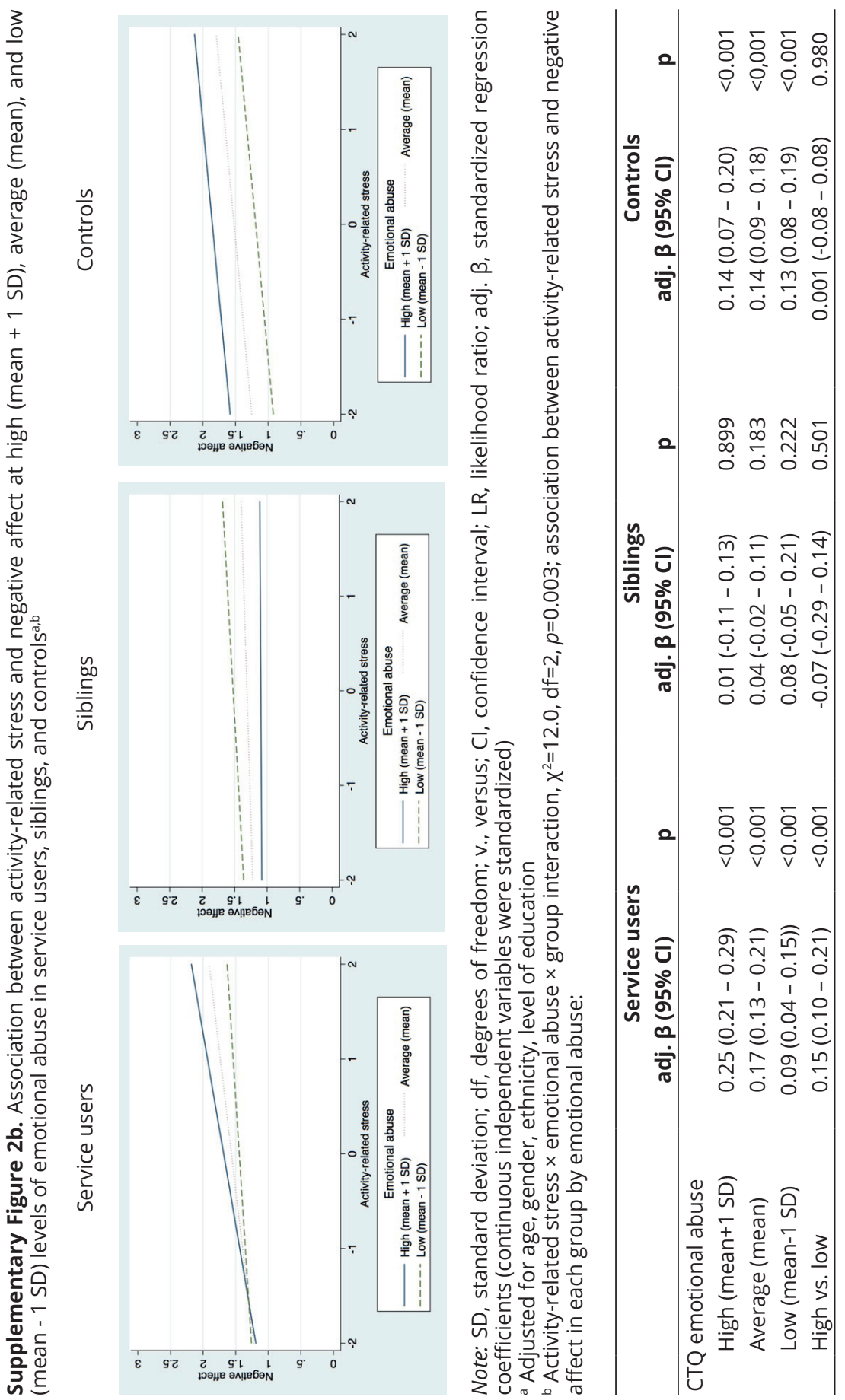

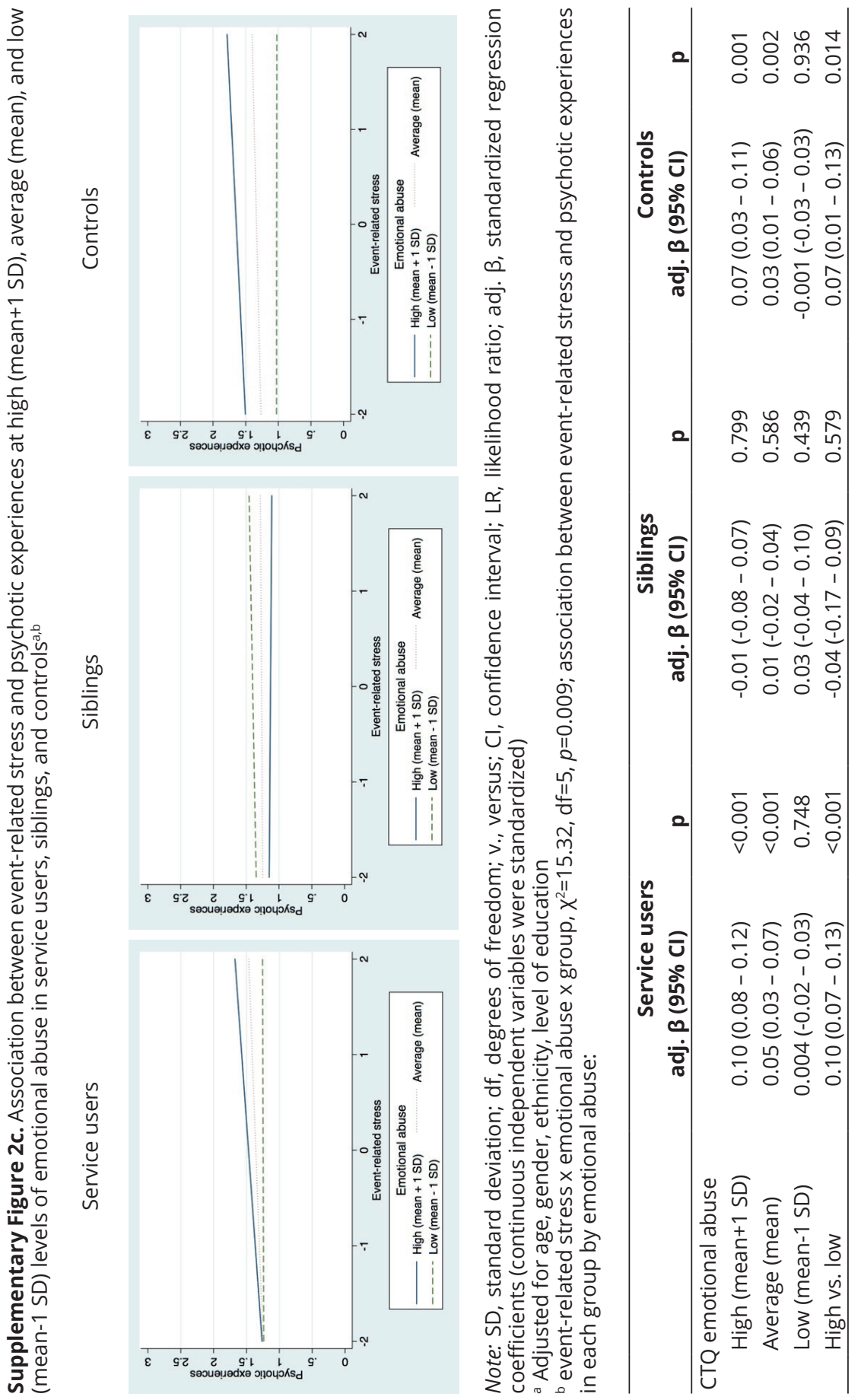

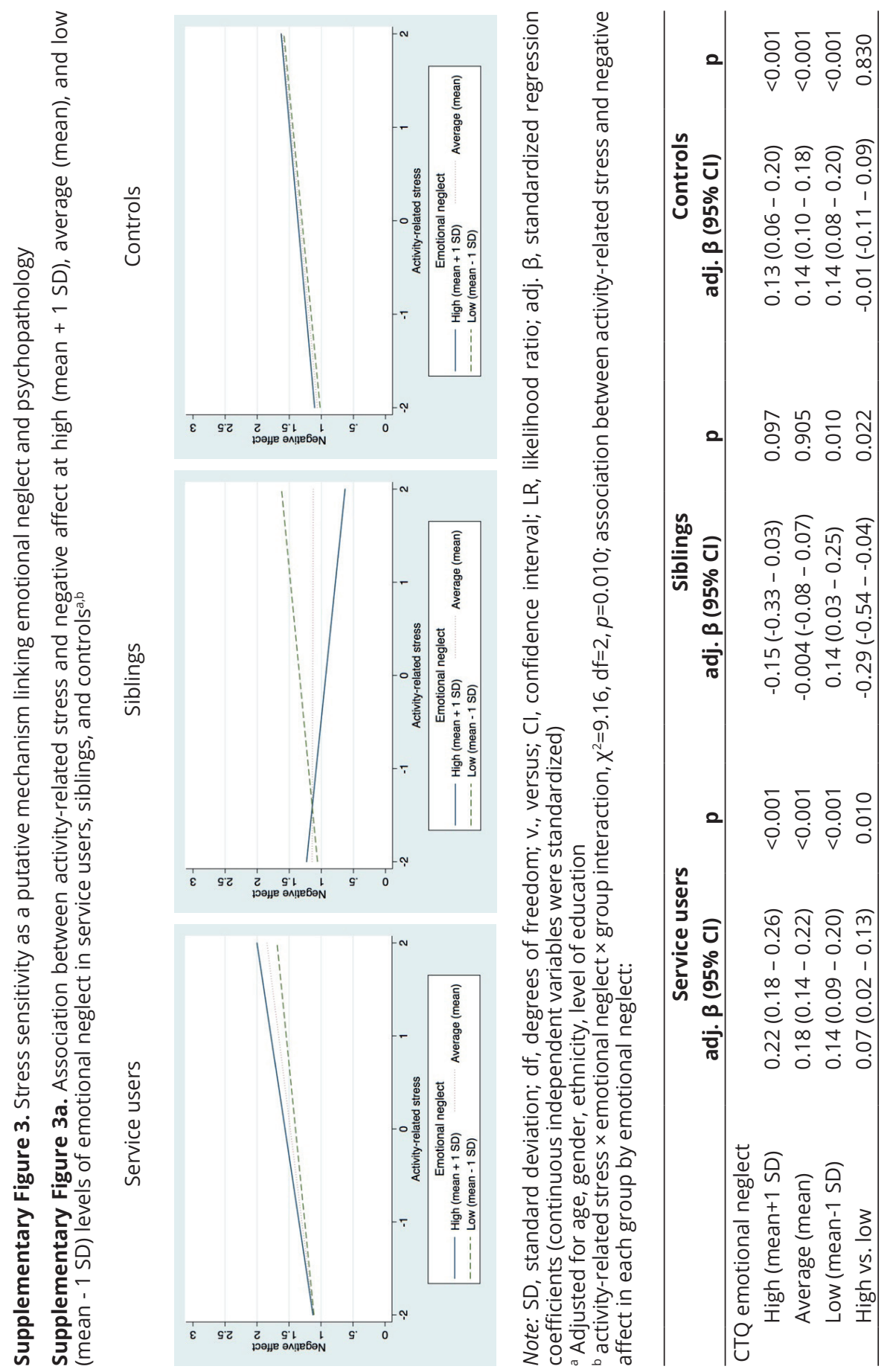


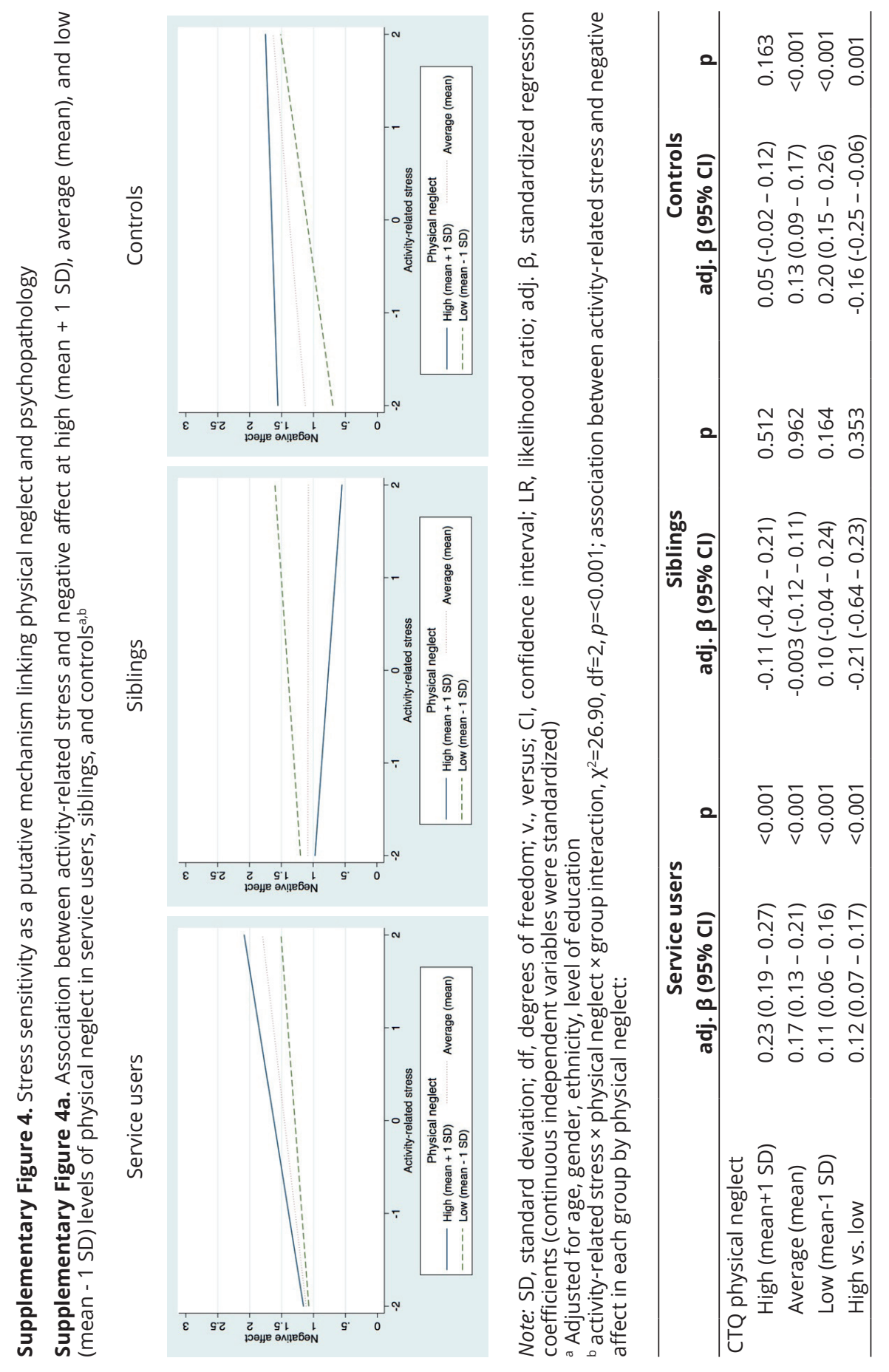



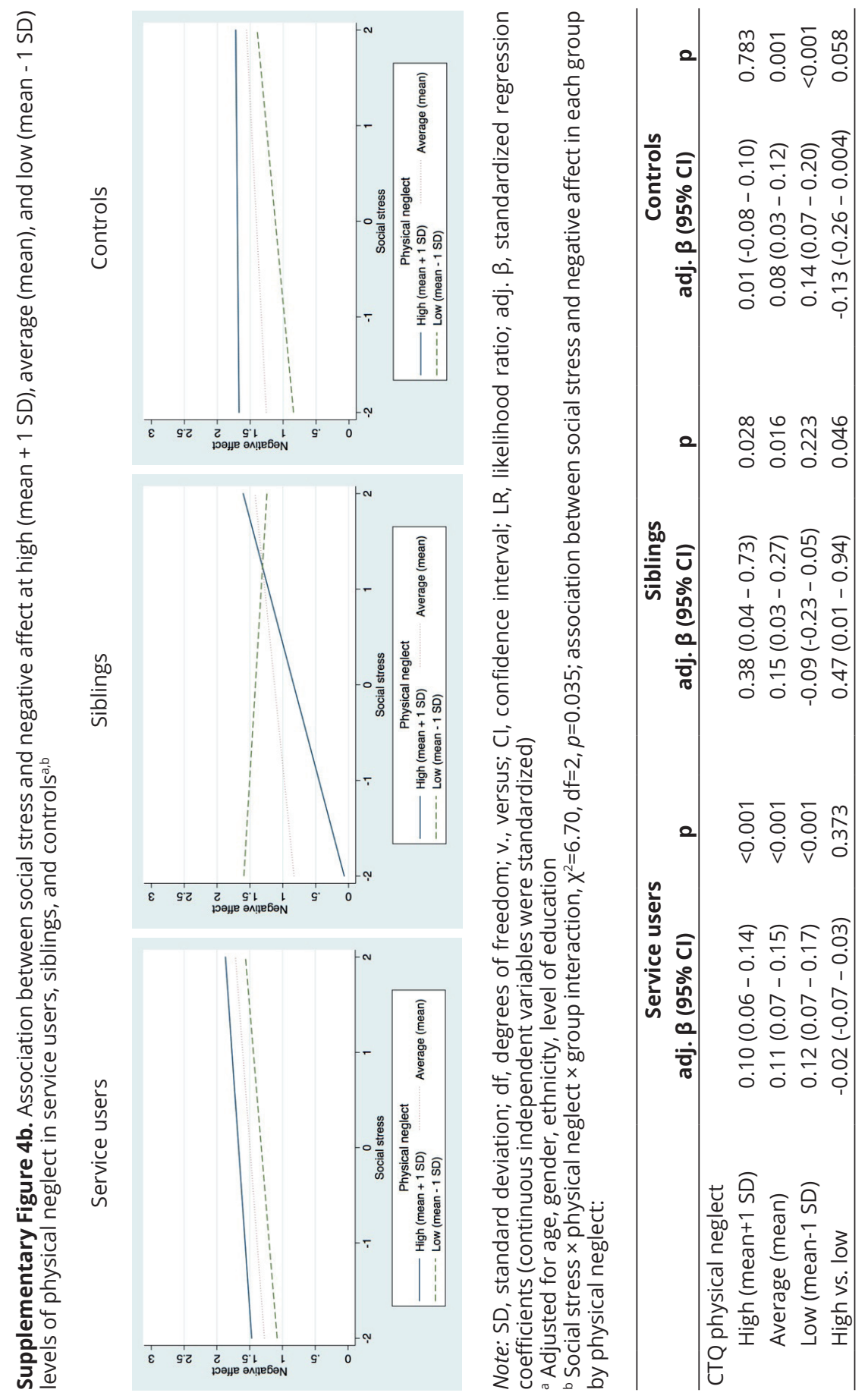



\section{CHAPTER 5}

\section{Bullying victimization and stress sensitivity in help-seeking youth: findings from an experience sampling study}




\section{Abstract}

Background: Bullying victimization confers risk for developing various mental disorders, but studies investigating candidate mechanisms remain scarce, especially in the realm of youth mental health. Elevated stress sensitivity may constitute a mechanism linking bullying victimization and mental health problems. In the current study, we aimed to investigate whether exposure to bullying victimization amplifies stress sensitivity in youth's daily life.

Methods: The Experience Sampling Method (ESM) was used to measure stress sensitivity (i.e. the association of momentary stress with (i) negative affect and (ii) psychotic experiences) in 42 help-seeking youths (service users), 17 siblings, and 40 comparison subjects (mean age 15 years). Before ESM assessments, bullying victimization at school as well as various psychopathological domains (i.e. depression, anxiety, psychosis) were assessed.

Results: Service users exposed to high levels of overall (primary hypotheses) as well as specific types (secondary hypotheses; physical and indirect, but not verbal) of bullying victimization experienced more intense negative affect and psychotic experiences in response to stress compared to those with low exposure levels (all $p<0.05$ ), whereas, in contrast, controls showed either less intense negative affect or no marked differences in stress sensitivity by exposure levels. In siblings, a less consistent pattern of findings was observed. Conclusion: Findings suggest that stress sensitivity may constitute a potential risk and resilience mechanism linking bullying victimization and youth mental health. Interventions that directly target individuals' reactivity to stress by providing treatment components in real-life using novel mHealth tools may be a promising novel therapeutic approach.

Keywords: Bullying; Stress, Psychological; Adolescent; Mental Health; Psychopathology; Ecological Momentary Assessment 


\section{Introduction}

Bullying victimization is defined as an intentional misuse of power in which an individual or a group of individuals engage in repeated hostile behavior against peers who have difficulties to defend themselves [1]. The experience of being bullied has long been seen to reflect a normal pattern of interaction between peers that is transitory and important for individuals' social development [2]. As a consequence, exposure to bullying has not been considered to represent a particularly stressful experience and, therefore, not to be an important risk factor involved in the development of mental health problems [2, 3].

However, evidence has accumulated that exposure to bullying victimization is associated with a range of mental disorders (e.g. depression, anxiety, psychosis), general psychopathology, self-harm, and suicidality, amongst others [4-13], and has been found to predict the use of mental health services [14]. These findings suggest that exposure to bullying victimization may be an important non-specific risk factor for mental health problems, which is consistent with the detrimental, but non-specific effects reported for other adverse childhood experiences (e.g. childhood maltreatment) [15]. Recent estimates from the World Health Organization [16] are alarming: around two in ten children and adolescents are being exposed to bullying victimization at school, although prevalence estimates differ considerably across countries (e.g. in Europe: 4\% in Italy, 30\% in Lithuania). These findings have contributed to formal recognition of bullying as a risk factor for mental health problems in the Global Burden of Disease Study 2017 [17].

In recent years, there has been an increasing focus on dimensional and transdiagnostic approaches to psychopathology [18-20], resulting in classification frameworks (e.g. HiTOP) [21] that are based on patterns of symptom co-occurrence, cutting across traditional diagnostic boundaries. In support of these efforts and also based on frequent co-occurrence of more common psychopathological domains (e.g. anxiety, depression) with psychotic experiences, an extended and transdiagnostic psychosis spectrum phenotype has been proposed that is temporally and phenomenologically continuous across psychotic and nonpsychotic disorders and shares socio-environmental risk factors, including bullying victimization [22]. 
Overall, high prevalence of bullying victimization in youth, and associations with immediate as well as prolonged mental health problems, which are often characterized by a number of co-occurring psychopathological domains (e.g. anxiety, depression, psychosis) [22, 23] that appear already early in life [24] underline the importance to develop early and transdiagnostic intervention strategies [25]. For this, an important step is to investigate candidate mechanisms that are relevant to linking exposure to bullying victimization and various mental health problems. Critically, however, the developmental processes and putative mechanisms involved remain largely under-researched, especially in the realm of youth mental health.

In contemporary models, exposure to socio-environmental risk (e.g., bullying victimization, childhood maltreatment, life events) is thought to impact on mental health through a progressive increase in individuals' stress response to subsequent adversity $[26,27]$. This has often been referred to as a process of sensitization [28] which is thought to be mediated by a number of biological and psychological factors [29-34]. Although evidence remains limited, there is an ongoing debate [15] regarding the extent to which specific forms of adversity may be more strongly associated with specific forms of mental health outcomes (e.g., whether more intrusive types of adversity are specifically associated with psychosis). The Experience Sampling Methodology (ESM), a structured self-report diary technique [35], is particularly well suited to test these propositions at a behavioral level by investigating whether overall as well as specific types of bullying victimization is associated with an increased sensitivity to specific types of minor stressors in daily life.

In most studies using ESM, individuals' stress sensitivity has been conceptualized as the association of minor stressors with (i) negative affect and (ii) psychotic experiences in daily life and, thus, has also been referred to individuals' affective and psychotic reactivity, respectively. These studies have consistently found an increased stress sensitivity in adults who were exposed to childhood trauma and adult life events, including individuals with depression, an at-risk mental state for psychosis, and psychosis spectrum disorders [36-39]. Thus, findings suggest that stress sensitivity in the flow of daily life may play an important non-specific and transdiagnostic role linking childhood adversity and mental disorders in help-seeking individuals. In a recent experience sampling study, derived from the 
same sample as the current study, exposure to childhood trauma was, similarly, associated with elevated stress sensitivity in help-seeking youth [40]. Also, there is some evidence of specificity as some studies have reported that more intrusive forms of childhood trauma (e.g. sexual and physical abuse as well as physical neglect) were most consistently associated with an elevated stress sensitivity in help-seeking individuals [38, 40]. Consequently, more intrusive forms of bullying (i.e., physical bullying) may be particularly associated with an increased stress sensitivity in help-seeking youth.

To date, however, only one study has reported elevated stress sensitivity in a non-clinical sample of young adults exposed to bullying [41] and, to the best of our knowledge, no study has investigated the impact of bullying victimization on individuals' affective and psychotic reactivity to stress in a sample of help-seeking youth and whether effects of bullying exposure on stress sensitivity differ across individuals at differing liability to mental health conditions. To address current knowledge gaps, a sample of adolescents and young adults receiving help from a secondary mental health service (service users), their biological siblings, and controls were recruited in the current study. We included siblings of service users as they have an increased risk for developing a mental disorder and, hence, reflect an intermediate risk group (compared with service users and controls) and also share genetic and socio-environmental risk factors with service users [42-44].

\section{Aims and hypotheses of the study}

The aim of the current study was to determine whether bullying victimization modifies sensitivity towards stress in a sample of help-seeking youth (service users), their biological siblings, and comparison subjects (controls). More specifically, the study aimed to investigate the following primary hypotheses: First, within groups (service users, siblings, and controls), stress sensitivity (i.e., the association between momentary stress and (i) negative affect and (ii) psychotic experiences) is modified by bullying victimization, with greater associations in individuals exposed to high vs. those exposed to low exposure levels of bullying victimization (H1); second, the effect of bullying victimization on stress sensitivity differs across groups at differing liability to mental health problems, with a greater impact in service users vs. controls, service users vs. siblings, and siblings vs. controls ( $\mathrm{H} 2)$. In addition, to investigate whether some bullying types are specifically modifying 
stress sensitivity, the following secondary hypotheses were tested: First, within groups, exposure to specific bullying types (i.e. physical, verbal, indirect bullying) impact on stress sensitivity, with greater associations when high vs. low exposure levels are compared (H3); second, across groups, the impact of specific types of bullying victimization on stress sensitivity is greater in service users vs. controls, service users vs. siblings, and siblings vs. controls (H4). Lastly, to test whether specific bullying types modify specific forms of affective and psychotic reactivity, the following exploratory analyses were conducted: Within groups, individuals' response to specific stressors in daily life (i.e. event-related, activity-related, social stress) is modified by specific types of bullying victimization (i.e. physical, verbal, indirect bullying), with greater associations in individuals exposed to high vs. low exposure levels (H5).

\section{Materials and methods}

\section{Sample}

Data were derived from the Youth Experience Study (YES), a study conducted to investigate candidate mechanisms involved in linking adverse childhood experiences and youth mental health. Dataset version 1.1 was used for the current analysis. This version differs from version 1.0 used in earlier work [40] in the group status used for one individual. A sample of help-seeking youth (service users) were recruited from the Mutsaers Foundation (MF) by treatment coordinators and leaflets and posters were distributed in waiting areas of all outpatient locations of MF. The MF offers secondary mental health services for young individuals in Limburg, the Netherlands. The following broad inclusion criteria were used: aged 12-20 years; currently receiving treatment from MF. Exclusion criteria were: being diagnosed with an Autistic Spectrum Disorder according to DSM-IV with the exception of Pervasive Developmental Disorder Not Otherwise Specified; intellectual disability (IQ score below 70); insufficient knowledge of the Dutch language. Further, we recruited siblings of service users. Inclusion criteria were as follows: aged 12-20; participation of a biological sibling who is receiving treatment from MF. Exclusion criteria were the same as for service users with the addition of a lifetime history of receiving treatment from a mental health service. Lastly, a control sample of non-help-seeking individuals was recruited through schools from the same catchment area as MF mental health services. These schools were 
asked for permission to conduct the study and a letter accompanied by a leaflet was sent to parents, asking them whether their child is allowed to participate in the study. The YES was also introduced in form of an information session in class. Inclusion criteria were: aged 12-20 years, attending a school in the same catchment area as MF mental health services. Exclusion criteria were the same as for siblings. If a participant was older than 18, he/she was allowed to give written informed consent without asking the parents. The study was approved by the Medical Ethics Review Committee of Maastricht University Medical Centre in Maastricht, the Netherlands (approval number: NL37420.068.11).

\section{Measures}

\section{Socio-demographic characteristics}

Socio-demographic data (i.e. age, sex, ethnicity, and level of education) was collected using a socio-demographic schedule.

\section{Bullying victimization}

The Retrospective Bullying Questionnaire (RBQ), a 44-item self-report questionnaire [45], was used to assess bullying victimization. The questionnaire measures exposure to bullying at primary and secondary school, while the precise timing of exposure prior to assessment is not specified. Three types of bullying were assessed: physical (hit/punched, stolen property), verbal (called names, threatened), and indirect (spread lies, excluded) bullying. In addition to assessing exposure to bullying victimization, the RBQ asks more general questions about individuals' experiences at school (e.g. whether individuals were happy), details about the bullying incident (e.g. the number of bullies involved, reasons individuals believe they were bullied), and also bullying experiences at the workplace. For this study, we used 2 items asking for frequency and intensity of each bullying type (physical, verbal, indirect) for primary as well as secondary school resulting in 12 items rated on a 5-point scale ranging from 1-5 [45]. Frequency was assessed by asking participants how often they were exposed to bullying ( $1=$ 'never', $5={ }^{\prime}$ constantly') and intensity was assessed by asking to evaluate the seriousness ( $1=$ 'not at all', $5=$ 'extremely serious'). For primary hypotheses, sum scores were calculated by adding items assessing the frequency and intensity of bullying experiences (12 items; range sum score, 12-60, Cronbach's alpha, $a=.90$ ) and, 
for secondary and exploratory hypotheses, sum scores were calculated for three specific bullying types (4 items; range sum score 4-20; physical bulling, $a=.77$; verbal bullying, $a=.84$; indirect bullying, $a=.87$ ), respectively. Good psychometric properties have been reported for this measure [45].

\section{Depressive, anxiety, and psychotic symptoms}

The Beck Depression Inventory (BDI-II), a well-established questionnaire consisting of 21 items, was completed to assess depressive symptoms over the past 2 weeks (4-point scale ranging from 0-3). A Dutch version of the State-Trait Anxiety Inventory (STAI) was used to assess state and trait anxiety. The first part (STAIDY1) measures trait (20 items) and the second part (STAI-DY2) assesses state (40 items) anxiety, both rated on a 4-point scale (ranging from 1-4; 1=not at all, 4=very much). The Community Assessment of Psychic Experiences (CAPE) was used to assess the frequency and distress of positive (20 items) and negative (14 items) sub-clinical psychotic and depressive (8 items) symptoms (rated on a 4-point scale ranging from 0-3; 0=not at all, 3=very much). For all measures, good psychometric properties have been [46-48] demonstrated.

\section{Momentary stress, negative affect, and psychotic experiences}

Momentary stress, negative affect, and psychotic experiences were assessed using the experience sampling method (ESM), an intensive self-assessment technique to assess subjective experiences and social contexts in real life, outside the research laboratory with high ecological validity [35]. A personal digital assistant (PsyMate) was used for data collection. In accordance with previous ESM studies [49, 50], the PsyMate beeped 10 times a day on 6 consecutive days at unpredictable moments between 7:30 am and 10:30 pm (scheduled at random within set time blocks of 90 minutes). Event-related, activity-related, and social stress were defined as unpleasant events, activities, and social situations occurring in daily life. Sufficient concurrent validity with other stress measures has been reported [51].

Momentary stress was calculated by computing the mean score of six items assessing event-related, activity-related, and social stress. Event-related stress was measured asking participants to report the pleasantness of the most important event that had happened since the last beep on a 7-point scale ranging 
from 'very unpleasant' (rating of -3) to 'very pleasant' (rating of 3). To ensure that higher ratings indicate higher levels of stress and pleasant events are excluded from analyses, the item was recoded (ratings of -3 were coded as $4,-2$ as $3,-1$ as 2 , and neutral events as 1 , while pleasant events were coded as 0 ). Activityrelated stress was assessed by asking 'What am I doing (just before the beep)' (e.g. being at work/school, doing household, eating/drinking) and three additional items ('I would prefer doing something else', 'This activity is difficult for me', 'I can do this well' [reversed]) ranging from 'not at all' (rating of 1) to 'very much' (rating of 7). Social stress was measured by asking participants about their current social situation (e.g. 'I am alone', 'I am with my family', 'I am with my friends') and to rate this using the items 'I find the people I am with pleasant' [reversed] (if with someone) or 'I like to be alone' [reversed] (if alone) ranging from 'not at all' (rating of 1) to 'very much' (rating of 7).

Negative affect was assessed using five items asking participants to report the degree of feeling anxious, lonely, insecure, irritated, and down. Psychotic experiences were measured using eight items ('I see things that aren't really there', 'I hear things that aren't really there', 'I feel suspicious/paranoid', 'I feel harried', 'I feel unreal', 'My thoughts are influenced by other', 'I can't get these thoughts out of my head', 'I feel like I am losing control'). All items were rated on a 7-point scale (1='not at all', 7='very much') and mean scores were calculated to compute both variables. High levels of internal consistency and good concurrent validity with interviewer-rated measures has been previously reported [38].

\section{Statistical analysis}

First, we compared socio-demographic characteristics and psychopathological domains (i.e. standardized BDI-II, STAI-DY1/DY2, and CAPE scores) across groups using linear regression and $\chi^{2}$-tests. Second, the MIXED command in Stata 15 was used to fit linear mixed models. This statistical modelling technique is needed as ESM data has a multilevel structure with multiple observations nested within participants. Maximum likelihood estimation of these models allows all available data to be used under the relatively unrestricted assumption that data is missing at random. We fitted models with momentary stress (event-related, activity-related, and social stress; primary and secondary hypotheses [H1-H4]: overall mean score including all stress items; exploratory analyses [H5]: mean score of specific 
stressors) as the continuous independent variable and (i) negative affect and (ii) psychotic experiences as the outcome variable, while controlling for potential confounders and variables associated with missing values (i.e. age, sex, ethnicity, level of education). To test whether associations between momentary stress and (i) negative affect and (ii) psychotic experiences are modified by exposure to bullying victimization at school (i.e. exposure at primary and secondary school combined; continuous total scores) and group (service users, siblings, and controls), twoway (stress $x$ bullying, stress $x$ group, bullying $x$ group) and three-way (stress $x$ bullying $x$ group) interaction terms were simultaneously added into models. Wald tests were performed using the TESTPARM command to evaluate significance of three-way interaction terms to the model. The continuous stress and continuous bullying variables were standardized (mean=0, S.D.=1) for interpreting significant three-way interaction terms [52] and the LINCOM command was used to compute linear combinations of coefficients to test the hypotheses that, within each group, the association of momentary stress with (i) negative affect and (ii) psychotic experiences was greater in individuals exposed to high vs. those exposed to low levels of bullying victimization (+/- 1 S.D. of standardized continuous bullying victimization total scores; primary hypotheses [H1]: exposure to overall bullying; secondary and exploratory hypothesis [H3 and $\mathrm{H} 5]$ : exposure to specific bullying types $[53,54]$. Lastly, we investigated whether the impact of bullying victimization on stress sensitivity differed across groups by comparing the differences in the magnitude of associations of momentary stress with (i) negative affect and (ii) psychotic experiences between those exposed to high vs. low levels of bullying victimization (primary hypotheses [H2]: exposure to overall bullying; secondary hypotheses [H4]: exposure to specific bullying types) in service users compared to controls, service users compared to siblings, and siblings compared to controls. Separate models for momentary stress (overall as well as three specific stressors) and bullying exposure (overall as well as three specific types) were calculated, resulting in 2 models for primary hypotheses, 6 models for secondary hypotheses, and 24 models for exploratory analyses. We adjusted significance levels of Wald tests for three-way interactions to correct for Type-1 error proliferation using family-wise error-corrected $p$ values ( $p$ FWE) by multiplying the unadjusted $p$-value by the total number of tests ( $N=8$ for primary and secondary analyses and $N=24$ for exploratory analyses). 


\section{Results}

\section{Basic sample and clinical characteristics}

In total, 109 individuals were eligible to participate. Of these, 99 youths (42 service users, 17 siblings, and 40 controls) completed the ESM with $\geq 20$ valid responses over the 6-day assessment period as well as the BDI-II, STAI-DY1/DY2, CAPE, and RBQ. Thus, a high proportion of those initially assessed were included in the analysis (i.e. $90.8 \%$ of 109). There were, within groups, no differences between individuals who completed ESM assessments and those who did not with regard to socio-demographic characteristics and other variables. Groups did not differ in age, sex, or ethnicity (Table 1). However, there was evidence for higher levels of depression (BDI-II: $\beta=0.71, p=0.001 ; B=1.08 p<0.001$ ), state $(\beta=0.50, p=0.025 ; \beta$ $=0.59, p=0.40)$ and trait $(\beta=0.59, p=0.004 ; B=0.95, p<0.001)$ anxiety, and negative ( $\beta=0.42, p=0.057 ; \beta=0.73, p=0.011)$ and positive $(\beta=0.75, p<0.001 ; \beta=0.84$, $p=0.002$ ) psychotic-like experiences in service users vs. controls and service users vs. siblings, respectively. As shown in Table 2, service users were exposed to higher overall levels of bullying victimization compared to controls ( $\beta=0.56$, $p=0.010)$ and siblings $(\beta=0.60, p=0.034)$, while, in contrast, no differences were found comparing siblings and controls $(\beta=-0.03, p=0.902)$. Further, service users reported higher levels of physical $(\beta=0.86, p<0.001 ; \beta=0.77, p=0.005)$, but not verbal $(\beta=0.29, p=0.188 ; \beta=0.42, p=0.150)$ and indirect $(\beta=0.37, p=0.092 ; \beta=0.39$, $p=0.169$ ) bullying compared to controls and siblings, respectively. Moreover, although not the primary aim of the current paper, it is worth mentioning that service users were more likely to report bullying-related mental health complaints, harmful behavior, and occupational problems when compared to controls and siblings (Table 2). 
Table 1. Basic sample characteristics

\begin{tabular}{|c|c|c|c|c|c|}
\hline & $\begin{array}{c}\text { Service } \\
\text { users } \\
(n=42)\end{array}$ & $\begin{array}{l}\text { Siblings } \\
(n=17)\end{array}$ & $\begin{array}{l}\text { Controls } \\
(n=40)\end{array}$ & Test statistic & p \\
\hline Age (years), mean (S.D.) & $15.4(1.4)$ & $15.3(2.3)$ & $15.6(2.0)$ & $\mathrm{F}=0.24, \mathrm{df}=2$ & 0.785 \\
\hline \multicolumn{6}{|l|}{ Sex, n (\%) } \\
\hline Female & $25(59.5)$ & $10(58.8)$ & $23(57.5)$ & $\chi^{2}=0.04, \mathrm{df}=2$ & 0.983 \\
\hline Male & $17(40.5)$ & $7(41.2)$ & $17(42.5)$ & & \\
\hline \multicolumn{6}{|l|}{ Ethnicity, n (\%)a } \\
\hline White Dutch & $26(61.9)$ & $11(64.7)$ & $25(64.1)$ & $\chi^{2}=0.06, d f=2$ & 0.970 \\
\hline Other & $16(38.1)$ & $6(35.3)$ & $14(35.9)$ & & \\
\hline \multicolumn{6}{|l|}{ Level of education ${ }^{b}, \mathrm{n}(\%)$} \\
\hline School & $30(71.4)$ & $7(41.2)$ & $17(42.5)$ & $\chi^{2}=10.48, \mathrm{df}=2$ & 0.033 \\
\hline Further & $12(28.6)$ & $8(47.1)$ & $20(50.0)$ & & \\
\hline Higher & - & $2(11.8)$ & $3(7.5)$ & & \\
\hline \multicolumn{6}{|l|}{ Cannabis use, n (\%) } \\
\hline 12-month & $9(21.4)$ & $1(5.9)$ & $4(10.0)$ & $\chi^{2}=3.36, d f=2$ & 0.187 \\
\hline lifetime & $9(21.4)$ & $2(11.8)$ & $5(12.5)$ & $\chi^{2}=1.50, d f=2$ & 0.473 \\
\hline \multicolumn{6}{|l|}{ Attempted suicide, n (\%) } \\
\hline During last year & $6(14.6)$ & - & - & - & - \\
\hline Before age 17 & $8(19.1)$ & - & - & & \\
\hline \multicolumn{6}{|l|}{ DSM-IV diagnoses, n (\%) } \\
\hline $\begin{array}{l}\text { Pervasive } \\
\text { developmental } \\
\text { disorders NOS }\end{array}$ & $10(23.8)$ & - & $5(12.5)$ & & \\
\hline $\begin{array}{l}\text { Attention-deficit and } \\
\text { disruptive behaviour }\end{array}$ & $6(14.3)$ & $3(17.6)$ & - & & \\
\hline Adjustment disorders & $4(9.5)$ & - & - & & \\
\hline Anxiety disorders & $2(4.8)$ & - & - & & \\
\hline Depressive disorders & $2(4.8)$ & - & - & & \\
\hline $\begin{array}{l}\text { Gender identity } \\
\text { disorders }\end{array}$ & $2(4.8)$ & - & - & & \\
\hline Learning disorders & - & - & $2(5.0)$ & & \\
\hline $\begin{array}{l}\text { Other disorders of } \\
\text { infancy, childhood, or } \\
\text { adolescence }\end{array}$ & $5(11.9)$ & - & - & & \\
\hline $\begin{array}{l}\text { Parent-child relational } \\
\text { problem }\end{array}$ & $5(11.9)$ & $1(5.9)$ & $1(2.5)$ & & \\
\hline Comorbid condition ${ }^{c}$ & $24(57.1)$ & $2(11.8)$ & - & & \\
\hline None & $6(14.3)$ & $13(76.5)$ & $32(80.0)$ & & \\
\hline 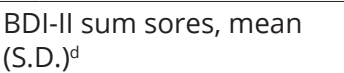 & $12.8(9.2)$ & $3.9(3.3)$ & $6.9(7.0)$ & $\mathrm{F}=10.5, \mathrm{df}=2$ & $<0.001$ \\
\hline
\end{tabular}


Table 1. (continued). Basic sample characteristics

\begin{tabular}{|c|c|c|c|c|c|}
\hline & $\begin{array}{c}\text { Service } \\
\text { users } \\
(n=42)\end{array}$ & $\begin{array}{l}\text { Siblings } \\
(\mathrm{n}=17)\end{array}$ & $\begin{array}{l}\text { Controls } \\
(n=40)\end{array}$ & Test statistic & p \\
\hline \multicolumn{6}{|l|}{ CAPE sum scores, mean (S.D. $)^{d}$} \\
\hline Positive & $10.0(9.4)$ & $3.9(3.2)$ & $4.6(3.9)$ & $\mathrm{F}=8.28, \mathrm{df}=2$ & $<0.001$ \\
\hline Negative & $9.9(6.7)$ & $5.6(3.8)$ & $7.4(4.8)$ & $F=3.88, d f=2$ & 0.024 \\
\hline Depressive & $7.7(4.0)$ & $4.2(1.8)$ & $4.7(3.4)$ & $\mathrm{F}=9.90, \mathrm{df}=2$ & $<0.001$ \\
\hline $\begin{array}{l}\text { STAI-DY1 (trait anxiety) }{ }^{a} \\
\text { sum scores, mean (S.D.) }\end{array}$ & $35.5(10.6)$ & $30.2(6.8)$ & $31.1(7.2)$ & $\mathrm{F}=3.47, \mathrm{df}=2$ & 0.035 \\
\hline $\begin{array}{l}\text { STAI-DY2 (state anxiety) } \\
\text { sum scores, mean (S.D.) }\end{array}$ & $85.6(20.8)$ & $67.1(9.2)$ & $74.1(16.4)$ & $F=8.12, d f=2$ & $<0.001$ \\
\hline $\begin{array}{l}\text { Number of valid beeps } \\
\text { Mean (range, min-max) }\end{array}$ & $44.16(25-59)$ & $43.4(23-57)$ & $44.9(24-58)$ & $F=0.27, d f=2$ & 0.754 \\
\hline
\end{tabular}

Note: S.D., standard deviation; $\mathrm{df}$, degrees of freedom; $\beta$, standardized regression coefficients (mean score differences); $\mathrm{Cl}$, confidence interval;

a Missing values: ethnicity $=1, \mathrm{BDI}=1, \mathrm{STAl}-\mathrm{DY} 1=1, \mathrm{STAI}-\mathrm{DY} 2=2,{ }^{\mathrm{b}}$ Categories defined as: school (primary education, LBO, MAVO, VMBO), further (MBO, HAVO, VWO), and higher (HBO, WO) of the Dutch educational system, ' Consisting of the following diagnostic categories in the case group: Additional codes (Parent-child relational problem, 33.3\%; Borderline intellectual functioning, 13.3\%; Neglect of child, 6.7\%), Attention-deficit and disruptive behaviour disorders (10\%), Learning disorders (10\%), Personality disorders (6.7\%), Mild mental retardation (6.7\%), Anxiety disorders (3.3\%), Dissociative disorders (3.3\%), Tic disorders (3.3\%), Amphetamine related disorders (3.3\%) ${ }^{\mathrm{d}}$ Standardized mean score differences across groups:

\begin{tabular}{lcccccc}
\hline & \multicolumn{2}{c}{ Cases v. controls } & \multicolumn{2}{c}{ Siblings v. controls } & \multicolumn{2}{c}{ Cases v. siblings } \\
& $\boldsymbol{\beta}(\mathbf{9 5 \%} \mathrm{CI})$ & $\mathbf{p}$ & $\boldsymbol{\beta}(\mathbf{9 5 \%} \mathrm{CI})$ & $\mathbf{p}$ & $\boldsymbol{\beta}(95 \% \mathrm{CI})$ & $\mathbf{p}$ \\
\hline BDI- II & $0.71(0.30-1.11)$ & 0.001 & $-0.37(-0.89-0.16)$ & 0.168 & $1.08(0.55-1.60)<0.001$ \\
\hline CAPE & & & & & & \\
Positive & $0.75(0.34-1.17)$ & $<0.001$ & $-0.09(-0.63-0.44)$ & 0.735 & $0.84(0.31-1.38)$ & 0.002 \\
Negative & $0.42(-0.01-0.85)$ & 0.057 & $-0.31(-0.87-0.25)$ & 0.269 & $0.73(0.17-1.29)$ & 0.011 \\
Depressive & $0.80(0.40-1.21)$ & $<0.001$ & $-0.12(-0.64-0.41)$ & 0.666 & $0.92(0.39-1.45)$ & 0.001 \\
\hline STAI-DY1 & $0.50(0.06-0.93)$ & 0.025 & $-0.09(-0.65-0.47)$ & 0.748 & $0.59(0.03-1.14)$ & 0.040 \\
\hline STAI-DY2 & $0.59(0.19-0.99)$ & 0.004 & $-0.36(-0.88-0.16)$ & 0.170 & $0.95(0.43-1.46)<0.001$ \\
\hline
\end{tabular}




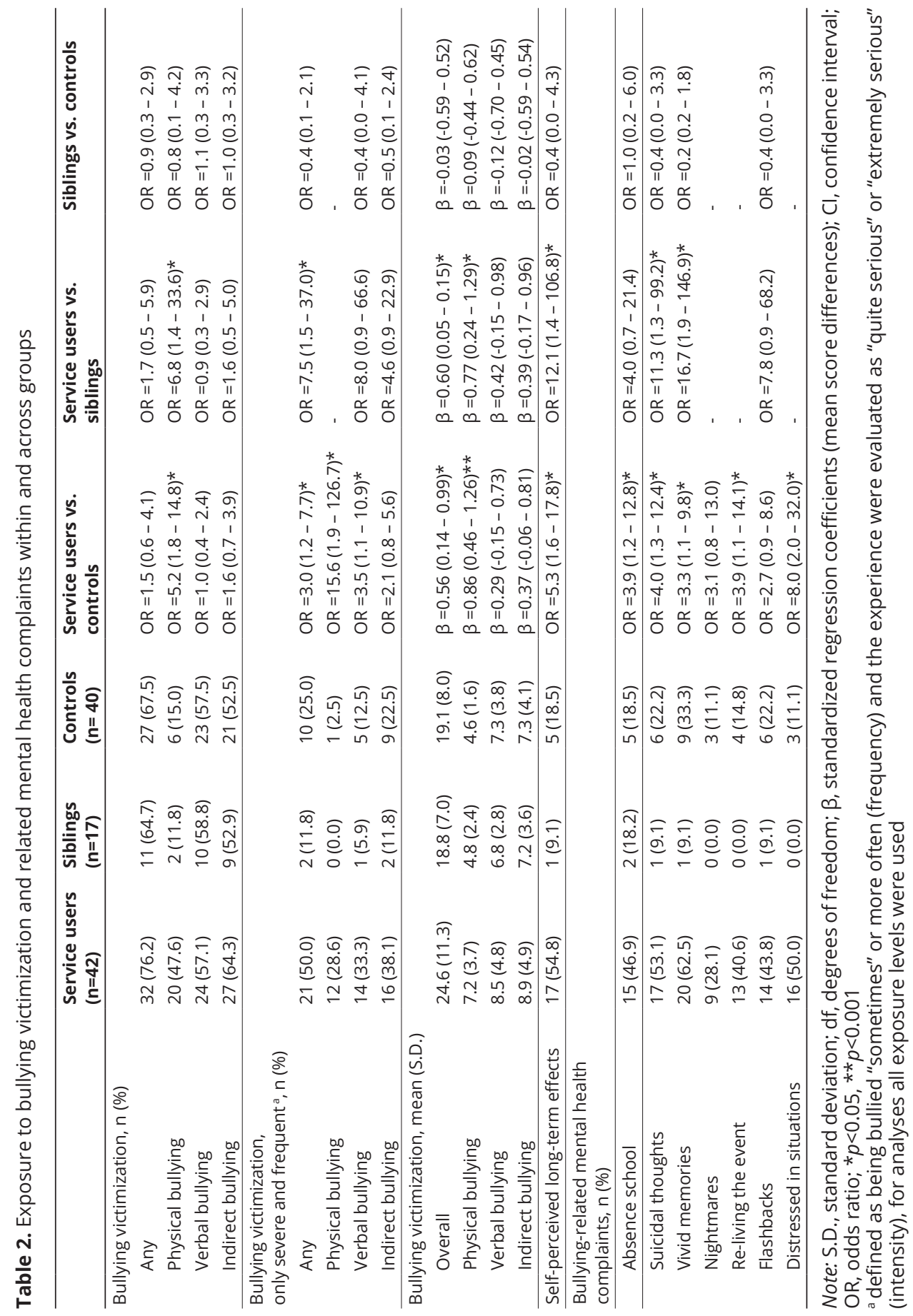




\section{Association between momentary stress and negative affect by bullying victimization and group}

There was evidence in support of primary and secondary hypotheses that exposure to overall bullying victimization as well as physical bullying, but not verbal and indirect bullying, modified the association of momentary stress with negative affect (Table 3). Evidence for effect modification by levels of bullying exposure within and across groups was evidenced by statistically significant 3-way interaction effects described below (Table 3).

\section{Within-group comparisons}

Within groups, momentary stress was associated with higher negative affect in service users (adj. $\beta=0.09, p=0.002$ ) and lower negative affect in controls (adj. $\beta=-0.11, p=0.024$ ) when high vs. low overall bullying victimization levels were compared, while no differences by exposure levels were found in siblings (adj. $\beta$ $=0.07, p=0.392$ ) (see Table 3 ). Analyses to test secondary hypotheses revealed that stress was associated with lower negative affect in controls comparing those with high vs. those with low physical bullying levels (adj. $\beta=-0.33, p<0.001$ ), whereas higher negative affect was observed in service-users (adj. $\beta=0.07, p=0.010$ ) and, at trend level, siblings ( $a d j . \beta=0.12, p=0.073$ ). There was no evidence that verbal and indirect bullying modified the affective reactivity to stress in daily life. Results of exploratory analyses that test effect modification by levels of bullying exposure for associations of specific stressors (event-related, activity-related, and social) with negative affect are provided in Supplement 1 and Table S1. 


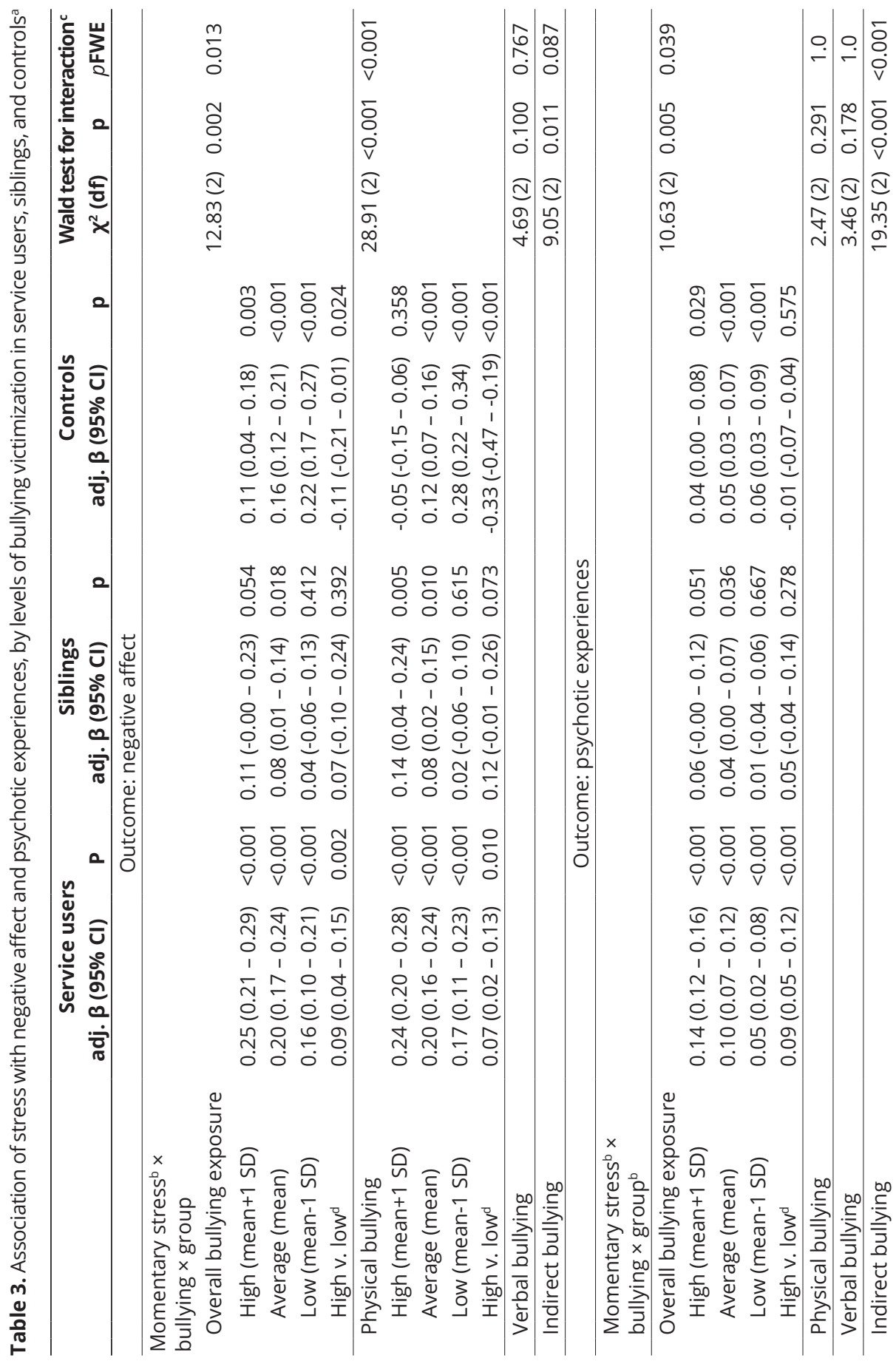




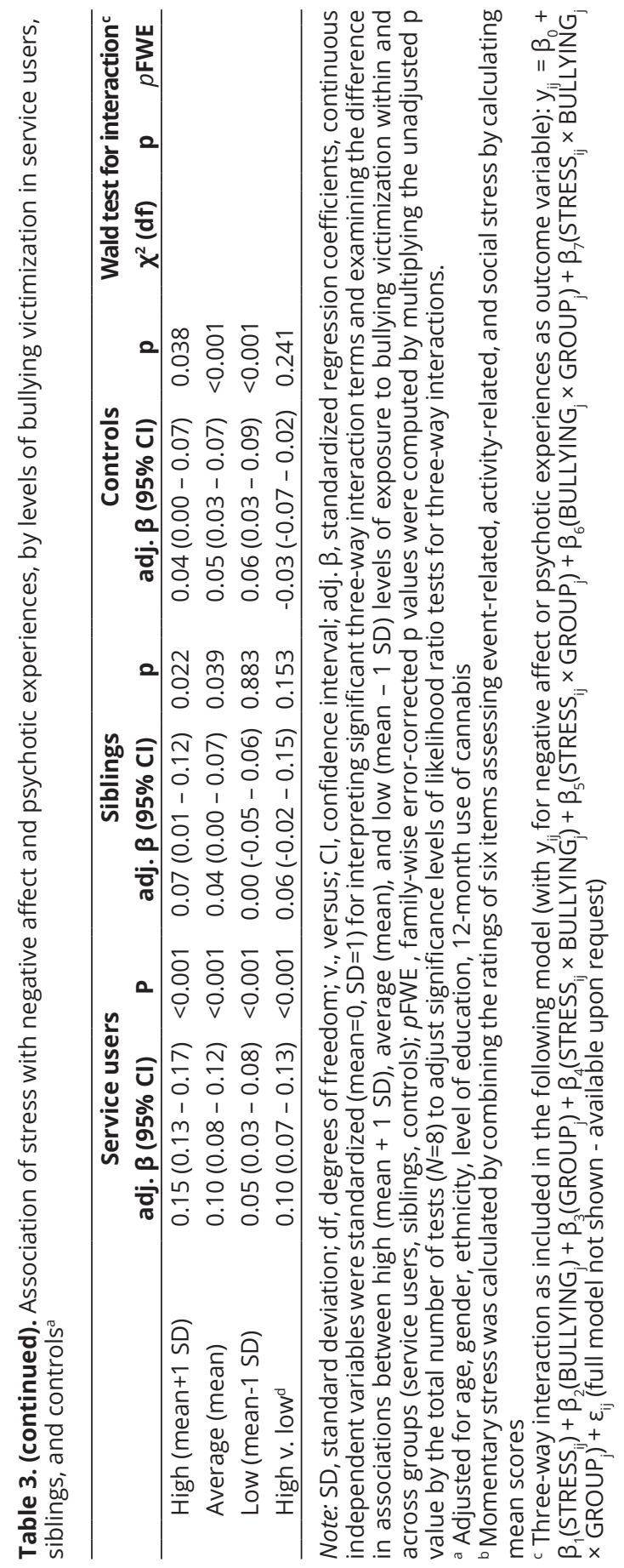




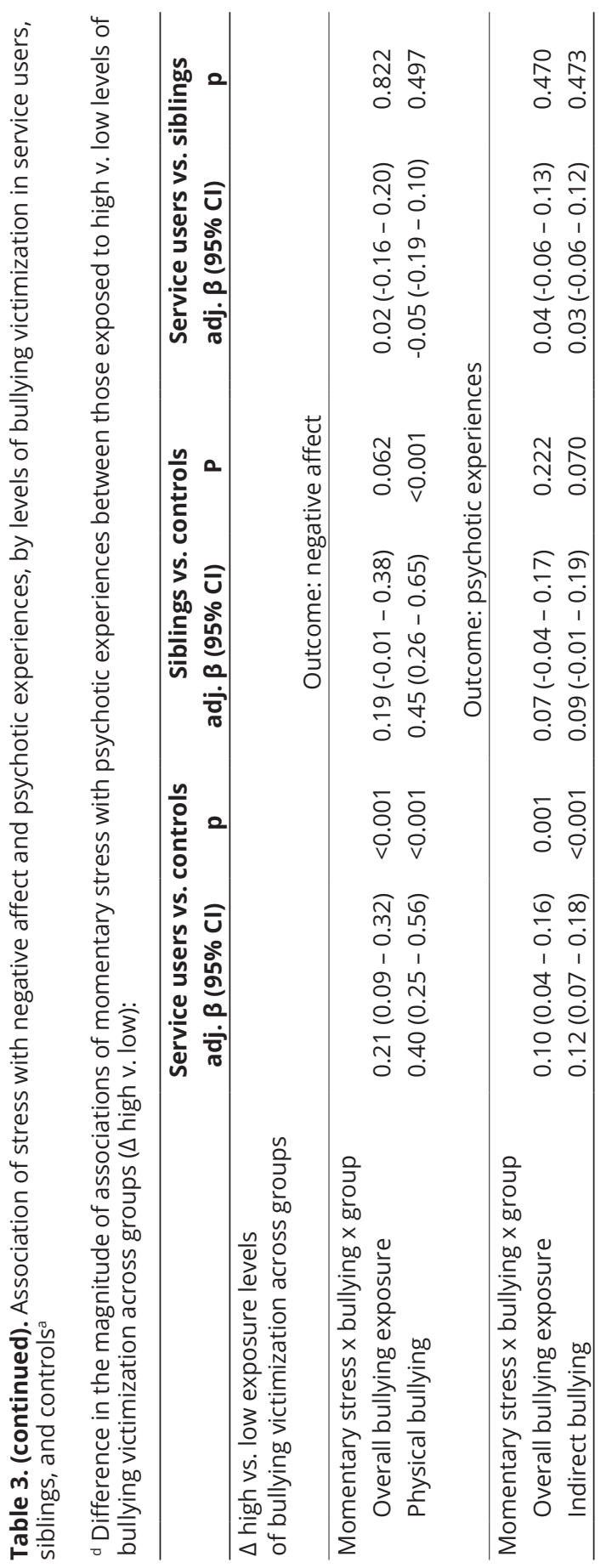




\section{Between-group comparisons}

To investigate whether the impact of exposure to bullying victimization on stress sensitivity differed across groups, differences in magnitude of associations between those exposed to high vs. low levels of bullying victimization were examined across groups. The difference in magnitude of associations between stress and negative affect was greater in service users than in controls when high vs. low levels of exposure to overall bullying victimization (adj. $\beta=0.21, p<0.001$ ) as well as physical (adj. $\beta=0.40, p<0.001$ ) bullying were compared. Further, there were differences in the magnitude of associations between stress and negative affect by physical (adj. $\beta=0.45, p<0.001$ ) and, at trend level, overall (adj. $\beta=0.19$, $p=0.062$ ) bullying comparing siblings vs. controls. No differences were found comparing service users vs. siblings.

\section{Association between stress and psychotic experiences by bullying victimization and group}

There was evidence that exposure to overall bullying victimization as well as indirect bullying, but not verbal and physical bullying, amplified the association of momentary stress with psychotic experiences, as evidenced by statistically significant 3-way interaction effects described below (Table 3).

\section{Within-group comparisons}

Within groups, momentary stress was associated with more intense psychotic experiences in service users (adj. $\beta=0.09, p<0.001$ ) exposed to high overall bullying victimization levels compared to those with low exposure levels, while no differences by bullying exposure were found in siblings (adj. $\beta=0.05, p=0.278$ ) and controls (adj. $\beta=-0.01, p=0.575$ ) (Table 3). Analyses of secondary hypotheses revealed that stress was associated with more intense psychotic experiences in service users ( $a d j . \beta=0.10, p<0.001$ ), but not in siblings ( $a d j . \beta=0.06, p=0.153$ ), and controls (adj. $\beta=-0.03, p=0.241$ ) comparing high vs. low levels of indirect bullying. There was no evidence that physical as well as verbal bullying modified the psychotic reactivity to stress in daily life. Results of exploratory analyses that test effect modification by levels of bullying exposure for associations of specific stressors (event-related, activity-related, and social) with psychotic experiences are provided in Supplement 1 and Table S2. 


\section{Between-group comparisons}

There were differences in the magnitude of associations between momentary stress and psychotic experiences in those exposed to high vs. low exposure levels to overall bullying victimization comparing service users and controls (adj. $\beta=0.10$, $p=0.001$ ), but not service users and siblings ( $\operatorname{adj} . \beta=0.04, p=0.470$ ) and siblings and controls (adj. $\beta=0.07, p=0.222$ ). Further, there was evidence for differences in the magnitude of associations between stress and psychotic experiences by exposure levels to indirect bullying comparing service users and controls (adj. $\beta$ $=0.12, p<0.001$ ), and, at trend level, siblings and controls (adj. $\beta=0.09, p=0.070$ ), but not service users and siblings ( $a d j$. $\beta=0.03, p=0.473$ ).

As groups differed considerably with regard to cannabis use, a sensitivity analysis is provided in Table S3 testing primary hypotheses while also controlling for 12-month prevalence of cannabis use. A similar pattern of findings emerged.

\section{Discussion}

\section{Main findings}

In line with primary and secondary hypotheses, our findings suggest that exposure to overall, as well as specific types of (i.e. physical and indirect, but not verbal), bullying victimization modifies individuals' affective and psychotic reactivity to minor stress in daily life. While there was strong evidence that service users who were exposed to high levels of bullying victimization reported more intense (i) negative affect and (ii) psychotic experience in response to stress compared to those with low exposure levels, controls showed either no marked differences or, intriguingly, less intense negative affect (evident for physical bullying) by exposure levels. In siblings, a less consistent pattern of findings was observed.

\section{Methodological considerations}

The current findings should be interpreted in light of potential limitations. First, bullying victimization was assessed retrospectively using a self-report measure. Thus, recall bias may have influenced reported findings [55] and recent studies also indicate that retrospectively assessed adverse childhood experiences, including bullying, may identify largely different groups of individuals [56]. However, potential effects may have been minimized due to the young age of the sample and 
the reduced time that had passed between exposure and assessment. Similarly, ESM measures were based on self-report. While this allows for ecologically valid assessment of experiences and contexts in real life, an important next step is to investigate how bullying victimization impacts on individuals' stress sensitivity on other levels of investigation, including biological markers [57-59] and passively assessed sensor data [60]. Second, due to potentially high assessment burden associated with ESM assessment for some participants, selection may have influenced our findings and possibly introduced bias, particularly if differential by bullying exposure. However, studies have shown that the ESM is a feasible and reliable assessment method in adolescent and adult populations [35]. In addition, extensive briefing on the ESM procedure resulted in a sufficient number of responses (i.e. $\geq 20$ valid responses with, on average, 45 observations in each group) in most participants (90.8\%). Also, there was no evidence that the number of valid responses differed across groups. Thus, as maximum likelihood estimations were used allowing for use of all available data, the potential impact of selection and sampling bias are kept at a minimum. Third, while we adjusted for potential confounders (i.e. age, sex, ethnicity, level of education), other unmeasured factors could have influenced reported findings (e.g. polygenetic risk for various psychopathologies and personality traits, other socio-environmental risk factors). Further, current mental health problems may influence reporting of bullying victimization and stress sensitivity and may, as a consequence, influence the interpretation of reported findings. Time-lagged analyses would be required to adjust for levels of symptoms in analyses of experience sampling data, which, in turn, would require a higher number of observations to conduct such analyses with sufficient power. Fourth, the sibling group was comparably small $(N=17)$ and inconsistent findings may have occurred due to sampling error. Fifth, experience sampling data over the 6-day assessment period was used for cross-sectional modelling. Thus, the temporal order of stress, negative affect, and psychotic experiences were not specifically investigated. We therefore cannot rule out that reverse causality may have affected our findings. Similarly, using the RBQ and combining total scores of bullying exposure at primary and secondary school did not allow us for investigating the precise timing of the effects of bullying exposure on stress sensitivity. Thus, we cannot rule out that some participants may have been exposed to bullying during ESM assessments and hence timing of exposure, mechanism, and outcome could not be established. Future studies 
may investigate the effects of timing of bulling exposure on stress sensitivity by using time-lagged analyses potentially in combination with multilevel moderated mediation models and a cohort design to test for temporality as an important criterion for establishing causality [61]. Sixth, we combined all stress items to test primary and secondary hypotheses. However, using a composite measure of minor stressors may require further scrutiny by psychometric experience sampling studies. Last, we decided to recode a bipolar scale assessing eventrelated stress from "very unpleasant" (coded as -3) to "very pleasant" (coded as 3) into a unipolar scale including only unpleasant and neutral events that have happened since the last beep (sores ranging from 1-4). This may have resulted in potential underrepresentation of event-related stress in the composite stress score. We computed sensitivity analyses including the bipolar event-related stress scale and found no marked differences for reported associations (see Table S4).

\section{Comparison with previous research}

In recent years, evidence has accumulated that exposure to adverse childhood experiences, including bullying victimization [4-12], is associated with an increased risk of developing mental health problems. However, our understanding of candidate mechanisms remains limited, especially in youth. A process of sensitization that may ultimately lead to lasting changes in individuals' responses to stress has been proposed to form a common mechanistic pathway that may partly explain associations between exposure to socio-environmental risk and psychopathology [28]. At a behavioral level, this proposition has been investigated using ESM and is largely supported by findings of an increased affective and psychotic reactivity in response to minor daily stressors in adults with various mental health problems and experiences of childhood trauma and adult life events [36-39].

In the current study, we found, for the first time, that young help-seeking individuals who were exposed to high levels of bullying victimization at elementary and/or secondary school responded with more intense negative affect and psychotic experiences to minor stress in their daily lives compared to those with low exposure levels. These findings are in accordance with reported effects of childhood trauma on stress sensitivity derived from the same sample [40] and may lend further support to behavioral sensitization as a process that emerges from 
adversity and that may contribute to push people along pathways to poor mental health outcomes in daily life in developmentally early stages of psychopathology.

In contrast, the response to stress was not differentially amplified by bullying exposure levels in controls. Specifically, controls exposed to high, but not low, exposure levels to physical bullying appeared to be resilient to its effects indicated by less intense negative affect in response to stress comparing high vs. low exposure levels. This is an interesting finding and parallels previous findings in which physical abuse and neglect [40] as well as sexual abuse [38] were found to be associated with lower negative affect in response to stress in controls. This may suggest that high levels of exposure to more intrusive forms of adversity may lead to the development of resilience towards subsequent stress in some individuals who do not develop help-seeking behavior, though some inconsistencies were observed in previous studies [38, 40] and direct replication studies are needed before firm conclusion can be drawn. We may speculate, however, that various protective factors may partly explain this finding, especially if they are differentially utilized and/or available in controls compared to service users, including good interpersonal relationships, social support, various personality characteristics, positive atmosphere at home, higher levels of neighborhood social cohesion, high self-esteem, low rumination tendencies, and low polygenetic risk [62,63]. While tempting, this explanation needs to be carefully tested in future studies. It also corroborates, more generally, previous research that has shown that a large proportion of individuals exposed to socio-environmental risk do show resilience to its detrimental effects on mental health [64].

In siblings, a less consistent pattern of findings was observed with no differences in individuals' affective and psychotic reactivity to stress by exposure levels except some evidence for more intense negative affect in response to stress for those exposed to high vs. low levels of physical bullying. These results, however, should be interpreted with caution as the sample size was small and, therefore, findings may have occurred due to sampling error. In addition, as siblings represent an intermediate risk group, it may be speculated that only a small proportion develop a heightened sensitivity to stress while others are more resilient which leads to inconsistent findings at the group level. Notably, we found no evidence that verbal bullying modified the affective and psychotic reactivity in response to stress in all groups. 
Interestingly, secondary analyses revealed that physical bullying at school was associated with more intense negative affect, whereas indirect bullying was associated with more intense psychotic experiences in response to stress in daily lives of help-seeking young individuals. These findings may be interpreted in light of cognitive models of psychosis $[65,66]$ in which various psychological factors and dysfunctional schemas are thought to be crucial in the development and maintenance of delusional ideations, one form of psychotic experiences. A core feature of delusions is thought to be an unfounded belief, and not a founded 'proof', that harm will occur from others. While speculative, indirect bullying may be more strongly associated with the development of persecutory beliefs of other people wanting to harm than other types of bullying which are more directly associated with physical violence, leading to an increased likelihood to respond with delusional ideations to daily life stressors. In following this line, one would expect that the psychotic reactivity to socially stressful situations is especially amplified by indirect bullying. This was, however, not the case in our exploratory analyses after we adjusted for multiple testing. Instead, indirect bullying was associated with an elevated psychotic reactivity to activity-related stress in service users. To the best of our knowledge, no study has specifically investigated differential associations of various bullying types with specific symptom domains of the psychosis spectrum.

Although strong evidence was found that bullying victimization modifies affective and psychotic reactivity to minor stress in help-seeking individuals, future studies should further investigate effects of poly- and re-victimization on stress sensitivity. Arguably, exposure to various adverse childhood experiences and other socio-environmental risk factors, the so-called exposome [67], may lead to an accumulation of risk by progressively increasing individuals' sensitivity to stress that may, in turn, contribute to the develop and maintenance of mental health problems. This approach would also account for findings that most risk factors are prone to cluster within a relatively small number of vulnerable individuals $[2$, $6]$ and tend to be associated with mental disorders in a dose-response fashion [15]. Additionally, more research is needed that focusses on investigating timing of bullying exposure, mechanism, and outcome by conducting well-controlled cohort studies to test whether elevated stress sensitivity mediates the association between exposure to and the onset of mental disorders. In using this study 
design, the potential buffering role of protective factors (e.g. number of close relationships, coping skills, personality traits) on stress sensitivity as well as potential complex interactions with other socio-environmental and genetic risk factors may be further investigated [62]. Lastly, in line with findings of frequently co-occurring psychopathological domains, especially at a developmentally early stage [23], we found high levels of depressive, anxiety, and psychotic symptoms and high proportions of non-specific diagnoses and comorbidity in service users. This further supports dimensional models of psychopathology [18, 19, 21] as well as notions of extended and transdiagnostic phenotypes [22].

\section{Conclusion}

Our findings suggest that individuals' response to minor stress in daily life may represent a putative risk or resilience mechanism through which exposure to bullying victimization may impact on mental health in youth. As dissipation of detrimental effects of bullying victimization on mental health has recently been reported to occur over time [7], programs that aim to prevent bullying victimization at school and inform teachers, parents, and the general public remain the ultimate goal. There is also a pressing need to directly assess bullying victimization in youth mental health services to integrate and directly tackle these adverse experiences in psychological interventions. Finally, to interrupt the process of prolonged sensitization to stress and alleviate individuals' mental health burden in daily life, novel mHealth tools (e.g. ecological momentary interventions) may be used to provide treatment components in real life using interactive delivery schemes to extend psychotherapy from clinical settings to individuals' everyday environment $[35,68,69]$. 


\section{References}

1. Olweus, D., Bullying at School: What We Know and What We Can Do. 1993: Wiley-Blackwell.

2. Arseneault, L., L. Bowes, and S. Shakoor, Bullying victimization in youths and mental health problems: 'much ado about nothing'? Psychol Med, 2010. 40(5): p. 717-29.

3. Tolan, P.H., International trends in bullying and children's health: giving them due consideration. Arch Pediatr Adolesc Med, 2004. 158(8): p. 831-2.

4. Copeland, W.E., et al., Adult psychiatric outcomes of bullying and being bullied by peers in childhood and adolescence. JAMA Psychiatry, 2013. 70(4): p. 419-26.

5. Lee, K.S. and T. Vaillancourt, Longitudinal Associations Among Bullying by Peers, Disordered Eating Behavior, and Symptoms of Depression During Adolescence. JAMA Psychiatry, 2018. 75(6): p. 605-612.

6. Lereya, S.T., et al., Adult mental health consequences of peer bullying and maltreatment in childhood: two cohorts in two countries. Lancet Psychiatry, 2015. 2(6): p. 524-31.

7. Singham, T., et al., Concurrent and Longitudinal Contribution of Exposure to Bullying in Childhood to Mental Health: The Role of Vulnerability and Resilience. JAMA Psychiatry, 2017. 74(11): p. 1112-1119.

8. Takizawa, R., B. Maughan, and L. Arseneault, Adult health outcomes of childhood bullying victimization: evidence from a five-decade longitudinal British birth cohort. Am J Psychiatry, 2014. 171(7): p. 777-84.

9. Varese, F., et al., Childhood adversities increase the risk of psychosis: a meta-analysis of patient-control, prospective- and cross-sectional cohort studies. Schizophr Bull, 2012. 38(4): p. 661-71.

10. Brunstein Klomek, A., et al., Bi-directional longitudinal associations between different types of bullying victimization, suicide ideation/attempts, and depression among a large sample of European adolescents. J Child Psychol Psychiatry, 2018.

11. Koyanagi, A., et al., Bullying Victimization and Suicide Attempt Among Adolescents Aged 12-15 Years From 48 Countries. J Am Acad Child Adolesc Psychiatry, 2019.

12. Riglin, L., et al., Developmental Contributions of Schizophrenia Risk Alleles and Childhood Peer Victimization to Early-Onset Mental Health Trajectories. Am J Psychiatry, 2019. 176(1): p. 36-43.

13. Forbes, M.K., N.R. Magson, and R.M. Rapee, Evidence that Different Types of Peer Victimization have Equivalent Associations with Transdiagnostic Psychopathology in Adolescence. J Youth Adolesc, 2020. 49(3): p. 590-604.

14. Sourander, A., et al., Association of Bullying Behavior at 8 Years of Age and Use of Specialized Services for Psychiatric Disorders by 29 Years of Age. JAMA Psychiatry, 2016. 73(2): p. 159-65.

15. Green, J.G., et al., Childhood adversities and adult psychiatric disorders in the national comorbidity survey replication I: associations with first onset of DSM-IV disorders. Arch Gen Psychiatry, 2010. 67(2): p. 113-23.

16. Currie, C., et al., Risk behaviours: being bullied and bullying others. Social Determinants of Health and Well-being Among Young People: Health Behaviour in School-Aged Children (HBSC) Study: International Report From the 2009/2010 Survey (Health Policy for Children and Adolescents, No. 6), ed. W.R.O.f. Europe. 2012, Copenhagen, Denmark: Word Health Organization (WHO). 
17. Stanaway, J.D., et al., Global, regional, and national comparative risk assessment of 84 behavioural, environmental and occupational, and metabolic risks or clusters of risks for 195 countries and territories, 1990-2017: a systematic analysis for the Global Burden of Disease Study 2017. The Lancet, 2018. 392(10159): p. 1923-1994.

18. Caspi, A., et al., The p Factor: One General Psychopathology Factor in the Structure of Psychiatric Disorders? Clin Psychol Sci, 2014. 2(2): p. 119-137.

19. Forbes, M.K., et al., Beyond comorbidity: Toward a dimensional and hierarchical approach to understanding psychopathology across the life span. Dev Psychopathol, 2016. 28(4pt1): p. 971-986.

20. Insel, T., et al., Research domain criteria (RDoC): toward a new classification framework for research on mental disorders. Am J Psychiatry, 2010. 167(7): p. 748-51.

21. Kotov, R., et al., The Hierarchical Taxonomy of Psychopathology (HiTOP): A dimensional alternative to traditional nosologies. J Abnorm Psychol, 2017. 126(4): p. 454-477.

22. van Os, J. and U. Reininghaus, Psychosis as a transdiagnostic and extended phenotype in the general population. World Psychiatry, 2016. 15(2): p. 118-24.

23. van Os, J., The dynamics of subthreshold psychopathology: implications for diagnosis and treatment. Am J Psychiatry, 2013. 170(7): p. 695-8.

24. Kessler, R.C., et al., Lifetime prevalence and age-of-onset distributions of DSM-IV disorders in the National Comorbidity Survey Replication. Arch Gen Psychiatry, 2005. 62(6): p. 593-602.

25. McGorry, P.D., et al., Beyond the "at risk mental state" concept: transitioning to transdiagnostic psychiatry. World Psychiatry, 2018. 17(2): p. 133-142.

26. Howes, O.D. and R.M. Murray, Schizophrenia: an integrated sociodevelopmentalcognitive model. Lancet, 2014. 383(9929): p. 1677-1687.

27. Bentall, R.P., et al., From adversity to psychosis: pathways and mechanisms from specific adversities to specific symptoms. Soc Psychiatry Psychiatr Epidemiol, 2014. 49(7): p. 1011-22.

28. Collip, D., I. Myin-Germeys, and J. Van Os, Does the concept of "sensitization" provide a plausible mechanism for the putative link between the environment and schizophrenia? Schizophr Bull, 2008. 34(2): p. 220-5.

29. Howes, O.D., et al., The Role of Genes, Stress, and Dopamine in the Development of Schizophrenia. Biol Psychiatry, 2017. 81(1): p. 9-20.

30. Koss, K.J. and M.R. Gunnar, Annual Research Review: Early adversity, the hypothalamicpituitary-adrenocortical axis, and child psychopathology. J Child Psychol Psychiatry, 2018. 59(4): p. 327-346.

31. Williams, J., et al., Psychological mediators of the association between childhood adversities and psychosis: A systematic review. Clin Psychol Rev, 2018. 65: p. 175-196.

32. du Plessis, M.R., et al., Bullying the Brain? Longitudinal Links Between Childhood Peer Victimization, Cortisol, and Adolescent Brain Structure. Front Psychol, 2018. 9: p. 2706.

33. Cristobal-Narvaez, P., et al., The Interaction between Childhood Bullying and the FKBP5 Gene on Psychotic-Like Experiences and Stress Reactivity in Real Life. PLoS One, 2016. 11(7): p. e0158809.

34. Lambe, L.J., W.M. Craig, and T. Hollenstein, Blunted Physiological Stress Reactivity among Youth with a History of Bullying and Victimization: Links to Depressive Symptoms. J Abnorm Child Psychol, 2019. 
35. Myin-Germeys, l., et al., Experience sampling methodology in mental health research: new insights and technical developments. World Psychiatry, 2018. 17(2): p. 123-132.

36. Lardinois, M., et al., Childhood trauma and increased stress sensitivity in psychosis. Acta Psychiatr Scand, 2011. 123(1): p. 28-35.

37. Myin-Germeys, I., et al., Do life events have their effect on psychosis by influencing the emotional reactivity to daily life stress? Psychological Medicine, 2003. 33(2): p. 327-333.

38. Reininghaus, U., et al., Psychological processes underlying the association between childhood trauma and psychosis in daily life: an experience sampling study. Psychol Med, 2016. 46(13): p. 2799-813.

39. Wichers, M., et al., Mechanisms of gene-environment interactions in depression: evidence that genes potentiate multiple sources of adversity. Psychol Med, 2009. 39(7): p. 1077-86.

40. Rauschenberg, C., et al., Stress sensitivity as a putative mechanism linking childhood trauma and psychopathology in youth's daily life. Acta Psychiatr Scand, 2017. 136(4): p. 373-388.

41. Cristobal-Narvaez, P., et al., Impact of Adverse Childhood Experiences on Psychotic-Like Symptoms and Stress Reactivity in Daily Life in Nonclinical Young Adults. PLoS One, 2016. 11(4): p. e0153557.

42. Lichtenstein, P., et al., Common genetic determinants of schizophrenia and bipolar disorder in Swedish families: a population-based study. Lancet, 2009. 373(9659): p. 2349.

43. van Os, J., B.P. Rutten, and R. Poulton, Gene-environment interactions in schizophrenia: review of epidemiological findings and future directions. Schizophr Bull, 2008. 34(6): p. 1066-82.

44. Shih, R.A., P.L. Belmonte, and P.P. Zandi, A review of the evidence from family, twin and adoption studies for a genetic contribution to adult psychiatric disorders. Int Rev Psychiatry, 2004. 16(4): p. 260-83.

45. Schafer, M., et al., Lonely in the crowd: Recollections of bullying. British Journal of Developmental Psychology, 2004. 22: p. 379-394.

46. Osman, A., et al., Reliability and validity of the Beck depression inventory--Il with adolescent psychiatric inpatients. Psychol Assess, 2004. 16(2): p. 120-32.

47. Konings, M., et al., Validity and reliability of the CAPE: a self-report instrument for the measurement of psychotic experiences in the general population. Acta Psychiatr Scand, 2006. 114(1): p. 55-61.

48. Van der Ploeg, H.M., De Zelf-Beoordelings Vragenlijst (STAI-DY). Tijdschrift voor psychiatrie, 1982. 24(9): p. 576-88.

49. Myin-Germeys, I., M. Birchwood, and T. Kwapil, From environment to therapy in psychosis: a real-world momentary assessment approach. Schizophr Bull, 2011. 37(2): p. 244-7.

50. Reininghaus, U., et al., Stress Sensitivity, Aberrant Salience, and Threat Anticipation in Early Psychosis: An Experience Sampling Study. Schizophr Bull, 2016. 42(3): p. 712-22.

51. Palmier-Claus, J.E., G. Dunn, and S.W. Lewis, Emotional and symptomatic reactivity to stress in individuals at ultra-high risk of developing psychosis. Psychol Med, 2012. 42(5): p. 1003-12. 
52. Dawson, J.F. and A.W. Richter, Probing three-way interactions in moderated multiple regression: development and application of a slope difference test. J Appl Psychol, 2006. 91(4): p. 917-26.

53. Aiken, L.S., West, S. G., Multiple regression: Testing and interpreting interactions. 1991, Thousand Oaks, CA: Sage Publications.

54. Cohen, J., Cohen, P, West, S, Aiken, L, Applied Multiple Regression/Correlation Analysis for the Behavioral Sciences. 2003, Mahwah, NJ: Erlbaum.

55. Reuben, A., et al., Lest we forget: comparing retrospective and prospective assessments of adverse childhood experiences in the prediction of adult health. J Child Psychol Psychiatry, 2016. 57(10): p. 1103-12.

56. Baldwin, J.R., et al., Agreement Between Prospective and Retrospective Measures of Childhood Maltreatment: A Systematic Review and Meta-analysis. JAMA Psychiatry, 2019.

57. Kasanova, Z., et al., Striatal dopaminergic modulation of reinforcement learning predicts reward-oriented behavior in daily life. Biol Psychol, 2017. 127: p. 1-9.

58. Vaessen, T., et al., Overall cortisol, diurnal slope, and stress reactivity in psychosis: An experience sampling approach. Psychoneuroendocrinology, 2018. 96: p. 61-68.

59. Ouellet-Morin, l., et al., A discordant monozygotic twin design shows blunted cortisol reactivity among bullied children. J Am Acad Child Adolesc Psychiatry, 2011. 50(6): p. 574-582 e3.

60. Onnela, J.P. and S.L. Rauch, Harnessing Smartphone-Based Digital Phenotyping to Enhance Behavioral and Mental Health. Neuropsychopharmacology, 2016. 41(7): p. 1691-6.

61. Hill, A.B., The Environment and Disease: Association or Causation? Proc R Soc Med, 1965. 58: p. 295-300.

62. Crush, E., et al., Protective Factors for Psychotic Symptoms Among Poly-victimized Children. Schizophr Bull, 2018. 44(3): p. 691-700.

63. Fritz, J., et al., A Systematic Review of Amenable Resilience Factors That Moderate and/ or Mediate the Relationship Between Childhood Adversity and Mental Health in Young People. Front Psychiatry, 2018. 9: p. 230.

64. Rutten, B.P., et al., Resilience in mental health: linking psychological and neurobiological perspectives. Acta Psychiatr Scand, 2013. 128(1): p. 3-20.

65. Freeman, D., Persecutory delusions: a cognitive perspective on understanding and treatment. Lancet Psychiatry, 2016. 3(7): p. 685-92.

66. Garety, P.A., et al., A cognitive model of the positive symptoms of psychosis. Psychol Med, 2001. 31(2): p. 189-95.

67. Guloksuz, S., J. van Os, and B.P.F. Rutten, The Exposome Paradigm and the Complexities of Environmental Research in Psychiatry. JAMA Psychiatry, 2018. 75(10): p. 985-986.

68. Myin-Germeys, I., et al., Ecological momentary interventions in psychiatry. Curr Opin Psychiatry, 2016. 29(4): p. 258-63.

69. Reininghaus, U., C.A. Depp, and I. Myin-Germeys, Ecological Interventionist Causal Models in Psychosis: Targeting Psychological Mechanisms in Daily Life. Schizophr Bull, 2016. 42(2): p. 264-9. 


\section{Supplementary material}

\section{Supplement 1: Results of exploratory analyses}

\section{Results}

\section{Association between momentary stressors and negative affect by bullying victimization and group}

We found no evidence that prior exposure to overall as well as specific types of bullying victimization modified the association of event-related with negative affect (Table S1). However, the association of activity-related stress and social stress with negative affect was amplified by physical bullying, but not by exposure to overall bullying victimization as well as other bullying types (i.e. verbal and indirect). Evidence for effect modification by levels of bullying exposure within and across groups was evidenced by statistically significant 3-way interaction effects (Table S1).

\section{Within-group comparisons}

Within groups, activity-related stress was associated with less intense negative affect in controls with high vs. low physical bullying levels (adj. $\beta=-0.28, p<0.001$ ), whereas no differences were observed in service-users ( $\operatorname{adj} . \beta=0.05, p=0.120$ ) and siblings (adj. $\beta=0.04, p=0.619$ ). In addition, social stress was associated with more intense negative affect in service users (adj. $\beta=0.08, p=0.004$ ) and less intense negative affect in controls (adj. $\beta=-0.26, p=0.004$ ), but no differences in siblings, when high vs. low physical bullying levels were compared.

\section{Between-group comparisons}

To investigate whether the impact of exposure to bullying victimization on stress sensitivity differed across groups, differences in magnitude of associations between those exposed to high vs. low levels of bullying victimization were examined across groups. The difference in magnitude of associations between activity-related stress and negative affect was greater in service users vs. controls (adj. $\beta=0.33, p<0.001$ ) and siblings vs. controls ( $a d j . \beta=0.32, p=0.002$ ), but not in service users vs. siblings (adj. $\beta=0.01, p=0.891$ ), when high vs. low levels of physical bullying were compared. In addition, there were significant differences in the 
magnitude of associations between social stress and negative affect by levels of physical bullying in service users vs. controls (adj. $\beta=0.34, p<0.001$ ) and siblings vs. controls (adj. $\beta=0.39, p=0.001$ ), but not service users vs. siblings ( $\operatorname{adj} . \beta=-0.05$, $p=0.522)$.

\section{Association between momentary stressors and psychotic experiences by bullying victimization and group}

Within-group comparisons

We found no evidence that prior exposure to overall as well as specific types of bullying victimization modified the association of event-related and social stress with psychotic experiences (Table S2). However, we found evidence that activityrelated stress was associated with more intense psychotic experiences in service users (adj. $\beta=0.09, p<0.001$ ), but not in siblings ( $a d j . \beta=0.04, p=0.417$ ) and controls (adj. $\beta=-0.02, p=0.367$ ) (Table S2) when high vs. low exposure levels to indirect bullying were compared, while no evidence for effect modification was found for overall bullying victimization as well as physical and verbal bullying.

\section{Between-group comparisons}

There were differences in the magnitude of associations of activity-related stress with psychotic experiences by high vs. low exposure levels to indirect bullying victimization comparing service users and controls (adj. $\beta=0.11, p<0.001$ ), but not service users and siblings (adj. $\beta=0.05, p=0.280$ ) and siblings and controls (adj. $\beta=0.06, p=0.249$ ). 


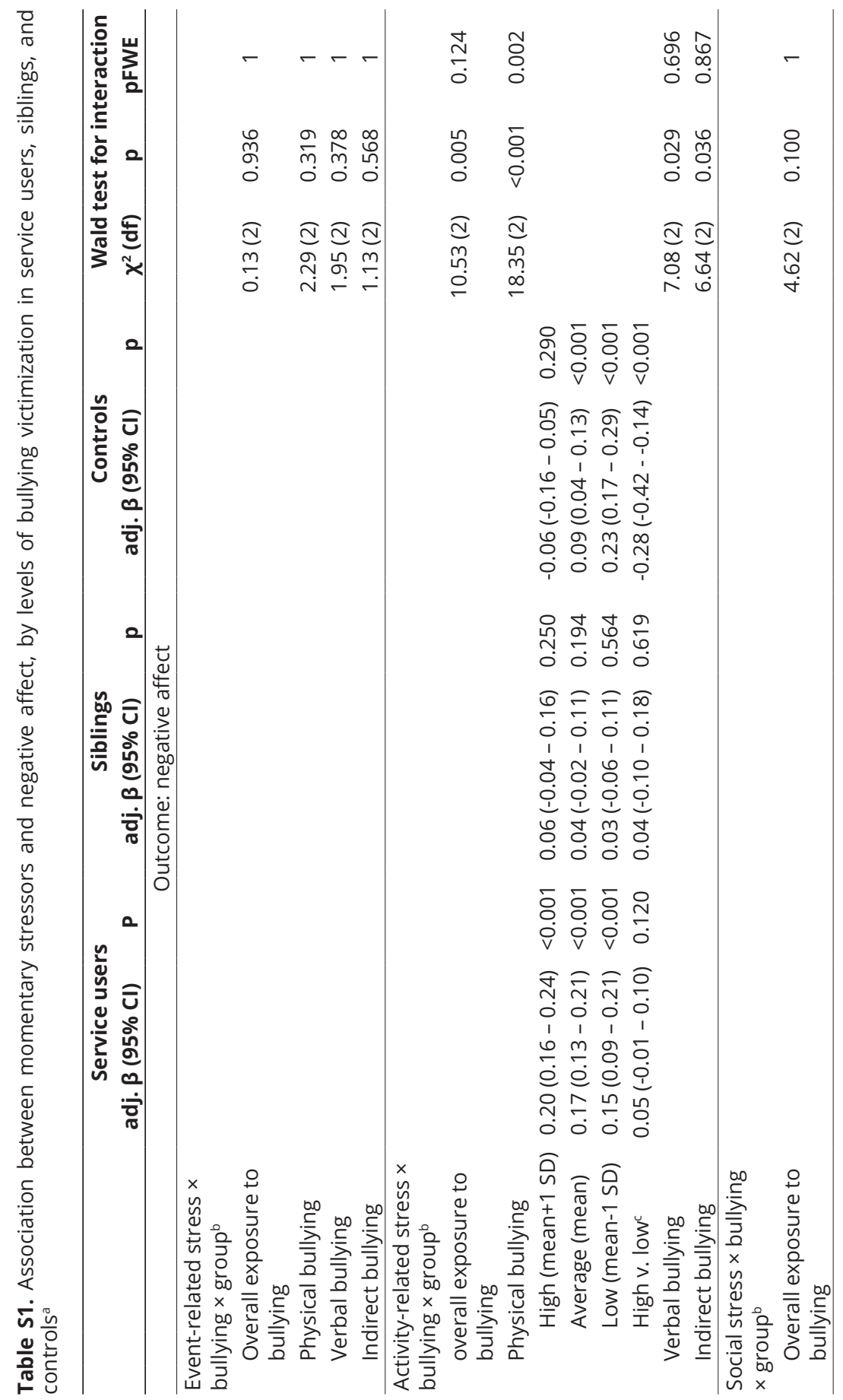




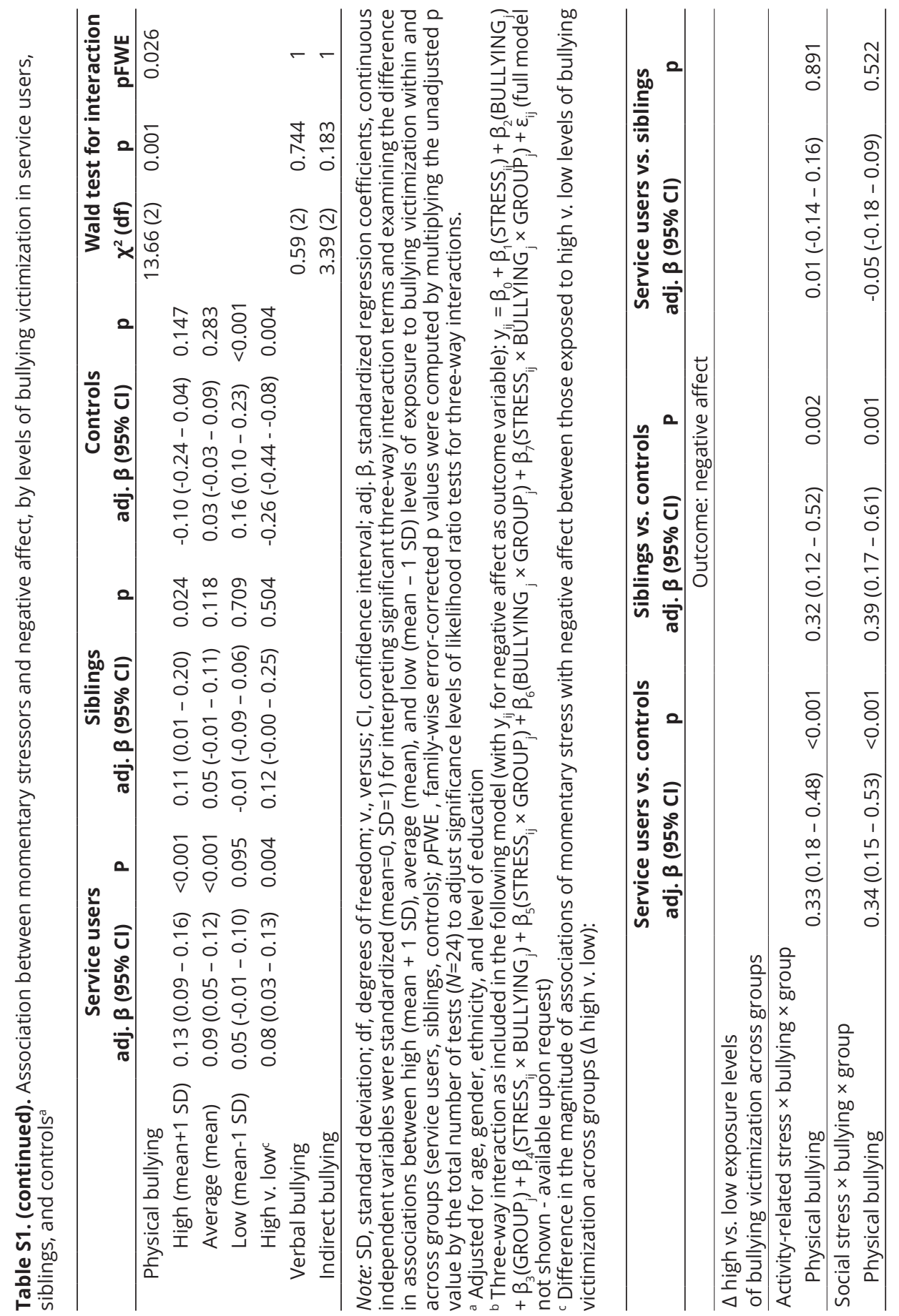




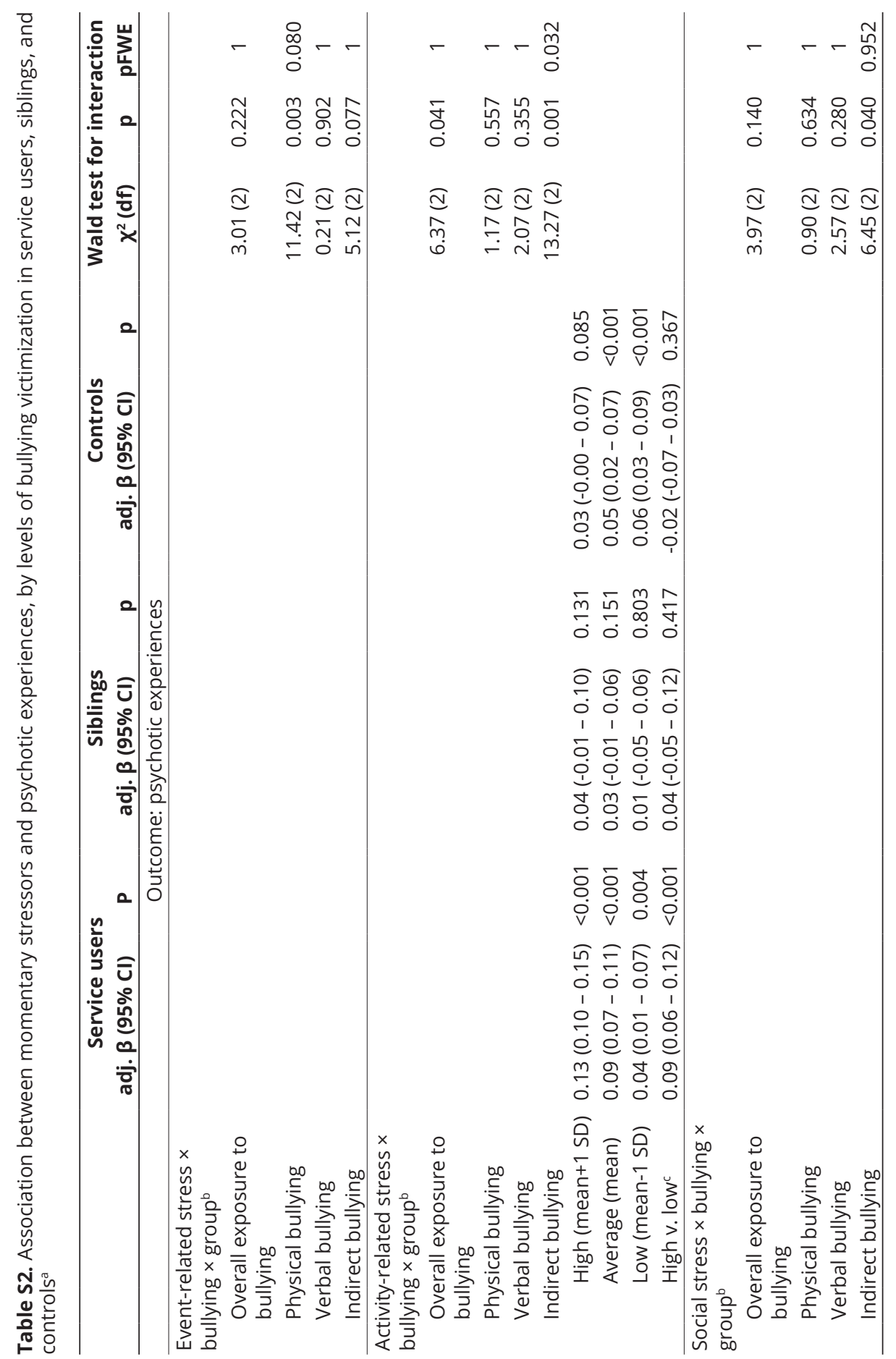




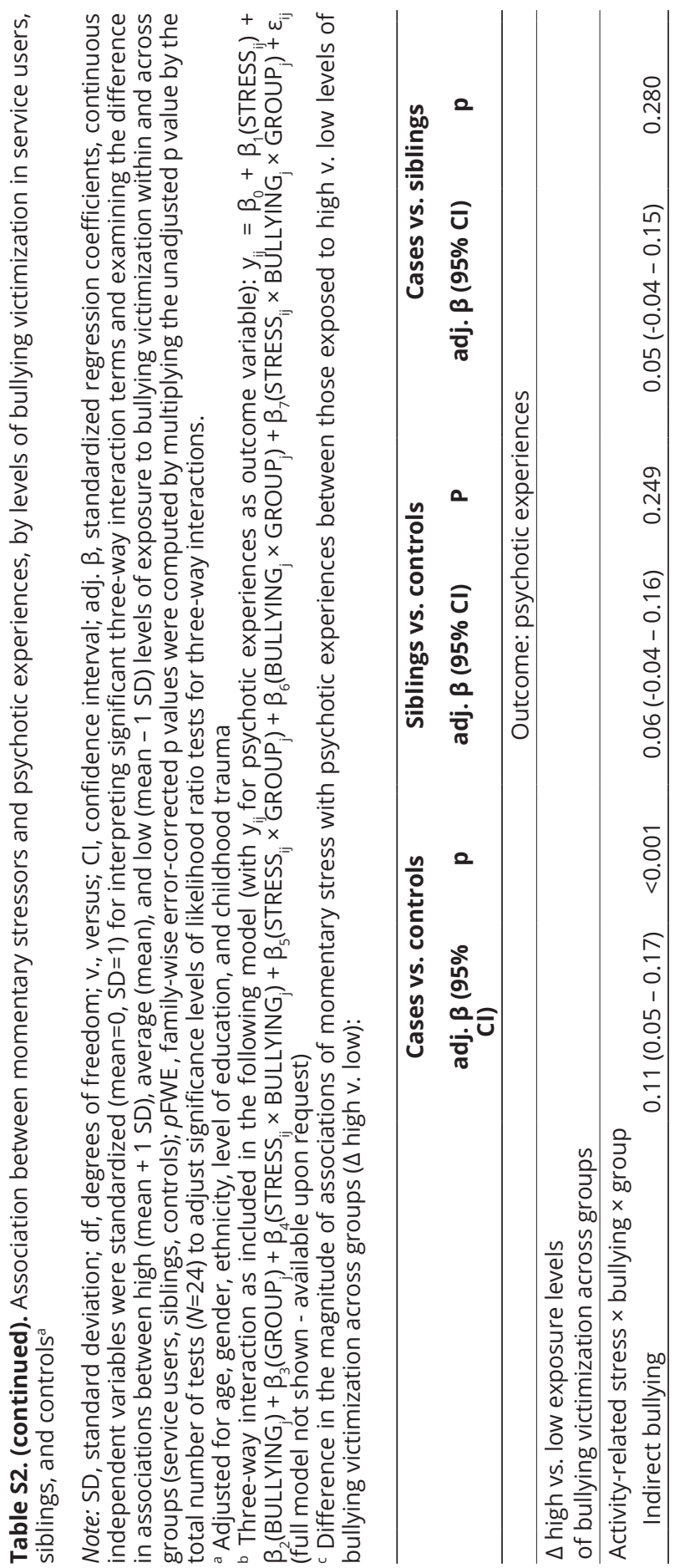




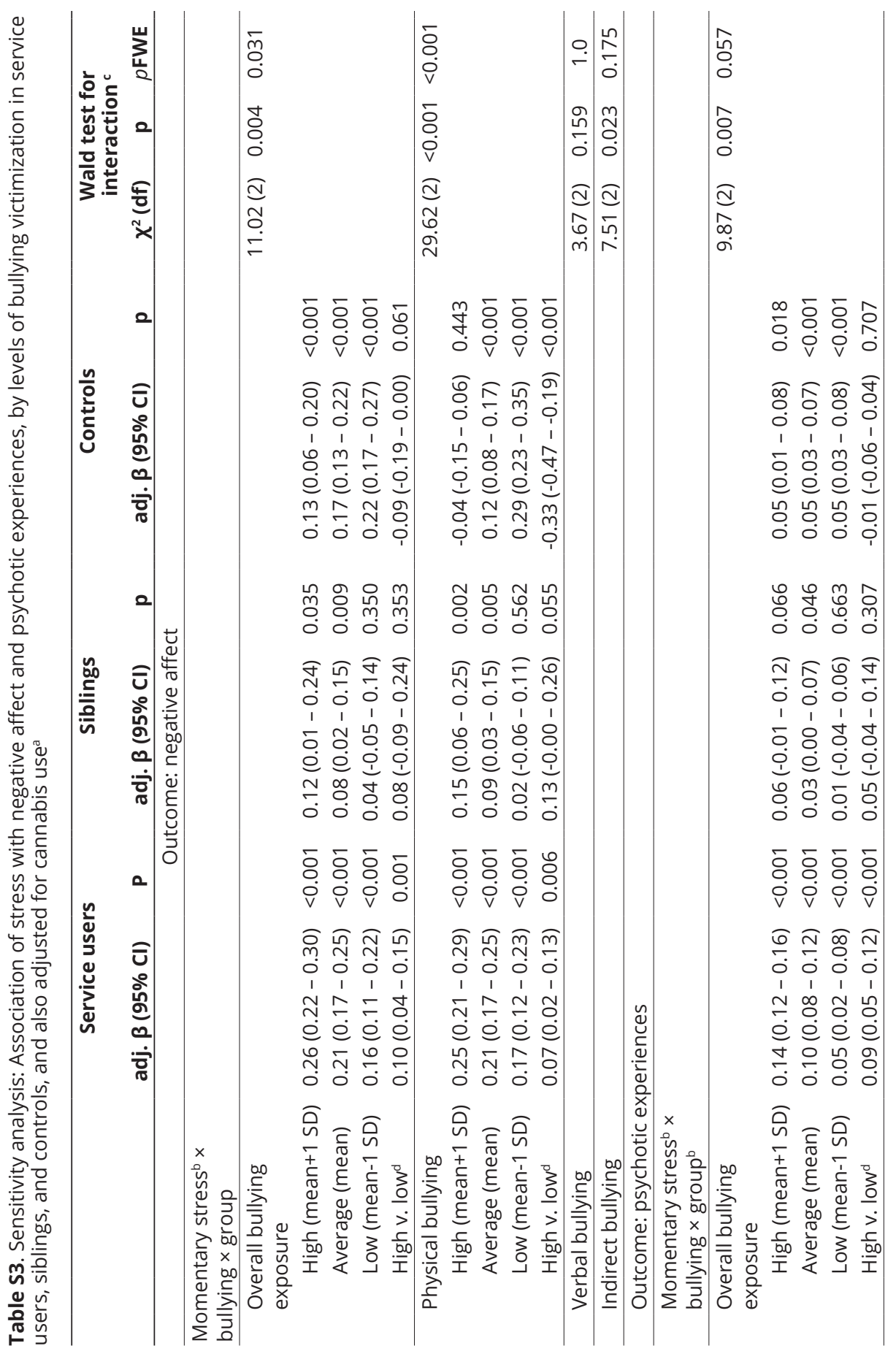




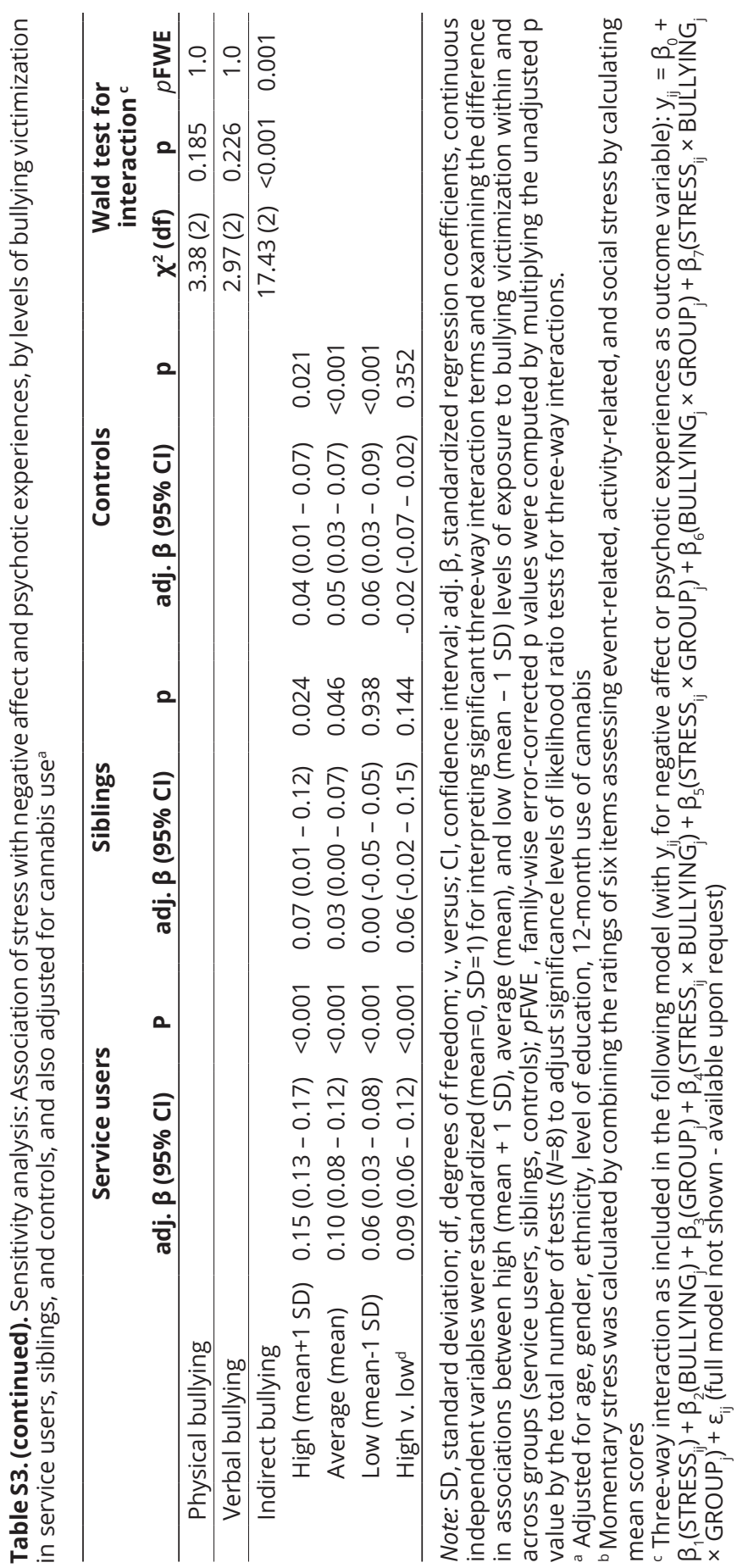




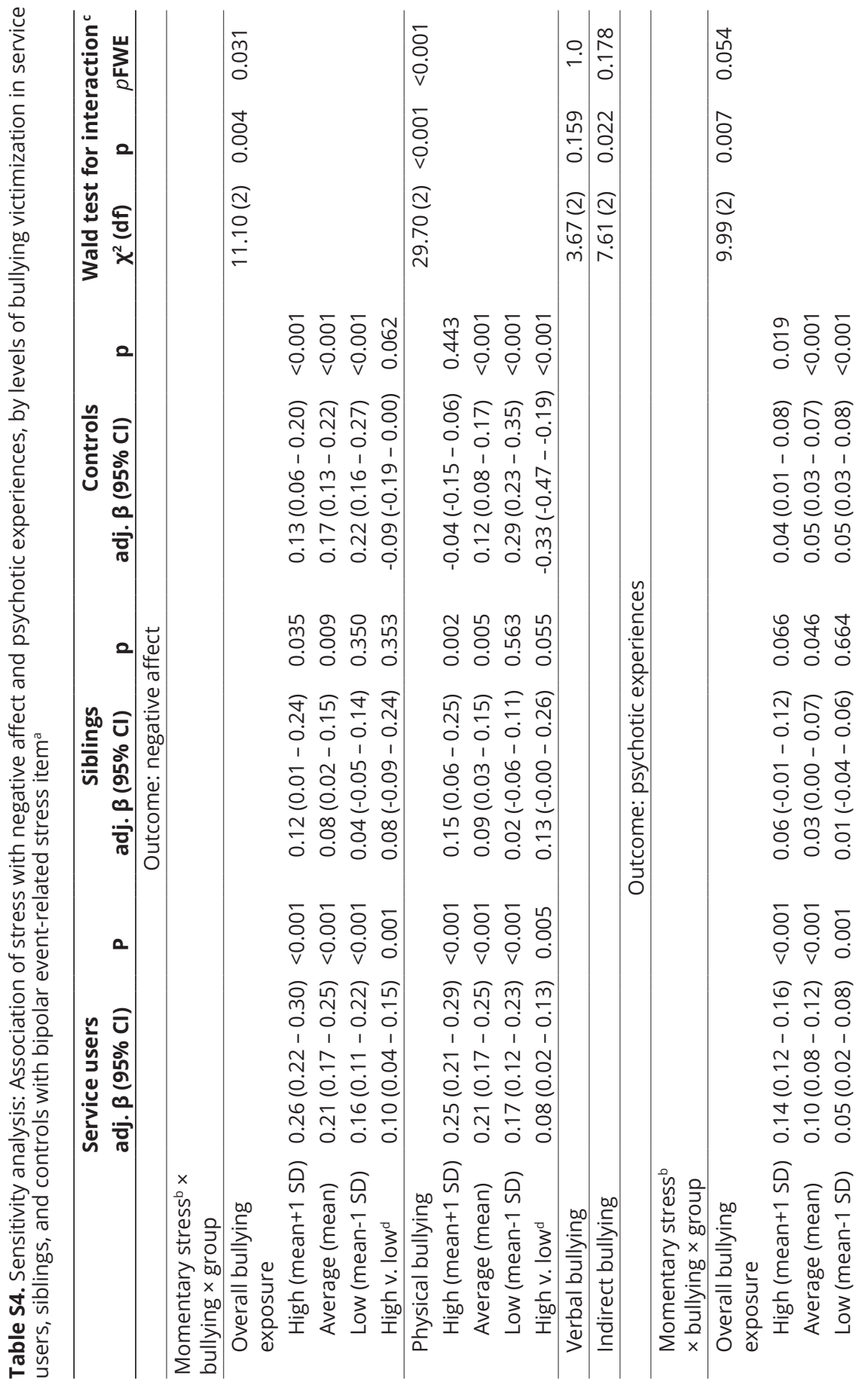




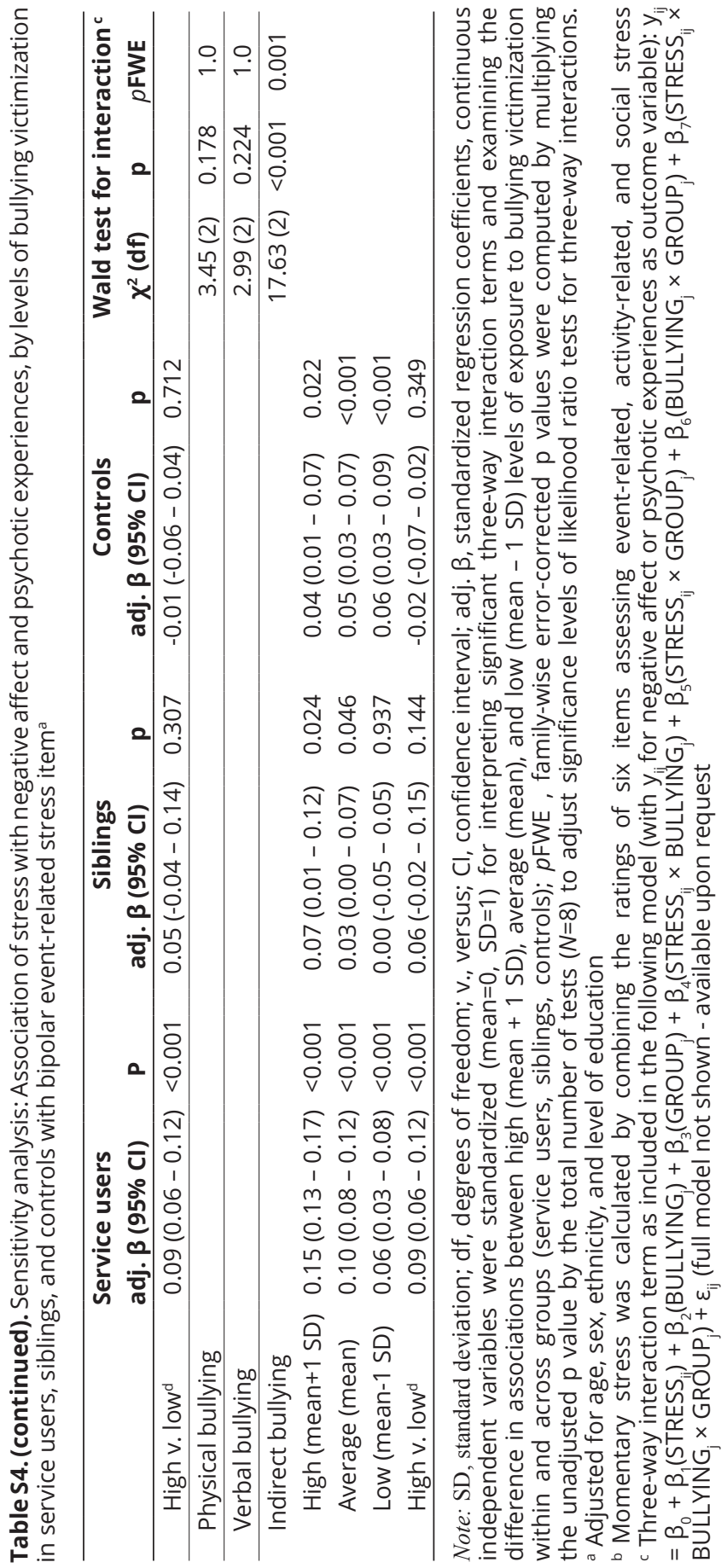





\section{CHAPTER 6}

\section{Negative life events and stress sensitivity in youth's daily life: an ecological momentary assessment study}




\section{Abstract}

Background: Negative life events (LEs) are associated with mental health problems in youth. However, little is known about underlying mechanisms. The aim of the study was to investigate whether exposure to LEs modifies stress sensitivity in youth's daily life.

Methods: Ecological Momentary Assessment (EMA) was used to assess stress sensitivity (i.e. association of momentary stress with (i) negative affect and (ii) psychotic experiences) in 99 adolescents and young adults (42 service users, 17 siblings, and 40 controls; $M_{\text {age }} 15$ years). Before EMA, exposure to LEs (e.g., intrusive threats, experience of loss, serious illness) was assessed.

Results: Lifetime as well as previous-year exposure to LEs modified stress sensitivity in service users: they experienced more intense negative affect and psychotic experiences in response to stress when high vs. low exposure levels were compared. In contrast, controls showed no differences in stress sensitivity by exposure levels. Looking at specific types of LEs, controls showed less intense negative affect in response to stress when high vs. low exposure levels to threatening events during the last year, but not lifetime exposure, were compared. In siblings, no evidence was found that LEs modified stress sensitivity.

Conclusion: Stress sensitivity may constitute a putative risk and protective mechanism linking LEs and mental health in help-seekingyouth, while unfavourable effects of LEs on stress sensitivity may attenuate over time or do not occur in controls and siblings. Targeting individuals' sensitivity to stress in daily life using novel digital interventions may be a promising approach towards improving youth mental health.

Keywords: Life Events; Stress Sensitivity; Youth Mental Health; Psychopathology; Ecological Momentary Assessment 


\section{Introduction}

Most mental disorders manifest early ( $75 \%$ by age 24 ) and often increase in severity and specificity over time [1]. The onset of many mental disorders - e.g., psychotic, anxiety, mood, personality, eating, and substance-use disorders - fall into discrete time periods spanning from early adolescence (before age 14 ) to early adulthood (before age 25) [2]. Consequently, there has been a reform of youth mental health services [3] aimed at disrupting illness trajectories at developmentally early stages [4]. However, development and implementation of early intervention strategies are complicated by high comorbidity rates [2], and limited knowledge of underlying mechanisms, especially in the realm of youth mental health.

Mental health problems (e.g., depression, anxiety, psychosis) frequently co-occur during early stages of psychopathology [4] and share socio-environmental (e.g., negative life events) and genetic risk. This supports the notion of transdiagnostic phenotypes, including the extended psychosis spectrum phenotype [5], which are characterized by temporal and phenomenological continuity across developmental stages of clinical and subclinical mental health problems crossing traditional diagnostic boundaries. For instance, individuals reporting psychotic experiences are at increased risk of developing psychotic and affective disorders and the presence of psychotic experiences has been shown to predict greater illness severity as well as poorer treatment outcomes [5]. Thus, psychotic experiences may represent a severity marker of psychopathology [5], and subclinical as well as clinical expressions of affective dysregulation and psychosis may help elucidate putative underlying mechanisms through which socio-environmental risk factors, such as negative life events, impact on poor mental health.Life events (LEs) are situations with clear beginnings and endings that generate positive or negative changes within personal circumstances, and/or contain an element of immediate threat [6]. Widely studied LEs include exposure to serious illness, death of a family member, financial hardship, intrafamilial conflict, relationship conflicts and divorce, occupational changes, and legal problems [6]. Increasing evidence suggests exposure to LEs is, as other forms of adverse experiences, non-specifically associated with psychopathology [6-8] such as depression [9], schizophrenia [10], anxiety [11], attention-deficit/hyperactivity disorder [12], and suicidality [13]. Moreover, studies suggest a dose-response relationship in which higher numbers of LEs and the co-occurrence with other socio-environmental risk factors are 
associated with increased severity of psychopathology, including psychosis [14]. This is in accordance with a recent study demonstrating that individuals with firstepisode psychosis (FEP) were almost four times more likely than healthy controls to have encountered both childhood and recent stressful LEs (e.g., death, divorce, sickness/accidents), in contrast to exposure to early LEs alone [15]. Considering that early adverse childhood adversities (ACEs) are linked to later psychopathology [2], and an increasing number of LEs exposure is suggested to increase odds of developing various mental health problems, including FEP [14], it is crucial to investigate LEs in the context of early-stage psychopathology.

Studies have also investigated associations of specific types of LEs with mental health problems. For instance, LeMoult and colleagues (2019) have shown that the death of a family member was associated with a higher risk of developing major depressive disorder before age 18. In youth, loss experiences have been linked to depressive symptoms, while events characterized by future threat were associated with anxiety [16]. Notably, there is a growing body of evidence suggesting the impact of LEs on mental health problems may be mediated by individuals' cognitive appraisal of and secondary to the event [17]. Thus, overall, there is evidence of an association between LEs exposure and mental health problems. Critically, however, underlying processes and mechanisms involved remain largely under-researched, especially in youth.

Elevated stress sensitivity in daily life has been proposed to be a transdiagnostic psychological mechanism contributing to the development and maintenance of mental health problems. Integrated models suggest that, as a consequence of exposure to socio-environmental risk (e.g., LEs), a process of gradual sensitization makes individuals more reactive to subsequent adversity as well as minor stressors in daily life (often referred to as elevated stress sensitivity or reactivity, which is partly related to other models, including the Diathesis-Stress Model or the Kindling hypothesis) $[10,18,19]$. It is thought that an increased sensitivity to minor stress in daily life may contribute to mental health problems and play a non-specific role in linking LEs and psychopathology in help-seeking individuals.

Context-sensitive ecological momentary assessment (EMA) - also known as experience sampling method - is a self-assessment diary technique that may be particularly well-suited to test the proposed role of stress sensitivity on a 
behavioural level by investigating whether exposure to LEs is associated with heightened stress sensitivity [20]. In recent EMA studies, derived from the same sample as the present study, exposure to childhood trauma and bullying victimization [21,22] was associated with elevated stress sensitivity in help-seeking youth. To our knowledge, however, no study has investigated the modifying effects of negative LEs on stress sensitivity in help-seeking youth. To investigate the role of stress sensitivity in linking exposure to LEs and mental disorders at a developmentally early stage of psychopathology, help-seeking adolescents and young adults (service users), their biological siblings, and control subjects were recruited.

The current study aimed to investigate whether exposure to negative LEs modifies stress sensitivity in youth's daily life. Stress sensitivity was conceptualized as the associations between momentary stress (i.e., event-, activity-related and social stress combined) and (i) negative affect and (ii) psychotic experiences. We aimed to test the following primary hypotheses: First, we investigated whether overall lifetime as well as previous-year exposure to negative LEs (i.e., all LE exposure types combined) modifies individuals' stress sensitivity within groups (service users, siblings, and control group) with greater associations when high vs. low exposure levels are compared. Second, we tested whether differences exist in the magnitude of associations when modifying effects of LEs exposure on stress sensitivity are compared across groups, with greater differences in service users vs. controls, service users vs. siblings, and siblings vs. controls. Next, as secondary hypotheses, we examined whether lifetime and previous-year exposure to specific types of LEs (e.g., illness) modifies stress sensitivity within groups and whether there were differences of modifying effects across groups.

\section{Materials and methods}

\section{Sample}

Overall, 99 adolescents and young adults (age range 12-20 years) were recruited, consisting of help-seeking service users, their biological siblings, and controls. The help-seeking individuals were recruited from secondary youth mental health services provided by the Mutsaers Foundation (MF) in Limburg, the Netherlands. Service users were included if they were currently receiving treatment from MF, and excluded if they had a DSM-IV autism spectrum disorder diagnosis with 
the exception of pervasive developmental disorder not otherwise specified, IQ under 70, or insufficient command of Dutch. Moreover, biological siblings of participating service users were recruited. The same exclusion criteria applied, with the addition of lifetime history of receiving treatment from a mental health service. Lastly, individuals attending a school in the same catchment area as MF services were recruited as control subjects. Exclusion criteria were the same as for biological siblings.

\section{Measures}

\section{Socio-demographic characteristics}

We collected data on age, sex, ethnicity, and education level using a sociodemographic schedule.

\section{Negative life events}

A modified version of the List of Threatening Experiences questionnaire (LTE) [23, 24] was used to assess negative LEs. In the current study, 19 differing LEs were grouped into eight frequently used categories, which were based on the type of LEs exposure, including exposure to serious illness (e.g., "hospitalisation or other medical treatments"), experiences of loss (e.g., "death of a family member or friend"), and threatening events (e.g., "involved in a serious accident in which you and/or someone else got hurt seriously"). All items are provided in supplementary Table S1. In addition to asking whether individuals were exposed to LEs (dichotomous item), appraisal of LEs was also included. If an event has occurred (yes/no), participants were asked to rate the degree to which they perceived it as "unpleasant" or "pleasant" on a 5-point scale (from 1 = very unpleasant; to $5=$ very pleasant). As our focus was on negative LEs, events appraised as neutral or pleasant were coded as 0 , while events appraised as unpleasant or very unpleasant were coded as 1 and 2, respectively. All LTE items were used to calculate a total score (i.e., overall exposure of LEs). In the current study, the LTE was modified to assess LEs from the previous 12 months as well as before age 17. For participants younger than 17 years, the latter assessed LEs from birth to current age, excluding the previous year. The LTE has good discriminating power and test-retest reliability [24]. 


\section{Depressive symptoms}

The 21-item Beck Depression Inventory (BDI-II) [25] was used to assess depressive symptoms from the past 2-weeks on a 4-point scale (from $0=$ not present; to 3 = severe). Good psychometric properties have been reported in clinical [26] and non-clinical [27] adolescent populations.

\section{Anxiety symptoms}

A Dutch version [28] of the State-Trait Anxiety Inventory (STAI-Y) [29] was used to measure state and trait anxiety. The STAI-DY consists of two parts and demonstrates good reliability and moderate validity [30]. The first 20-item part (STAI-DY1) assesses state anxiety (current intensity; ranging from $1=$ not at all; 4 = very much), whereas the second 40-item part (STAI-DY2) assesses trait anxiety (pervasive frequency; ranging from 1 = rarely or never; to 4 = almost always).

\section{Psychotic symptoms}

The 42-item Community Assessment of Psychic Experiences (CAPE) was used to assess the frequency (from 0 = never; to 3 = nearly always) and distress (from $0=$ not distressed; to 3 = very distressed) of negative (14 items), positive (20 items), and depressive (8 items) dimensions of non-clinical psychotic symptoms. CAPE has demonstrated good psychometric properties [31].

\section{EMA measures}

Daily changes in momentary stress (i.e., event-related, activity-related, and social stress), negative affect, and psychotic experiences were assessed using EMAs. This intensive self-assessment diary technique measures daily fine-grained subjective and social experiences with high ecological validity [20]. Data was collected outside the laboratory using a personal digital assistant (PDA), the "PsyMate", which prompted participants with beeps ten times per day (between 7:30 am to 10:30 pm) at random intervals in set blocks of time for six consecutive days. A detailed description of used EMA items is shown in Table 1. 
Table 1. EMA measures of stress, negative affect, and psychotic experiences

\begin{tabular}{|c|c|}
\hline Domain & EMA Measure \\
\hline Momentary stress & $\begin{array}{l}\text { Mean scores of event-related, activity-related, and social stress } \\
\text { items were calculated and combined in form of a composite } \\
\text { stress score to represent individuals' momentary stress. Adequate } \\
\text { concurrent validity with different stress measures has been } \\
\text { reported [23]. }\end{array}$ \\
\hline Event & $\begin{array}{l}\text { For event-related stress, participants had to rate the most } \\
\text { important event since the last beep on a } 7 \text {-point scale (from }-3= \\
\text { very unpleasant; to }+3=\text { very pleasant). The item was reverse-coded } \\
\text { such that higher ratings reflect higher levels of stress }(-3 \text { coded as } \\
7 ;+3 \text { coded as } 1) \text {. }\end{array}$ \\
\hline Activity & $\begin{array}{l}\text { Activity-related stress was assessed by asking participants to } \\
\text { identify what they were doing just before the beep (e.g., work } \\
\text { or study, resting) and, subsequently, by asking whether they } \\
\text { would "rather be doing something else", whether "this activity is } \\
\text { difficult" for them, and whether they believe they "can do this well" } \\
\text { [reversed] on a 7-point scale (from } 1 \text { = not at all; to } 7 \text { = very much). }\end{array}$ \\
\hline Social & $\begin{array}{l}\text { Participants were asked about their current social context (e.g., "I } \\
\text { am alone", “I am with colleagues", "I am with friends"). Social stress } \\
\text { was assessed by asking participants to rate the items "I find the } \\
\text { people I am with pleasant" [reversed; if with someone] or "I like to } \\
\text { be alone" [reversed; if alone] on 7-point scale (from } 1 \text { = not at all; to } \\
7 \text { = very much). }\end{array}$ \\
\hline Negative affect & $\begin{array}{l}\text { Participants reported the degree to which they felt anxious, lonely, } \\
\text { down, irritated, and insecure on a } 7 \text {-point scale (from } 1 \text { = not at } \\
\text { all; to } 7=\text { very much). The mean of these five items constitute the } \\
\text { negative affect score. Good psychometric properties have been } \\
\text { reported for the EMA measure of negative affect [24]. }\end{array}$ \\
\hline $\begin{array}{l}\text { Psychotic } \\
\text { experiences }\end{array}$ & $\begin{array}{l}\text { The mean scores of eight items about mental states related to } \\
\text { psychotic experiences were used (7-point scale from } 1 \text { = not at } \\
\text { all; to } 7 \text { = very much). Participants were asked about the presence } \\
\text { and intensity of hallucinations (e.g., "I hear things that aren't really } \\
\text { there"), thought problems (e.g., "My thoughts are influenced by } \\
\text { others", "It's hard to express my thoughts in words"), delusional } \\
\text { ideations and other states (e.g., "I feel suspicious/paranoid", "I } \\
\text { feel unreal", "I feel harried"). The EMA measure for psychotic } \\
\text { experiences has demonstrated good concurrent validity [24]. }\end{array}$ \\
\hline
\end{tabular}

\section{Statistical analysis}

In line with previous studies [21, 22] we first compared socio-demographic characteristics and standardized baseline scores (BDI-II, STAI-DY1/STAI-DY2, and CAPE) between groups (service users, biological siblings, and controls) using linear regression and $\chi^{2}$-tests. To account for statistical dependencies in EMA data resulting from the multilevel data structure (multiple observations nested within participants), linear mixed models were computed using the MIXED command 
in STATA 15. Momentary stress and group status were added as independent variables and (i) negative affect and (ii) psychotic experiences as outcome variables, while controlling for age, sex, ethnicity, and level of education. To test primary and secondary hypotheses, we added two-way (StressXLE, StressXGroup, LEXGroup) and three-way (StressXLEXGroup) interaction terms into the models. After the three-way interaction terms were added, increase in model fit was tested using Wald tests (TESTPARM command). To account for multiple testing, the $p$-values of Wald tests were multiplied by the total number of tests to calculate familywise error-corrected $p$-values ( $p$ FWE). Next, we computed linear combinations of coefficients with the LINCOM command to test whether, within-groups, associations between momentary stress and (i) negative affect and (ii) psychotic experiences were greater in individuals exposed to high vs. low levels of exposure to LEs (by calculating standardized LEs scores: +/- $1 S D, M=0$ ). Lastly, we explored whether effect moderation of LEs on stress sensitivity differs across groups by comparing differences in the magnitude of associations between momentary stress and (i) negative affect and (ii) psychotic experiences between individuals exposed to high vs. low levels of LEs exposure in service users vs. controls, service users vs. siblings, and siblings vs. controls.

\section{Results}

\section{Sample characteristics}

In total, 109 individuals were eligible to participate. Of these, 99 youths (42 service users, 17 siblings, and 40 controls) completed the EMA with $\geq 20$ valid responses over the 6-day assessment period as well as the LTE, BDI-II, CAPE, and STAI-DY1/ DY2. Groups did not significantly differ on age, sex, or ethnicity (Table 2). However, there was evidence for higher levels of depression, state and trait anxiety, and negative and positive psychotic experiences in service users vs. controls and service users vs. siblings, respectively. As shown in Table 3, service users were exposed to higher overall levels of LEs during the last year compared to controls and siblings, while no differences were found comparing siblings and controls. A similar pattern of findings was demonstrated for overall LEs before age 17, with service users exposed to higher overall levels of LEs as compared to controls and siblings, while no significant differences were found comparing siblings and controls. A similar pattern of findings was evident by looking at specific types of LEs during the last year and before the age of 17 . 
Table 2. Sample characteristics

\begin{tabular}{|c|c|c|c|c|c|}
\hline & $\begin{array}{c}\text { Service } \\
\text { users } \\
(n=42)\end{array}$ & $\begin{array}{l}\text { Siblings } \\
(n=17)\end{array}$ & $\begin{array}{c}\text { Controls } \\
(n=40)\end{array}$ & Test statistic & $p$ \\
\hline Age (years), mean (S.D.) & $15.4(1.4)$ & $15.3(2.3)$ & $15.6(2.0)$ & $\mathrm{F}=0.24, \mathrm{df}=2$ & 0.785 \\
\hline \multicolumn{6}{|l|}{ Sex, n (\%) } \\
\hline Female & $25(59.5)$ & $10(58.8)$ & $23(57.5)$ & $\chi^{2}=0.04, \mathrm{df}=2$ & 0.983 \\
\hline Male & $17(40.5)$ & $7(41.2)$ & $17(42.5)$ & & \\
\hline \multicolumn{6}{|l|}{ Ethnicity, n (\%)a } \\
\hline White Dutch & $26(61.9)$ & $11(64.7)$ & $25(64.1)$ & $\chi^{2}=0.06, \mathrm{df}=2$ & 0.970 \\
\hline Other & $16(38.1)$ & $6(35.3)$ & $14(35.9)$ & & \\
\hline \multicolumn{6}{|l|}{ Level of education, $\mathrm{n}(\%)^{\mathrm{c}}$} \\
\hline School & $30(71.4)$ & $7(41.2)$ & $17(42.5)$ & $\chi^{2}=10.48, \mathrm{df}=2$ & 0.033 \\
\hline Further & $12(28.6)$ & $8(47.1)$ & $20(50.0)$ & & \\
\hline Higher & - & $2(11.8)$ & $3(7.5)$ & & \\
\hline \multicolumn{6}{|l|}{ Cannabis use, n (\%) } \\
\hline 12-months & $9(21.4)$ & $1(5.9)$ & $4(10.0)$ & $\chi^{2}=3.36, \mathrm{df}=2$ & 0.187 \\
\hline Lifetime & $9(21.4)$ & $2(11.8)$ & $5(12.5)$ & $\chi^{2}=1.50, d f=2$ & 0.473 \\
\hline \multicolumn{6}{|l|}{ Attempted suicide, n (\%) } \\
\hline During last year & $6(14.6)$ & - & - & - & - \\
\hline Before age 17 & $8(19.1)$ & - & - & - & - \\
\hline \multicolumn{6}{|l|}{ DSM-IV diagnoses, n (\%) } \\
\hline $\begin{array}{l}\text { Pervasive } \\
\text { developmental } \\
\text { disorders NOS }\end{array}$ & $10(23.8)$ & - & $5(12.5)$ & - & - \\
\hline $\begin{array}{l}\text { Attention-deficit and } \\
\text { disruptive behaviour }\end{array}$ & $6(14.3)$ & $3(17.6)$ & - & - & - \\
\hline Adjustment disorders & $4(9.5)$ & - & - & - & - \\
\hline Anxiety disorders & $2(4.8)$ & - & - & - & - \\
\hline Depressive disorders & $2(4.8)$ & - & - & - & - \\
\hline $\begin{array}{l}\text { Gender identity } \\
\text { disorders }\end{array}$ & $2(4.8)$ & - & - & - & - \\
\hline Learning disorders & - & - & $2(5.0)$ & - & - \\
\hline $\begin{array}{l}\text { Other disorders of } \\
\text { infancy, childhood, or } \\
\text { adolescence }\end{array}$ & $5(11.9)$ & - & - & - & - \\
\hline $\begin{array}{l}\text { Parent-child relational } \\
\text { problem }\end{array}$ & $5(11.9)$ & $1(5.9)$ & $1(2.5)$ & - & - \\
\hline Comorbid condition ${ }^{b}$ & $24(57.1)$ & $2(11.8)$ & - & - & - \\
\hline None & $6(14.3)$ & $13(76.5)$ & $32(80.0)$ & - & - \\
\hline $\begin{array}{l}\text { BDI-II sum sores, mean } \\
\text { (S.D. })^{\mathrm{a}, \mathrm{d}}\end{array}$ & $12.8(9.2)$ & $3.9(3.3)$ & $6.9(7.0)$ & $F=10.5, d f=2$ & $<0.001$ \\
\hline
\end{tabular}


Table 2. (continued). Sample characteristics

\begin{tabular}{|c|c|c|c|c|c|}
\hline & $\begin{array}{c}\text { Service } \\
\text { users } \\
(n=42)\end{array}$ & $\begin{array}{l}\text { Siblings } \\
(n=17)\end{array}$ & $\begin{array}{l}\text { Controls } \\
(n=40)\end{array}$ & Test statistic & $p$ \\
\hline \multicolumn{6}{|l|}{$\begin{array}{l}\text { CAPE sum scores, mean } \\
(\text { S.D. })^{d}\end{array}$} \\
\hline Positive & $10.0(9.4)$ & $3.9(3.2)$ & $4.6(3.9)$ & $F=8.28, d f=2$ & $<0.001$ \\
\hline Negative & $9.9(6.7)$ & $5.6(3.8)$ & $7.4(4.8)$ & $F=3.88, d f=2$ & 0.024 \\
\hline Depressive & $7.7(4.0)$ & $4.2(1.8)$ & $4.7(3.4)$ & $F=9.90, d f=2$ & $<0.001$ \\
\hline $\begin{array}{l}\text { STAI-DY1 (state anxiety) } \\
\text { sum scores, mean (S.D.) }\end{array}$ & $35.5(10.6)$ & $30.2(6.8)$ & $31.1(7.2)$ & $F=3.47, d f=2$ & 0.035 \\
\hline $\begin{array}{l}\text { STAI-DY2 (trait anxiety) }^{\text {a }} \\
\text { sum scores, mean (S.D.) }\end{array}$ & $85.6(20.8)$ & $67.1(9.2)$ & $74.1(16.4)$ & $F=8.12, d f=2$ & $<0.001$ \\
\hline
\end{tabular}

SD: standard deviation; df: degrees of freedom; $\beta$ : standardized regression coefficients (mean score differences); vs.: versus; Cl: confidence interval.

${ }^{\text {a }}$ Missing values: ethnicity=1, BDI=1, STAI-DY1=1, STAI-DY2=2.

${ }^{\mathrm{b}}$ Consisting of the following diagnostic categories in the service users group: Additional codes (Parent-child relational problem, 33.3\%; Borderline intellectual functioning, 13.3\%; Neglect of child, 6.7\%), Attention-deficit and disruptive behaviour disorders (10\%), Learning disorders $(10 \%)$, Personality disorders (6.7\%), Mild mental retardation (6.7\%), Anxiety disorders (3.3\%), Dissociative disorders (3.3\%), Tic disorders (3.3\%), Amphetamine related disorders (3.3\%).

'Categories defined as: school (primary education, LBO, MAVO, VMBO), further (MBO, HAVO, VWO), and higher (HBO, WO) education of the Dutch educational system.

d Standardized mean score differences across groups:

\begin{tabular}{lcccccc}
\hline & \multicolumn{2}{c}{ Service users vs. controls } & \multicolumn{2}{c}{ Siblings vs. controls } & \multicolumn{2}{c}{ Service users vs. siblings } \\
& $\boldsymbol{\beta}(\mathbf{9 5 \%} \mathrm{Cl})$ & $\mathbf{p}$ & $\boldsymbol{\beta}(\mathbf{9 5 \%} \mathrm{Cl})$ & $\mathbf{p}$ & $\boldsymbol{\beta}(\mathbf{9 5 \%} \mathrm{Cl})$ & $\mathbf{p}$ \\
\hline BDI- II & $0.71(0.30-1.11)$ & 0.001 & $-0.37(-0.89-0.16)$ & 0.168 & $1.08(0.55-1.60)<0.001$ \\
\hline CAPE & \multicolumn{1}{l}{} & & & \\
\hline Positive & $0.75(0.34-1.17)<0.001$ & $-0.09(-0.63-0.44)$ & 0.735 & $0.84(0.31-1.38)$ & 0.002 \\
\hline Negative & $0.42(-0.01-0.85)$ & 0.057 & $-0.31(-0.87-0.25)$ & 0.269 & $0.73(0.17-1.29)$ & 0.011 \\
\hline Depressive & $0.80(0.40-1.21)<0.001$ & $-0.12(-0.64-0.41)$ & 0.666 & $0.92(0.39-1.45)$ & 0.001 \\
\hline STAI-DY1 & $0.50(0.06-0.93)$ & 0.025 & $-0.09(-0.65-0.47)$ & 0.748 & $0.59(0.03-1.14)$ & 0.040 \\
\hline STAI-DY2 & $0.59(0.19-0.99)$ & 0.004 & $-0.36(-0.88-0.16)$ & 0.170 & $0.95(0.43-1.46)<0.001$ \\
\hline
\end{tabular}




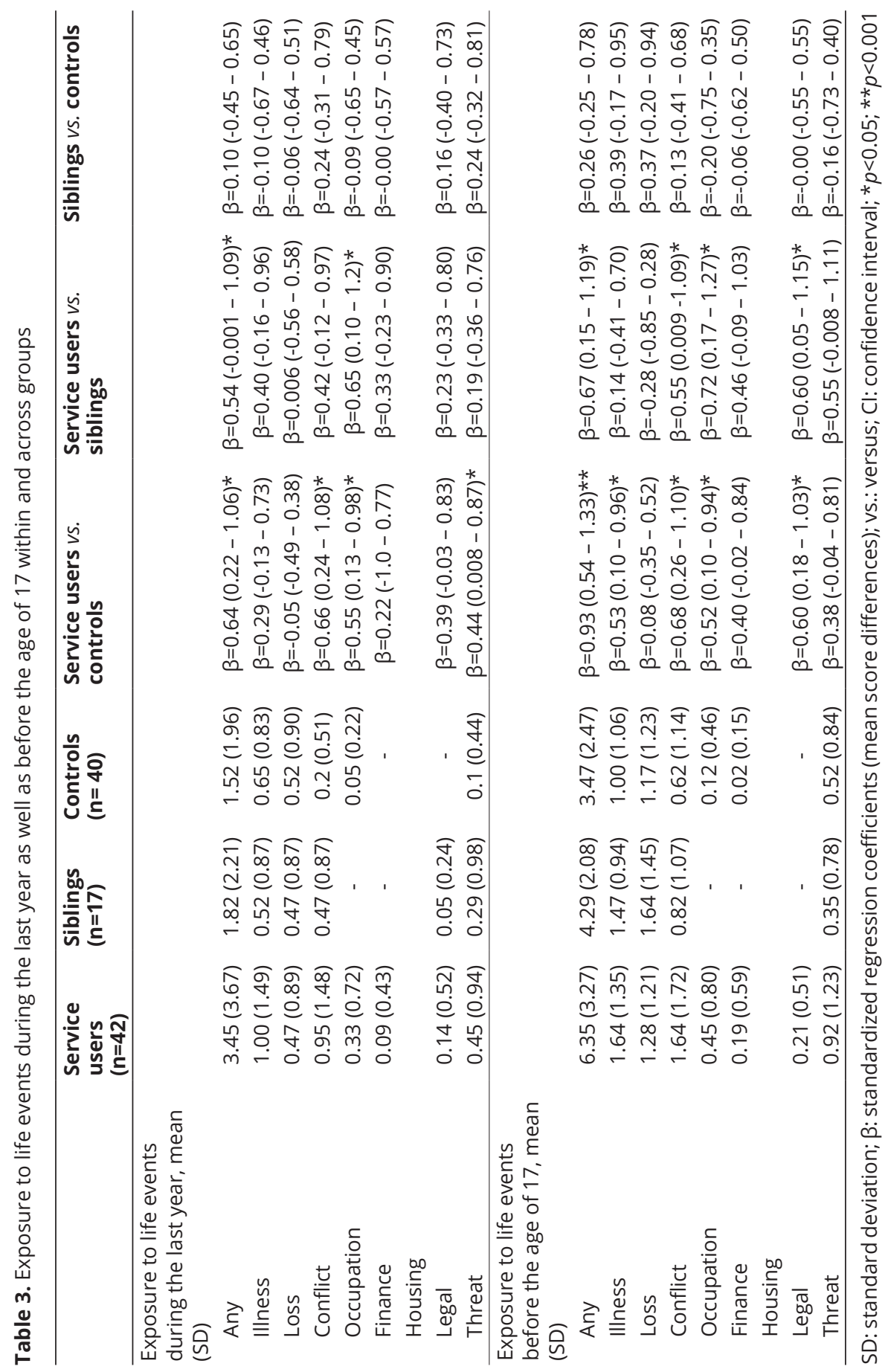




\section{Association between momentary stress and negative affect by LEs exposure and group}

There was evidence in support of the primary hypotheses that overall lifetime as well as previous-year exposure to LEs modified the association between momentary stress and negative affect (Table 4), as indicated by significant threeway interaction effects described below.

\section{Within-group comparisons}

Momentary stress was associated with increased negative affect in service users (adj. $\beta=0.18, p<0.001$ ) when high vs. low levels of exposure to overall LEs during the last year were compared, while no significant differences by exposure levels were found in siblings and controls. When comparing high vs. low levels of exposure to overall LEs before age 17, momentary stress was associated with increased negative affect in service users (adj. $\beta=0.16, p<0.001$ ), while no significant differences were found in siblings and controls. Analyses to test secondary hypotheses (Table 5) revealed that momentary stress was associated with lower negative affect in the control group when comparing high vs. low levels of exposure to threatening events ( $\operatorname{adj} . \beta=-0.14, p=0.012$ ). In contrast, increased negative affect in response to stress was observed in service users comparing high vs. low levels of exposure to loss experiences ( $\operatorname{adj} . \beta=0.21, p<0.001$ ), conflict events ( $\operatorname{adj} . \beta=0.17, p<0.001$ ), and threatening events (adj. $\beta=0.09, p<0.001$ ), while no significant differences were demonstrated in siblings.

\section{Between-group comparisons}

To investigate whether the modifying effects of exposure to LEs on stress sensitivity differed between groups, differences in magnitude of associations between those exposed to high vs. low levels of LEs were examined across groups. Specifically, the difference in magnitude of associations between stress and negative affect was greater in service users than in controls when comparing high vs. low levels of exposure to overall LEs during the last year ( $\operatorname{adj} . \beta=0.21, p<0.001)$ and before age 17 (adj. $\beta=0.16, p=0.011$ ). Further, in testing secondary hypotheses (Table 5), we found significant differences in the magnitude of associations between those exposed to high vs. low levels of experience of loss ( $\operatorname{adj} . \beta=0.26, p<0.001$ ), conflict events ( $\operatorname{adj} . \beta=0.18, p=0.026$ ), and threatening events ( $\operatorname{adj} . \beta=0.23, p<0.001$ ) during 
the last year comparing service users vs. controls. Moreover, the difference in magnitude of associations between stress and negative affect was greater in service users than siblings comparing high vs. low exposure to conflict events during the last year (adj. $\beta=0.26, p=0.002$ ), and threatening events (adj. $\beta=0.26$, $p=0.001$ ) and, at trend level, experiences of loss (adj. $\beta=0.13, p=0.054$ ) before age 17. No significant differences were found comparing siblings vs. controls.

\section{Association between stress and psychotic experiences by LEs exposure and group}

There was evidence in support of the primary hypotheses that exposure to overall LEs modified the association between momentary stress and psychotic experiences (Table 4), as indicated by significant three-way interaction effects described below.

\section{Within-group comparisons}

Momentary stress was associated with more intense psychotic experiences in service users ( $\operatorname{adj}$. $\beta=0.09, p<0.001$ ) exposed to high levels of overall LEs compared to those with low exposure levels during the last year, while no differences were found in siblings and controls. When considering overall exposure to LEs before age 17 , momentary stress was associated with more intense psychotic experiences in service users ( $a d j . \beta=0.10, p<0.001$ ) when high vs. low overall LEs levels were compared, while no significant differences were found in siblings and controls. Analyses of secondary hypotheses (Table 5 ) revealed that stress was significantly associated with more intense psychotic experiences during the last year when comparing service users exposed to high vs. low levels of experiences of loss (adj. $\beta=0.10, p<0.001$ ) and threatening events (adj. $\beta=0.07, p<0.001)$. A similar pattern of findings was evident in service users with high vs. low exposure to threatening events ( $\operatorname{adj} . \beta=0.06, p<0.001$ ) before age 17 . In contrast, stress was not significantly associated with more intense psychotic experiences across any type or level of LEs in neither the control group nor the sibling group (Table 5).

\section{Between-group comparisons}

There were differences in the magnitude of associations between momentary stress and psychotic experiences in those exposed to high vs. low levels of overall 
LEs comparing service users vs. controls during the last year ( $\operatorname{adj} . \beta=0.08, p=0.010$ ) and before age 17 ( $a d j . \beta=0.08, p=0.018$ ). Moreover, the difference in magnitude of associations between momentary stress and psychotic experiences was greater in service users than in siblings when high vs. low levels of exposure to overall LEs during the last year (adj. $\beta=0.12, p=0.012$ ) as well as before age 17 (adj. $\beta=0.11$, $p=0.031$ ) were compared (Table 4). However, there was no difference when high vs. low levels of exposure to overall LEs were compared in siblings vs. controls. Analyses of secondary hypotheses (Table 5) showed differences in magnitude of associations between momentary stress and psychotic experiences comparing high vs. low exposure levels to experiences of loss ( $\operatorname{adj} . \beta=0.10, p<0.001$ ), but not threatening events ( $a d j . \beta=0.04, p=0.211$ ), during the last year in service users vs. controls. However, service users marginally differed from controls in the magnitude of associations between momentary stress and psychotic experiences by exposure levels to threatening events before age 17 (adj. $\beta=0.06, p=0.053$ ). Lastly, service users differed significantly from siblings in magnitude of associations between momentary stress and psychotic experiences comparing high vs. low levels of exposure to experiences of loss ( $\operatorname{adj} . \beta=0.10, p=0.003)$ and threatening events (adj. $\beta=0.09, p=0.004$ ) during the last year. No significant differences were found comparing siblings vs. controls. 


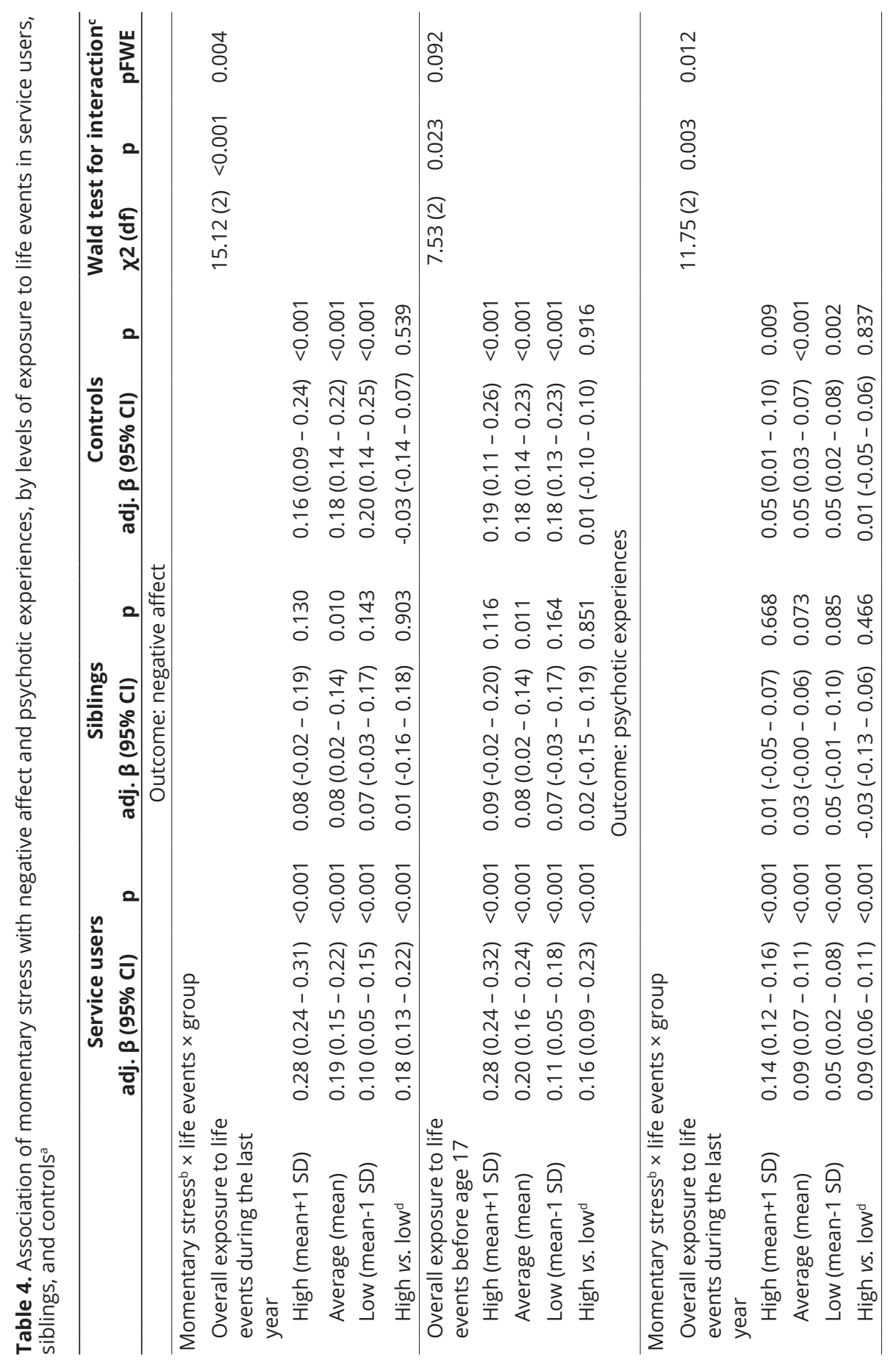




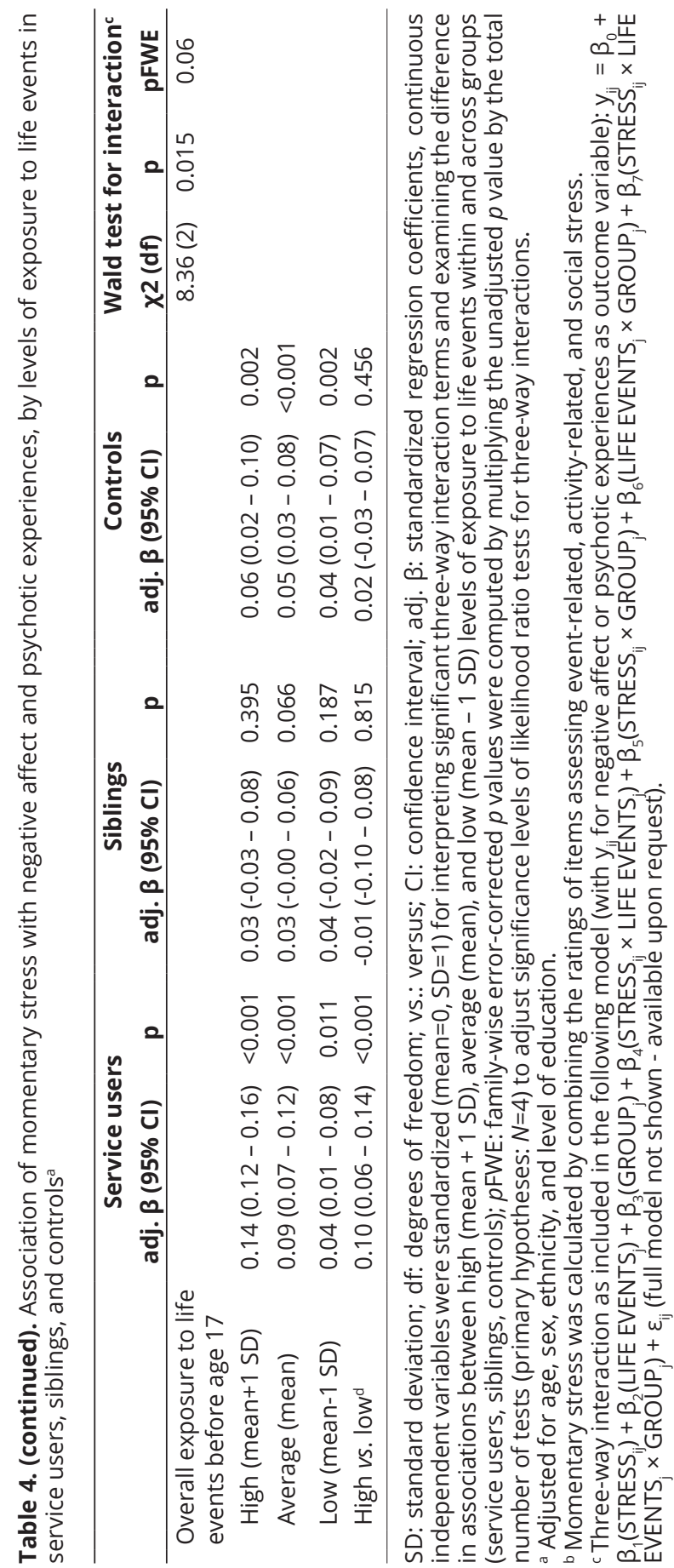




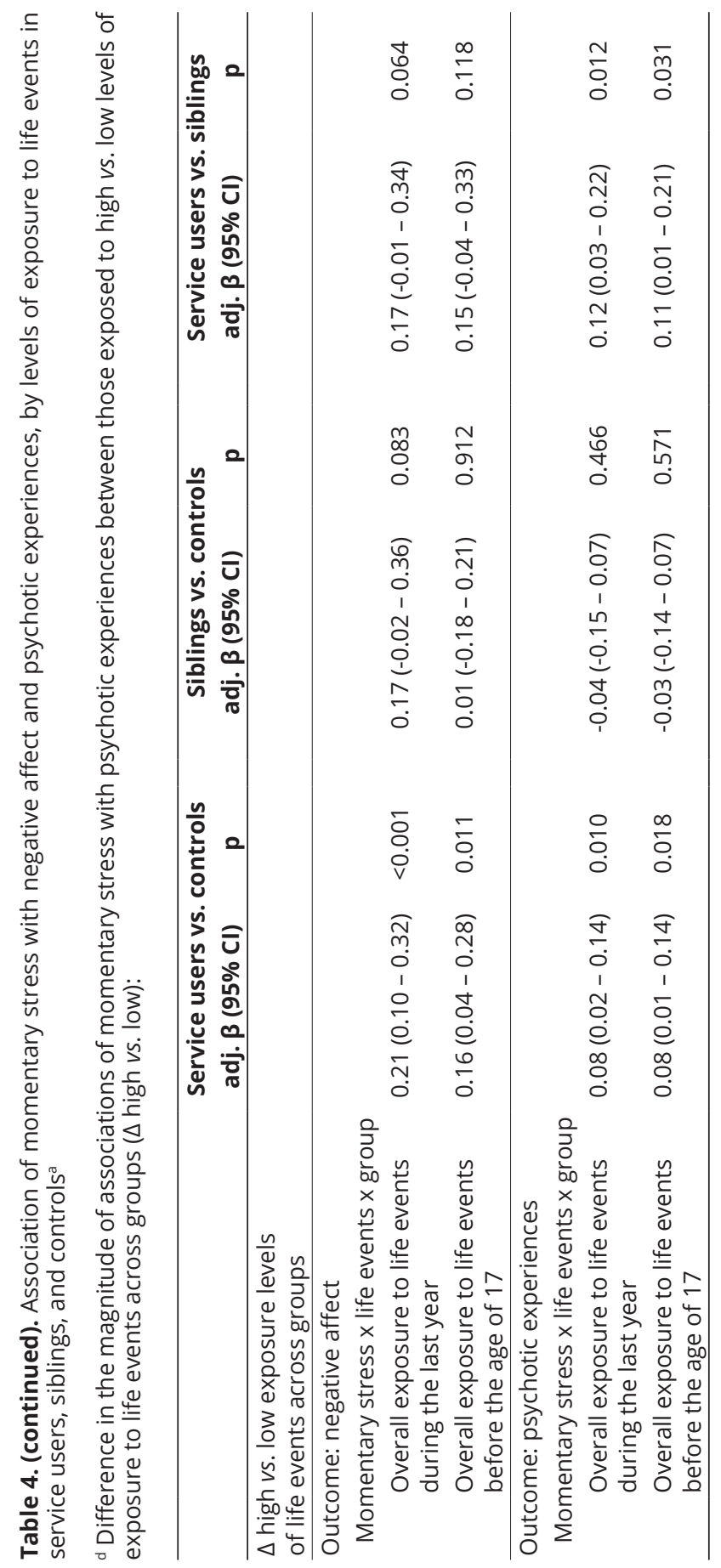




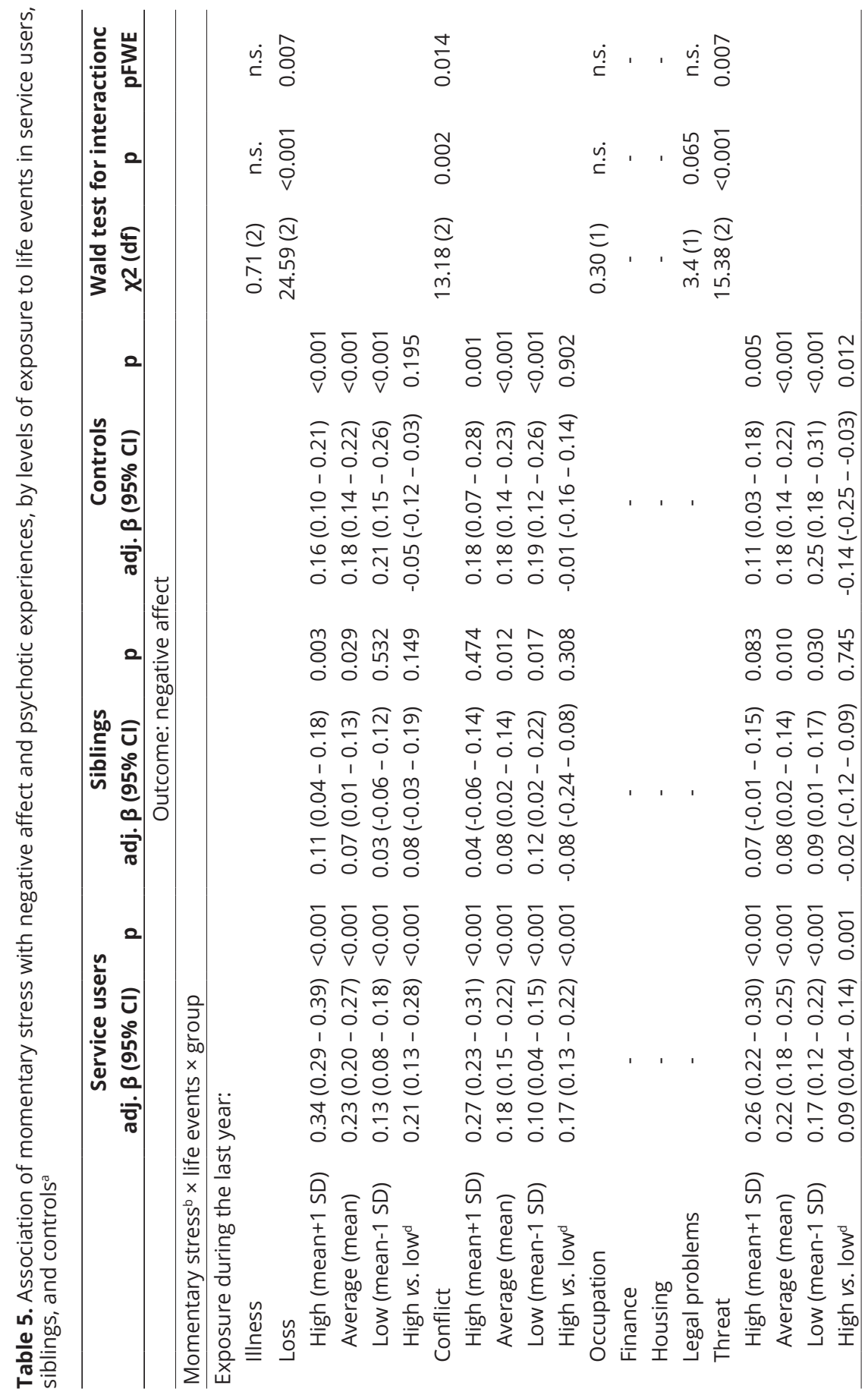




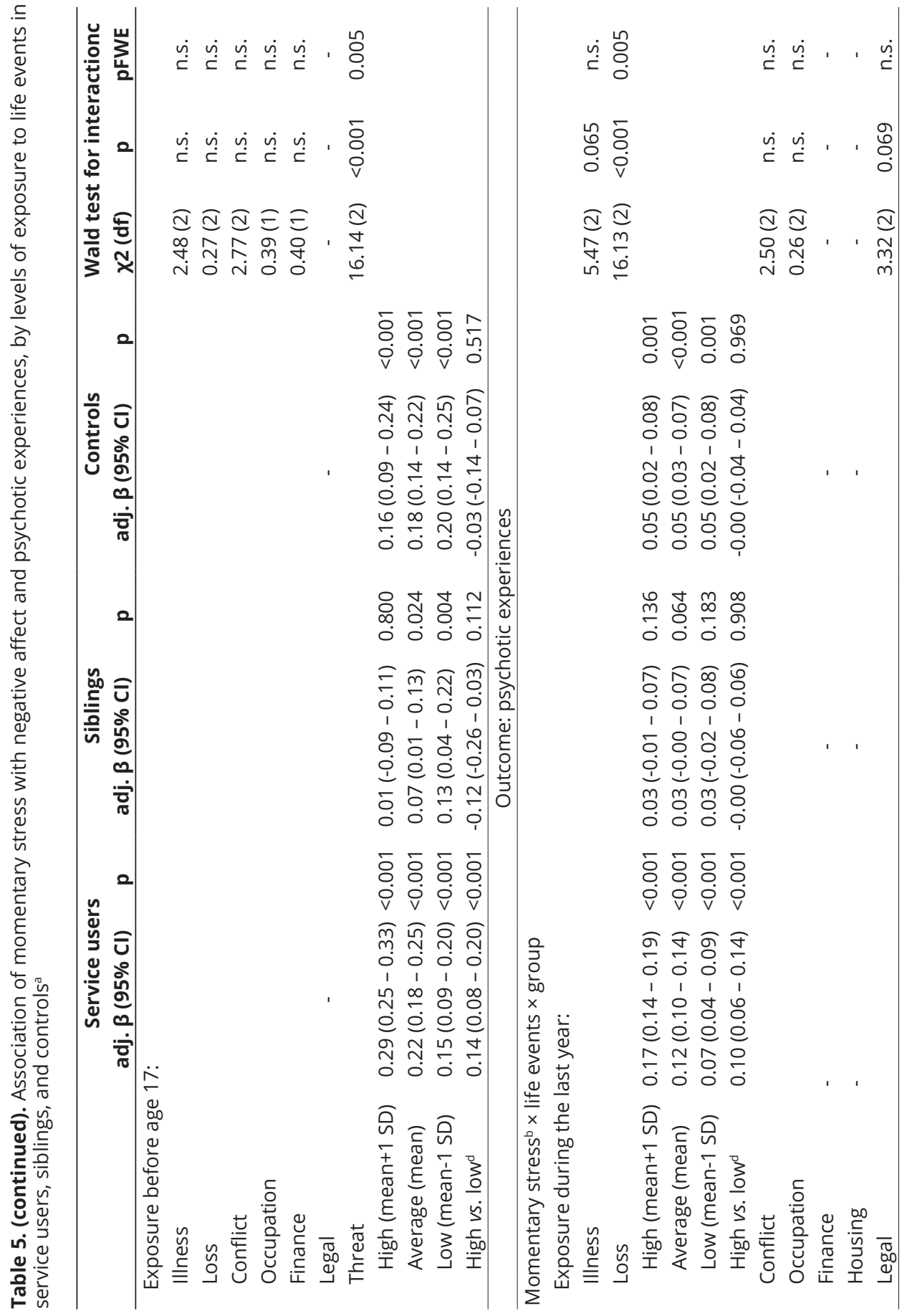




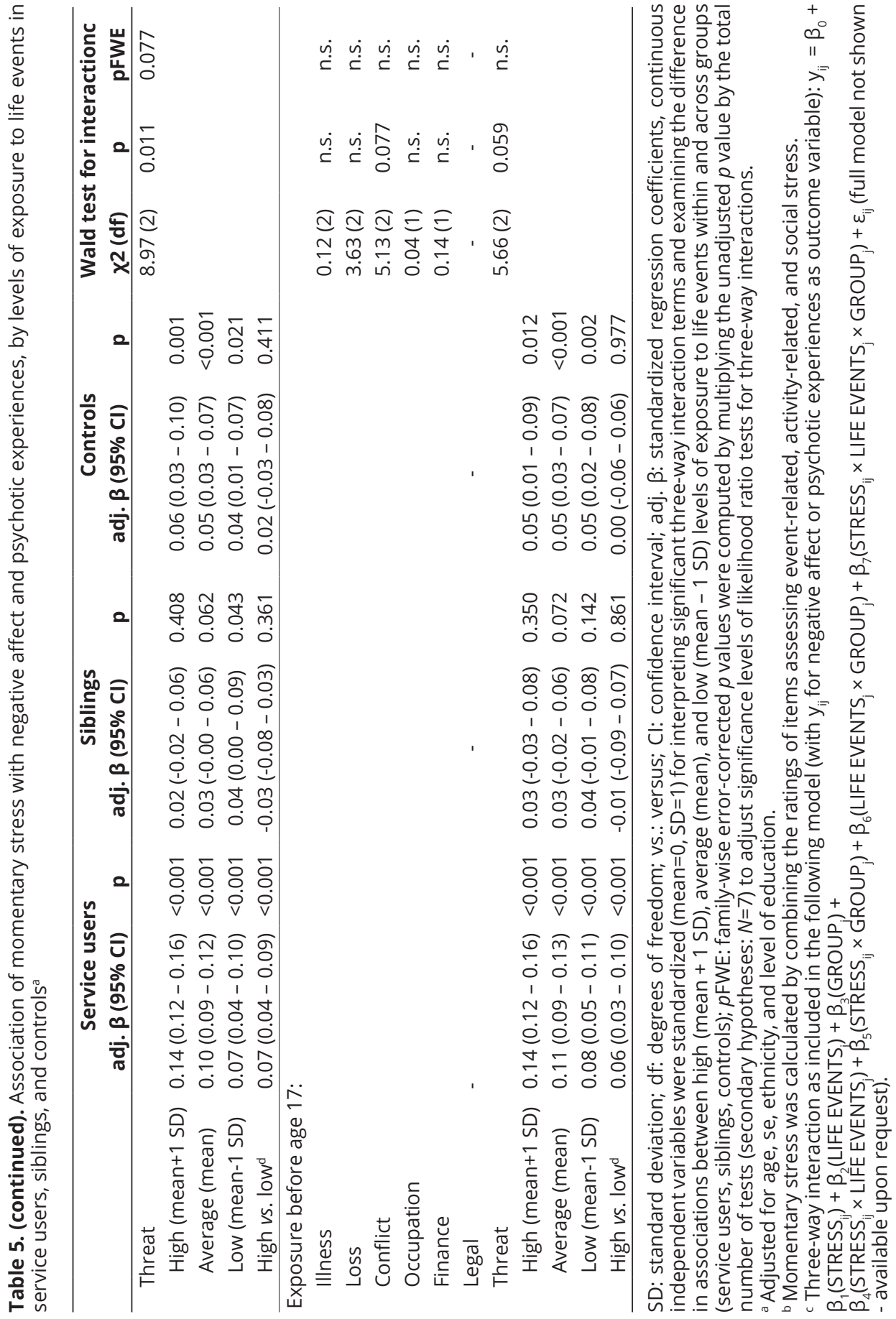




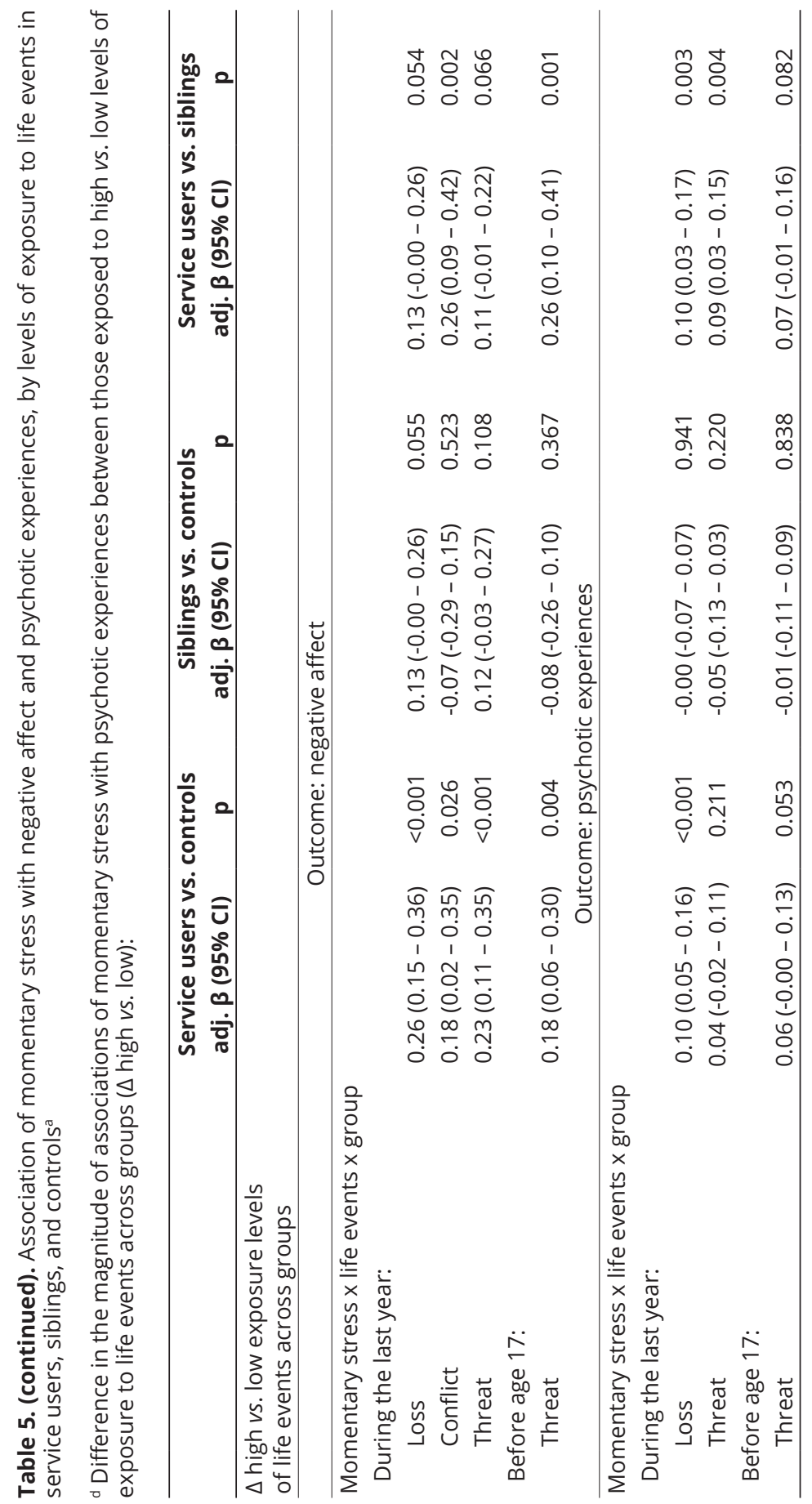




\section{Discussion}

\section{Main findings}

There was strong evidence that lifetime as well as previous-year exposure to overall as well as specific types of LEs, modified stress sensitivity in help-seeking service users. Service users experienced increased negative affect and more intense psychotic experiences in response to minor daily stress when high vs. low exposure levels were compared. In siblings, however, we found no evidence that overall exposure to as well as specific types of LEs modified stress sensitivity. In controls, however, secondary analyses revealed decreased negative affect in response to stress comparing those exposed to high vs. low levels of threatening experiences. Thus, our findings tentatively suggest that individuals' response to minor stress in daily life represents a putative risk and resilience mechanism through which exposure to LEs may influence youth mental health.

\section{Methodological considerations}

The reported findings should be interpreted in view of potential limitations. First, the LTE is a retrospective measure of self-reported LEs. Consequently, recall bias and cognitive distortion may have influenced reported findings [25]. Moreover, it may have been difficult for participants to differentiate LEs from within the last 12 months from LEs before age 17 . However, the low sample age likely minimized the impact of biases on reported LEs, as the time between exposure to and time of assessments of LEs was limited. Similarly, EMAs were based on self-report. While this allows for ecologically valid measurements of momentary stress, context, and experiences on the behavioural level, future research should consider triangulating the impact of LEs on individuals' stress sensitivity across other levels of investigation, such as biological markers [26] and passively collected digital sensor data [27]. Secondly, some of the LEs included in the current study (e.g., housing, financial, legal problems) were only prevalent in few or none of participating individuals. Consequently, we were not able to test modifying effects of all specific types of LEs on stress sensitivity in secondary analyses. Third, assessment burden associated with EMAs may have introduced selection bias. However, studies have demonstrated that EMAs are reliable and feasible in adult as well as adolescent clinical and community populations [20]. Additionally, extensive briefing on the "PsyMate" and EMA procedure ensured a high number of valid responses (90.8\%). 
Fourth, the group of siblings was relatively small $(n=17)$ and findings that LEs did not modify stress sensitivity in this intermediate risk group may have occurred because of sampling error. Fifth, despite adjustment for potential confounders (i.e., age, sex, education level, and ethnicity), other unmeasured factors such as personality traits and polygenetic risk for psychopathologies could have influenced reported findings [28]. Lastly, the data collected over 6 days with EMA was modelled cross-sectionally and temporality of stress, negative affect, and psychotic experiences was not specifically investigated. It is recommended that future studies consider time-lagged and moderated mediation models to further investigate temporality as one important criterion important to inferring causality.

\section{Comparison with previous research}

Previous research suggests exposure to ACEs, including LEs [6-9, 11-14, 16, 29], is associated with an increased risk of developing mental health problems. However, candidate mechanisms remain poorly understood, particularly in youth. By contributing to lasting changes to the way an individual responds to stress, stress sensitization has been proposed to be a common mechanistic pathway that may help explain the relation between exposure to socio-environmental risk and psychopathology [18]. Notably, this proposition has already been investigated in adult populations. Adults with mental disorders (e.g., depression, psychosis) and a history of childhood trauma and LEs were found to have elevated affective and psychotic reactivity in response to minor daily stressors $[24,30]$. Thus, stress sensitization may represent a putative mechanism underlying exposure to LEs and psychopathology. However, whether these findings can be generalized to young help-seeking individuals remained under-researched.

The current study is the first to report that young help-seeking individuals who were exposed to high levels of LEs responded to minor stressors in daily life with increased negative affect and psychotic experiences as compared to those with low exposure levels. Interestingly, these findings are in line with reported effects of childhood trauma [21] and bullying victimization [22] on stress sensitivity derived from the same sample, and with findings including individuals with at-risk mental state (ARMS) for psychosis and FEP exposed to childhood trauma [24]. Thus, in line with previous research, the present study suggests, although not directly tested, that behavioural sensitization in form of an increased stress sensitivity in 
daily life may be associated with exposure to ACEs and may have downstream contributions along multiple pathways leading to poor mental health.

In contrast, exposure to LEs before age 17 did not modify individuals' stress sensitivity in control subjects. However, in secondary analyses, controls exposed to high levels of more intrusive LEs (i.e., threatening experiences, serious accident) during the previous year responded with decreased negative affect as compared to those with low exposure to LEs. In other words, controls appeared to be more resilient to the detrimental effects of high exposure to more recent threatening LES when compared to low exposure levels. This is interesting as it mirrors previous findings in which physical abuse and neglect [21], physical bullying [22], and sexual abuse [24] were associated with decreased negative affect in response to stress in controls. Taken together, high levels of exposure to more intrusive ACEs may result in resilience against subsequent minor stressors in daily life in individuals who do not develop help-seeking behaviour.

In biological siblings of service users, we found no evidence that exposure to LEs modifies stress sensitivity. Specifically, when comparing high vs. low exposure to LEs, siblings did not respond to minor stressors in daily life with an increased negative affect and psychotic experiences. However, with a small sample size, we have to be cautious when interpreting these findings. Moreover, findings at the group-level are possibly influenced by mixed resiliency to stress sensitivity at the person-level considering that siblings form an intermediate risk group and have a higher liability to psychopathology. Consequently, it may be that only some siblings developed an increased sensitivity to stress as service users, which may be not detectable at the group-level.

Secondary analyses revealed that, similar to exposure to overall LEs, loss, threat, and conflict events modified stress sensitivity in service users. In line with earlier findings reporting associations between the experience of loss and depressive symptoms in youth [16], we found that exposure to loss within the last year increased help-seeking individuals' negative affect in response to stress. Similarly, conflict events during the past year were associated with higher negative affect in response to stress in service users, while, in siblings, these effects were not found. This contrast between service users and their siblings is particularly interesting as these groups have probably been exposed to comparable levels of several 
assessed LEs (e.g., parental death, serious persistent quarrels with members within the family). Notably, service users and siblings do not only share genetic risk but also exposure to some socio-environmental risk.

The findings that exposure to LEs did not modify stress sensitivity in siblings and controls or resulted in lower affective reactivity to stress in controls may be partly explained by various protective factors. These may be differentially available in and/or used by controls and siblings compared to help-seeking individuals. Accumulating evidence suggests that social support, optimism, higher self-esteem, family/neighbourhood cohesion, parental involvement, positive atmosphere at home, low polygenetic risk, and low rumination tendencies contribute to helping individuals in light of ACEs [31-35]. It may be speculated that these processes protect individuals from an increased stress sensitivity by supporting helpful coping strategies and cognitive factors (e.g., greater cognitive flexibility [36]). A recent study demonstrated that psychological flexibility moderates the association between LEs and depressive symptoms and can therefore be considered a "buffer" against unfavourable impacts that LEs have on mental health [37].

Future studies may further explore the proposed transdiagnostic risk mechanism by considering the role of cognitive factors in individuals' stress response (e.g., cognitive appraisal [17,38], aberrant salience, jumping-to-conclusions bias, and theory of mind). Moreover, it is important to investigate whether the effects of ACEs on stress sensitivity accumulate over time (e.g., by using cohort designs and, for instance, calculating individuals' environmental load) [39]. Lastly, exploring the contribution of stress sensitivity in symptom progression and persistence over time by using longitudinal EMA designs may be an important next step. In accordance with prior work that psychiatric symptoms frequently co-occur during developmentally early stages of psychopathology [4], high proportions of comorbid depressive, anxiety, and psychotic symptoms in service users further supports dimensional models of psychopathology [40] as well as transdiagnostic phenotypes, including an extended psychosis spectrum phenotype [5]. 


\section{Conclusion}

Our results suggest that stress sensitivity may reflect an important risk and resilience mechanism through which LEs negatively impact mental health in helpseeking youth. While we found no unfavourable effects of exposure to LEs on stress sensitivity in controls and siblings, service users appeared to be at greater risk of experiencing elevated stress sensitivity. Targeting sensitivity to stress in daily life with novel mHealth tools (e.g., ecological momentary interventions) by focusing on emotion regulation skills (e.g., mindfulness-based or compassionfocused therapies) may be a promising preventive as well as intervention strategy helping adolescents and young adults with mental health problems. 


\section{References}

1. McGorry, P.D., et al., Clinical staging of psychiatric disorders: a heuristic framework for choosing earlier, safer and more effective interventions. Australian and New Zealand Journal of Psychiatry, 2006. 40(8): p. 616-622.

2. Kessler, R.C., et al., Lifetime prevalence and age-of-onset distributions of DSM-IV disorders in the National Comorbidity Survey Replication. Archives of general psychiatry, 2005. 62(6): p. 593-602.

3. Malla, A., et al., From early intervention in psychosis to youth mental health reform: a review of the evolution and transformation of mental health services for young people. Social psychiatry and psychiatric epidemiology, 2016. 51(3): p. 319-326.

4. McGorry, P.D. and J. van Os, Redeeming diagnosis in psychiatry: timing versus specificity. The Lancet, 2013. 381(9863): p. 343-345.

5. van Os, J. and U. Reininghaus, Psychosis as a transdiagnostic and extended phenotype in the general population. World Psychiatry, 2016. 15(2): p. 118-124.

6. Beards, S., et al., Life events and psychosis: a review and meta-analysis. Schizophrenia bulletin, 2013. 39(4): p. 740-747.

7. Jenness, J.L., et al., Dynamic associations between stressful life events and adolescent internalizing psychopathology in a multiwave longitudinal study. Journal of abnormal psychology, 2019. 128(6): p. 596.

8. March-Llanes, J., et al., Stressful life events during adolescence and risk for externalizing and internalizing psychopathology: a meta-analysis. European child \& adolescent psychiatry, 2017. 26(12): p. 1409-1422.

9. Boardman, J.D., K.B. Alexander, and M.C. Stallings, Stressful life events and depression among adolescent twin pairs. Biodemography and social biology, 2011. 57(1): p. 53-66.

10. Pries, L.-K., et al., Association of recent stressful life events with mental and physical health in the context of genomic and exposomic liability for schizophrenia. JAMA psychiatry, 2020.

11. McLaughlin, K.A., et al., Childhood adversities and first onset of psychiatric disorders in a national sample of US adolescents. Archives of general psychiatry, 2012. 69(11): p. 1151-1160.

12. Humphreys, K.L., et al., Stressful life events, ADHD symptoms, and brain structure in early adolescence. Journal of abnormal child psychology, 2019. 47(3): p. 421-432.

13. Liu, R.T. and I. Miller, Life events and suicidal ideation and behavior: a systematic review. Clinical psychology review, 2014. 34(3): p. 181-192.

14. Beards, S., et al., Threatening Life Events and Difficulties and Psychotic Disorder. Schizophrenia Bulletin, 2020.

15. AyesaロArriola, R., et al., The synergetic effect of childhood trauma and recent stressful events in psychosis: associated neurocognitive dysfunction. Acta Psychiatrica Scandinavica, 2020. 141(1): p. 43-51.

16. Elmore, A.L. and E. Crouch, The Association of Adverse Childhood Experiences With Anxiety and Depression for Children and Youth, 8 to 17 Years of Age. Academic Pediatrics, 2020. 
17. Schwager, S. and K. Rothermund, Counter-regulation triggered by emotions: Positive/ negative affective states elicit opposite valence biases in affective processing. Cognition \& Emotion, 2013. 27(5): p. 839-855.

18. Collip, D., I. Myin-Germeys, and J. Van Os, Does the concept of "sensitization" provide a plausible mechanism for the putative link between the environment and schizophrenia? Schizophrenia bulletin, 2008. 34(2): p. 220-225.

19. Howes, O.D. and R.M. Murray, Schizophrenia: an integrated sociodevelopmentalcognitive model. The Lancet, 2014. 383(9929): p. 1677-1687.

20. Myin-Germeys, I., et al., Experience sampling methodology in mental health research: new insights and technical developments. World Psychiatry, 2018. 17(2): p. 123-132.

21. Rauschenberg, C., et al., Stress sensitivity as a putative mechanism linking childhood trauma and psychopathology in youth's daily life. Acta Psychiatrica Scandinavica, 2017. 136(4): p. 373-388.

22. Rauschenberg, C., et al., Bullying victimization and stress sensitivity in help-seeking youth: findings from an experience sampling study. European Child \& Adolescent Psychiatry., 2020.

23. Palmier-Claus, J., G. Dunn, and S. Lewis, Emotional and symptomatic reactivity to stress in individuals at ultra-high risk of developing psychosis. Psychological medicine, 2012. 42(5): p. 1003-1012.

24. Reininghaus, U., et al., Psychological processes underlying the association between childhood trauma and psychosis in daily life: an experience sampling study. Psychological medicine, 2016. 46(13): p. 2799-2813.

25. Reuben, A., et al., Lest we forget: comparing retrospective and prospective assessments of adverse childhood experiences in the prediction of adult health. Journal of Child Psychology and Psychiatry, 2016. 57(10): p. 1103-1112.

26. Vaessen, T., et al., Overall cortisol, diurnal slope, and stress reactivity in psychosis: An experience sampling approach. Psychoneuroendocrinology, 2018. 96: p. 61-68.

27. Onnela, J.-P. and S.L. Rauch, Harnessing smartphone-based digital phenotyping to enhance behavioral and mental health. Neuropsychopharmacology, 2016. 41(7): p. 1691-1696.

28. McLaughlin, K.A., Early life stress and psychopathology, in The Oxford Handbook of Stress and Mental Health. 2019.

29. LeMoult, J., et al., Meta-analysis: exposure to early life stress and risk for depression in childhood and adolescence. Journal of the American Academy of Child \& Adolescent Psychiatry, 2019.

30. Lardinois, M., et al., Childhood trauma and increased stress sensitivity in psychosis. Acta Psychiatrica Scandinavica, 2011. 123(1): p. 28-35.

31. Askeland, K.G., et al., Life events and adolescent depressive symptoms: Protective factors associated with resilience. Plos one, 2020. 15(6): p. e0234109.

32. Crush, E., et al., Protective factors for psychotic symptoms among poly-victimized children. Schizophrenia bulletin, 2018. 44(3): p. 691-700.

33. Fritz, J., et al., A systematic review of amenable resilience factors that moderate and/ or mediate the relationship between childhood adversity and mental health in young people. Frontiers in psychiatry, 2018. 9: p. 230. 
34. Kuranova, A., et al., Don't worry, be happy: Protective factors to buffer against distress associated with psychotic experiences. Schizophrenia Research, 2020.

35. Perchtold, C.M., et al., Humorous cognitive reappraisal: More benign humour and less" dark" humour is affiliated with more adaptive cognitive reappraisal strategies. PloS one, 2019. 14(1).

36. Kashdan, T.B. and J. Rottenberg, Psychological flexibility as a fundamental aspect of health. Clinical psychology review, 2010. 30(7): p. 865-878.

37. Fonseca, S., et al., The buffer role of psychological flexibility against the impact of major life events on depression symptoms. Clinical Psychologist, 2020. 24(1): p. 82-90.

38. Lazarus, R.S. and S. Folkman, Coping and adaptation. The handbook of behavioral medicine, 1984. 282325.

39. Vassos, E., et al., The Maudsley environmental risk score for psychosis. Psychological Medicine, 2019: p. 1-8.

40. Kotov, R., et al., The Hierarchical Taxonomy of Psychopathology (HiTOP): a dimensional alternative to traditional nosologies. Journal of abnormal psychology, 2017. 126(4): p. 454. 


\section{Supplementary material}

Table S1. Items used in the modified List of Threatening Events (LTE) questionnaire

\begin{tabular}{lll}
\hline Types of negative life events & \\
\hline Illness & LE_001 & $\begin{array}{l}\text { "Serious illness, injury or assault to yourself?" } \\
\text { "Serious illness, injury or assault to a family member or } \\
\text { friend?" } \\
\end{array}$ \\
& LE_018 & "Hospitalisation or other medical treatments?" \\
\hline Loss & LE_003 & "Death of a parent, partner, or child?" \\
& LE_004 & "Death of a family member or friend?" \\
& LE_015 & "Loss or theft of anything of value?" \\
\hline
\end{tabular}

Interpersonal/

Intra-familial conflicts

LE_005 "Break-up with partner (with whom you lived together)/ from parents?"

LE_006 "Break-up committed relationship (not living together)/ of a parent?"

LE_007 "Divorce/ of parents?"

LE_008 "Cheating of spouse (one of the parents)?"

LE_009 "Serious persistent problems (quarrel) with members within the family?"

LE_010 "A serious problem with a friend, neighbour, or family member?"

Occupational conflicts

LE_011 "Serious problems at school/work (being suspended/ stopped/ fired/ finding no work)?"

Financial problems

LE_012 "Serious financial problems within the family (very serious debt, bankruptcy)?"

Housing problems

LE_013 "Serious housing problems (including homelessness)?"

Legal conflicts

LE_014 "A problem with the police and/or law (violation, court, prison / community service)?"

Threatening/

Intrusive incidents

LE_016 "Victim of threats, robbery, or burglary?"

LE_017 "Witness to serious threat or another traumatic event?"

LE_021 "Involved in a serious accident in which you and/or someone else got hurt seriously?" 



\section{PART 2}

Digital interventions in public mental health provision 



\section{CHAPTER 7}

\section{An ecological momentary compassion-focused intervention for enhancing resilience in help-seeking youth: a pilot study}




\section{Abstract}

Background: Digital interventions offer new avenues for low-threshold prevention and treatment in youth. Ecological momentary interventions (EMIs) represent a powerful approach that allows for adaptive, real-time, and real-world delivery of intervention components in daily life by real-time processing of multimodal Ecological Momentary Assessment (EMA) data. Compassion-focused interventions (CFIs) may be particularly amenable to translation into an EMI to strengthen emotional resilience and modifying putative risk mechanisms, including stress sensitivity, in daily lives of young help-seeking individuals.

Objective: We aimed to investigate feasibility, safety, and initial therapeutic effects of a novel, accessible, transdiagnostic, ecological momentary, compassionfocused intervention for improving emotional resilience to stress ('EMIcompass').

Methods: In an uncontrolled pilot study, help-seeking youth with psychotic, depressive, and/or anxiety symptoms were offered the EMIcompass intervention in addition to treatment as usual. EMIcompass consisted of three sessions with a trained psychologist (i.e., training session of CFI exercises, follow-up 'booster' session, and review session) and a 3-week EMI (i.e., consisting of enhancing, consolidating, and EMA-informed interactive tasks) administered through a mobile health (mHealth) app.

Results: In total, ten individuals (Mean age=20.3 years, range 14-25 years) were included in the study. Most participants were satisfied (80\%) and reported low burden of app usage. No adverse events were observed. In around 1/3 of all EMAs, individuals scored high on stress, negative affect, or threat anticipation during the intervention period, resulting in real-time delivery of CFI intervention components in addition to weekly enhancing and daily consolidating tasks. Although findings should be interpreted with caution due to the small sample size, reduced stress sensitivity, momentary negative affect, and psychotic experiences as well as increased positive affect were found at post-intervention and 4-week follow-up. Further, reductions in psychotic, anxiety, and depressive symptoms were found $(r=0.30-0.65)$. 
Conclusions: Our findings provide evidence on feasibility and safety of the EMlcompass intervention in help-seeking youth and promising evidence of beneficial effects on stress sensitivity and psychopathological outcomes. An exploratory randomized controlled trial is warranted to establish feasibility and preliminary evidence of efficacy.

Keywords: Mental Health; Adolescent Psychopathology; Digital Interventions; Mobile Health; Self-Compassion; Ecological Momentary Assessment 


\section{Introduction}

Most mental disorders first emerge in adolescence and young adulthood (three fourths by age 24 [1]), with an estimated lifetime prevalence of around $50 \%$ of any mental disorder in young age groups [1-5]. Further, the Global Burden of Disease study has reported that mental and substance use disorders in children and youth aged 10-24 years were the leading cause of overall disease burden in high-income countries [6-8]. Evidence further suggests that most mental disorders are continuous - phenomenologically and temporarily - and, in their early stages, non-specific in nature, often evolving in the form of transdiagnostic phenotypes associated with a range of exit psychopathologies [9-16]. Consequently, clinical staging models as an adjunct to formal diagnoses have been introduced [17-19], which highlight the importance of transdiagnostic (indicated) prevention and early intervention [20-24].

Recent transformations in our understanding of phenomenology, aetiology, and early course of mental disorders have contributed to a move towards early detection and prevention [10-13, 20, 25-31]. While conventional mental health services offer a range of therapeutic options, it has been widely documented that psychological help remains difficult to access, especially for young individuals in the early stages of mental health problems [21, 22, 32, 33]. Further, tailoring of therapeutic options to specific needs and preferences of youth remains a challenge [32-36] and likely contributes to the problem that only a fraction of young people in need of help access any mental health service. Hence, young individuals often experience a long duration of untreated mental health problems which has been identified an important marker of poor course and outcome [32].

There is increasing interest in using digital tools to deliver mental health services [37], which may help to extend access to and personalisation of mental health care $[38,39]$. This has driven the development of novel mobile health (mHealth) interventions for various mental health problems [40-42], of which ecological momentary interventions (EMIs) [23, 34, 38, 39, 43], such as Acceptance and Commitment Therapy in Daily Life (ACT-DL) [34-36, 44], represent a very powerful approach. EMIs allow for adaptive, real-time, and real-world transfer of intervention components in individuals' daily lives. Thus, EMIs provide a unique opportunity to deliver personalized, precision interventions that are tailored to what young 
individuals need in a given moment and context through interactive sampling in real-time and the real-world. They are based on fine-grained ecological momentary assessment (EMA) data acquired through cutting-edge digital technology [21, 23, $24,38,39,45,46]$. More recently, some authors have started to use the term Just-in-Time Adaptive Interventions (IITAIs) which emphasize EMI's capability of adapting the delivery of intervention components to person and context based on experience sampling or other, e.g. sensing data [47, 48].

One tangible prevention and early intervention strategy using digital tools is to identify and target transdiagnostic psychological mechanisms in daily life, which have been shown to be involved in the development of mental health problems [23, 38]. In recent years, research using EMA, a structured diary technique also known as Experience Sampling Methodology [43], has contributed to a better understanding of putative underlying mechanisms likely to be involved in impacting at different stages on, and increasing intensity of, mental health problems in individuals' daily lives, in real time and outside the research laboratory [21-23, 29, 43, 49, 50]. To date, the psychological mechanism most widely studied in daily life is elevated stress sensitivity, characterized by more intense negative affective and psychotic experiences in response to minor stressors and routine daily hassles [22, 24, 29, 43]. Findings from previous studies suggest that stress sensitivity is elevated in individuals with (1) higher familial or psychometric risk, (2) an Ultra-High Risk (UHR) state for psychosis, (3) other early mental health problems, (4) first-episode psychosis (FEP), (5) severe and enduring psychosis, and (6) depressive disorder $[21,22,24,28,50-58]$. In addition, heightened interpersonal sensitivity and threat anticipation have previously been reported to represent further candidate mechanisms in individuals with UHR, paranoia, and psychotic disorder [24, 29, 30 , 59-62] as well as individuals with depression and anxiety [63-66]. Taken together, these transdiagnostic mechanisms reflect candidate targets to be modified by EMIs [21, 22, 24, 29].

Compassion-focused interventions (CFIs) are considered an important strand of transdiagnostic interventions for modifying emotion regulation systems $[67,68]$. CFIs form part of third-wave Cognitive Behavioural Therapy (CBT) and previous meta-analytic evidence on third-wave CBT, including CFIs [69-73], suggests that these types of interventions may yield improvements in mental health outcomes of moderate to large effect size. CFIs have been successfully administered to 
and appraised positively by help-seeking individuals, including individuals with depression, anxiety, and psychosis [74-77]. Further, CFIs have been shown to induce reductions in negative affect and paranoia in moments of high stress in prior experimental work in the research lab [78, 79]. Further, positive imagery, an important component of CFls, have been found to be effective for reducing various mental health problems, including depression, anxiety, and psychosis $[76,80,81]$ as well as for increasing positive affect, optimism, and behavioural activation [79, 82-84]. Thus, CFIs are particularly well placed to be administered as an EMI to strengthen emotional resilience and modify putative risk mechanism of poor mental health in young individuals with psychological distress [72, 78, 85], including stress sensitivity and threat anticipation [21, 22]. However, the use of conventional CFIs under real-world conditions remains very limited [86].

As young individuals are 'digital natives', translating CFI components into an EMI administered through an mHealth app may be a particularly promising approach and offers entirely new avenues for low-threshold prevention and intervention in youth. As such, EMIs are fundamentally translational as they directly build on evidence on underlying momentary mechanisms in daily life and translate this into the development and evaluation of novel digital interventions by targeting these mechanisms in real-time and in the real-world, outside the research lab or clinic $[23,39,43]$. However, it remains to be established whether evidence on reductions in negative affect and paranoia in moments of high stress carried out in the research laboratory as well as effects on other mental health outcomes can indeed be translated to real-world and real-time delivery of EMIs that harness compassion-focused intervention techniques, especially in young help-seeking individuals, where accessible, youth-friendly translation of prevention and early intervention principles reflects a particular challenge.

\section{The present study}

This study aimed to establish the clinical feasibility, safety, and initial therapeutic effects of a novel, accessible, transdiagnostic, ecological momentary, compassionfocused intervention for improving emotional resilience to stress ('EMIcompass') in an uncontrolled phase I pilot study in help-seeking youth with psychotic, depressive, and/or anxiety symptoms. The EMlcompass intervention consisted of three sessions with a trained psychologist (i.e., training session, 'booster' 
session, review session) and a 3-week EMI. More specifically, the intervention offered widely used CFI techniques (e.g., compassionate and positive imagery, and writing; emotion as a wave). To facilitate the interactive, real-time, and real-world translation of the therapeutic content and techniques used in the initial training and booster sessions into individuals' daily lives, the EMI was administered through an mHealth app on a smartphone. The EMI consisted of i) enhancing, ii) consolidating, and iii) interactive EMI tasks that aim at ecological translation of CFI principles and techniques to daily life: participants were required to complete one 'enhancing task' per week, which allowed them to practice new compassionfocused exercises that were then extended throughout the study period. Additionally, they were required to practice learned CFI components once a day by completing 'consolidating tasks'. Each time an enhancing task was presented, the intervention components covered by consolidating tasks were expanded. Participants were also offered 'interactive tasks' if they scored high on stress, negative affect, or threat anticipation in daily EMA.

The primary objective of this study was to (1) assess the clinical feasibility of delivering the EMIcompass intervention to help-seeking youth based on successful recruitment, assessment of outcomes, compliance, satisfaction, and acceptability as well as safety by carefully documenting any serious adverse events throughout the entire study period. The secondary objectives were to examine (2) initial therapeutic effects of EMlcompass on reducing stress sensitivity, negative affect, and psychotic experiences, and increasing positive affect in daily life at the end of the 3-week intervention period ('post-intervention'), and after a 4-week followup period ('follow-up') and (3) the initial therapeutic effects of EMlcompass on reducing threat anticipation, psychotic, depressive, and anxiety symptoms as well as general psychopathology.

\section{Methods}

\section{Study design}

In an uncontrolled, phase I pilot study, help-seeking individuals with psychotic, depressive, and/or anxiety symptoms aged 14-25 referred to secondary mental health services in the Netherlands (i.e., Mondriaan Mental Health Trust; Virenze Mental Health Care) received the EMIcompass intervention in addition to treatment as usual. Data were collected before the intervention ('baseline'), at 
the end of the 3-week intervention period ('post-intervention'), and after a 4-week follow-up period ('follow-up'). Particularly close attention was paid to establishing clinical feasibility (e.g., pragmatic inclusion and exclusion criteria based on routine assessments) and safety (i.e., documentation of any serious adverse events) of this study. Our recruitment strategy drew on our previous and ongoing work with youth [22, 24, 29, 34-36, 44] as well as guidance for pragmatic randomised controlled trials [87] and, hence, was geared to reflect the heterogeneity of the population commonly encountered in routine care.

\section{Sample}

We recruited young individuals with psychotic, depressive, and/or anxiety symptoms who were seeking help from two secondary mental services (i.e., Mondriaan Mental Health Trust; Virenze Mental Health Care). The inclusion and exclusion criteria were equivalent in principle across the two services but were purposefully selected to be pragmatic and, hence, based on routine assessments for screening, diagnosis, formulation, and outcome measurement, which differed between the two services (see Table 1). This approach was adopted to ensure the aim of establishing of feasibility reflected the population actually encountered in clinical practice (rather than imposed by researchers) whilst keeping assessment burden at a minimum. The study was approved by the Ethics Review Committee Mondriaan Mental Health Trust and the Ethics Review Committee Psychology and Neuroscience, Maastricht University. A study flowchart is provided in Figure 1.

At Virenze, the Prodromal Questionnaire (PQ) $[88,89]$, which has been reported to be a very good screening measure in routine mental health services [89, 90], was used to screen for psychotic symptoms. Also, the Brief Symptom Inventory (BSI) $[91,92]$ was used to screen for anxiety, depressive and psychotic symptoms. At Mondriaan, the Symptom Questionnaire-48 (SQ-48) [93] was used in addition to the PQ to screen for anxiety and depressive symptoms. 
Table 1. Inclusion and exclusion criteria by participating mental health services

\begin{tabular}{lll}
\hline & Mondriaan & Virenze \\
\hline Inclusion criteria: & Aged between & Aged between \\
& 18 and 25 years & 14 and 25 \\
\hline & PQ: & PQ: \\
& score of 6 or above & score of 6 or above \\
and/or & and/or \\
& SQ-48: & BSI: \\
& score of 9 or above on social & T-score of 63 or above \\
& phobia subscale; or score of 8 or & \\
& above on depression subscale; or & \\
& score of 11 or above on anxiety & \\
& subscale & \\
& Willingness to participate in the compassion-focused ecological \\
& momentary intervention. & \\
& & \\
& Ability to give written informed consent independently, without \\
& help from others & \\
\hline & Insufficient command of Dutch, primary clinical diagnosis of \\
& alcohol or substance dependency, \\
& severe endocrine, cardiovascular or organic brain disease
\end{tabular}

Notes: PQ, Prodromal Questionnaire; SQ49, Symptom Questionnaire-48; BSI, Brief Symptom Inventory 


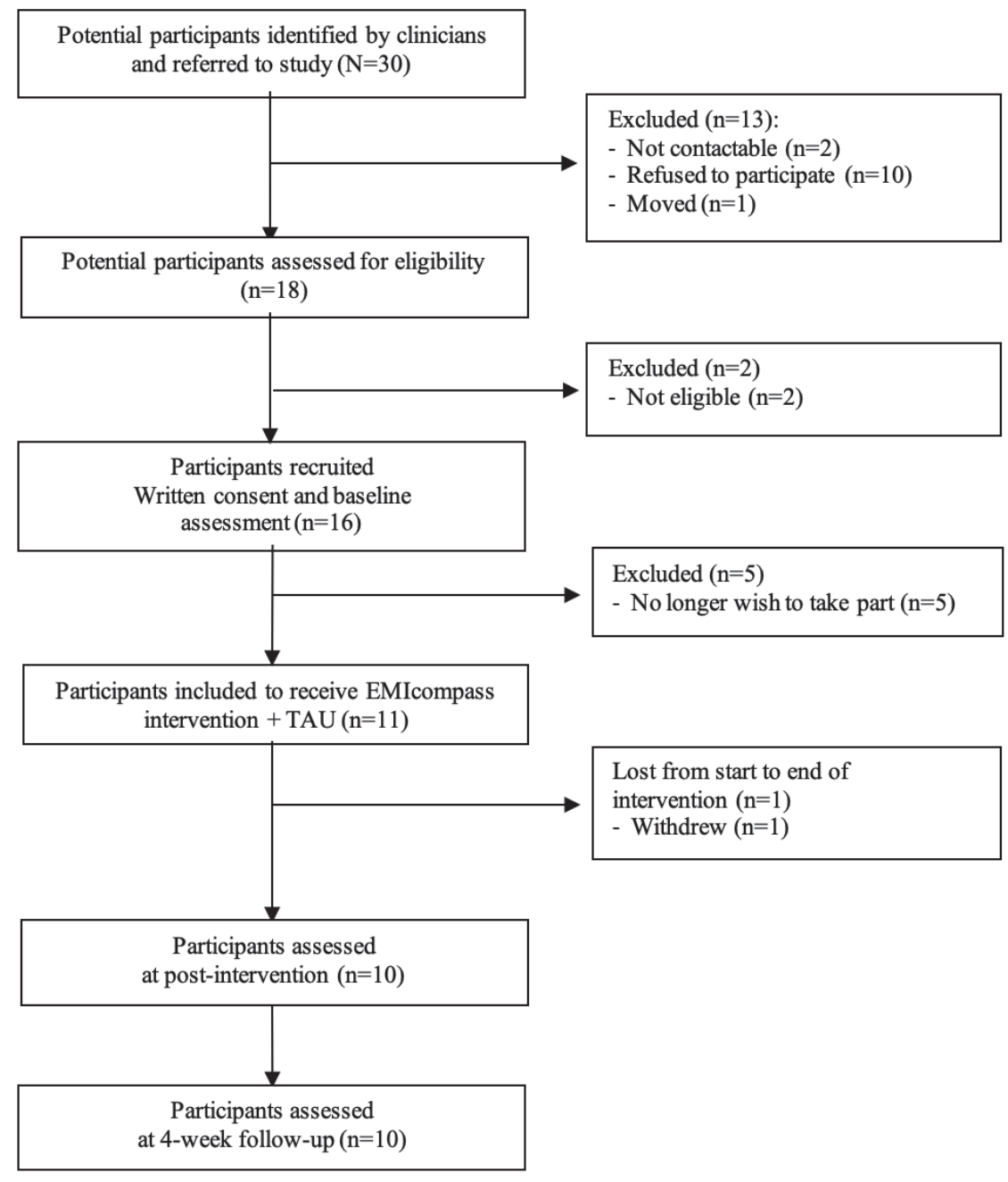

Figure 1. Study flowchart

\section{The EMIcompass intervention}

\section{Development of the manual}

The intervention was structured and manualised in order to ensure consistent delivery of the intervention. The manual was based on widely used CFI techniques (e.g., compassionate and positive imagery, and writing; emotion as a wave) and developed following a process of reviewing existing manuals and the extant CFI 
literature $[67,68,73,74,78,80]$, through the team's clinical experience of working with these approaches with clients, and through consultation with local experts in CFI and the wider research team. The intervention was designed based on principles of EMIs [23, 34-36, 39, 43, 44].

\section{EMIcompass intervention + treatment as usual}

In the current study, participants were offered the EMIcompass intervention in addition to treatment as usual, which included all the treatment they received prior to the start of the study (i.e., good standard care delivered according to local and national guidelines by their general practitioner, psychiatrist, and other health care professionals. This may include CBT, third-wave CBT, DBT, and other psychological interventions). The EMIcompass intervention consisted of three face-to-face sessions (one training session, one follow-up 'booster' session, and one review session) given by a trained psychologist, who was supervised by an expert clinical psychologist in compassion-focused therapy, and a 3-week EMI administered through an mHealth app on a smartphone (PsyMateTM). In addition, participants were offered on-demand e-mail and/or phone contact during the intervention period.

At the beginning of the 3-week intervention period, an initial face-to-face training session was offered to participants. This session was fully manualized based on previous research using compassion-focused interventions [67, 68, 74, 78, 94]. The goal of the first session was to train individuals in how to cope with negative emotions by applying a personal compassionate image that conveys compassion, care, and warmth to them based on Gilbert's (2010, pp. 187-189) descriptions and as applied by Lincoln et al. (2013). This was followed by inducing negative emotions using in-sensu exposure to a personally relevant social situation that participants remember having experienced as distressing. This method has been safely applied in individuals with mental health problems $[74,78]$ without any adverse consequences or health-related risks. Following induction of negative emotions, participants were asked to practice a 5-minute application of the compassionate image participants were trained in at the beginning of the session $[67,68,78]$. This step of actively using compassionate imagery subsequent to inducing negative emotions is considered essential for compassion-focused therapy to be efficacious in reducing stress sensitivity, threat anticipation, and psychotic, depressive and/or 
anxiety symptoms in daily life $[67,68]$. Training the use of compassionate imagery was repeated and extended to imagery involving a 'compassionate self' [68] and 'emotion as a wave' [94] in the following booster session two weeks after the initial training session. In the review session at the end of the 3-week intervention period, the smartphone was returned, progress and satisfaction with, and acceptability of, the intervention were reviewed and assessed.

Table 2. Components of the EMIcompass intervention

\begin{tabular}{|c|c|c|c|}
\hline & Week 1 & Week 2 & Week 3 \\
\hline $\begin{array}{l}\text { Compassion- } \\
\text { focused training } \\
\text { sessions }\end{array}$ & $\begin{array}{l}\text { Training session } \\
\text { (compassionate } \\
\text { image) }\end{array}$ & $\begin{array}{l}\text { Booster session (day 11-15) } \\
\text { (compassionate self-training, } \\
\text { 'emotion as a wave') }\end{array}$ & $\begin{array}{l}\text { Review session } \\
\text { (after day 20) }\end{array}$ \\
\hline Enhancing tasks & $\begin{array}{l}\text { Task } 1 \text { (day } 3 \text { or } 4 \text { ): } \\
\text { Compassionate self- } \\
\text { validation }\end{array}$ & $\begin{array}{l}\text { Task } 2 \text { (day } 9 \text { or } 10) \text { : } \\
\text { 'Emotion as a wave' }\end{array}$ & $\begin{array}{l}\text { Task } 3 \text { (day } 15 \text { or } 16 \text { ): } \\
\text { Self-compassionate } \\
\text { writing }\end{array}$ \\
\hline $\begin{array}{l}\text { Consolidating } \\
\text { tasks }\end{array}$ & $\begin{array}{l}\text { Compassionate self- } \\
\text { validation } \\
\text { (from day } 5, \\
\text { following enhancing } \\
\text { EMI task 1) }\end{array}$ & $\begin{array}{l}\text { Compassionate self- } \\
\text { validation } \\
\text { 'Emotion as a wave' } \\
\text { (from day 11, following } \\
\text { enhancing EMI task 2) }\end{array}$ & $\begin{array}{l}\text { Compassionate self- } \\
\text { validation } \\
\text { 'Emotion as a wave' } \\
\text { Self-compassionate } \\
\text { writing } \\
\text { (from day 17, } \\
\text { following enhancing } \\
\text { EMI task 3) }\end{array}$ \\
\hline Interactive tasks & $\begin{array}{l}\text { Compassionate } \\
\text { image } \\
\text { Compassionate self- } \\
\text { validation } \\
\text { (from day 5, } \\
\text { following enhancing } \\
\text { EMI task 1) }\end{array}$ & $\begin{array}{l}\text { Compassionate image } \\
\text { Compassionate self- } \\
\text { validation } \\
\text { 'Emotion as a wave' } \\
\text { (from day 11, following } \\
\text { enhancing EMI task 2) }\end{array}$ & $\begin{array}{l}\text { Compassionate } \\
\text { image } \\
\text { Compassionate self- } \\
\text { validation } \\
\text { ‘Emotion as a wave' } \\
\text { Self-compassionate } \\
\text { writing } \\
\text { (from day 17, } \\
\text { following enhancing } \\
\text { EMI task 3) }\end{array}$ \\
\hline
\end{tabular}

Note: EMI, ecological momentary interventions

To allow for interactive, real-time, and real-world translation of the therapeutic content and techniques of initial and booster sessions into individuals' daily lives, participants were additionally offered a 3-week EMI delivered through an 
mHealth app. During the 3-week intervention period, the smartphone prompted a signalling sound from the smartphone seven times per day on six consecutive days per week to reduce burden associated with app usage. At each beep, participants were asked to complete a brief EMA on momentary stress, positive and negative affect, and threat anticipation in daily life (see section on used EMA measures). EMA was scheduled at random within set blocks of time. The EMI consisted of three different types of tasks (see Table 2): participants were asked to complete one 'enhancing task' per week, allowing them to practice new compassion-focused exercises, which were subsequently extended over the study period (e.g., discovering their own compassionate self, experiencing emotions as a wave). In addition, they were asked to practice learned CFI components once a day by completing 'consolidating tasks' at a predefined time. The components covered by consolidating tasks were extended each time an enhancing task was presented. Further, 'interactive tasks' were offered if participants scored high on stress, negative affect, or threat anticipation in the EMA (i.e., scores higher than 4 on a 7-point Likert scale). Given an essential element of compassion-focused therapy is for individuals to use compassionate imagery in moments of high stress, negative affect, or threat anticipation, these interactive tasks are thought to reflect a core active component of the 3-week compassion-focused EMI.

\section{Measures}

\section{Socio-demographic characteristics}

A socio-demographic schedule was used to assess age, gender, occupation, and level of education.

\section{Clinical feasibility and safety}

Feasibility was assessed based on successful recruitment, assessment of outcomes, compliance with the manual, satisfaction, and acceptability. For some of the domains of feasibility, a debriefing scale was used. Reasons for participants to decline to take part in the study were carefully recorded, and completeness of outcomes at each time point was documented. Acceptability was assessed in the review session of the EMlcompass intervention together with the trained psychologist by asking participants to complete a feedback form about the EMI tasks and sessions and to rate the extent to which they feel they benefit from, and 
are satisfied with, the intervention $[74,78]$. In addition, the trained psychologist asked participants in the review session to report whether they perceived the face-to-face sessions, the compassion-focused exercises, and the EMI tasks as helpful. App usability was assessed by asking participants to rate the readability of the text shown on the screen, any difficulties to operate the app or technical problems, the clarity of provided instructions, and whether the app was perceived as burdensome. All items were rated on a 7-point Likert scale ranging from 'not at all' (rating of 1) to 'moderate' (rating of 4) and 'very' (rating of 7) which were subsequently grouped into three categories of 'not' (rating of 3 or lower), 'moderate' (rating of 4 or 5), and 'very' (rating of 6 or 7) for sake of interpretability of findings (given small numbers in each cell). Safety was assessed by carefully documenting any serious adverse events throughout the entire study period as well as potential negative effects of app usage on mental health in participants.

\section{Stress sensitivity, negative and positive affect, and psychotic experiences in daily life}

EMA was used to assess stress sensitivity, negative and positive affect, psychotic experiences, and threat anticipation in daily life. For this, the same app was used as for the EMlcompass intervention (PsyMateTM) and assessments were completed at baseline, post-intervention, and 4-week follow-up for a period of 6 consecutive days following the protocol from previous EMA studies [22, 24, 29, 46, 49]. Stress was operationalised as minor disturbances and distinctive unpleasant events, activities, and social situations that occur in the flow of daily life. Eventrelated stress was measured with one item asking participants to rate the most important event that has happened since the last beep on a 7-point Likert scale ranging from 'very unpleasant' (rating of -3) to 'very pleasant' (rating of 3) [54]. The item was recoded that higher ratings indicate higher levels of stress (with ratings of -3 coded as 7 and ratings of 3 coded as 1). Activity-related stress was measured by asking participants, first, to specify their current activity (e.g. resting, watching TV), which was followed by asking to rate the pleasantness of this activity on a 7-point Likert scale (1='very unpleasant', 7='very pleasant'). Social stress was measured by asking participants to specify categorically with whom they were spending time (e.g. nobody, partner, family) and to appraise the current social context using the items 'I find being with these people pleasant' [reversed], 'I feel 
accepted' [reversed], and 'I feel excluded (if with someone) or 'I find it pleasant to be alone' [reversed] and 'I would prefer to have company' (if alone) ranging from 'not at all' (rating of 1) to 'very much' (rating of 7). Good concurrent validity of these EMA stress measures has been reported [54, 55]. Further, a composite stress score was calculated by using the mean score of all seven stress items [21, 95]. Negative affect was assessed using five items by asking participants to rate the extent to which they feel anxious, down, insecure, uncomfortable, and guilty at each entry point [54] and positive affect was assessed by asking participants to rate the extent to which they feel cheerful and relaxed, all rated on a 7-point Likert scale ranging from 'not at all' (rating of 1) to 'very much' (rating of 7) $[54,55,96]$. Psychotic experiences were assessed using seven items ('I see things that aren't really there', 'I hear things that aren't really there', 'I feel suspicious/paranoid', 'I feel unreal', 'My thoughts are influenced by other', 'I can't get these thoughts out of my head', 'I feel like I am losing control') rated on a 7-point Likert scale ranging from 1 ('not at all') to 7 ('very much') [55, 96]. Threat anticipation was assessed by asking participants to think of what might happen in the next few hours and to rate the item 'I think that something unpleasant will happen' on a 7-point Likert scale (ranging from 1 ('not at all') to 7 ('very much')) [24, 29]. Negative and positive affect, psychotic experiences, and threat anticipation scores were assessed by computing mean scores, respectively. In line with earlier studies [22, 24, 29, 46, 49], items on stress, negative affect, and psychotic experiences were used as a proxy for individuals' stress sensitivity in daily life by modelling the association between stress and (i) negative affect and (ii) psychotic experiences. Thus, we conceptualized stress sensitivity in daily life as individuals' affective and psychotic reactivity to minor daily stressors.

\section{Psychotic, depressive, and anxiety symptoms as well as general psychopathology}

We used non-EMA outcome measures to assess psychotic, depressive, and anxiety symptoms as well as general psychopathology. First, the BSI was used to assess depressive and anxiety symptoms (based on the respective BSI subscales) and general psychopathology by computing the Global Severity Index (GSI; based on $53 \mathrm{BSI}$ items). Participants rated each item on a 5-point scale ranging from 0 ('not at all') to 4 ('extremely') [91, 92]. Second, the Green et al. Paranoid Thoughts Scale 
(GPTS), a reliable and valid scale, was used to assess psychosis [97]. The GPTS was modified to ask participants about paranoid ideation over the past week rather than the past month given the intervention period was only 3 weeks. A total score was computed using all 32 items (both with a 5-point scale; 1='not at all', 3='somewhat', $5=$ 'totally'). Third, the Threat anticipation measure (TAM) [98] was employed to measure threat anticipation by asking participants to estimate the future likelihood of a list of threatening, neutral, and positive events happening to themselves and to other people $[62,98,99]$. Items for threating and neutral events were used to compute total scores. Each event was rated separately for the likelihood that it will happen to oneself and to another person on a 7-point scale ( $1=$ 'not at all', $7=$ 'very likely'), resulting in four total sum scores (i.e., threat anticipation-self, threat anticipation-other, neutral anticipation-self, neutral anticipation-other) where higher scores indicate higher probability estimates. Finally, the Prodromal Questionnaire (PQ) $[88,89]$ was used to assess the presence of prodromal and attenuated psychotic symptoms (i.e., positive symptoms, disorganized symptoms, negative symptoms, and general symptoms). This measure consists of 16 items that assess the presence of psychotic symptoms ( $0=$ false, $1=$ true) which were used to compute a total score (range 0-16). Good psychometric properties have been reported for these measures $[88,97,98,100,101]$.

\section{Statistical analysis}

STATA 15.1 was used to analyse the data. First, descriptive statistics was used, and confidence intervals constructed, as appropriate, to summarize findings on feasibility and safety. Second, as EMA data have a multilevel structure, such that multiple observations (level-1) are nested within subjects (level-2), linear mixed models were used to control for within-subject clustering of multiple observations using the "mixed" command in STATA. Thus, to examine the effects of the EMlcompass intervention on reducing stress sensitivity, EMA stress variables and time point were included as independent variables and (i) negative affect and (ii) psychotic experiences as the outcome variable in linear mixed models, which were fitted separately for each outcome variable. We then added twoway interaction terms for stress $\times$ time and used likelihood ratio tests ("Irtest" command) to evaluate improvement in model fit as well as the "lincom" command to compute linear combinations of coefficients for testing our hypotheses whether 
stress sensitivity was reduced at post-intervention and 4-week follow-up. We standardized continuous ESM and variables (mean = 0, S.D. = 1) for interpreting significant interaction terms. Family-wise error-corrected $p$-values were computed to control for multiple testing by multiplying the unadjusted p-values of the twoway interaction effects by the total number of tests $(\mathrm{N}=4)$ for each outcome. Third, to examine the effects of the EMIcompass intervention on other EMA outcome measures, time point was included as independent variable and negative affect, positive affect, psychotic experiences and threat anticipation as the outcome variable in separate linear mixed models. All models were controlled for potential confounders (i.e. age, gender, level of education). Lastly, we used Wilcoxon signed rank tests to examine the effects of EMIcompass on non-EMA outcome measures of threat anticipation, psychotic, depressive, and anxiety symptoms as well as general psychopathology at post-intervention and 4-week follow-up. Resulting Z-scores were used to calculate effect sizes displayed in $r$ as described by Rosenthal [102].

\section{Results}

\section{Socio-demographic characteristics}

A study flowchart is shown in Figure 1. In total, 30 potential participants aged 14-25 years were referred to the study by clinicians from the two participating mental health services. Of these, 16 provided written informed consent and were eligible, of whom eleven completed the baseline assessment and were included to receive the EMIcompass intervention. One participant was lost during the 3-week intervention period, whereas 10 participants (mean age 20.3 years, range 14-24) completed the EMIcompass intervention and both post-intervention and 4-week follow-up assessments. The majority of participants were female $(7 / 10 ; 70 \%)$ and currently at school/university $(6 / 10 ; 60 \%)$. Half of the participants had a clinical diagnosis of major depressive disorder (5/10; 50\%) and met criteria for a comorbid mental health condition. Most participants were of white Dutch ethnic background and some reported having used cannabis during the last 12 months (3/10; 30\%). 
Table 3. Basic sample characteristics

\begin{tabular}{|c|c|}
\hline & Service users $(n=10)$ \\
\hline $\begin{array}{l}\text { Age, mean } \\
\text { (S.D.; range) }\end{array}$ & $\begin{array}{c}20.3 \\
(3.8 ; 14-25)\end{array}$ \\
\hline \multicolumn{2}{|l|}{ Sex, n (\%) } \\
\hline Female & $7(70.0)$ \\
\hline Male & $3(30.0)$ \\
\hline \multicolumn{2}{|l|}{ Ethnicity, n (\%) } \\
\hline White Dutch & $6(60.0)$ \\
\hline Other & $1(10.0)$ \\
\hline Missing value & $3(30.0)$ \\
\hline \multicolumn{2}{|l|}{ Level of education, $\mathbf{n}(\%)^{a}$} \\
\hline School & $2(20.0)$ \\
\hline Further & $4(40.0)$ \\
\hline Higher & $4(40.0)$ \\
\hline \multicolumn{2}{|l|}{ Occupation, n (\%) } \\
\hline School / Education & $6(60.0)$ \\
\hline Employed (full-/part-time) & $3(30.0)$ \\
\hline Unstructured activities & $1(10.0)$ \\
\hline \multicolumn{2}{|l|}{ Cannabis use ${ }^{\mathrm{a}}, \mathrm{n}(\%)$} \\
\hline 12-months & $3(30.0)$ \\
\hline Lifetime & $4(40.0)$ \\
\hline \multicolumn{2}{|l|}{ DSM-IV diagnosis, n (\%) } \\
\hline Major depressive disorder & $5(50.0)$ \\
\hline Attention-deficit hyperactivity disorder & $1(10.0)$ \\
\hline Reactive attachment disorder & $2(20.0)$ \\
\hline None & $2(20.0)$ \\
\hline Comorbid condition ${ }^{b}$ & $5(50.0)$ \\
\hline
\end{tabular}

Notes: S.D., standard deviation

${ }^{a}$ Categories defined as school (primary education, LBO, MAVO, VMBO), further (MBO, HAVO, VWO) and higher (HBO, HO, WO) of the Dutch educational system.

a Based on CIDI-L: Defined as having used cannabis more than 5 times on own initiative during the last 12-month or lifetime.

b Consisting of the following diagnostic categories: Panic disorder, Attention-deficit hyperactivity disorder, Intermittent explosive disorder, Borderline personality disorder, Parent-child relational problem.

\section{Clinical feasibility and safety}

Findings on clinical feasibility and safety are shown in Table 4. Almost all individuals $(90 \%, 9 / 10)$ reported that taking part in the study did not interfere with their daily activities. Most individuals reported to be very (40-50\%) or moderately satisfied (40-50\%) with tasks delivered through the EMlcompass app as well as moderately $(20 \%-30 \%)$ or very $(60 \%)$ satisfied across face-to-face sessions. The 
majority of participants was also very $(50 \%, 5 / 10)$ or moderately $(20 \%, 2 / 10)$ successful in imagining a compassionate image. Some individuals reported that the intervention positively influenced social contacts (30\%, 3/10 (ratings of 'moderate' and 'very' combined)) and levels of activity (40\%, 4/10). All individuals were very satisfied with the face-to-face contact sessions with, and felt they were understood by, the trained psychologists. While all participants reported that they were able to follow the instructions shown on the screen, observer-ratings by trained psychologists, who also delivered the face-to-face sessions, indicated that some individuals may have had problems with this (10\% at session 1 and $20 \%$ at session 3). Findings on app usability was satisfactory and the burden associated with app usage was perceived to be low or very low across all time points (70$90 \%)$, although some individuals $(30 \%, 3 / 10)$ found the number of signals per day moderately burdensome. Also, some individuals perceived the items used in the PsyMateTM app as difficult or unclear (20\%, 2/10). There were no severe adverse events recorded during the study period.

In-app usage data during the interventions period suggests high completion rates of EMA assessments. More specifically, the EMIcompass app triggered, in sum, 1260 signals asking participants to complete brief EMA assessments (126 for each person). Of these, individuals reacted to 467 (37.1\%), although high variability between individuals was found (range $17 \%$ to $67 \%$ ). In total, individuals scored high on stress, negative affect, or threat anticipation in 150 out of 467 EMA assessments (32.1\%), resulting in real-time delivery of CFI intervention components in around $1 / 3$ of all EMA assessments. When considering assessment of outcomes during the baseline, post-intervention, and follow-up, we found satisfactory completions rates (no missing data for outcome measures filled in in person as well as at least $30 \%$ of all EMA assessments). Thus, when combining self-reports and in-app usage data, assessment of outcomes and compliance with the manual was considered satisfactory. What is more, the conversation rate of recruitment was 3:1 (i.e., from identified to included individuals; see Figure 1), which is in line with previous research and considered successful recruitment. 
Table 4. Findings on safety, feasibility, and app usability of the EMIcompass intervention

\begin{tabular}{|c|c|c|c|}
\hline & Very $^{a}$ & Moderate $^{\text {a }}$ & Not ${ }^{a}$ \\
\hline \multicolumn{4}{|l|}{ Safety and feasibility } \\
\hline Interference of study participation with daily activities & - & $1(10 \%)$ & $9(90 \%)$ \\
\hline Satisfaction with face-to-face sessions & $6(60 \%)$ & $2(20 \%)$ & $2(20 \%)$ \\
\hline $\begin{array}{l}\text { Session 1: compassionate image; inducing negative } \\
\text { emotions }\end{array}$ & $6(60 \%)$ & $3(30 \%)$ & $1(10 \%)$ \\
\hline Session 2: compassionate self; emotion as a wave & $6(60 \%)$ & $3(30 \%)$ & $1(10 \%)$ \\
\hline Session 3: review session & $6(60 \%)$ & $3(30 \%)$ & $1(10 \%)$ \\
\hline \multicolumn{4}{|l|}{ Satisfaction with tasks } \\
\hline Task 1: compassionate self-validation & $4(40 \%)$ & $5(50 \%)$ & $1(10 \%)$ \\
\hline Task 2: emotion as a wave & $5(50 \%)$ & $3(30 \%)$ & $2(20 \%)$ \\
\hline Task 3: self-compassionate writing & $5(50 \%)$ & $3(30 \%)$ & $2(20 \%)$ \\
\hline $\begin{array}{l}\text { Self-reported success in making a compassionate } \\
\text { image }\end{array}$ & $5(50 \%)$ & $3(30 \%)$ & $2(20 \%)$ \\
\hline $\begin{array}{l}\text { Taking part in the study positively } \\
\text { affected activities }^{\text {b }}\end{array}$ & $2(20 \%)$ & $2(20 \%)$ & $5(50 \%)$ \\
\hline \multicolumn{4}{|l|}{ Taking part in the study affected social contacts } \\
\hline Positively & $1(10 \%)$ & $2(20 \%)$ & $7(70 \%)$ \\
\hline Negatively & - & - & $10(100 \%)$ \\
\hline Satisfaction with contact with trained psychologist ${ }^{b}$ & $9(100 \%)$ & - & - \\
\hline Participant felt understood by trained psychologist ${ }^{b}$ & $9(100 \%)$ & - & - \\
\hline $\begin{array}{l}\text { Self-reported level of understanding of instructions } \\
\text { provided by trained psychologist? }{ }^{b}\end{array}$ & $9(100 \%)$ & - & - \\
\hline \multicolumn{4}{|l|}{ Observer-rating by trained psychologists } \\
\hline Compliance in session 1 & $7(70 \%)$ & $2(20 \%)$ & $1(10 \%)$ \\
\hline Compliance in session 2 & $7(70 \%)$ & $3(30 \%)$ & - \\
\hline Compliance in session 3 & $6(60 \%)$ & $2(20 \%)$ & $2(20 \%)$ \\
\hline \multicolumn{4}{|l|}{ EMIcompass app usability } \\
\hline Readability of text on screen & $10(100 \%)$ & - & - \\
\hline Difficulties to operate the app & - & - & $10(100 \%)$ \\
\hline Clarity of instructions given on screen & $10(100 \%)$ & - & - \\
\hline Difficulties understanding used items & - & $2(20 \%)$ & $8(80 \%)$ \\
\hline \multicolumn{4}{|l|}{ EMIcompass app perceived as burdensome } \\
\hline In terms of the number of signals per day & - & $3(30 \%)$ & $7(70 \%)$ \\
\hline In terms of the number of items asked per signal & - & $1(10 \%)$ & $9(90 \%)$ \\
\hline In terms of the signal sound & $1(10 \%)$ & $1(10 \%)$ & $8(80 \%)$ \\
\hline Technical problems & - & $1(10 \%)$ & $9(90 \%)$ \\
\hline
\end{tabular}




\section{Initial therapeutic effects}

\section{Stress sensitivity, negative and positive affect, and psychotic experiences in daily life}

Findings on initial therapeutic effects of the EMlcompass intervention on stress sensitivity are provided in Table 5. We found preliminary evidence that participants experienced less intense negative affect in response to event-related and activityrelated stress at post-intervention as well as in response to overall, event-related, activity-related, and social stress at follow-up than at baseline, as indicated by statistically significant two-way interaction effects for stress $\times$ time point. Further, participants reported less intense psychotic experiences in response to minor stressors in daily life (i.e., overall as well as specific types of stressors) at postintervention and follow-up than at baseline.

Further, Table 6 shows findings of initial effects of EMlcompass on momentary negative affect, psychotic experiences, and positive affect. There was preliminary evidence that participants experienced less intense negative affect and psychotic experiences as well as more intense positive affect in daily life at post-intervention and 4-week follow-up than at baseline. There was also some evidence that individuals anticipated fewer threatening events in their daily lives at postintervention and 4-week follow-up than at baseline. 
Table 5. Initial therapeutic effects of EMIcompass on stress sensitivity in daily life

\begin{tabular}{|c|c|c|c|c|c|c|c|c|}
\hline & \multicolumn{2}{|c|}{$\begin{array}{l}\text { Post-intervention } \\
\text { vs. baseline }\end{array}$} & \multirow{2}{*}{$\begin{array}{c}\begin{array}{c}\text { Follow-up } \\
\text { vs. baseline }\end{array} \\
\text { adj. } \beta \\
(95 \% \mathrm{CI})\end{array}$} & \multicolumn{3}{|c|}{$\begin{array}{l}\text { Follow-up } \\
\text { vs. post- } \\
\text { intervention }\end{array}$} & \multicolumn{2}{|c|}{$\begin{array}{l}\text { LR test for } \\
\text { interaction }^{\mathrm{a}}\end{array}$} \\
\hline & $\begin{array}{c}\text { adj. } \beta \\
(95 \% \mathrm{CI})\end{array}$ & $\mathbf{p}$ & & $\mathbf{p}$ & $\begin{array}{l}\text { adj. } \beta \\
(95 \% \mathrm{CI})\end{array}$ & $\mathbf{p}$ & $\chi^{2}(\mathrm{df})$ & pFWE \\
\hline \multicolumn{9}{|c|}{ Outcome: Negative affect } \\
\hline \multicolumn{9}{|l|}{ Stress } \\
\hline Overall & $\begin{array}{c}-0.12 \\
(-0.27-0.03)\end{array}$ & 0.110 & $\begin{array}{c}-0.51 \\
(-0.63--0.40)\end{array}$ & $<0.001$ & $\begin{array}{c}-0.39 \\
(-0.55--0.23)\end{array}$ & $<0.001$ & $72.6(2)$ & $<0.001$ \\
\hline $\begin{array}{l}\text { Event- } \\
\text { related }\end{array}$ & $\begin{array}{c}-0.41 \\
(-0.56--0.25)\end{array}$ & $<0.001$ & $\begin{array}{c}-0.39 \\
(-0.51--0.27)\end{array}$ & $<0.001$ & $\begin{array}{c}0.02 \\
(-0.14-0.18)\end{array}$ & 0.831 & $51.6(2)$ & $<0.001$ \\
\hline $\begin{array}{l}\text { Activity- } \\
\text { related }\end{array}$ & $\begin{array}{c}-0.25 \\
(-0.40--0.09)\end{array}$ & 0.002 & $\begin{array}{c}-0.35 \\
(-0.47--0.23)\end{array}$ & $<0.001$ & $\begin{array}{c}-0.10 \\
(-0.27-0.06)\end{array}$ & 0.216 & $32.5(2)$ & $<0.001$ \\
\hline Social & $\begin{array}{c}0.05 \\
(-0.10-0.20) \\
\end{array}$ & 0.502 & $\begin{array}{c}-0.41 \\
(-0.53--0.28) \\
\end{array}$ & $<0.001$ & $\begin{array}{c}-0.46 \\
(-0.62--0.29)\end{array}$ & $<0.001$ & $47.6(2)$ & $<0.001$ \\
\hline \multicolumn{9}{|c|}{ Outcome: Psychotic experiences } \\
\hline \multicolumn{9}{|l|}{ Stress } \\
\hline Overall & $\begin{array}{c}-0.15 \\
(-0.25--0.04)\end{array}$ & 0.005 & $\begin{array}{c}-0.28 \\
(-0.36--0.20)\end{array}$ & $<0.001$ & $\begin{array}{c}-0.14 \\
(-0.25--0.03)\end{array}$ & 0.013 & $48.7(2)$ & $<0.001$ \\
\hline $\begin{array}{l}\text { Event- } \\
\text { related }\end{array}$ & $\begin{array}{c}-0.29 \\
(-0.39--0.19)\end{array}$ & $<0.001$ & $\begin{array}{c}-0.19 \\
(-0.27--0.11)\end{array}$ & $<0.001$ & $\begin{array}{c}0.10 \\
(-0.01-0.20)\end{array}$ & 0.080 & $40.6(2)$ & $<0.001$ \\
\hline $\begin{array}{l}\text { Activity- } \\
\text { related }\end{array}$ & $\begin{array}{c}-0.25 \\
(-0.35--0.14)\end{array}$ & $<0.001$ & $\begin{array}{c}-0.20 \\
(-0.28--0.12)\end{array}$ & $<0.001$ & $\begin{array}{c}0.05 \\
(-0.06-0.16)\end{array}$ & 0.399 & $33.3(2)$ & $<0.001$ \\
\hline Social & $\begin{array}{c}-0.01 \\
(-0.11-0.09)\end{array}$ & 0.863 & $\begin{array}{c}-0.24 \\
(-0.32--0.16)\end{array}$ & $<0.001$ & $\begin{array}{c}-0.23 \\
(-0.34--0.12)\end{array}$ & $<0.001$ & $36.3(2)$ & $<0.001$ \\
\hline
\end{tabular}

Note: adj. $\beta$, standardized regression coefficients [continuous independent variables were standardized (mean = 0, S.D. = 1) for interpreting interaction terms; Cl, confidence interval; df, degrees of freedom, LR, likelihood ratio test; pFWE, family-wise error-corrected $p$ values were computed by multiplying the unadjusted $p$ value by the total number of tests for each outcome $(N=4)$ to adjust significance levels of likelihood ratio tests for two-way interactions. a Likelihood ratio test for stress $\times$ time interaction after inclusion in the following model: (for $\mathrm{y}_{\mathrm{i}}$ negative affect, psychotic experiences or positive affect as outcome variable):

$y_{i j}=\beta_{0}+\beta_{1}\left(\right.$ STRESS $\left._{i j}\right)+\beta_{2}\left(\right.$ TIME $\left._{j}\right)+\beta_{3}\left(\right.$ STRESS $_{\mathrm{ij}} \times$ TIME $\left._{\mathrm{j}}\right)+\varepsilon_{\mathrm{ij}}$. 
Table 6. Initial therapeutic effects of EMlcompass on individuals' momentary stress, negative affect, psychotic experiences, positive affect, and threat anticipation

\begin{tabular}{lccccccc}
\hline & Baseline & $\begin{array}{c}\text { Post- } \\
\text { inter- } \\
\text { vention }\end{array}$ & $\begin{array}{c}\text { Follow- } \\
\text { up }\end{array}$ & $\begin{array}{c}\text { Post-intervention } \\
\text { vs. baseline }\end{array}$ & \multicolumn{2}{c}{$\begin{array}{c}\text { Follow-up } \\
\text { vs. baseline }\end{array}$} \\
\cline { 2 - 9 } & $\begin{array}{c}\text { Mean } \\
\text { (S.D.) }\end{array}$ & $\begin{array}{c}\text { Mean } \\
\text { (S.D.) }\end{array}$ & $\begin{array}{c}\text { Mean } \\
\text { (S.D.) }\end{array}$ & $\begin{array}{c}\text { B } \\
(\mathbf{9 5 \% ~ C l )}\end{array}$ & $\mathbf{p}$ & $\begin{array}{c}\text { B } \\
(\mathbf{9 5 \%} \text { Cl) }\end{array}$ & $\mathbf{p}$ \\
\hline Positive & 3.9 & 4.5 & 4.3 & 0.39 & 0.001 & 0.31 & 0.004 \\
affect & $(1.8)$ & $(1.5)$ & $(1.6)$ & $(0.16-0.62)$ & & $(0.10-0.52)$ & \\
\hline Negative & 2.2 & 1.8 & 1.4 & -0.44 & $<0.001$ & -0.59 & $<0.001$ \\
affect & $(1.3)$ & $(1.1)$ & $(0.7)$ & $(-0.59--0.30)$ & & $(-0.72--0.46)$ & \\
\hline Psychotic & 1.7 & 1.4 & 1.3 & -0.25 & $<0.001$ & -0.36 & $<0.001$ \\
experiences & $(0.8)$ & $(0.9)$ & $(0.6)$ & $(-0.34--0.16)$ & & $(-0.44--0.28)$ & \\
\hline Threat & 2.7 & 2.2 & 1.6 & -0.61 & $<0.001$ & -0.96 & $<0.001$ \\
anticipation & $(1.9)$ & $(1.3)$ & $(1.1)$ & $(-0.83--0.39)$ & & $(-1.15--0.76)$ & \\
\hline
\end{tabular}

Notes: S.D., standard deviation, $\mathrm{Cl}$, confidence interval

\section{Psychotic, depressive, and anxiety symptoms as well as general psychopathology}

Findings on the initial therapeutic effects of EMIcompass on non-EMA outcome measures are presented in Table 7. Overall, reductions in threat anticipation, psychotic, depressive, and anxiety symptoms as well as general psychopathology (as indexed by the GSI) of moderate to large effect size were found at the end of the 3-week intervention period ('post-intervention'), and after a 4-week follow-up period ( $r=0.30-0.65)$. There was initial evidence, despite small sample size and, hence, limited statistical power, that these reductions were beyond what would be expected by chance alone for psychotic symptoms at post-intervention and 4-week follow-up as well as, at trend level, for anxiety symptoms (post-intervention, 4-week follow-up) and anticipation of a positive future self (4-week follow-up). The intervention effects on depressive symptoms and general psychopathology were also of medium to large effect size but fell short of statistical significance. Reductions in threat anticipation (self, other) were only of small to moderate effect size and did not reach conventional levels of statistical significance. 
Table 7. Initial therapeutic effects of EMlcompass intervention on psychotic, depressive and anxiety symptoms, general psychopathology, and threat anticipation

\begin{tabular}{|c|c|c|c|c|c|c|c|c|c|}
\hline & \multirow{3}{*}{ Baseline } & \multicolumn{2}{|c|}{$\begin{array}{l}\text { edian scores } \\
\text { (range) }\end{array}$} & \multicolumn{6}{|c|}{$\begin{array}{l}\text { Paired Wilcoxon signed ranks test } \\
(n=10)\end{array}$} \\
\hline & & \multirow{2}{*}{$\begin{array}{c}\text { Post- } \\
\text { inter- } \\
\text { vention }\end{array}$} & \multirow{2}{*}{$\begin{array}{c}\text { Follow } \\
\text {-up }\end{array}$} & \multicolumn{2}{|c|}{$\begin{array}{c}\text { Post- } \\
\text { intervention } \\
\text { vs. baseline }\end{array}$} & \multicolumn{2}{|c|}{$\begin{array}{l}\text { Follow- } \\
\text { up vs. } \\
\text { baseline }\end{array}$} & \multicolumn{2}{|c|}{$\begin{array}{l}\text { Follow-up } \\
\text { vs. post- } \\
\text { intervention }\end{array}$} \\
\hline & & & & $\mathbf{Z}$ & $\begin{array}{l}\text { Effect } \\
\text { size (ra) }\end{array}$ & Z & $\begin{array}{c}\text { Effect } \\
\text { size (ra) }\end{array}$ & $\mathbf{Z}$ & $\begin{array}{l}\text { Effect } \\
\text { size (ra) }\end{array}$ \\
\hline \multicolumn{10}{|l|}{$\overline{B S I}$} \\
\hline $\begin{array}{l}\text { Global } \\
\text { Severity } \\
\text { Index }\end{array}$ & $\begin{array}{c}81 \\
(22-146)\end{array}$ & $\begin{array}{c}68.5 \\
(5-158)\end{array}$ & $\begin{array}{c}51 \\
(7-142)\end{array}$ & -1.02 & -0.32 & -1.17 & -0.37 & -1.53 & -0.48 \\
\hline Depression & $\begin{array}{c}13.5 \\
(1-23)\end{array}$ & $\begin{array}{c}12 \\
(0-23)\end{array}$ & $\begin{array}{c}7 \\
(1-21)\end{array}$ & -1.02 & -0.33 & -1.03 & -0.33 & -1.38 & -0.44 \\
\hline Anxiety & $\begin{array}{c}11.5 \\
(4-16)\end{array}$ & $\begin{array}{c}9.5 \\
(0-17)\end{array}$ & $\begin{array}{c}7 \\
(2-14)\end{array}$ & -1.74 & $-0.55 t$ & -1.79 & $-0.57 \dagger$ & -0.82 & -0.26 \\
\hline \multicolumn{10}{|l|}{ GPTS } \\
\hline Total score & $\begin{array}{c}41 \\
(32-73)\end{array}$ & $\begin{array}{c}46.5 \\
(32-83)\end{array}$ & $\begin{array}{c}38 \\
(32-70)\end{array}$ & 1.94 & 0.61 * & -1.74 & $-0.55 t$ & -2.50 & $-0.79 *$ \\
\hline \multicolumn{10}{|l|}{$P Q$} \\
\hline Total score & $\begin{array}{c}5 \\
(1-10)\end{array}$ & $\begin{array}{c}5 \\
(0-9)\end{array}$ & $\begin{array}{c}2 \\
(0-10)\end{array}$ & -1.32 & -0.42 & -2.05 & $-0.65^{\star}$ & -1.34 & -0.42 \\
\hline \multicolumn{10}{|l|}{ TAM } \\
\hline $\begin{array}{l}\text { Future self } \\
\text { (positive) }\end{array}$ & $\begin{array}{c}26.5 \\
(17-37)\end{array}$ & $\begin{array}{c}27 \\
(16-37)\end{array}$ & $\begin{array}{c}33 \\
(7-42)\end{array}$ & 0.41 & 0.13 & 1.89 & $0.60 \dagger$ & 1.79 & $0.57 \dagger$ \\
\hline $\begin{array}{l}\text { Future self } \\
\text { (threatening) }\end{array}$ & $\begin{array}{c}15.5 \\
(11-25)\end{array}$ & $\begin{array}{c}16.5 \\
(7-24)\end{array}$ & $\begin{array}{c}13 \\
(7-34)\end{array}$ & -0.46 & -0.15 & -1.28 & -0.40 & -0.52 & -0.16 \\
\hline $\begin{array}{l}\text { Future others } \\
\text { (positive) }\end{array}$ & $\begin{array}{c}31.5 \\
(19-45)\end{array}$ & $\begin{array}{c}31 \\
(27-42)\end{array}$ & $\begin{array}{c}33.5 \\
(22-44)\end{array}$ & 0.21 & 0.07 & 1.33 & 0.42 & 1.74 & $0.55 t$ \\
\hline $\begin{array}{l}\text { Future others } \\
\text { (threatening) }\end{array}$ & s $\begin{array}{c}15.5 \\
(7-37)\end{array}$ & $\begin{array}{c}14 \\
(8-36)\end{array}$ & $\begin{array}{c}13.5 \\
(7-32)\end{array}$ & -0.78 & -0.25 & -0.77 & -0.24 & -0.21 & -0.07 \\
\hline
\end{tabular}

Notes: $t p<0.10$; $*$ p 0.05; BSI, Brief Symptom Inventory; GBTS, Green et al. Paranoid Thoughts Scale; PQ, Prodromal Questionnaire; IPSM, Interpersonal Sensitivity Measure; TAM, Threat Anticipation Measure

a effect size estimates are based on $r$ described by Rosenthal, 2001 using the following formula: $r=Z / \sqrt{ }$ number of pairs. 


\section{Discussion}

\section{Principal findings}

Findings of this uncontrolled phase I pilot study suggest initial findings on feasibility, safety, and preliminary therapeutic effects of a compassion-focused ecological momentary transdiagnostic intervention designed to improve emotional resilience to stress ('EMlcompass') in help-seeking youth with psychotic, depressive, and/or anxiety symptoms. First, individuals were satisfied with faceto-face and app-based intervention components, interference with daily activities was low, and observer-rated compliance with the treatment was high. Indicators of app usability were satisfactory. Also, no adverse effects were reported. Second, there was preliminary evidence of decreased stress sensitivity, negative affect, and psychotic experiences as well as increased positive affect in daily life at the end of the 3-week intervention period ('post-intervention'), and after a 4-week followup period ('follow-up') as compared to baseline. Third, there was initial evidence, despite the small sample size and limited statistical power, for reductions in threat anticipation, psychotic, anxiety, and depressive symptoms of medium to large effect size ( $r=0.30-0.65)$. Overall, this reflects promising preliminary evidence of clinical feasibility and safety of the EMIcompass intervention in help-seeking youth and some evidence on initial therapeutic effects, although findings on clinical outcomes should be interpreted with caution considering the small sample size of this pilot study.

\section{Strengths and limitations}

The strength of the current study is that principles of CFIs were, for the first time, translated into an EMI administered through an mHealth app as a new avenue for real-world and real-time prevention and intervention in youth. Further, EMlcompass transforms evidence on putative underlying mechanisms into an intervention that directly targets these mechanisms in daily life and, hence, is, at heart, translational. However, there are a number of limitations that have to be considered in interpreting our findings. First, in line with state-of-the-art guidance on developing and evaluating complex interventions [103], mHealth interventions in particular [104], the sample size $(\mathrm{N}=10)$ of this pilot study was selected to be small. Thus, the primary focus of this study was on investigating feasibility and safety as well as estimating the effect size of initial therapeutic effects rather than 
statistical significance to provide the basis for a feasibility randomised controlled trial (RCT) [105]. Nonetheless, while considering low statistical power and limitations associated with a small sample size, we found preliminary evidence (in terms of statistical significance) on effects of the EMlcompass intervention on stress sensitivity. These are promising findings as stress sensitivity is the primary target of this emotion regulation-focused intervention. Second, data on feasibility and acceptability were assessed together with or by the trained psychologist and not an independent person. Thus, we cannot rule out biases and underreporting of unhelpful experiences. Third, we used a debriefing scale to assess domains of feasibility. Thus, no established measure was used for classifying and assessing the quality of the EMIcompass app (e.g., MARS which is used to assess app engagement, functionality, aesthetics, and information quality) which limits reported findings. Fourth, due to the absence of a waiting-list or active control group, we cannot rule out that there may be no additive therapeutic effects of the EMIcompass intervention over and above the therapeutic effects of the faceto-face sessions with the trained psychologists or other therapeutic interventions participants received during the intervention period in form of treatment as usual. However, again, the primary aim of this pragmatic phase I pilot study was to provide the basis of a feasibility RCT by investigating feasibility and safety as well as generating initial effect sizes. It is now urgently warranted to further examine the efficacy of the EMIcompass intervention. Third, the majority of participants were female, and half of participants suffered from depression which may limit generalisability of findings as selection bias may have operated on our sampling procedure. Fifth, after written informed consent was obtained and baseline assessments were completed, five individuals decided not to participate in the study. The reasons for exclusion were not assessed which limits our findings on feasibility. Finally, the complex nature of investigated constructs, sample size, and study design exclude any form of causal inference.

\section{Ideas for future work}

The EMIcompass intervention aimed to augment current treatment options for young individuals seeking help for mental health problems. Most individuals reported to be satisfied with the intervention. While the small sample size has to be considered when interpreting findings, the preliminary therapeutic effects on candidate psychological mechanisms, including stress sensitivity, and on other 
psychopathological outcomes were promising. Importantly, no adverse effects have been reported and taking part in the study did not hinder individuals in their daily activities. Thus, overall, findings on feasibility, safety, and initial therapeutic effects may be considered to be encouraging.

This is one of the first studies to develop and pilot an ecological momentary intervention that incorporates an adaptive and context-dependent delivery scheme of intervention components in youth with mental health problems. The 'interactive-tasks' were triggered in around 1/3 of all EMA assessments when individuals experienced elevated levels of negative affect (e.g. feeling anxious, insecure, down; i.e. scores higher than 4 on a 7-point Likert scale) or momentary stress. Thus, real-time data processing was successfully applied based on EMA data to determine delivery of compassion-focused intervention components. This may represent not only an important step towards ecologically more valid and accessible psychological interventions in youth, but also a more personalised and contextualised clinical and preventive approach. In other words, principles of EMIs allow not only to translate intervention components targeting candidate momentary mechanisms and contexts to individuals' daily lives, but take also a personalized, adaptive approach informed by fine-grained real-time EMA data to produce sustainable change in the real world. Although a feasibility RCT is needed as a significant next step to investigate the efficacy of the intervention and feasibility as a basis for a confirmatory RCT [23, 34], the current pilot study of this novel EMI reflects an important steppingstone towards more personalized and accessible youth mental health care. Furthermore, in-app data analytics revealed a high variability in compliance between individuals. This suggests, that for some individuals the number of signals per day were too high (i.e., seven times per day on six consecutive days per week).

These findings hint towards potential avenues for improvement of the EMIcompass intervention to be iteratively incorporated. First, future versions of the EMIcompass intervention may offer adaptive intervention trajectories that vary in type of exercises depending on individual needs and preferences. Importantly, in doing so, potentially influencing factors (e.g. educational level, language skills, cultural peculiarities, subjective preferences) should be considered at an early stage of the design process and taken into account in optimizing EMIs further. Co-production with young service users is essential during these developmental processes 
[106]. Second, sustained engagement in using digital tools remains an important challenge [107], which may be addressed through the use of gamification elements, especially in youth [108, 109]. However, in the current study, burden associated with app usage was low so far and problems with engagement have mainly been reported for standalone mHealth apps without components of blended care [110]. Third, in working towards more personalised mHealth apps, more sophisticated methods may be used to inform the timing and context of when intervention components are offered (e.g. by using mobile sensing data) and a broader range of intervention components delivered over a longer intervention period may help enhance effects of EMIcompass further and achieve sustainable change in individuals' daily life. Fourth, the type of intervention components may be personalised further by assessing effects of specific intervention components on individuals' mental health at the person-level. Fifth, it should be further examined whether and, if so, how the therapeutic alliance can be strengthened in the light of a limited number of face-to face sessions [111]. Finally, the number of signals per day triggered by the smartphone were perceived as burdensome by some participants. Thus, future versions of the EMIcompass app may lower the number of signals per day and/or shortening the number of items per signal [112].

\section{Conclusions}

Taken together, evidence on feasibility and safety as well as preliminary evidence on therapeutic effects of the EMlcompass intervention suggest that translating compassion-focused intervention components into individuals' daily life through an EMI delivered by an mHealth app may be a promising novel, accessible, and transdiagnostic treatment approach in help-seeking youth by strengthening emotional resilience and directly targeting candidate psychological mechanisms. As an important next step, an exploratory randomised controlled trial is warranted to demonstrate feasibility and preliminary evidence of efficacy of the EMIcompass intervention. 


\section{References}

1. Kessler RC, Berglund P, Demler O, Jin R, Merikangas KR, Walters EE. Lifetime prevalence and age-of-onset distributions of DSM-IV disorders in the National Comorbidity Survey Replication. Arch Gen Psychiatry. 2005 Jun;62(6):593-602.

2. Beesdo-Baum K, Knappe S, Asselmann E, Zimmermann P, Bruckl T, Hofler M, et al. The 'Early Developmental Stages of Psychopathology (EDSP) study': a 20-year review of methods and findings. Soc Psychiatry Psychiatr Epidemiol. 2015 Jun;50(6):851-66.

3. Perala J, Suvisaari J, Saarni SI, Kuoppasalmi K, Isometsa E, Pirkola S, et al. Lifetime prevalence of psychotic and bipolar I disorders in a general population. Arch Gen Psychiatry. 2007 Jan;64(1):19-28.

4. Merikangas KR, HeJP, Burstein M, Swanson SA, Avenevoli S, Cui L, et al. Lifetime prevalence of mental disorders in U.S. adolescents: results from the National Comorbidity Survey Replication--Adolescent Supplement (NCS-A). J Am Acad Child Adolesc Psychiatry. 2010 Oct;49(10):980-9.

5. Ormel J, Raven D, van Oort F, Hartman CA, Reijneveld SA, Veenstra R, et al. Mental health in Dutch adolescents: a TRAILS report on prevalence, severity, age of onset, continuity and co-morbidity of DSM disorders. Psychol Med. 2015 Jan;45(2):345-60.

6. Harhay $\mathrm{MO}$, King $\mathrm{CH}$. Global burden of disease in young people aged 10-24 years. Lancet. 2012:379(9810):27-8; author reply 8. PMID: 22225664. doi: 10.1016/S01406736(12)60019-1.

7. Erskine HE, Moffitt TE, Copeland WE, Costello EJ, Ferrari AJ, Patton G, et al. A heavy burden on young minds: the global burden of mental and substance use disorders in children and youth. Psychol Med. 2015 May;45(7):1551-63.

8. Gore FM, Bloem PJ, Patton GC, Ferguson J, Joseph V, Coffey C, et al. Global burden of disease in young people aged 10-24 years: a systematic analysis. Lancet. 2011 Jun 18;377(9783):2093-102.

9. van Os J. The dynamics of subthreshold psychopathology: implications for diagnosis and treatment. Am J Psychiatry. 2013 Jul;170(7):695-8.

10. van Os J, Reininghaus U. Psychosis as a transdiagnostic and extended phenotype in the general population. World Psychiatry. 2016 Jun;15(2):118-24.

11. Reininghaus $U$, Priebe $S$, Bentall RP. Testing the psychopathology of psychosis: evidence for a general psychosis dimension. Schizophr Bull. 2013 Jul;39(4):884-95.

12. Reininghaus U, Bohnke JR, Hosang G, Farmer A, Burns T, McGuffin P, et al. Evaluation of the validity and utility of a transdiagnostic psychosis dimension encompassing schizophrenia and bipolar disorder. Br J Psychiatry. 2016 Aug;209(2):107-13.

13. Reininghaus $U$, Bohnke JR, Chavez-Baldini $U$, Gibbons R, Ivleva E, Clementz BA, et al. Transdiagnostic dimensions of psychosis in the Bipolar-Schizophrenia Network on Intermediate Phenotypes (B-SNIP). World Psychiatry. 2019 Feb;18(1):67-76.

14. Kotov R, Jonas KG, Carpenter WT, Dretsch MN, Eaton NR, Forbes MK, et al. Validity and utility of Hierarchical Taxonomy of Psychopathology (HiTOP): I. Psychosis superspectrum. World Psychiatry. 2020 Jun;19(2):151-72.

15. Krueger RF, Kotov R, Watson D, Forbes MK, Eaton NR, Ruggero CJ, et al. Progress in achieving quantitative classification of psychopathology. World Psychiatry. 2018 Oct;17(3):282-93. 
16. Rutigliano G, Valmaggia L, Landi P, Frascarelli M, Cappucciati M, Sear V, et al. Persistence or recurrence of non-psychotic comorbid mental disorders associated with 6-year poor functional outcomes in patients at ultra high risk for psychosis. J Affect Disord. 2016 Oct;203:101-10.

17. Iorfino F, Scott EM, Carpenter JS, Cross SP, Hermens DF, Killedar M, et al. Clinical Stage Transitions in Persons Aged 12 to 25 Years Presenting to Early Intervention Mental Health Services With Anxiety, Mood, and Psychotic Disorders. JAMA Psychiatry. 2019 Nov 1;76(11):1167-75.

18. Hickie IB, Scott EM, Hermens DF, Naismith SL, Guastella AJ, Kaur M, et al. Applying clinical staging to young people who present for mental health care. Early Interv Psychiatry. 2013 Feb;7(1):31-43.

19. Hartmann JA, Nelson B, Spooner R, Paul Amminger G, Chanen A, Davey CG, et al. Broad clinical high-risk mental state (CHARMS): Methodology of a cohort study validating criteria for pluripotent risk. Early Interv Psychiatry. 2019 Jun;13(3):379-86.

20. McGorry PD, HartmannJA, Spooner R, Nelson B. Beyond the "at risk mental state" concept: transitioning to transdiagnostic psychiatry. World Psychiatry. 2018 Jun;17(2):133-42.

21. Rauschenberg C, van Os J, Goedhart M, Schieveld JNM, Reininghaus U. Bullying victimization and stress sensitivity in help-seeking youth: findings from an experience sampling study. Eur Child Adolesc Psychiatry. 2020 May 13.

22. Rauschenberg C, van Os J, Cremers D, Goedhart M, Schieveld JNM, Reininghaus U. Stress sensitivity as a putative mechanism linking childhood trauma and psychopathology in youth's daily life. Acta Psychiatr Scand. 2017 Oct;136(4):373-88.

23. Reininghaus U, Depp CA, Myin-Germeys I. Ecological Interventionist Causal Models in Psychosis: Targeting Psychological Mechanisms in Daily Life. Schizophr Bull. 2016 Mar;42(2):264-9.

24. Reininghaus U, Kempton MJ, Valmaggia L, Craig TK, Garety P, Onyejiaka A, et al. Stress Sensitivity, Aberrant Salience, and Threat Anticipation in Early Psychosis: An Experience Sampling Study. Schizophr Bull. 2016 May;42(3):712-22.

25. Quattrone D, Di Forti M, Gayer-Anderson C, Ferraro L, Jongsma HE, Tripoli G, et al. Transdiagnostic dimensions of psychopathology at first episode psychosis: findings from the multinational EU-GEl study. Psychol Med. 2019 Jun;49(8):1378-91.

26. European Network of National Networks studying Gene-Environment Interactions in S, van Os J, Rutten BP, Myin-Germeys I, Delespaul P, Viechtbauer W, et al. Identifying geneenvironment interactions in schizophrenia: contemporary challenges for integrated, large-scale investigations. Schizophr Bull. 2014 Jul;40(4):729-36.

27. Morgan C, Reininghaus U, Fearon P, Hutchinson G, Morgan K, Dazzan P, et al. Modelling the interplay between childhood and adult adversity in pathways to psychosis: initial evidence from the AESOP study. Psychol Med. 2014 Jan;44(2):407-19.

28. Klippel A, Myin-Germeys I, Chavez-Baldini U, Preacher KJ, Kempton M, Valmaggia L, et al. Modeling the Interplay Between Psychological Processes and Adverse, Stressful Contexts and Experiences in Pathways to Psychosis: An Experience Sampling Study. Schizophr Bull. 2017 Mar 1;43(2):302-15.

29. Reininghaus U, Gayer-Anderson C, Valmaggia L, Kempton MJ, Calem M, Onyejiaka A, et al. Psychological processes underlying the association between childhood trauma and psychosis in daily life: an experience sampling study. Psychol Med. 2016 Oct;46(13):2799813. 
30. Reininghaus U, Oorschot M, Moritz S, Gayer-Anderson C, Kempton MJ, Valmaggia L, et al. Liberal Acceptance Bias, Momentary Aberrant Salience, and Psychosis: An Experimental Experience Sampling Study. Schizophr Bull. 2019 Jun 18;45(4):871-82.

31. Hermans K, Myin-Germeys I, Gayer-Anderson C, Kempton MJ, Valmaggia L, McGuire P, et al. Elucidating negative symptoms in the daily life of individuals in the early stages of psychosis. Psychol Med. 2020 May 22:1-11.

32. Malla A, lyer S, McGorry P, Cannon M, Coughlan H, Singh S, et al. From early intervention in psychosis to youth mental health reform: a review of the evolution and transformation of mental health services for young people. Soc Psychiatry Psychiatr Epidemiol. 2016 Mar;51(3):319-26.

33. McGorry $\mathrm{P}$, Bates $\mathrm{T}$, Birchwood M. Designing youth mental health services for the 21st century: examples from Australia, Ireland and the UK. Br J Psychiatry Suppl. 2013 Jan;54:s30-5.

34. Reininghaus $U$, Klippel $A$, Steinhart $H$, Vaessen $T$, van Nierop $M$, Viechtbauer $W$, et al. Efficacy of Acceptance and Commitment Therapy in Daily Life (ACT-DL) in early psychosis: study protocol for a multi-centre randomized controlled trial. Trials. 2019 Dec 26;20(1):769.

35. van Aubel E, Bakker JM, Batink T, Michielse S, Goossens L, Lange I, et al. Blended care in the treatment of subthreshold symptoms of depression and psychosis in emerging adults: A randomised controlled trial of Acceptance and Commitment Therapy in DailyLife (ACT-DL). Behav Res Ther. 2020 May;128:103592.

36. Vaessen T, Steinhart H, Batink T, Klippel A, Van Nierop M, Reininghaus U, et al. ACT in daily life in early psychosis: an ecological momentary intervention approach. Psychosis. 2019 Apr 3;11(2):93-104.

37. Bhugra D, Tasman A, Pathare S, Priebe S, Smith S, Torous J, et al. The WPA-Lancet Psychiatry Commission on the Future of Psychiatry. Lancet Psychiatry. 2017 Oct;4(10):775-818.

38. Reininghaus U. [Ecological Momentary Interventions in Psychiatry: The Momentum for Change in Daily Social Context]. Psychiatr Prax. 2018 Mar;45(2):59-61. PMID: 29495051. doi: 10.1055/s-0044-101986.

39. Myin-Germeys I, Klippel A, Steinhart H, Reininghaus U. Ecological momentary interventions in psychiatry. Curr Opin Psychiatry. 2016 Jul;29(4):258-63.

40. Miralles I, Granell C, Diaz-Sanahuja L, Van Woensel W, Breton-Lopez J, Mira A, et al. Smartphone Apps for the Treatment of Mental Disorders: Systematic Review. JMIR Mhealth Uhealth. 2020 Apr 2;8(4):e14897.

41. McKay FH, Wright A, Shill J, Stephens H, Uccellini M. Using Health and Well-Being Apps for Behavior Change: A Systematic Search and Rating of Apps. JMIR Mhealth Uhealth. 2019 Jul 4;7(7):e11926.

42. Seppala J, De Vita I, Jamsa T, Miettunen J, Isohanni M, Rubinstein K, et al. Mobile Phone and Wearable Sensor-Based mHealth Approaches for Psychiatric Disorders and Symptoms: Systematic Review. JMIR Ment Health. 2019 Feb 20;6(2):e9819.

43. Myin-Germeys I, Kasanova Z, Vaessen T, Vachon H, Kirtley O, Viechtbauer W, et al. Experience sampling methodology in mental health research: new insights and technical developments. World Psychiatry. 2018 Jun;17(2):123-32. 
44. Batink T, Bakker J, Vaessen T, Kasanova Z, Collip D, van Os J, et al. Acceptance and Commitment Therapy in Daily Life Training: A Feasibility Study of an mHealth Intervention. JMIR Mhealth Uhealth. 2016 Sep 15;4(3):e103.

45. Heron KE, Smyth JM. Ecological momentary interventions: incorporating mobile technology into psychosocial and health behaviour treatments. $\mathrm{Br} J$ Health Psychol. 2010 Feb;15(Pt 1):1-39.

46. Myin-Germeys I, Birchwood M, Kwapil T. From environment to therapy in psychosis: a real-world momentary assessment approach. Schizophr Bull. 2011 Mar;37(2):244-7.

47. Nahum-Shani I, Smith SN, Spring BJ, Collins LM, Witkiewitz K, Tewari A, et al. Just-in-Time Adaptive Interventions (IITAIs) in Mobile Health: Key Components and Design Principles for Ongoing Health Behavior Support. Ann Behav Med. 2018 May 18;52(6):446-62.

48. Bidargaddi N, Schrader G, Klasnja P, Licinio J, Murphy S. Designing m-Health interventions for precision mental health support. Transl Psychiatry. 2020 Jul 7;10(1):222.

49. Myin-Germeys I, Oorschot M, Collip D, Lataster J, Delespaul P, van Os J. Experience sampling research in psychopathology: opening the black box of daily life. Psychol Med. 2009 Sep;39(9):1533-47.

50. Oorschot M, Kwapil T, Delespaul P, Myin-Germeys I. Momentary assessment research in psychosis. Psychol Assess. 2009 Dec;21(4):498-505.

51. Collip D, Nicolson NA, Lardinois M, Lataster T, van Os J, Myin-Germeys I, et al. Daily cortisol, stress reactivity and psychotic experiences in individuals at above average genetic risk for psychosis. Psychol Med. 2011 Nov;41(11):2305-15.

52. Lataster $T$, Wichers $M$, Jacobs N, Mengelers R, Derom C, Thiery E, et al. Does reactivity to stress cosegregate with subclinical psychosis? A general population twin study. Acta Psychiatr Scand. 2009 Jan;119(1):45-53.

53. Myin-Germeys I, van Os J. Stress-reactivity in psychosis: evidence for an affective pathway to psychosis. Clin Psychol Rev. 2007 May;27(4):409-24.

54. Myin-Germeys I, van Os J, Schwartz JE, Stone AA, Delespaul PA. Emotional reactivity to daily life stress in psychosis. Arch Gen Psychiatry. 2001 Dec;58(12):1137-44.

55. Palmier-Claus JE, Dunn G, Lewis SW. Emotional and symptomatic reactivity to stress in individuals at ultra-high risk of developing psychosis. Psychol Med. 2012 May;42(5):100312.

56. Wichers M, Schrijvers D, Geschwind N, Jacobs N, Myin-Germeys I, Thiery E, et al. Mechanisms of gene-environment interactions in depression: evidence that genes potentiate multiple sources of adversity. Psychol Med. 2009 Jul;39(7):1077-86.

57. Vaessen T, Kasanova Z, Hernaus D, Lataster J, Collip D, van Nierop M, et al. Overall cortisol, diurnal slope, and stress reactivity in psychosis: An experience sampling approach. Psychoneuroendocrinology. 2018 Oct;96:61-8.

58. Klippel A, Viechtbauer W, Reininghaus U, Wigman J, van Borkulo C, Merge, et al. The Cascade of Stress: A Network Approach to Explore Differential Dynamics in Populations Varying in Risk for Psychosis. Schizophr Bull. 2018 Feb 15;44(2):328-37.

59. Bell V, Freeman D. A pilot trial of cognitive behavioural therapy for interpersonal sensitivity in individuals with persecutory delusions. J Behav Ther Exp Psychiatry. 2014 Dec;45(4):441-6. 
60. Freeman D, Pugh K, Vorontsova N, Antley A, Slater M. Testing the continuum of delusional beliefs: an experimental study using virtual reality. J Abnorm Psychol. 2010 Feb;119(1):83-92.

61. Masillo A, Day F, Laing J, Howes O, Fusar-Poli P, Byrne M, et al. Interpersonal sensitivity in the at-risk mental state for psychosis. Psychol Med. 2012 Sep;42(9):1835-45.

62. Freeman D, Dunn G, Fowler D, Bebbington P, Kuipers E, Emsley R, et al. Current paranoid thinking in patients with delusions: the presence of cognitive-affective biases. Schizophr Bull. 2013 Nov;39(6):1281-7.

63. Harb GC, Heimberg RG, Fresco DM, Schneier FR, Liebowitz MR. The psychometric properties of the Interpersonal Sensitivity Measure in social anxiety disorder. Behav Res Ther. 2002 Aug;40(8):961-79.

64. Wilhelm K, Boyce P, Brownhill S. The relationship between interpersonal sensitivity, anxiety disorders and major depression. J Affect Disord. 2004 Apr;79(1-3):33-41.

65. Bentall RP, de Sousa P, Varese F, Wickham S, Sitko K, Haarmans M, et al. From adversity to psychosis: pathways and mechanisms from specific adversities to specific symptoms. Soc Psychiatry Psychiatr Epidemiol. 2014 Jul;49(7):1011-22.

66. Freeman D. Persecutory delusions: a cognitive perspective on understanding and treatment. Lancet Psychiatry. 2016 Jul;3(7):685-92.

67. Gilbert P. Introducing compassion-focused therapy. Advances in Psychiatric Treatment. 2009;15(3):199-208.

68. Gilbert P. Compassion Focused Therapy (CBT Distinctive Features). London; New York: Routledge; 2010.

69. Hunot V, Moore TH, Caldwell DM, Furukawa TA, Davies P, Jones $H$, et al. 'Third wave' cognitive and behavioural therapies versus other psychological therapies for depression. Cochrane Database Syst Rev. 2013 Oct 18(10):CD008704.

70. Johns LC, Oliver JE, Khondoker M, Byrne M, Jolley S, Wykes T, et al. The feasibility and acceptability of a brief Acceptance and Commitment Therapy (ACT) group intervention for people with psychosis: The 'ACT for life' study. J Behav Ther Exp Psychiatry. 2016 Mar;50:257-63.

71. Shawyer F, Farhall J, Thomas N, Hayes SC, Gallop R, Copolov D, et al. Acceptance and commitment therapy for psychosis: randomised controlled trial. Br J Psychiatry. 2017 Feb;210(2):140-8.

72. Tai S, Turkington D. The evolution of cognitive behavior therapy for schizophrenia: current practice and recent developments. Schizophr Bull. 2009 Sep;35(5):865-73.

73. Kirby JN, Tellegen CL, Steindl SR. A Meta-Analysis of Compassion-Based Interventions: Current State of Knowledge and Future Directions. Behav Ther. 2017 Nov;48(6):778-92.

74. Ascone L, Sundag J, Schlier B, Lincoln TM. Feasibility and Effects of a Brief CompassionFocused Imagery Intervention in Psychotic Patients with Paranoid Ideation: A Randomized Experimental Pilot Study. Clin Psychol Psychother. 2017 Mar;24(2):348-58.

75. Heriot-Maitland C, McCarthy-Jones S, Longden E, Gilbert P. Compassion Focused Approaches to Working With Distressing Voices. Front Psychol. 2019;10:152.

76. Leaviss J, Uttley L. Psychotherapeutic benefits of compassion-focused therapy: an early systematic review. Psychol Med. 2015 Apr;45(5):927-45. 
77. Cuppage J, Baird K, Gibson J, Booth R, Hevey D. Compassion focused therapy: Exploring the effectiveness with a transdiagnostic group and potential processes of change. $\mathrm{Br} J$ Clin Psychol. 2018 Jun;57(2):240-54.

78. Lincoln TM, Hohenhaus F, Hartmann M. Can Paranoid Thoughts be Reduced by Targeting Negative Emotions and Self-Esteem? An Experimental Investigation of a Brief Compassion-Focused Intervention. Cogn Ther Res. 2013;37:390-402.

79. Pearson J, Naselaris T, Holmes EA, Kosslyn SM. Mental Imagery: Functional Mechanisms and Clinical Applications. Trends Cogn Sci. 2015 Oct;19(10):590-602.

80. Holmes EA, Mathews A. Mental imagery in emotion and emotional disorders. Clin Psychol Rev. 2010 Apr;30(3):349-62.

81. Braehler C, Gumley A, Harper J, Wallace S, Norrie J, Gilbert P. Exploring change processes in compassion focused therapy in psychosis: results of a feasibility randomized controlled trial. Br J Clin Psychol. 2013 Jun;52(2):199-214.

82. Holmes EA, Blackwell SE, Burnett Heyes S, Renner F, Raes F. Mental Imagery in Depression: Phenomenology, Potential Mechanisms, and Treatment Implications. Annu Rev Clin Psychol. 2016;12:249-80.

83. Blackwell SE, Holmes EA. Brightening the Day With Flashes of Positive Mental Imagery: A Case Study of an Individual With Depression. J Clin Psychol. 2017 May;73(5):579-89.

84. Renner F, Ji JL, Pictet A, Holmes EA, Blackwell SE. Effects of Engaging in Repeated Mental Imagery of Future Positive Events on Behavioural Activation in Individuals with Major Depressive Disorder. Cognit Ther Res. 2017;41(3):369-80.

85. Galante J, Dufour G, Vainre M, Wagner AP, Stochl J, Benton A, et al. A mindfulnessbased intervention to increase resilience to stress in university students (the Mindful Student Study): a pragmatic randomised controlled trial. Lancet Public Health. 2018 Feb;3(2):e72-e81. PMID: 29422189. doi: 10.1016/S2468-2667(17)30231-1.

86. Linardon J. Can Acceptance, Mindfulness, and Self-Compassion Be Learned by Smartphone Apps? A Systematic and Meta-Analytic Review of Randomized Controlled Trials. Behav Ther. 2020 Jul;51(4):646-58.

87. Hotopf M. The pragmatic randomised controlled trial. Advances in Psychiatric Treatment. 2018;8(5):326-33.

88. Loewy RL, Bearden CE, Johnson JK, Raine A, Cannon TD. The prodromal questionnaire $(\mathrm{PQ})$ : preliminary validation of a self-report screening measure for prodromal and psychotic syndromes. Schizophr Res. 2005 Nov 1;79(1):117-25.

89. Ising HK, Veling W, Loewy RL, Rietveld MW, Rietdijk J, Dragt S, et al. The validity of the 16-item version of the Prodromal Questionnaire (PQ-16) to screen for ultra high risk of developing psychosis in the general help-seeking population. Schizophr Bull. 2012 Nov;38(6):1288-96.

90. Savill M, D'Ambrosio J, Cannon TD, Loewy RL. Psychosis risk screening in different populations using the Prodromal Questionnaire: A systematic review. Early Interv Psychiatry. 2018 Feb;12(1):3-14.

91. Derogatis LR. BSI, Brief Symptom Inventory: Administration, scoring \& procedures manual. Minneapolis, MN: National Computer Systems; 1993.

92. Derogatis LR, Melisaratos N. The Brief Symptom Inventory: an introductory report. Psychol Med. 1983 Aug;13(3):595-605. 
93. Carlier I, Schulte-Van Maaren Y, Wardenaar K, Giltay E, Van Noorden M, Vergeer P, et al. Development and validation of the 48-item Symptom Questionnaire (SQ-48) in patients with depressive, anxiety and somatoform disorders. Psychiatry Res. 2012 Dec 30;200(23):904-10.

94. Leahy RL, Tirch D, Napolitano LA. Emotion Regulation in Psychotherapy: A Practitioner's Guide: Guilford Publications; 2011.

95. Pries LK, Klingenberg B, Menne-Lothmann C, Decoster J, van Winkel R, Collip D, et al. Polygenic liability for schizophrenia and childhood adversity influences daily-life emotion dysregulation and psychosis proneness. Acta Psychiatr Scand. 2020 May;141(5):465-75.

96. Myin-Germeys I, Marcelis M, Krabbendam L, Delespaul P, van Os J. Subtle fluctuations in psychotic phenomena as functional states of abnormal dopamine reactivity in individuals at risk. Biol Psychiatry. 2005 Jul 15;58(2):105-10.

97. Green CE, Freeman D, Kuipers E, Bebbington P, Fowler D, Dunn G, et al. Measuring ideas of persecution and social reference: the Green et al. Paranoid Thought Scales (GPTS). Psychol Med. 2008 Jan;38(1):101-11.

98. Corcoran R, Cummins S, Rowse G, Moore R, Blackwood N, Howard R, et al. Reasoning under uncertainty: heuristic judgments in patients with persecutory delusions or depression. Psychol Med. 2006 Aug;36(8):1109-18.

99. Bentall RP, Rowse G, Shryane N, Kinderman P, Howard R, Blackwood N, et al. The cognitive and affective structure of paranoid delusions: a transdiagnostic investigation of patients with schizophrenia spectrum disorders and depression. Arch Gen Psychiatry. 2009 Mar;66(3):236-47.

100. Boulet J, Boss MW. Reliability and validity of the Brief Symptom Inventory. Psychological Assessment: A Journal of Consulting and Clinical Psychology. 1991;3(3):433-7. doi: 10.1037/1040-3590.3.3.433.

101. Freeman D, Loe BS, Kingdon D, Startup H, Molodynski A, Rosebrock L, et al. The revised Green et al., Paranoid Thoughts Scale (R-GPTS): psychometric properties, severity ranges, and clinical cut-offs. Psychol Med. 2019 Nov 20:1-10.

102. Rosenthal R, DiMatteo MR. Meta-analysis: recent developments in quantitative methods for literature reviews. Annu Rev Psychol. 2001;52:59-82.

103. Anderson R. New MRC guidance on evaluating complex interventions. BMJ. 2008 Oct 22;337:a1937.

104. Collins LM, Murphy SA, Strecher V. The multiphase optimization strategy (MOST) and the sequential multiple assignment randomized trial (SMART): new methods for more potent eHealth interventions. Am J Prev Med. 2007 May;32(5 Suppl):S112-8.

105. Eldridge SM, Lancaster GA, Campbell MJ, Thabane L, Hopewell S, Coleman CL, et al. Defining Feasibility and Pilot Studies in Preparation for Randomised Controlled Trials: Development of a Conceptual Framework. PLoS One. 2016;11(3):e0150205.

106. Bucci S, Schwannauer M, Berry N. The digital revolution and its impact on mental health care. Psychol Psychother. 2019 Jun;92(2):277-97.

107. Torous J, Lipschitz J, Ng M, Firth J. Dropout rates in clinical trials of smartphone apps for depressive symptoms: A systematic review and meta-analysis. J Affect Disord. 2020 Feb 15;263:413-9. 
108. Cheng VWS, Davenport T, Johnson D, Vella K, Hickie IB. Gamification in Apps and Technologies for Improving Mental Health and Well-Being: Systematic Review. JMIR Ment Health. 2019 Jun 26;6(6):e13717.

109. Christie GI, Shepherd M, Merry SN, Hopkins S, Knightly S, Stasiak K. Gamifying CBT to deliver emotional health treatment to young people on smartphones. Internet Interv. 2019 Dec;18:100286.

110. Lattie EG, Adkins EC, Winquist N, Stiles-Shields C, Wafford QE, Graham AK. Digital Mental Health Interventions for Depression, Anxiety, and Enhancement of Psychological Well-Being Among College Students: Systematic Review. J Med Internet Res. 2019 Jul 22;21(7):e12869.

111. Hollis C, Sampson S, Simons L, Davies EB, Churchill R, Betton V, et al. Identifying research priorities for digital technology in mental health care: results of the James Lind Alliance Priority Setting Partnership. Lancet Psychiatry. 2018 Oct;5(10):845-54.

112. Eisele G, Vachon H, Lafit G, Kuppens P, Houben M, Myin-Germeys I, et al. The Effects of Sampling Frequency and Questionnaire Length on Perceived Burden, Compliance, and Careless Responding in Experience Sampling Data in a Student Population. Assessment. 2020 Sep 10:1073191120957102. 



\section{CHAPTER 8}

\section{Digital interventions for psychosis}

Christian Rauschenberg, Mar Rus-Calafell, Ulrich Reininghaus, Lucia Valmaggia Comprehensive Clinical Psychology (2nd ed.). Amsterdam, Netherlands: Elsevier Ltd. Doi: 10.1016/B978-0-12-818697-8.00070-4 


\section{Abstract}

Digital technologies such as smartphones, smartwatches and head-mounted displays are increasingly available at low cost and have witnessed an unparalleled gain in computational power over the recent years. This has resulted in an increasing interest in, and steep upsurge in the possibilities of, using these technologies to deliver personalized, engaging and adaptive digital interventions for individuals with psychosis. In this chapter, different types of digital interventions will be described, recent examples of cutting-edge internet-based eHealth, appbased mHealth and virtual reality-based interventions provided and important challenges discussed. The chapter will conclude by discussing promising avenues for the future and clinical implications.

Keywords: Telemedicine; Mobile Health; Virtual Reality; Technology; Digital Psychiatry; Digital Intervention; eHealth; Monitoring; Feedback; Ecological Momentary Assessment; Ecological Momentary Interventions; Ecological Translation; Psychosis; Schizophrenia 


\section{Introduction}

Although current routine mental health services offer various treatment options for individuals with psychotic symptoms, specialized support remains difficult to access and tailoring of interventions to service users' needs continues to be problematic. These limitations of existing mental health services likely contribute to an increased burden for individuals and their caregivers [1], considerably high dropout and relapse rates [2], treatment resistance [3] and low medication adherence [4]. Further, perceived stigma associated with mental health conditions, psychosis in particular [5], remains to be high and has been found to negatively influence individuals willingness to seeking help. This may ultimately lead to long durations of untreated psychosis [6, 7], an important predictor of poor prognosis and outcome [8]. Thus, modifying illness trajectories at early stages of psychosis is important $[9,10]$ and finding novel, easy-to-access and more engaging forms of delivering mental health services are urgently needed [11].

This chapter examines the clinical potential of emerging digital technologies for helping individuals with subclinical expressions of psychosis as well as psychosis spectrum disorders. For this, a selection of recently published studies and metaanalyses will be used to exemplary summarize recent developments. First, different types of digital interventions will be described, and examples of stateof-the-art applications provided. This will be followed by recent developments and studies that explore the potential of digital interventions to specifically target potential mechanisms underpinning psychosis. The chapter will conclude by discussing promising avenues for the future and clinical implications derived from the available evidence. Overall, the aim of the current chapter is to give interested readers a review on how digital interventions are currently being used to help individuals with psychosis at different clinical stages.

\section{Differing types of digital interventions}

Rapid technological advances, such as smartphones, smartwatches, fitness trackers and head-mounted displays, are increasingly available at low cost and have witnessed an unparalleled gain in computational power over the recent years. This has resulted in an increasing interest in, and steep upsurge in the possibilities of, using digital tools to deliver pioneering and truly integrative mental health 
services for individuals with psychosis and other mental disorders [12, 13]. In many countries, people have access to various of these information and communication technologies (e.g. mobile phone ownership rate among individuals with psychosis has been reported to be above $80 \%$ [14]) and are already accustomed to frequently use their wide-ranging functionalities. Consequently, digital interventions have great potential of bringing personalized, engaging and adaptive interventions to help individuals with mental health conditions, including psychosis [15-18].

There are differing types of digital interventions which are currently most widely studied for helping individuals with psychosis that can be roughly categorized in telemedical and internet-based (eHealth) [19, 20], app-based mobile health (mHealth) $[15,18,21,22]$ and virtual reality-based (VR) interventions [23, 24]. These digital tools offer a plethora of novel possibilities researchers and clinicians are only just beginning to explore, with the ultimate goal of enhancing mental health care. These tools differ greatly in terms of their content, complexity and functionality ranging from simple prompts sent via SMS or other messaging services, with the goal to remind users to do certain therapeutic tasks at home, to technologically very sophisticated interventions that include avatars - computer-generated virtual representation of humans - in VR environments that have the ability to respond to clients' verbal responses by using speech-recognition techniques and may even help individuals to guide through virtual therapy sessions. Importantly, digital interventions can either be used to supplement traditional face-to-face treatment (e.g. use of an mHealth app to practice certain intervention components to augment traditional cognitive behavior therapy) or to offer stand-alone services.

Digital interventions that have been developed and tested to support individuals with psychosis have primarily focused on five domains [25]:

(1) enabling remote communication and social interactions among service users, carers and mental health professionals (e.g. moderated online forums or online peer-support);

(2) allowing easy access to evidence-based information (e.g. modules delivered through mHealth apps that offer psychoeducation);

(3) supporting real-time and real-world self-monitoring and management of symptoms (e.g. use of mood trackers which are based on frequent self-reports on momentary mental states); 
(4) delivery of person-tailored feedback (e.g. the use of fine-grained real-time data on behaviors, mental states and minor stressors that are actively used by clinicians during therapy sessions to help service users to gain meaningful insights about their mental health problems, including important determinants);

(5) delivery of psychological interventions in individuals' daily lives or in immersive virtual environments (e.g. exposure to fearful social stimuli to lower social anxiety in an interactive three-dimensional virtual environment).

In the following subsections, cutting-edge examples of eHealth platforms, mHealth apps and VR interventions will be discussed that offer one or more of aforementioned domains to help individuals with psychosis and are largely based on findings reported in recently published reviews [15, 17, 20, 22, 24, 26-33].

\section{eHealth and mHealth interventions for psychosis}

Studies have found that the frequency, availability and flexibility of contact with mental health professionals as well as peer-support are effective components of psychological interventions, which can be decisive for the reduction of relapse [34]. Digital interventions may help to increase the flexibility of contact with mental health professionals and peers through online chat, video calls and online platforms and may thereby optimize both treatment continuity and cost-effectiveness. In addition, giving individuals the right digital tools to inform themselves and others about their experiences, and to develop skills and strategies to better cope with mental health conditions they experience, have been found to contribute to empowering those in need of care, which may also support a more active role of individuals' recovery processes [35]. There are numerous digital interventions that improve communication among service users and health professionals and purposefully include components of peer-support components, easy-to-access evidence-based information and intervention components in form of online eHealth platforms or mHealth apps. In the following paragraphs, recent examples of cutting-edge applications will be provided (see also Table 1 and Table 2).

Schizophrenia Online Access to Resources ('SOAR') [36] is a web-based platform that gives individuals with psychosis spectrum disorders and their families access to modules on psychoeducation as well as modules that focus on practicing problem solving skills, coping strategies and promoting self-efficacy, amongst others. SOAR also includes moderated online support with trained health professionals and a 
peer discussion forum and has been found to reduce positive psychotic symptoms and improve knowledge about schizophrenia of medium to large effect when compared to treatment as usual (TAU). Another web-based and mobile platform is Moderated Online Social Therapy ('MOST') that offers continuous, integrated faceto-face and digital care to young people which has been pioneered by the eOrygen team at Orygen, the National Centre of Excellence in Youth Mental Health, Australia. The elements of the MOST conceptual model have been applied to different interventions: 'HORYZON' [37] includes an extensive psychosocial intervention that aims to increase social functioning in individuals with first episode psychosis, and has later been extended to helping individuals ultra-high-risk for psychosis ('MOMENTUM') [38]. These interventions were developed to increase self-efficacy, positive emotions and social support through online social environments. The interventions consist of an interactive online social therapy, peer-to-peer online social networking and expert and peer moderation. It also includes modules on helping individuals in defining their strength and doing mindfulness exercises to improve social functioning. In an uncontrolled single-group phase I pilot study, MOMENTUM has been found to be safe, feasible and engaging for young individuals with "ultra-high-risk" (UHR) state for psychosis. When comparing baseline and follow-up, an increased use of strengths, mindfulness skills and aspects of social support have been reported which was, in turn, associated with self-efficacy, life satisfaction and lower loneliness and depression scores, respectively. Overall, there was a large increase in social functioning [37, 38].

Table 1. Recent examples of eHealth applications with published feasibility/efficacy results.

\begin{tabular}{lllll}
\hline Study & Name & $\begin{array}{l}\text { Country of } \\
\text { Origen }\end{array}$ & Targeted population & $\begin{array}{l}\text { Main therapeutical } \\
\text { targets }\end{array}$ \\
\hline $\begin{array}{l}\text { Rotondi et al., } \\
\text { (2010) }\end{array}$ & SOAR & USA & $\begin{array}{l}\text { Psychosis spectrum } \\
\text { disorders }\end{array}$ & $\begin{array}{l}\text { - Problem solving } \\
\text { skills } \\
\text { - Coping strategies - } \\
\text { Self-efficacy }\end{array}$ \\
\hline $\begin{array}{l}\text { Alvarez-Jimenez } \\
\text { et al., 2013 }\end{array}$ & HORYZONS & Australia & FEP & $\begin{array}{l}\text { - Social engagement } \\
\text { - Self-efficacy } \\
\text { - Wellbeing }\end{array}$ \\
\hline $\begin{array}{l}\text { Alvarez-Jimenez } \\
\text { et al., 2018 }\end{array}$ & MOMENTUM & Australia & CHR & $\begin{array}{l}\text { - Social engagement } \\
\text { - Self-efficacy } \\
\text { - Wellbeing }\end{array}$ \\
\hline Laine et al., 2019 & Mental-net & Finland & $\begin{array}{l}\text { Psychosis spectrum } \\
\text { disorders }\end{array}$ & $\begin{array}{l}\text { - Psychoeducation - } \\
\text { Self-efficacy }\end{array}$ \\
\hline
\end{tabular}

Note: FEP: First Episode of Psychosis; CHR: Clinical High Risk of psychosis. 
Table 2. Recent examples of mHealth (smartphone) applications with published feasibility/ efficacy results

\begin{tabular}{|c|c|c|c|c|}
\hline Study & Name & $\begin{array}{l}\text { Country of } \\
\text { Origen }\end{array}$ & $\begin{array}{l}\text { Targeted } \\
\text { population }\end{array}$ & $\begin{array}{l}\text { Main therapeutical } \\
\text { targets }\end{array}$ \\
\hline $\begin{array}{l}\text { Ben-Zeev et al., } \\
2014\end{array}$ & FOCUS & USA & $\begin{array}{l}\text { Psychosis spectrum } \\
\text { disorders }\end{array}$ & $\begin{array}{l}\text { - Coping strategies } \\
\text { - Anxiety and depression } \\
\text { management } \\
\text { - Sleep disturbances } \\
\text { - Social functioning } \\
\text { - Medication } \\
\text { management }\end{array}$ \\
\hline $\begin{array}{l}\text { Schlosser et al., } \\
2016 \text { and } 2018\end{array}$ & PRIME & USA & EIS individuals & $\begin{array}{l}\text { - Reward-processing } \\
\text { impairments }\end{array}$ \\
\hline Kim et al., 2018 & HYM & South Korea & $\begin{array}{l}\text { Psychosis spectrum } \\
\text { disorders }\end{array}$ & $\begin{array}{l}\text { - Symptom monitoring } \\
\text { (follow-up after CBT } \\
\text { intervention) }\end{array}$ \\
\hline $\begin{array}{l}\text { Niendam et al., } \\
2018\end{array}$ & Ginger.io & USA & EIS individuals & $\begin{array}{l}\text { - Assessment. } \\
\text { - Symptom monitoring }\end{array}$ \\
\hline $\begin{array}{l}\text { Bucci et al., } \\
2018\end{array}$ & Actissist & $\begin{array}{l}\text { United } \\
\text { Kingdom }\end{array}$ & EIS individuals & $\begin{array}{l}\text { - Psychoeducation } \\
\text { - Symptom self- } \\
\text { management skills }\end{array}$ \\
\hline $\begin{array}{l}\text { Depp et al., } \\
2019\end{array}$ & CBT2go & USA & $\begin{array}{l}\text { Psychosis spectrum } \\
\text { disorders }\end{array}$ & $\begin{array}{l}\text { - Negative beliefs about } \\
\text { the self/others } \\
\text { - Attributions }\end{array}$ \\
\hline Lim et al., 2019 & +Connect & Australia & EIS & - Loneliness \\
\hline $\begin{array}{l}\text { Garety et al., } \\
2017\end{array}$ & $\begin{array}{l}\text { SlowMo } \\
\text { (blended } \\
\text { therapy) }\end{array}$ & $\begin{array}{l}\text { United } \\
\text { Kingdom }\end{array}$ & $\begin{array}{l}\text { Non-affective } \\
\text { psychosis }\end{array}$ & $\begin{array}{l}\text { - Reasoning biases } \\
\text { - Paranoid attributions }\end{array}$ \\
\hline
\end{tabular}

Note: EIS: Early intervention services; FEP: First Episode of Psychosis; CHR: Clinical High Risk of psychosis.

'Mental-Net' [39], a web-based patient education intervention, offers a number of psychoeducational material and an online discussion forum. These psychoeducational modules are divided into 5 themes: information on mental disorders, evidence-based treatment options, well-being, patients' rights and ways to cope with challenges experienced in daily life (e.g. economic support, taking care of home). In a feasibility pilot study, service users diagnosed with schizophrenia accessed the website together with a mental health professional once per week (45-60 minutes). The acceptability has been found to be high as users were satisfied with the offered intervention components and also felt that provided information were useful to better cope with their mental health problems. In comparison to individuals in a waiting list control condition (TAU), the intervention group has also been found to have significantly higher scores in self-efficacy and lower scores in perceived hopelessness. 
In addition to web-based eHealth platforms, there has been a recent increase of app-based digital interventions designed to help individuals with psychosis. 'FOCUS' [40] is a multimodal digital intervention for people with serious mental illness, including psychosis spectrum disorders and consists of an mHealth app as well as a dashboard that can be assessed by clinicians. The app includes components which primarily target 5 domains, that is, (1) strategies to cope with auditory hallucinations (e.g. distraction, cognitive restructuring and hypothesis testing techniques); (2) managing anxiety and depression (e.g. relaxation techniques, behavioral activation and other supportive content); (3) sleep (e.g. sleep hygiene, wellness psychoeducation); (4) social functioning (e.g. anger management, activity scheduling); and (5) medication (e.g. reminders and psychoeducation). FOCUS has been found to be feasible and acceptable and initial therapeutic effects have been demonstrated in individuals with psychosis [40,41], including those who were recently discharged [42]. Another pioneering mHealth app is Personalized RealTime Intervention for Motivation Enhancement ('PRIME') [43]. PRIME is designed to target reward-processing impairments in individuals with recent onset schizophrenia and includes selecting goals that are being monitored over the course of the treatment, daily challenges, and social components with a trained online coach who assists users in the use of the app. Compared to a waitlist control condition, individuals in the PRIME condition showed greater improvements in depression, defeatist beliefs, self-efficacy and components of motivation [44].

Heal your Mind ('HYM') has been used after the completion of group-based Cognitive Behavioral Therapy for Psychosis to allow on-demand personal advice and support based on real-time monitoring of symptoms [45]. A case manager accessed users' responses and was able to provide real-time feedback which has been found to help personalizing case-management. In a survey study, including 24 people with early psychosis, more than $80 \%$ of the participants reported that the app was easy to use and around $70 \%$ perceived some benefit from having used it [45]. 'Ginger.io' [46], a mobile app which works in combination with a webbased dashboard, has been used as part of early psychosis outpatient care. Ginger. io allows the assessment and monitoring of symptoms as well as passive data (e.g. physical movement, calls and texting pattern) and a clinician dashboard to provide feedback and alerts sent when responses indicate worsening of symptoms [46]. Longitudinal data (14 months) from 76 individuals from Early Intervention Services showed good feasibility of the integration of the app and the dashboard as a self- 
reported assessment of symptoms, providing data comparable to clinician-rate assessment ratings [46].

'Actissist' is an mHealth intervention that delivers cognitive behavioral therapy (CBT) through a smartphone app for individuals with early psychosis. It incorporates adaptive delivery of intervention components and does thus purposefully incorporate individuals' responses to short questionnaires that are prompted three times per day to ask participants about their current mental states. In addition, users also have the ability to access the therapeutic content of the app on demand [47]. Similarly, 'My Journey 3' [48] is a smartphone delivered self-management tool for individuals with first episode psychosis recruited from Early Intervention in Psychosis services. The app includes key elements of self-management, including the ability to create a relapse prevention plan (e.g. identification of potential triggers, early warning signs, coping strategies), definition of recovery goals, listing of actions that can increase well-being and reminders to engage in these activities. Another mHealth app that has been recently developed and evaluated is 'CBT2go' [49]. Again, this digital intervention is based on principles of CBT and consists of a single face-to-face session which is supplemented by an mHealth app. The app provides real-time thought-provoking interventions focusing on specific symptom domains (e.g. voice hearing) and beliefs which have been identified during face-to-face therapy session. The app also prompts individuals to complete modules on cognitive restructuring and other tasks. In a three-arm (i.e. CBT2go, self-monitoring, TAU) randomized single-blind controlled trial including 255 individuals with psychosis spectrum disorders, CBT2go has been found to be feasible and to have small to moderate effects on global psychopathology and community function as compared to TAU. However, CBT2go was not found to be more effective when compared to the active control condition (i.e. self-monitoring) for most of the primary and secondary outcomes [49]. Finally, the mobile app '+Connect' $[50,51]$ has been designed based on principles of positive psychology and focusses on the reduction of loneliness. The app offers peer and expert videos on demand, mood tracking and gamified challenges. Preliminary evidence of this mobile app showed significant reductions in loneliness (main intervention target) at post-treatment and in gain maintenance at 3-month follow-up [50]. See also Rus-Calafell and Schneider (2020) for a specific review on eHealth and mHealth applications designed to help people at early psychosis stages. 


\section{Future outlook on eHealth and mHealth interventions}

In this section, a future outlook on eHealth and mHealth interventions is provided. For this, a selection of recently published trial protocols with pending efficacy or feasibility results is summarized.

A recent example of blended therapy, where face-to-face sessions with a therapist are combined with a mobile app, is the SlowMo Therapy [52]. SlowMo is a digital approach which employs technology to target the fast-thinking tendencies that have been associated with paranoia (e.g. jumping to conclusions, belief inflexibility). The therapy consists of eight individual, face-to-face sessions, delivered by trained therapists, assisted by a webapp with interactive personal accounts and daily scenarios. A smartphone app helps the person embed strategies into everyday life. As such, the SlowMo approach attempts to combine the best of existing talking therapies with novel digital technology to increase engagement, effectiveness and generalization to everyday life [53,54]. Efficacy results from a large controlled randomized study $(\mathrm{N}=350)$ are expected to be published early 2021 [52].

'EviBaS' [55] is an internet-based 8-week online self-help intervention for people with psychosis that includes psychoeducational modules on delusional ideations, voice hearing, social competence and mindfulness. Early signs Monitoring to Prevent relapse in psychosis and prOmote Well-being, Engagement and Recovery ('EMPOWER') $[56,57]$ is a digital mHealth intervention which will be offered as a supplement to TAU. EMPOWER focusses on early signs monitoring to prevent relapse in psychosis and promote well-being. 'EMBRACE' [58] is a moderated 12-week online intervention to treat social anxiety in individuals with FEP and is, similar to MOMENTUM and HORYZONS, based on elements of the MOST conceptual model. 'Robin' [59] integrates content of face-to-face treatment sessions with an mHealth app for individuals with clinical high risk for psychosis. The 'momentum trial' [60] is an mHealth app that aims to support self-perceived patient activation and shared decision making for people with psychosis spectrum disorders in outpatient treatment settings. Finally, in the context of symptomfocused interventions where a single-symptom of psychosis is the main target of the psychological therapy, a Dutch group has developed 'Temstem' [61]. It is a mobile app that aims to help individuals who are hearing voices by offering motoric language games, tasks to improve self-esteem and imagery exercises. 
In summary, there has been a recent surge of eHealth interventions and mHealth apps to help individuals with psychosis. Early pioneering work laid the foundation for a now rapidly evolving field, resulting in increasingly adaptive, multimodal digital interventions which aim to be engaging and tailored to what a person needs in a given moment and context. In doing so, a range of therapeutic approaches - most commonly CBT and third-wave CBT - have been used. In this section of the chapter, a selection of advanced eHealth and mHealth interventions were described and a future outlook provided to exemplify the current state of development. Overall, these interventions have been found to offer psychoeducational material and modalized digital interventions targeting established risk and resilience factors for psychosis as well as symptom domains (e.g. social functioning, self-efficacy, physical activity, auditory hallucinations, delusional ideations). Some interventions also enabled users to actively manage and monitor their symptoms in real-time, access broad set of skill trainings (e.g. relaxation techniques, helpful coping strategies) and to engage in various forms of remote communication with peers and service providers. Accumulating evidence suggests the feasibility, acceptability, and therapeutic effects of eHealth and mHealth interventions for psychosis. However, our understanding on candidate mechanisms, long-term effects, and cost-effectiveness remains very limited and the current use and implementation of digital tools in routine mental health services, outside the research context, is very limited.

\section{VR interventions for psychosis}

In addition to eHealth interventions and mHealth apps, the use of VR has great potential to offer completely new avenues for mental health care. Immersive VR in particular allows users to interact with a three-dimensional computer-generated virtual environment that is completely controllable by researchers or clinicians [62]. There has been a long tradition to use immersive VR environments to assess mental health problems as well as processes and mechanisms which have been found to be important for the development and persistence of psychosis in ecologically more valid ways with high external validity as compared to other lab-based assessment methods [23, 24, 33, 63-65]. More recently, VR has been used for the treatment of various mental health problems, including attention deficit hyperactivity disorder, autism, post-traumatic stress disorder and anxiety 
disorders [24, 29, 65-69]. VR-based interventions that are specifically designed to help individuals with psychosis are scarce, although, over the recent years, they have gained traction. Originally, VR interventions were mostly focusing on prevocational training and job interview training and have thus not been directly targeting psychosis symptomatology [24, 29, 33]. More recently, however, several studies have demonstrated initial therapeutic effects of VR intervention on putative mechanisms of symptoms of psychosis (see also Table 3).

One of the first studies considered to test a VR-based intervention for psychosis, is the one published by Freeman and colleagues (2016). This study included 30 individuals with psychosis who were randomized to VR-CBT or VR exposure. The VR-CBT condition was focusing on enabling patients to drop safety-seeking behaviors in computerized versions of feared social situations in virtual reality environments, while in the exposure condition individuals had solely to move in a VR environment while the number of avatars was increasing. In comparison with VR exposure, VR-CBT was found to reduce delusional conviction and real-world distress [70].

Table 3. Recent examples of Virtual Reality applications with published feasibility/efficacy results

\begin{tabular}{lllll}
\hline Study & Name & $\begin{array}{l}\text { Country of } \\
\text { Origen }\end{array}$ & $\begin{array}{l}\text { Targeted } \\
\text { population }\end{array}$ & $\begin{array}{l}\text { Main therapeutical } \\
\text { targets }\end{array}$ \\
\hline $\begin{array}{l}\text { Freeman et } \\
\text { al., 2016 }\end{array}$ & VR-CBT & $\begin{array}{l}\text { United } \\
\text { Kingdom }\end{array}$ & $\begin{array}{l}\text { Non-affective } \\
\text { psychosis }\end{array}$ & $\begin{array}{l}\text { - Safety behaviours } \\
\text { - Social avoidance }\end{array}$ \\
\hline $\begin{array}{l}\text { Pot-Kolder et } \\
\text { al., } 2018\end{array}$ & VR based CBT & Netherlands & $\begin{array}{l}\text { Psychosis spectrum } \\
\text { disorders }\end{array}$ & $\begin{array}{l}\text { - Paranoid attributions } \\
\text {-Social engagement }\end{array}$ \\
\hline $\begin{array}{l}\text { Nijman et al., } \\
2019\end{array}$ & DiSCoVR & Netherlands & $\begin{array}{l}\text { Psychosis spectrum } \\
\text { disorders }\end{array}$ & $\begin{array}{l}\text { - Social cognition } \\
\text { - General } \\
\text { psychopathology }\end{array}$ \\
\hline $\begin{array}{llll}\text { Vass et al., } \\
2019\end{array}$ & VR-ToMIS & Hungary & $\begin{array}{l}\text { Psychosis spectrum } \\
\text { disorders }\end{array}$ & $\begin{array}{l}\text { - Negative symptoms } \\
\text { - Theory of Mind }\end{array}$ \\
& & & - Language skills \\
\hline $\begin{array}{l}\text { Craig et al., } \\
2018\end{array}$ & $\begin{array}{l}\text { AVATAR } \\
\text { therapy }\end{array}$ & $\begin{array}{l}\text { United } \\
\text { Kingdom }\end{array}$ & $\begin{array}{l}\text { Non-affective } \\
\text { psychosis }\end{array}$ & $\begin{array}{l}\text { - Distressing auditory } \\
\text { hallucinations }\end{array}$ \\
\hline $\begin{array}{l}\text { duSert et al., } \\
2018\end{array}$ & $\begin{array}{l}\text { Avatar therapy } \\
\text { (immersive) }\end{array}$ & Canada & $\begin{array}{l}\text { Psychosis spectrum } \\
\text { disorders }\end{array}$ & $\begin{array}{l}\text { - Distressing auditory } \\
\text { hallucination }\end{array}$ \\
\hline
\end{tabular}

Note: VR: Virtual Reality. 
A larger randomized study has found that a CBT-based VR intervention, aimed to improve paranoid thoughts and social involvement in people with psychosis, lowered paranoid ideation and anxiety as compared to TAU at post-treatment [71]. However, groups did not differ with regard to the amount of time spend with other people at post-treatment (i.e. increase of social activities). The treatment effects on paranoia were maintained at 6-month follow-up. Interestingly, individuals' safety behaviors and social cognition were found to mediate treatments effects on paranoid ideation [71]. The Dynamic Interactive Social Cognition Training in Virtual Reality ('DiSCoVR') was tested in a single-group feasibility pilot study investigating initial therapeutic effects of a 16-session training on social cognition and psychiatric symptoms [72]. In total, 17 individuals with psychosis spectrum disorders and problems in social cognition were included in this pilot study. Acceptability (e.g. satisfaction) has been reported to be high and most participants explicitly mentioned that the intervention was helpful because of the possibility to practice social interactions in VR environments. While the study has demonstrated changes in emotion perception of moderate effect, no effects were found on other outcomes measures such as social cognition and self-esteem [73]. In another randomized-controlled phase I feasibility pilot study [74], a VR-based intervention was designed to target Theory of Mind in outpatients with schizophrenia ('VRToMIS') [74]. The intervention consisted of 9-sessions of either VR-ToMIS or passive VR. In the passive VR condition, patients were able to freely explore the virtual environments, but not to contact any avatars. In the intervention group, improvements on negative symptoms, theory of mind and language skills were found as compared to the passive VR condition, but no differences on quality of life [74].

AVATAR therapy is an example of a more symptom-focused VR-based intervention for psychosis $[75,76]$. The therapy allows a 'face-to-face' dialogue between the person and a computerized representation of their voice (or auditory hallucination). Although AVATAR therapy as originally developed by Leff and colleagues (2013) is not delivered using a complex immersive environment, the platform uses VR technology to create a virtual embodiment of the experience of hearing voices and to re-enact the relationship with the voice within a real-time dialogue (see also [77] for an independent pilot using a head mounted display to deliver the therapy). The embodiment of the voice is enhanced by the use of direct verbatim speech, and enactment of the ascribed character and background of the voice. 
The efficacy of the therapy has now been demonstrated in two independent pilot studies $[76,77]$ and in a large fully-powered randomized controlled trial comparing AVATAR therapy and Supportive Counselling that showed AVATAR therapy to be more effective post-therapy in terms of reductions in the frequency, distress and omnipotence of voices after an average of 6 therapy sessions [75]. Interestingly, the interaction of sense of voice presence and reduction of anxiety was associated with two of the significant therapy outcomes (total severity and frequency of voices, see [78]).

\section{Future outlook on VR interventions}

Freeman and colleagues are currently working on automated psychological treatments delivered using VR [79]. In the 'gameChange' trial, 432 patients with psychosis and avoidance of social situations will be recruited and randomized to receive either VR cognitive treatment (gameChange) additionally to their treatment as usual (TAU) or to continue solely with TAU. The intervention aims to reduce avoidance and to help individuals to feel more comfortable around other people by working on fear expectations and relearning feeling safe in social situations. In total, the intervention lasts for up to 6 sessions of 30 minutes and a computerized virtual coach will guide participants through 6 VR environments that include gamified social challenges of increasing difficulty. First results are expected to be published around mid 2021 [79].

THerapeutic Realistic Immersive Virtual Environments 'THRIVE' [80] is another automated VR intervention that is currently investigated by the same research group and forms the basis of gameChange VR. Thus, it also focusses on individuals with psychosis and avoidance of social situations, although in this particular case the VR cognitive treatment will be compared with an active treatment condition (VR mental relaxation). Secondary outcomes include real-world distress, suicidal ideation and quality of life [80]. Further research on VR-based interventions for psychosis is currently being carried out at Dr Valmaggia's VR Lab at the Institute of Psychiatry, Psychology and Neuroscience (IoPPN, King's College London). Among other work, this research group is testing the efficacy of a new VR-assisted therapy to help people improve social performance and manage paranoia thoughts while participating in social interactions (see [81] for a validation of the virtual environment designed for this purpose). An ongoing small feasibility trial is being 
carried out including people at first-episode psychosis (FEP). The same research group is working on the design and testing of a novel VR-based intervention to improve negative symptoms in people with psychosis (VR Therapy for Psychosis Negative Symptoms, 'V-NeST') (this work is led by Dr Matteo Cella).

Overall, VR interventions for psychosis have gained a great momentum. Similar to most eHealth and mHealth interventions, VR interventions often build on techniques commonly used in CBT as they are particularly amendable to translation into computer-generated virtual environments. So far, most VR interventions for psychosis aim to target factors which have been found to contribute to psychosis progression and persistence, including social anxiety and associated safety-seeking behaviors and theory of mind impairments as well as positive psychotic symptoms. Importantly, there is a growing number of high-end, all-in-one portable VR headsets available on the consumer market that can be used without expensive setups. This will likely further accelerate the use of VR intervention in clinical settings, outside the research laboratory.

\section{From mechanistic research to novel treatment targets}

As described earlier, digital interventions are well positioned to not only target psychotic symptoms in daily life, but also putative risk and resilience mechanisms which have been found to be important in the development and persistence of psychosis [18, 23, 82]. For instance, studies using Ecological Momentary Assessment (EMA) or synonymously Experience Sampling Methodology, a structured diary technique [83], have robustly found an elevated sensitivity towards stress in daily lives of individuals with an at-risk mental state for psychosis as well as psychosis spectrum disorders $[84,85]$. Thus, these findings support the notion that individuals' stress sensitivity may constitute an important candidate psychological mechanism involved in the formation and maintenance of psychosis that could be a promising novel treatment target of digital interventions [18].

Building on advances in the field of mHealth interventions, an ecological interventionist causal model approach for targeting psychological mechanisms in daily life has been proposed [18]. This approach draws on Ecological Momentary Interventions (EMIs) which represent a promising digital intervention approach for targeting risk and resilience mechanisms as well as symptoms domains in the real- 
world and in real-time through cutting-edge mobile technologies $[15,18,86]$. EMIs allow the transfer of evidence-based intervention components (e.g. therapeutic techniques of cognitive-behavioral therapy, CBT, or third-wave CBT) that are particularly suitable to target putative mechanisms in daily lives of individuals with psychosis or individuals with a known clinical risk for developing psychosis. Two studies have recently been published that used principles of EMIs to target risk mechanisms, symptom domains and promoted helpful cognitions and behaviors. In the Acceptance and Commitment Therapy in Daily Life ('ACT-DL') trial [87, 88], principles and exercises of ACT were translated into an EMI and delivered through an mHealth app which aimed to target putative psychological mechanisms, including stress sensitivity, psychological flexibility and reward experience in individuals with UHR/FEP. ACT-DL includes modules on creative hopelessness, acceptance, cognitive difusion, self as context, committed action and defining values. The feasibility of the treatment protocol and evidence of efficacy has recently been demonstrated [88]. Further, in an uncontrolled pilot study [89], an ecological momentary, compassion-focused intervention for improving emotional resilience ('EMIcompass') was investigated including help-seeking youth with psychotic, depressive and/or anxiety symptoms. The intervention consisted of three sessions with a trained psychologist and a 3-week EMI administered through an mHealth app. Preliminary findings of this phase I pilot study suggest that using compassion-focused intervention components (e.g. compassionate image and writing, emotion as a wave, breathing exercises) delivered using an mHealth app following principles of EMIs may reduce stress sensitivity in daily life and various psychopathological domains in help-seeking youth [89]. The EMlcompass intervention is currently tested in an exploratory randomized controlled trial to establish clinical feasibility, candidate underlying mechanisms, and initial signals of efficacy. The results are expected to be published in 2021 [90].

Similarly, VR enables new opportunities for improving well established cognitive and behavioral techniques in an engaging and tailored way, as well as providing novel therapeutic contexts within which core psychological processes (Valmaggia et al., 2016) can be targeted in real time with immediate feedback. For example, in people with paranoia, VR scenarios are designed in which the degree of hostility that the virtual characters display can be manipulated (for example to be neutral, benign or hostile). This allows a more refined assessment of paranoia than selfreport measures, at the same time that offers the opportunity to the person, for 
example, to drop key safety behaviors in a controlled and predictable setting (see Freeman et al., 2016 and Pot-Kolder et al., 2018) and to learn new emotional and behavioral responses to feared situations [81]. Crucially VR environments allow 'embodied' cognitive processes to be targeted 'in action', potentially matching the dynamic conceptualization of appraisals (e.g. attributions of power and control about voices) with the ultimate aim that improvements made in VR environments will generalize to real-life contexts.

\section{Quality from the user perspective}

Findings from digital interventions included in this chapter suggest moderate to high acceptability and feasibility of eHealth, mHealth and VR interventions. However, some of the included studies did not specifically report data on users' perspective. Notably, acceptability as well as effectiveness on various healthrelated outcomes have been described to be particularly high if interventions were embedded in a therapeutic context and include some form of social contact with mental health professionals - commonly referred to as the blended care approach $[14,91,92]$. Furthermore, studies have shown that perceived quality increased if digital intervention include possibilities to socially interact with peers [93] and implement strategies to promote user engagement like gamification strategies [94].

\section{Future research directions}

Digital mental health services for psychosis, especially mHealth apps and VR interventions, are still at an early stage of their development and many interventions have only been tested in small feasibility trials. Thus, more wellpowered randomized controlled trials are urgently needed to further investigate their efficacy, long-term effects, processes and mechanisms of action and costeffectiveness. Furthermore, after careful testing, efforts should be made to bring evidence-based digital interventions into clinical practice as well as making them publicly available. In addition, most digital interventions which are currently developed and tested do arguably not fully harness the full potential of more recent technological advances. For example, integrating other technologies (e.g. sensors such as accelerometer, gyroscope, GPS to passively assess psychophysiological markers) may have additive effects on treatment outcomes and may also be used 
to further personalize the timing and context of when intervention components are delivered. Also, as engagement remains to be a problem [95], gamification features as well as other elements that aim to improve service users' motivation to use digital tools should be further investigated and incorporated [96]. In addition, it has become increasingly clear that fostering principles of co-design and coproduction at an early stage of the developmental process of digital interventions is crucial for satisfying specific needs and preferences of service users as well as mental health professionals $[13,53]$ and has been found to be beneficial for implementing these tools into diverse clinical settings [94]. Finally, an important next step is to systematically investigate the role of relational components like the digital therapeutic alliance on health-related outcomes [97] and how to strengthen rapport and other unspecific treatment effects in light of potentially declining numbers of face-to face sessions $[98,99]$.

\section{Clinical applications and recommendations}

The use of telemedical and internet-based (eHealth), app-based mobile health (mHealth) and virtual reality (VR) interventions have been found to be safe among individuals with psychosis. Recent findings indicate feasibility and high acceptability ratings, and evidence on effectiveness is very promising although long-term effects have to be further investigated. Importantly, acceptability, feasibility and effectiveness are particularly high if digital interventions are used in clinical settings and thus include some form of social contact with a mental health professional. Thus, clinicians interested in the diverse possibilities of digital intervention may start using these tools in their everyday therapeutic work to gain important first-hand experience of the unique opportunities and current limitations of these tools and to supplement face-to-face treatment sessions.

Digital interventions hold great potential of bringing person-centered and adaptive interventions into individuals' everyday lives and, thereby, help to ecologically translate treatment components to contexts outside clinicians' office or specialized clinics. However, digital interventions developed by research groups are mostly neither routinely offered by health care professionals, nor integrated in established psychological treatments, and do also very scarcely find their way into major app stores. This strongly limits the current potential of digital interventions to alleviate mental health burden associated with psychosis and other mental 
and somatic health complaints. To address this problem, there have been recent efforts to systematically evaluate currently available digital interventions based on established criteria of digital mental health and mental health services research (e.g. National Health Service Apps Library in the UK; Platform for Digital health applications (DiGA) in Germany; App Evaluation Database by the Division of Digital Psychiatry, Beth Israel Deaconess Medical Center in the USA). Additionally, researchers and policy makers are working on national implementation strategies with the ultimate goal of making evidence-based digital interventions clinically and publicly available, and incorporating them in existing healthcare systems by, for example, enabling "health apps on prescription". These efforts allow individuals with psychosis and clinicians making more informed decisions on the quality of currently available digital interventions and to stay up to date in this rapidly evolving field.

Lastly, clinicians should be aware of possible barriers as well as social inequalities (e.g. disproportional distribution of ownership of required technologies, language skills, motor, or cognitive deficits) that must be taken into account when they are planning to use digital tools to supplement their services that may impact the access and abilities to use digital interventions in individuals with psychosis [100, 101].

\section{Conclusion}

This chapter explored the use of internet-based eHealth, app-based mHealth and virtual reality-based digital interventions to help individuals with psychosis. These differing types of digital interventions have been found to offer wide-ranging functionalities and therapeutic components, including remote communication, access to evidence-based information, digital self-monitoring and feedback as well as real-world and real-time delivery of psychological interventions. Overall, there is accumulating evidence on feasibility, acceptability and therapeutic effects of digital interventions for psychosis, especially if interventions are embedded in a therapeutic context (i.e. blended care approach). Thus, digital interventions signal great promise to help individuals who are experiencing psychotic symptoms and other commonly co-occurring mental health problems.

For most digital interventions described in this chapter, the theoretical base has been clearly stated and recent empirical evidence provided. Most interventions 
included intervention components that were informed by evidence-based recommendations and clinical guidelines (e.g. published by the National Institute for Health and Excellence, NICE) and primarily offered psycho-social interventions such as CBT, third-wave CBT and ACT. There were also a number of studies explicitly targeting cognitive factors described in integrated models of psychosis $[82,102-104]$. Nevertheless, despite the promising results from clinical research on the use of these technologies described in this chapter, there are still important challenges to overcome. Lack of large randomized controlled trials, efforts to bridge the gap between research and clinical practice and the inclusion of the users' experience as an essential part of the interventions' evaluation, are among these challenges for the successful implementation of digital interventions for psychosis. To overcome these challenges, more efforts from the clinical research community and mental health services are needed in order to train clinicians to routinely offer, use and master digital interventions. There is also a need to further expand our knowledge on long-term effects, cost-effectiveness and the theoretical foundation of digital interventions, particularly on putative mediators and mechanisms of change and their potential causal link in the development and persistence of psychosis. 


\section{References}

1. Charlson, F.J., et al., Global Epidemiology and Burden of Schizophrenia: Findings From the Global Burden of Disease Study 2016. Schizophr Bull, 2018. 44(6): p. 1195-1203.

2. Fusar-Poli, P., P.D. McGorry, and J.M. Kane, Improving outcomes of first-episode psychosis: an overview. World Psychiatry, 2017. 16(3): p. 251-265.

3. Legge, S.E., et al., Association of Genetic Liability to Psychotic Experiences With Neuropsychotic Disorders and Traits. JAMA Psychiatry, 2019.

4. Kane, J.M., T. Kishimoto, and C.U. Correll, Non-adherence to medication in patients with psychotic disorders: epidemiology, contributing factors and management strategies. World Psychiatry, 2013. 12(3): p. 216-26.

5. Franz, L., et al., Stigma and treatment delay in first-episode psychosis: a grounded theory study. Early Interv Psychiatry, 2010. 4(1): p. 47-56.

6. Clement, S., et al., What is the impact of mental health-related stigma on help-seeking? A systematic review of quantitative and qualitative studies. Psychol Med, 2015. 45(1): p. 11-27.

7. Gronholm, P.C., et al., Mental health-related stigma and pathways to care for people at risk of psychotic disorders or experiencing first-episode psychosis: a systematic review. Psychol Med, 2017. 47(11): p. 1867-1879.

8. Penttila, M., et al., Duration of untreated psychosis as predictor of long-term outcome in schizophrenia: systematic review and meta-analysis. Br J Psychiatry, 2014. 205(2): p. 88-94.

9. McGorry, P.D., A. Ratheesh, and B. O'Donoghue, Early Intervention-An Implementation Challenge for 21st Century Mental Health Care. JAMA Psychiatry, 2018. 75(6): p. 545546.

10. van Os, J. and U. Reininghaus, Psychosis as a transdiagnostic and extended phenotype in the general population. World Psychiatry, 2016. 15(2): p. 118-24.

11. Malla, A., et al., From early intervention in psychosis to youth mental health reform: a review of the evolution and transformation of mental health services for young people. Soc Psychiatry Psychiatr Epidemiol, 2016. 51(3): p. 319-26.

12. Bhugra, D., et al., The WPA-Lancet Psychiatry Commission on the Future of Psychiatry. Lancet Psychiatry, 2017. 4(10): p. 775-818.

13. Bucci, S., M. Schwannauer, and N. Berry, The digital revolution and its impact on mental health care. Psychol Psychother, 2019. 92(2): p. 277-297.

14. Firth, J., et al., Mobile Phone Ownership and Endorsement of "mHealth" Among People With Psychosis: A Meta-analysis of Cross-sectional Studies. Schizophr Bull, 2016. 42(2): p. 448-55.

15. Myin-Germeys, I., et al., Ecological momentary interventions in psychiatry. Curr Opin Psychiatry, 2016. 29(4): p. 258-63.

16. Hariman, K., A. Ventriglio, and D. Bhugra, The Future of Digital Psychiatry. Curr Psychiatry Rep, 2019. 21(9): p. 88.

17. Rus-Calafell, M. and S. Schneider, Are we there yet?!-a literature review of recent digital technology advances for the treatment of early psychosis. Mhealth, 2020. 6: p. 3. 
18. Reininghaus, U., C.A. Depp, and I. Myin-Germeys, Ecological Interventionist Causal Models in Psychosis: Targeting Psychological Mechanisms in Daily Life. Schizophr Bull, 2016. 42(2): p. 264-9.

19. Mohr, D.C., et al., The behavioral intervention technology model: an integrated conceptual and technological framework for eHealth and mHealth interventions. J Med Internet Res, 2014. 16(6): p. e146.

20. Alvarez-Jimenez, M., et al., Online, social media and mobile technologies for psychosis treatment: a systematic review on novel user-led interventions. Schizophr Res, 2014. 156(1): p. 96-106.

21. Smoktunowicz, E., et al., Consensus statement on the problem of terminology in psychological interventions using the internet or digital components. Internet Interv, 2020. 21: p. 100331

22. Miralles, I., et al., Smartphone Apps for the Treatment of Mental Disorders: Systematic Review. JMIR Mhealth Uhealth, 2020. 8(4): p. e14897.

23. Valmaggia, L.R., F. Day, and M. Rus-Calafell, Using virtual reality to investigate psychological processes and mechanisms associated with the onset and maintenance of psychosis: a systematic review. Soc Psychiatry Psychiatr Epidemiol, 2016. 51(7): p. 921-36.

24. Freeman, D., et al., Virtual reality in the assessment, understanding, and treatment of mental health disorders. Psychol Med, 2017. 47(14): p. 2393-2400.

25. Rauschenberg, C., et al., [Innovative digital forms of service delivery for personalized crisis resolution and home treatment for people with severe mental health problems]. Nervenarzt, 2021.

26. Bell, I.H. and M. Alvarez-Jimenez, Digital Technology to Enhance Clinical Care of Early Psychosis. Current Treatment Options in Psychiatry, 2019. 6(3): p. 256-270.

27. Pennou, A., et al., Mobile Intervention for Individuals With Psychosis, Dual Disorders, and Their Common Comorbidities: A Literature Review. Front Psychiatry, 2019. 10: p. 302.

28. Clarke, S., et al., A systematic review and meta-analysis of digital health technologies effects on psychotic symptoms in adults with psychosis. Psychosis, 2019. 11(4): p. 362373.

29. Valmaggia, L.R., et al., Virtual reality in the psychological treatment for mental health problems: An systematic review of recent evidence. Psychiatry Res, 2016. 236: p. 189195.

30. Riva, G. and S. Serino, Virtual Reality in the Assessment, Understanding and Treatment of Mental Health Disorders. J Clin Med, 2020. 9(11).

31. Rauschenberg, C., et al., Digital interventions to mitigate the negative impact of the COVID-19 pandemic on public mental health: a rapid meta-review. J Med Internet Res, 2021. 23: e23365.

32. Camacho, E., L. Levin, and J. Torous, Smartphone Apps to Support Coordinated Specialty Care for Prodromal and Early Course Schizophrenia Disorders: Systematic Review. J Med Internet Res, 2019. 21(11): p. e16393.

33. Rus-Calafell, M., et al., Virtual reality in the assessment and treatment of psychosis: a systematic review of its utility, acceptability and effectiveness. Psychol Med, 2018. 48(3): p. 362-391. 
34. Spaniel, F., et al., ITAREPS: information technology aided relapse prevention programme in schizophrenia. Schizophr Res, 2008. 98(1-3): p. 312-7.

35. Bucci, S., et al., Early Psychosis Service User Views on Digital Technology: Qualitative Analysis. JMIR Ment Health, 2018. 5(4): p. e10091.

36. Rotondi, A.J., et al., Web-Based Psychoeducational Intervention for Persons With Schizophrenia and Their Supporters: One-Year Outcomes. Psychiatr Serv, 2010. 61(11): p. 1099-1105.

37. Alvarez-Jimenez, M., et al., On the HORYZON: moderated online social therapy for longterm recovery in first episode psychosis. Schizophr Res, 2013. 143(1): p. 143-9.

38. Alvarez-Jimenez, M., et al., Enhancing social functioning in young people at Ultra High Risk (UHR) for psychosis: A pilot study of a novel strengths and mindfulness-based online social therapy. Schizophr Res, 2018. 202: p. 369-377.

39. Laine, A., et al., Feasibility, Acceptability, and Preliminary Impacts of Web-Based Patient Education on Patients With Schizophrenia Spectrum Disorder: Quasi-Experimental Cluster Study. J Med Internet Res, 2019. 21(10): p. e13073.

40. Ben-Zeev, D., et al., Feasibility, Acceptability, and Preliminary Efficacy of a Smartphone Intervention for Schizophrenia. Schizophrenia Bulletin, 2014. 40(6): p. 1244-1253.

41. Ben-Zeev, D., et al., Mobile Health (mHealth) Versus Clinic-Based Group Intervention for People With Serious Mental Illness: A Randomized Controlled Trial. Psychiatr Serv, 2018. 69(9): p. 978-985.

42. Ben-Zeev, D., et al., mHealth for Schizophrenia: Patient Engagement With a Mobile Phone Intervention Following Hospital Discharge. JMIR Ment Health, 2016. 3(3): p. e34.

43. Schlosser, D., et al., Feasibility of PRIME: A Cognitive Neuroscience-Informed Mobile App Intervention to Enhance Motivated Behavior and Improve Quality of Life in Recent Onset Schizophrenia. JMIR Res Protoc, 2016. 5(2): p. e77.

44. Schlosser, D.A., et al., Efficacy of PRIME, a Mobile App Intervention Designed to Improve Motivation in Young People With Schizophrenia. Schizophr Bull, 2018. 44(5): p. $1010-$ 1020.

45. Kim, S.W., et al., Development and feasibility of smartphone application for cognitivebehavioural case management of individuals with early psychosis. Early Interv Psychiatry, 2018. 12(6): p. 1087-1093.

46. Niendam, T.A., et al., Enhancing early psychosis treatment using smartphone technology: A longitudinal feasibility and validity study. J Psychiatr Res, 2018. 96: p. 239-246.

47. Bucci, S., et al., Actissist: Proof-of-Concept Trial of a Theory-Driven Digital Intervention for Psychosis. Schizophr Bull, 2018. 44(5): p. 1070-1080.

48. Steare, T., et al., Smartphone-delivered self-management for first-episode psychosis: the ARIES feasibility randomised controlled trial. BMJ Open, 2020. 10(8): p. e034927.

49. Depp, C.A., et al., Single-Session Mobile-Augmented Intervention in Serious Mental Illness: A Three-Arm Randomized Controlled Trial. Schizophr Bull, 2019. 45(4): p. $752-$ 762.

50. Lim, M.H., et al., A pilot digital intervention targeting loneliness in young people with psychosis. Soc Psychiatry Psychiatr Epidemiol, 2020. 55(7): p. 877-889.

51. Lim, M.H., et al., A Pilot Digital Intervention Targeting Loneliness in Youth Mental Health. Front Psychiatry, 2019. 10: p. 604. 
52. Garety, P.A., et al., SlowMo, a digital therapy targeting reasoning in paranoia, versus treatment as usual in the treatment of people who fear harm from others: study protocol for a randomised controlled trial. Trials, 2017. 18(1): p. 510.

53. Hardy, A., et al., How Inclusive, User-Centered Design Research Can Improve Psychological Therapies for Psychosis: Development of SlowMo. JMIR Ment Health, 2018. 5(4): p. e11222.

54. Garety, P.A., T. Ward, and M. Rus-Calafell, Chapter 25 - Beyond Belief-New Approaches to the Treatment of Paranoia, in A Clinical Introduction to Psychosis, J.C. Badcock and G. Paulik, Editors. 2020, Academic Press. p. 591-613.

55. Ruegg, N., et al., An internet-based intervention for people with psychosis (EviBaS): study protocol for a randomized controlled trial. BMC Psychiatry, 2018. 18(1): p. 102.

56. Gumley, A., et al., Early Signs Monitoring to Prevent Relapse in Psychosis and Promote Well-Being, Engagement, and Recovery: Protocol for a Feasibility Cluster Randomized Controlled Trial Harnessing Mobile Phone Technology Blended With Peer Support. JMIR Res Protoc, 2020. 9(1): p. e15058.

57. Allan, S., et al., Understanding Implementation of a Digital Self-Monitoring Intervention for Relapse Prevention in Psychosis: Protocol for a Mixed Method Process Evaluation. JMIR Res Protoc, 2019. 8(12): p. e15634.

58. McEnery, C., et al., Development of a Moderated Online Intervention to Treat Social Anxiety in First-Episode Psychosis. Front Psychiatry, 2019. 10: p. 581.

59. Traber-Walker, N., et al., Evaluation of the Combined Treatment Approach "Robin" (Standardized Manual and Smartphone App) for Adolescents at Clinical High Risk for Psychosis. Front Psychiatry, 2019. 10: p. 384.

60. Vitger, T., et al., The Momentum trial: the efficacy of using a smartphone application to promote patient activation and support shared decision making in people with a diagnosis of schizophrenia in outpatient treatment settings: a randomized controlled single-blind trial. BMC Psychiatry, 2019. 19(1): p. 185.

61. Jongeneel, A., et al., Reducing distress and improving social functioning in daily life in people with auditory verbal hallucinations: study protocol for the 'Temstem' randomised controlled trial. BMJ Open, 2018. 8(3): p. e020537.

62. Bell, I.H., et al., Virtual reality as a clinical tool in mental health research and practice. Dialogues Clin Neurosci, 2020. 22(2): p. 169-177.

63. Valmaggia, L.R., et al., Virtual reality and paranoid ideations in people with an 'at-risk mental state' for psychosis. Br J Psychiatry Suppl, 2007. 51: p. s63-8.

64. Riches, S., et al., Subjective experience of paranoid ideation in a virtual reality social environment: A mixed methods cross-sectional study. Clin Psychol Psychother, 2020. 27(3): p. 337-345.

65. Riva, G., Virtual Reality in Clinical Psychology, in Reference Module in Neuroscience and Biobehavioral Psychology. 2022.

66. Deng, W., et al., The efficacy of virtual reality exposure therapy for PTSD symptoms: A systematic review and meta-analysis. J Affect Disord, 2019. 257: p. 698-709.

67. Clus, D., et al., The Use of Virtual Reality in Patients with Eating Disorders: Systematic Review. J Med Internet Res, 2018. 20(4): p. e157. 
68. Park, M.J., et al., A Literature Overview of Virtual Reality (VR) in Treatment of Psychiatric Disorders: Recent Advances and Limitations. Front Psychiatry, 2019. 10: p. 505.

69. van Bennekom, M.J., P.P. de Koning, and D. Denys, Virtual Reality Objectifies the Diagnosis of Psychiatric Disorders: A Literature Review. Front Psychiatry, 2017. 8: p. 163.

70. Freeman, D., et al., Virtual reality in the treatment of persecutory delusions: randomised controlled experimental study testing how to reduce delusional conviction. $\mathrm{Br}$ J Psychiatry, 2016. 209(1): p. 62-7.

71. Pot-Kolder, R., et al., Virtual-reality-based cognitive behavioural therapy versus waiting list control for paranoid ideation and social avoidance in patients with psychotic disorders: a single-blind randomised controlled trial. Lancet Psychiatry, 2018. 5(3): p. 217-226.

72. Nijman, S.A., et al., Dynamic Interactive Social Cognition Training in Virtual Reality (DiSCoVR) for social cognition and social functioning in people with a psychotic disorder: study protocol for a multicenter randomized controlled trial. BMC Psychiatry, 2019. 19(1): p. 272.

73. Nijman, S.A., et al., Dynamic Interactive Social Cognition Training in Virtual Reality (DiSCoVR) for People With a Psychotic Disorder: Single-Group Feasibility and Acceptability Study. JMIR Ment Health, 2020. 7(8): p. e17808.

74. Vass, E., et al., A novel virtual reality-based Theory of Mind intervention for outpatients with schizophrenia: a proof of concept pilot study. Clin Psychol Psychother, 2020.

75. Craig, T.K.J., et al., AVATAR therapy for auditory verbal hallucinations in people with psychosis: a single-blind, randomised controlled trial. The Lancet Psychiatry, 2018. 5(1): p. 31-40.

76. Leff,J., et al., Computer-assisted therapy for medication-resistant auditory hallucinations: proof-of-concept study. Br J Psychiatry, 2013. 202: p. 428-33.

77. du Sert, O.P., et al., Virtual reality therapy for refractory auditory verbal hallucinations in schizophrenia: A pilot clinical trial. Schizophr Res, 2018. 197: p. 176-181.

78. Rus-Calafell, M., et al., The Role of Sense of Voice Presence and Anxiety Reduction in AVATAR Therapy. J Clin Med, 2020. 9(9).

79. Freeman, D., et al., Automated virtual reality (VR) cognitive therapy for patients with psychosis: study protocol for a single-blind parallel group randomised controlled trial (gameChange). BMJ Open, 2019. 9(8): p. e031606.

80. Freeman, D., et al., Automated psychological therapy using virtual reality (VR) for patients with persecutory delusions: study protocol for a single-blind parallel-group randomised controlled trial (THRIVE). Trials, 2019. 20(1): p. 87.

81. Riches, S., et al., Using Virtual Reality to Assess Associations Between Paranoid Ideation and Components of Social Performance: A Pilot Validation Study. Cyberpsychol Behav Soc Netw, 2019. 22(1): p. 51-59.

82. Freeman, D., Persecutory delusions: a cognitive perspective on understanding and treatment. Lancet Psychiatry, 2016. 3(7): p. 685-92.

83. Myin-Germeys, I., et al., Experience sampling methodology in mental health research: new insights and technical developments. World Psychiatry, 2018. 17(2): p. 123-132.

84. Myin-Germeys, I. and J. van Os, Stress-reactivity in psychosis: evidence for an affective pathway to psychosis. Clin Psychol Rev, 2007. 27(4): p. 409-24. 
85. Reininghaus, U., et al., Stress Sensitivity, Aberrant Salience, and Threat Anticipation in Early Psychosis: An Experience Sampling Study. Schizophr Bull, 2016. 42(3): p. 712-22.

86. Heron, K.E. and J.M. Smyth, Ecological momentary interventions: incorporating mobile technology into psychosocial and health behaviour treatments. $\mathrm{Br} J$ Health Psychol, 2010. 15(Pt 1): p. 1-39.

87. Reininghaus, U., et al., Efficacy of Acceptance and Commitment Therapy in Daily Life (ACT-DL) in early psychosis: study protocol for a multi-centre randomized controlled trial. Trials, 2019. 20(1): p. 769.

88. Vaessen, T., et al., ACT in daily life in early psychosis: an ecological momentary intervention approach. Psychosis, 2019. 11(2): p. 93-104.

89. Rauschenberg, C., et al., An ecological momentary compassion-focused intervention for enhancing resilience in help-seeking youths: a pilot study. JMIR Mental Health, 2021. 8(8): p. e25650.

90. Schick, A., et al., The effects of a novel, accessible, transdiagnostic ecological momentary intervention for improving resilience in youth (EMIcompass): study protocol for a randomized controlled trial. 2020: PsyArXiv.

91. Fairburn, C.G. and V. Patel, The impact of digital technology on psychological treatments and their dissemination. Behav Res Ther, 2017. 88: p. 19-25.

92. Erbe, D., et al., Blending Face-to-Face and Internet-Based Interventions for the Treatment of Mental Disorders in Adults: Systematic Review. J Med Internet Res, 2017. 19(9): p. e306.

93. Biagianti, B., S.H. Quraishi, and D.A. Schlosser, Potential Benefits of Incorporating Peerto-Peer Interactions Into Digital Interventions for Psychotic Disorders: A Systematic Review. Psychiatr Serv, 2018. 69(4): p. 377-388.

94. Aref-Adib, G., et al., Factors affecting implementation of digital health interventions for people with psychosis or bipolar disorder, and their family and friends: a systematic review. Lancet Psychiatry, 2019. 6(3): p. 257-266.

95. Torous, J., et al., Dropout rates in clinical trials of smartphone apps for depressive symptoms: A systematic review and meta-analysis. J Affect Disord, 2020. 263: p. 413419.

96. Edwards, E.A., et al., Gamification for health promotion: systematic review of behaviour change techniques in smartphone apps. BMJ Open, 2016. 6(10): p. e012447.

97. van Os, J., et al., The evidence-based group-level symptom-reduction model as the organizing principle for mental health care: time for change? World Psychiatry, 2019. 18(1): p. 88-96.

98. Hollis, C., et al., Identifying research priorities for digital technology in mental health care: results of the James Lind Alliance Priority Setting Partnership. Lancet Psychiatry, 2018. 5(10): p. 845-854.

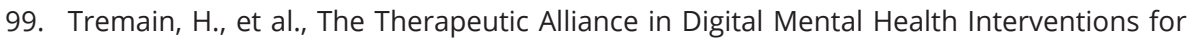
Serious Mental Illnesses: Narrative Review. JMIR Ment Health, 2020. 7(8): p. e17204.

100. Luther, L., et al., Examining Potential Barriers to mHealth Implementation and Engagement in Schizophrenia: Phone Ownership and Symptom Severity. Journal of Technology in Behavioral Science, 2020. 
101. Bernard, R., C. Sabariego, and A. Cieza, Barriers and Facilitation Measures Related to People With Mental Disorders When Using the Web: A Systematic Review.J Med Internet Res, 2016. 18(6): p. e157.

102. Garety, P.A., et al., Implications for neurobiological research of cognitive models of psychosis: a theoretical paper. Psychol Med, 2007. 37(10): p. 1377-91.

103. Howes, O.D. and R.M. Murray, Schizophrenia: an integrated sociodevelopmentalcognitive model. Lancet, 2014. 383(9929): p. 1677-1687.

104. Morrison, A.P., The Interpretation of Intrusions in Psychosis: An Integrative Cognitive Approach to Hallucinations and Delusions. Behavioural and Cognitive Psychotherapy, 2002. 29(3): p. 257-276. 



\section{CHAPTER 9}

\section{Social isolation, mental health, and use of digital interventions in youth during the COVID-19 pandemic: a nationally representative survey}

Christian Rauschenberg*, Anita Schick*, Christian Goetzl, Susanne Roehr, Steffi G. Riedel-Heller, Georgia Koppe, Daniel Durstewitz, Silvia Krumm, Ulrich Reininghaus

* These authors share first authorship European Psychiatry. 2021; 64(1), E20. Doi: 10.1192/j.eurpsy.2021.17 


\section{Abstract}

Background: Public health measures to curb SARS-CoV-2 transmission rates may have negative psychosocial consequences in youth. Digital interventions may help to mitigate these effects. We investigated the associations between social isolation, COVID-19-related cognitive preoccupation, worries, and anxiety, objective social risk indicators, psychological distress as well as use of, and attitude towards, mobile health (mHealth) interventions in youth.

Methods: Data were collected as part of the 'Mental Health And Innovation During COVID-19 Survey' - a cross-sectional panel study including a representative sample of individuals aged 16 to 25 years $\left(N=666 ; M_{\text {age }} 21 \cdot 3\right)$ (assessment period: 07.05.16.05.2020).

Results: Overall, $38 \%$ of youth met criteria for moderate or severe psychological distress. Social isolation worries and anxiety, and objective risk indicators were associated with psychological distress, with evidence of dose-response relationships for some of these associations. For instance, psychological distress was progressively more likely to occur as levels of social isolation increased (reporting 'never' as reference group: 'occasionally': adjusted odds ratio [aOR] 9.1, 95\% confidence interval [CI] 4.3 - 19.1, $p<0.001$; 'often': aOR 22.2, Cl 9.8 $50.2, p<0.001$;'very often': aOR 42.3, Cl 14.1 - 126.8, $p<0.001)$. There was evidence that psychological distress, worries, and anxiety were associated with a positive attitude towards using mHealth interventions, whereas psychological distress, worries, and anxiety were associated with actual use.

Conclusions: Public health measures during pandemics may be associated with poor mental health outcomes in youth. Evidence-based digital interventions may help mitigate the negative psychosocial impact without risk of viral infection given there is an objective need and subjective demand.

Keywords: COVID-19; Youth Mental Health; mHealth; Social isolation; Social risk 


\section{Introduction}

As of March 2020, most European countries have adopted a range of public health measures to lower the transmission of SARS-CoV-2 coronavirus. Physical distancing and quarantine have been amongst the most important non-pharmacological measures to reduce infection rates of coronavirus disease 2019 (COVID-19). These preventive measures, however, may have a profound impact on public mental health. Studies investigating the psychosocial impact of earlier pandemics (e.g. SARS, MERS) have shown that physical distancing and quarantine have immediate as well as prolonged effects on individuals' mental health, including depression, anxiety, psychosis, and perceived stress [1-5]. Further, it has been found that these safety measures are associated with an increase of more distal risk factors for poor mental health such as social isolation, risk behaviours (e.g. cannabis and alcohol misuse), and lowered physical activity [1]. In line with findings on earlier outbreaks, accumulating evidence suggests negative psychosocial consequences of the current COVID-19 pandemic on public mental health, including increased levels of depression, anxiety, self-harm, and loneliness [6-27]. Although the increasing number of approved vaccines and potential breakthroughs in the pharmacological treatment of COVID-19 are reasons for optimism, health related outcomes may worsen at any time due to new virus variants (e.g. lineage B.1.1.7 or B.1.351) as well as economic uncertainties and recession which may occur secondary to the pandemic.

There is also evidencethat the detrimental effects of pandemicsare disproportionally distributed across communities: societal inequalities have been found to increase the risk of COVID-19 on various health domains. Those with inferior social position, for instance, have been found to have increased disease fatality and hospital admission rates as well as to experience more severe psychosocial and economic consequences [28] and initial findings from the UK suggest inequalities in adverse experiences during the early weeks of the lockdown [29]. Other studies have found that individuals with histories of migration and unemployment experience more severe depressive and anxiety symptoms, especially in youth [30].

Information and communication technologies may be particularly important in alleviating COVID-19-related psychosocial consequences [31]. For instance, smartphone applications (apps) help individuals to remotely interact with others 
(e.g. by using video conferencing software) and digital interventions, which do not require face-to-face contact (e.g. internet-based interventions [eHealth] and mobile health applications [mHealth apps]), may help to increase public mental health during health crises [31]. Previous studies have shown that digital tools available in major app stores, especially mHealth apps, are already frequently being used although most developers do not provide information on their evidence base, safety, and effectiveness [32-34]. While, in contrast, eHealth and mHealth interventions that have been developed and evaluated by research groups signal great promise on their safety, acceptability, and effectiveness across the whole spectrum of public mental health provision (i.e., mental health promotion, prevention and treatment of mental disorders), especially if embedded in social and therapeutic contexts (e.g. peer-support, blended care) $[31,35]$. Thus, although mHealth apps available in app stores should be used with caution, digital interventions may be used to mitigate the negative impact of the COVID-19 pandemic [31]. If used purposefully, these tools may help to provide lowthreshold, timely, and personalized public mental health care and can be tailored to the individual needs - even under the restrictive conditions of the COVID-19 pandemic and without the risk of viral infection [31, 36-38]. However, to the best of our knowledge, there has been no study to date which has specifically investigated the role of publicly available mHealth apps during public health crises, including the current COVID-19 pandemic, and the available evidence on the occurrence of psychological distress in young individuals and important correlates remain very limited.

In the current study, we aimed to investigate the associations between social isolation, COVID-19-related cognitive preoccupation, worries, and anxiety, objective social risk indicators and psychological distress as well as use of, and attitude towards, digital mHealth apps in a representative sample of youth aged 16-25 from the general population during the COVID-19 pandemic. Specifically, we sought to test the following hypotheses: First, (a) social isolation and lack of company, (b) COVID-19-related cognitive preoccupation, worries, and anxiety, and (c) objective indicators of social risk (e.g. unemployment, migrant or ethnic minority group position) are associated with occurrence of psychological distress. Second, these associations are consistent with a dose-response pattern. Third, current use of, and positive attitudes towards, mHealth apps are more common in those who experience psychological distress, more frequent social isolation and 
lack of company, COVID-19-related preoccupation, worries, and anxiety, and who are exposed to more objective indicators of social risk.

\section{Methods}

\section{Design and participants}

Data were drawn from the 'Mental Health And Innovation During COVID-19 Survey' - a cross-sectional panel study. This study was conducted as part of a living lab entitled "AI4U - Artificial Intelligence for personalized digital mental health promotion and prevention in youth", which aims to develop, optimize, evaluate, and implement digital Al-based interventions in routine public mental health provision by adopting a transdisciplinary approach involving users from the target population and relevant stakeholders in all stages of the research process. We recruited a representative sample of youth aged 16-25 from the German general population. The study commenced on May $7^{\text {th }}$ and was completed on May $16^{\text {th }}, 2020$. Thus, data were collected at times of active lockdown measures to lower transmission rates. More specifically, during this time period, regionspecific measures were enacted to prevent the spread of SARS-CoV-2, including the closure of schools, kindergartens, playgrounds, zoos, churches, sports clubs, services that require close physical contact (e.g. hairdressers), and non-essential shops. In addition, it was forbidden to leave the house without a good reason, and it was only allowed to have contact with one other person not living in the same household. Furthermore, keeping a physical distance of $1.5 \mathrm{~m}$ and wearing face masks in public places as well as in public transportation was obligatory. Also, in order to reduce the effects of these measures on the population and the economy, many companies received state aid to be able to pay for running costs (e.g. personnel costs, rent).

For data collection, we used the Norstatpanel by Norstat Deutschland GmbH [39], which consists of a group of registered internet users who have agreed to take part in surveys and opinion polls and is certified according to ISO 26362 and ISO 9001 standards. To ensure the high quality of the panel, various quality assurance measures have been implemented and are frequently evaluated, such as, random selection, representativeness, diversified sources, and active recruitment of panellists as well as the absence of a public registration page, profile validation, plausibility testing, and cheater detection. The online panel operates in accordance 
with the applicable data protection laws (i.e., EU General Data Protection Regulation (GDPR); Federal Data Protection Act (BDSG)). Prior to assessments, informed consent was obtained from participants by Norstat in this general population sample. Participants were registered members of the Norstatpanel and selected at random. Selected individuals were invited by email to participate in the online survey. To ensure representativeness of the sample, individuals were stratified by gender, education, and population density data published by the Federal Statistical Office of Germany. Participation was incentivised through payments (i.e., around $0.10 €$ per minute) and other benefits (e.g. discounts). All procedures performed in studies involving human participants were in accordance with the ethical standards of the institutional and/or national research cofmmittee and with the 1964 Helsinki Declaration and its later amendments or comparable ethical standards. The study was approved by the Medical Ethics Review Committee II of Heidelberg University (Medical Faculty Mannheim; Ref. No. 529-20).

\section{Measures}

\section{Social isolation/lack of company}

Social isolation and lack of company were assessed using two items of Three-Item Loneliness Scale which has been developed based on the 20-item Revised UCLA Loneliness Scale [40] and has been specifically developed to assess loneliness in large-scale surveys [41]. However, as we were interested in measuring social isolation and lack of company, we excluded one item assessing the feeling of being left out. Subjective experiences of social isolation ('How often do you feel socially isolated?') and lack of company ('How often do you feel that you lack the company of others?') were both rated on a 6-point Likert scale ranging from 1 to 6 (1='never', $3={ }^{\prime}$ rarely', $6={ }^{\prime}$ very often'). A high internal consistency has been demonstrated for different versions of the UCLA Loneliness Scale, including the Three-Item Loneliness Scale [41, 42].

\section{COVID-19-related cognitive preoccupation, worries, and anxiety}

COVID-19-related cognitive preoccupation, worries, and anxiety were assessed using modified items from the COVID-19 Snapshot MOnitoring (COSMO) [30] survey in Germany. First, worries were assessed using 10 items introduced by the following sentence: 'On a scale from 1 (no worries at all) to 7 (a lot of worries), how 
often did you worry last week that...' which was followed by differing types of worries (e.g. about financial difficulties). For current analyses, we computed the overall mean score (Cronbach's alpha, $a=0.80$ ). We also dichotomized the continuous score using median split of the continuous variable: $<50$. percentile was coded as 0 and $\geq 50$. percentile coded as 1 ). Second, preoccupation and anxiety with the COVID-19 pandemic were assessed using three separate items rated on a 5-point scale ('The novel coronavirus is something I...', with ratings ranging from 1 '...never think of' to 5 '...keep thinking about'; 'The novel coronavirus is...', with ratings ranging from 1 '...not scary at all' to 5 '....scary'; 'The novel coronavirus is...', with ratings ranging from 1 '...not worrying' to 5 '...worrying').

\section{Objective social risk indicators}

Data on objective indicators of individuals' social circumstances and migrant/ ethnic minority group position were assessed using a modified version of the Medical Research Council (MRC) Sociodemographic Schedule [43]. In total, six domains of social risk were included in the current study: (1) employment, (2) education, (3) relationship status, (4) living arrangements, (5) parental educational level as a proxy for lower socioeconomic status, (6) migrant or ethnic minority group position. To investigate the impact of social risk, we built on the work by Morgan et al.[44], and created an index by dichotomizing variables from each of the six domains to define the presence or absence of well-established indicators of social risk (i.e., [1] unemployment, unable to work, early retirement $=1$, other $=0$; [2] lower educational level [i.e., secondary school, no school-leaving qualification]=1, other=0; [3] being single=1, other=0; [4] living alone or alone with children=1, other=0; [5] lower parental educational level as a proxy for lower socioeconomic status [i.e., secondary school, no school-leaving qualification of both parents]=1, other $=0$; [6] foreign born or second generation migrant $=1$, other $=0$ ). This generated an index ranging from 0 to 6 .

\section{Current use of, and attitudes towards, mHealth apps}

After providing a definition of mHealth apps participants were asked whether they are already using mHealth apps by asking the following question 'Do you already use mHealth apps (e.g. to relax or increase physical activity)?'. This item was rated on a 6 -point Likert scale and dichotomized (a rating of $1=$ 'never' coded as 0 , and 
ratings of 2 ='very rarely', $4==^{\prime}$ occasionally' to $6==^{\prime}$ very often' were coded as 1 ). This item was followed by an item assessing the positive, negative, or neutral attitude towards the use of mHealth apps to help cope with the COVID-19 pandemic ('Do you think that an mHealth app could help you deal better with the corona situation?'). This item was rated on a 5-point Likert scale and dichotomized (with ratings of $1=$ 'strongly disagree' to $3={ }^{\prime}$ neither/nor' coded as 0 , and ratings of $4={ }^{\prime a g r e e}$ to $5=$ 'strongly agree' coded as 1 ).

\section{Psychological distress}

The Kessler-10 (K10) [45], a well-established screening instrument for mental disorder in the general population, was used to assess psychological distress. The questionnaire was modified to assess psychological distress during the COVID-19 pandemic. That is, instead of asking about psychological distress experienced in the past 30 days, psychological distress experienced since the beginning of the pandemic was assessed ('How often did you feel since the coronavirus outbreak...' followed e.g. by '...tired out for no good reason?). The 10 items were rated on a 5-point scale ( $1={ }^{\prime}$ none of the time', $3=$ 'some of the time', $5=$ 'all of the time'), yielding a minimum score of 10 and a maximum score of 50 (Cronbach's alpha, $a=0.93$ ). For analyses including psychological distress as dependent variable, we dichotomized continuous distress scores based on an established cut-off score (absence of psychological distress: scores from 10-19 were coded as 0; presence of mild, moderate or severe psychological distress: scores from 20-50 were coded as 1). Good psychometric properties have been reported for this measure [46]. For analyses in which psychological distress was used as independent variable we used a categorical variable with four levels: 1) likely to be well (range score: 10 to 19), 2) mild mental disorder (range score: 20-24), 3) moderate mental disorder (range score: 25-29), 4) severe mental disorder (range score: 30-50), again, based on established and validated cut-off scores $[45,47]$.

\section{Analysis}

Descriptive statistics were used to report on basic sample characteristics. Logistic regression was used to, first, quantify the association of (1) social isolation/lack of company, (2) COVID-19-related cognitive preoccupation, worrying, and anxiety, and (3) objective indicators of social risk (separate as well as combined in form 
of a social risk index) as independent variables with psychological distress as outcome variable (hypotheses 1). This approach allows for examining doseresponse relationships (hypotheses 2). Second, we investigated whether (1) psychological distress, (2) social isolation/lack of company, (3) COVID-19-related preoccupation, worries, and anxiety, and (4) the objective indicators of social risk as well as the social risk index are associated with the current use of, and attitude towards, mHealth apps (hypotheses 3). In all analyses, we adjusted for potential confounders (i.e., age, gender, educational level, migrant/ethnic minority group position, employment status), except in models that included social risk indicators as independent variable. Here, we adjusted for age and gender. We adjusted significance levels for Type-1 error proliferation using family-wise error-corrected $p$-values ( $p$ FWE) by multiplying the unadjusted $p$-value by the total number of independent variables ( $N=7$ for models with psychological distress as dependent variable; $N=8$ for models with the use of, or attitude towards, mHealth apps as dependent variable). All analyses were performed using STATA version 15.1.

\section{Results}

\section{Sample characteristics}

In total, 1006 individuals were invited by email to participate. Of these, 685 youths completed the online survey and 19 individuals had to be excluded after completion of quality control checks (e.g. implausible response time and pattern of responses). Thus, 666 individuals were included in current analyses. There were no differences in variables between individuals with and without sufficient data quality (data not shown, available upon request). The sample characteristics are presented in Table 1, including frequencies of all assessed variables.

\section{Social isolation and COVID-19-related preoccupation, worries, and anxiety by psychological distress}

Individuals who reported subjective experiences of social isolation, lack of company, and COVID-19-related worries and anxiety were more likely to experience psychological distress during the COVID-19 pandemic. As shown in Table 2, there was evidence for dose-response relationships in that psychological distress was progressively more likely to occur as levels of reported social isolation, lack of company, and COVID-19-related worries and anxiety increased. For example, those 
Table 1. Sample characteristics, psychological distress, and risk $(N=666)$

\begin{tabular}{|c|c|}
\hline $\begin{array}{l}\text { Age, mean } \\
\text { (S.D.; range) }\end{array}$ & $\begin{array}{c}21 \cdot 3 \\
(2 \cdot 6 ; 16-25)\end{array}$ \\
\hline \multicolumn{2}{|l|}{ Gender, n (\%) } \\
\hline Female & $318(47 \cdot 8)$ \\
\hline Male & $346(52 \cdot 0)$ \\
\hline Divers & $2(0 \cdot 3)$ \\
\hline \multicolumn{2}{|l|}{ Educational level, n (\%)a } \\
\hline Low & $135(20 \cdot 3)$ \\
\hline Middle & $358(53 \cdot 7)$ \\
\hline High & $173(26 \cdot 0)$ \\
\hline \multicolumn{2}{|l|}{ Migrant/ethnic minority group position, $\mathrm{n}(\%)$} \\
\hline $1^{\text {st }}$ generation migrant & $53(7 \cdot 9)$ \\
\hline $2^{\text {nd }}$ generation migrant & $156(23 \cdot 4)$ \\
\hline \multicolumn{2}{|l|}{ Psychological distress ${ }^{\mathrm{b}}, \mathrm{n}(\%)$} \\
\hline Likely to be well & $287(43 \cdot 1)$ \\
\hline Likely to have a mild disorder & $130(19 \cdot 5)$ \\
\hline Likely to have a moderate disorder & $104(15 \cdot 6)$ \\
\hline Likely to have a severe disorder & $145(21 \cdot 8)$ \\
\hline $\begin{array}{l}\text { Psychological distress, } \\
\text { mean (S.D.; range) }\end{array}$ & $\begin{array}{c}22 \cdot 0 \\
(8 \cdot 6 ; 10-50)\end{array}$ \\
\hline \multicolumn{2}{|l|}{ Social isolation, $\mathrm{n}(\%)$} \\
\hline Never & $61(9 \cdot 2)$ \\
\hline Very rarely & $65(9 \cdot 8)$ \\
\hline Rarely & $135(20 \cdot 3)$ \\
\hline Occasionally & $212(31 \cdot 8)$ \\
\hline Often & $136(20 \cdot 4)$ \\
\hline Very often & $57(8 \cdot 6)$ \\
\hline \multicolumn{2}{|l|}{ Lack of company, n (\%) } \\
\hline Never & $34(5 \cdot 1)$ \\
\hline Very rarely & $43(6 \cdot 5)$ \\
\hline Rarely & $81(12 \cdot 2)$ \\
\hline Occasionally & $245(36 \cdot 8)$ \\
\hline Often & $178(26 \cdot 7)$ \\
\hline Very often & $85(12 \cdot 3)$ \\
\hline \multicolumn{2}{|l|}{ COVID-19-related worries ', n (\%) } \\
\hline$<50^{\text {th }}$ percentile & $350(52 \cdot 6)$ \\
\hline$\geq 50^{\text {th }}$ percentile & $316(47 \cdot 4)$ \\
\hline Mean (S.D.; range) & $3 \cdot 6(1 \cdot 2 ; 1-7)$ \\
\hline \multicolumn{2}{|l|}{$\begin{array}{l}\text { Cognitive preoccupation with COVID-19 } \\
\text { Item: 'The novel coronavirus is something I...', n (\%) }\end{array}$} \\
\hline ... never think of & $20(3 \cdot 0)$ \\
\hline ... don't think about very often & $115(17 \cdot 3)$ \\
\hline$\ldots$ think in part & $245(36 \cdot 8)$ \\
\hline ... think about a lot & $245(36 \cdot 8)$ \\
\hline ... keep thinking about & $41(6 \cdot 2)$ \\
\hline
\end{tabular}


Table 1. (continued). Sample characteristics, psychological distress, and risk ( $N=666)$

COVID-19-related anxiety

Item: 'The novel coronavirus is...', n (\%)

... not scary at all
... rather not scary
... partly scary
... rather scary
... scary

$105(15 \cdot 8)$

$187(28 \cdot 1)$

$219(32 \cdot 9)$

$133(20 \cdot 0)$

$22(3 \cdot 3)$

COVID-19-related worrying

Item: 'The novel coronavirus is...', n (\%)

... not worrying

$70(10 \cdot 5)$

... rather not worrying

$142(21 \cdot 3)$

... partly worrying

$207(31 \cdot 1)$

... rather worrying

$207(31 \cdot 1)$

... worrying

$40(6 \cdot 0)$

Social risk index ${ }^{\mathrm{d}}$

0

$160(24 \cdot 0)$

1

$270(40 \cdot 5)$

2

$174(26 \cdot 1)$

$3+$

$62(9 \cdot 3)$

Current use of mHealth apps ${ }^{e}$

Yes

$473(71 \cdot 0)$

No

$193(29 \cdot 0)$

Attitude towards the use of mHealth apps

during the COVID-19 pandemic ${ }^{f}$

Positive

$170(25 \cdot 5)$

Neutral/Negative

$496(74 \cdot 5)$

Notes: S.D., standard deviation.

a Educational levels were defined as follows: 'low' (i.e. lower secondary school certificate, secondary school certificate, no school-leaving qualification, or visiting respective school types), 'middle' (i.e. high-school diploma, completed vocational training, or visiting respective school type/doing an apprenticeship), 'high' (i.e. bachelor's, master's degree, or currently studying).

${ }^{b}$ The following K10 cut-offs were used to categories severity levels of psychological distress: 'none' (range score: 10 to 19); 'mild' (range score: 20-24); 'moderate' (range score: 25-29); 'severe' (range score: 30-50).

'Based on 10 items asking for potential worries related to the COVID-19 pandemic (e.g., health system overloaded, financial difficulties, completion of education/school) during the last week rated on a 7-point Likert scale ( $1=$ 'no worries at all'; $7=^{\prime}$ a lot of worries'). Items were dichotomized (i.e. median split of the continues variable: $<50$. percentile was coded as 0 and $\geq 50$. percentile coded as 1 ).

${ }^{d}$ Defined as the number of objective indicators of social risk (i.e. unemployment/unable to work/early retirement; low education of both parents as a proxy for low socio-economic status; foreign born/second generation migration; living alone/alone with kids; being single; range social risk index: 0-6).

e Based on the following item 'Do you already use mHealth apps (e.g. to relax or increase physical activity)?' binary outcome variable: answering 'never' were coded as 0 , whereas answering 'very rarely', 'rarely', 'occasionally', 'often', or 'very often' were coded as 1.

${ }^{f}$ Based on the following item 'Do you think that an mHealth app could help you deal better with the corona situation?'. This item was rated on a 5-point Likert scale and dichotomized (with ratings of $1=$ 'strongly disagree' to $3={ }^{\prime}$ neither/nor' coded as 0 , and ratings of $4={ }^{\prime}$ agree' to $5=$ 'strongly agree' coded as 1 ). 


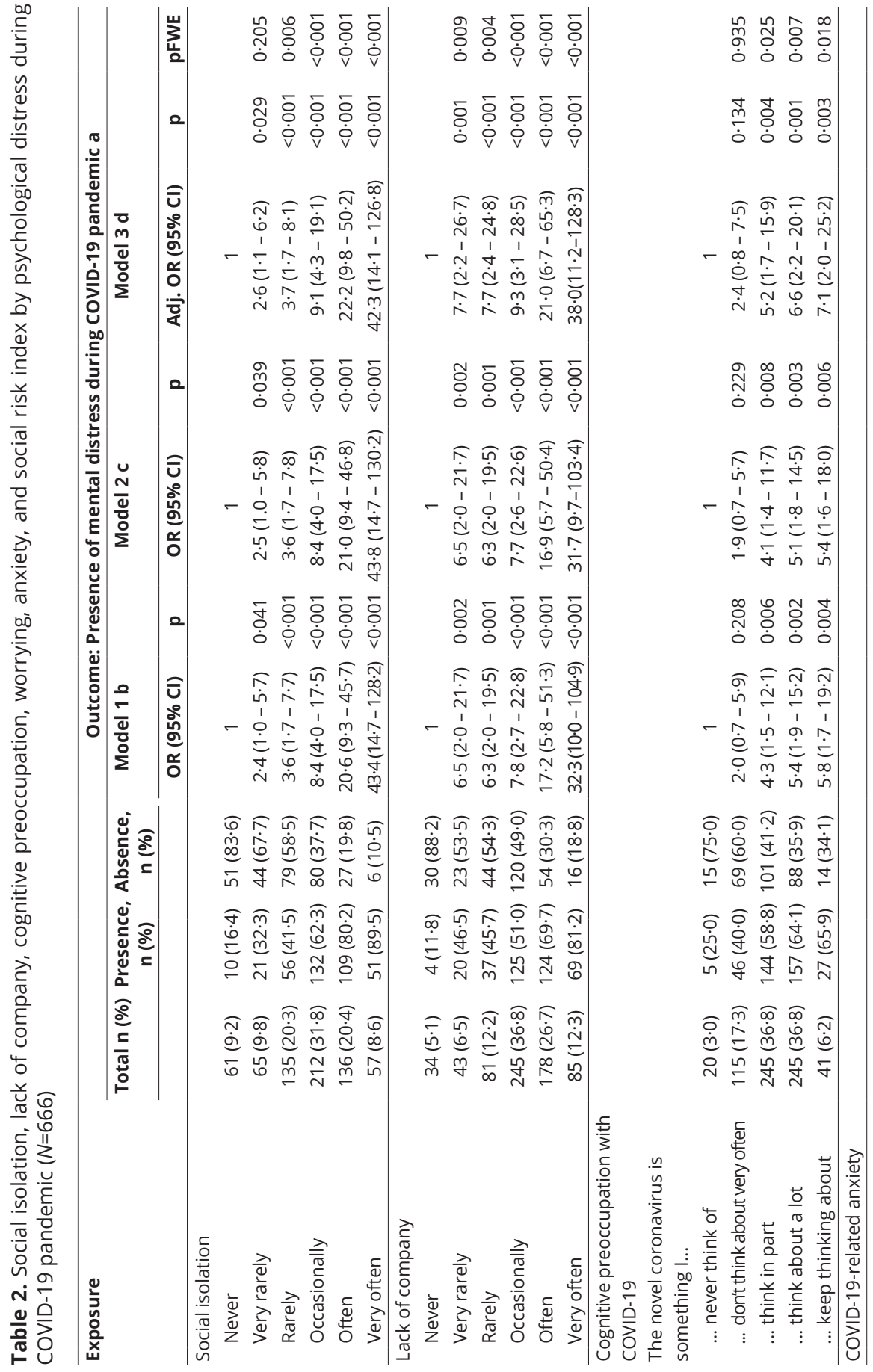




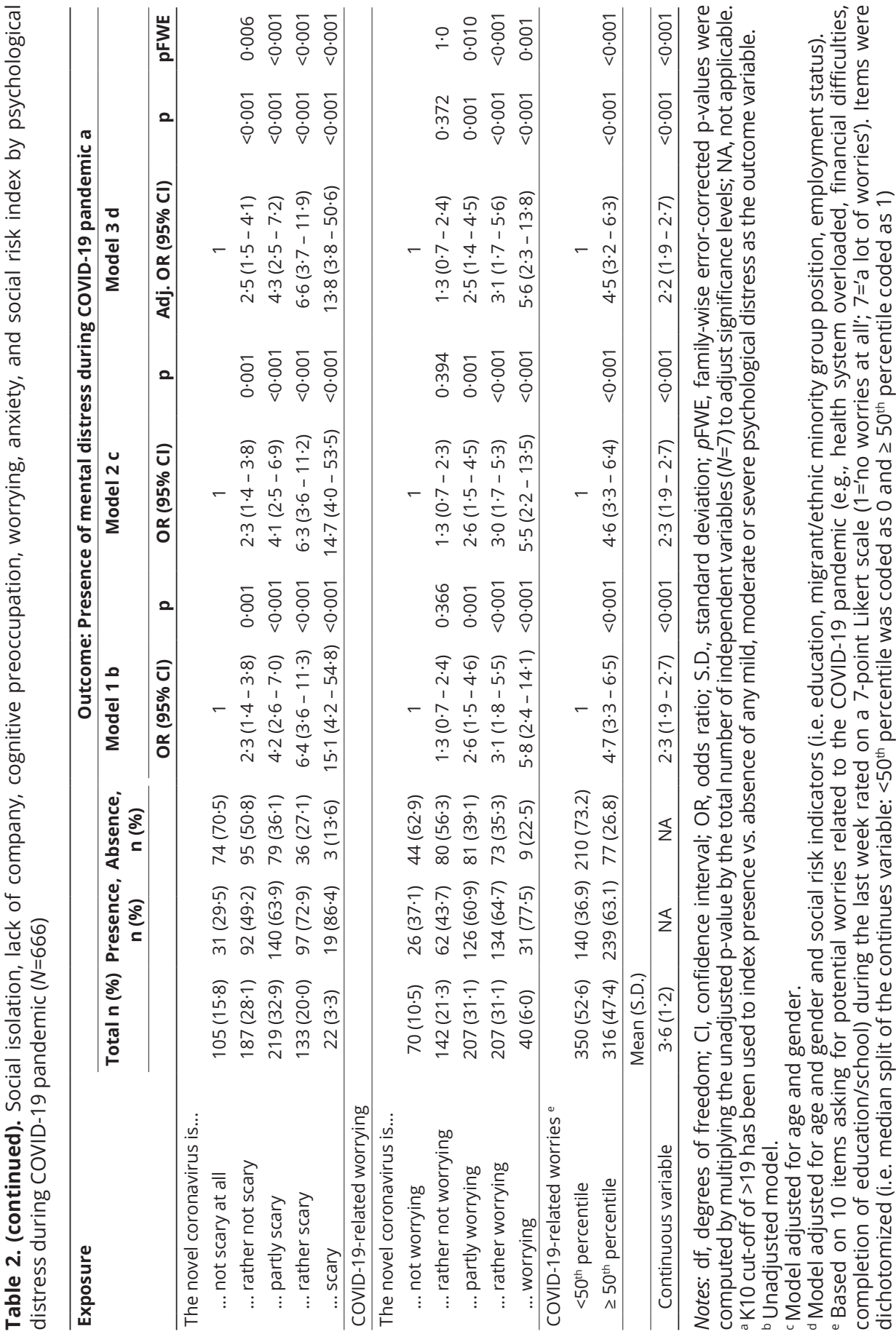


who reported to be 'rarely', 'occasionally', 'often', and 'very often' socially isolated were around four, nine, 22 and 42 times, respectively, more likely to experience psychological distress (adjusted odds ratio [aOR] 3.7, 95\% confidence interval [CI] 1.7 - 8.1, $p=0.006 ;$ aOR 9.1, Cl 4.3 - 19.1, $p<0.001$; aOR 22.2, Cl 9.8 - 50.2, $p<0 \cdot 001$; aOR $42 \cdot 3, \mathrm{Cl} 14 \cdot 1-126 \cdot 8, p<0 \cdot 001$; respectively) as compared to those who reported to be 'never' socially isolated (see Figure 1 ).

\section{Social risk indicators by psychological distress}

We next investigated whether objective indicators of social risk were associated with psychological distress in young individuals during the COVID-19 pandemic. First, we investigated associations of all individual indicators of social risk and migrant/ethnic minority group position with psychological distress. We found that individuals from migrant and ethnic minority groups were more likely to experience psychological distress compared to those from the ethnic majority group (aOR $1 \cdot 7$, $\mathrm{Cl} 1 \cdot 2-2 \cdot 4, p=0 \cdot 041)$. However, after adjustment for multiple testing, there was no evidence that unemployment, being single, lower educational level, parental educational level, or living arrangements were associated with psychological distress (see Table 3). In testing associations between the social risk index and psychological distress we found that, compared to individuals in whom objective social risk indicators were absent, individuals with two objective indicators were at an increased risk to experience psychological distress during the COVID-19 pandemic (presence of two indicators: aOR 1.9, Cl 1.2 - 3.0, p=0.034). By contrast, there was no strong evidence that, after adjustment for multiple testing, those exposed to only one social risk indicator or three or more indicators were at an increased risk for psychological distress (aOR 1.3, Cl $0.9-1.9, p=1.0$; aOR 2.1, Cl $1 \cdot 1-3 \cdot 9, p=0 \cdot 113$, respectively). There was also no evidence of a dose-response relationship.

\section{Psychological distress, COVID-19-related preoccupation, worries, anxiety, and social isolation by current mHealth app use}

There was some evidence that psychological distress, perceived social isolation and lack of company as well as COVID-19-related cognitive preoccupation, worries, and anxiety were associated with current use of mHealth apps (Table 4). 


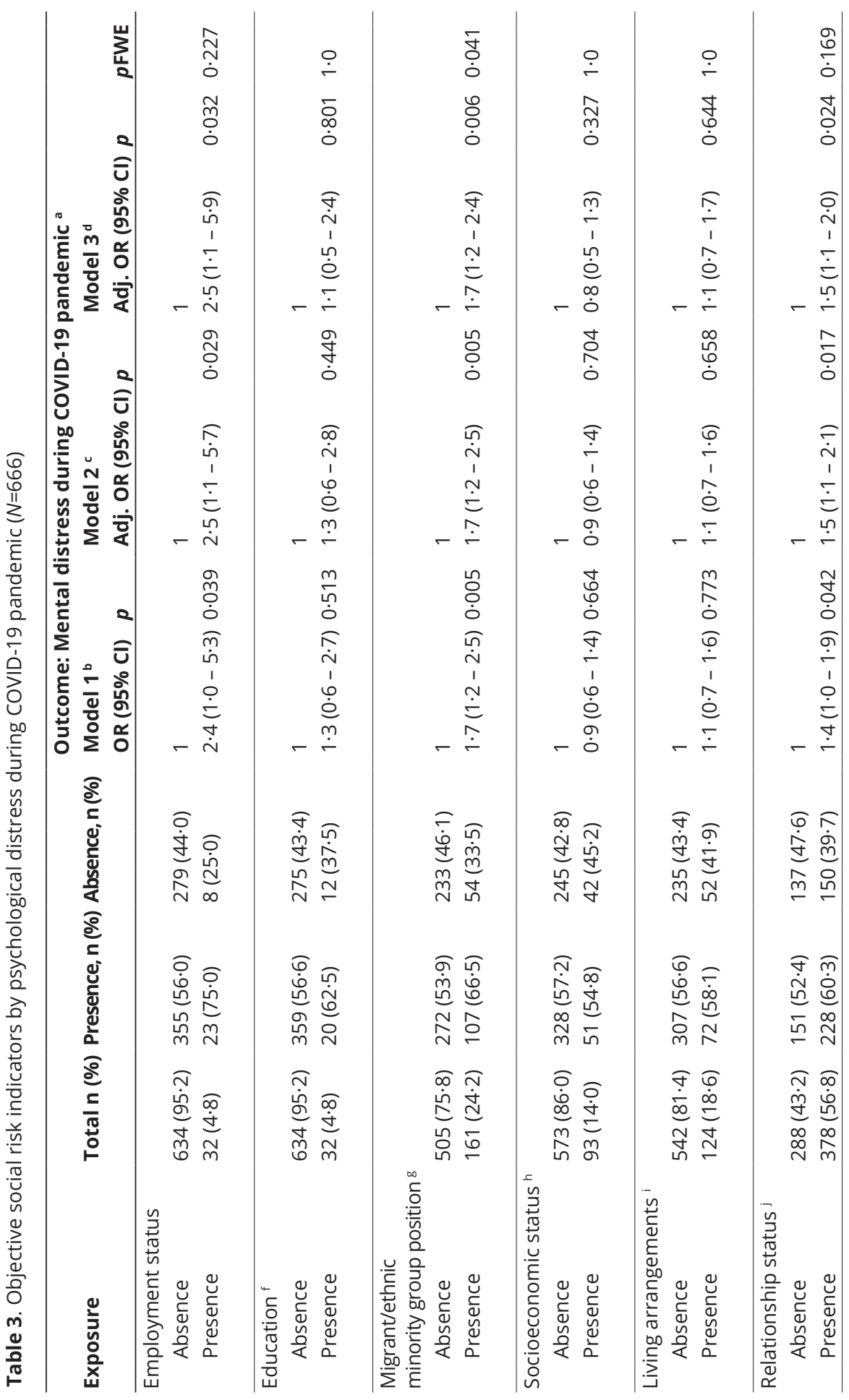




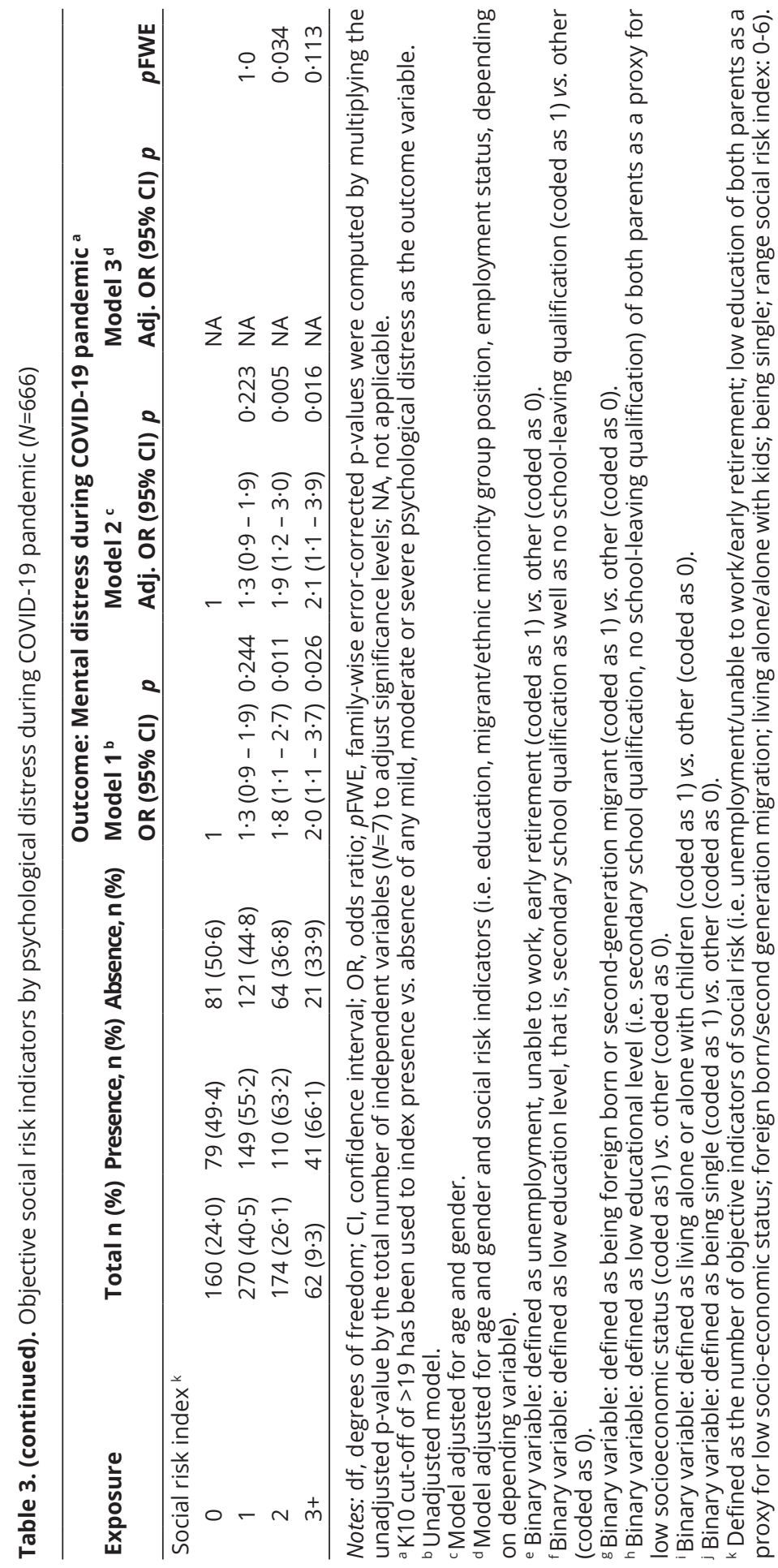




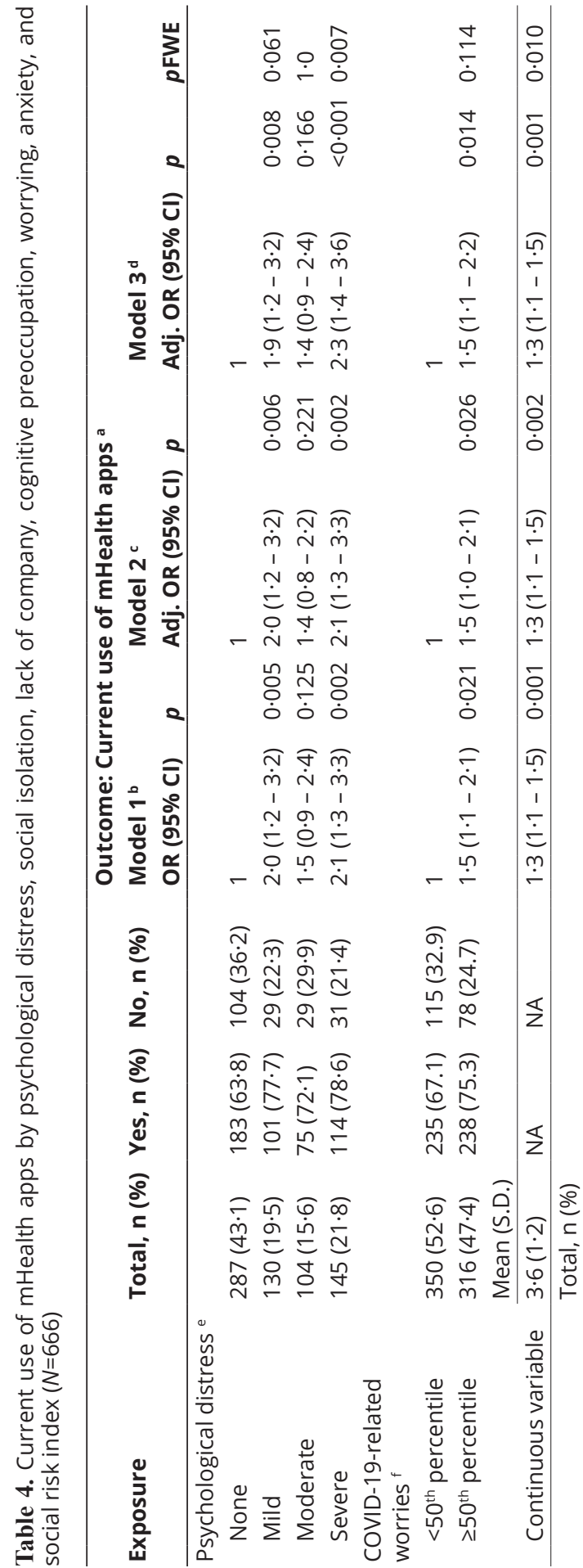

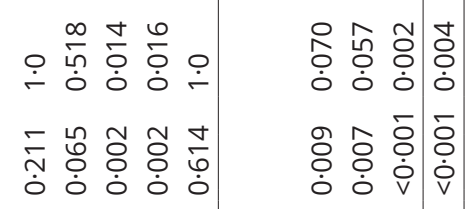

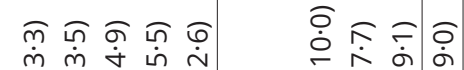

$$
\begin{aligned}
& \begin{array}{lllllllll}
1 & 1 & 1 & 1 & 1 & 1 & 1 & 1
\end{array}
\end{aligned}
$$

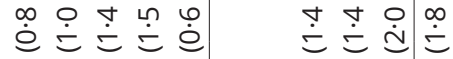

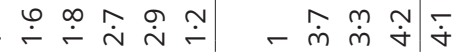

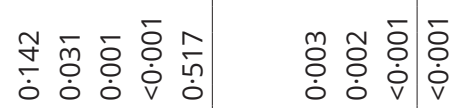

$$
\begin{aligned}
& \text { อิ }
\end{aligned}
$$

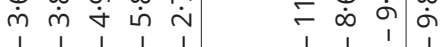

$$
\begin{aligned}
& \begin{array}{ccccc|ccc}
1 & 1 & 1 & 1 & 1 & 1 & 1 & 1 \\
0 & - & 1 & 0 & 0 & 0 & 0 & -
\end{array}
\end{aligned}
$$

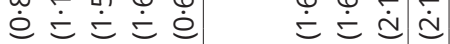

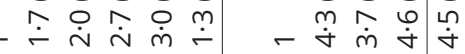

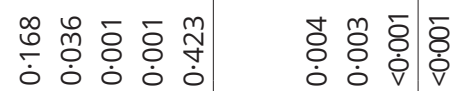

$$
\begin{aligned}
& \text { ช } \\
& \dot{m} \dot{m} \text { iो } \dot{1} \dot{\sim} \quad \therefore \dot{\sigma} \dot{0} \\
& \text { । } 1 \text { । } 1 \\
& \infty 0 \text { i } 60 \\
& \dot{0} \doteq \dot{\ominus} \doteq \dot{\ominus} \\
& \text { - } \\
& \text { ล } \\
& \text { क ลิ ลิก } \\
& \text { 守 } \ddot{m} \text { 官 }
\end{aligned}
$$

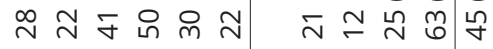

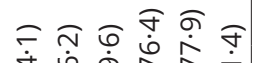

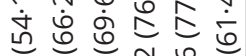

$$
\begin{aligned}
& \text { m m }
\end{aligned}
$$

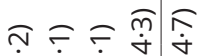

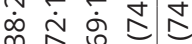

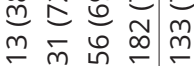

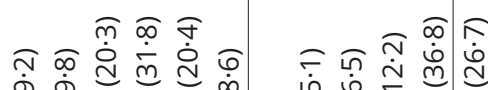

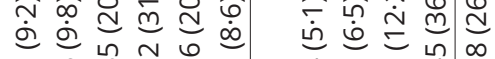

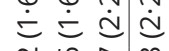

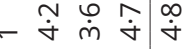




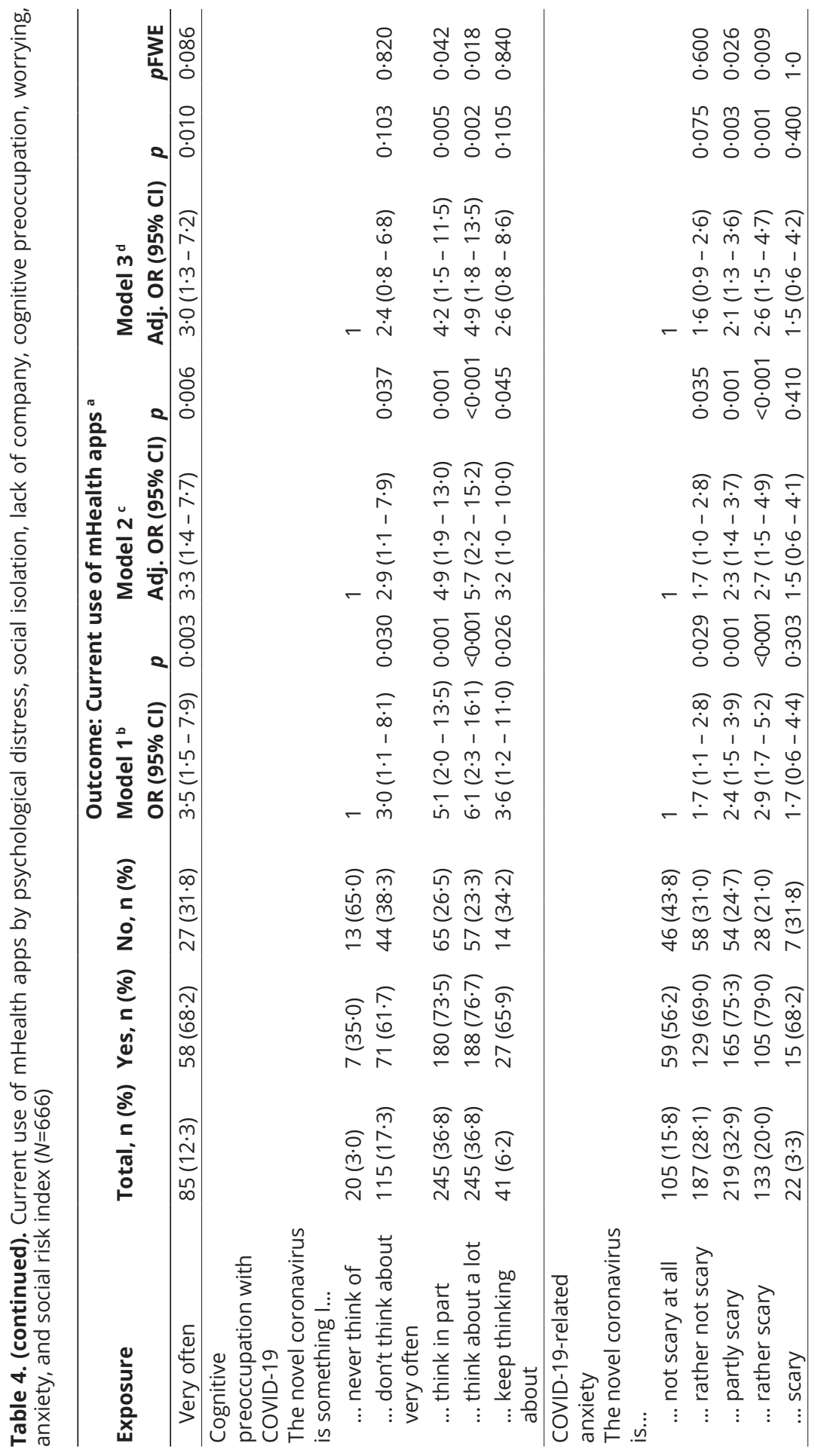




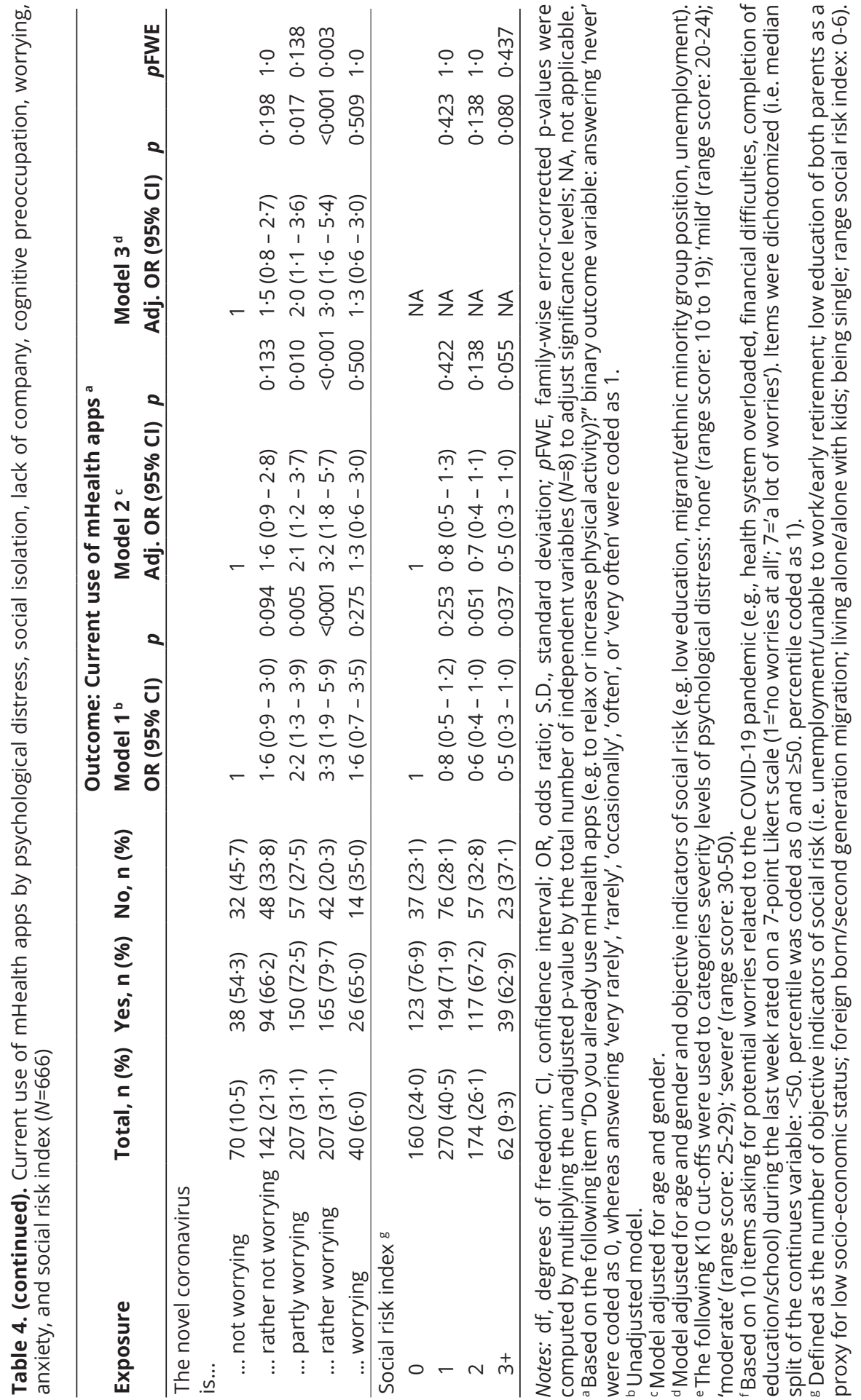




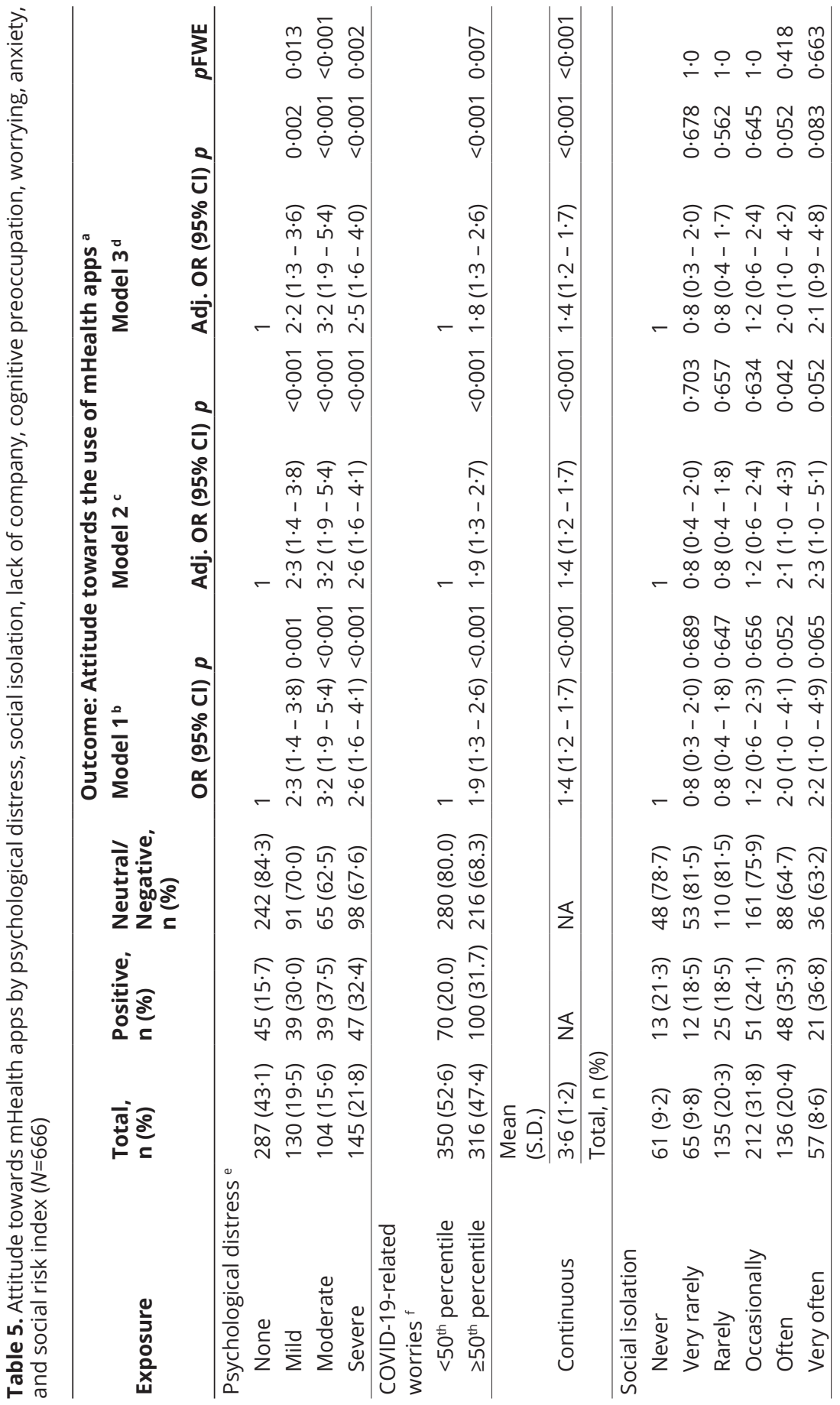




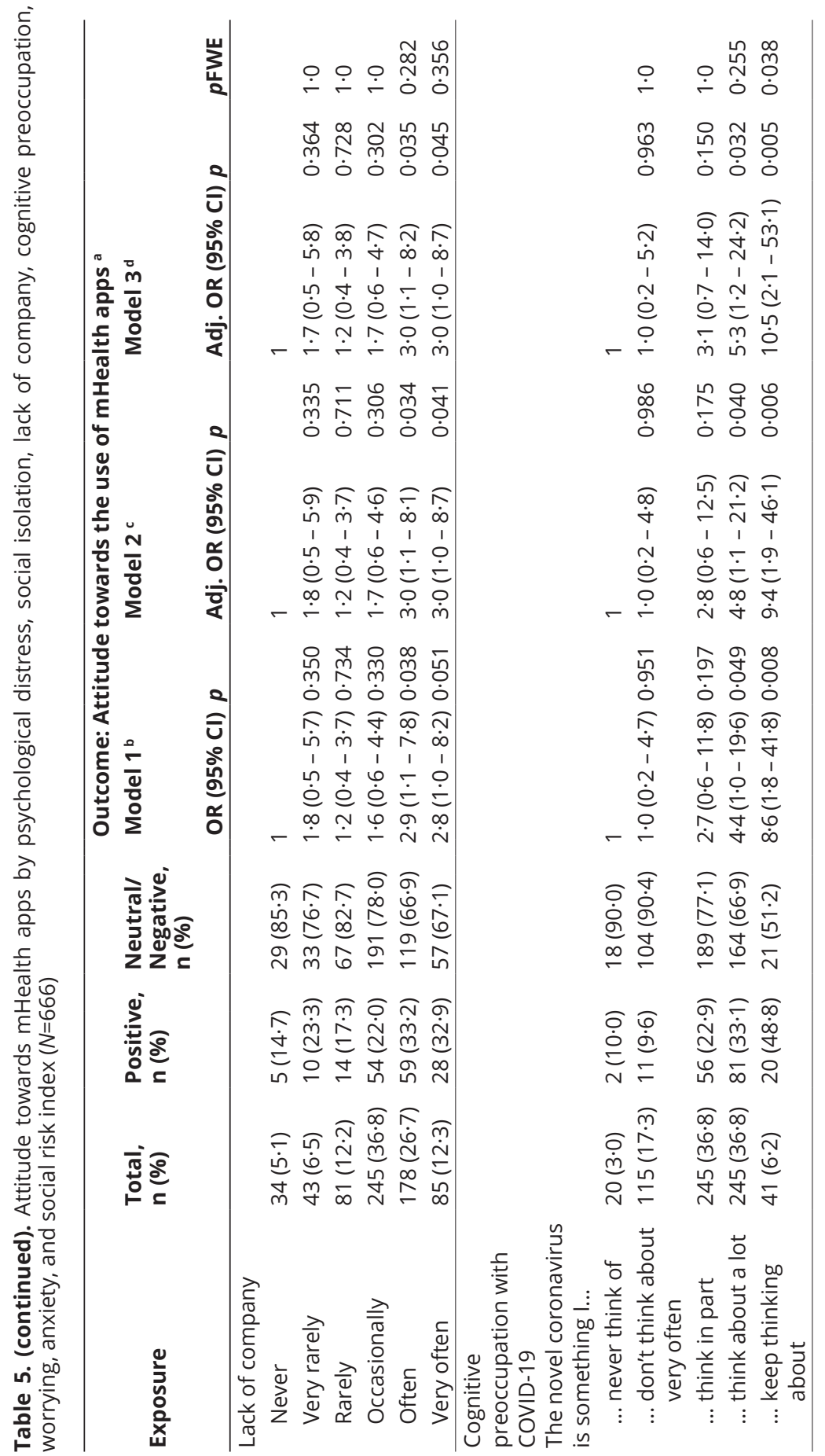




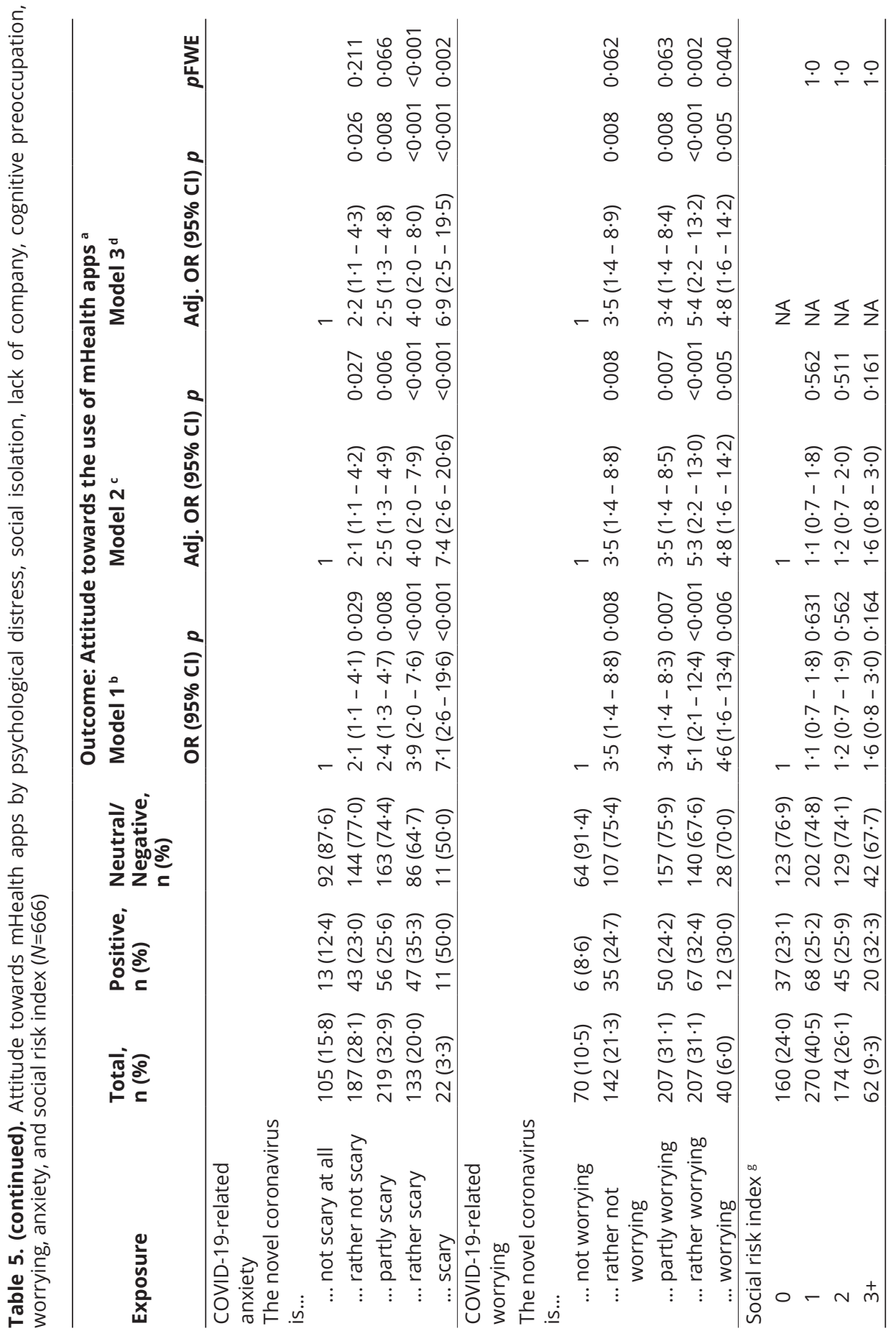




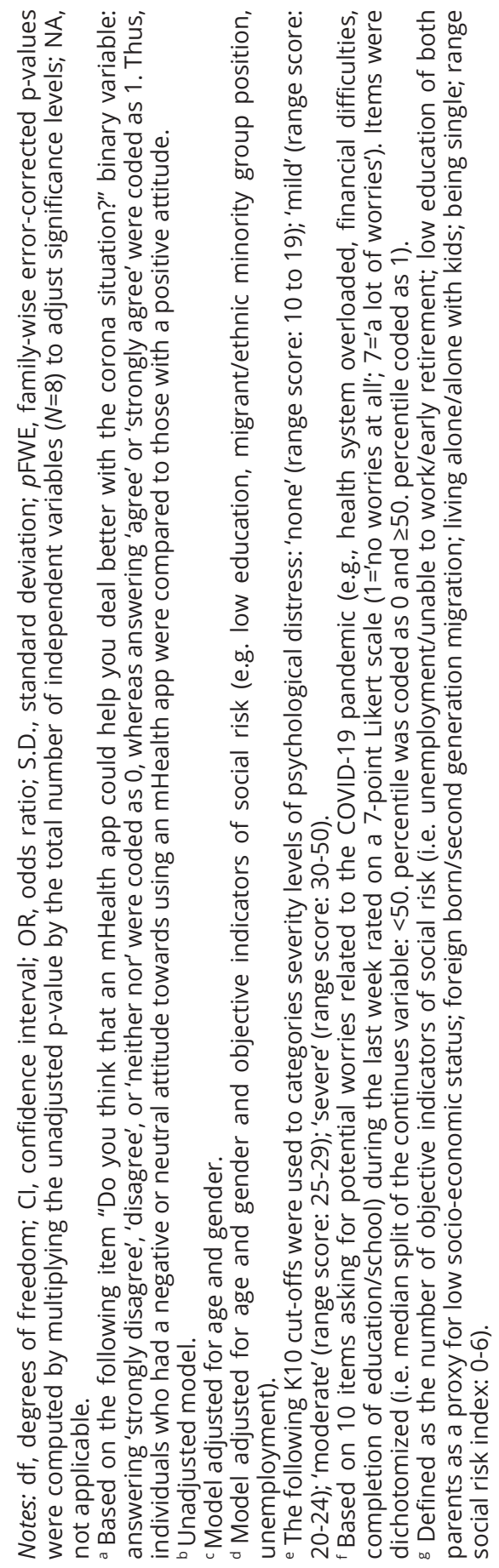




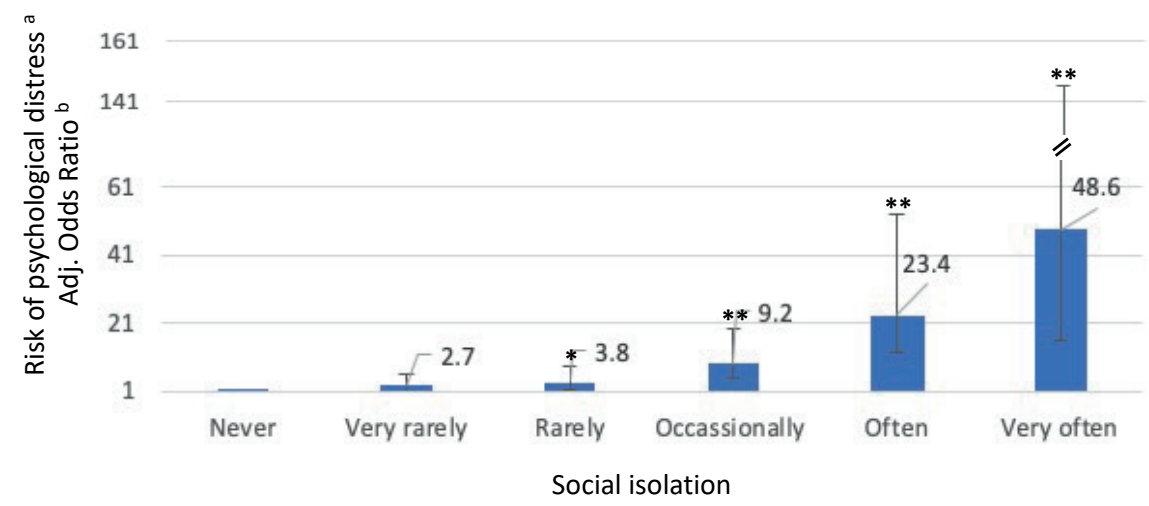

Figure 1. Associations of social isolation with psychological distress

Notes: Odds ratios and 95\% confidence intervals are shown.

$* p<0.05, * * p<0.001$

a K10 cut-off of $>19$ has been used to index presence vs. absence of any mild, moderate or severe psychological distress as the outcome variable.

b Model adjusted for age, gender, educational level, migrant/ethnic minority group position, employment status.

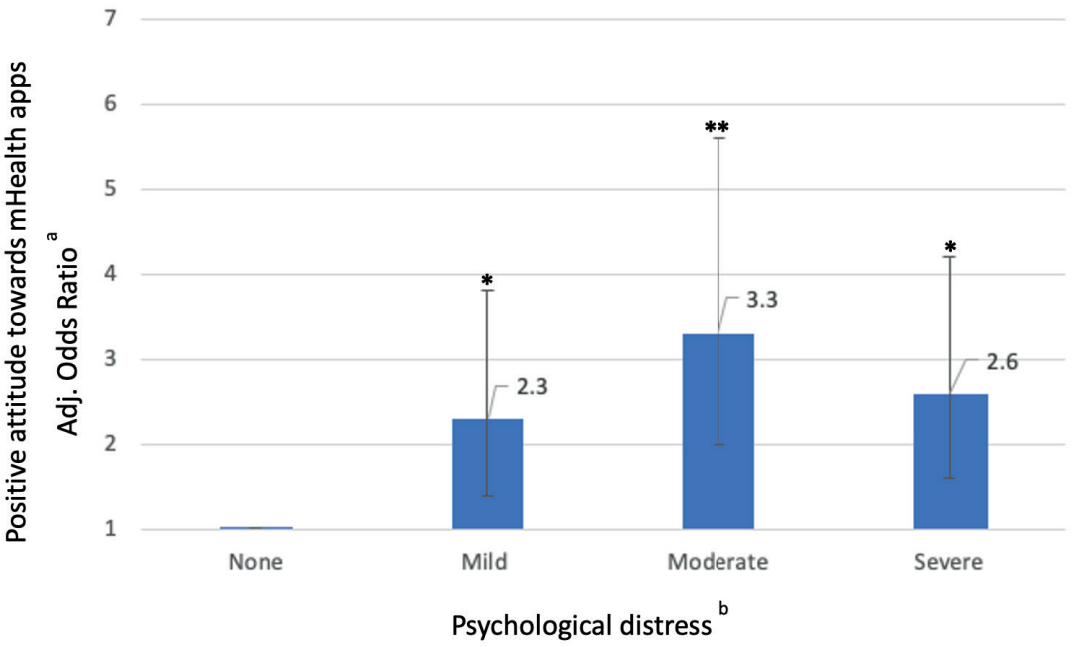

Figure 2. Associations of psychological distress with the positive attitude towards using mHealth apps

Notes: Odds ratios and $95 \%$ confidence intervals are shown.

$* p<0.05, * * p<0.001$

a Model adjusted for age and gender and social risk indicators (i.e. education, migrant/ethnic minority group position, employment status).

b The following K10 cut-offs were used to categories severity levels of psychological distress: 'none' (range score: 10 to 19); 'mild' (range score: 20-24); 'moderate' (range score: 25-29); 'severe' (range score: 30-50). 
For example, those with severe levels of psychological distress were two times more likely to use $\mathrm{mHealth}$ apps compared to those without psychological distress (aOR 2·3, Cl 1·4 - 3·6, $p=0 \cdot 007$ ). However, those with mild and moderate levels of psychological distress were as likely to use mHealth apps as those without psychological distress after adjustments for multiple testing. Further, youth who perceived a lack of company were more likely to use mHealth apps ('occasionally': aOR 4.2, Cl 2.0 - 9.1, $p=0 \cdot 002$; 'often': aOR 4.1, Cl 1.8 - 9.0, $p=0 \cdot 004$; respectively) as compared to those who reported to 'never' experience a lack of company during the COVID-19 pandemic although some inconsistencies were found. In contrast, there was no evidence that objective indicators of social risk were associated with the use of mHealth apps.

\section{Psychological distress, COVID-19-related worries, anxiety, and social isolation by attitude towards mHealth apps}

As shown in Table 5 and Figure 2, individuals who experienced psychological distress were, across all levels of severity, more likely to report a positive attitude towards the use of mHealth apps (mild psychological distress: aOR 2.2, Cl 1.34 3.6, $p=0 \cdot 013$; moderate: aOR 3.2, Cl 1.9 - 5.4, $p<0 \cdot 001$; severe: aOR 2.5, Cl 1.6 - 4.0, $p=0.002$ ) than those who did not report psychological distress. Similarly, those with more pronounced COVID-19-related worries ( 250 . percentile : aOR $1 \cdot 8, \mathrm{Cl} 1 \cdot 3$ - 2.6, $p=0 \cdot 007$ ), anxiety ('The novel coronavirus is rather scary': aOR 4.0, $\mathrm{Cl} 2 \cdot 0$ 8.0, $p<0.001$; 'The novel coronavirus is scary': aOR 6.9, Cl 2.5 - 19.5, $p=0.002$ ), or high levels of cognitive preoccupation with COVID-19 ('The novel coronavirus is something I keep thinking about': aOR 10.5, Cl 2.1 - 53.1, p=0.038) were more likely to report a positive attitude towards the use of mHealth apps. However, social isolation, lack of company, and objective indicators of social risk were not associated with individuals' attitudes towards the use of mHealth apps to address psychosocial consequences of the COVID-19 pandemic after adjustments for multiple testing.

\section{Discussion}

This study investigated whether social isolation, lack of company, COVID-19-related worries and anxiety as well as objective social risk indicators were associated with psychological distress during the COVID-19 pandemic in a representative 
sample of adolescents and young adults. In addition, associations with current use of, and attitude towards, mHealth apps were investigated. First, there was evidence that social isolation, lack of company, and COVID-19-related cognitive preoccupation, worries, and anxiety were associated with psychological distress. Second, we found evidence of dose-response relationships as psychological distress was progressively more likely to occur as the level of reported social isolation, lack of company, and COVID-19-related preoccupation, anxiety, and worrying increased - although some inconsistencies were observed. Third, an association between migrant/ethnic minority group position and psychological distress was found, while other objective indicators of social risk were not associated with psychological distress. Similarly, associations of levels of the social risk index and psychological distress were inconsistent. Fourth, there was evidence that psychological distress and high levels of COVID-19-related cognitive preoccupation, worries, and anxiety were associated with a more positive attitude towards the use of mHealth apps to help overcome negative consequences of the COVID-19 pandemic. Finally, the actual use of mHealth apps was more likely to be evident in those with severe psychological distress, frequent social isolation and lack of company as well as COVID-19-related preoccupation, anxiety, and worries, though some inconsistencies were found by levels of respective variables.

An important strength of this study is that findings are based on a representative sample of adolescents and young adults who participated in this survey during active lockdown in Germany. However, several limitations should be taken into account before interpreting reported findings. First, the cross-sectional design of the study did not allow us to investigate temporal order and, thus, we cannot rule out that reverse causality may have operated on our findings and, importantly, the complex nature of investigated constructs and the study design exclude any form of causal inference [48]. Also, as we have not assessed variables before the pandemic, we are not able to disentangle the unique additive effects of the pandemic on reported associations. However, longitudinal cohort studies have found that the prevalence of psychological distress and various mental health conditions was considerably higher during the pandemic as compared to time periods before the pandemic [18, 20, 23, 26, 27, 45], although some inconsistencies were reported [21]. Also, participants were explicitly asked to report levels of psychological distress during the COVID-19 pandemic. Second, the very dynamic 
development of the pandemic may limit the generalizability of findings to later stages of the ongoing pandemic or subsequent pandemics. Although strong evidence was found that social isolation, worrying, and other psychosocial factors related to public health measures for minimising transmission rates were strongly associated with psychological distress, it is possible that the withdrawal of restrictions quickly decreases subjective feelings of social isolation and worrying and, thus, may contribute to a reduction of psychological distress for most individuals [20]. However, the survey was conducted after the peak of new cases per day had occurred during the first wave of the pandemic in Germany and some infection control measures were already beginning to be lifted. Thus, our findings may also underestimate prevalence of psychological distress as compared to moments of strict lockdown and high rates of new cases. That said, mental health outcomes may worsen due to ongoing and expected economic uncertainties and, hence, it may be argued that further negative psychosocial consequences are yet to come. Furthermore, we used a conservative method to minimize type I error rate inflation, which further supports robustness of our findings. Third, some of the indicators used to conceptualize social risk may - although frequently being used in social epidemiological studies - apply to young people only to a limited extent. For instance, living alone may not be perceived as indexing social adversity. Also, some social risk indicators may only be contributing to poor mental health later in life (e.g. lower educational level). Fourth, we used a short screening measure (i.e. K10) to assess psychological distress. As the K10 is arguably largely focussing on depressive symptoms (e.g. feeling hopeless/worthless), other potentially important psychopathological domains (e.g. positive psychotic symptoms) have been largely neglected. Lastly, due to time constraints, the study was not preregistered before data collection and data on the psychometric properties of COVID-19 related measures (i.e., COSMO worry scale) is very limited. However, we tested a-priori defined hypotheses and findings on internal consistency are reported.

Overall, there is accumulating evidence on the negative consequences of the COVID-19 pandemic on public mental health. A number of cross-sectional and longitudinal cohort studies have found detrimental effects of the pandemic on various mental health domains, including psychological distress, depression, anxiety, and an increase of more distal risk factors such as cannabis and alcohol 
misuse and loneliness [6-24]. These findings are largely in line with findings from this nationally representative survey, i.e., high levels of social isolation, lack of company, COVID-19-related worrying, and anxiety have been reported and found to impact psychological distress in youth during active lockdown in Germany. Although migrant/ethnic minority group position was found to be associated with psychological distress, we found no evidence that an increased number of social risk indicators was associated with increased levels of psychological distress. Thus, our findings partly differ from other studies which have found that psychosocial consequences of the current pandemic are disproportionally distributed in the society and may especially affect those with more inferior social positions or minority status. However, our findings are in line with findings demonstrating more pronounced effects in youth $[23,24]$ and one study has shown associations between loneliness and COVID-19 related distress [25]. Further, positive attitudes towards the use of mHealth apps to help alleviate the psychosocial consequences of the pandemic was highly prevalent and associated with an objective need (e.g. more severe levels of psychological distress, higher levels of worrying). There have been also other studies which have reported that individuals have a positive attitude towards, and increasingly use, digital interventions during the current COVID-19 pandemic across the whole spectrum of public mental health provision (i.e., mental health promotion, prevention and treatment of mental disorders) [36-38, 49,50], and alterations of telemedicine regulations have been reported [51]. However, mHealth apps provide the opportunity of delivering low-threshold, personalized mental health care in daily life.

The present findings suggest that there is a pressing demand for evidence-based public mental health interventions that aim to specifically target the negative consequences of the COVID-19 pandemic [1]. Digital interventions, including eHealth interventions and mHealth apps, may help to mitigate the negative psychosocial consequences by providing evidence-based information, reliably monitoring symptoms, or delivering intervention components in individual 's daily lives [31, 36, 37, 52, 53]. Furthermore, digital interventions may be used to ensure continuity of care in the provision of mental health services in case of repeated outbreaks and lockdowns during the pandemic, and for providing and extending digital interventions to the area of mental health promotion and prevention to mitigate the negative impact of the pandemic especially in youth. Digital mHealth 
interventions may be particularly suited to help achieve this goal, as they have the potential, once developed and evaluated, to be scaled up and broadly offered at the population level.

To conclude, digital interventions may help to mitigate the negative impact of the COVID-19 pandemic on youth mental health as there is a subjective demand and objective need. Smartphone-based mHealth apps are particularly suited to provide low-threshold and timely public mental health care in times of physical distancing and quarantine. As the quality of evidence of currently available apps in major app stores is often unknown or very limited [54-59] there is an urgent need to (1) develop and evaluate digital interventions specifically designed to address social isolation and poor mental health to actively prepare for a potential worsening of the current pandemic as well as future health crises, (2) make these evidence-based digital interventions publicly available to improve public mental health, and (3) develop digital strategies for continued mental health care as well as mental health promotion and prevention of mental disorders. Finally, decision-makers and stakeholders in the area of public mental health should work on systematically evaluating currently available digital interventions to support young users to find evidenced-based digital tools which are most helpful for their individual preferences and current needs [60-62]. 


\section{References}

1. Holmes, E.A., et al., Multidisciplinary research priorities for the COVID-19 pandemic: a call for action for mental health science. Lancet Psychiatry, 2020. 7(6): p. 547-560.

2. Kozloff, N., et al., The COVID-19 Global Pandemic: Implications for People With Schizophrenia and Related Disorders. Schizophr Bull, 2020. 46(4): p. 752-757.

3. Brown, E., et al., The potential impact of COVID-19 on psychosis: A rapid review of contemporary epidemic and pandemic research. Schizophr Res, 2020. 222: p. 79-87.

4. Jeong, H., et al., Mental health status of people isolated due to Middle East Respiratory Syndrome. Epidemiol Health, 2016. 38: p. e2016048.

5. Leigh-Hunt, N., et al., An overview of systematic reviews on the public health consequences of social isolation and loneliness. Public Health, 2017. 152: p. 157-171.

6. Smith, L., et al., Correlates of symptoms of anxiety and depression and mental wellbeing associated with COVID-19: a cross-sectional study of UK-based respondents. Psychiatry Res, 2020. 291: p. 113138.

7. Huang, Y. and N. Zhao, Generalized anxiety disorder, depressive symptoms and sleep quality during COVID-19 outbreak in China: a web-based cross-sectional survey. Psychiatry Res, 2020. 288: p. 112954.

8. Zhou, S.J., et al., Prevalence and socio-demographic correlates of psychological health problems in Chinese adolescents during the outbreak of COVID-19. Eur Child Adolesc Psychiatry, 2020. 29(6): p. 749-758.

9. Yao, H., J.H. Chen, and Y.F. Xu, Patients with mental health disorders in the COVID-19 epidemic. Lancet Psychiatry, 2020. 7(4): p. e21.

10. Li, L.Z. and S. Wang, Prevalence and predictors of general psychiatric disorders and loneliness during COVID-19 in the United Kingdom. Psychiatry Res, 2020. 291: p. 113267.

11. Liang, L., et al., The Effect of COVID-19 on Youth Mental Health. Psychiatr Q, 2020. 91(3): p. 841-852.

12. Shanahan, L., et al., Emotional distress in young adults during the COVID-19 pandemic: evidence of risk and resilience from a longitudinal cohort study. Psychol Med, 2020: p. 1-10.

13. Power, E., et al., Youth mental health in the time of COVID-19. Ir J Psychol Med, 2020: p. 1-5.

14. Clemens, V., et al., Potential effects of "social" distancing measures and school lockdown on child and adolescent mental health. Eur Child Adolesc Psychiatry, 2020. 29(6): p. 739742.

15. Pierce, M., et al., Mental health before and during the COVID-19 pandemic: a longitudinal probability sample survey of the UK population. Lancet Psychiatry, 2020. 7(10): p. 883892.

16. Salari, N., et al., Prevalence of stress, anxiety, depression among the general population during the COVID-19 pandemic: a systematic review and meta-analysis. Global Health, 2020. 16(1): p. 57.

17. Hwang, T.J., et al., Loneliness and social isolation during the COVID-19 pandemic. Int Psychogeriatr, 2020. 32(10): p. 1217-1220. 
18. Kwong, A.S.F., et al., Mental health before and during the COVID-19 pandemic in two longitudinal UK population cohorts. Br J Psychiatry, 2020: p. 1-10.

19. Iob, E., A. Steptoe, and D. Fancourt, Abuse, self-harm and suicidal ideation in the UK during the COVID-19 pandemic. Br J Psychiatry, 2020. 217(4): p. 543-546.

20. Chandola, T., et al., The mental health impact of COVID-19 and lockdown-related stressors among adults in the UK. Psychol Med, 2020: p. 1-10.

21. McGinty, E.E., et al., Psychological Distress and COVID-19-Related Stressors Reported in a Longitudinal Cohort of US Adults in April and July 2020. JAMA, 2020. 324(24): p. 25552557.

22. Holland, K.M., et al., Trends in US Emergency Department Visits for Mental Health, Overdose, and Violence Outcomes Before and During the COVID-19 Pandemic. JAMA Psychiatry, 2021.

23. Daly, M., A.R. Sutin, and E. Robinson, Longitudinal changes in mental health and the COVID-19 pandemic: evidence from the UK Household Longitudinal Study. Psychol Med, 2020: p. 1-10.

24. Twenge, J.M. and T.E. Joiner, Mental distress among U.S. adults during the COVID-19 pandemic. J Clin Psychol, 2020. 76(12): p. 2170-2182.

25. Liu, S., et al., Increased Psychological Distress, Loneliness, and Unemployment in the Spread of COVID-19 over 6 Months in Germany. Medicina (Kaunas), 2021. 57(1).

26. Charles, N.E., et al., Increased mood disorder symptoms, perceived stress, and alcohol use among college students during the COVID-19 pandemic. Psychiatry Res, 2021. 296: p. 113706.

27. Ravens-Sieberer, U., et al., Impact of the COVID-19 pandemic on quality of life and mental health in children and adolescents in Germany. Eur Child Adolesc Psychiatry, 2021.

28. Gayer[Anderson, C., et al. Impacts of social isolation among disadvantaged and vulnerable groups during public health crises. 2020 22.06.2020]; Available from: https:// esrc.ukri.org/files/news-events-and-publications/evidence-briefings/impacts-of-socialisolation-among-disadvantaged-and-vulnerable-groups-during-public-health-crises/.

29. Wright, L., A. Steptoe, and D. Fancourt, Are we all in this together? Longitudinal assessment of cumulative adversities by socioeconomic position in the first 3 weeks of lockdown in the UK. J Epidemiol Community Health, 2020. 74(9): p. 683-688.

30. Betsch, C., Korn, L., Felgendreff, L., Eitze, S., Schmid, P., Sprengholz, P., Wieler, L., Schmich, P., Stollorz, V., Ramharter, M., Bosnjak, M., Omer, S. B., Thaiss, H., De Bock, F., Von Rüden, U., Lieb, K., \& Thrull, J., German COVID-19 Snapshot Monitoring (COSMO) Welle 5 (31.03.2020). PsychArchives.

31. Rauschenberg, C., et al., Evidence synthesis of digital interventions to mitigate the negative impact of the COVID-19 pandemic on public mental health: a rapid metareview. J Med Internet Res, 2021. 23: e23365.

32. Larsen, M.E., et al., Using science to sell apps: Evaluation of mental health app store quality claims. NPJ Digit Med, 2019. 2(18): p. 18.

33. Marshall, J.M., D.A. Dunstan, and W. Bartik, Apps With Maps-Anxiety and Depression Mobile Apps With Evidence-Based Frameworks: Systematic Search of Major App Stores. JMIR Ment Health, 2020. 7(6): p. e16525. 
34. Baumel, A., et al., There is a non-evidence-based app for that: A systematic review and mixed methods analysis of depression- and anxiety-related apps that incorporate unrecognized techniques. J Affect Disord, 2020. 273: p. 410-421.

35. Erbe, D., et al., Blending Face-to-Face and Internet-Based Interventions for the Treatment of Mental Disorders in Adults: Systematic Review. J Med Internet Res, 2017. 19(9): p. e306.

36. Baumgart, D.C., Digital advantage in the COVID-19 response: perspective from Canada's largest integrated digitalized healthcare system. NPJ Digit Med, 2020. 3: p. 114.

37. Miu, A.S., et al., Teletherapy with serious mental illness populations during COVID-19: telehealth conversion and engagement. Counselling Psychology Quarterly, 2020: p. 1-18.

38. Marques, G., et al., Impact of COVID-19 on the psychological health of university students in Spain and their attitudes toward Mobile mental health solutions. Int J Med Inform, 2021. 147: p. 104369.

39. Nostat Panel. 20.05.20)]; Available from: https://norstat.de.

40. Russell, D., L.A. Peplau, and C.E. Cutrona, The revised UCLA Loneliness Scale: Concurrent and discriminant validity evidence. Journal of Personality and Social Psychology, 1980. 39(3): p. 472-480.

41. Hughes, M.E., et al., A Short Scale for Measuring Loneliness in Large Surveys: Results From Two Population-Based Studies. Res Aging, 2004. 26(6): p. 655-672.

42. Vassar, M. and J.W. Crosby, A reliability generalization study of coefficient alpha for the UCLA loneliness scale. J Pers Assess, 2008. 90(6): p. 601-7.

43. Mallet, R., MRC Sociodemographic Schedule 1997, Section of Social Psychiatry, Institute of Psychiatry, London, UK.

44. Morgan, C., et al., Cumulative social disadvantage, ethnicity and first-episode psychosis: a case-control study. Psychol Med, 2008. 38(12): p. 1701-15.

45. Slade, T., R. Grove, and P. Burgess, Kessler Psychological Distress Scale: normative data from the 2007 Australian National Survey of Mental Health and Wellbeing. Aust N Z J Psychiatry, 2011. 45(4): p. 308-16.

46. Kessler, R.C., et al., Short screening scales to monitor population prevalences and trends in non-specific psychological distress. Psychol Med, 2002. 32(6): p. 959-76.

47. Andrews, G. and T. Slade, Interpreting scores on the Kessler Psychological Distress Scale (K10). Aust N Z J Public Health, 2001. 25(6): p. 494-7.

48. Hill, A.B., The Environment and Disease: Association or Causation? Proc R Soc Med, 1965. 58: p. 295-300.

49. Guinart, D., et al., Mental Health Care Providers' Attitudes Toward Telepsychiatry: A Systemwide, Multisite Survey During the COVID-19 Pandemic. Psychiatr Serv, 2021: p. appips202000441.

50. Kaess, M., et al., A plea for the sustained implementation of digital interventions for young people with mental health problems in the light of the COVID-19 pandemic. J Child Psychol Psychiatry, 2020.

51. Kinoshita, S., et al., Changes in telepsychiatry regulations during the COVID-19 pandemic: 17 countries and regions' approaches to an evolving healthcare landscape. Psychol Med, 2020: p. 1-8. 
52. Rauschenberg, C., et al., [Digital forms of service delivery for personalized crisis resolution and home treatment]. Nervenarzt, 2021.

53. van Agteren, J., et al., Using Internet-Based Psychological Measurement to Capture the Deteriorating Community Mental Health Profile During COVID-19: Observational Study. JMIR Ment Health, 2020. 7(6): p. e20696.

54. Alyami, M., et al., Social anxiety apps: a systematic review and assessment of app descriptors across mobile store platforms. Evid Based Ment Health, 2017. 20(3): p. 6570.

55. Sander, L.B., et al., 'Help for trauma from the app stores?' A systematic review and standardised rating of apps for Post-Traumatic Stress Disorder (PTSD). Eur J Psychotraumatol, 2020. 11(1): p. 1701788.

56. Sucala, M., et al., Anxiety: There is an app for that. A systematic review of anxiety apps. Depress Anxiety, 2017. 34(6): p. 518-525.

57. Terhorst, Y., et al., «Hilfe aus dem App-Store?»: Eine systematische Übersichtsarbeit und Evaluation von Apps zur Anwendung bei Depressionen. Verhaltenstherapie, 2018. 28(2): p. 101-112.

58. Ilagan, G.S., et al., Smartphone applications targeting borderline personality disorder symptoms: a systematic review and meta-analysis. Borderline Personal Disord Emot Dysregul, 2020. 7(1): p. 12.

59. Miralles, I., et al., Smartphone Apps for the Treatment of Mental Disorders: Systematic Review. JMIR Mhealth Uhealth, 2020. 8(4): p. e14897.

60. Federal Institute for Drugs and Medical Devices

(BfArM), Germany: Digital health applications (DiGA). 29.06.2020)]; Available from: https:// www.bfarm.de/EN/MedicalDevices/DiGA/_node.html.

61. United Kingdom National Health Service (NHS): Apps Library - Category Mental Health. 29.06.2020)]; Available from: https://www.nhs.uk/apps-library/ filter/?categories=Mental\%20health.

62. Division of Digital Psychiatry, Beth Israel Deaconess Medical Center, USA: App Evaluation Database. 2020 29.06.2020)]; Available from: https://apps.digitalpsych.org. 



\title{
CHAPTER 10
}

\author{
Evidence synthesis of digital \\ Interventions to mitigate the \\ negative impact of the COVID-19 \\ pandemic on public mental health: a \\ rapid meta-review
}




\section{Abstract}

Background: Accumulating evidence suggests negative effects of the COVID-19 pandemic on public mental health. Digital interventions that have been developed and evaluated in recent years may be used to mitigate negative consequences of the COVID-19 pandemic. However, evidence-based recommendations on the use of existing telemedicine and internet-based (eHealth) and app-based mobile Health (mHealth) interventions are lacking.

Objective: The aim was to investigate the theoretical and empirical base, user perspective, safety, effectiveness, and cost effectiveness of digital interventions in public mental health provision (i.e. mental health promotion, prevention and treatment of mental disorders) that may help to reduce the consequences of the current COVID-19 pandemic.

Methods: A rapid meta-review was conducted. MEDLINE, PsycINFO, and CENTRAL databases were searched on May 11, 2020. Study inclusion criteria were broad and considered systematic reviews and meta-analyses that investigated digital tools for health promotion, prevention, or treatment of mental health conditions likely affected by the COVID-19 pandemic.

Results: Overall, 815 peer-reviewed systematic reviews and meta-analysis were identified of which 83 met inclusion criteria. The present findings suggest that there is good evidence on the usability, safety, acceptance/satisfaction, and effectiveness of eHealth interventions while evidence on mHealth apps is promising, especially if social components (e.g. blended care) and strategies to promote adherence are incorporated. Although most digital interventions focus on the prevention or treatment of mental disorders, there is some evidence on mental health promotion. However, evidence on process quality, cost-effectiveness, and long-term effects is very limited.

Conclusions: There is evidence that digital interventions are particularly suited to mitigating psychosocial consequences at the population level. In times of physical distancing, quarantine, and restrictions on social contacts, decision-makers should develop digital strategies for continued mental health care and invest time and efforts in the development and implementation of mental health promotion and prevention programs.

Keywords: COVID-19; mHealth; eHealth; prevention; mental health promotion; intervention; digital mental health; telemedicine 


\section{Introduction}

Measures to prevent and control infections in the COVID-19 pandemic such as physical distancing, quarantine, and restrictions on social contacts can have a negative impact on public mental health [1]. This includes an increase in depression, anxiety, loneliness, and perceived stress [2] as well as in risk behaviours, such as cannabis and alcohol use [3] in the population. In addition to the immediate effects of the infection control measures, further negative consequences for mental health are to be expected due to more direct deleterious effects of COVID-19 (e.g. illness anxiety, contamination fears) and the economic downturn and recession [4]. Recently reported restrictions in access to, and continuity of, care for individuals with mental disorder caused by infection prevention and control measures in some countries are an additional cause for concern $[3,5,6]$.

Digital interventions, which do not require face-to-face contact, may play an important role in improving public mental health at times of infection prevention and control measures. They can be broadly grouped in telemedicine and internetbased interventions (hereafter eHealth intervention) [7], and app-based mobile Health (mHealth) interventions delivered using smartphones or other mobile devices [8]. These interventions provide a unique opportunity for delivering lowthreshold, public mental health care tailored to individual needs and contexts in daily life, outside the clinic [9], even under the restrictive conditions of the COVID-19 pandemic. As smartphones are mostly in close proximity to users, and accessible whenever and wherever convenient, the use of mHealth apps in particular represents a powerful approach that allows the real-time and realworld delivery of intervention components in individuals daily lives.

Digital tools may help to mitigate the negative psychosocial consequences most effectively if intervention strategies are not only targeted at vulnerable individuals with a clinically high-risk state or mental disorder, but also at the population level. More specifically, following the seminal 'population strategy' advocated by Geoffrey Rose [10], even a small shift in the population mean of mental health, which is continuously distributed in the population, may lead to a substantial reduction of the prevalence of mental health problems. If applied to the current pandemic, a scalable, digital public mental health approach may contribute to lower rates of mental disorders by targeting important determinants and shifting the mean level of mental health in the population. 
In order to minimize the negative impact of the COVID-19 pandemic on mental health of the population, digital interventions can be used in the following areas of public mental health provision: primary prevention strategies, including a) mental health promotion and literacy at the population level; b) indicated, selective, or universal prevention targeting high-risk individuals, subpopulations, or the entire population, respectively as well as secondary and tertiary prevention strategies, including c) treatment and preventive services for people with mental disorders. Indeed, evidence from ad-hoc surveys suggests that digital interventions for improving public mental health are urgently needed to address the psychosocial consequences of the COVID-19 pandemic [1-3, 11, 12]. For example, findings from the German COVID-19 Snapshot Monitoring (COSMO [13]), a repeated representative cross-sectional survey, suggest strong concerns about the economy, social inequalities, and the healthcare system as well as high levels of psychological distress in the adult general population, and in young people in particular $[14,15]$. Another representative survey (Norstatpanel) found that a staggering $39 \%$ of youth met criteria for moderate mental health problems, even after the most restrictive infection control measures had been lifted [16]. Furthermore, the reported social isolation during the COVID-19 pandemic was associated with levels of psychological distress in a dose-response fashion [16]. Recent evidence also suggests a high subjective demand for digital mental health interventions in the general population and people with mental disorder [17, 18 , which is matched with a high and rapidly growing number of mHealth apps available in major app stores, with the strongest growth having been noted for mHealth apps[19]. It has further been reported that the demand for mHealth apps has increased globally by $49 \%$ during the COVID-19 pandemic [20], with $73 \%$ of psychologically distressed and socially isolated youth in the Norstatpanel survey indicating the use of mHealth apps to be helpful in coping with the ongoing COVID-19 pandemic [16].

Taken together, based on the evidence presented, there is an urgent need for, and high potential in, using digital interventions to improve public mental health and mitigate the negative psychosocial impact of the COVID-19 pandemic. However, evidence-based recommendations for the use of digital interventions during public health crises, including this ongoing pandemic, is currently lacking. The present meta-review aimed to synthesise the available evidence on the theoretical and empirical base of interventions, quality from the user perspective (i.e., 
acceptability, usability, satisfaction), safety, effectiveness, and cost effectiveness of digital interventions in the area of public mental health provision (i.e., mental health promotion, prevention of, and treatment for mental disorder).

\section{Methods}

A rapid meta-review of systematic reviews on digital public mental health interventions was conducted. For this, the Preferred Reporting Items for Systematic Reviews and Meta-Analyses (PRISMA;[21]) was used as a guideline for reporting findings. In line with the current state of the art in the development and evaluation of complex digital mental health interventions [8], the following criteria to review the available evidence were used: theoretical and evidence base, quality from the user perspective (i.e., acceptability, usability, satisfaction), safety, effectiveness, and cost effectiveness.

\section{Search strategy and selection criteria}

The databases Medical Literature Analysis and Retrieval System Online (MEDLINE), PsycINFO, and The Cochrane Central Register of Controlled Trials (CENTRAL) were searched for systematic reviews and meta-analyses published in English and German language from inception to April 2020. An extensive search of bibliographic databases was performed using queries that combined search terms on mental health, public mental health provision, digital eHealth/mHealth interventions (see Multimedia Appendix 1) and high-quality reviews (i.e. systematic review, metaanalysis) using logical operators. In doing so, database-specific queries were used to ensure semantic equivalence. The queries were launched on May 11, 2020, covering results until April 2020. The results were obtained and duplicates were removed. References written in English and German language were included. No other filters or restrictions were applied.

The search criteria were purposefully broad and considered systematic reviews that investigated digital tools for health promotion, prevention, or treatment of mental health conditions and determinants likely affected by the COVID-19 pandemic (e.g. depression, anxiety, psychosis, substance misuse, self-harm, wellbeing, quality of life, self-esteem, loneliness). Titles and abstracts were screened for inclusion by one reviewer (NM). Studies were included if they were published in a peer-reviewed journal, contained original findings examining the theoretical 


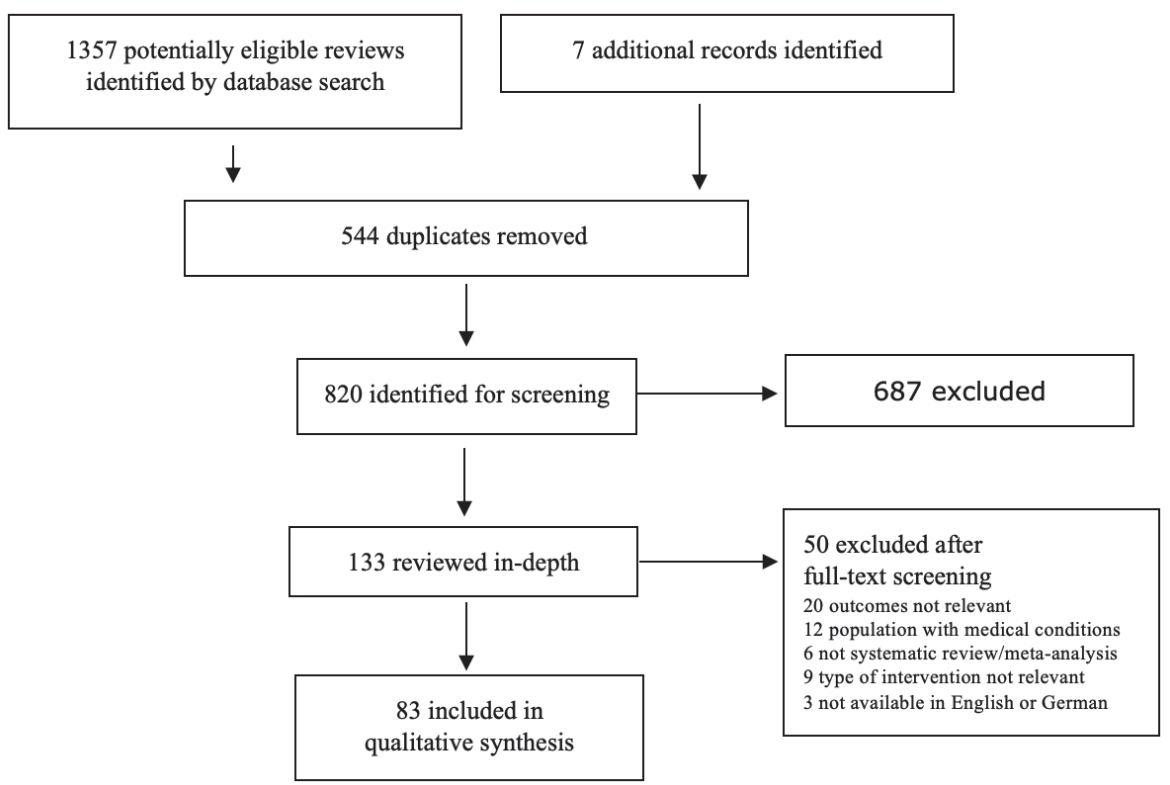

Figure 1. Study selection

and evidence base, quality from the user perspective (i.e., acceptability, usability, satisfaction), safety, effectiveness, or cost effectiveness of digital mHealth and eHealth interventions. Due to the rapid meta-review format of the current study, conclusions drawn by the included authors are reported. The included articles had to be systematic reviews and/or meta-analysis that followed established reporting guidelines (e.g. PRISMA; [21]). Because of time constraints and the rapid meta-review format of the current study, a second reviewer (CR) independently screened a randomly selected subset (40\%) of identified studies. The references were categorized as 'eligible', 'query', and 'not eligible'. Inclusion and exclusion criteria were applied to references that were queried or eligible. Reviewers were blinded and potential discrepancies in selection decisions were discussed with another member of the research team. There has been a pilot screening of a randomly selected subset of identified studies (around 5\%) to discuss decisions on categorising studies at an early stage. As inclusion criteria were purposefully broad, discrepancies between the reviewers (CR and NM) were very low. Full texts of potentially relevant articles were obtained, read, and assessed by one reviewer (CR), and data extraction was performed by three reviewers (CR as well as research assistants NM and VP under close supervision by CR; see acknowledgments). 
Reviews and meta-analysis on preprint servers and grey literature were not included. EndNote reference management software [22] was used to record reviewers' decisions, including reasons for exclusion. The study selection process was documented using the PRISMA flow diagram (see Figure 1).

\section{Results}

The search strategy of our meta-review on digital interventions yielded 815 peerreviewed systematic reviews and meta-analysis (Figure 1). Of these, 83 references were included in the meta-review (Table 1-3). Overall, 44 of the included reviews have summarized findings on eHealth interventions of which 19 focused on interventions targeting depression, 22 focused on anxiety, 11 focused on problematic substance use and two focused on eating disorders. Several reviews included interventions that targeted several mental health problems (see Table 1). In total, 16 reviews have summarized findings on mHealth interventions. Of these, two focused on depression, one focused on anxiety and one focused on problematic substance use and another on eating disorder. Similarly, the majority of included reviews focused on various mental health domains (see Table 2). Furthermore, 23 of the included reviews jointly reported effects of eHealth and mHealth interventions on various mental health outcomes (see Table 3). A complete summary of included reviews on eHealth, mHealth, and mixed interventions are shown in the Supplements, including secondary outcomes, quality from the user perspective, safety, and cost effectiveness. Here, findings on intervention components, theoretical and evidence base on process and outcomes, primary outcomes, and quality of evidence are shown.

\section{Quality from the user perspective}

Evidence from the included systematic reviews suggest moderate to high levels of acceptance, feasibility and user satisfaction of eHealth and mHealth interventions for mental health promotion and prevention $[30,31]$ as well as the treatment of mental health problems [32-41]. This applies, in particular, for interventions including social components [32,42], strategies to promote user adherence [33, 43], symptom monitoring [44, 45], or a blended care approach [46].

Safety: Data sharing and data safety regulations as well as aspects of eHealth/ $\mathrm{mHealth}$ and clinical safety of interventions were often not explicitly reported 
or systematically investigated in the identified systematic reviews [47-49]. The descriptions of many eHealth interventions do not make explicit reference to prevailing regulations and clinical guidelines [50]. Furthermore, there is evidence that $\mathrm{mHealth}$ apps available in major app stores use problematic data sharing and privacy practices (e.g. monetization of sensitive user data through analytics and advertising) $[8,27,28]$.

\section{Effectiveness of eHealth interventions}

There was good evidence on the effectiveness of telemedical and other eHealth interventions in the field of mental health promotion and prevention as well as the treatment of mental health conditions.

\section{Mental health promotion and prevention}

There have been a number of systematic reviews that aimed to investigate the effectiveness of telemedical and eHealth interventions for mental health promotion and prevention. These interventions have primarily been shown to improve mental health [34], physical activity [34, 35], well-being [36, 37], stress $[23,38]$, depression $[23,36,38,51,52]$, anxiety $[23,36,38,51,52]$, alcohol use [24, 53-56] and cannabis use $[57,58]$ in the general population as well as dysfunctional cognition and self-esteem in at-risk populations $[59,60]$. Importantly, effectiveness has been demonstrated across differing age groups, including adults [24, 54, 59] as well as adolescents from the general population [34, 52, 56, 61-63], and effect sizes mostly ranged from small to medium. However, evidence on the use of eHealth intervention for the elderly is scarce, although findings from the identified reviews indicated some evidence on the effectiveness of eHealth interventions for reducing social isolation and increasing social participation of people aged 65 and older [64] which may be of particular interest for the current COVID-19 pandemic.

\section{Treatment of mental health conditions}

There was also strong evidence on the effectiveness of telemedical and eHealth interventions in the provision of treatment and services for people with mental disorder. 
This included anxiety disorders [65-68], depression [60, 61, 65-67, 69-73], substance abuse [54, 74-76], eating disorders [77], and severe mental illness [78], with overall small to medium effect sizes, not only with regard to the reduction of relevant symptoms but also the improvement of dysfunctional cognition [60], self-esteem [60] and quality of life [66]. Some of the identified studies have even reported medium to large effect sizes for cognitive-behavioural eHealth interventions that aimed to reduce symptoms of depression [79].

The effectiveness of telemedicine interventions which use videoconference tools or the telephone has also been well documented in depressive [80-83], anxiety $[80,83-85]$ and psychotic disorders [86], with comparable effects of online group and individual therapy sessions [87, 88], compared with conventional (offline) therapy sessions. Superior effectiveness was observed for interventions adopting a blended-care approach combining eHealth with conventional intervention components $[46,54,71]$.

Overall, findings suggest that the evidence on long-term effects and non-inferiority compared to conventional therapy and active control conditions remains limited $[79,81,82,86,87]$ and there is only limited evidence of telemedical and eHealth interventions on underlying processes and mechanisms of action [89].

\section{Effectiveness of mHealth interventions}

While there is some, initial evidence on the effectiveness of mHealth interventions to improve physical activity [90-95], stress appraisal [96, 97], depression [26, 96$100]$, anxiety [25, 26, 96, 97] and alcohol and substance use [55, 96, 98, 101-103], with small to medium effect sizes in all areas of public mental health provision, the amount of research to investigate this issue remains, overall, limited [104108]. Only a minority of mHealth interventions were found to use more advanced techniques (accelerometer, GPS) to inform the delivery of intervention components $[25,89,92]$. In addition, a substantial difference was found between mHealth apps available in major app stores, for which there is no or only very limited evidence on their effectiveness [29, 108-111], and mHealth interventions developed by research groups. As for eHealth interventions, evidence on long-term effects and on underlying processes and mechanisms of action remains very limited. 


\section{Cost effectiveness}

There is some evidence on the cost effectiveness of eHealth interventions for depression and anxiety in primary care settings when compared to care as usual and waiting list control conditions [51] as well as for a range of mental disorders when compared to conventional CBT $[112,113]$. However, as only a few systematic reviews have systematically investigated the cost-effectiveness of digital interventions to date, these findings should be interpreted with caution. While there is some evidence on the cost effectiveness of mHealth interventions (e.g. for digital monitoring and feedback in depression) from individual studies [18], evidence summarized at the level of systematic reviews is very limited.

Table 1. Summary of included reviews on eHealth interventions ${ }^{\text {a }}$

\begin{tabular}{|c|c|c|c|c|c|}
\hline Reference & $\begin{array}{l}\text { Meta } \\
\text { analyses }\end{array}$ & Population & $\begin{array}{l}\text { Intervention } \\
\text { components }\end{array}$ & $\begin{array}{l}\text { Theoretical and } \\
\text { evidence base on } \\
\text { process/outcomes }\end{array}$ & $\begin{array}{l}\text { Primary } \\
\text { outcomes \& } \\
\text { quality of evidence }\end{array}$ \\
\hline \multicolumn{6}{|c|}{ Mental Health Promotion and Prevention } \\
\hline $\begin{array}{l}\text { Alkhaldi et al. } \\
(2016)^{33}\end{array}$ & Yes & $\begin{array}{l}\text { Adult general } \\
\text { population }\end{array}$ & $\begin{array}{l}\text { Technology-based } \\
\text { engagement promotion } \\
\text { strategies (e.g. text } \\
\text { messages, self-monitoring, } \\
\text { F, support) }\end{array}$ & Not reported & $\begin{array}{l}\text { Small to moderate } \\
\text { positive effects } \\
\text { on technology- } \\
\text { based strategies } \\
\text { for promoting } \\
\text { engagement with } \\
\text { digital interventions } \\
\text { as compared to no } \\
\text { strategy; quality of } \\
\text { evidence: moderate } \\
\text { (assessed using } \\
\text { Cochrane risk-of-bias } \\
\text { tool) }\end{array}$ \\
\hline $\begin{array}{l}\text { Elaheebocus et } \\
\text { al. }(2018)^{32}\end{array}$ & No & $\begin{array}{l}\text { Adult general } \\
\text { population }\end{array}$ & $\begin{array}{l}\text { Identity representation, } \\
\text { communication, peer } \\
\text { grouping, data sharing, } \\
\text { competition/gamification, } \\
\text { activity data viewing, online } \\
\text { social network }\end{array}$ & $\begin{array}{l}\text { Social Behavior } \\
\text { Change Technique }\end{array}$ & $\begin{array}{l}\text { Positive effects } \\
\text { on outcomes (e.g. } \\
\text { increased PA, lower } \\
\text { alcohol intake) in } \\
\text { most behavioral } \\
\text { interventions that } \\
\text { included social } \\
\text { media features, } 28 \% \\
\text { no effect; quality } \\
\text { of evidence: low } \\
\text { (e.g. small samples, } \\
\text { heterogeneous } \\
\text { interventions); no } \\
\text { standardized approach } \\
\text { was used to assess } \\
\text { the quality of included } \\
\text { studies }\end{array}$ \\
\hline
\end{tabular}


Table 1. (continued). Summary of included reviews on eHealth interventions ${ }^{a}$

\begin{tabular}{|c|c|c|c|c|c|}
\hline Reference & $\begin{array}{l}\text { Meta } \\
\text { analyses }\end{array}$ & Population & $\begin{array}{l}\text { Intervention } \\
\text { components }\end{array}$ & $\begin{array}{l}\text { Theoretical and } \\
\text { evidence base on } \\
\text { process/outcomes }\end{array}$ & $\begin{array}{l}\text { Primary } \\
\text { outcomes \& } \\
\text { quality of evidence }\end{array}$ \\
\hline $\begin{array}{l}\text { Cotie et al. } \\
(2018)^{35}\end{array}$ & Yes & $\begin{array}{l}\text { Adult general } \\
\text { population }\end{array}$ & $\begin{array}{l}\text { Multimodal/unimodal } \\
\text { websites: information, } \\
\text { PA tracking tools, online } \\
\text { discussion forums; } \\
\text { prompts (e.g. reminders to } \\
\text { exercise, track movement) }\end{array}$ & $\begin{array}{l}\text { Social Cognitive } \\
\text { Theory, } \\
\text { Transtheoretical } \\
\text { Behavioral Theory }\end{array}$ & $\begin{array}{l}\text { Moderate } \\
\text { improvement in PA; } \\
\text { no improvement } \\
\text { in obesity-related } \\
\text { outcomes; quality of } \\
\text { evidence: moderate } \\
\text { (assessed using the } \\
\text { GRADE approach) }\end{array}$ \\
\hline $\begin{array}{l}\text { Deady et al. } \\
\text { (2017) }{ }^{59}\end{array}$ & Yes & $\begin{array}{l}\text { Adult general } \\
\text { population }\end{array}$ & $\begin{array}{l}\text { Behavioral and } \\
\text { problem-solving therapy, } \\
\text { mindfulness, situational } \\
\text { analysis, skills program, } \\
\text { relaxation self-help } \\
\text { program, persuasive } \\
\text { framing, PE }\end{array}$ & $\begin{array}{l}\text { CBT, third wave } \\
C B T, A C T, P E\end{array}$ & $\begin{array}{l}\text { Small positive effects } \\
\text { on depression and } \\
\text { anxiety; similar effect } \\
\text { sizes for universal and } \\
\text { indicated/selective } \\
\text { interventions; quality } \\
\text { of evidence: moderate } \\
\text { (assessed using the } \\
\text { Downs and Black } \\
\text { checklist) }\end{array}$ \\
\hline $\begin{array}{l}\text { Heber et al. } \\
(2017)^{23}\end{array}$ & Yes & $\begin{array}{l}\text { Adult general } \\
\text { population }\end{array}$ & $\begin{array}{l}\text { Mindfulness, stress and } \\
\text { mood management, } \\
\text { problem solving, ER, coping } \\
\text { with stress, ACT, skills, } \\
\text { relaxation }\end{array}$ & $\begin{array}{l}\text { CBT, third wave } \\
\text { CBT, ALT }\end{array}$ & $\begin{array}{l}\text { Small effect on } \\
\text { stress, depression } \\
\text { and anxiety; guided } \\
\text { interventions } \\
\text { more effective } \\
\text { than unguided } \\
\text { interventions; small } \\
\text { effects for short } \\
\text { interventions ( } \leq 4 \\
\text { weeks), moderate } \\
\text { effects for medium- } \\
\text { long interventions (5-8 } \\
\text { weeks; small-moderate } \\
\text { effect for CBT and } \\
\text { third wave CBT; small } \\
\text { effect for alternative } \\
\text { interventions; small- } \\
\text { moderate effect up to } \\
6 \text { months follow-up } \\
\text { for computer-based } \\
\text { stress-management } \\
\text { interventions; quality } \\
\text { of evidence: low } \\
\text { (assessed using the } \\
\text { Cochrane risk-of-bias } \\
\text { tool) }\end{array}$ \\
\hline
\end{tabular}


Table 1. (continued). Summary of included reviews on eHealth interventions ${ }^{\text {a }}$

\begin{tabular}{|c|c|c|c|c|c|}
\hline Reference & $\begin{array}{l}\text { Meta } \\
\text { analyses }\end{array}$ & Population & $\begin{array}{l}\text { Intervention } \\
\text { components }\end{array}$ & $\begin{array}{l}\text { Theoretical and } \\
\text { evidence base on } \\
\text { process/outcomes }\end{array}$ & $\begin{array}{l}\text { Primary } \\
\text { outcomes \& } \\
\text { quality of evidence }\end{array}$ \\
\hline $\begin{array}{l}\text { Ennis et al. } \\
(2018)^{31}\end{array}$ & No & $\begin{array}{l}\text { Trauma- } \\
\text { exposed } \\
\text { individuals }\end{array}$ & $\begin{array}{l}\text { PE, interactive games, } \\
\text { cognitive and behavioral } \\
\text { principles, peer support, MI }\end{array}$ & CBT & $\begin{array}{l}\text { No effects on various } \\
\text { symptom domains for } \\
\text { selected interventions, } \\
\text { significant effects for } \\
\text { indicated interventions } \\
\text { as compared to active } \\
\text { control conditions; } \\
\text { quality of evidence: } \\
\text { fair to good (assessed } \\
\text { using the Downs and } \\
\text { Black checklist) }\end{array}$ \\
\hline $\begin{array}{l}\text { Pennant et al. } \\
(2015)^{52}\end{array}$ & Yes & $\begin{array}{l}\text { Young } \\
\text { individuals } \\
\text { (aged 5-25 } \\
\text { years) with risk } \\
\text { of developing } \\
\text { anxiety disorder } \\
\text { or depression; } \\
\text { young } \\
\text { individuals from } \\
\text { the general } \\
\text { population }\end{array}$ & $\begin{array}{l}\text { Supportive text, } \\
\text { animation, photographs, } \\
\text { videos, rewards, games, } \\
\text { homework, web pages } \\
\text { with reading, quizzes, F, } \\
\text { phone calls, interactive } \\
\text { fantasy adventure game, } \\
\text { depression monitor, diary, } \\
\text { counter-thought generator }\end{array}$ & CBT & $\begin{array}{l}\text { Medium effects } \\
\text { on anxiety and } \\
\text { depression in } \\
\text { populations at } \\
\text { risk; small effects } \\
\text { on anxiety and } \\
\text { depression in } \\
\text { general population; } \\
\text { inconsistent findings } \\
\text { in children; quality } \\
\text { of evidence: low } \\
\text { (assessed using the } \\
\text { GRADE approach) }\end{array}$ \\
\hline $\begin{array}{l}\text { Fleming et al. } \\
(2014)^{61}\end{array}$ & No & $\begin{array}{l}\text { Young } \\
\text { individuals } \\
\text { (aged 9-25 } \\
\text { years) with } \\
\text { depressive } \\
\text { symptoms }\end{array}$ & $\begin{array}{l}\text { Serious games: supported } \\
\text { and fully self-help } \\
\text { interventions; F, conflict/ } \\
\text { competition, interaction, PE }\end{array}$ & CBT & $\begin{array}{l}\text { Small treatment } \\
\text { effects on depressive } \\
\text { symptoms; quality } \\
\text { of evidence: low } \\
\text { (e.g. small samples, } \\
\text { heterogeneous } \\
\text { interventions); no } \\
\text { standardized approach } \\
\text { was used to assess } \\
\text { the quality of included } \\
\text { studies }\end{array}$ \\
\hline $\begin{array}{l}\text { Flujas-Contreras } \\
\text { et al. (2019) }{ }^{37}\end{array}$ & Yes & $\begin{array}{l}\text { Parents of } \\
\text { children with } \\
\text { mental or } \\
\text { physical health } \\
\text { problems }\end{array}$ & $\begin{array}{l}\text { PE, self-care, positive } \\
\text { parenting, coping } \\
\text { strategies, children's } \\
\text { contingency management, } \\
\text { problem solving games }\end{array}$ & CBT, Triple P & $\begin{array}{l}\text { Moderate effect sizes } \\
\text { on various mental } \\
\text { health domains; } \\
\text { small effect size on } \\
\text { parental self-efficacy, } \\
\text { no effects on parental } \\
\text { stress; Triple P: small } \\
\text { effect size; quality } \\
\text { of evidence: high } \\
\text { (assessed using the } \\
\text { Cochrane risk-of-bias } \\
\text { tool and the Moncrieff } \\
\text { Scale) }\end{array}$ \\
\hline
\end{tabular}


Table 1. (continued). Summary of included reviews on eHealth interventions ${ }^{a}$

\begin{tabular}{|c|c|c|c|c|c|}
\hline Reference & $\begin{array}{l}\text { Meta } \\
\text { analyses }\end{array}$ & Population & $\begin{array}{l}\text { Intervention } \\
\text { components }\end{array}$ & $\begin{array}{l}\text { Theoretical and } \\
\text { evidence base on } \\
\text { process/outcomes }\end{array}$ & $\begin{array}{l}\text { Primary } \\
\text { outcomes \& } \\
\text { quality of evidence }\end{array}$ \\
\hline $\begin{array}{l}\text { Boumparis et al. } \\
(2019)^{57}\end{array}$ & Yes & $\begin{array}{l}\text { School and high } \\
\text { school students } \\
\text { (aged 12-20); } \\
\text { problematic } \\
\text { cannabis users } \\
\text { (aged 16-40) }\end{array}$ & $\begin{array}{l}\text { Parent-involvement- } \\
\text { program, climate } \\
\text { school course, MI, brief } \\
\text { interventions, solution } \\
\text { focused approaches, } \\
\text { skills-based prevention } \\
\text { programs, community } \\
\text { reinforcement approach }\end{array}$ & $\begin{array}{l}\text { CBT, Person- } \\
\text { centered Therapy, } \\
\text { Social Influence } \\
\text { Theory }\end{array}$ & $\begin{array}{l}\text { Small effects on } \\
\text { cannabis use in students } \\
\text { and problematic } \\
\text { cannabis users as } \\
\text { compared to controls; } \\
\text { effects maintained in } \\
\text { students at 12-month } \\
\text { follow-up but not in } \\
\text { problematic cannabis } \\
\text { users; quality of } \\
\text { evidence: low (assessed } \\
\text { using the Cochrane risk- } \\
\text { of-bias tool) }\end{array}$ \\
\hline $\begin{array}{l}\text { Hadjistav- } \\
\text { ropoulos et al. } \\
(2020)^{54}\end{array}$ & Yes & $\begin{array}{l}\text { Adults general } \\
\text { population; } \\
\text { service users } \\
\text { with alcohol } \\
\text { use disorder } \\
\text { recruited from } \\
\text { specialized } \\
\text { mental health } \\
\text { services }\end{array}$ & $\begin{array}{l}\text { Information on alcohol, } \\
\text { preparing for change } \\
\text { exercises, skills training, } \\
\text { drinking diary, online } \\
\text { discussion forum, } \\
\text { automated motivational } \\
\text { text or email prompts, } \\
\text { blood-alcohol- } \\
\text { concentration calculator }\end{array}$ & CBT & $\begin{array}{l}\text { Self-guided iCBT } \\
\text { significantly more } \\
\text { effective in reducing } \\
\text { alcohol consumption } \\
\text { when compared to } \\
\text { information about } \\
\text { alcohol and waitlist } \\
\text { control conditions; } \\
\text { therapist-guided iCBT } \\
\text { more effective as } \\
\text { compared to waitlist } \\
\text { (medium-large effect) } \\
\text { control condition; } \\
\text { quality of evidence: } \\
\text { moderate (assessed } \\
\text { using the Cochrane } \\
\text { risk-of-bias tool) }\end{array}$ \\
\hline Tait et al. $(2010)^{56}$ & No & $\begin{array}{l}\text { Young people } \\
\text { with substance } \\
\text { misuse }\end{array}$ & $\begin{array}{l}\text { Interactive assignments, } \\
\text { video clips, personalized } \\
\text { normative F, information, } \\
\text { interactive online alcohol } \\
\text { education, assessment }\end{array}$ & Not reported & $\begin{array}{l}\text { Small effect } \\
\text { in preventing } \\
\text { development of } \\
\text { alcohol-related } \\
\text { problems among } \\
\text { people who were non- } \\
\text { drinkers at baseline; } \\
\text { effects similar to brief } \\
\text { in-person interventions; } \\
\text { quality of evidence: } \\
\text { low (e.g. small sample } \\
\text { sizes, mostly short-term } \\
\text { effects investigated, } \\
\text { considerable } \\
\text { heterogeneity), no } \\
\text { standardized approach } \\
\text { was used to assess } \\
\text { the quality of included } \\
\text { studies }\end{array}$ \\
\hline
\end{tabular}


Table 1. (continued). Summary of included reviews on eHealth interventions ${ }^{\circ}$

\begin{tabular}{|c|c|c|c|c|c|}
\hline Reference & $\begin{array}{l}\text { Meta } \\
\text { analyses }\end{array}$ & Population & $\begin{array}{l}\text { Intervention } \\
\text { components }\end{array}$ & $\begin{array}{l}\text { Theoretical and } \\
\text { evidence base on } \\
\text { process/outcomes }\end{array}$ & $\begin{array}{l}\text { Primary } \\
\text { outcomes \& } \\
\text { quality of evidence }\end{array}$ \\
\hline \multicolumn{6}{|l|}{ Treatment } \\
\hline $\begin{array}{l}\text { Arguel et al. } \\
(2018)^{50}\end{array}$ & Yes & $\begin{array}{l}\text { Adults with } \\
\text { various mental } \\
\text { disorders }\end{array}$ & $\begin{array}{l}\text { Social network } \\
\text { interventions (e.g. social } \\
\text { networks and forums, } \\
\text { discussion groups) }\end{array}$ & $\begin{array}{l}\text { Social Cognitive } \\
\text { Theory; Theory of } \\
\text { Planned Behavior, } \\
\text { Technology } \\
\text { Acceptance Model. } \\
\text { Perceived Risk } \\
\text { Influence }\end{array}$ & $\begin{array}{l}\text { Most common theory } \\
\text { for online social } \\
\text { network interventions } \\
\text { is Social Cognitive } \\
\text { Theory, quality } \\
\text { of evidence: low } \\
\text { (assessed using the } \\
\text { Cochrane risk-of-bias } \\
\text { tool) }\end{array}$ \\
\hline Lin et al. (2019) ${ }^{75}$ & No & $\begin{array}{l}\text { General } \\
\text { population, } \\
\text { patients with } \\
\text { substance } \\
\text { use disorder, } \\
\text { primary care } \\
\text { patients }\end{array}$ & $\begin{array}{l}\text { Psychotherapy: SC, MI, } \\
\text { individual counselling } \\
\text { sessions, group } \\
\text { therapy focused on } \\
\text { relapse prevention, } \\
\text { ME, combination of } \\
\text { videoconference \& } \\
\text { methadone treatment, PE }\end{array}$ & $\mathrm{CBT}, \mathrm{CBI}$ & $\begin{array}{l}\text { Superior treatment } \\
\text { retention for } \\
\text { telemedicine in one } \\
\text { study; lower dropout } \\
\text { rate in one study; } \\
\text { quality of evidence: } \\
\text { moderate (e.g. } \\
\text { retrospective studies, } \\
\text { moderate risk of bias) } \\
\text { (assessed using the } \\
\text { Cochrane risk-of-bias } \\
\text { tool) }\end{array}$ \\
\hline $\begin{array}{l}\text { Simon et al. } \\
(2019)^{39}\end{array}$ & No & $\begin{array}{l}\text { Adults with } \\
\text { PTSD }\end{array}$ & $\begin{array}{l}\mathrm{PE} \text {; stress management } \\
\text { techniques; cognitive } \\
\text { restructuring/trauma } \\
\text { processing; relapse } \\
\text { prevention. }\end{array}$ & CBT & $\begin{array}{l}\text { High levels of } \\
\text { acceptability; quality } \\
\text { of evidence: moderate } \\
\text { (assessed using the } \\
\text { Cochrane risk-of-bias } \\
\text { tool) }\end{array}$ \\
\hline $\begin{array}{l}\text { Richards et al. } \\
(2012)^{70}\end{array}$ & Yes & $\begin{array}{l}\text { Adults with } \\
\text { symptoms of } \\
\text { depression }\end{array}$ & $\begin{array}{l}\text { CBT-ID, Beating the Blues } \\
\text { (BTB), MoodGym, Sadness } \\
\text { Program, Overcoming } \\
\text { Depression on the Internet } \\
\text { (ODIN), Color your Life } 5\end{array}$ & CBT & $\begin{array}{l}\text { Overall medium effect } \\
\text { size; large effect and } \\
\text { greater retention for } \\
\text { therapist-supported } \\
\text { studies; small effect } \\
\text { for studies without } \\
\text { support; quality of } \\
\text { evidence: high, but } \\
\text { high risk of missing } \\
\text { data (assessed using } \\
\text { the Cochrane risk-of- } \\
\text { bias tool) }\end{array}$ \\
\hline
\end{tabular}


Table 1. (continued). Summary of included reviews on eHealth interventions ${ }^{a}$

\begin{tabular}{|c|c|c|c|c|c|}
\hline Reference & $\begin{array}{l}\text { Meta } \\
\text { analyses }\end{array}$ & Population & $\begin{array}{l}\text { Intervention } \\
\text { components }\end{array}$ & $\begin{array}{l}\text { Theoretical and } \\
\text { evidence base on } \\
\text { process/outcomes }\end{array}$ & $\begin{array}{l}\text { Primary } \\
\text { outcomes \& } \\
\text { quality of evidence }\end{array}$ \\
\hline $\begin{array}{l}\text { Sierra et al. } \\
(2018)^{79}\end{array}$ & Yes & $\begin{array}{l}\text { Individuals } \\
\text { with } \\
\text { depression }\end{array}$ & $\begin{array}{l}\text { Behavioral activation, } \\
\text { acceptance and } \\
\text { commitment therapy }\end{array}$ & third wave CBT & $\begin{array}{l}\text { Medium to large } \\
\text { effect sizes comparing } \\
\text { online intervention } \\
\text { vs. waitlist or TAU; } \\
\text { quality of evidence: } \\
\text { low (e.g. high attrition } \\
\text { to post-measure in } \\
\text { some studies, high } \\
\text { variability in sample } \\
\text { size), no standardized } \\
\text { approach was used to } \\
\text { assess the quality of } \\
\text { included studies }\end{array}$ \\
\hline $\begin{array}{l}\text { Erbe et al. } \\
(2017)^{46}\end{array}$ & No & $\begin{array}{l}\text { Adults with } \\
\text { depression, } \\
\text { anxiety, and/ } \\
\text { or substance } \\
\text { abuse }\end{array}$ & $\begin{array}{l}\text { Blended Interventions: } \\
\text { treatment programs that } \\
\text { use elements of both } \\
\text { face-to-face and internet- } \\
\text { based interventions; } \\
\text { web-based programs with } \\
\text { modules such as cognitive, } \\
\text { behavioral and emotion- } \\
\text { focused interventions, email } \\
\text { support, PE, group chats }\end{array}$ & CBT & $\begin{array}{l}\text { Blended interventions } \\
\text { are feasible and more } \\
\text { effective than waiting } \\
\text { list control conditions; } \\
\text { quality of evidence: } \\
\text { low; no standardized } \\
\text { approach was used to } \\
\text { assess the quality of } \\
\text { included studies }\end{array}$ \\
\hline Lau et al. (2016) ${ }^{62}$ & Yes & $\begin{array}{l}\text { Population } \\
\text { (aged 7-80) with } \\
\text { symptoms of } \\
\text { depression, } \\
\text { PTSD, autism, } \\
\text { ADHD, alcohol } \\
\text { use disorder }\end{array}$ & $\begin{array}{l}\text { Goal-oriented, cognitive } \\
\text { training games (physical, } \\
\text { emotional, cognition, } \\
\text { skills), PE }\end{array}$ & $\begin{array}{l}\text { Goal-oriented, } \\
\text { Problem-Solving, } \\
\text { Cognition Training, } \\
\text { and Games }\end{array}$ & $\begin{array}{l}\text { Moderate effect on } \\
\text { various symptom } \\
\text { domains; quality of } \\
\text { evidence: moderate, } \\
\text { risk of bias unclear } \\
\text { (incomplete reporting) } \\
\text { (assessed using the } \\
\text { Cochrane risk-of-bias } \\
\text { tool) }\end{array}$ \\
\hline $\begin{array}{l}\text { Irvine et al. } \\
(2020)^{88}\end{array}$ & Yes & $\begin{array}{l}\text { Hetero-genous } \\
\text { sample (e.g. } \\
\text { students, } \\
\text { adults with } \\
\text { and without } \\
\text { diagnoses } \\
\text { of mental } \\
\text { disorders, } \\
\text { adults referred } \\
\text { to mental } \\
\text { health services) }\end{array}$ & $\begin{array}{l}\text { Counselling, CBT } \\
\text { techniques used by } \\
\text { therapist, solution focused } \\
\text { therapy, peer counselling, } \\
\text { Employee Assistant } \\
\text { Program }\end{array}$ & CBT & $\begin{array}{l}\text { Telephone sessions } \\
\text { shorter than face-to-face } \\
\text { sessions; no significant } \\
\text { difference in therapeutic } \\
\text { alliance, disclosure, } \\
\text { empathy, attentiveness; } \\
\text { more active participation } \\
\text { in telephone mode; } \\
\text { quality of evidence: } \\
\text { low (e.g. small sample } \\
\text { sizes, observational } \\
\text { studies, high levels } \\
\text { of inconsistency), no } \\
\text { standardized approach } \\
\text { was used to assess } \\
\text { the quality of included } \\
\text { studies }\end{array}$ \\
\hline
\end{tabular}


Table 1. (continued). Summary of included reviews on eHealth interventions ${ }^{\text {a }}$

\begin{tabular}{|c|c|c|c|c|c|}
\hline Reference & $\begin{array}{l}\text { Meta } \\
\text { analyses }\end{array}$ & Population & $\begin{array}{l}\text { Intervention } \\
\text { components }\end{array}$ & $\begin{array}{l}\text { Theoretical and } \\
\text { evidence base on } \\
\text { process/outcomes }\end{array}$ & $\begin{array}{l}\text { Primary } \\
\text { outcomes \& } \\
\text { quality of evidence }\end{array}$ \\
\hline $\begin{array}{l}\text { Gentry et al. } \\
(2019)^{87}\end{array}$ & No & $\begin{array}{l}\text { Veterans with } \\
\text { PTSD, opioid- } \\
\text { dependents; } \\
\text { women with HIV; } \\
\text { cancer patients, } \\
\text { inmates in } \\
\text { seclusion, } \\
\text { smokers, } \\
\text { diabetes } \\
\text { patients }\end{array}$ & $\begin{array}{l}\text { Group therapy via VTC } \\
\text { services e.g. relapse } \\
\text { control therapy, coping } \\
\text { skills, healthy relationship } \\
\text { educational program, } \\
\text { cognitive processing } \\
\text { therapy, relaxation } \\
\text { response resilience } \\
\text { program, mindfulness, } \\
\text { SC, self-management } \\
\text { education, chat group, } \\
\text { video group, psychosocial } \\
\text { support }\end{array}$ & CBT, ACT, PE & $\begin{array}{l}\text { No differences } \\
\text { between VTC and } \\
\text { face-to-face group } \\
\text { sesstions on treatment } \\
\text { outcomes (e.g. PTSD } \\
\text { symptoms); effects of } \\
\text { VTC comparable to } \\
\text { face-to-face treatment; } \\
\text { mild decreases of } \\
\text { therapeutic alliance } \\
\text { in VTC; quality of } \\
\text { evidence: moderate } \\
\text { (assessed using the } \\
\text { quality of evidence } \\
\text { criteria of the US } \\
\text { Preventive Services } \\
\text { Task Force) }\end{array}$ \\
\hline $\begin{array}{l}\text { Grist et al. } \\
(2013)^{65}\end{array}$ & Yes & $\begin{array}{l}\text { Participants } \\
\text { with common } \\
\text { mental } \\
\text { disorders }\end{array}$ & $\begin{array}{l}\text { e.g. Beating the Blues, } \\
\text { MoodGYM, Panic Online, } \\
\text { Color Your Life, DE-STRESS, } \\
\text { FearFighter, Coping With } \\
\text { Depression; support via } \\
\text { email, phone, web forum, } \\
\text { text messages }\end{array}$ & CBT & $\begin{array}{l}\text { Medium effect size of } \\
\text { iCBT on various mental } \\
\text { health outcomes, } \\
\text { comparable to face } \\
\text { to face CBT; iCBT } \\
\text { significantly more } \\
\text { effective than waitlist } \\
\text { and active control; } \\
\text { quality of evidence: } \\
\text { moderate (assessed } \\
\text { using the Cochrane } \\
\text { risk-of-bias tool) }\end{array}$ \\
\hline $\begin{array}{l}\text { Axelsson et al., } \\
(2019)^{112}\end{array}$ & Yes & $\begin{array}{l}\text { Adult patients } \\
\text { with health } \\
\text { anxiety }\end{array}$ & $\begin{array}{l}\text { Cognitive restructuring, } \\
\text { exposure-based techniques }\end{array}$ & CBT & $\begin{array}{l}\text { Moderate to large } \\
\text { effects of iCBT } \\
\text { on health anxiety } \\
\text { (superior to active } \\
\text { controls in two } \\
\text { studies), small effect } \\
\text { on QoL; comparable } \\
\text { effects of iCBT to } \\
\text { face-to-face-CBT; } \\
\text { quality of evidence: } \\
\text { moderate (e.g. small } \\
\text { effect of publication } \\
\text { bias, substantial } \\
\text { heterogeneity), low } \\
\text { risk of bias (assessed } \\
\text { using the Cochrane } \\
\text { risk-of-bias tool) }\end{array}$ \\
\hline
\end{tabular}


Table 1. (continued). Summary of included reviews on eHealth interventions ${ }^{a}$

\begin{tabular}{|c|c|c|c|c|c|}
\hline Reference & $\begin{array}{l}\text { Meta } \\
\text { analyses }\end{array}$ & Population & $\begin{array}{l}\text { Intervention } \\
\text { components }\end{array}$ & $\begin{array}{l}\text { Theoretical and } \\
\text { evidence base on } \\
\text { process/outcomes }\end{array}$ & $\begin{array}{l}\text { Primary } \\
\text { outcomes \& } \\
\text { quality of evidence }\end{array}$ \\
\hline $\begin{array}{l}\text { Berryhill et al. } \\
(2019)^{80}\end{array}$ & No & $\begin{array}{l}\text { Adult patients } \\
\text { with anxiety } \\
\text { disorder }\end{array}$ & $\begin{array}{l}\text { Video-conferencing } \\
\text { psychological therapy }\end{array}$ & $\begin{array}{l}\text { CBT, ACT, } \\
\text { Metacognitive } \\
\text { Therapy }\end{array}$ & $\begin{array}{l}\text { Small to large } \\
\text { improvements on } \\
\text { anxiety; quality of } \\
\text { evidence: moderate } \\
\text { (assessed using the } \\
\text { Effective Public Health } \\
\text { Practice Project quality } \\
\text { assessment tool) }\end{array}$ \\
\hline $\begin{array}{l}\text { Bolton et al. } \\
(2015)^{82}\end{array}$ & Yes & $\begin{array}{l}\text { Adults with } \\
\text { traumatic } \\
\text { experience }\end{array}$ & $\begin{array}{l}\text { Hybrid design: internet } \\
\text { and real-time (telephone } \\
\text { calls, initial face-to-face } \\
\text { introductory meeting) } \\
\text { delivery of intervention } \\
\text { components or } \\
\text { asynchronous (email) } \\
\text { communication }\end{array}$ & CBT & $\begin{array}{l}\text { Medium-large } \\
\text { improvements } \\
\text { in cognitive and } \\
\text { behavioral symptoms } \\
\text { of depression, } \\
\text { generalized anxiety } \\
\text { and posttraumatic } \\
\text { stress; quality of } \\
\text { evidence: moderate } \\
\text { (e.g. underpowered, } \\
\text { rare blinded group } \\
\text { allocation) (assessed } \\
\text { using the Quality } \\
\text { Index) }\end{array}$ \\
\hline $\begin{array}{l}\text { Castro et al. } \\
(2020)^{81}\end{array}$ & Yes & $\begin{array}{l}\text { Depressed } \\
\text { adults with } \\
\text { no comorbid } \\
\text { somatic } \\
\text { disorders }\end{array}$ & $\begin{array}{l}\text { Workbook including } \\
\text { exercises, skills trainings } \\
\text { and coping strategies, } \\
\text { information about local } \\
\text { mental health services, } \\
\text { pedometer }\end{array}$ & CBT & $\begin{array}{l}\text { Large reduction in } \\
\text { depressive symptoms } \\
\text { compared to control; } \\
\text { non-significant small } \\
\text { effect of telephone- } \\
\text { administered } \\
\text { psychotherapy } \\
\text { compared to active } \\
\text { comparators; quality } \\
\text { of evidence: low } \\
\text { (assessed using the } \\
\text { Cochrane risk-of-bias } \\
\text { tool) }\end{array}$ \\
\hline
\end{tabular}


Table 1. (continued). Summary of included reviews on eHealth interventions ${ }^{\text {a }}$

\begin{tabular}{|c|c|c|c|c|c|}
\hline Reference & $\begin{array}{l}\text { Meta } \\
\text { analyses }\end{array}$ & Population & $\begin{array}{l}\text { Intervention } \\
\text { components }\end{array}$ & $\begin{array}{l}\text { Theoretical and } \\
\text { evidence base on } \\
\text { process/outcomes }\end{array}$ & $\begin{array}{l}\text { Primary } \\
\text { outcomes \& } \\
\text { quality of evidence }\end{array}$ \\
\hline $\begin{array}{l}\text { Stech et al. } \\
(2020)^{68}\end{array}$ & Yes & $\begin{array}{l}\text { Adults with an } \\
\text { at-risk mental } \\
\text { state or a } \\
\text { diagnosis of } \\
\text { panic disorder }\end{array}$ & $\begin{array}{l}\mathrm{PE} \text {, cognitive restructuring, } \\
\text { relapse prevention, } \\
\text { exposure techniques }\end{array}$ & CBT & $\begin{array}{l}\text { Large improvements } \\
\text { for panic and } \\
\text { agoraphobia severity } \\
\text { for iCBT compared } \\
\text { to waitlist and } \\
\text { information controls; } \\
\text { similar results of iCBT } \\
\text { and face-to-face CBT } \\
\text { in reducing panic } \\
\text { and agoraphobia } \\
\text { symptoms; large } \\
\text { within-group } \\
\text { improvements for } \\
\text { panic, medium for } \\
\text { agoraphobia symptom } \\
\text { severity; quality of } \\
\text { evidence: low- } \\
\text { moderate (assessed } \\
\text { using the Cochrane } \\
\text { risk-of-bias tool and } \\
\text { ROBINS-I tool) }\end{array}$ \\
\hline $\begin{array}{l}\text { Rees et al. } \\
(2015)^{83}\end{array}$ & No & $\begin{array}{l}\text { Participants } \\
\text { with anxiety } \\
\text { disorders } \\
\text { (e.g., PTSD, } \\
\text { obsessive- } \\
\text { compulsive, } \\
\text { social phobia) } \\
\text { and/or } \\
\text { depression }\end{array}$ & $\begin{array}{l}\text { Treatment components } \\
\text { based on CBT principles } \\
\text { delivered using video } \\
\text { conferencing software }\end{array}$ & CBT & $\begin{array}{l}\text { Treatment effective } \\
\text { in reducing anxiety } \\
\text { symptoms (moderate } \\
\text { - large effect sizes), } \\
\text { comparable to face-to- } \\
\text { face treatment; quality } \\
\text { of evidence: low (e.g. } \\
\text { partly no controls or } \\
\text { case studies, small } \\
\text { sample sizes), no } \\
\text { standardized approach } \\
\text { was used to assess } \\
\text { the quality of included } \\
\text { studies }\end{array}$ \\
\hline
\end{tabular}


Table 1. (continued). Summary of included reviews on eHealth interventions ${ }^{a}$

\begin{tabular}{|c|c|c|c|c|c|}
\hline Reference & $\begin{array}{l}\text { Meta } \\
\text { analyses }\end{array}$ & Population & $\begin{array}{l}\text { Intervention } \\
\text { components }\end{array}$ & $\begin{array}{l}\text { Theoretical and } \\
\text { evidence base on } \\
\text { process/outcomes }\end{array}$ & $\begin{array}{l}\text { Primary } \\
\text { outcomes \& } \\
\text { quality of evidence }\end{array}$ \\
\hline $\begin{array}{l}\text { Richardson et al. } \\
(2010)^{60}\end{array}$ & No & $\begin{array}{l}\text { Children and } \\
\text { adolescents } \\
\text { with depression }\end{array}$ & $\begin{array}{l}\text { Stressbusters; Master } \\
\text { your Mood online (group } \\
\text { therapy via online chat } \\
\text { room), Catch It (CBT } \\
\text { principles with aspects } \\
\text { of interpersonal therapy } \\
\text { and behavioral activation); } \\
\text { MoodGYM (CBT- based, } \\
\text { delivered online); iCBT: } \\
\text { Cool Teens, BRAVE; } \\
\text { including intervention } \\
\text { components such as } \\
\text { PE, quizzes, homework } \\
\text { assignments, case } \\
\text { vignettes, narration, } \\
\text { cartoons, educational and } \\
\text { training videos }\end{array}$ & CBT & $\begin{array}{l}30 \% \text { - } 78 \% \text { of included } \\
\text { participants no longer } \\
\text { met diagnostic criteria } \\
\text { for primary diagnoses; } \\
\text { improvements in } \\
\text { depressive symptoms } \\
\text { only in participants } \\
\text { completing } \geq 3 \\
\text { sessions; quality of } \\
\text { evidence: low (e.g. } \\
50 \% \text { case studies, } \\
\text { studies without control } \\
\text { groups, possibility of } \\
\text { publication bias, small } \\
\text { number of databases), } \\
\text { no standardized } \\
\text { approach was used to } \\
\text { assess the quality of } \\
\text { included studies }\end{array}$ \\
\hline $\begin{array}{l}\text { Rost et al. } \\
(2017)^{41}\end{array}$ & No & $\begin{array}{l}\text { Individuals } \\
\text { with } \\
\text { depression }\end{array}$ & $\begin{array}{l}\text { Mindfulness-based } \\
\text { cognitive therapy, } \\
\text { behavioral activation, MI }\end{array}$ & CBT & $\begin{array}{l}\text { Effectiveness of digital } \\
\text { interventions on } \\
\text { depressive symptoms; } \\
\text { drop-out rates } \\
\text { comparable to face-to- } \\
\text { face treatment; quality } \\
\text { of evidence: low (e.g. } \\
\text { high heterogeneity, } \\
\text { possibility of biases), } \\
\text { no standardized } \\
\text { approach was used to } \\
\text { assess the quality of } \\
\text { included studies due } \\
\text { to high variability in } \\
\text { study type }\end{array}$ \\
\hline $\begin{array}{l}\text { Pasarelu et al. } \\
(2017)^{66}\end{array}$ & Yes & $\begin{array}{l}\text { Non-clinical } \\
\text { and clinical } \\
\text { population } \\
\text { (depressive } \\
\text { and anxiety } \\
\text { symptoms) }\end{array}$ & $\begin{array}{l}\text { iCBT: transdiagnostic/ } \\
\text { tailored, clinician guided } \\
\text { with interview/ self-guided } \\
\text { with interview/ self-guided, } \\
\text { 4-25 modules }\end{array}$ & CBT & $\begin{array}{l}\text { Medium-large effect } \\
\text { for iCBT on anxiety } \\
\text { and depression } \\
\text { outcomes; medium } \\
\text { effect on QoL; large } \\
\text { effect on generic } \\
\text { outcome measures; no } \\
\text { differences on anxiety } \\
\text { and QoL compared } \\
\text { to disorder-specific } \\
\text { treatments; quality of } \\
\text { evidence: moderate to } \\
\text { high (assessed using } \\
\text { the Cochrane risk-of- } \\
\text { bias tool) }\end{array}$ \\
\hline
\end{tabular}


Table 1. (continued). Summary of included reviews on eHealth interventions ${ }^{\text {a }}$

\begin{tabular}{|c|c|c|c|c|c|}
\hline Reference & $\begin{array}{l}\text { Meta } \\
\text { analyses }\end{array}$ & Population & $\begin{array}{l}\text { Intervention } \\
\text { components }\end{array}$ & $\begin{array}{l}\text { Theoretical and } \\
\text { evidence base on } \\
\text { process/outcomes }\end{array}$ & $\begin{array}{l}\text { Primary } \\
\text { outcomes \& } \\
\text { quality of evidence }\end{array}$ \\
\hline $\begin{array}{l}\text { Pittock et al. } \\
(2018)^{77}\end{array}$ & No & $\begin{array}{l}\text { Patients with } \\
\text { symptoms } \\
\text { of bulimia } \\
\text { nervosa }\end{array}$ & $\begin{array}{l}\text { Weekly emails, F, tasks, } \\
\text { manuals, email support, } \\
\text { messaging with coach, } \\
\text { face-to-face evaluations, } \\
\text { group online setting }\end{array}$ & CBT & $\begin{array}{l}\text { Large effects for iCBT } \\
\text { in binge eating and } \\
\text { purging reduction; } \\
\text { sustained at follow-up; } \\
\text { no overall significant } \\
\text { superiority to controls; } \\
\text { quality of evidence: } \\
\text { moderate (assessed } \\
\text { using the Cochrane } \\
\text { risk-of-bias tool) }\end{array}$ \\
\hline $\begin{array}{l}\text { Massoudi et al. } \\
(2019)^{51}\end{array}$ & Yes & $\begin{array}{l}\text { Patients } \\
\text { in primary } \\
\text { care with } \\
\text { depressive/ } \\
\text { anxiety } \\
\text { symptoms/ } \\
\text { disorders }\end{array}$ & $\begin{array}{l}\text { Monitoring, PE, relaxation, } \\
\text { behavioral activation, } \\
\text { intensive disease } \\
\text { management, mindfulness, } \\
\text { ACT, medication } \\
\text { management, assessment } \\
\text { of symptoms, email } \\
\text { reminder }\end{array}$ & $\begin{array}{l}\text { CBT, Trans- } \\
\text { theoretical Model of } \\
\text { Behavioural Change }\end{array}$ & $\begin{array}{l}\text { Small effect of e-health } \\
\text { interventions for } \\
\text { depression compared } \\
\text { to control groups/ } \\
\text { TAS, moderate effect } \\
\text { compared to waitlist; } \\
\text { effects maintained in } \\
\text { long-term; no evidence } \\
\text { for effectiveness } \\
\text { for anxiety; quality } \\
\text { of evidence: low- } \\
\text { moderate (assessed } \\
\text { using the Cochrane } \\
\text { risk-of-bias tool) }\end{array}$ \\
\hline $\begin{array}{l}\text { Lewis et al. } \\
\text { (2019) }^{85}\end{array}$ & Yes & $\begin{array}{l}\text { Adults with } \\
\text { PTSD }\end{array}$ & $\begin{array}{l}\text { PTSD-Coach, trauma- } \\
\text { focused PE, skills training, } \\
\text { imaginal exposure, } \\
\text { cognitive restructuring }\end{array}$ & CBT & $\begin{array}{l}\text { Clinically relevant } \\
\text { reduction in PTSD } \\
\text { symptoms as } \\
\text { compared to waiting } \\
\text { list control; quality } \\
\text { of evidence: low } \\
\text { (assessed using } \\
\text { Cochrane risk-of-bias } \\
\text { tool) }\end{array}$ \\
\hline $\begin{array}{l}\text { Harrer et al. } \\
(2019)^{38}\end{array}$ & Yes & $\begin{array}{l}\text { University } \\
\text { students with } \\
\text { symptoms of } \\
\text { depression, } \\
\text { anxiety, } \\
\text { stress, sleep } \\
\text { problems, } \\
\text { and eating } \\
\text { disorders }\end{array}$ & $\begin{array}{l}\text { Mindfulness based } \\
\text { intervention, Talk to Me, } \\
\text { eating disorder prevention, } \\
\text { Moodgym, problem solving } \\
\text { simulator, PE, present } \\
\text { control intervention, eating } \\
\text { and stress management, } \\
\text { ACT based intervention, } \\
\text { cognitive bias modification }\end{array}$ & $\begin{array}{l}\text { CBT, CBM, } \\
\text { third wave } \\
\text { CBT, Emotional } \\
\text { Disclosure, } \\
\text { Skills Training, } \\
\text { Personalized F }\end{array}$ & $\begin{array}{l}\text { Small effect on } \\
\text { depression, stress and } \\
\text { anxiety; moderate } \\
\text { effect on eating } \\
\text { disorder symptoms } \\
\text { and role functioning; } \\
\text { no significant effect on } \\
\text { wellubeing; quality of } \\
\text { evidence: moderate, } \\
\text { high risk of bias in } 50 \% \\
\text { of studies (assessed } \\
\text { using the Cochrane } \\
\text { risk-of-bias tool) }\end{array}$ \\
\hline
\end{tabular}


Table 1. (continued). Summary of included reviews on eHealth interventions ${ }^{a}$

\begin{tabular}{|c|c|c|c|c|c|}
\hline Reference & $\begin{array}{l}\text { Meta } \\
\text { analyses }\end{array}$ & Population & $\begin{array}{l}\text { Intervention } \\
\text { components }\end{array}$ & $\begin{array}{l}\text { Theoretical and } \\
\text { evidence base on } \\
\text { process/outcomes }\end{array}$ & $\begin{array}{l}\text { Primary } \\
\text { outcomes \& } \\
\text { quality of evidence }\end{array}$ \\
\hline $\begin{array}{l}\text { Hedman et al. } \\
(2012)^{113}\end{array}$ & No & $\begin{array}{l}\text { Adult patients } \\
\text { (e.g. with } \\
\text { depression, } \\
\text { social phobia, } \\
\text { panic disorder, } \\
\text { PTSD) }\end{array}$ & $\begin{array}{l}\text { Online bibliotherapy, } \\
\text { therapist contact over the } \\
\text { internet (text messages, } \\
\text { email), PE }\end{array}$ & CBT & $\begin{array}{l}\text { Large effect sizes on } \\
\text { reducing depression, } \\
\text { panic disorder, social } \\
\text { phobia, small-moderate } \\
\text { effect sizes on lowering } \\
\text { chronic pain; quality } \\
\text { of evidence: high for } \\
\text { depression, panic } \\
\text { disorder and social } \\
\text { phobia (assessed } \\
\text { with APA criteria for } \\
\text { evidence) }\end{array}$ \\
\hline $\begin{array}{l}\text { Grist et al. } \\
(2019)^{63}\end{array}$ & Yes & $\begin{array}{l}\text { Children and } \\
\text { adolescents } \\
\text { with anxiety } \\
\text { and depression } \\
\text { symptoms }\end{array}$ & $\begin{array}{l}\text { films, text, animations, } \\
\text { interactive fantasy } \\
\text { game, computerized } \\
\text { spider exposure } \\
\text { therapy, interactive } \\
\text { presentation, workbook, } \\
\text { self-help manual, anxiety } \\
\text { management, dot probe } \\
\text { task, problem solving }\end{array}$ & $\begin{array}{l}\text { CBT, computer- } \\
\text { delivered Attention } \\
\text { Bias Modification } \\
\text { programs (ABM), } \\
\text { Cognitive Bias } \\
\text { Modification } \\
\text { programs (CBM), } \\
\text { video games utilizing } \\
\text { Neurofeedback, } \\
\text { Biofeedback, ER } \\
\text { Training, internet- } \\
\text { based ACT program, } \\
\text { Problem-Solving } \\
\text { Therapy }\end{array}$ & $\begin{array}{l}\text { Small effect of } \\
\text { technology delivered } \\
\text { interventions } \\
\text { compared to waitlist; } \\
\text { CBT medium effect, } \\
\text { ABM small effect; no } \\
\text { significant benefit over } \\
\text { control groups; quality } \\
\text { of evidence: high risk } \\
\text { of detection bias, low } \\
\text { risk of attrition bias } \\
\text { (assessed using the } \\
\text { Cochrane risk-of-bias } \\
\text { tool) }\end{array}$ \\
\hline $\begin{array}{l}\text { Davies et al. } \\
(2014)^{36}\end{array}$ & Yes & $\begin{array}{l}\text { University } \\
\text { students with } \\
\text { symptoms of } \\
\text { de-pression, } \\
\text { anxiety, psy- } \\
\text { chological } \\
\text { distress }\end{array}$ & $\begin{array}{l}\text { Stress management, } \\
\text { improving relationship } \\
\text { functioning, decreasing } \\
\text { elevated levels of } \\
\text { perfectionism, mindfulness, } \\
\text { social support, increasing } \\
\text { use of lucid dreaming, }\end{array}$ & $\begin{array}{l}\text { CBT, Mindfulness, } \\
\text { Stress Management } \\
\text { Theory, Cognitive } \\
\text { Learning Theory, } \\
\text { Lucid Dreaming }\end{array}$ & $\begin{array}{l}\text { Improvements in } \\
\text { anxiety, depression, } \\
\text { stress compared to } \\
\text { inactive control; no } \\
\text { support for either } \\
\text { condition for anxiety } \\
\text { or depression } \\
\text { compared to active } \\
\text { controls; quality } \\
\text { of evidence: low } \\
\text { (e.g. skewed data), } \\
\text { moderate risk of bias } \\
\text { (assessed using the } \\
\text { Cochrane risk-of-bias } \\
\text { tool) }\end{array}$ \\
\hline
\end{tabular}


Table 1. (continued). Summary of included reviews on eHealth interventions ${ }^{\text {a }}$

\begin{tabular}{|c|c|c|c|c|c|}
\hline Reference & $\begin{array}{l}\text { Meta } \\
\text { analyses }\end{array}$ & Population & $\begin{array}{l}\text { Intervention } \\
\text { components }\end{array}$ & $\begin{array}{l}\text { Theoretical and } \\
\text { evidence base on } \\
\text { process/outcomes }\end{array}$ & $\begin{array}{l}\text { Primary } \\
\text { outcomes \& } \\
\text { quality of evidence }\end{array}$ \\
\hline $\begin{array}{l}\text { Coughtrey et al. } \\
(2018)^{82}\end{array}$ & No & $\begin{array}{l}\text { Population with } \\
\text { depression and } \\
\text { other medical } \\
\text { conditions } \\
\text { (e.g. HIV/ } \\
\text { AIDS, multiple } \\
\text { sclerosis, } \\
\text { parkinson's } \\
\text { disease) }\end{array}$ & $\begin{array}{l}\text { Telephone-based } \\
\text { Interventions, cognitive and } \\
\text { behavioral components }\end{array}$ & $\begin{array}{l}\text { CBT, Interpersonal } \\
\text { Psychotherapy, } \\
\text { Behavioral } \\
\text { Activation, Exposure } \\
\text { and Response } \\
\text { Prevention, Applied } \\
\text { Relaxation }\end{array}$ & $\begin{array}{l}\text { Small to high effects } \\
\text { on reducing symptoms } \\
\text { of depression or } \\
\text { anxiety (greater } \\
\text { for uncontrolled } \\
\text { compared to } \\
\text { controlled studies); } \\
\text { but significant change } \\
\text { in only } 4 / 14 \text { studies; } \\
\text { quality of evidence: } \\
\text { moderate to high } \\
\text { (assessed using the } \\
\text { Effective Public Health } \\
\text { Practice Project Quality } \\
\text { Assessment Tool) }\end{array}$ \\
\hline $\begin{array}{l}\text { Asuzu et al. } \\
(2019)^{58}\end{array}$ & Yes & $\begin{array}{l}\text { Adolescents } \\
\text { and young } \\
\text { adults (<24 } \\
\text { years) with } \\
\text { cannabis } \\
\text { misuse }\end{array}$ & $\begin{array}{l}\text { Cannabis specific and } \\
\text { non-specific; prevention } \\
\text { program tailored to } \\
\text { mother-daughter-dyad, } \\
\text { RealTeen, Climate } \\
\text { Schools, Computer brief } \\
\text { intervention }\end{array}$ & $\begin{array}{l}\text { Family Interaction } \\
\text { Theory; } \\
\text { Personalized } \\
\text { and Corrective } \\
\text { Normative F; Harm } \\
\text { Reduction; Social } \\
\text { Competency and } \\
\text { Skills Building; } \\
\text { Motivational } \\
\text { Enhancement } \\
\text { Model }\end{array}$ & $\begin{array}{l}\text { Small-medium effects } \\
\text { in reduction of } \\
\text { cannabis use (7/11); } \\
\text { quality of evidence: } \\
\text { low (assessed using } \\
\text { the National Institute } \\
\text { of Health quality rating } \\
\text { tool) }\end{array}$ \\
\hline $\begin{array}{l}\text { Baker et al. } \\
(2018)^{86}\end{array}$ & Yes & $\begin{array}{l}\text { Adults with } \\
\text { psychotic } \\
\text { disorders }\end{array}$ & $\begin{array}{l}\text { Telephone Intervention: } \\
\text { relapse prevention, } \\
\text { medication adherence, } \\
\text { reduction of smoking and } \\
\text { cardiovascular disease risk } \\
\text { behaviors }\end{array}$ & $\begin{array}{l}\text { Not reported; } \\
\text { person-delivered } \\
\text { interventions } \\
\text { (spoken word } \\
\text { and psychological } \\
\text { strategies) }\end{array}$ & $\begin{array}{l}\text { Relapse prevention } \\
\text { ( } n=5 / 8) \text {, medication } \\
\text { adherence ( } n=1 / 3 \text { ); } \\
\text { at least half of } \\
\text { outcomes in favor } \\
\text { of the telephone } \\
\text { intervention, } \\
\text { comparable levels of } \\
\text { improvement; quality } \\
\text { of evidence: low } \\
\text { (e.g., high variation } \\
\text { in quality, many } \\
\text { uncontrolled studies), } \\
\text { no standardized } \\
\text { approach was used to } \\
\text { assess the quality of } \\
\text { included studies }\end{array}$ \\
\hline
\end{tabular}


Table 1. (continued). Summary of included reviews on eHealth interventions ${ }^{a}$

\begin{tabular}{|c|c|c|c|c|c|}
\hline Reference & $\begin{array}{l}\text { Meta } \\
\text { analyses }\end{array}$ & Population & $\begin{array}{l}\text { Intervention } \\
\text { components }\end{array}$ & $\begin{array}{l}\text { Theoretical and } \\
\text { evidence base on } \\
\text { process/outcomes }\end{array}$ & $\begin{array}{l}\text { Primary } \\
\text { outcomes \& } \\
\text { quality of evidence }\end{array}$ \\
\hline $\begin{array}{l}\text { Chebli et al. } \\
(2016)^{53}\end{array}$ & No & $\begin{array}{l}\text { Pathological } \\
\text { gamblers, } \\
\text { problem } \\
\text { drinkers, } \\
\text { smokers, opioid } \\
\text { dependent } \\
\text { outpatients }\end{array}$ & $\begin{array}{l}\text { Support calls via telephone } \\
\text { (positive F, encouragement, } \\
\text { answer questions), SMS, } \\
\text { email prompts, voice } \\
\text { respond messages; peer } \\
\text { based social support; } \\
\text { behavioral approaches, } \\
\text { internet-based modules/ } \\
\text { assignments, face-to-face } \\
\text { meetings, internet-based } \\
\text { forum, information, self- } \\
\text { help kit }\end{array}$ & CBT, MI & $\begin{array}{l}\text { Consistent evidence } \\
\text { for positive treatment } \\
\text { outcomes for addictive } \\
\text { behavior (effect size } \\
\text { not reported); positive } \\
\text { behavioral changes } \\
\text { through reduction of } \\
\text { problematic behaviors; } \\
\text { quality of evidence: } \\
\text { moderate (assessed } \\
\text { using the Downs and } \\
\text { Black checklist) }\end{array}$ \\
\hline $\begin{array}{l}\text { Danielsson et al. } \\
(2014)^{74}\end{array}$ & Yes & $\begin{array}{l}\text { Adults with } \\
\text { substance } \\
\text { misuse (i.e. } \\
\text { cigarettes, } \\
\text { alcohol) and/ } \\
\text { or pathological } \\
\text { gambling }\end{array}$ & $\begin{array}{l}\text { E.g. Online SC training, } \\
\text { digital multimedia } \\
\text { intervention, emails, } \\
\text { SMS; Gambling \& } \\
\text { Alcohol misuse: Internet } \\
\text { intervention with emails, } \\
\text { telephone helplines, } \\
\text { drinking journal, decision } \\
\text { making modules, } \\
\text { individual/group } \\
\text { intervention, self-help } \\
\text { booklet, craving helpline }\end{array}$ & $\mathrm{MI}, \mathrm{F}, \mathrm{PE}, \mathrm{CBT}$ & $\begin{array}{l}\text { Telephone helplines } \\
\text { can reduce tobacco } \\
\text { smoking; inconsistent } \\
\text { effects for alcohol } \\
\text { use and gambling; all } \\
\text { together inconsistent } \\
\text { evidence of eHealth } \\
\text { on tobacco/ alcohol/ } \\
\text { gambling; quality of } \\
\text { evidence: low (e.g. } \\
\text { lack of controls, very } \\
\text { high attrition rates), no } \\
\text { standardized approach } \\
\text { was used to assess } \\
\text { the quality of included } \\
\text { studies }\end{array}$ \\
\hline $\begin{array}{l}\text { Gilmore et al. } \\
(2017)^{76}\end{array}$ & No & $\begin{array}{l}\text { Patients with } \\
\text { co-occurring } \\
\text { PTSD/ trauma } \\
\text { symptoms and } \\
\text { substance use } \\
\text { disorder }\end{array}$ & $\begin{array}{l}\text { Web-based modules, } \\
\text { telehealth treatment, } \\
\text { skill training, symptom } \\
\text { management, Ml, expert } \\
\text { advice }\end{array}$ & Behavioral therapy & $\begin{array}{l}\text { eHealth efficacious in } \\
\text { reducing substance } \\
\text { use and trauma } \\
\text { symptoms: significant } \\
\text { decrease in trauma } \\
\text { symptoms in } 3 / 4 \\
\text { studies; significant } \\
\text { decrease in substance } \\
\text { use in } 4 / 6 \text { studies; } \\
\text { quality of evidence: } \\
\text { moderate-high } \\
\text { (assessed using the } \\
\text { GRADE approach) }\end{array}$ \\
\hline
\end{tabular}

Notes: $\mathrm{CBT}=$ Cognitive Behavioral Therapy; $\mathrm{PE}=$ psychoeducation; $\mathrm{PA}=$ physical activity; $\mathrm{F}=$ feedback; $\mathrm{ME}=$ motivation enhancement; $\mathrm{Ml}=$ motivational interviewing; $\mathrm{ER}=$ emotion regulation; $\mathrm{SC}=$ smoking cessation; $\mathrm{Q} \mathrm{L}=$ =quality of life; $\mathrm{TAU}=$ treatment as usual; $\mathrm{VTC}=\mathrm{video}$ teleconferencing.

a Complete summary of included reviews on eHealth and mHealth are shown in the Supplements, including findings on secondary outcomes, quality from the user perspective, safety, and cost effectiveness. 
Table 2. Summary of included studies on mHealth interventions ${ }^{\text {a }}$

\begin{tabular}{|c|c|c|c|c|c|}
\hline Reference & $\begin{array}{l}\text { Meta } \\
\text { analyses }\end{array}$ & Population & $\begin{array}{l}\text { Intervention } \\
\text { components }\end{array}$ & $\begin{array}{l}\text { Theoretical and } \\
\text { evidence base on } \\
\text { process/outcomes }\end{array}$ & $\begin{array}{l}\text { Primary Outcome \& } \\
\text { quality of evidence }\end{array}$ \\
\hline \multicolumn{6}{|c|}{ Mental Health Promotion and Prevention } \\
\hline $\begin{array}{l}\text { Sucala et al. } \\
(2016)^{110}\end{array}$ & No & Not reported & $\begin{array}{l}\text { Meditation, breathing } \\
\text { exercises, digital diary, } \\
\text { cognitive restructuring, } \\
\text { emotional ratings, } \\
\text { problem solving, rational } \\
\text { statements, goal setting, } \\
\text { hypnosis, physical } \\
\text { exercise }\end{array}$ & $\begin{array}{l}\text { No information for } \\
63,5 \% \text { of the apps, } \\
26,9 \% \text { with Cognitive } \\
\text { Behavioral Approach, } \\
7,7 \% \text { using mixed } \\
\text { approach }\end{array}$ & $\begin{array}{l}\text { Great majority of the } \\
\text { mHealth apps do not } \\
\text { offer evidence-based } \\
\text { interventions; } \\
\text { quality of evidence: low } \\
\text { (e.g. no information on } \\
\text { evidence-base in } 96,2 \% \\
\text { of the apps; none of the } \\
14 \text { apps reported studies } \\
\text { on effectiveness); no } \\
\text { standardized approach } \\
\text { was used to assess } \\
\text { the quality of included } \\
\text { studies/apps }\end{array}$ \\
\hline $\begin{array}{l}\text { Edwards et } \\
\text { al. }(2016)^{114}\end{array}$ & No & Not reported & $\begin{array}{l}\text { Gamification: } F \text {, } \\
\text { monitoring, reward } \\
\text { and threat, goals } \\
\text { and planning; } \\
\text { individual techniques: } \\
\text { self-monitoring of } \\
\text { behavior, non-specific } \\
\text { reward, social support } \\
\text { unspecified, non-specific } \\
\text { incentive and focus on } \\
\text { past success }\end{array}$ & $\begin{array}{l}\text { Behavior Change } \\
\text { Technique }\end{array}$ & $\begin{array}{l}\text { Median number of } \\
\text { techniques per app } \\
\text { was 14; Common } \\
\text { combinations: goal } \\
\text { setting, self-monitoring, } \\
\text { non-specific reward } \\
\text { and non-specific } \\
\text { incentive; goal setting, } \\
\text { self-monitoring and } \\
\text { focus on past success; } \\
\text { no correlation between } \\
\text { number of techniques and } \\
\text { user ratings or price; no } \\
\text { standardized approach } \\
\text { was used to assess } \\
\text { the quality of included } \\
\text { studies/apps }\end{array}$ \\
\hline $\begin{array}{l}\text { Böhm et al. } \\
\text { (2019) })^{90}\end{array}$ & No & $\begin{array}{l}\text { Healthy } \\
\text { Children and } \\
\text { adolescents } \\
\text { (aged 6-18 } \\
\text { years) }\end{array}$ & $\begin{array}{l}\text { F, self-monitoring, goal } \\
\text { setting, strategies to } \\
\text { overcome barriers, } \\
\text { information }\end{array}$ & $\begin{array}{l}\text { Social Cognitive } \\
\text { Theory, Behavior } \\
\text { Change Technique, }\end{array}$ & $\begin{array}{l}\text { No statistically significant } \\
\text { effects on PA-related } \\
\text { outcomes with mHealth } \\
\text { tools; quality of evidence: } \\
\text { low (e.g. small number } \\
\text { of studies, inadequate } \\
\text { use of validated } \\
\text { measures, missing } \\
\text { RCTs, heterogeneity } \\
\text { of interventions); no } \\
\text { standardized approach } \\
\text { was used to assess the } \\
\text { quality of included apps/ } \\
\text { studies }\end{array}$ \\
\hline
\end{tabular}


Table 2. (continued). Summary of included studies on mHealth interventions a

\begin{tabular}{|c|c|c|c|c|c|}
\hline Reference & $\begin{array}{l}\text { Meta } \\
\text { analyses }\end{array}$ & Population & $\begin{array}{l}\text { Intervention } \\
\text { components }\end{array}$ & $\begin{array}{l}\text { Theoretical and } \\
\text { evidence base on } \\
\text { process/outcomes }\end{array}$ & $\begin{array}{l}\text { Primary Outcome \& } \\
\text { quality of evidence }\end{array}$ \\
\hline $\begin{array}{l}\text { Kim et al. } \\
(2019)^{93}\end{array}$ & Yes & $\begin{array}{l}\text { Adults (e.g. } \\
\text { overweight } \\
\text { university staff } \\
\text { and students) }\end{array}$ & $\begin{array}{l}\text { Lifestyle intervention, } \\
\text { education and coaching } \\
\text { to promote PA, dietary } \\
\text { counseling, education } \\
\text { related to health } \\
\text { behavior, F }\end{array}$ & $\begin{array}{l}\text { Transtheoretical } \\
\text { model, Theory of } \\
\text { Planned Behavior }\end{array}$ & $\begin{array}{l}\text { Significant increase in PA, } \\
\text { significant weight loss in } \\
\text { the intervention groups; } \\
\text { quality of evidence: } \\
\text { moderate (low risk of } \\
\text { bias assessed using the } \\
\text { Cochrane risk-of-bias } \\
\text { tool), but only } 5 \text { studies } \\
\text { included }\end{array}$ \\
\hline $\begin{array}{l}\text { Song et al. } \\
(2019)^{102}\end{array}$ & No & $\begin{array}{l}\text { General } \\
\text { population } \\
\text { with unhealthy } \\
\text { alcohol use }\end{array}$ & $\begin{array}{l}\text { Motivation (encouraging } \\
\text { messages, peer } \\
\text { support, monetary } \\
\text { compensation), general } \\
\text { and personalized } \\
\text { information, reminder, } \\
\text { and warning }\end{array}$ & $\begin{array}{l}\text { Behavioral change } \\
\text { theories (e.g. Theory } \\
\text { of Planned Behavior, } \\
\text { Health Belief Model, } \\
\text { Theory of Reasoned } \\
\text { Action, Social Learning, } \\
\text { Theory) }\end{array}$ & $\begin{array}{l}63 \% \text { mHealth } \\
\text { interventions brought } \\
\text { significant positive } \\
\text { outcomes in improving } \\
\text { participants' health } \\
\text { compared to traditional } \\
\text { methods; quality of } \\
\text { evidence: high (assessed } \\
\text { using Mixed Methods } \\
\text { Appraisal Tool) }\end{array}$ \\
\hline $\begin{array}{l}\text { Feter et al. } \\
(2019)^{92}\end{array}$ & Yes & Not reported & $\begin{array}{l}\text { PA interventions with } \\
\text { SMS or App promotion } \\
\text { using accelerometer, } \\
\text { pedometer, } \\
\text { questionnaire, daily } \\
\text { diary, }\end{array}$ & Not reported & $\begin{array}{l}\text { Efficient in increasing } \\
\text { minutes and steps per day } \\
\text { in adults when compared } \\
\text { to baseline; quality of } \\
\text { evidence: moderate- } \\
\text { high (assessed using the } \\
\text { Downs and Black scale) }\end{array}$ \\
\hline $\begin{array}{l}\text { Bort-Roig et } \\
\text { al. }(2014)^{91}\end{array}$ & No & $\begin{array}{l}\text { General } \\
\text { population, } \\
\text { special } \\
\text { populations } \\
\text { (e.g. obese } \\
\text { patients) }\end{array}$ & $\begin{array}{l}\text { Smartphone strategies } \\
\text { to influence PA; PA } \\
\text { profiles, goal setting, } \\
\text { real-time F, social } \\
\text { support networking, } \\
\text { and online expert } \\
\text { consultation }\end{array}$ & $\begin{array}{l}\text { Behavior Change } \\
\text { Theories, including } \\
\text { Social Cognitive } \\
\text { Theory and the Trans- } \\
\text { theoretical Model }\end{array}$ & $\begin{array}{l}\text { PA increases and one } \\
\text { study reported PA } \\
\text { maintenance over } 3 \\
\text { months; quality of } \\
\text { evidence: low (e.g. small } \\
\text { sample sizes, short study } \\
\text { periods), no standardized } \\
\text { approach was used to } \\
\text { assess the quality of } \\
\text { included studies }\end{array}$ \\
\hline $\begin{array}{l}\text { Muntaner et } \\
\text { al. }(2016)^{95}\end{array}$ & No & $\begin{array}{l}\text { General } \\
\text { population }\end{array}$ & $\begin{array}{l}\text { Monitoring, F, } \\
\text { information, interactive } \\
\text { voice response system, } \\
\text { questionnaire, messages } \\
\text { with helpful hints, goal } \\
\text { setting, motivational } \\
\text { messages, reminders }\end{array}$ & $\begin{array}{l}\text { Social Cognitive } \\
\text { Theory, Protection } \\
\text { Motivation Theory, } \\
\text { Transtheoretical } \\
\text { Model, Theory of } \\
\text { Planned Behavior, } \\
\text { Goal Setting Theory, } \\
\text { Problem Solving } \\
\text { Theory }\end{array}$ & $\begin{array}{l}\text { 6/12 studies reported } \\
\text { significant increases in PA } \\
\text { levels; quality of evidence: } \\
\text { low-moderate (e.g. lack of } \\
\text { information provided by } \\
\text { studies) (assessed using } \\
\text { the Downs and Black } \\
\text { scale) }\end{array}$ \\
\hline
\end{tabular}


Table 2. (continued). Summary of included studies on mHealth interventions a

\begin{tabular}{|c|c|c|c|c|c|}
\hline Reference & $\begin{array}{l}\text { Meta } \\
\text { analyses }\end{array}$ & Population & $\begin{array}{l}\text { Intervention } \\
\text { components }\end{array}$ & $\begin{array}{l}\text { Theoretical and } \\
\text { evidence base on } \\
\text { process/outcomes }\end{array}$ & $\begin{array}{l}\text { Primary Outcome \& } \\
\text { quality of evidence }\end{array}$ \\
\hline $\begin{array}{l}\text { Rathbone et } \\
\text { al. (2017) }{ }^{99}\end{array}$ & No & $\begin{array}{l}\text { General } \\
\text { population }\end{array}$ & $\begin{array}{l}\text { Self-monitoring, SMS, PE, } \\
\text { podcast, journal }\end{array}$ & CBT & $\begin{array}{l}\text { Significant decrease } \\
\text { in anxiety and stress } \\
\text { with medium to large } \\
\text { effect sizes, quality of } \\
\text { evidence: low to moderate } \\
\text { (assessed using Cochrane } \\
\text { risk-of-bias tool); } 8 \text { of the } \\
\text { studies without control } \\
\text { groups }\end{array}$ \\
\hline $\begin{array}{l}\text { Alyami et al. } \\
(2017)^{29}\end{array}$ & No & Not reported & $\begin{array}{l}\mathrm{PE} \text {, symptom } \\
\text { management, } \\
\text { therapeutic treatment, } \\
\text { self-assessment, } \\
\text { supportive resources, } \\
\text { multi-purpose }\end{array}$ & $\begin{array}{l}\text { CBT, mobile-based } \\
\text { Interpersonal } \\
\text { Psychotherapy, } \\
\text { smartphone-based } \\
\text { CBM for Attention }\end{array}$ & $\begin{array}{l}\text { Over } 60 \% \text { of apps } \\
\text { exclusively focused on } \\
\text { social anxiety, remainder } \\
\text { targeted social anxiety } \\
\text { and related conditions; } \\
\text { most developers did not } \\
\text { provide information on } \\
\text { organizational affiliations } \\
\text { or content source; most } \\
\text { apps used multimedia } \\
\text { while } 17 \text { apps used text } \\
\text { only; quality of evidence } \\
\text { not reported }\end{array}$ \\
\hline
\end{tabular}

\begin{tabular}{|c|c|c|c|c|}
\hline Treatment & & & & \\
\hline $\begin{array}{l}\text { Sander et al. No } \\
(2020)^{109}\end{array}$ & $\begin{array}{l}\text { Soldiers or } \\
\text { veterans, } \\
\text { family } \\
\text { members } \\
\text { (of people } \\
\text { with PTSD, } \\
\text { Clinicians, } \\
\text { Children, Police } \\
\text { officers or } \\
\text { public safety } \\
\text { professionals) }\end{array}$ & $\begin{array}{l}63.8 \% \text { of apps offered } \\
\text { elements of } \\
\text { mindfulness, relaxation, } \\
\text { breathing, or body } \\
\text { exercises; information/ } \\
\text { PE, assessment, } \\
\text { monitoring and } \\
\text { tracking, F, skill training, } \\
\text { exposure, mindfulness, } \\
\text { relaxation, breathing, } \\
\text { body exercises, resource } \\
\text { orientation, tips and } \\
\text { advices }\end{array}$ & $\begin{array}{l}\text { CBT, Behavior } \\
\text { Therapy, Systemic } \\
\text { Therapy, third wave } \\
\text { CBT, Psychodynamic } \\
\text { therapy }\end{array}$ & $\begin{array}{l}\text { Overall app quality based } \\
\text { on the MARS was medium. } \\
\text { Most offered a wide range } \\
\text { of content, including } \\
\text { established psychological } \\
\text { PTSD treatment methods } \\
\text { (processing of trauma- } \\
\text { related emotions and } \\
\text { beliefs, relaxation } \\
\text { exercises, and PE); quality } \\
\text { of evidence not reported }\end{array}$ \\
\hline $\begin{array}{l}\text { Terhorst et } \\
\text { al. }(2018)^{111}\end{array}$ & $\begin{array}{l}\text { Patients with } \\
\text { depression }\end{array}$ & $\begin{array}{l}\text { Assessment, } \mathrm{PE}, \\
\text { monitoring and tracking }\end{array}$ & $\begin{array}{l}\text { third wave CBT, CBT, } \\
\text { Behavioral Therapy, } \\
\text { Alternative Medicine }\end{array}$ & $\begin{array}{l}\text { Overall quality was } \\
\text { average. Four high-quality } \\
\text { apps were identified } \\
\text { and recommended with } \\
\text { reservations for practical } \\
\text { use; quality of evidence } \\
\text { not reported }\end{array}$ \\
\hline
\end{tabular}


Table 2. (continued). Summary of included studies on mHealth interventions a

\begin{tabular}{|c|c|c|c|c|c|}
\hline Reference & $\begin{array}{l}\text { Meta } \\
\text { analyses }\end{array}$ & Population & $\begin{array}{l}\text { Intervention } \\
\text { components }\end{array}$ & $\begin{array}{l}\text { Theoretical and } \\
\text { evidence base on } \\
\text { process/outcomes }\end{array}$ & $\begin{array}{l}\text { Primary Outcome \& } \\
\text { quality of evidence }\end{array}$ \\
\hline $\begin{array}{l}\text { Ilagan et al. } \\
(2020)^{108}\end{array}$ & Yes & $\begin{array}{l}\text { Adults with } \\
\text { borderline } \\
\text { personality } \\
\text { disorder (BPD) }\end{array}$ & $\begin{array}{l}\text { Positive stimuli paired } \\
\text { with self, personalized } \\
\text { safety-plan, PE on } \\
\text { suicidal thoughts, } \\
\text { symptom monitoring, } \\
\text { digital hope kit, } \\
\text { self-help skills, anger } \\
\text { management exercises, } \\
\text { mindfulness meditation } \\
\text { exercises }\end{array}$ & CBT & $\begin{array}{l}\text { No significant effect } \\
\text { of apps on BPD } \\
\text { symptoms and general } \\
\text { psychopathology, quality } \\
\text { of evidence: moderate } \\
\text { (assessed using Cochrane } \\
\text { Collaboration Risk of Bias } \\
\text { Assessment Tool) }\end{array}$ \\
\hline $\begin{array}{l}\text { Loo Gee et al. } \\
(2016)^{97}\end{array}$ & No & $\begin{array}{l}\text { General } \\
\text { population and } \\
\text { patients (aged } \\
17-55 \text { years) }\end{array}$ & $\begin{array}{l}\text { Integrative EMIs: } \\
\text { self-monitoring of } \\
\text { symptoms, delivery of } \\
\text { automated or therapist- } \\
\text { delivered psychotherapy } \\
\text { content }\end{array}$ & CBT & $\begin{array}{l}\text { EMIs may be a promising } \\
\text { treatment for generalized } \\
\text { anxiety and may be } \\
\text { effective for reducing } \\
\text { stress; quality of evidence: } \\
\text { low-moderate (assessed } \\
\text { using the Cochrane } \\
\text { Effective Practice and } \\
\text { Organization of Care } \\
\text { Group criteria) }\end{array}$ \\
\hline $\begin{array}{l}\text { Rootes- } \\
\text { Murdy et al. } \\
(2018)^{100}\end{array}$ & No & $\begin{array}{l}\text { Patients } \\
\text { with mood } \\
\text { disorders }\end{array}$ & $\begin{array}{l}\text { Apps, phone calls, SMS, } \\
\text { mobile web-based } \\
\text { surveys; electronic pill } \\
\text { dispenser, face-to-face } \\
\text { conversation with } \\
\text { animated agent }\end{array}$ & Not reported & $\begin{array}{l}\text { Overall satisfaction and } \\
\text { feasibility of mobile } \\
\text { technology, reduction } \\
\text { in mood symptoms; few } \\
\text { examined effectiveness } \\
\text { of mobile technology } \\
\text { improving medication } \\
\text { adherence through } \\
\text { RCTs; results represent } \\
\text { approximately 10\% } \\
\text { higher mean medication } \\
\text { adherence rates } \\
\text { when compared to } \\
\text { observational studies; } \\
\text { quality of evidence: low } \\
\text { (e.g. mostly observational } \\
\text { studies, technologies only } \\
\text { used for short period), no } \\
\text { standardized approach } \\
\text { was used to assess the } \\
\text { quality of included studies }\end{array}$ \\
\hline
\end{tabular}


Table 2. (continued). Summary of included studies on mHealth interventions a

\begin{tabular}{|c|c|c|c|c|c|}
\hline Reference & $\begin{array}{l}\text { Meta } \\
\text { analyses }\end{array}$ & Population & $\begin{array}{l}\text { Intervention } \\
\text { components }\end{array}$ & $\begin{array}{l}\text { Theoretical and } \\
\text { evidence base on } \\
\text { process/outcomes }\end{array}$ & $\begin{array}{l}\text { Primary Outcome \& } \\
\text { quality of evidence }\end{array}$ \\
\hline $\begin{array}{l}\text { Miralles et al. } \\
(2020)^{25}\end{array}$ & No & Not reported & $\begin{array}{l}\text { Psychological } \\
\text { interventions for mental } \\
\text { disorders delivered via } \\
\text { smartphone, plus SMS } \\
\text { and phone calls }\end{array}$ & $\begin{array}{l}\text { CBT, Behavior } \\
\text { Therapy, third wave } \\
\text { CBT }\end{array}$ & $\begin{array}{l}72.7 \% \text { of the papers } \\
\text { focused on six mental } \\
\text { disorders: depression, } \\
\text { anxiety, trauma and } \\
\text { stressor-related, } \\
\text { substance-related and } \\
\text { addiction, schizophrenia } \\
\text { spectrum, and other } \\
\text { psychotic disorders, or a } \\
\text { combination of disorders; } \\
\text { quality of evidence: low } \\
\text { (e.g. little percentage of } \\
\text { RCTs, few studies asses } \\
\text { effect of mHealth on } \\
\text { symptomatology), no } \\
\text { standardized approach } \\
\text { was used to assess the } \\
\text { quality of included studies }\end{array}$ \\
\hline
\end{tabular}

Notes: CBT=Cognitive Behavioral Therapy; PE=psychoeducation; PA=physical activity; $\mathrm{F}=$ feedback; EMIs=ecological momentary interventions.

a Complete summary of included reviews on eHealth and mHealth are shown in the Supplements, including findings on secondary outcomes, quality from the user perspective, safety, and cost effectiveness. 
Table 3. Summary of included reviews on interventions including eHealth and mHealth interventions (mixed) ${ }^{a}$

\begin{tabular}{|c|c|c|c|c|c|}
\hline Reference & $\begin{array}{l}\text { Meta } \\
\text { analyses }\end{array}$ & Population & Intervention components & $\begin{array}{l}\text { Theoretical and } \\
\text { evidence base on } \\
\text { process/outcomes }\end{array}$ & $\begin{array}{l}\text { Primary outcomes \& } \\
\text { quality of evidence }\end{array}$ \\
\hline \multicolumn{6}{|c|}{ Mental Health Promotion and Prevention } \\
\hline $\begin{array}{l}\text { Rose et al. } \\
(2017)^{94}\end{array}$ & No & $\begin{array}{l}\text { General } \\
\text { population of } \\
\text { adolescents } \\
\text { (aged 10-19 } \\
\text { years); } \\
\text { specific at-risk } \\
\text { populations }\end{array}$ & $\begin{array}{l}\text { Diet and PA education, } \\
\text { goal setting, monitoring, } \\
\text { parental involvement; } \\
\text { counselling; health } \\
\text { information, assessment } \\
\text { of behavior, skill } \\
\text { building, reward system, } \\
\text { F, pedometer, self- } \\
\text { monitoring, cycling video } \\
\text { game, fruit promotion via } \\
\text { email, family involvement }\end{array}$ & Not reported & $\begin{array}{l}\text { Significant } \\
\text { improvements } \\
\text { in behaviour in } \\
\text { 8/22; significant } \\
\text { improvement in diet } \\
\text { and/or PA for majority } \\
\text { of interventions } \\
\text { with goal setting; } \\
\text { self-monitoring less } \\
\text { effective without goal } \\
\text { setting; significant } \\
\text { improvement in } \\
\text { behaviour in most } \\
\text { interventions with } \\
\text { family involvement; } \\
\text { quality of evidence: } \\
\text { low-moderate (e.g. } \\
\text { poor handling of } \\
\text { confounding factors, } \\
\text { participant selection } \\
\text { bias), assessed using } \\
\text { a checklist based on } \\
\text { quality assessment } \\
\text { criteria by the Centre } \\
\text { for Reviews and } \\
\text { Dissemination }\end{array}$ \\
\hline $\begin{array}{l}\text { Baker et al. } \\
(2018)^{64}\end{array}$ & No & Older adults & $\begin{array}{l}\text { ICT based interventions: } \\
\text { using devices with } \\
\text { touchscreen, social } \\
\text { network services }\end{array}$ & $\begin{array}{l}\text { Social concepts often } \\
\text { poorly defined }\end{array}$ & $\begin{array}{l}\text { No evaluation of } \\
\text { efficacy due to } \\
\text { insufficient attention } \\
\text { to social concepts; } \\
\text { quality of evidence: low } \\
\text { (e.g. very small sample } \\
\text { sizes, poorly defined } \\
\text { outcomes), Multiple } \\
\text { tools were used to } \\
\text { assess the quality of } \\
\text { included studies (i.e. } \\
\text { Cochrane Collaboration } \\
\text { Risk of Bias Assessment } \\
\text { Tool; PEDro scale, } \\
\text { Downs and Black scale) }\end{array}$ \\
\hline
\end{tabular}


Table 3. (continued). Summary of included reviews on interventions including eHealth and mHealth interventions (mixed) ${ }^{\text {a }}$

\begin{tabular}{|c|c|c|c|c|c|}
\hline Reference & $\begin{array}{l}\text { Meta } \\
\text { analyses }\end{array}$ & Population & Intervention components & $\begin{array}{l}\text { Theoretical and } \\
\text { evidence base on } \\
\text { process/outcomes }\end{array}$ & $\begin{array}{l}\text { Primary outcomes \& } \\
\text { quality of evidence }\end{array}$ \\
\hline $\begin{array}{l}\text { Kreuze et al. } \\
(2017)^{106}\end{array}$ & No & $\begin{array}{l}\text { Hetero- } \\
\text { geneous, } \\
\text { including } \\
\text { adults, } \\
\text { adolescents, } \\
\text { university } \\
\text { students }\end{array}$ & $\begin{array}{l}\text { Virtual Hope Box (support, } \\
\text { comfort, distract or relax } \\
\text { using audio, video, pictures, } \\
\text { games, mindfulness } \\
\text { exercises, messages, } \\
\text { inspirational quotes and } \\
\text { coping statements), coping } \\
\text { strategies, behavioural } \\
\text { activation, interpersonal } \\
\text { psychotherapy, community } \\
\text { resiliency concepts, } \\
\text { problem solving, F }\end{array}$ & $\begin{array}{l}\text { CBT, Cognitive Therapy, } \\
\text { Dialectic Behaviour } \\
\text { Therapy, Mindfulness- } \\
\text { based Cognitive } \\
\text { Therapy, Problem- } \\
\text { solving Therapy }\end{array}$ & $\begin{array}{l}\text { Participants } \\
\text { improved significantly } \\
\text { on depression, anxiety, } \\
\text { hopelessness, self- } \\
\text { esteem, and negative } \\
\text { automatic } \\
\text { thoughts; promising } \\
\text { evidence for reduction } \\
\text { of suicidal ideation } \\
\text { and mental health } \\
\text { co-morbidities; quality } \\
\text { of evidence: low (e.g. } \\
\text { unprecise study effects, } \\
\text { small sample sizes, } \\
\text { low engagement); no } \\
\text { standardized approach } \\
\text { was used to assess } \\
\text { the quality of included } \\
\text { studies }\end{array}$ \\
\hline $\begin{array}{l}\text { Lau et al. } \\
(2011)^{34}\end{array}$ & No & $\begin{array}{l}\text { Children and } \\
\text { adolescents }\end{array}$ & $\begin{array}{l}\text { Goal setting, tips, comic } \\
\text { stories, social support } \\
\text { emails, self-monitoring, } \\
\text { quizzes, games, charts to } \\
\text { plan activities, motivational } \\
\text { reminder, F, tailored } \\
\text { information, online } \\
\text { counselling }\end{array}$ & $\begin{array}{l}\text { Health Behaviour } \\
\text { Change Theory, Social } \\
\text { Cognitive Theory, } \\
\text { Transtheoretical Model, } \\
\text { Relapse Prevention } \\
\text { Model }\end{array}$ & $\begin{array}{l}\text { Evidence supporting } \\
\text { efficacy of interventions } \\
\text { on improving } \\
\text { psychosocial outcomes } \\
\text { vs. conventional } \\
\text { or no treatment, } \\
\text { less consistent for } \\
\text { behavioural outcomes; } \\
\text { quality of evidence: } \\
\text { 7/9 studies good } \\
\text { methodological quality; } \\
\text { assessed using the } \\
\text { Cochrane risk-of-bias } \\
\text { tool }\end{array}$ \\
\hline $\begin{array}{l}\text { Yonker et al. } \\
(2015)^{103}\end{array}$ & No & $\begin{array}{l}\text { Adolescents } \\
\text { and young } \\
\text { adults (aged } \\
11-25 \text { years) }\end{array}$ & $\begin{array}{l}\text { Observation, providing } \\
\text { health information, } \\
\text { engaging in community, } \\
\text { recruiting research } \\
\text { participants }\end{array}$ & Not reported & $\begin{array}{l}\text { Positive impact } \\
\text { on mental health } \\
\text { outcomes; mixed } \\
\text { results for community } \\
\text { engagement; quality } \\
\text { of evidence: low (e.g. } \\
62 \% \text { of studies with } \\
\text { sampling bias, } 24 \% \text { with } \\
\text { incomplete datasets, } \\
19 \% \text { with small sample } \\
\text { size); no standardized } \\
\text { approach was used to } \\
\text { assess the quality of } \\
\text { included studies }\end{array}$ \\
\hline
\end{tabular}


Table 3. (continued). Summary of included reviews on interventions including eHealth and mHealth interventions (mixed) ${ }^{\text {a }}$

\begin{tabular}{|c|c|c|c|c|c|}
\hline Reference & $\begin{array}{l}\text { Meta } \\
\text { analyses }\end{array}$ & Population & Intervention components & $\begin{array}{l}\text { Theoretical and } \\
\text { evidence base on } \\
\text { process/outcomes }\end{array}$ & $\begin{array}{l}\text { Primary outcomes \& } \\
\text { quality of evidence }\end{array}$ \\
\hline \multicolumn{6}{|l|}{ Treatment } \\
\hline $\begin{array}{l}\text { Berry et al. } \\
(2016)^{115}\end{array}$ & No & $\begin{array}{l}\text { Individuals } \\
\text { with severe } \\
\text { mental health } \\
\text { problems } \\
\text { (e.g. bipolar } \\
\text { disorder, } \\
\text { schizoaffective } \\
\text { disorder, } \\
\text { psychotic } \\
\text { disorder, } \\
\text { schizophrenia) }\end{array}$ & $\begin{array}{l}\text { Medication reminders, } \\
\text { information, advice, } \\
\text { helpline for crisis } \\
\text { intervention, decision } \\
\text { making tools, coping skills, } \\
\text { check-ins }\end{array}$ & CBT & $\begin{array}{l}\text { High acceptability of } \\
\text { online and mobile } \\
\text { phone-delivered } \\
\text { interventions; } \\
\text { acceptability was } \\
\text { higher for interventions } \\
\text { delivered via mobile } \\
\text { phones as compared } \\
\text { to online formats; } \\
\text { quality of evidence: low } \\
\text { (e.g. varied findings, } \\
\text { limited number of } \\
\text { studies, inaccurate } \\
\text { measurement of } \\
\text { acceptability), no } \\
\text { standardized approach } \\
\text { was used to assess } \\
\text { the quality of included } \\
\text { studies }\end{array}$ \\
\hline $\begin{array}{l}\text { Walsh et al. } \\
(2016)^{47}\end{array}$ & No & $\begin{array}{l}\text { Population } \\
\text { with variety } \\
\text { of mental } \\
\text { illnesses } \\
\text { e.g. anxiety, } \\
\text { depression, } \\
\text { bipolar } \\
\text { disorder, } \\
\text { eating } \\
\text { disorder }\end{array}$ & $\begin{array}{l}\text { Technology based } \\
\text { symptom-monitoring, F }\end{array}$ & Self-monitoring, CBT & $\begin{array}{l}\text { Acceptability of } \\
\text { monitoring is related } \\
\text { to perceived validity, } \\
\text { ease of practice, } \\
\text { convenient technology, } \\
\text { appropriate frequency, } \\
\text { helpfulness of F, } \\
\text { impact of monitoring } \\
\text { on participants' ability } \\
\text { to manage health and } \\
\text { personal relationships; } \\
\text { quality of evidence: low } \\
\text { (e.g. descriptive results, } \\
\text { high heterogeneity), no } \\
\text { standardized approach } \\
\text { was used to assess } \\
\text { the quality of included } \\
\text { studies due to low } \\
\text { quality of data }\end{array}$ \\
\hline
\end{tabular}


Table 3. (continued). Summary of included reviews on interventions including eHealth and mHealth interventions (mixed) ${ }^{\text {a }}$

\begin{tabular}{|c|c|c|c|c|c|}
\hline Reference & $\begin{array}{l}\text { Meta } \\
\text { analyses }\end{array}$ & Population & Intervention components & $\begin{array}{l}\text { Theoretical and } \\
\text { evidence base on } \\
\text { process/outcomes }\end{array}$ & $\begin{array}{l}\text { Primary outcomes \& } \\
\text { quality of evidence }\end{array}$ \\
\hline $\begin{array}{l}\text { Rice et al. } \\
(2014)^{69}\end{array}$ & No & $\begin{array}{l}\text { Young } \\
\text { people with } \\
\text { depression }\end{array}$ & $\begin{array}{l}\text { PE, behavioural activation, } \\
\text { thought monitoring, skills } \\
\text { training, online support } \\
\text { group postings }\end{array}$ & CBT & $\begin{array}{l}\text { Most intervention } \\
\text { studies demonstrated } \\
\text { superiority of online } \\
\text { intervention vs. } \\
\text { comparison treatment } \\
\text { (treatment as usual, } \\
\text { waitlist, brief PE); no } \\
\text { standardized approach } \\
\text { was used to assess } \\
\text { the quality of included } \\
\text { studies }\end{array}$ \\
\hline $\begin{array}{l}\text { Aref-Adib et } \\
\text { al. }(2019)^{43}\end{array}$ & No & $\begin{array}{l}\text { Patients } \\
\text { with schizo- } \\
\text { phreniform } \\
\text { disorders and/ } \\
\text { or bipolar } \\
\text { disorder }\end{array}$ & $\begin{array}{l}\text { Web-based computer } \\
\text { program, Telecare, online } \\
\text { platform, mHealth, peer- } \\
\text { run web-based computer } \\
\text { program; e.g. mindfulness, } \\
\text { shared decision making, } \\
\text { aid to build relationships } \\
\text { and to communicate. }\end{array}$ & Not reported & $\begin{array}{l}\text { Factors that affect } \\
\text { implementation: e.g. } \\
\text { lack of motivation, poor } \\
\text { information technology } \\
\text { skills, language } \\
\text { problems, poor mental } \\
\text { state, labour-intensive } \\
\text { scheduling, availability } \\
\text { of telehealth space, } \\
\text { equipment, attitudes/ } \\
\text { beliefs about digital } \\
\text { interventions; quality } \\
\text { of evidence: low, no } \\
\text { standardized approach } \\
\text { was used to assess } \\
\text { the quality of included } \\
\text { studies as research is in } \\
\text { early stages }\end{array}$ \\
\hline $\begin{array}{l}\text { Biagianti et } \\
\text { al. }(2018)^{42}\end{array}$ & No & $\begin{array}{l}\text { Adults with } \\
\text { psychotic } \\
\text { disorder }\end{array}$ & $\begin{array}{l}\text { Internet peer-support, } \\
\text { peer-support bulletin } \\
\text { board, PE, therapy groups, } \\
\text { moderated peer discussion } \\
\text { forums, psychosocial } \\
\text { interventions, social } \\
\text { networking, SMS based } \\
\text { motivational coaching, } \\
\text { goal setting, computerized } \\
\text { social cognition training, } \\
\text { group texting }\end{array}$ & $\begin{array}{l}\text { Social Cognition } \\
\text { Training }\end{array}$ & $\begin{array}{l}\text { Digital interventions+ } \\
\text { peer-to-peer } \\
\text { communication } \\
\text { associated with good } \\
\text { retention rates (78,4\%), } \\
\text { + mental health } \\
\text { providers improved } \\
\text { engagement, peer-to- } \\
\text { peer communication } \\
\text { highly engaging; quality } \\
\text { of evidence: low (e.g. } \\
\text { small samples, no } \\
\text { control groups); no } \\
\text { standardized approach } \\
\text { was used to assess the } \\
\text { quality of the included } \\
\text { studies }\end{array}$ \\
\hline
\end{tabular}


Table 3. (continued). Summary of included reviews on interventions including eHealth and mHealth interventions (mixed) ${ }^{a}$

\begin{tabular}{|c|c|c|c|c|c|}
\hline Reference & $\begin{array}{l}\text { Meta } \\
\text { analyses }\end{array}$ & Population & Intervention components & $\begin{array}{l}\text { Theoretical and } \\
\text { evidence base on } \\
\text { process/outcomes }\end{array}$ & $\begin{array}{l}\text { Primary outcomes \& } \\
\text { quality of evidence }\end{array}$ \\
\hline $\begin{array}{l}\text { Faurholt- } \\
\text { Jepsen et al. } \\
(2016)^{45}\end{array}$ & No & $\begin{array}{l}\text { In- and } \\
\text { outpatients } \\
\text { with bipolar } \\
\text { disorder } \\
\text { type } 1\end{array}$ & $\begin{array}{l}\text { Computer, ChronoRecord, } \\
\text { PDA, Prism, Smartphone, } \\
\text { MONARCA, Personal Life } \\
\text { Chart App, Paper Pencil, } \\
\text { Moodswings, Websites } \\
\text { on healthy lifestyle; } \\
\text { self-monitoring of mood, } \\
\text { medications, self- } \\
\text { management, PE, F }\end{array}$ & CBT & $\begin{array}{l}\text { Consistent evidence } \\
\text { for validity of electronic } \\
\text { self-monitored mood } \\
\text { for depression; no } \\
\text { consistent evidence for } \\
\text { validity of electronic } \\
\text { self-monitored mood } \\
\text { for mania; quality of } \\
\text { evidence: moderate } \\
\text { (e.g. possible risks of } \\
\text { biases) (assessed using } \\
\text { the Cochrane risk-of- } \\
\text { bias tool) }\end{array}$ \\
\hline $\begin{array}{l}\text { Goldberg et } \\
\text { al., }(2018)^{44}\end{array}$ & No & $\begin{array}{l}\text { Patients } \\
\text { with mental } \\
\text { disorders (e.g. } \\
\text { depression, } \\
\text { bulimia, } \\
\text { psychotic } \\
\text { disorder, } \\
\text { substance } \\
\text { use, bipolar } \\
\text { disorder) }\end{array}$ & $\begin{array}{l}\text { Remote measurement- } \\
\text { based care as part of } \\
\text { treatment e.g. biofeedback, } \\
\text { self-guided intervention, } \\
\text { psychotherapy, remote } \\
\text { therapist support }\end{array}$ & $\begin{array}{l}\text { Remote measurement- } \\
\text { based care (RMBC) }\end{array}$ & $\begin{array}{l}\text { Positive effects of } \\
\text { the intervention } \\
\text { for RMBC as part } \\
\text { of multicomponent } \\
\text { intervention; } \\
\text { inconsistent evidence } \\
\text { for clinical effectiveness } \\
\text { of intervention alone } \\
\text { (effective in 1/3 studies, } \\
\text { but not as effective as } \\
\text { part of multicomponent } \\
\text { intervention); quality } \\
\text { of evidence: low } \\
\text { (meta-analysis could } \\
\text { not be conducted due } \\
\text { to high heterogeneity } \\
\text { of outcomes and } \\
\text { study design), no } \\
\text { standardized approach } \\
\text { was used to assess } \\
\text { the quality of included } \\
\text { studies }\end{array}$ \\
\hline $\begin{array}{l}\text { Perry et al. } \\
(2016)^{107}\end{array}$ & No & $\begin{array}{l}\text { Young people } \\
\text { (aged } 12-25 \\
\text { years) with } \\
\text { suicidal } \\
\text { thoughts }\end{array}$ & $\begin{array}{l}\text { Cognitive restructuring, } \\
\text { behavioural activation, } \\
\text { focus on problem solving } \\
\text { around suicidal ideation, } \\
\text { video diaries, message } \\
\text { board }\end{array}$ & CBT & $\begin{array}{l}\text { Significant reductions in } \\
\text { suicidality, depression } \\
\text { and hopelessness } \\
\text { (small-moderate } \\
\text { effect sizes); quality of } \\
\text { evidence: low (only one } \\
\text { study, small sample, } \\
\text { high attrition rate, } \\
\text { no control group); no } \\
\text { standardized approach } \\
\text { was used to assess the } \\
\text { quality of the included } \\
\text { studies }\end{array}$ \\
\hline
\end{tabular}


Table 3. (continued). Summary of included reviews on interventions including eHealth and mHealth interventions (mixed) ${ }^{\text {a }}$

\begin{tabular}{|c|c|c|c|c|c|}
\hline Reference & $\begin{array}{l}\text { Meta } \\
\text { analyses }\end{array}$ & Population & Intervention components & $\begin{array}{l}\text { Theoretical and } \\
\text { evidence base on } \\
\text { process/outcomes }\end{array}$ & $\begin{array}{l}\text { Primary outcomes \& } \\
\text { quality of evidence }\end{array}$ \\
\hline $\begin{array}{l}\text { Wright et al. } \\
(2019)^{71}\end{array}$ & Yes & $\begin{array}{l}\text { Population } \\
\text { ( } \geq 16 \\
\text { years) with } \\
\text { diagnosed } \\
\text { depression }\end{array}$ & $\begin{array}{l}\text { Multimedia, text (with } \\
\text { images) via computer } \\
\text { or app }\end{array}$ & CBT & $\begin{array}{l}\text { Small to moderate } \\
\text { effects of ICBT } \\
\text { compared to control } \\
\text { conditions; significantly } \\
\text { larger effects for } \\
\text { studies with support } \\
\text { from clinician } \\
\text { (moderate effect) than } \\
\text { for studies without } \\
\text { support (small effect); } \\
\text { quality of evidence: } \\
\text { moderate-high } \\
\text { (assessed using the } \\
\text { CLEAR NPT) }\end{array}$ \\
\hline $\begin{array}{l}\text { Xiang et al. } \\
(2019)^{73}\end{array}$ & No & $\begin{array}{l}\text { Older } \\
\text { adults ( } \geq 50 \\
\text { years) with } \\
\text { symptoms or } \\
\text { diagnosis of } \\
\text { depression }\end{array}$ & $\begin{array}{l}\text { Therapist- or self-guided } \\
\text { CBT: Beating the Blues, } \\
\text { Sadness Program, Manage } \\
\text { your Mood, Wellbeing Plus } \\
\text { Course, MoodTech, }\end{array}$ & CBT & $\begin{array}{l}\text { Large within-group } \\
\text { and between-group } \\
\text { effect sizes; quality of } \\
\text { evidence: Iow (assessed } \\
\text { using the Cochrane } \\
\text { risk-of-bias tool) }\end{array}$ \\
\hline $\begin{array}{l}\text { Alvarez- } \\
\text { Jimenes et al. } \\
(2014)^{40}\end{array}$ & No & $\begin{array}{l}\geq 80 \% \text { of } \\
\text { participants } \\
\text { diagnosed } \\
\text { with } \\
\text { schizophrenia- } \\
\text { spectrum } \\
\text { disorders }\end{array}$ & $\begin{array}{l}\text { Web-based PE; moderated } \\
\text { forums for patients and } \\
\text { supporters; integrated } \\
\text { web-based therapy, social } \\
\text { networking and peer } \\
\text { and expert moderation; } \\
\text { personalized advice based } \\
\text { on clinical monitoring; text } \\
\text { messaging interventions }\end{array}$ & CBT & $\begin{array}{l}\text { Efficient use in about } \\
80 \% \text { of patients; high } \\
\text { perception as positive } \\
\text { and useful, little } \\
\text { dropout in follow-up } \\
\text { ( } \leq 30 \% \text { ); quality of } \\
\text { evidence: low (e.g. } \\
\text { poor description of } \\
\text { methodology/results) } \\
\text { (assessed using the } \\
\text { Cochrane risk-of-bias } \\
\text { tool) }\end{array}$ \\
\hline $\begin{array}{l}\text { Arshad et al. } \\
(2020)^{104}\end{array}$ & Yes & $\begin{array}{l}\text { Adults with } \\
\text { suicide } \\
\text { attempts/ } \\
\text { meeting } \\
\text { criteria for } \\
\text { NSSI disorder, } \\
\text { PTSD, } \\
\text { depression, } \\
\text { suicidal } \\
\text { ideation }\end{array}$ & $\begin{array}{l}\text { Dialectical behavioral } \\
\text { therapy techniques, } \\
\text { individual ER therapy, } \\
\text { toolbox of support (e.g. } \\
\text { coping skills, strategies, } \\
\text { crisis support) }\end{array}$ & $\begin{array}{l}\text { CBT, DBT, individual ER } \\
\text { therapy }\end{array}$ & $\begin{array}{l}\text { Limited evidence for } \\
\text { efficacy of internet- } \\
\text { and mobile-based } \\
\text { interventions for self- } \\
\text { injurious thoughts and } \\
\text { behaviours, reductions } \\
\text { in SB in single-arm } \\
\text { noncontrolled studies; } \\
\text { beneficial effect on } \\
\text { suicidal ideation } \\
\text { compared to TAU, } \\
\text { not when compared } \\
\text { to active controls; } \\
\text { quality of evidence: low } \\
\text { (assessed using the } \\
\text { Cochrane risk-of-bias } \\
\text { tool) }\end{array}$ \\
\hline
\end{tabular}


Table 3. (continued). Summary of included reviews on interventions including eHealth and mHealth interventions (mixed) ${ }^{\text {a }}$

\begin{tabular}{|c|c|c|c|c|c|}
\hline Reference & $\begin{array}{l}\text { Meta } \\
\text { analyses }\end{array}$ & Population & Intervention components & $\begin{array}{l}\text { Theoretical and } \\
\text { evidence base on } \\
\text { process/outcomes }\end{array}$ & $\begin{array}{l}\text { Primary outcomes \& } \\
\text { quality of evidence }\end{array}$ \\
\hline $\begin{array}{l}\text { Domhardt et } \\
\text { al. }(2020)^{26}\end{array}$ & Yes & $\begin{array}{l}\text { Children and } \\
\text { adolescents } \\
\text { with mental } \\
\text { and somatic } \\
\text { disorders }\end{array}$ & $\begin{array}{l}\text { Internet- and mobile } \\
\text { based Interventions (IMIs): } \\
\text { webpages, email, chat, } \\
\text { videoconferencing, mobile } \\
\text { app, instant messaging; } \\
\text { psychotherapeutic } \\
\text { orientation }\end{array}$ & CBT & $\begin{array}{l}\text { Large effect for } \\
\text { IMIs across mental } \\
\text { disorders, medium } \\
\text { effect of IMIs for } \\
\text { somatic conditions } \\
\text { compared to nonactive } \\
\text { controls; quality of } \\
\text { evidence: moderate } \\
\text { (assessed using the } \\
\text { AMSTAR-2 checklist) }\end{array}$ \\
\hline $\begin{array}{l}\text { Meyer et al. } \\
(2018)^{78}\end{array}$ & Yes & $\begin{array}{l}\text { Population } \\
\text { with major } \\
\text { depressive } \\
\text { disorder, } \\
\text { borderline } \\
\text { personality } \\
\text { disorder, } \\
\text { variety of } \\
\text { diagnoses }\end{array}$ & $\begin{array}{l}\text { Monitoring, adherence } \\
\text { promotion, } \mathrm{PE} \text {, self- } \\
\text { management, relapse } \\
\text { prevention }\end{array}$ & CBT & $\begin{array}{l}\text { Larger RCTs showed } \\
\text { beneficial effects } \\
\text { on symptoms and } \\
\text { functioning (effect size } \\
\text { not reported); quality of } \\
\text { evidence: low (e.g. small } \\
\text { sample sizes, unreliable } \\
\text { differentiation between } \\
\text { on- and offline } \\
\text { available software), no } \\
\text { standardized approach } \\
\text { was used to assess } \\
\text { the quality of included } \\
\text { studies }\end{array}$ \\
\hline $\begin{array}{l}\text { Ye et al. } \\
(2014)^{67}\end{array}$ & Yes & $\begin{array}{l}\text { Children and } \\
\text { adolescents } \\
\text { with anxiety } \\
\text { and/or } \\
\text { depressions }\end{array}$ & $\begin{array}{l}\text { Self-monitoring of moods, } \\
\text { stress, alcohol and } \\
\text { cannabis use, SMS and } \\
\text { phone call support, online } \\
\text { self-help sessions, email, } \\
\text { SMS, phone calls, family/ } \\
\text { group/ teacher support }\end{array}$ & CBT & $\begin{array}{l}\text { Medium effect size } \\
\text { in reducing anxiety } \\
\text { symptom severity } \\
\text { compared to waitlist } \\
\text { control, increased } \\
\text { remission rate; non- } \\
\text { significant reduction of } \\
\text { depression symptom } \\
\text { severity; no difference } \\
\text { in anxiety/ depression } \\
\text { symptoms between } \\
\text { internet-based } \\
\text { intervention and face- } \\
\text { to-face intervention; } \\
\text { no superiority of } \\
\text { usual care; quality of } \\
\text { evidence: moderate- } \\
\text { high (assessed using } \\
\text { a modified version of } \\
\text { Quality Assessment } \\
\text { Tool for Quantitative } \\
\text { Studies) }\end{array}$ \\
\hline
\end{tabular}


Table 3. (continued). Summary of included reviews on interventions including eHealth and mHealth interventions (mixed) ${ }^{\text {a }}$

\begin{tabular}{|c|c|c|c|c|c|}
\hline Reference & $\begin{array}{l}\text { Meta } \\
\text { analyses }\end{array}$ & Population & Intervention components & $\begin{array}{l}\text { Theoretical and } \\
\text { evidence base on } \\
\text { process/outcomes }\end{array}$ & $\begin{array}{l}\text { Primary outcomes \& } \\
\text { quality of evidence }\end{array}$ \\
\hline $\begin{array}{l}\text { O.Rourke et } \\
\text { al. }(2016)^{55}\end{array}$ & No & $\begin{array}{l}\text { Hazardous } \\
\text { young } \\
\text { drinkers }\end{array}$ & $\begin{array}{l}\text { Behavioural interventions; } \\
\text { web-based, email, text } \\
\text { messages, social network } \\
\text { sites; personalized F, } \\
\text { monitoring, social norms } \\
\text { F (SNS), PE, improving } \\
\text { knowledge, self-efficacy } \\
\text { and awareness of social } \\
\text { norms }\end{array}$ & Not reported & $\begin{array}{l}\text { Interactive approaches } \\
\text { (text messaging, email, } \\
\text { SNS) significantly } \\
\text { reduce frequency of } \\
\text { drinking; personalized } \\
\text { electronic F reduces } \\
\text { alcohol consumption, } \\
\text { frequency of binge } \\
\text { drinking, and drinking } \\
\text { in a non-risky way; } \\
\text { quality of evidence: } \\
\text { moderate (assessed } \\
\text { using the Cochrane } \\
\text { risk-of-bias tool) }\end{array}$ \\
\hline $\begin{array}{l}\text { Dick et al. } \\
\text { (2019) }{ }^{101}\end{array}$ & No & $\begin{array}{l}\text { Third level } \\
\text { students }\end{array}$ & $\begin{array}{l}\text { F, descriptive norm } \\
\text { correction, self-affirmation } \\
\text { manipulation, theory- } \\
\text { based messages, } \\
\text { implementation intention } \\
\text { tasks, motivational brief } \\
\text { intervention, messages } \\
\text { based on social cognitive } \\
\text { theory }\end{array}$ & $\begin{array}{l}\text { Social Cognitive Theory, } \\
\text { little to no detail } \\
\text { about design and } \\
\text { development process } \\
\text { of the intervention }\end{array}$ & $\begin{array}{l}\text { Reduction of substance } \\
\text { misuse and initiation } \\
\text { (effect size not } \\
\text { reported); quality of } \\
\text { evidence: low (assessed } \\
\text { using the Quality } \\
\text { Assessment Tool for } \\
\text { Quantitative Studies } \\
\text { by the Effective Public } \\
\text { Health Practice Project) }\end{array}$ \\
\hline $\begin{array}{l}\text { Giroux et al. } \\
(2017)^{24}\end{array}$ & No & $\begin{array}{l}\text { Adults with } \\
\text { high school } \\
\text { education and } \\
\text { high risk or } \\
\text { problematic } \\
\text { alcohol/drug } \\
\text { use }\end{array}$ & $\begin{array}{l}\text { PE, F, Craving and relapse } \\
\text { management, behaviour } \\
\text { change, alcohol use } \\
\text { journal, control strategies, } \\
\text { social skills learning, chat } \\
\text { with therapist }\end{array}$ & $\begin{array}{l}\text { CBT, Theory of Planned } \\
\text { Behaviour, Solution } \\
\text { focused, Self-control, } \\
\text { Relapse Prevention }\end{array}$ & $\begin{array}{l}\text { Significant decrease in } \\
\text { substance use in } 3 / 4 \\
\text { of studies; long term } \\
\text { (12-month follow-up, } \\
\text { investigated by only } \\
2 \text { studies): effects } \\
\text { maintained for women; } \\
\text { quality of evidence: } \\
\text { moderate (assessed } \\
\text { using the Cochrane } \\
\text { criteria checklist) }\end{array}$ \\
\hline
\end{tabular}

Notes: CBT=Cognitive Behavioural Therapy; DBT=Dialectical behaviour therapy; $\mathrm{PE}=$ psychoeducation; $\mathrm{PA}=$ physical activity; $\mathrm{F}=$ feedback; $\mathrm{ER}=$ emotion regulation; TAU=treatment as usual.

a Complete summary of included reviews on eHealth and mHealth are shown in the Supplements, including findings on secondary outcomes, quality from the user perspective, safety, and cost effectiveness. 


\section{Discussion}

\section{Research in context}

\section{Evidence before this study}

Digital interventions may help to mitigate the negative psychosocial impact of pandemics. We therefore searched for original papers investigating the use of digital interventions to help alleviate negative psychosocial consequences in earlier coronavirus and influenza outbreaks. On May 3, 2020, we searched Medline, CENTRAL, and PsycINFO with no date and language restrictions using the following keywords: Previous epi-/pandemics (e.g. 'Corona' OR 'SARS' OR 'MERS' OR 'Influenza') AND mental health (e.g. 'Mental dis ${ }^{\star \prime}$ OR 'Depress ${ }^{\star \prime}$ ) OR social isolation ('Lonel*') AND digital interventions (e.g. 'mHealth' OR 'eHealth' OR 'Telemed ${ }^{\star \prime}$ ). A priori defined inclusion criteria were applied (e.g. papers on need, acceptability, usability, safety, and effectiveness). 264 articles were identified and none of the studies met inclusion criteria. Thus, we found no studies on the use of digital interventions during earlier outbreaks.

\section{Added value of this study}

For the first time, this meta-review systematically examined the current evidence on feasibility, safety, and effectiveness of digital interventions relevant for improving public mental health by mitigating reported negative psychosocial consequences of epi-/pandemics. It shows that the evidence on eHealth interventions is robust and mHealth interventions very promising if developed and evaluated in context of scientific research projects but very low or non-existing for mHealth apps available in major app Stores.

\section{Implications of all the available evidence}

Evidence-based digital interventions may help to mitigate the negative impact of the COVID-19 pandemic on public mental health. Digital interventions are particularly suited to provide low-threshold and timely public mental health care in times of physical distancing and quarantine. As the quality of evidence of currently available apps in app Stores is often unknown or very limited, there is an urgent need to 1) develop and evaluate digital interventions specifically designed to address social isolation and poor mental health during public health crises and 
2) make evidence-based digital interventions publicly available to improve public mental health.

\section{Principal findings}

Evidence-based eHealth and mHealth interventions may play a central role in areas of public mental health provision (i.e., mental health promotion, prevention of, and treatment for mental disorder) to mitigate the negative consequences of the current COVID-19 pandemic. To date, however, evidence-based recommendations on existing digital interventions that have been developed and evaluated in recent years were still lacking. This meta-review was the first to review the available evidence on the theoretical and empirical base, quality from the user perspective (i.e., acceptability, usability, satisfaction), safety, effectiveness, and cost effectiveness of digital interventions in the area of public mental health provision, that is, mental health promotion at the population level, indicated, selective, or universal prevention targeting at-risk, sub-, or the entire population as well as treatment and services for people with mental disorders.

First, there was robust evidence on effectiveness of telemedical internet-based or eHealth interventions and initial evidence on the effectiveness of mHealth interventions in relation to mental health outcomes likely affected by the current COVID-19 pandemic (e.g., anxiety, depression), especially if interventions are informed by clinical guidelines and co-designed by service users and mental health professionals. Second, effectiveness, acceptability, feasibility, and user satisfaction have been described to be particularly high if digital interventions are embedded in a therapeutic context and include some form of social interaction with a mental health professional (blended-care approach). Third, some of the included systematic reviews and meta-analyses suggest non-inferiority of effectiveness for some eHealth interventions as compared to traditional face-to-face therapy, but further replication is needed before firm conclusions can be drawn. Thus, in order to exclude the risk of infection in the current public health crisis, clinicians and other health professionals may consider combining differing types of digital interventions (e.g. counselling or psychotherapy using videoconference software augmented by a smartphone-based mHealth app) as this approach may be particularly promising given the current evidence base and reflects a novel digital version of the blended-care approach. However, more research is needed to 
investigate long-term treatment effects and effects of symptom monitoring on mental health outcomes. Notably, the evidence on the use of digital interventions for elderly as well as children is very limited. This is an important finding as these age groups may be more strongly challenged by the current pandemic. Fourth, most studies to date do not specifically investigate the additive effects on healthrelated outcomes when using more advanced techniques (e.g., accelerometer, GPS) to further personalize the delivery of intervention components, gamification elements as well as the integration of other technologies such as wearables although it has been described to be potentially beneficial in some of the included reviews $[25,89,92,114]$. Fifth, the theoretical basis of most digital interventions that have been described in previous reviews were found to be CBT or third-wave CBT as they may be particularly amendable to translation into digital intervention components $[23,25,26]$. Thus, clinicians with an expertise in CBT techniques may find it easier to purposefully incorporate intervention components delivered using digital tools in their daily clinical routines. However, findings suggest that there is a need to further improve the theoretical foundation of digital intervention, particularly mHealth interventions publicly available in major app stores. Sixth, the data available on process quality and cost-effectiveness of eHealth and mHealth interventions is very limited. Seventh, users frequently report concerns about data safety and privacy [115]. While eHealth and mHealth interventions developed and evaluated by research groups generally comply with the General Data Protection Regulation (GDPR; in European countries) and work in accordance with Good Clinical Practice (GCP) standards, the contents of many mHealth apps currently available in major app stores, do not explicitly refer to existing clinical guidelines and recommendation by learned societies $[50,116]$. There are a number of reviews that have concluded that $\mathrm{mHealth}$ apps use problematic data sharing and privacy practices $[8,27,28]$ and that there may not only be a lack of quality of offered content but even harmful intervention components. Also, although not specifically reported in included systematic reviews and meta-analysis, the recent surge in the use of popular and freely available platforms (e.g. Zoom, Skype) to provide online mental health services rather than secured platforms may be another cause of concern [117] as these platforms do mostly not comply with national standards for sensitive patient data protection. In order to demonstrate user safety, clinical guidelines should be explicitly taken into account and advice by mental health professionals, learned societies, and IT professionals actively incorporated. 
Overall, existing apps available in app stores should be used with caution due to the existing risks in data and clinical safety as well as lack of evidence on their effectiveness.

\section{Limitations}

This meta-review has several limitations. Because of time constraints and the rapid meta-review format of the current study, the quality of included systematic reviews was not evaluated using established assessment tools (e.g. AMSTAR 2 checklist [118]). Along similar lines, the conclusions drawn in this meta-review on the quality of evidence are largely based on quality assessments undertaken in the included systematic reviews and meta-analysis. However, if the quality of evidence was not systematically evaluated using a standardized approach it is indicated in Tables 1-3. Also, only one reviewer screened identified articles while a second reviewer independently screened a randomly selected subset (40\%) of studies. However, this meta-review was conducted in line with the state of the art of conducting rapid reviews [119]. Furthermore, the World Health Organization has explicitly recommended rapid reviews for evidence synthesis during the ongoing public health crisis, given these are urgently needed for policy makers and the public [120].

In considering the urgent need of continued access to mental health care for vulnerable individuals during the COVID-19 pandemic, and the importance of developing and implementing public mental health prevention and promotion strategies, digital interventions should be provided by public health services and routinely offered during infection control measures of pandemics. Since there is currently no direct evidence on digital interventions that aim to minimize the psychosocial impact of previous corona and influenza virus outbreaks, digital interventions should be developed and evaluated by research groups in close collaboration with relevant stakeholders to ensure established standards for investigating quality from the user perspective, effectiveness, and cost effectiveness are met. Importantly, evidence-based digital interventions are rapidly deliverable and scalable at the population level. This may facilitate delivering personalized care and minimizing the negative impact of the COVID-19 pandemic on public mental health. 


\section{Conclusions}

Decision-makers and stakeholders, including policymakers, technology companies, and public health professionals, should join forces to develop evidence-based strategies for mental health care in the area of public mental health provision, especially in moments of public health crises. As studies from previous pandemics as well as accumulating evidence from the COVID-19 pandemic suggest a negative impact on public mental health, the development and implementation of mental health promotion and prevention strategies at the population level may be an important measure to improve public mental health. As shown in earlier studies, digital interventions which incorporate contact with mental health staff in a blended-care approach may be particularly suited to alleviate mental health burden in help-seeking individuals. At times of COVID-19 and physical distancing measures, this may be translated into a digital blended care approach by combining telemedical with internet-based eHealth or smartphone-based mHealth interventions. Furthermore, efforts should be made to systematically evaluate currently available digital interventions based on established criteria of digital mental health and mental health services research (e.g., National Health Service Apps Library in the UK; Platform for Digital health applications in Germany; App Evaluation Database provided by the Division of Digital Psychiatry, Beth Israel Deaconess Medical Center in the USA) [121-123]. This would systematize the search for evidence-based mHealth apps and thus allow clinicians and interested users to make more informed decisions on the quality of currently available digital interventions. There is also a need to carefully examine the role of social inequalities and the related digital divide as well as possible barriers (e.g. disproportional access to necessary technologies, educational requirements, language skills, cultural peculiarities, motor, or cognitive impairments), which can influence the access to and use of information platforms of digital mental health interventions. 


\section{References}

1. Roehr, S., et al., [Psychosocial Impact of Quarantine Measures During Serious Coronavirus Outbreaks: A Rapid Review]. Psychiatr Prax, 2020. 47(4): p. 179-189.

2. Brooks, S.K., et al., The psychological impact of quarantine and how to reduce it: rapid review of the evidence. The Lancet, 2020. 395(10227): p. 912-920.

3. Holmes, E.A., et al., Multidisciplinary research priorities for the COVID-19 pandemic: a call for action for mental health science. Lancet Psychiatry, 2020. 7(6): p. 547-560.

4. McKibbin, W.J. and R. Fernando, The Global Macroeconomic Impacts of COVID-19: Seven Scenarios. SSRN Electronic Journal, 2020.

5. Luykx, J.J., C.H. Vinkers, and J.K. Tijdink, Psychiatry in Times of the Coronavirus Disease 2019 (COVID-19) Pandemic: An Imperative for Psychiatrists to Act Now. JAMA Psychiatry, 2020.

6. Yao, H., J.H. Chen, and Y.F. Xu, Patients with mental health disorders in the COVID-19 epidemic. Lancet Psychiatry, 2020. 7(4): p. e21.

7. Mohr, D.C., et al., The behavioral intervention technology model: an integrated conceptual and technological framework for eHealth and mHealth interventions. J Med Internet Res, 2014. 16(6): p. e146.

8. Kramer, U., et al., [DNVF-Memorandum - Health and Medical Apps]. Gesundheitswesen, 2019. 81(10): p. e154-e170.

9. Myin-Germeys, I., et al., Ecological momentary interventions in psychiatry. Curr Opin Psychiatry, 2016. 29(4): p. 258-63.

10. Rose, G., Sick individuals and sick populations. Int J Epidemiol, 2001. 30(3): p. 427-32; discussion 433-4.

11. Wright, L., A. Steptoe, and D. Fancourt, Are we all in this together? Longitudinal assessment of cumulative adversities by socioeconomic position in the first 3 weeks of lockdown in the UK. J Epidemiol Community Health, 2020.

12. Pierce, M., et al., Mental health before and during the COVID-19 pandemic: a longitudinal probability sample survey of the UK population. The Lancet Psychiatry, 2020.

13. Betsch, C., et al., Germany COVID-19 Snapshot MOnitoring (COSMO Germany): Monitoring knowledge, risk perceptions, preventive behaviours, and public trust in the current coronavirus outbreak in Germany, in PsychArchives. 2020.

14. Kwong, A.S.F., et al., Mental health during the COVID-19 pandemic in two longitudinal UK population cohorts. medRxiv, 2020.

15. Betsch, C., Korn, L., Felgendreff, L., Eitze, S., Schmid, P., Sprengholz, P., Wieler, L., Schmich, P., Stollorz, V., Ramharter, M., Bosnjak, M., Omer, S. B., Thaiss, H., De Bock, F., Von Rüden, U., Lieb, K., \& Thrull, J., German COVID-19 Snapshot Monitoring (COSMO) Welle 5 (31.03.2020). 2020: PsychArchives.

16. Rauschenberg, C., et al., Social isolation, mental health, and use of digital interventions in youth during the COVID-19 pandemic: A nationally representative survey. European Psychiatry 64, e20.

17. Torrent-Sellens, J., et al., Modelling and Predicting eHealth Usage in Europe: A Multidimensional Approach From an Online Survey of 13,000 European Union Internet Users. J Med Internet Res, 2016. 18(7): p. e188. 
18. Simons, C.J.P., et al., Economic evaluation of an experience sampling method intervention in depression compared with treatment as usual using data from a randomized controlled trial. BMC Psychiatry, 2017. 17(1): p. 415.

19. Safety, E.C.D.-G.f.H.a.F. Market study on telemedicine. 20182018 14.07.2020)]; Available from: https://ec.europa.eu/health/sites/health/files/ehealth/docs/2018_provision_ marketstudy_telemedicine_en.pdf.

20. Briskman, J. Sensor Tower's Q1 2020 Data Digest: Exploring COVID-19's Impact on the Global App Ecosystem. 20202020 14.07.2020)]; Available from: https://sensortower. com/blog/q1-2020-data-digest.

21. Moher, D., et al., Preferred Reporting Items for Systematic Reviews and Meta-Analyses: The PRISMA Statement. PLoS Medicine, 2009. 6(7): p. e1000097.

22. Team, T.E., Endnote. 2013, Clarivate Analytics: Philadelphia, PA.

23. Heber, E., et al., The Benefit of Web- and Computer-Based Interventions for Stress: A Systematic Review and Meta-Analysis. J Med Internet Res, 2017. 19(2): p. e32.

24. Giroux, I., et al., Online and Mobile Interventions for Problem Gambling, Alcohol, and Drugs: A Systematic Review. Front Psychol, 2017. 8: p. 954.

25. Miralles, I., et al., Smartphone Apps for the Treatment of Mental Disorders: Systematic Review. JMIR Mhealth Uhealth, 2020. 8(4): p. e14897.

26. Domhardt, M., L. Steubl, and H. Baumeister, Internet- and Mobile-Based Interventions for Mental and Somatic Conditions in Children and Adolescents. Z Kinder Jugendpsychiatr Psychother, 2020. 48(1): p. 33-46.

27. Larsen, M.E., et al., Using science to sell apps: Evaluation of mental health app store quality claims. NPJ Digit Med, 2019. 2(18): p. 18.

28. Mercurio, M., et al., Longitudinal trends in the quality, effectiveness and attributes of highly rated smartphone health apps. Evid Based Ment Health, 2020.

29. Alyami, M., et al., Social anxiety apps: a systematic review and assessment of app descriptors across mobile store platforms. Evid Based Ment Health, 2017. 20(3): p. 6570.

30. Loucas, C., et al., E-therapies for mental health problems in children and young people: a systematic review and focus group investigation. Archives of disease in childhood, 2014. 99.

31. Ennis, N., I. Sijercic, and C.M. Monson, Internet-Delivered Early Interventions for Individuals Exposed to Traumatic Events: Systematic Review. J Med Internet Res, 2018. 20(11): p. e280.

32. Elaheebocus, S., et al., Peer-Based Social Media Features in Behavior Change Interventions: Systematic Review. J Med Internet Res, 2018. 20(2): p. e20.

33. Alkhaldi, G., et al., The Effectiveness of Prompts to Promote Engagement With Digital Interventions: A Systematic Review. J Med Internet Res, 2016. 18(1): p. e6.

34. Lau, P.W., et al., A systematic review of information and communication technologybased interventions for promoting physical activity behavior change in children and adolescents. J Med Internet Res, 2011. 13(3): p. e48.

35. Cotie, L.M., et al., The effectiveness of eHealth interventions on physical activity and measures of obesity among working-age women: a systematic review and metaanalysis. Obes Rev, 2018. 19(10): p. 1340-1358. 
36. Davies, E.B., R. Morriss, and C. Glazebrook, Computer-delivered and web-based interventions to improve depression, anxiety, and psychological well-being of university students: a systematic review and meta-analysis. J Med Internet Res, 2014. 16(5): p. e130.

37. Flujas-Contreras, J.M., A. Garcia-Palacios, and I. Gomez, Technology-based parenting interventions for children's physical and psychological health: a systematic review and meta-analysis. Psychol Med, 2019. 49(11): p. 1787-1798.

38. Harrer, M., et al., Internet interventions for mental health in university students: A systematic review and meta-analysis. Int J Methods Psychiatr Res, 2019. 28(2): p. e1759.

39. Simon, N., et al., Acceptability of internet-based cognitive behavioural therapy (i-CBT) for post-traumatic stress disorder (PTSD): a systematic review. Eur J Psychotraumatol, 2019. 10(1): p. 1646092.

40. Alvarez-Jimenez, M., et al., Online, social media and mobile technologies for psychosis treatment: a systematic review on novel user-led interventions. Schizophr Res, 2014. 156(1): p. 96-106.

41. Rost, T., et al., User Acceptance of Computerized Cognitive Behavioral Therapy for Depression: Systematic Review. J Med Internet Res, 2017. 19(9): p. e309.

42. Biagianti, B., S.H. Quraishi, and D.A. Schlosser, Potential Benefits of Incorporating Peerto-Peer Interactions Into Digital Interventions for Psychotic Disorders: A Systematic Review. Psychiatr Serv, 2018. 69(4): p. 377-388.

43. Aref-Adib, G., et al., Factors affecting implementation of digital health interventions for people with psychosis or bipolar disorder, and their family and friends: a systematic review. Lancet Psychiatry, 2019. 6(3): p. 257-266.

44. Goldberg, S.B., et al., Measuring Psychiatric Symptoms Remotely: a Systematic Review of Remote Measurement-Based Care. Curr Psychiatry Rep, 2018. 20(10): p. 81.

45. Faurholt-Jepsen, M., et al., Electronic self-monitoring of mood using IT platforms in adult patients with bipolar disorder: A systematic review of the validity and evidence. BMC Psychiatry, 2016. 16: p. 7.

46. Erbe, D., et al., Blending Face-to-Face and Internet-Based Interventions for the Treatment of Mental Disorders in Adults: Systematic Review. J Med Internet Res, 2017. 19(9): p. e306.

47. Walsh, S., E. Golden, and S. Priebe, Systematic review of patients' participation in and experiences of technology-based monitoring of mental health symptoms in the community. BMJ Open, 2016. 6(6): p. e008362.

48. Pravettoni, G. and S. Triberti, P5 eHealth: An Agenda for the Health Technologies of the Future. 2020.

49. Hutton, L., et al., Assessing the Privacy of mHealth Apps for Self-Tracking: Heuristic Evaluation Approach. JMIR Mhealth Uhealth, 2018. 6(10): p. e185.

50. Arguel, A., et al., Theoretical approaches of online social network interventions and implications for behavioral change: a systematic review. J Eval Clin Pract, 2018. 24(1): p. 212-221.

51. Massoudi, B., et al., The effectiveness and cost-effectiveness of e-health interventions for depression and anxiety in primary care: A systematic review and meta-analysis. J Affect Disord, 2019. 245: p. 728-743. 
52. Pennant, M.E., et al., Computerised therapies for anxiety and depression in children and young people: a systematic review and meta-analysis. Behav Res Ther, 2015. 67: p. 1-18.

53. Chebli, J.L., A. Blaszczynski, and S.M. Gainsbury, Internet-Based Interventions for Addictive Behaviours: A Systematic Review. J Gambl Stud, 2016. 32(4): p. 1279-1304.

54. Hadjistavropoulos, H.D., et al., A systematic review of internet-delivered cognitive behavior therapy for alcohol misuse: study characteristics, program content and outcomes. Cogn Behav Ther, 2020. 49(4): p. 327-346.

55. O.Rourke, L., G. Humphris, and A. Baldacchino, Electronic communication based interventions for hazardous young drinkers: A systematic review. Neurosci Biobehav Rev, 2016. 68: p. 880-890.

56. Tait, R.J. and H. Christensen, Internet-based interventions for young people with problematic substance use: a systematic review. Med J Aust, 2010. 192(S11): p. S15-21.

57. Boumparis, N., et al., Short- and long-term effects of digital prevention and treatment interventions for cannabis use reduction: A systematic review and meta-analysis. Drug Alcohol Depend, 2019. 200: p. 82-94.

58. Asuzu, K., et al., Electronically-Delivered Interventions to Reduce Cannabis Use in Adolescents: A Systematic Review. Adolescent Psychiatry, 2019. 8(3): p. 195-213.

59. Deady, M., et al., eHealth interventions for the prevention of depression and anxiety in the general population: a systematic review and meta-analysis. BMC Psychiatry, 2017. 17(1): p. 310.

60. Richardson, T., P. Stallard, and S. Velleman, Computerised cognitive behavioural therapy for the prevention and treatment of depression and anxiety in children and adolescents: a systematic review. Clin Child Fam Psychol Rev, 2010. 13(3): p. 275-90.

61. Fleming, T.M., et al., Serious games for the treatment or prevention of depression: A systematic review. Revista de Psicopatologia y Psicologia Clinica, 2014. 19(3): p. 227-242.

62. Lau, H.M., et al., Serious Games for Mental Health: Are They Accessible, Feasible, and Effective? A Systematic Review and Meta-analysis. Front Psychiatry, 2016. 7: p. 209.

63. Grist, R., et al., Technology Delivered Interventions for Depression and Anxiety in Children and Adolescents: A Systematic Review and Meta-analysis. Clin Child Fam Psychol Rev, 2019. 22(2): p. 147-171.

64. Baker, S., et al., Combatting social isolation and increasing social participation of older adults through the use of technology: A systematic review of existing evidence. Australas J Ageing, 2018. 37(3): p. 184-193.

65. Grist, R. and K. Cavanagh, Computerised Cognitive Behavioural Therapy for Common Mental Health Disorders, What Works, for Whom Under What Circumstances? A Systematic Review and Meta-analysis. Journal of Contemporary Psychotherapy, 2013. 43(4): p. 243-251.

66. Pasarelu, C.R., et al., Internet-delivered transdiagnostic and tailored cognitive behavioral therapy for anxiety and depression: a systematic review and meta-analysis of randomized controlled trials. Cogn Behav Ther, 2017. 46(1): p. 1-28.

67. Ye, X., et al., Effectiveness of internet-based interventions for children, youth, and young adults with anxiety and/or depression: a systematic review and meta-analysis. BMC Health Serv Res, 2014. 14: p. 313. 
68. Stech, E.P., et al., Internet-delivered cognitive behavioral therapy for panic disorder with or without agoraphobia: a systematic review and meta-analysis. Cogn Behav Ther, 2020. 49(4): p. 270-293.

69. Rice, S.M., et al., Online and social networking interventions for the treatment of depression in young people: A systematic review. Journal of Medical Internet Research, 2014. Vo 16(9): p. 190-200.

70. Richards, D. and T. Richardson, Computer-based psychological treatments for depression: a systematic review and meta-analysis. Clin Psychol Rev, 2012. 32(4): p. 32942.

71. Wright, J.H., et al., Computer-Assisted Cognitive-Behavior Therapy for Depression: A Systematic Review and Meta-Analysis. J Clin Psychiatry, 2019. 80(2): p. 19.

72. Sikorski, C., et al., [Computer-aided cognitive behavioral therapy for depression]. Psychiatr Prax, 2011. 38(2): p. 61-8.

73. Xiang, X., et al., Internet-delivered cognitive behavioral therapies for late-life depressive symptoms: a systematic review and meta-analysis. Aging Ment Health, 2019: p. 1-11.

74. Danielsson, A.K., A.K. Eriksson, and P. Allebeck, Technology-based support via telephone or web: a systematic review of the effects on smoking, alcohol use and gambling. Addict Behav, 2014. 39(12): p. 1846-68.

75. Lin, L.A., et al., Telemedicine-delivered treatment interventions for substance use disorders: A systematic review. J Subst Abuse Treat, 2019. 101: p. 38-49.

76. Gilmore, A.K., et al., A systematic review of technology-based interventions for cooccurring substance use and trauma symptoms. J Telemed Telecare, 2017. 23(8): p. 701-709.

77. Pittock, A., L. Hodges, and S.M. Lawrie, The effectiveness of internet-delivered cognitive behavioural therapy for those with bulimic symptoms: a systematic review : A review of iCBT treatment for bulimic symptoms. BMC Res Notes, 2018. 11(1): p. 748.

78. Meyer, T.D., et al., Novel technology as platform for interventions for caregivers and individuals with severe mental health illnesses: A systematic review. J Affect Disord, 2018. 226: p. 169-177.

79. Sierra, M.A., F.J. Ruiz, and C.L. Flórez, A Systematic Review and Meta-Analysis of ThirdWave Online Interventions for Depression. Revista Latinoamericana de Psicología, 2018. 50(2): p. 126-135.

80. Berryhill, M.B., et al., Videoconferencing psychological therapy and anxiety: a systematic review. Fam Pract, 2019. 36(1): p. 53-63.

81. Castro, A., et al., Effectiveness and adherence of telephone-administered psychotherapy for depression: A systematic review and meta-analysis. J Affect Disord, 2020. 260: p. 514-526.

82. Coughtrey, A.E. and N. Pistrang, The effectiveness of telephone-delivered psychological therapies for depression and anxiety: A systematic review. J Telemed Telecare, 2018. 24(2): p. 65-74.

83. Rees, C.S. and E. Maclaine, A Systematic Review of Videoconference-Delivered Psychological Treatment for Anxiety Disorders. Australian Psychologist, 2015. 50(4): p. 259-264. 
84. Bolton, A.J. and D.S. Dorstyn, Telepsychology for Posttraumatic Stress Disorder: A systematic review. J Telemed Telecare, 2015. 21(5): p. 254-67.

85. Lewis, C., et al., Internet-delivered cognitive behavioural therapy for post-traumatic stress disorder: systematic review and meta-analysis. Acta Psychiatr Scand, 2019. 140(6): p. 508-521.

86. Baker, A.L., et al., Telephone-delivered psychosocial interventions targeting key health priorities in adults with a psychotic disorder: systematic review. Psychol Med, 2018. 48(16): p. 2637-2657.

87. Gentry, M.T., et al., Evidence for telehealth group-based treatment: A systematic review. J Telemed Telecare, 2019. 25(6): p. 327-342.

88. Irvine, A., et al., Are there interactional differences between telephone and face-to-face psychological therapy? A systematic review of comparative studies. J Affect Disord, 2020. 265: p. 120-131.

89. Baker, T.B., D.H. Gustafson, and D. Shah, How can research keep up with eHealth? Ten strategies for increasing the timeliness and usefulness of eHealth research. J Med Internet Res, 2014. 16(2): p. e36.

90. Boehm, B., et al., Effects of Mobile Health Including Wearable Activity Trackers to Increase Physical Activity Outcomes Among Healthy Children and Adolescents: Systematic Review. JMIR Mhealth Uhealth, 2019. 7(4): p. e8298.

91. Bort-Roig, J., et al., Measuring and influencing physical activity with smartphone technology: a systematic review. Sports Med, 2014. 44(5): p. 671-86.

92. Feter, N., et al., What is the role of smartphones on physical activity promotion? A systematic review and meta-analysis. Int J Public Health, 2019. 64(5): p. 679-690.

93. Kim, H.N. and K. Seo, Smartphone-Based Health Program for Improving Physical Activity and Tackling Obesity for Young Adults: A Systematic Review and Meta-Analysis. Int J Environ Res Public Health, 2019. 17(1): p. 18.

94. Rose, T., et al., A Systematic Review of Digital Interventions for Improving the Diet and Physical Activity Behaviors of Adolescents. J Adolesc Health, 2017. 61(6): p. 669-677.

95. Muntaner, A., J. Vidal-Conti, and P. Palou, Increasing physical activity through mobile device interventions: A systematic review. Health Informatics J, 2016. 22(3): p. 451-69.

96. Donker, T., et al., Smartphones for smarter delivery of mental health programs: a systematic review. J Med Internet Res, 2013. 15(11): p. e247.

97. Loo Gee, B., K.M. Griffiths, and A. Gulliver, Effectiveness of mobile technologies delivering Ecological Momentary Interventions for stress and anxiety: a systematic review. J Am Med Inform Assoc, 2016. 23(1): p. 221-9.

98. Holmes, N.A., J.E. van Agteren, and D.S. Dorstyn, A systematic review of technologyassisted interventions for co-morbid depression and substance use.J Telemed Telecare, 2019. 25(3): p. 131-141.

99. Rathbone, A.L. and J. Prescott, The Use of Mobile Apps and SMS Messaging as Physical and Mental Health Interventions: Systematic Review. J Med Internet Res, 2017. 19(8): p. e295.

100. Rootes-Murdy, K., et al., Mobile technology for medication adherence in people with mood disorders: A systematic review. J Affect Disord, 2018. 227: p. 613-617. 
101. Dick, S., et al., A systematic review of the effectiveness of digital interventions for illicit substance misuse harm reduction in third-level students. BMC Public Health, 2019. 19(1): p. 1244.

102. Song, T., S. Qian, and P. Yu, Mobile Health Interventions for Self-Control of Unhealthy Alcohol Use: Systematic Review. JMIR Mhealth Uhealth, 2019. 7(1): p. e10899.

103. Yonker, L.M., et al., "Friending" teens: systematic review of social media in adolescent and young adult health care. J Med Internet Res, 2015. 17(1): p. e4.

104. Arshad, U., et al., A Systematic Review of the Evidence Supporting Mobile- and InternetBased Psychological Interventions For Self-Harm. Suicide Life Threat Behav, 2020. 50(1): p. 151-179.

105. Clarke, S., et al., A systematic review and meta-analysis of digital health technologies effects on psychotic symptoms in adults with psychosis. Psychosis, 2019. 11(4): p. 362373.

106. Kreuze, E., et al., Technology-enhanced suicide prevention interventions: A systematic review. J Telemed Telecare, 2017. 23(6): p. 605-617.

107. Perry, Y., et al., Web-Based and Mobile Suicide Prevention Interventions for Young People: A Systematic Review. J Can Acad Child Adolesc Psychiatry, 2016. 25(2): p. 73-9.

108. Ilagan, G.S., et al., Smartphone applications targeting borderline personality disorder symptoms: a systematic review and meta-analysis. Borderline Personal Disord Emot Dysregul, 2020. 7(1): p. 12.

109. Sander, L.B., et al., 'Help for trauma from the app stores?' A systematic review and standardised rating of apps for Post-Traumatic Stress Disorder (PTSD). Eur J Psychotraumatol, 2020. 11(1): p. 1701788.

110. Sucala, M., et al., Anxiety: There is an app for that. A systematic review of anxiety apps. Depress Anxiety, 2017. 34(6): p. 518-525.

111. Terhorst, Y., et al., «Hilfe aus dem App-Store?»: Eine systematische Übersichtsarbeit und Evaluation von Apps zur Anwendung bei Depressionen. Verhaltenstherapie, 2018. 28(2): p. 101-112.

112. Axelsson, E. and E. Hedman-Lagerlof, Cognitive behavior therapy for health anxiety: systematic review and meta-analysis of clinical efficacy and health economic outcomes. Expert Rev Pharmacoecon Outcomes Res, 2019. 19(6): p. 663-676.

113. Hedman, E., B. Ljotsson, and N. Lindefors, Cognitive behavior therapy via the Internet: a systematic review of applications, clinical efficacy and cost-effectiveness. Expert Rev Pharmacoecon Outcomes Res, 2012. 12(6): p. 745-64.

114. Edwards, E.A., et al., Gamification for health promotion: systematic review of behaviour change techniques in smartphone apps. BMJ Open, 2016. 6(10): p. e012447.

115. Berry, N., et al., Acceptability of Interventions Delivered Online and Through Mobile Phones for People Who Experience Severe Mental Health Problems: A Systematic Review. J Med Internet Res, 2016. 18(5): p. e121.

116. Mantovani, E., et al., Towards a Code of Conduct on Privacy for mHealth to Foster Trust Amongst Users of Mobile Health Applications, in Data Protection and Privacy: (In) visibilities and Infrastructures, R. Leenes, et al., Editors. 2017, Springer International Publishing: Cham. p. 81-106. 
117. Soron, T.R., et al., The hope and hype of telepsychiatry during the COVID-19 pandemic. The Lancet Psychiatry, 2020. 7(8).

118. Shea, B.J., et al., AMSTAR 2: a critical appraisal tool for systematic reviews that include randomised or non-randomised studies of healthcare interventions, or both. BMJ, 2017. 358: p. j4008.

119. Tricco, A.C., et al., A scoping review of rapid review methods. BMC Med, 2015. 13: p. 224.

120. Rapid reviews to strengthen health policy and systems: a practical guide. 2020 29.06.2020)]; Available from: https://www.who.int/alliance-hpsr/resources/ publications/rapid-review-guide/en/.

121. Federal Institute for Drugs and Medical Devices

(BfArM), Germany: Digital health applications (DiGA). 29.06.2020)]; Available from: https:// www.bfarm.de/EN/MedicalDevices/DiGA/_node.html.

122. United Kingdom National Health Service (NHS): Apps Library - Category Mental Health. 29.06.2020)]; Available from: https://www.nhs.uk/apps-library/ filter/?categories=Mental\%20health.

123. Division of Digital Psychiatry, Beth Israel Deaconess Medical Center, USA: App Evaluation Database. 2020 29.06.2020)]; Available from: https://apps.digitalpsych.org. 


\section{Supplementary material}

\section{Supplement 1. Search strategy: MEDLINE}

Database: Ovid MEDLINE(R) <1946 to April Week 4 2020>

Search Strategy:

1 exp Mental Health/ (37303)

2 exp Psychopathology/ (7194)

3 exp Mental Disorders/ (1225111)

4 "mental health".tw. (113359)

5 "mental dis*".tw. (37183)

6 psychopatholog*.tw. (35952)

7 "mental ill*".tw. (25880)

8 "psychiatric dis*".tw. (41201)

9 SMD.tw. (6004)

10 exp Depression/ (116762)

11 exp Depression, Postpartum/ (5418)

12 exp Depressive Disorder, Major/ (29572)

13 exp Depressive Disorder/ (107891)

14 exp Dysthymic Disorder/ (1135)

15 exp Mood Disorders/ (120656)

$16 \exp$ Affect/ (32941)

17 exp Emotions/ (237356)

18 exp Emotional Regulation/ (195)

19 depress*.tw. (394544)

20 dysthymi*.tw. (2850)

21 melanchol*.tw. (2736)

22 mood*.tw. (63196)

23 affect*.tw. (1563493)

24 emotion*.tw. (160488)

25 sad.tw. (8270)

26 lonel*.tw. (5604)

27 exp Anxiety/ (83635)

28 exp Anxiety Disorders/ (78672)

29 exp Phobic Disorders/ (11222)

30 exp Agoraphobia/ (2581)

31 exp Performance Anxiety/ (140)

32 exp Panic Disorder/ (6843)

33 exp Panic/ (2598)

34 exp Fear/ (32507)

35 anxi*.tw. (172074)

36 agoraphobi*.tw. (3157)

37 GAD.tw. (7875)

38 panic*.tw. (16859)

39 fear*.tw. (68150)

40 phobi*.tw. (10167)

41 fright*.tw. (2288)

42 exp Obsessive Behavior/ (1404)

43 exp Obsessive-Compulsive Disorder/ (14429) 


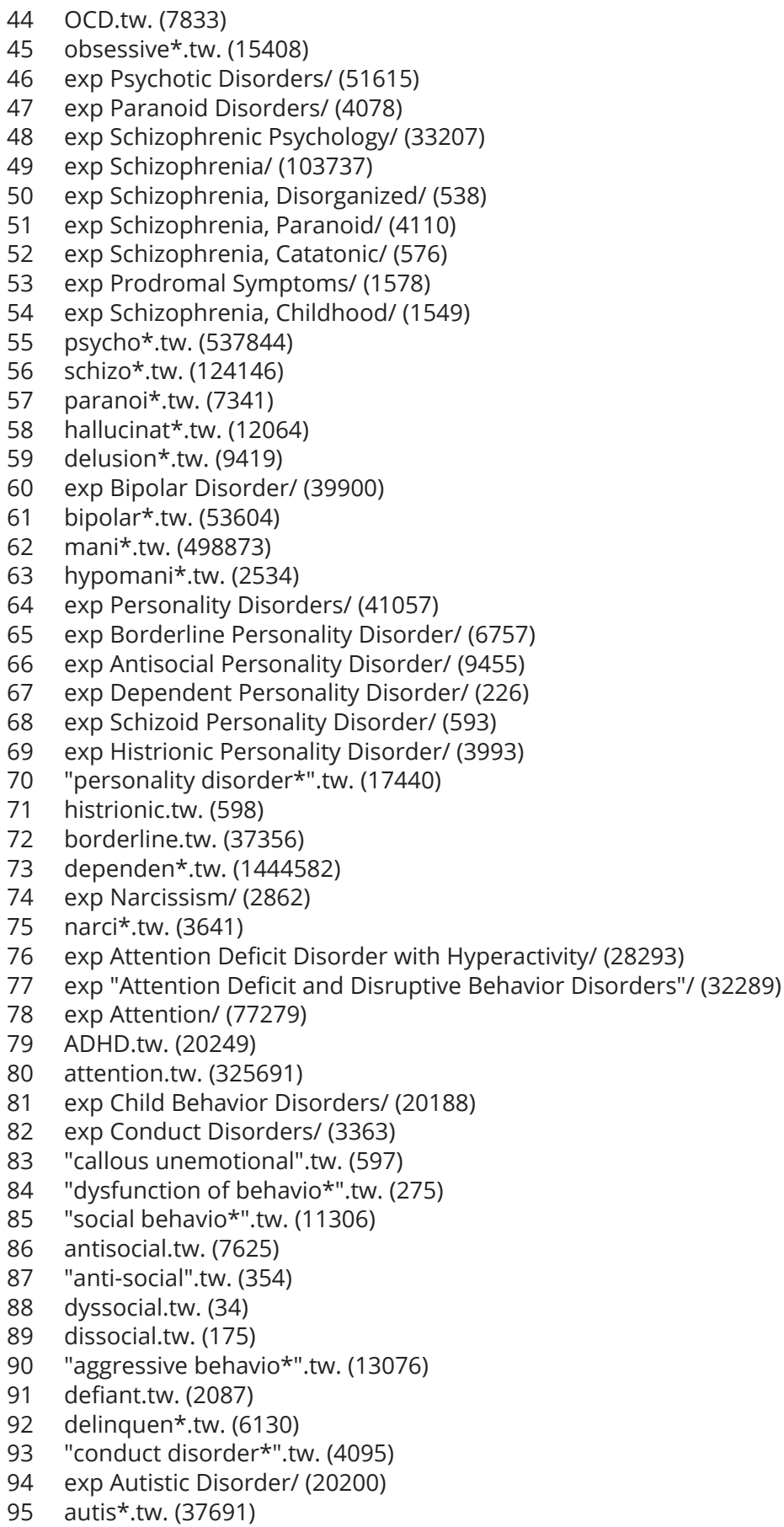


96 exp Adjustment Disorders/ (4204)

97 exp Social Adjustment/ (23204)

98 adjustment.tw. (139354)

99 exp Cognition Disorders/ (92327)

100 exp Intellectual Disability/ (95241)

101 cognition*.tw. (57240)

102 intellect*.tw. (34165)

103 disabilit*.tw. (153891)

104 learning.tw. (225885)

105 exp "Sleep Initiation and Maintenance Disorders"/ (13034)

106 exp Sleep Disorders, Circadian Rhythm/ (2269)

107 exp Sleep/ (78550)

108 sleep.tw. (138836)

109 insomnia.tw. (16769)

110 nightmares.tw. (1547)

111 exp Stress Disorders, Traumatic/ (35714)

112 exp Stress Disorders, Post-Traumatic/ (32062)

113 exp Stress, Psychological/ (129346)

114 exp Stress, Physiological/ (214074)

115 stress.tw. (611314)

116 distress*.tw. (103640)

117 "post-trauma*".tw. (26218)

118 PTSD.tw. (19272)

119 exp Substance-Related Disorders/ (275494)

120 "substance disorder*".tw. (310)

121 (substance adj3 abus*).tw. (23317)

122 addict*.tw. (53307)

123 cannabis.tw. (13262)

124 tobacco.tw. (84037)

$125 \exp$ Alcoholism/ (74611)

126 exp Alcohol Drinking/ (68175)

127 alcohol*.tw. (279470)

128 amphetamine.tw. (20830)

129 hallucinogens.tw. (1264)

130 exp "Feeding and Eating Disorders"/ (30305)

131 exp Anorexia Nervosa/ (12849)

132 exp Binge-Eating Disorder/ (1420)

133 exp Bulimia Nervosa/ (2321)

134 exp Eating/ (71950)

135 exp Bulimia/ (5444)

136 exp Anorexia/ (4933)

137 "eating disorder*".tw. (16385)

138 "body-imag*".tw. (10541)

139 "binge-eating".tw. (4751)

140 bulimi*.tw. (7600)

141 anorexi*.tw. (29319)

142 exp Sexual Dysfunctions, Psychological/ (26480)

143 sexual.tw. (166394)

144 orgasm.tw. (2590)

145 desire.tw. (27818)

146 erectile.tw. (18047)

147 ejaculation.tw. (6367) 
148 dyspareunia.tw. (3342)

149 exp Self-Injurious Behavior/ (69868)

150 "self-injur*".tw. (3839)

151 "self-harm".tw. (4156)

152 suicid*.tw. (66400)

153 "at risk*".tw. (147796)

154 "high risk*".tw. (236307)

155 ARMS.tw. (49756)

156 UHR.tw. (712)

157 vulnerab*.tw. (107836)

158 exp Anhedonia/ (959)

159 anhedon*.tw. (3456)

160 exp Motivation/ (167764)

161 exp Reward/ (21181)

162 motivation.tw. (49856)

163 exp Developmental Disabilities/ (19858)

164 exp Personality Development/ (153603)

165 exp Adaptation, Psychological/ (125798)

166 development*.tw. (2017494)

167 internali*.tw. (54249)

168 externali*.tw. (12204)

169 "quality of life".tw. (225450)

170 happiness.tw. (5276)

171 satisfact*.tw. (227385)

172 "social support".tw. (31355)

173 pleasure.tw. (5782)

174 gratitude.tw. (1050)

175 compassion*.tw. (7850)

176 well-being.tw. (60119)

177 resilien*.tw. (22733)

178 or/1-177 (8415847)

179 exp Telemedicine/ (27662)

180 exp Internet-Based Intervention/ (89)

181 internet.tw. (39914)

182 ehealth.tw. (1775)

183 "web-based".tw. (23543)

184 "e-health".tw. (1709)

185 telemed*.tw. (8716)

186 telehealth*.tw. (2984)

187 teletherap*.tw. (1266)

188 uhealth.tw. (1)

189 "u-health".tw. (19)

190 eTherap*.tw. (9)

191 "e-Therap*".tw. (373)

192 exp mobile application/ (5560)

193 exp Smartphone/ (4124)

194 "mobile health".tw. (1928)

195 mHealth.tw. (1600)

196 m-health.tw. (279)

197 app.tw. (19176)

198 "app-based".tw. (242)

199 "mobile app*".tw. (2150) 
200 "mobile-based".tw. (245)

201 "phone-based".tw. (773)

202 smartphone.tw. (5176)

203 "smartphone-based".tw. (910)

204 "digital tool".tw. (45)

205 "digital assist*".tw. (965)

206 apps.tw. (3316)

207 or/179-206 (115779)

208 exp Primary Prevention/ (150004)

209 exp Secondary Prevention/ (20087)

210 exp Health Promotion/ (75852)

211 exp Early Intervention, Educational/ (2987)

212 exp Internet-Based Intervention/ (89)

213 exp Early Medical Intervention/ (2920)

214 prevention.tw. (462307)

215 intervention*.tw. (812225)

216 "health promotion*".tw. (25265)

217 treatment*.tw. (3843362)

218 "health service".tw. (39081)

219 therap*.tw. (2375507)

220 counselling.tw. (22841)

221 counseling.tw. (56370)

222 or/208-221 (6099759)

223 "systematic review".ti. (90092)

224178 and 207 and 222 and 223 (825) 


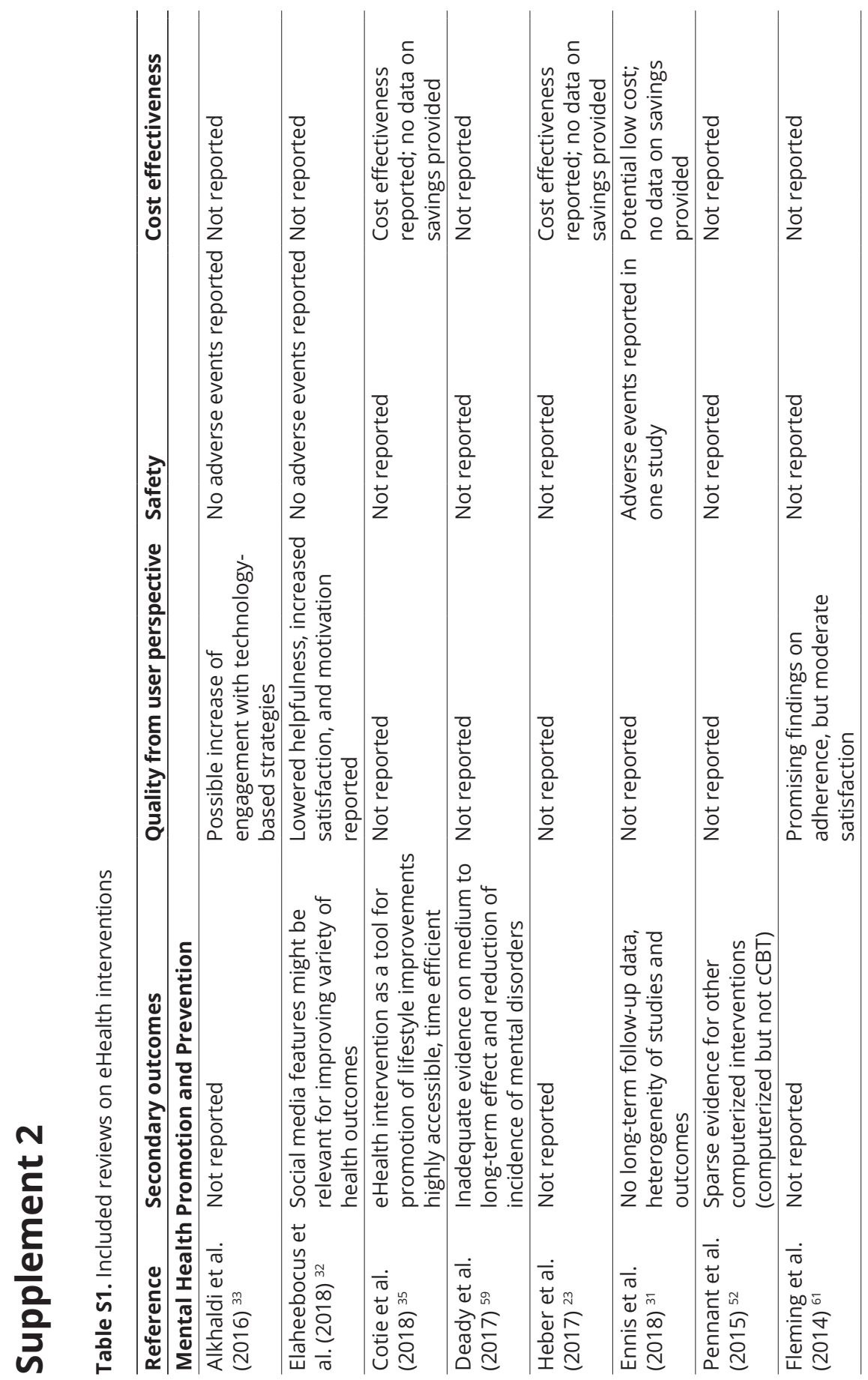




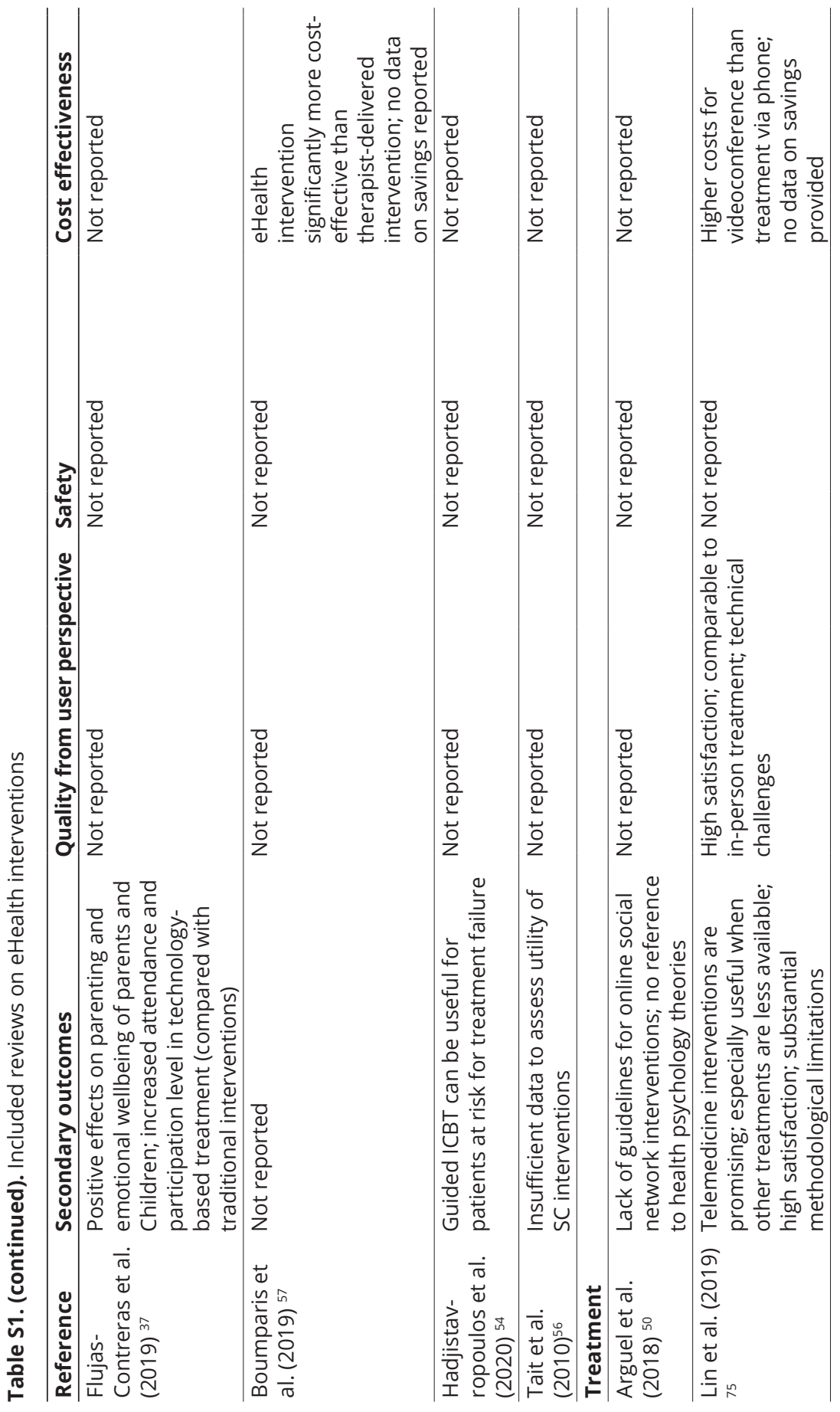




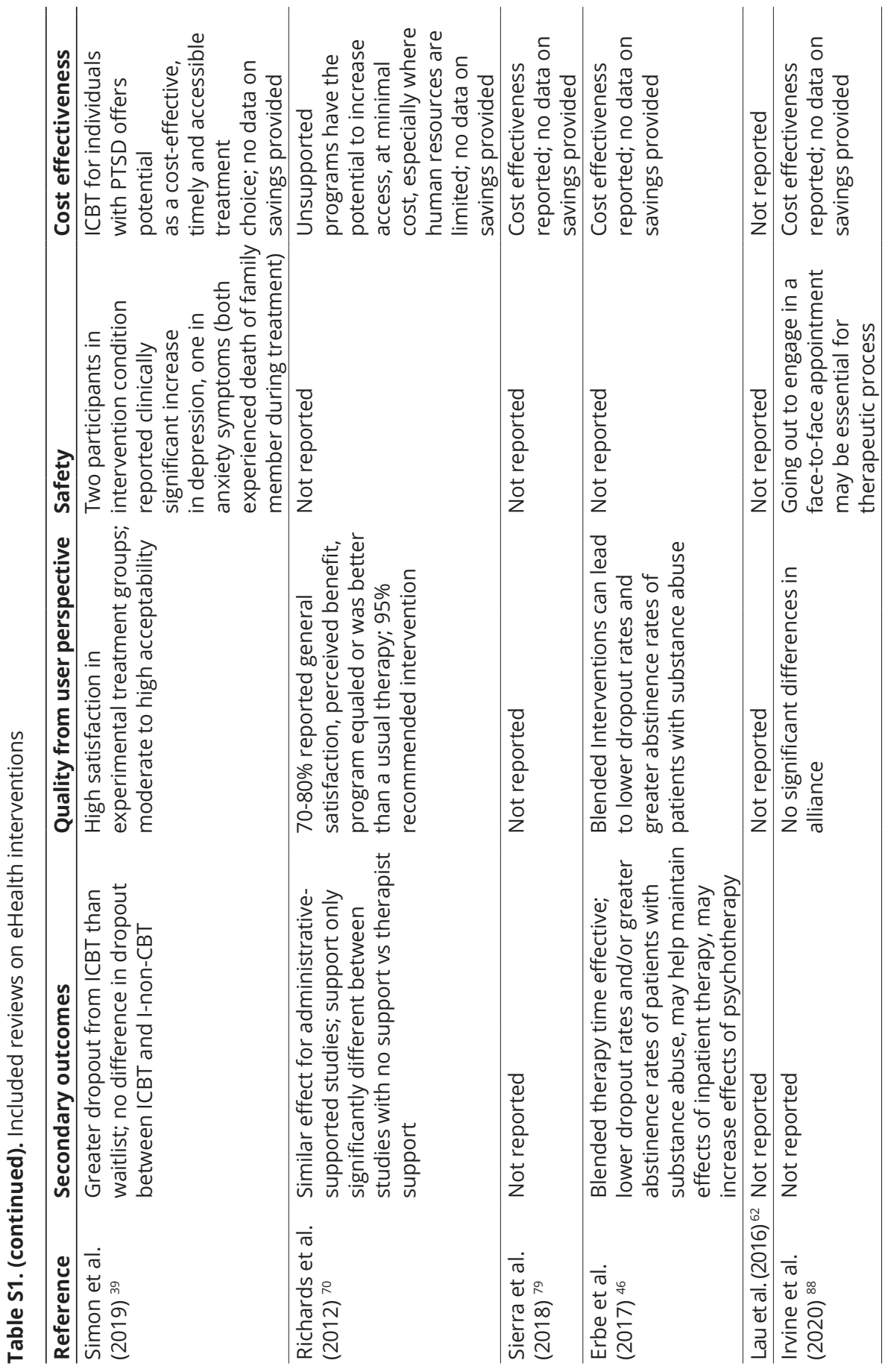




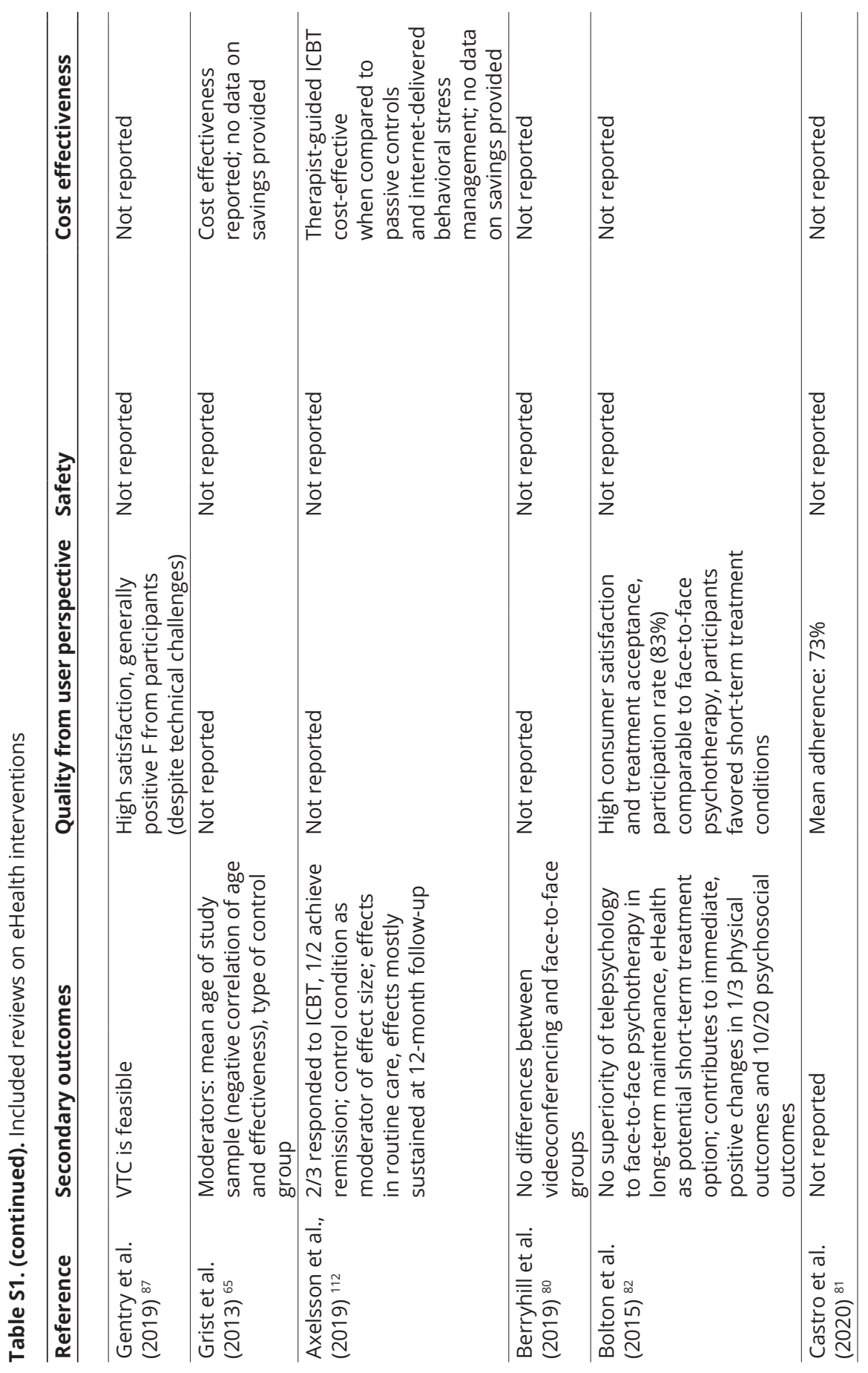




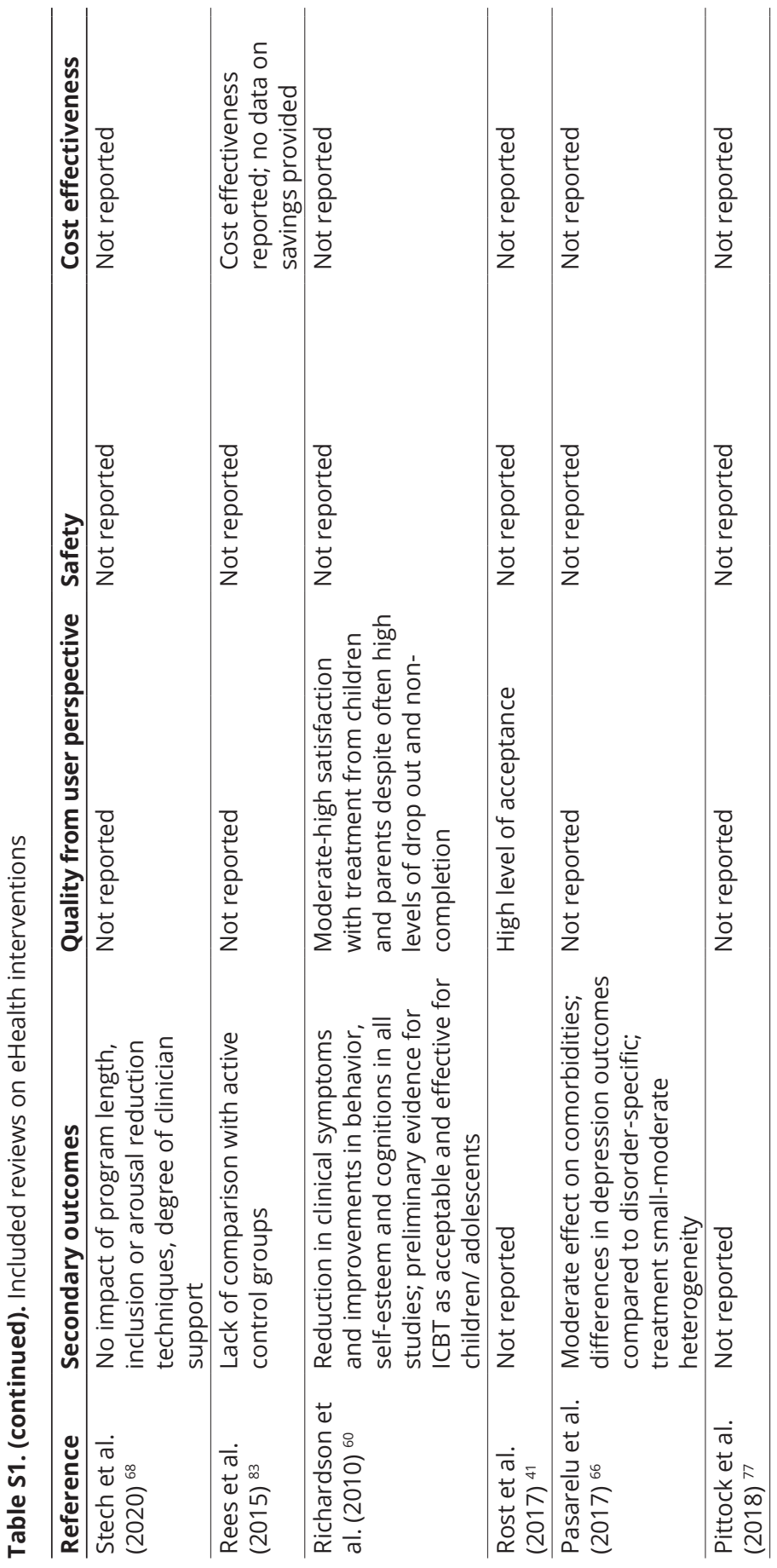




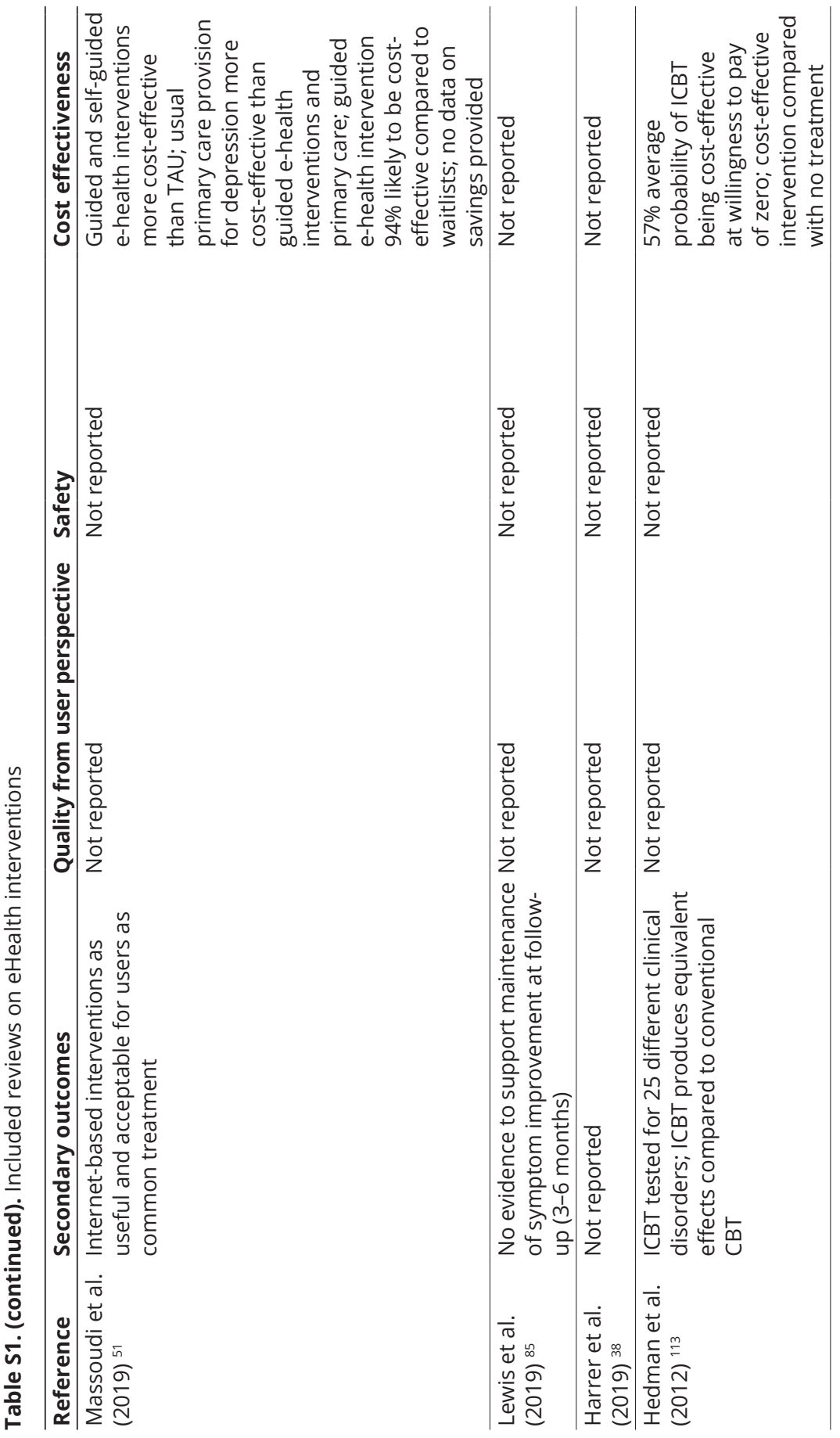




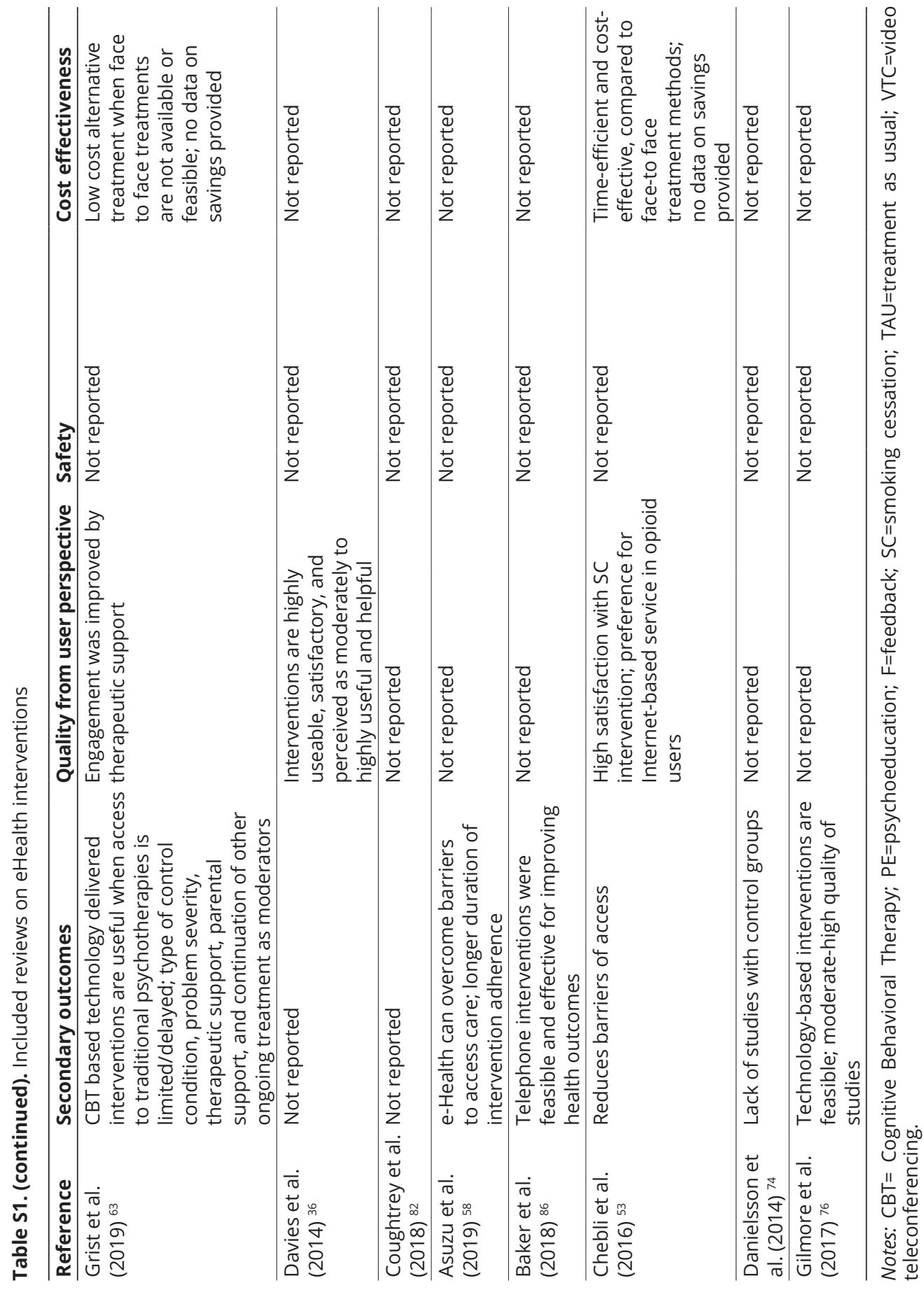




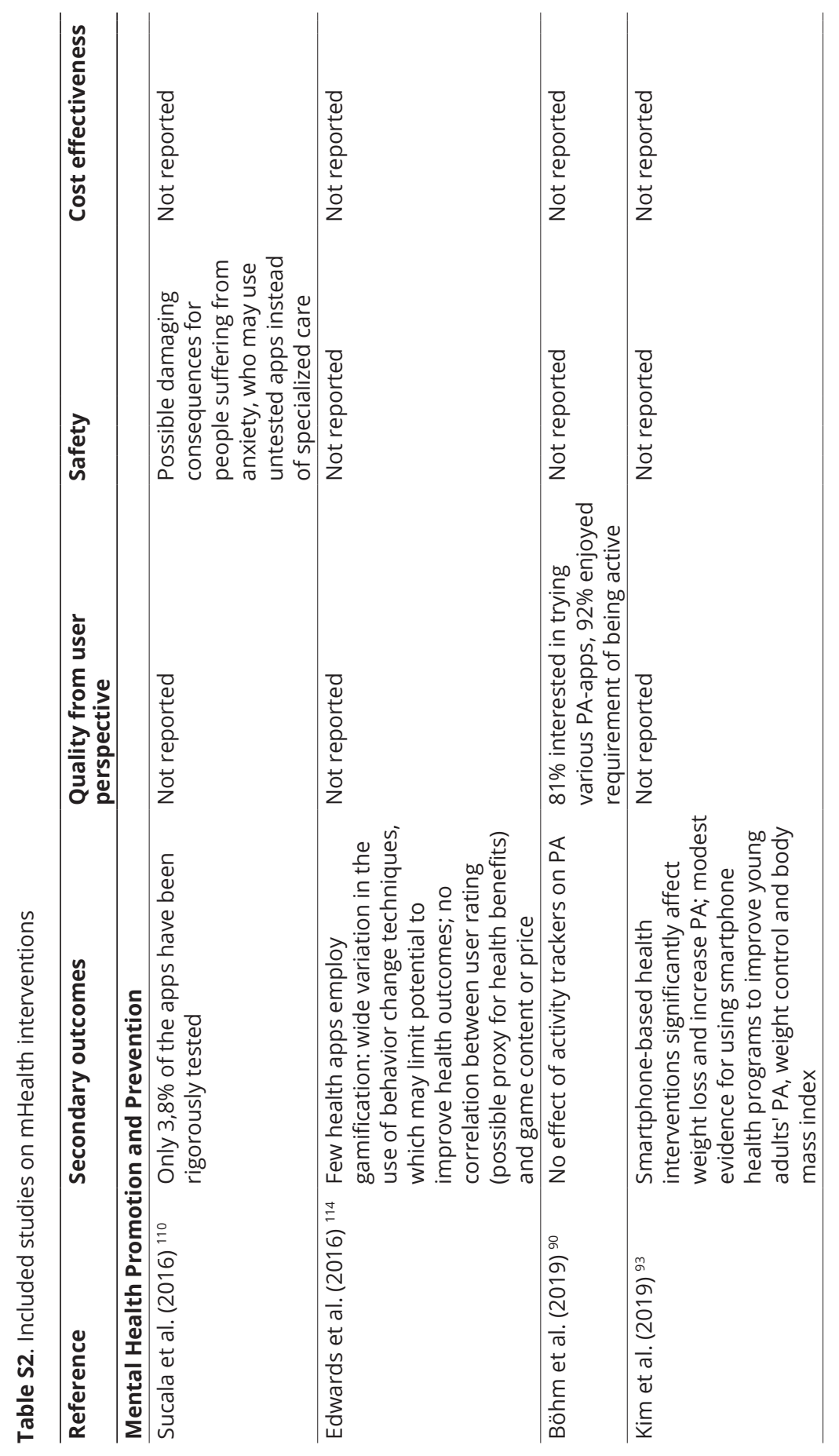




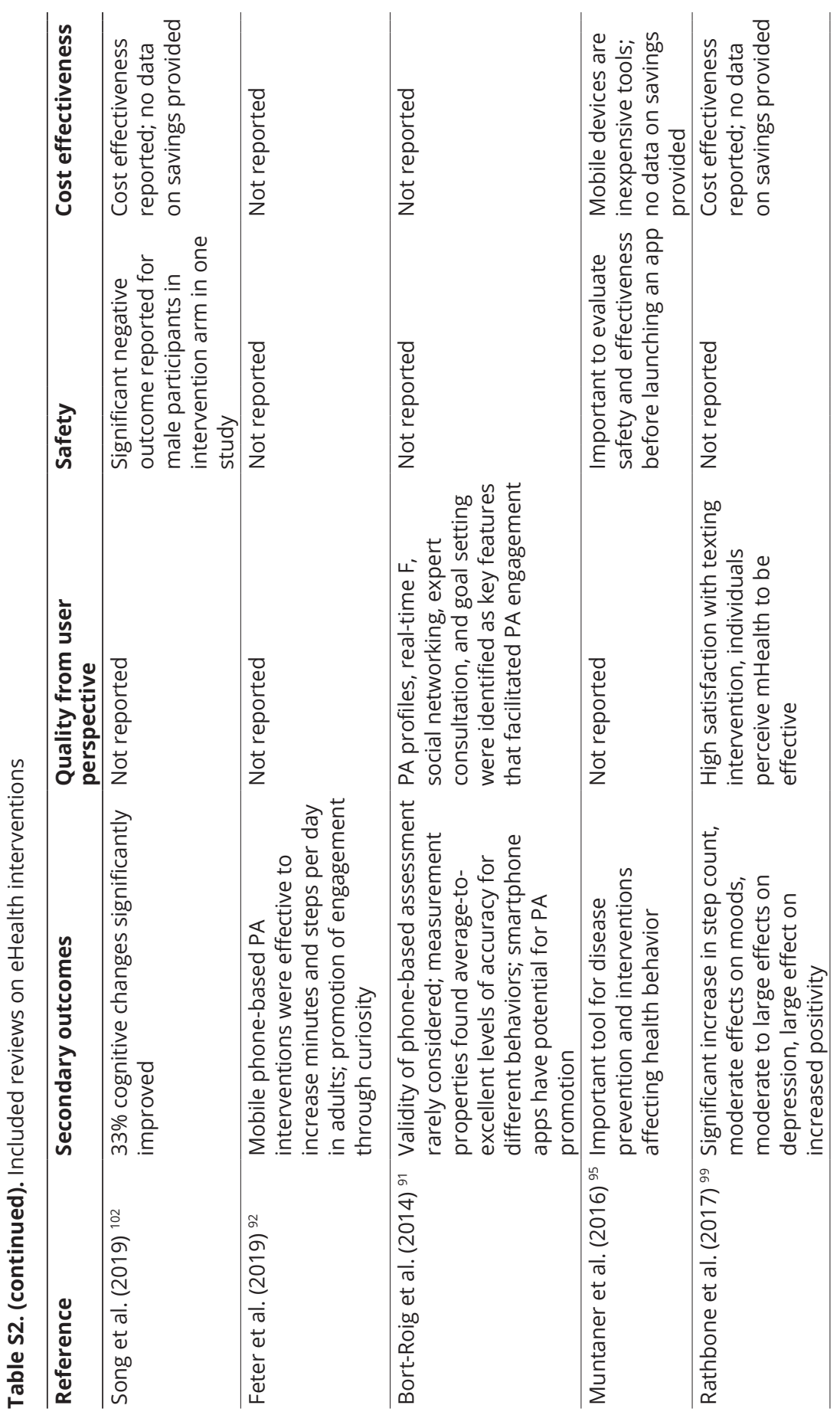




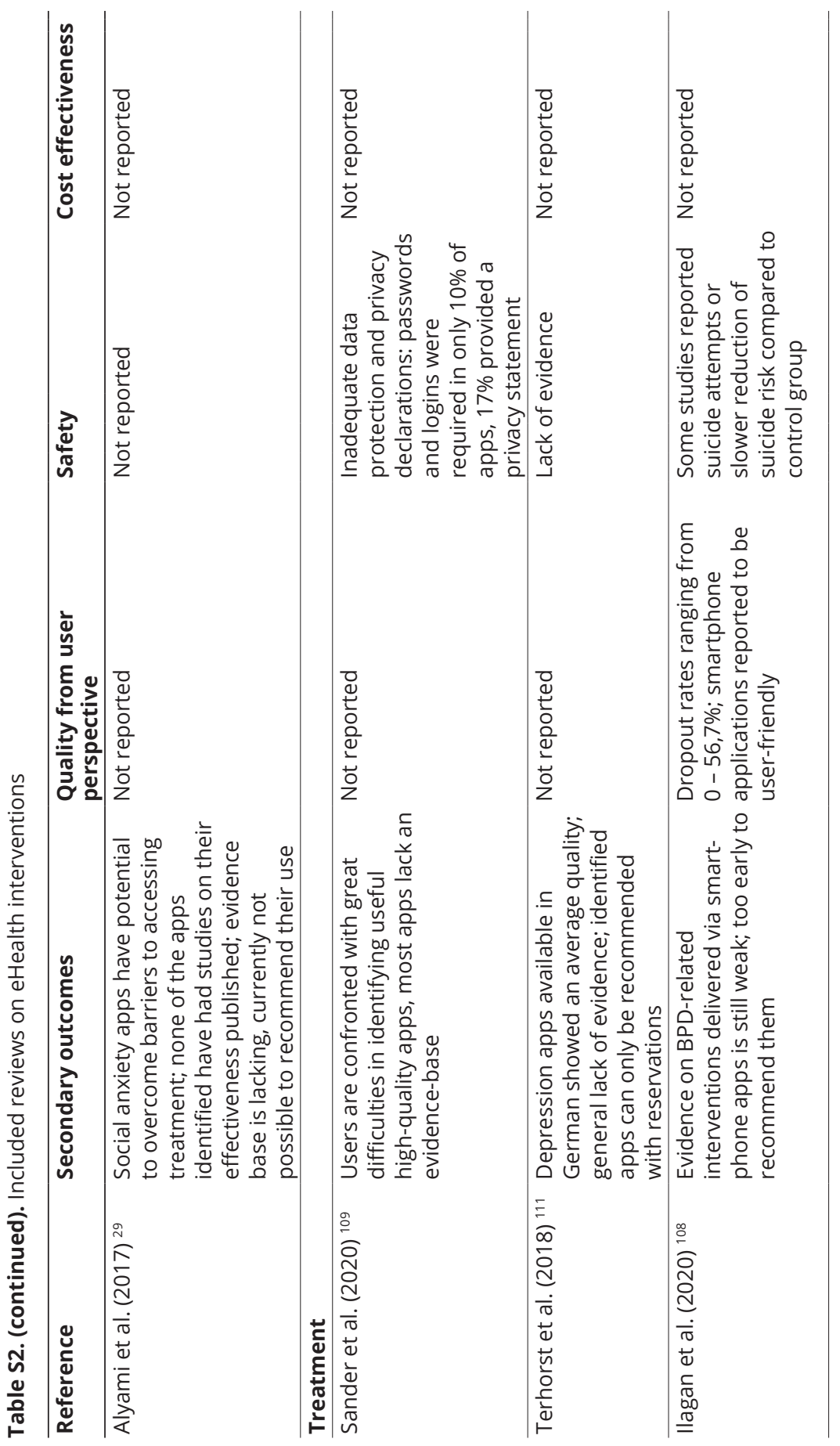




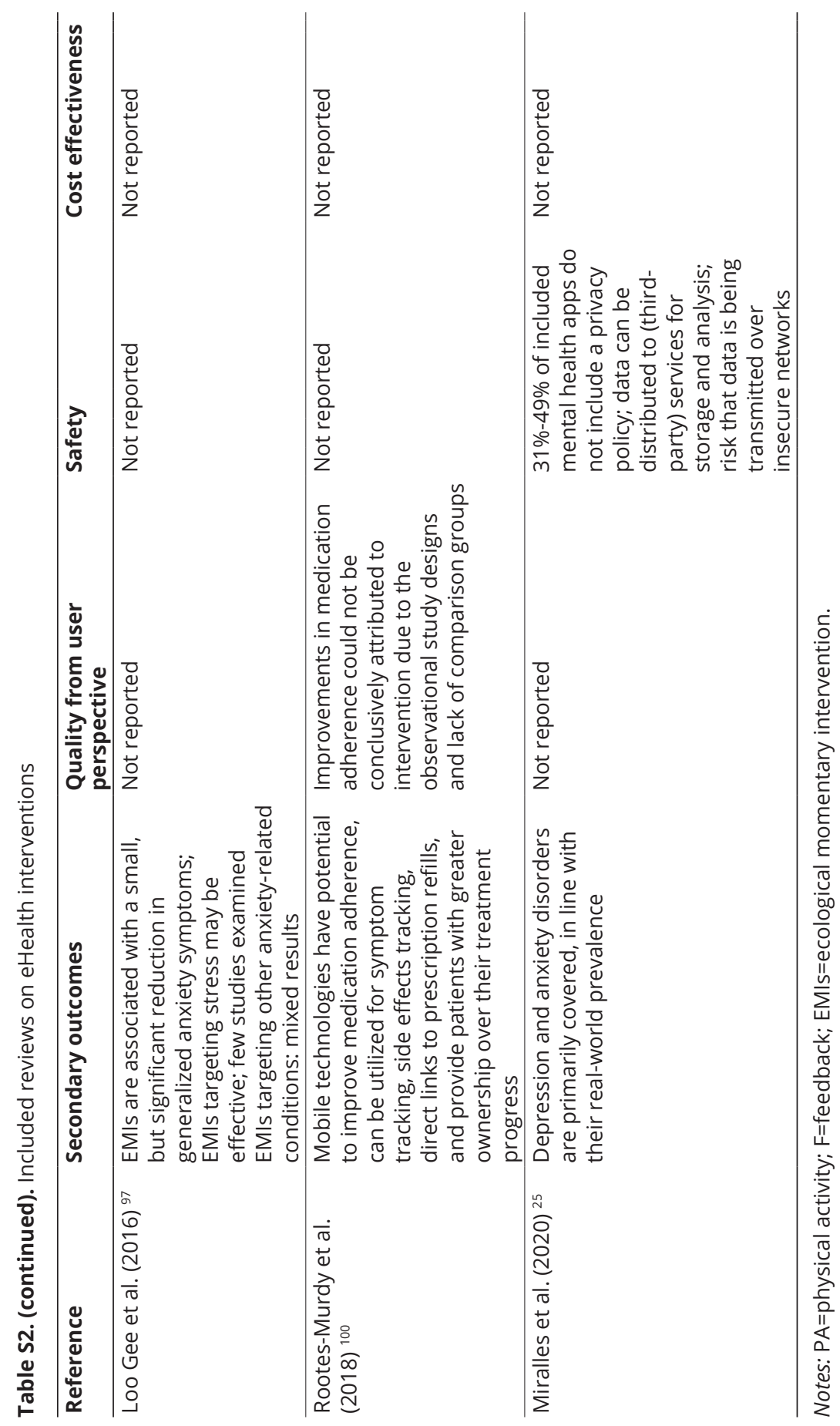




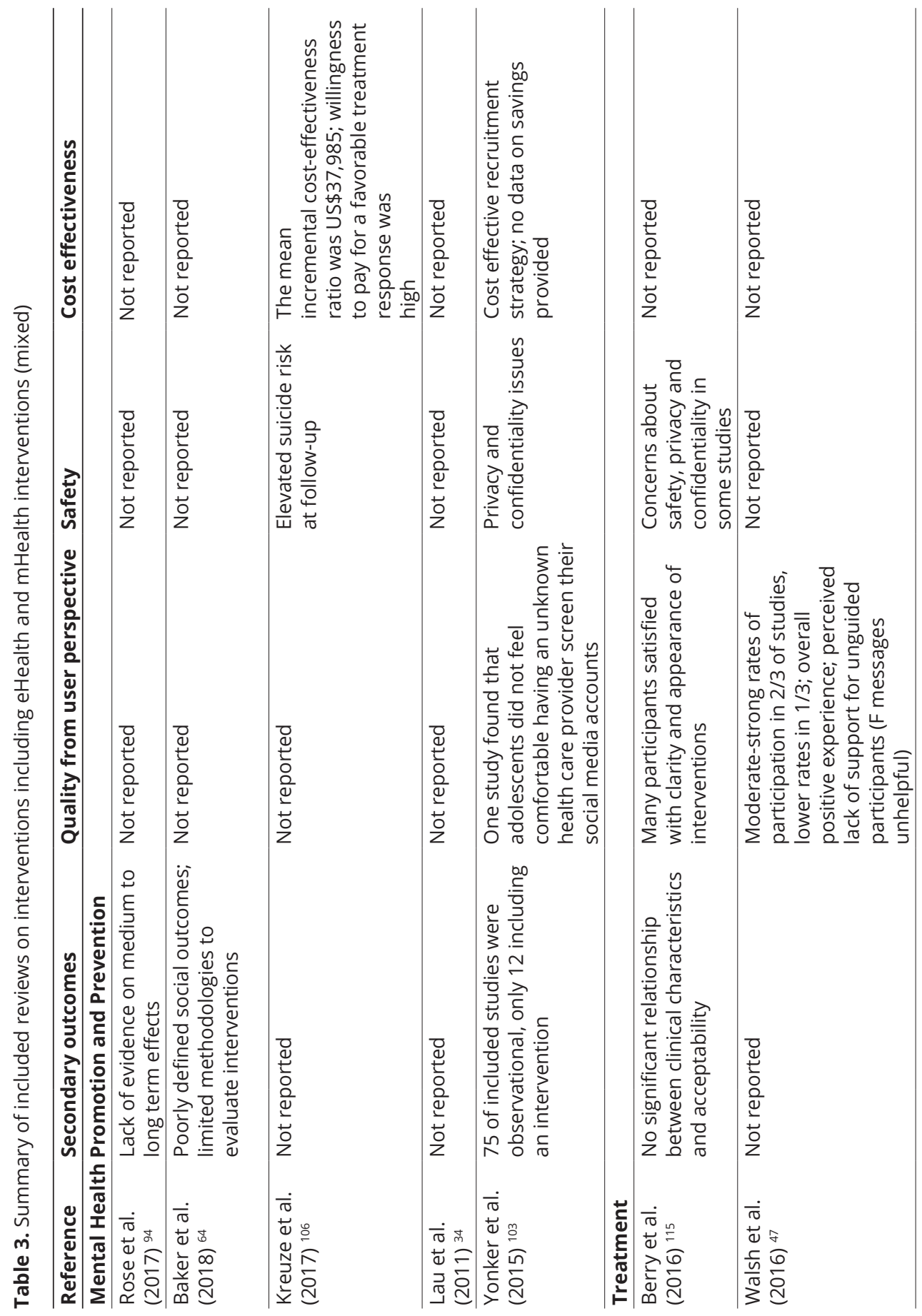




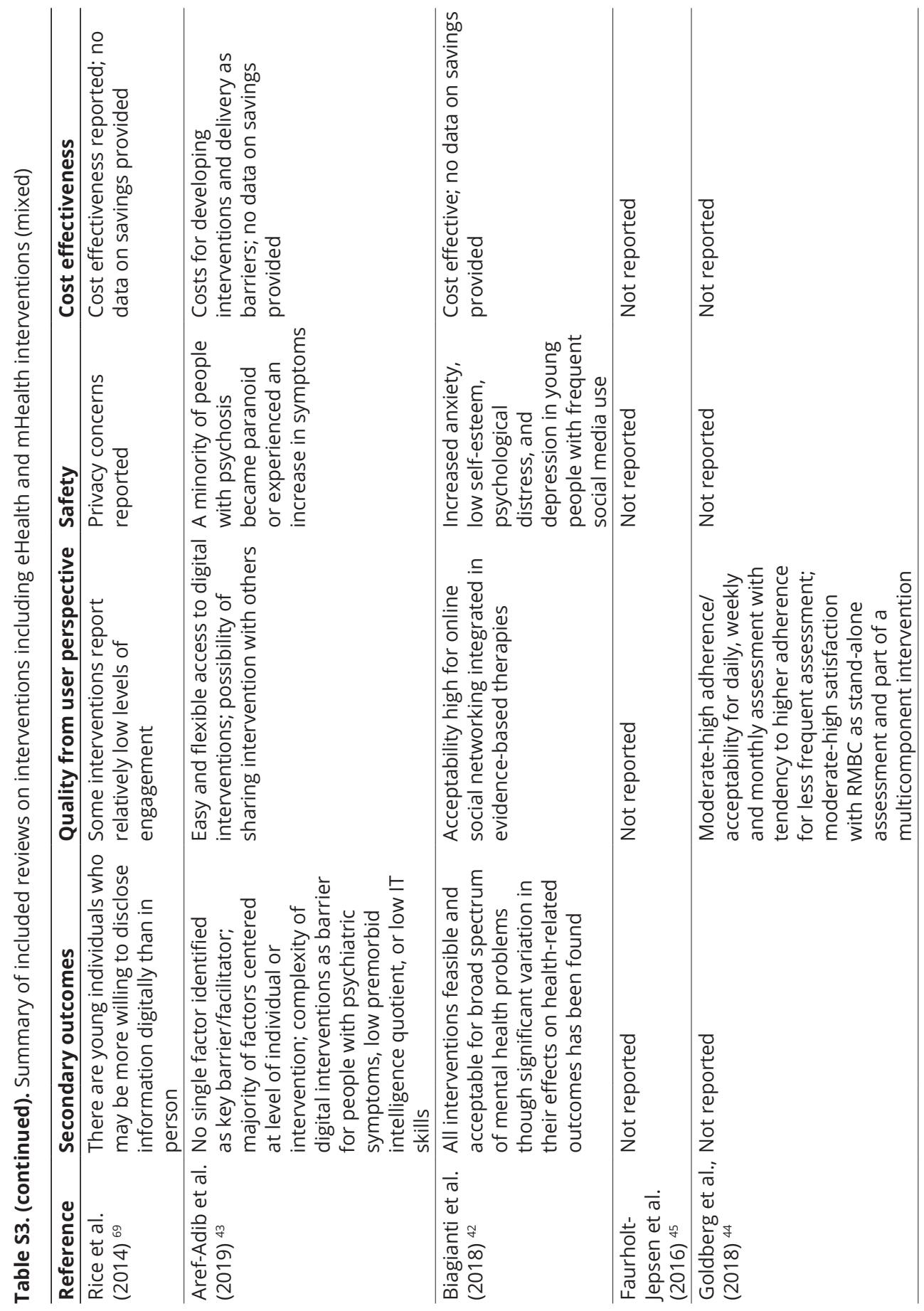




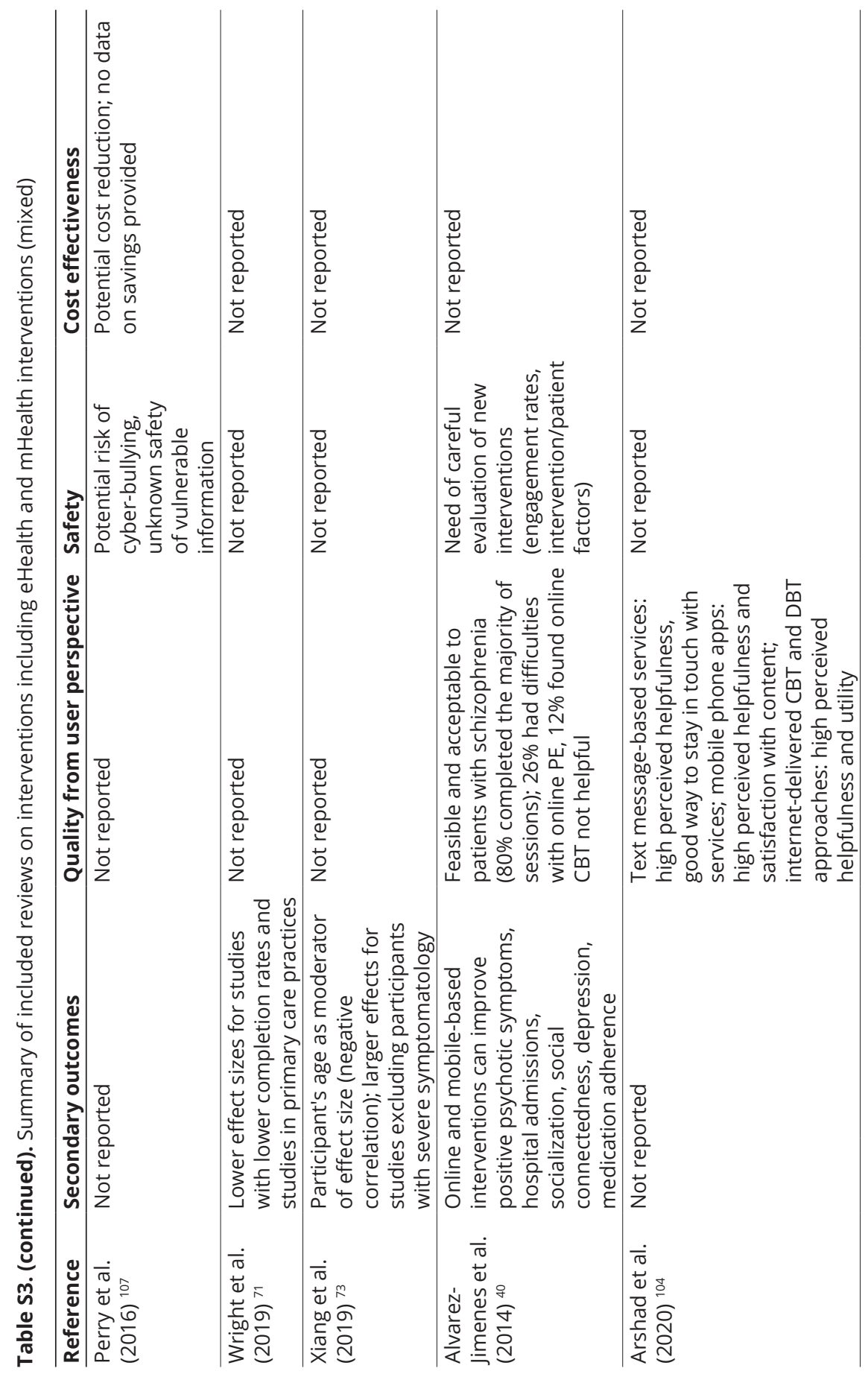




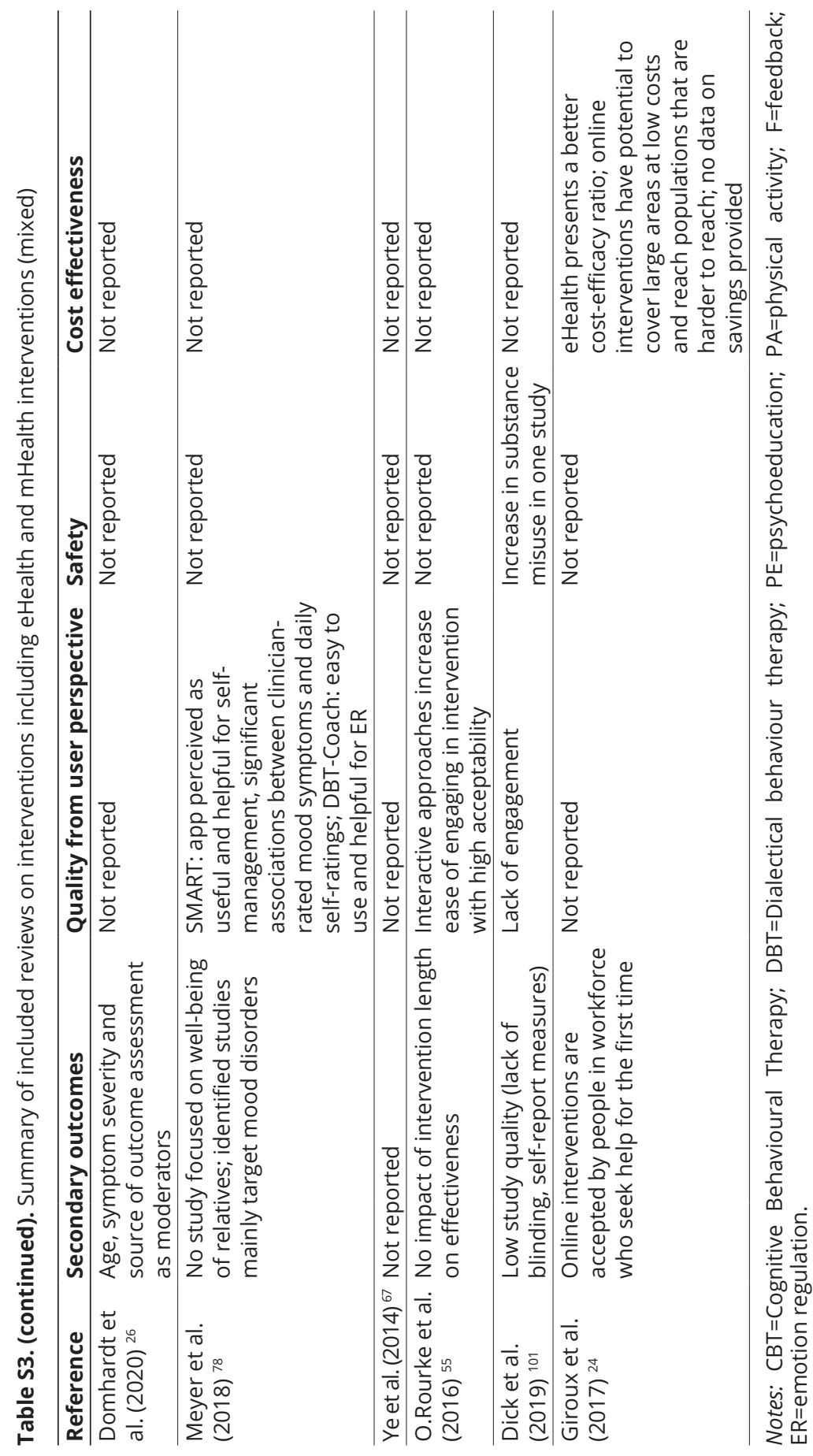



CHAPTER 11

General discussion 
This thesis builds upon the available literature by (1) examining the role of the jumping to conclusions reasoning (JTC) bias and working memory performance (WMP) in the development and persistence of an extended transdiagnostic psychosis phenotype in individuals with co-occurring affective dysregulation and psychotic experiences (PEs) in the general population (Part 1; chapters 2-3), (2) investigating stress sensitivity in daily life as a putative transdiagnostic mechanism involved in linking adverse childhood experiences (ACEs) and mental health outcomes in a sample of young service users with depression, anxiety, and psychosis using an experience sampling study design (Part 1; chapters 4-6), (3) exploring new avenues for digital interventions, such as smartphone-based ecological momentary interventions (EMIs) and other mobile health (mHealth) intervention, internet-based (eHealth) interventions, and virtual-reality (VR) based interventions, in diverse settings across the whole spectrum of public mental health (Part 2; chapters 7-8), (4) investigating the effects of the COVID-19 pandemic on youth mental health as well as young individuals' use of, and attitude towards, digital interventions in a nationally representative sample, and (5) examining the potential use of existing digital intervention for mitigating negative consequences of the COVID-19 pandemic (Part 2; chapters 9-10).

This concluding chapter will be centered around the aims of this thesis specified in the introduction and a critical appraisal of all chapters' principal findings and methodological considerations. This will be followed by a broader discussion on future directions and potential clinical implications in light of existing and newly generated evidence.

\section{Cognitive factors and the extended transdiagnostic psychosis phenotype}

There has been an increasing interest in investigating transdiagnostic phenotypes as evidence suggests that many mental disorders, including psychosis spectrum and affective disorders, are considerably overlapping on a genetic, biological, and symptom level. However, whether risk and resilience factors that have been proposed to be involved in the development and maintenance of specific psychopathological domains in various aetiological models generalise to transdiagnostic phenotypes remained largely unknown. 
The aim of chapter 2 was to investigate whether well-established cognitive risk factors proposed in contemporary models of psychosis [1-3], that is, the JTC bias and lowered WMP, are associated with a transdiagnostic phenotype of cooccurring affective disorders and PEs in the general population. In line with a priori defined hypotheses, we found that the JTC bias was more likely to occur in individuals with co-occurring affective dysregulation and PEs. This suggests, for the first time, that the role of the JTC bias extends to transdiagnostic phenotypes if they are accompanied by PEs. Second, a lower WMP was found in individuals who reported any mental health problems, including the sole presence, or cooccurrence, of affective dysregulation and PEs. Third, there was some evidence of dose-response relationships, as the JTC bias and decreased WMP were more likely to be present in individuals with affective dysregulation as the level of PEs increased or psychosis-related help-seeking behaviour was reported, although some inconsistencies were observed. Overall, these findings support previous evidence on the specificity of the JTC bias in subclinical and clinical expressions of psychosis which appears to be independent from the presence or absence of other co-occurring mental health problems. Moreover, as has been shown in previous studies, lowered WMP has been found to be a more broadly distributed cognitive risk factor across various psychopathological domains.

In chapter 3, we sought to investigate whether the JTC bias contributes to psychosis progression and persistence over time, building on cross-sectional associations demonstrated in chapter 2. In accordance with recent aetiological models [1, 2], we found some evidence that individuals with low levels of PEs (i.e., conceptualized to represent a state of aberrant salience) were more likely to progress to high levels of psychosis (i.e., conceptualized to represent frank psychosis) over a period of around 3 years if the JTC bias was present. We also found that the JTC bias may not only be associated with the progression, but also the persistence of high levels of psychosis over time. This suggests, to some degree, that the JTC bias may contribute to the progression and persistence of psychosis in individuals with a transdiagnostic psychosis phenotype. It is quite unlikely, however, that the presence of the JTC bias does inevitably lead to higher levels of psychosis in individuals with affective dysregulation, given the high prevalence of the JTC bias in clinical and general population samples. It is more likely that the JTC bias combines with, or elevates the risk of, other biopsychosocial cognitive and socioenvironmental risk factors, including belief inflexibility, negative attribution bias, 
bias against disconfirmatory evidence, maladaptive cognitive schemas, prior exposure to adversity, and high polygenetic risk [2, 4, 5]. Although speculative, the JTC bias may contribute to the development of full-threshold paranoia by influencing individuals' immediate response to ambiguous or neutral stimuli in the environment (e.g., a passing car), making it more likely to be interpreted as dangerous if pre-existing vulnerabilities and tendencies to perceive the world as threatening exist. In other words, the tendency to make hasty decisions based on insufficient information in a standardized cognitive test (e.g., the beads task) may manifest in real-world settings by influencing how external stimuli are interpreted, and, when combined with malfunctional schemas, biases, and other biopsychosocial risk factors, may contribute to transitional processes from subclinical to clinical severity. Although intriguing, these propositions have never been tested, and additional research is needed to elucidate the role of JTC bias in the development and persistence of psychosis spectrum disorder.

There are important methodological considerations that must be taken into account. First, significance levels remained below conventional alpha for some of the tested hypotheses. In particular, the prospective modelling applied in chapter 3 led to low statistical power and imprecise estimates. Thus, the results should be interpreted with caution and replication is strongly needed before firm conclusions on the role of the JTC bias on psychosis progression and persistence can be drawn. Additionally, there were conceptual limitations regarding the transdiagnostic psychosis phenotype, states of aberrant salience, and frank psychosis. A more sophisticated methodological approach would be desirable, which may include the use of recently proposed dimensional models $[6,7]$ or psychosis spectra [8]. Recent efforts have been made to develop novel quantitative classification frameworks that are more data-driven and are specifically based on patterns of symptom co-occurrence (e.g., the Hierarchical Taxonomy Of Psychopathology; НіTOP $[9,10])$.

\section{Stress sensitivity as a candidate mechanism linking adversity and youth mental health}

In the chapters 4,5, and 6 we have investigated whether exposure to ACEs, including childhood trauma, bullying victimisation, and negative life events, amplifies individuals' sensitivity to stress in a sample of young help-seeking 
individuals with high levels of depressive, anxiety and psychotic symptoms, their biological sibling, and comparison subjects. We found consistent evidence that service users exposed to high levels of various types of ACEs experienced more intense negative affect and psychotic experiences in response to stress compared to those with low exposure levels. Thus, young help-seeking individuals were found to be more sensitive towards minor stressors in daily life if they were exposed to ACEs. Contrary to these findings, controls showed less intense negative affect or no differences in stress sensitivity by levels of exposure to ACEs in some of the tested comparisons, while findings in biological siblings remained inconclusive. This suggests, as demonstrated in samples of help-seeking adults [11-13], that stress sensitivity may constitute a putative risk and resilience mechanism linking ACEs and youth mental health. Thus, targeting individuals' stress sensitivity may be a promising novel intervention strategy in youth with the goal of interrupting the process of stress sensitization and thereby alleviating individuals' mental health burden and enhancing emotional resilience in daily life.

There are several methodological limitations that must be considered when interpreting these chapters of the thesis. Most importantly, the effects of ACEs on stress sensitivity were examined independently. Given the high prevalence of poly-victimization $[14,15]$, an important next step is to determine whether ACEs have an accumulating effect in modifying stress sensitivity when the number of exposures increases. Furthermore, due to the study design, we were unable to examine whether stress sensitivity contributes to poor mental health outcomes over time [16]. There are also a number of limitations to retrospectively assessing ACEs, including recall bias [17]. Thus, there is an urgent need to conduct additional research on putative underlying mechanisms that influence mental health outcomes in youth daily lives, outside of the research laboratory, with a high degree of ecological validity.

\section{From candidate mechanism to novel treatment targets}

In chapter 7, we aimed to investigate the feasibility, safety, and preliminary therapeutic effects of a transdiagnostic, ecological momentary, compassionfocused intervention for enhancing emotional resilience to stress ('EMIcompass') in a phase I pilot study in young help-seeking individuals with psychotic, depressive, 
and/or anxiety symptoms. Our findings provide evidence on the feasibility and safety of the EMIcompass intervention in help-seeking adolescents as well as initial effects on stress sensitivity and various psychopathological domains. More specifically, as hypothesized, we found some evidence that an EMI may be wellsuited to target candidate mechanisms in daily life and that delivering compassionfocused intervention components to help-seeking youth via a smartphone is feasible and safe.

This study is among the first to develop and pilot an EMI in youth with mental health problems that actively incorporates an adaptive and context-sensitive delivery scheme for intervention components [18]. Interestingly, approximately a third of all EMA assessments in daily life triggered the delivery of 'interactive tasks'. This type of task was only offered if individuals experienced elevated levels of negative affect (e.g., feeling anxious, insecure, or down), threat anticipation, or momentary stress. Thus, these findings suggest that young help-seeking individuals frequently encounter difficult moments throughout the day and that real-time processing of EMA data may successfully be used to determine when intervention components are most needed. This could be a significant step toward developing more ecologically valid and accessible psychological interventions for youth, as well as more individualized contextualized clinical and preventive care $[18,19]$.

However, these findings must be interpreted within the context of methodological limitations, including the pilot study's small sample size $(N=10)$, the absence of a waiting list or active control group, and the fact that some of the findings on initial therapeutic effects on psychopathological domains remained below conventional alpha. A feasibility RCT is now required, and is currently underway [20], to ascertain the interventions' efficacy and feasibility, and to lay the groundwork for a confirmatory RCT.

\section{Digital intervention in public mental health provision}

The rapidly growing body of evidence on the use of digital technologies in the treatment of individuals suffering from mental health conditions, as well as areas of mental health promotion and prevention, is remarkable. In chapters 8, 9, and 10 , we examined these recent developments from a variety of perspectives. First, 
we narratively reviewed the available evidence and clinical potential of emerging digital technologies (i.e., mHealth, eHealth, and VR intervention delivered via smartphones, wearables, and head-mounted displays) for helping individuals experiencing subclinical expressions of psychosis as well as psychosis spectrum disorder. Second, we examined whether public health measures to reduce SARSCoV-2 infection rates had a negative effect on youth mental health in a general population sample and whether there was a subjective demand for using digital tools to improve mental health during the COVID-19 pandemic. Finally, we conducted a rapid meta-review to investigate the theoretical and empirical foundations, user perspectives, safety, effectiveness, and cost-effectiveness of digital interventions in public mental health provision (i.e., mental health promotion, prevention, and treatment of mental disorders) that may aid in mitigating the negative psychosocial consequences of the COVID-19 pandemic.

Overall, the evidence reported in chapters 8 and 10 suggests high acceptability and feasibility of eHealth, mHealth, and VR interventions in areas of public mental health provision. There was convincing evidence for the effectiveness of telemedical and other eHealth interventions in promoting and preventing mental health problems, as well as in treating mental health conditions, including psychosis spectrum disorder. There was, in contrast, preliminary, but very promising, evidence on the effectiveness of mHealth interventions [21]. More generally, the effectiveness of digital interventions utilizing a blended-care approach was found to be superior as compared to stand-alone digital interventions, especially in individuals with more severe mental health problems $[22,23]$ and the evidence for long-term effects and noninferiority to standard therapy and active control conditions have been found to be limited. Similarly, there is a paucity of evidence regarding the underlying processes and mechanisms of change, which largely parallels findings in other areas of intervention research. The theoretical and empirical foundations for most eHealth, mHealth, and VR interventions that have been developed by research groups are explicitly stated, whereas popular mHealth apps available in major app stores (e.g., Google Play Store, Apple App Store) as well as eHealth platforms do frequently not disclose the theoretical or empirical foundations and evidencebase of their content, while those who do only cite uncontrolled pilot studies. The publicly available apps have also been found to employ questionable data sharing and privacy practices for monetarizing user data [24, 25]. 
Most digital interventions that have been developed to date were found to primarily focus on four overarching domains of technology enabled mental health services $[18,26]$, that is, (1) remote communication, continuity of care, and flexibility through online chat and video call (i.e., telehealth); (2) monitoring of symptoms and behaviours in daily life; (3) personalized feedback on subjective experience and behavioural patterns as well as (4) static (e.g., on-demand access to psychoeducational modules) and adaptive interventions (e.g., EMIs) that apply real-time processing of data to inform the delivery of intervention components in daily life.

In chapter 9, we investigated whether public health measures implemented during the COVID-19 pandemic, such as physical distancing and quarantine, had a negative effect on youth mental health and young individuals' use of, and attitude towards, digital interventions during this unprecedented public health crisis. We have found strong evidence that social isolation, COVID-19-related cognitive preoccupation, worries, and anxiety were associated with psychological distress in youth. There was also evidence of dose-response relationships for some of these associations, as psychological distress was found to be more likely as reported social isolation and COVID-19-related preoccupation, anxiety, and worrying increased - although some inconsistencies were observed.

In line with hypotheses, there was also some evidence that psychological distress and high levels of COVID-19-related cognitive preoccupation, worries, and anxiety were associated with a more favourable attitude toward, and the current use of, mHealth apps to assist in overcoming the pandemic's negative consequences. This suggests that there is an objective need and subjective demand for digital intervention during public health crises, and that young people already use digital tools to work on their mental and physical health.

There are various methodological considerations which are important to be considered when interpreting this thesis' findings on digital interventions. First, in chapter 8 and 10, we conducted a narrative review and rapid meta-review, respectively. As a result, the representativeness of identified studies may be limited, as standard procedures for conducting systematic-reviews and meta-analyses were not followed (e.g., two independent reviewers who are blinded to each other's decisions). Second, in chapter 9, a questionnaire to assess psychological distress was used (i.e., Kessler-10) which has been developed as a brief screening tool to 
identify levels of distress. Thus, no validated semi-structured clinical interview was used to determine individual's subclinical and clinical symptoms. Further, the findings were based on an online panel which consisted of a group of registered internet users who had agreed to take part in surveys. Thus, selection bias cannot be ruled out. Further, the cross-sectional design of the survey did not allow us to compare findings on psychological distress and other variables with times before the COVID-19 pandemic.

\section{Concluding remarks}

There has been a recent shift towards a more dimensional understanding of mental disorders, crossing traditional diagnostic boundaries. This includes studying concepts of mental ill-health continua, spectra, and transdiagnostic phenotypes, as well as identifying transdiagnostic candidate mechanisms affecting mental health in everyday life, outside the research laboratory, and developing, evaluating, and implementing transdiagnostic intervention strategies. This thesis contributes to the body of knowledge by taking a transdiagnostic approach to mental health and linking adversity, cognition, candidate mechanisms, and novel digital intervention.

Taken together, the findings presented in this thesis suggest that stress sensitivity may be a critical transdiagnostic risk or resilience mechanism linking ACEs and youth mental health. While primary prevention of ACEs, including childhood trauma, bullying victimization, and negative life events, remains the most important goal, there is an urgent need to carefully assess ACEs in youth mental health services and to continue strengthening the evidence on their impact on youth mental health (e.g., their cumulative and long-term effects via stress sensitivity) as a basis for developing and evaluating evidence-based treatments in order to tackle their negative consequences. Building on these findings, we demonstrated that translating compassion-focused intervention components into individuals' daily life through an EMI delivered by an mHealth app may be a promising novel, accessible, and transdiagnostic treatment approach in help-seeking youth by strengthening emotional resilience and directly targeting candidate mechanisms in daily life. Our findings also underscore the critical need for additional research into the extent to which risk and resilience factors associated with the development and persistence of specific psychopathological domains generalise to transdiagnostic phenotypes to improve contemporary etiological models [27]. 
The use of digital mental health services in routine mental health care as well as mental health promotion and prevention of mental disorders is still in its infancy. There is a need to conduct well-powered randomized controlled trials to further investigate their efficacy, long-term effects, and processes and mechanisms of change before they can be recommended for widespread use in routine clinical care [21]. Additionally, it has become increasingly clear that early adoption of co-design and co-production principles is critical for meeting the unique needs and preferences of users and mental health professionals [21, 28, 29]. However, findings indicate that digital interventions have the potential to bring personcentered and adaptive interventions into individuals' daily lives, enabling the ecological translation of evidence-based interventions across areas of public mental health provision $[18,21]$. There is also a strong need to conduct systematic evaluations of currently available digital interventions in major app stores using established criteria of mental health services research. There have been global initiatives (e.g., the National Health Service Apps Library in the United Kingdom; the Platform for Digital Health Applications in Germany; and the Division of Digital Psychiatry's App Evaluation Database in the USA) with the goal of evaluating and making evidence-based digital interventions clinically and publicly available, as well as integrating them into existing healthcare systems. These efforts must be accelerated in the coming years to enable individuals with a range of needs and preferences to access the most appropriate evidence-based digital mental health service. 


\section{References}

1. Howes, O.D. and R.M. Murray, Schizophrenia: an integrated sociodevelopmentalcognitive model. Lancet, 2014. 383(9929): p. 1677-1687.

2. Freeman, D., Persecutory delusions: a cognitive perspective on understanding and treatment. Lancet Psychiatry, 2016. 3(7): p. 685-92.

3. Freeman, D., Improving cognitive treatments for delusions. Schizophr Res, 2011. 132(23): p. 135-9.

4. Ward, T. and P.A. Garety, Fast and slow thinking in distressing delusions: A review of the literature and implications for targeted therapy. Schizophr Res, 2019. 203: p. 80-87.

5. Garety, P.A., et al., A cognitive model of the positive symptoms of psychosis. Psychol Med, 2001. 31(2): p. 189-95.

6. Reininghaus, U., et al., Evaluation of the validity and utility of a transdiagnostic psychosis dimension encompassing schizophrenia and bipolar disorder. Br J Psychiatry, 2016. 209(2): p. 107-13.

7. Quattrone, D., et al., Transdiagnostic dimensions of psychopathology at first episode psychosis: findings from the multinational EU-GEl study. Psychol Med, 2019. 49(8): p. 1378-1391.

8. Kotov, R., et al., Validity and utility of Hierarchical Taxonomy of Psychopathology (HiTOP): I. Psychosis superspectrum. World Psychiatry, 2020. 19(2): p. 151-172.

9. Kotov, R., et al., The Hierarchical Taxonomy of Psychopathology (HiTOP): A dimensional alternative to traditional nosologies. J Abnorm Psychol, 2017. 126(4): p. 454-477.

10. Ruggero, C.J., et al., Integrating the Hierarchical Taxonomy of Psychopathology (HiTOP) into clinical practice. J Consult Clin Psychol, 2019. 87(12): p. 1069-1084.

11. Wichers, M., et al., Mechanisms of gene-environment interactions in depression: evidence that genes potentiate multiple sources of adversity. Psychol Med, 2009. 39(7): p. 1077-86.

12. Lardinois, M., et al., Childhood trauma and increased stress sensitivity in psychosis. Acta Psychiatr Scand, 2011. 123(1): p. 28-35.

13. Reininghaus, U., et al., Psychological processes underlying the association between childhood trauma and psychosis in daily life: an experience sampling study. Psychol Med, 2016. 46(13): p. 2799-813.

14. Finkelhor, D., R.K. Ormrod, and H.A. Turner, Poly-victimization: a neglected component in child victimization. Child Abuse Negl, 2007. 31(1): p. 7-26.

15. Turner, H.A., D. Finkelhor, and R. Ormrod, Poly-victimization in a national sample of children and youth. Am J Prev Med, 2010. 38(3): p. 323-30.

16. Collip, D., I. Myin-Germeys, and J. Van Os, Does the concept of "sensitization" provide a plausible mechanism for the putative link between the environment and schizophrenia? Schizophr Bull, 2008. 34(2): p. 220-5.

17. Baldwin, J.R., et al., Agreement Between Prospective and Retrospective Measures of Childhood Maltreatment: A Systematic Review and Meta-analysis. JAMA Psychiatry, 2019. 76(6): p. 584-593.

18. Reininghaus, U., [Ecological Momentary Interventions in Psychiatry: The Momentum for Change in Daily Social Context]. Psychiatr Prax, 2018. 45(2): p. 59-61. 
19. Reininghaus, U., C.A. Depp, and I. Myin-Germeys, Ecological Interventionist Causal Models in Psychosis: Targeting Psychological Mechanisms in Daily Life. Schizophr Bull, 2016. 42(2): p. 264-9.

20. Schick, A., et al., The effects of a novel, accessible, transdiagnostic ecological momentary intervention for improving resilience in youth (EMIcompass): study protocol for a randomized controlled trial. 2020: PsyArXiv.

21. Reininghaus, U. and C. Rauschenberg, Digitale Public Mental Health Ansätze zur Verminderung der psychosozialen Folgen der COVID-19 Pandemie. 2020: Policy Brief, COVID-19 Public Health Network.

22. Fairburn, C.G. and V. Patel, The impact of digital technology on psychological treatments and their dissemination. Behav Res Ther, 2017. 88: p. 19-25.

23. Erbe, D., et al., Blending Face-to-Face and Internet-Based Interventions for the Treatment of Mental Disorders in Adults: Systematic Review. J Med Internet Res, 2017. 19(9): p. e306.

24. Larsen, M.E., et al., Using science to sell apps: Evaluation of mental health app store quality claims. NPJ Digit Med, 2019. 2: p. 18.

25. Minen, M.T., et al., The Functionality, Evidence, and Privacy Issues Around Smartphone Apps for the Top Neuropsychiatric Conditions. J Neuropsychiatry Clin Neurosci, 2021. 33(1): p. 72-79.

26. Rauschenberg, C., et al., [Digital forms of service delivery for personalized crisis resolution and home treatment]. Nervenarzt, 2021.

27. Reininghaus, $U$. and C. Morgan, Integrated models in psychiatry: the state of the art. Soc Psychiatry Psychiatr Epidemiol, 2014. 49(1): p. 1-2.

28. Bucci, S., M. Schwannauer, and N. Berry, The digital revolution and its impact on mental health care. Psychol Psychother, 2019. 92(2): p. 277-297.

29. Hardy, A., et al., How Inclusive, User-Centered Design Research Can Improve Psychological Therapies for Psychosis: Development of SlowMo. JMIR Ment Health, 2018. 5(4): p. e11222. 

CHAPTER 12

Summary 
The current thesis adopted a transdiagnostic approach to mental health, with the objectives of (1) examining the role of the jumping to conclusions reasoning (JTC) bias and working memory performance (WMP) in the development and persistence of an extended transdiagnostic psychosis phenotype, (2) determining whether individuals' stress sensitivity in daily life may form a transdiagnostic candidate mechanism involved in linking adverse childhood experiences (ACEs) and mental health outcomes in young service users, (3) exploring new avenues for digital interventions, such as smartphone-based ecological momentary interventions (EMIs) and other mobile health (mHealth) intervention, internet-based (eHealth) interventions, and virtual-reality (VR) based interventions, in diverse clinical and non-clinical settings across the whole spectrum of public mental health provision, (4) examining the effects of the COVID-19 pandemic on youth mental health as well as young individuals' use of, and attitude towards, digital interventions, and (5) investigating the potential use of available digital intervention for mitigating negative consequences of the COVID-19 pandemic in youth.

In chapter $\mathbf{2}$ we investigated whether well-established cognitive risk factors, such as the JTC bias and a decreased WMP, are associated with a transdiagnostic phenotype of co-occurring affective disorders and psychotic experiences (PEs) in the general population. We found that the JTC bias was more likely to occur in individuals with co-occurring affective dysregulation and PEs. There was also some evidence of dose-response relationships, as the JTC bias and decreased WMP were more likely to be present in individuals with affective dysregulation when levels of PEs increased or psychosis-related help-seeking behavior was reported. These findings corroborate previous research demonstrating the JTC bias's specificity in subclinical and clinical manifestations of psychosis, which appears to be independent of the presence or absence of other co-occurring mental health problems. Chapter 3 builds on these findings and examined whether the JTC bias contributes to the progression and persistence of psychosis over time. We found some evidence that individuals with low levels of PEs were more likely to progress to high levels of psychosis over a three-year period if the JTC bias was present. Additionally, we found that the JTC bias may be associated with not only the progression of psychosis, but also with the persistence of high levels of psychosis over time. This suggests that the JTC bias may play a role in the progression and persistence of psychosis in individuals with a transdiagnostic psychosis phenotype. 
In chapters 4, 5, and 6, we examined whether exposure to ACEs, such as childhood trauma, bullying victimization, and negative life events, increases individuals' sensitivity to stress in a sample of young help-seeking individuals with high levels of depressive, anxiety, and psychotic symptoms, as well as their biological sibling and comparison subjects. We found consistent evidence that service users exposed to high levels of various ACEs had more intense negative affect and psychotic experiences in response to stress than those exposed to low levels. Thus, when exposed to ACEs, young help-seeking individuals were found to be more sensitive to minor stressors in daily life. In contrast to these findings, controls demonstrated less intense negative affect or no differences in stress sensitivity when ACE exposure levels were compared, while findings in biological siblings remained inconclusive. This suggests that stress sensitivity may serve as a putative risk and resilience mechanism linking ACEs and poor mental health in youth.

Chapter 7 explored the feasibility, safety, and preliminary therapeutic effects of a transdiagnostic, ecological momentary, compassion-focused intervention for enhancing emotional resilience to stress ('EMlcompass') in a phase I pilot study with young help-seeking individuals experiencing psychotic, depressive, or anxiety symptoms. The findings suggest EMIcompass intervention's feasibility and safety in help-seeking adolescents, as well as its initial effects on stress sensitivity and a variety of psychopathological domains. There was, therefore, some evidence that an EMI may be well-suited to directly target candidate mechanisms in daily life.

The body of evidence on the use of digital technologies to assist people with mental illnesses, as well as in areas of mental health promotion and prevention, is remarkable. We studied these recent developments from a number of angles in chapters $\mathbf{8}, \mathbf{9}$, and $\mathbf{1 0}$. To begin, in chapter $\mathbf{8}$, we summarized the available evidence and clinical potential of emerging digital technologies (i.e., mobile health, eHealth, and virtual reality interventions delivered via smartphones, wearables, and head-mounted displays) for supporting individuals experiencing subclinical manifestations of psychosis as well as psychosis spectrum disorder. Second, in chapter 9, we investigated if public health measures to lower SARS-CoV-2 infection rates had a detrimental effect on youth mental health in a general population sample and whether there was a subjective need for using digital technologies to promote mental health during the COVID-19 pandemic. Finally, in chapter 
10, we conducted a rapid meta-review to examine the theoretical and empirical foundations, user perspectives, safety, effectiveness, and cost-effectiveness of digital interventions in public mental health provision that may help mitigate the negative psychosocial consequences of the COVID-19 pandemic.

Overall, the findings described in chapters 8 and 10 indicate that eHealth, mHealth, and VR interventions in the domains of public mental health provision are feasible and accepted by most users. Telemedical and other eHealth treatments were found to be beneficial in promoting and preventing mental health problems, as well as treating mental health conditions. In contrast, there was preliminary, but highly encouraging, evidence on the efficacy of mHealth interventions. Furthermore, the effectiveness of hybrid digital interventions was found to be superior to stand-alone interventions, especially in people with more severe mental health problems, and the evidence for long-term effects and non-inferiority to standard therapy and active control conditions was found to be limited.

In chapter 9, we found evidence that social isolation, cognitive preoccupation, fears, and worry were all associated with psychological distress in adolescents during the COVID-19 pandemic. There was also evidence of dose-response relationships for some of these associations, with psychological distress becoming more likely as reported social isolation and COVID-19-related preoccupation, anxiety, and worrying increased. Additionally, there was some evidence that psychological distress and high levels of cognitive preoccupation, and worry about COVID-19 were associated with a more favorable attitude towards, and use of, mHealth apps. This indicates, to some degree, that there was an objective need and subjective demand for digital interventions during a public health crisis, and that young people are already utilizing digital technologies to manage their mental and physical health. 
sumany

${ }_{417}$ 



\title{
APPENDIX
}

\author{
Impact paragraph \\ Curriculum Vitae \\ List of publications \\ Acknowledgements
}




\section{Impact paragraph}

In this section, the scientific and societal impact of the research presented in this thesis will be discussed.

\section{Scientific impact}

Despite extensive research on mental health, significant individual suffering, and high societal costs, treatment effectiveness has remained relatively stable in recent years, especially for severe mental disorders [1]. These findings contrast sharply with significant advances in other medical disciplines and a wide variety of somatic diseases, where treatment options and effectiveness have significantly improved. Some of the current difficulties in mental health research in finding better treatment options may partly be explained by fundamental difficulties in applying the dominant traditional medical model to mental health conditions. The long-held belief that mental disorders are distinct disease entities with unambiguous phenotypic representation and biological correlates as well as disease-specific mechanisms and risk factors is increasingly being challenged by mounting evidence indicating a high degree of overlap between mental disorders on the genetic [2], neuroscientific [3], and behavioural level [4]. This implies that studying mental disorders in isolation introduces inherent problems that may obstruct significant progress towards developing novel therapeutic approaches as well as understanding important determinants of poor mental health and underlying mechanisms.

The primary objective of this thesis was to take a transdiagnostic approach to mental health by examining how cognitive factors, adverse childhood experiences, and candidate mechanisms contribute to an increased risk for developing transdiagnostic phenotypes of depression, anxiety, and psychosis. The thesis also sought to determine the extent to which digital interventions have the potential to target transdiagnostic candidate mechanisms and outcomes, as well as to alleviate mental health burden in various areas of public mental health provision (i.e., mental health promotion, prevention of, and treatment for mental disorder).

We found that the jumping to conclusions (JTC) reasoning bias - the most widely studied cognitive bias in psychosis - was more likely to occur and associated with an increased risk for psychosis progression and persistence in individuals with a transdiagnostic phenotype of co-occurring affective dysregulation and 
psychotic experiences in a large prospective cohort study. This suggests, for the first time, that the JTC bias extends to transdiagnostic phenotypes. Moreover, stress sensitivity has been found to constitute a putative transdiagnostic risk and resilience mechanism linking adverse childhood experiences and mental health in youth at a developmental early stage of psychopathology using an ecologically valid experience sampling study design. We have also demonstrated that a compassionfocused ecological momentary intervention may be effective in directly targeting candidate mechanisms, including stress sensitivity, and strengthening resilience in young help-seeking service users. Finally, digital interventions have been found to increasingly being used, and hold great promise for, mental health promotion and the prevention and treatment of mental disorders.

These findings emphasize the importance of conducting additional research to determine whether psychological processes and mechanisms involved in the development and maintenance of psychopathologies extend to transdiagnostic phenotypes in order to overcome current limitations in mental health research and to corroborate contemporary aetiological models (e.g., the integrated sociodevelopmental-cognitive model of psychosis [5]). This may ultimately improve prediction of onset, course, and outcome, and to help develop and implement more effective and person-tailored interventions. Studies that do not exclude but purposefully allow for comorbidities and multidimensional psychopathology are urgently needed to advance progress in research, treatment, and aetiological models as well as dimensional and transdiagnostic approaches to mental health $[6,7]$.

\section{Anticipated societal impact}

"The time will come when diligent research over long periods will bring to light things which now lie hidden. A single lifetime, even though entirely devoted to the sky, would not be enough for the investigation of so vast a subject (...) And so this knowledge will be unfolded only through long successive ages. There will come a time when our descendants will be amazed that we did not know things that are so plain to them (...) Many discoveries are reserved for ages still to come, when memory of us will have been effaced."

Lucius Annaeus Seneca (c. $4 B C-A D$ 65), Naturales quaestiones. 
According to the Global Burden of Disease study, an estimated 792 million people worldwide suffered from a mental disorder in 2017 [8]. This is slightly more than one in every ten people on the planet (10.7 percent). The majority of these individuals suffered from anxiety or depression. When substance use disorders are included, the numbers rise even higher: approximately one-in-seven people (15 percent) worldwide. There is strong evidence that individuals with mental health or substance use disorders are at an increased risk of committing suicide [9] and the immense individual suffering associated with mental illness can hardly be expressed in numbers. The economic cost of psychopathologies has been estimated to be 600 billion euros a year, or more than 4\% of GDP, in the European Union. This figure includes 190 billion euros (1.3 percent of GDP) for direct care, 170 billion euros (1.2 percent) for social security programs, and 260 billion euros (1.6 percent) for indirect public spending on unemployment and decreased productivity among people with mental disorders [10].

These staggeringly high rates of mental disorders and tremendous costs clearly demonstrate the critical need to improve support for people experiencing mental health difficulties, identify important risk factors early, and strengthen the population's resilience. The thesis contributes to a better understanding of how exposure to adversity, which millions of people face on a daily basis, and cognitive factors increase the risk of developing multidimensional mental health problems. Moreover, reported findings on the COVID-19 pandemic indicate that public health measures to reduce infection rates had detrimental effects on youth mental health. This is a significant finding that requires careful consideration when making decisions about how to properly manage this and future pandemics effectively.

The reported findings on the use of digital interventions are encouraging. We demonstrated that digital intervention, mHealth apps in particular, hold great potential to enable the delivery of highly personalized interventions that are tailored to an individual's specific needs in a given moment and context. They are especially well-suited for improving public mental health because they are easily scaled up and the devices required to deliver evidence-based interventions in daily life are readily available to most people. Additionally, the thesis may aid in a better understanding of the current state of evidence-based mental health services and in assisting decision makers in developing and implementing digital strategies during public health crises. 
The research topics covered in this thesis have the potential to enhance the reputation and impact of the regional academic community in the field of mental health. The findings from the studies presented in this thesis have been featured in news outlets, and they may help service users, the public, and policymakers make more informed decisions about the use of technology to enable mental health services, as well as contribute to a broader discussion about their potential and limitations.

However, as Seneca so beautifully stated, scientific discoveries typically take time to reach their full potential for societal benefit, and the findings presented in this thesis represent yet another step forward in the long process of improving societies' mental health. 


\section{References}

1. Kilbourne, A.M., et al., Measuring and improving the quality of mental health care: a global perspective. World Psychiatry, 2018. 17(1): p. 30-38.

2. Anttila, V., et al., Analysis of shared heritability in common disorders of the brain. Science, 2018. 360(6395).

3. Janiri, D., et al., Shared Neural Phenotypes for Mood and Anxiety Disorders: A Metaanalysis of 226 Task-Related Functional Imaging Studies. JAMA Psychiatry, 2020. 77(2): p. 172-179.

4. van Os, J. and U. Reininghaus, Psychosis as a transdiagnostic and extended phenotype in the general population. World Psychiatry, 2016. 15(2): p. 118-24.

5. Howes, O.D. and R.M. Murray, Schizophrenia: an integrated sociodevelopmentalcognitive model. Lancet, 2014. 383(9929): p. 1677-1687.

6. Reininghaus, U., et al., Evaluation of the validity and utility of a transdiagnostic psychosis dimension encompassing schizophrenia and bipolar disorder. Br J Psychiatry, 2016. 209(2): p. 107-13.

7. van Os, J., et al., The evidence-based group-level symptom-reduction model as the organizing principle for mental health care: time for change? World Psychiatry, 2019. 18(1): p. 88-96.

8. Global Burden of Disease Study 2019, (GBD 2019). Data Input Sources Tool. [cited 2021 01.05.2021]; Available from: http://ghdx.healthdata.org/gbd-2019/data-input-sources.

9. Prince, M., et al., No health without mental health. The Lancet, 2007. 370(9590): p. 859-877.

10. World Health Organization, Health at a Glance: Europe. 2018. 


\section{Curriculum Vitae}

Christian Rauschenberg was born in Bad Nauheim, Germany, on 25 June 1990. After completing his secondary school education (2006) and a three-year training as an administrative assistant, he decided to return to school to complete his high school education. He graduated from the Berta Jourdan School in Frankfurt a.M. (2010) and completed his civilian service at Bürgerhilfe Sozialpsychiatrie Frankfurt e.V., a non-profit

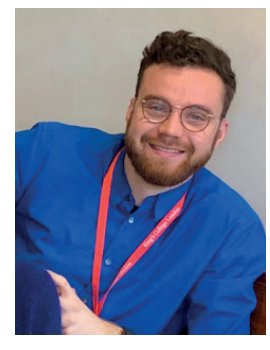
organization that helps people with enduring mental health conditions. He then studied psychology (B.Sc.) at the Justus Liebig University Giessen, Germany, from 2011-2015. As part of his studies, he spent one semester at the Fatih Universitesi in Istanbul. During the time of his undergraduate studies, he gained valuable experiences through a variety of internships (including at the Free University of Berlin and the Bender Institute of Neuroimaging) and positions as a student assistant (including the Departments of Psychotherapy and Systems Neuroscience, Biological Psychology and Developmental Psychology at the University of Giessen). He also served as chairperson of STOFF e.V. from 2012 to 2015, a non-profit organisation dedicated to organizing lectures, workshops, and the acquisition of academic books. After graduating in Psychology, he studied a Research Master in Cognitive and Clinical Neuroscience with the specialisation in Psychopathology at Maastricht University, which he completed in 2017. During this time, he was able to lay the groundwork for his research interests during a research internship at the School for Mental Health and Neuroscience, Department of Psychiatry and Neuropsychology, Maastricht University, which resulted in a subsequent position as PhD Candidate under the supervision of Prof Jim van Os and Prof Ulrich Reininghaus. He spent four months as a Visiting Researcher at King's College London's Virtual Reality Lab, where he worked under the supervision of Dr Lucia Valmaggia. Christian Rauschenberg began working part-time in February 2020 and full-time in May 2021 in the Department of Public Mental Health at the Central Institute of Mental Health, Heidelberg University, where he is conducting research on the use of digital monitoring, feedback, and ecological momentary interventions in areas of public mental health provision. This includes working on the research projects "Living lab AI4U - artificial intelligence for personalized digital mental health promotion in youth" and "DiSERVE@home digital forms of service delivery in crisis resolution and home treatment for people with severe mental health problems". He will continue to work on these projects as a postdoctoral research fellow. 


\section{List of publications}

\section{Scientific publications in international peer-reviewed journals}

Schick, A., Paetzold, I., Rauschenberg, C., Hirjak, D., Banaschewski, T., MeyerLindenberg, A., Boehnke, J. R., Boecking, B., Reininghaus, U. (2021). The effects of a novel, transdiagnostic, hybrid ecological momentary intervention for improving resilience in youth (EMIcompass): study protocol for an exploratory randomized controlled trial. JMIR Research Protocols.

Rauschenberg. C., Boecking, B., Paetzold I., Schruers, K., Schick, A., van Amelsvoort, T., Reininghaus, U. (2021). A Compassion-Focused Ecological Momentary Intervention for Enhancing Resilience in Help-Seeking Youth: Uncontrolled Pilot Study. JMIR Mental Health 8(8): p. e25650.

Rauschenberg, C.*, Schick, A.*, Goetzl, C., Roehr, S., Riedel-Heller, S. G., Koppe, G., Durstewitz, D., Krumm, S. \& Reininghaus, U. (2021). Social isolation, mental health, and use of digital interventions in youth during the COVID-19 pandemic: A nationally representative survey. European Psychiatry 64, e20.

Rauschenberg, C. ${ }^{*}$, Hirjak, D. *, Ganslandt, T., Schulte-Strathaus, J. C. C., Schick, A., Meyer Lindenberg, A. \& Reininghaus, U. (2021). [Digital forms of service delivery for personalized crisis resolution and home treatment]. Nervenarzt.

Rauschenberg, C.*, Schick, A.*, Hirjak, D., Seidler, A., Paetzold, I., Apfelbacher, C., Riedel-Heller, S. G. \& Reininghaus, U. (2021). Evidence Synthesis of Digital Interventions to Mitigate the Negative Impact of the COVID-19 Pandemic on Public Mental Health: Rapid Meta-review. Journal of Medical Internet Research 23, e23365.

Rauschenberg, C., van Os, J., Goedhart, M., Schieveld, J. N. \& Reininghaus, U. (2021). Bullying victimization and stress sensitivity in help-seeking youth: findings from an experience sampling study. European Child and Adolescent Psychiatry 30, 591-605.

Klippel, A., Schick, A., Myin-Germeys I., Rauschenberg, C., Vaessen T., Reininghaus, U. (2021) Modeling the temporal interplay between stress and affective disturbances in pathways to psychosis: an experience sampling study. Psychological Medicine. 
Henquet, C., van Os, J., Pries, L. K., Rauschenberg, C., Delespaul, P., Kenis, G., ... \& Gülöksüz, S. (2020). A replication study of JTC bias, genetic liability for psychosis and delusional ideation. Psychological Medicine.

Rauschenberg, C., Reininghaus, U., ten Have, M., de Graaf, R., van Dorsselaer, S., Simons, C. J., Gunther, N., Henquet, C., Pries, L.-K. \& Guloksuz, S. (2020). The jumping to conclusions reasoning bias as a cognitive factor contributing to psychosis progression and persistence: findings from NEMESIS-2. Psychological Medicine, 51, 1696-1703.

Reininghaus, U.*, Rauschenberg, C.*, Ten Have, M., de Graaf, R., van Dorsselaer, S., Simons, C. J., Gunther, N., Pries, L.-K., Guloksuz, S. \& Radhakrishnan, R. (2019). Reasoning bias, working memory performance and a transdiagnostic phenotype of affective disturbances and psychotic experiences in the general population. Psychological Medicine 49, 1799-1809.

Radhakrishnan, R., Guloksuz, S., Ten Have, M., de Graaf, R., van Dorsselaer, S., Gunther, N., Rauschenberg, C., Reininghaus, U., Pries, L.-K. \& Bak, M. (2019). Interaction between environmental and familial affective risk impacts psychosis admixture in states of affective dysregulation. Psychological Medicine 49, 18791889.

Pries, L.-K., Guloksuz, S., Ten Have, M., De Graaf, R., Van Dorsselaer, S., Gunther, N., Rauschenberg, C., Reininghaus, U., Radhakrishnan, R. \& Bak, M. (2018). Evidence that environmental and familial risks for psychosis additively impact a multidimensional subthreshold psychosis syndrome. Schizophrenia Bulletin 44, 710-719.

Rauschenberg, C., van Os, J., Cremers, D., Goedhart, M., Schieveld, J. \& Reininghaus, U. (2017). Stress sensitivity as a putative mechanism linking childhood trauma and psychopathology in youth's daily life. Acta Psychiatrica Scandinavica 136, 373-388.

\section{Submitted and in progress articles}

Rauschenberg, C.*, Schulte-Strathaus, J.C.C.*, van Os, J., Goedhart, M., Schieveld, J. N. M. \& Reininghaus, U. Negative life events and stress sensitivity in youth's daily life: an ecological momentary assessment study. 
Goetzl, C., Hiller, S., Rauschenberg, C., Schick, A., Koppe, G., Durstewitz, D., Reininghaus, U., Krumm, S. Al-Based Mobile Mental Health Apps for Young People: A Mixed-Methods Approach on Users' and Stakeholders' Perspectives.

Schick, A., Rauschenberg, C., Ader, L., Daemen, M., Wieland, L., Paetzold, I., Postma, M. R., Schulte-Strathaus, J.C.C., Reininghaus, U. Methods for gathering intensive time series data in mental health research: Scoping review of a rapidly evolving field (invited review, Psychological Medicine).

Paetzold, I., Schick, A., Rauschenberg, C., Hirjak, D., Banaschewski, T., MeyerLindenberg, A., Boehnke, J. R., Boecking, B., Reininghaus, U. Exploring putative therapeutic mechanisms and processes of change in a hybrid compassion-focused, ecological momentary intervention: Findings from the EMIcompass study.

Paetzold, I., Schick, A., Rauschenberg, C., Hirjak, D., Banaschewski, T., MeyerLindenberg, A., Boehnke, J. R., Boecking, B., Reininghaus, U. An ecological momentary compassion-focused intervention for enhancing resilience in helpseeking youths: Findings on the effects of baseline characteristics from the EMlcompass trial.

* These authors contributed equally.

\section{Book chapters}

Schulte-Strathaus, J.C.C., Rauschenberg, C., Baumeister, H., Reininghaus, U. (2022). Ecological Momentary Interventions in Public Mental Health Provision. In: Baumeister, H. \& Montag C., ed. Digital Phenotyping and Mobile Sensing: New Developments in Psychoinformatics. 2nd ed. Cham, Switzerland: Springer Nature Switzerland.

Rauschenberg C., Rus-Calafell M., Reininghaus U., Valmaggia L. (2022). Digital Interventions for Psychosis. In: Asmundson GJ, ed. Comprehensive Clinical Psychology. 2nd ed. Amsterdam, Netherlands: Elsevier Ltd.

\section{Other publications}

Reininghaus U. \& Rauschenberg C. (2020) Digitale Public Mental Health Ansätze zur Verminderung der psychosozialen Folgen der COVID-19 Pandemie (Policy 
Brief; carried out as part of the COVID-19 Public Health Research Network, an ad hoc consortium of more than 25 scientific societies and organizations that are active in the field of public health).

\section{Scientific communications related to this thesis}

Oral presentation. 14th European Public Health Conference (Online, 2021).

Oral presentation. Kongress der Deutschen Gesellschaft für Psychiatrie und Psychotherapie, Psychosomatik und Nervenheilkunde (DGPPN) (Berlin, 2021).

Oral presentation. 24. Bundeskongress für Schulpsychologie, Sektion Schulpsychologie des Berufsverbands Deutscher Psychologinnen und Psychologen (BDP) (Online, 2021).

Oral presentation. Jahrestagung der Landesarbeitsgemeinschaft für Erziehungsberatung Baden-Württemberg (Online, 2021).

Oral presentation. 8th European Conference on Schizophrenia Research (Online, 2021).

Oral presentation. 56. Jahrestagung der Deutschen Gesellschaft für Sozialmedizin und Prävention (DGSMP) (Online, 2021).

Oral presentation. 19. Deutscher Kongress für Versorgungsforschung (Online, 2021).

Oral presentation. 25. Mallorca-Treffen deutschsprachiger SozialpsychiaterInnen (Online, 2021).

Oral presentation. 1st ESRC Centre for Society and Mental Health (King's College London) annual conference (Online, 2021).

Poster presentation. 38. Symposium der Fachgruppe Klinische Psychologie und Psychotherapie der Deutschen Gesellschaft für Psychologie (DGPs) (Online, 2021).

Oral presentation. 36th San Diego International Conference on Child and Family Maltreatment (San Diego, 2020).

Poster presentation. NIHR MindTech MedTech Co-operative National Symposium: New Frontiers for Digital Mental Health: Implementation \& Impact (London, 2019). 
Oral presentation. 7th European Conference on Schizophrenia Research (Berlin, 2019).

Oral presentation. Annual Meeting of the Belgian Association for Psychological Sciences (Liège, 2019).

Poster presentation. 6th Biennial Schizophrenia International Research Society Conference (Florence, 2018).

Oral presentation. 2nd meeting of Belgian-Dutch ESM network (Groningen, 2017).

Poster presentation. 5th conference of the Society for Ambulatory Assessment (Luxembourg, 2017). 


\section{Acknowledgements}

It's impossible to put into words the luck I've had in my life thus far, meeting so many unique people who have helped me figure out who I am, what I'm interested in, and what path I've chosen - and who have ultimately created the conditions for me to pursue a PhD. It is still important for me to name some of the most influential individuals who inspired and guided me.

First and foremost, I'd like to thank my fantastic promoter team. Thank you, Uli and Jim, for your faith in my abilities and your unwavering support over the years. The completion of my PhD would not have been possible without your help. There are numerous steps that must be taken before a student can be considered an independent researcher. You have always accompanied me on this journey of intellect and constant reflection, and you have done everything possible to ensure that I have everything I need. You have given me a once-in-a-lifetime opportunity to mature and have given me space to find my own way of thinking, and to bring myself and my ideas forward - even though I am still at the very beginning.

Uli, in some of the articles we have written together, it appeared to me that time had stopped for a moment and the night and day had been resolved. Working on all these projects was a very demanding, but ultimately fulfilling experience for me. To be absorbed in a subject is, in my view, one of the highest and greatest human states and abilities. The intensive exchange, your unique scholarly thinking style, and your motivating and supportive words in the many moments when I felt I couldn't go on have left a lasting impression on me and will always accompany me on my future path. I thank you from the bottom of my heart for your patience and your boundless energy, which you have invested in my supervision. This is not a given, and I consider myself extremely fortunate to have received such enthusiastic support from you.

Jim, I'll never forget how curious I was about you as a person and whether my English was adequate as we held our first meeting as part of the Research Masters Mentoring Program. When we talked about the brain, philosophical issues, and the current state of psychiatric care when we met for the first time, I knew I was going to learn a lot from you as a mentor and, later, as a doctoral student. Your foresight and many of your articles have deeply influenced me and given my thinking an unknown and long-lasting direction. In the often frigid world of 
research, I will never forget your comprehensive, humanistic perspectives. Thank you for always dreaming big, for recognising opportunities rather than obstactles, and for constantly motivating me.

I'd also like to express my gratitude to you, Nathalie, Mirjam, Jan, Matthieu, and the rest of the team for your work on the Youth Experience Study, and all the researchers who work on NEMESIS-2 at the Trimbos institute in Utrecht. This PhD would not have been possible without you, and I will be forever grateful.

My heartfelt gratitude goes to my family. I thank you, my dear parents, Heike and Reinhard, for your boundless love and countless forms of support, which you have extended to me throughout my entire life. I'd also like to thank you for your patience with me over the last few years; I wasn't always calm and I couldn't be present at all family gatherings. I'd also like to thank my wonderful siblings, Andrea and Stefan, as well as my grandparents, aunts, uncles, and cousins. You, my family, have laid a solid foundation for me, giving me the strength and resources I needed to get through this, the most difficult test of my life.

But what would life be without intimate friendships, without people who are able to bring out the best in you? Without any doubt, I would never have decided to go back to school or to study without the support of my friends from my hometown Rosbach v.d.Höhe. I simply wouldn't have thought I could cope with the high demands. I will never forget how you, Alex, Cenk, David, Julian, Okan, and Philip, advised me in many situations to embark on an uncertain path after my secondary education and to obtain my university entrance qualification. Countless inspiring conversations and your firm belief that I should not spend my life working in a small administration and instead find something that brings me great joy and that I am passionate about continue to motivate me to this day. I'd also like to thank you, Anne, Elli, and Manu, for the innumerable imprints of your colourful being and your deep friendship towards me that I've been given the opportunity to experience. I am who I am today because of your way of life and thinking. I also appreciate your patience as I have had limited time over the last few years. I can't tell you how many birthdays and holidays I've had to cancel on short notice, or how many promised meetings I've had to cancel. You're a big part of my life, and I'm excited to seeing how we will continue to evolve over the next few years.

Simon and Lukas, I'll never forget our pseudo-intellectual debates during our studies in Giessen, and your unique - and wonderfully different - thinking style 
will live on in my heart. You've left an indelible mark on me, even if we're only rarely able to see each other in person. Simon, our time in Istanbul was fantastic, mashallah! I am so proud of you for defending your PhD so successfully! You're a fabulous badass who knows what he's doing. Teresa and Christopher, thank you for your numerous discussions and support throughout the course of our undergraduate studies. The attitude of being able to accomplish more together than alone has stayed with me to this day. Our learning group was superior :D:D:D! Teresa, I'll never forget this potato dish you used to make, and Christoph, I'Il never forget the weird sausages you brought.

Lars, you are a wonderful and generous person. I'll never forget how you welcomed me into the Scharnerweg in Maastricht. Even though my English was still quite rusty, you welcomed me with open arms and made it very easy for me to feel at ease in a new city. Your personality is one-of-a-kind, and the many special moments we've shared have given me a lot of strength during my studies and doctorate.

Diana, CIAO00000000000000000000000000000000000!! Cooking for you and giggling on Maatricht's streets was a blast. In a few years, I hope to meet you at the International Court of Human Rights and discuss famous German politicians with unbearable names. Thank you for all your support over the years, as well as your vivid personality, which brightens even the darkest rooms. Clara, I'm glad our paths crossed and that I was able to talk to you about everything down to the smallest detail. You were always my "Fels in der Brandung", the calm in the midst of the chaos of the research world. I would not have made it through the difficult times if we hadn't shared meals, gone for a nice walk, or cycled together. Lex, you're simply Gucci. Thank you for your genuine, loving, and truthful nature. Conversations with you have always left me feeling very calm and reminded me that, despite stressful situations, there must always be time for friends and pleasant moments. What wonderful times we had at university, Arne and Chris. I never thought l'd be able to finish this Master's degree. Thanks to you and your monsterkill brilliance, I was able to gradually learn the necessary skills. I'll never forget our time together and the endless Gelaber.

I'd also like to thank my Maastricht colleagues. Thank you for allowing me to share the office with you, Sophie, and for all of your pleasant conversations, as well as your ability to restore calm in stressful and emotional situations. I'd like to thank 
both "old" (Annelie, Thomas, Esther, Iris, Stijn, Jindra, Yori, Hennie, Simone, Boris, Ozan) and "new" PhD candidates (Lotta, Mary Rose, Naomi, Stella, Samantha, Nikita, Maud). We were a fantastic team, and I was fortunate to be able to learn from you. It was amazing to see how we were always able to openly discuss the issues that were bothering us in the SLAM and other contexts. There are many more people at Vijverdal who assisted me in numerous ways (Trees, Truda, Nele, Karel, Dafne, Ron, Jo, Marjan, Wolfgang, Thérèse, Maarten, Sinan, and many other wonderful colleagues). It's been a pleasure working with you, and all I can say is THANK YOU!

I'd also like to thank my wonderful Mannheim colleagues (Anita, Isa, Ulrike, Leonie, Julia, and Antonia) for all of your small and large contributions to my dissertation, as well as your very warm welcome to \#TeamPMH last year. I am looking forward to working with you in the coming years.

Finally, I'd like to thank you for your unfailing patience and support in the final stages of my PhD, Floriana. The months leading up to my dissertation's submission were difficult, and I couldn't have done it without you. In these trying times, your love and thoughts about our future have given me a lot of strength. I'm glad we went through this together, and that I found someone in my life who gives me confidence, strength, and inner peace. 